2. To: (Receiving Organization)

Solid Waste Management

Facility Engineering, Rust

Federal Services

5. Proj./Prog./Dept./Div.:

CWC

8. Originator Renarks:

This EDT is for the release of HNF-SD-WM-RPT-282, Rev. 0 , Report on Sampling and Analysis of Ambient Air at the Central Waste Complex

11. Receiver Remarks: 11A. Design Baseline Document? [] Yes [X] No

Special Analytical Support, Numatec Hanford Company

6. Design Authority/ Design Agent/Cog.

Engr.:

Markus Stauffer
3. From: (Originating Organization) 4. Related EDT No.:

$N / A$

7. Purchase Order No.:

$N / A$

9. Equip./Component No.: $N / A$

10. System/Bldg./Facility: CWC

12. Major Assm. Dwg. No.: $N / A$

13. Permit/Permit Application No.: N/A

14. Required Response Date: $N / A$

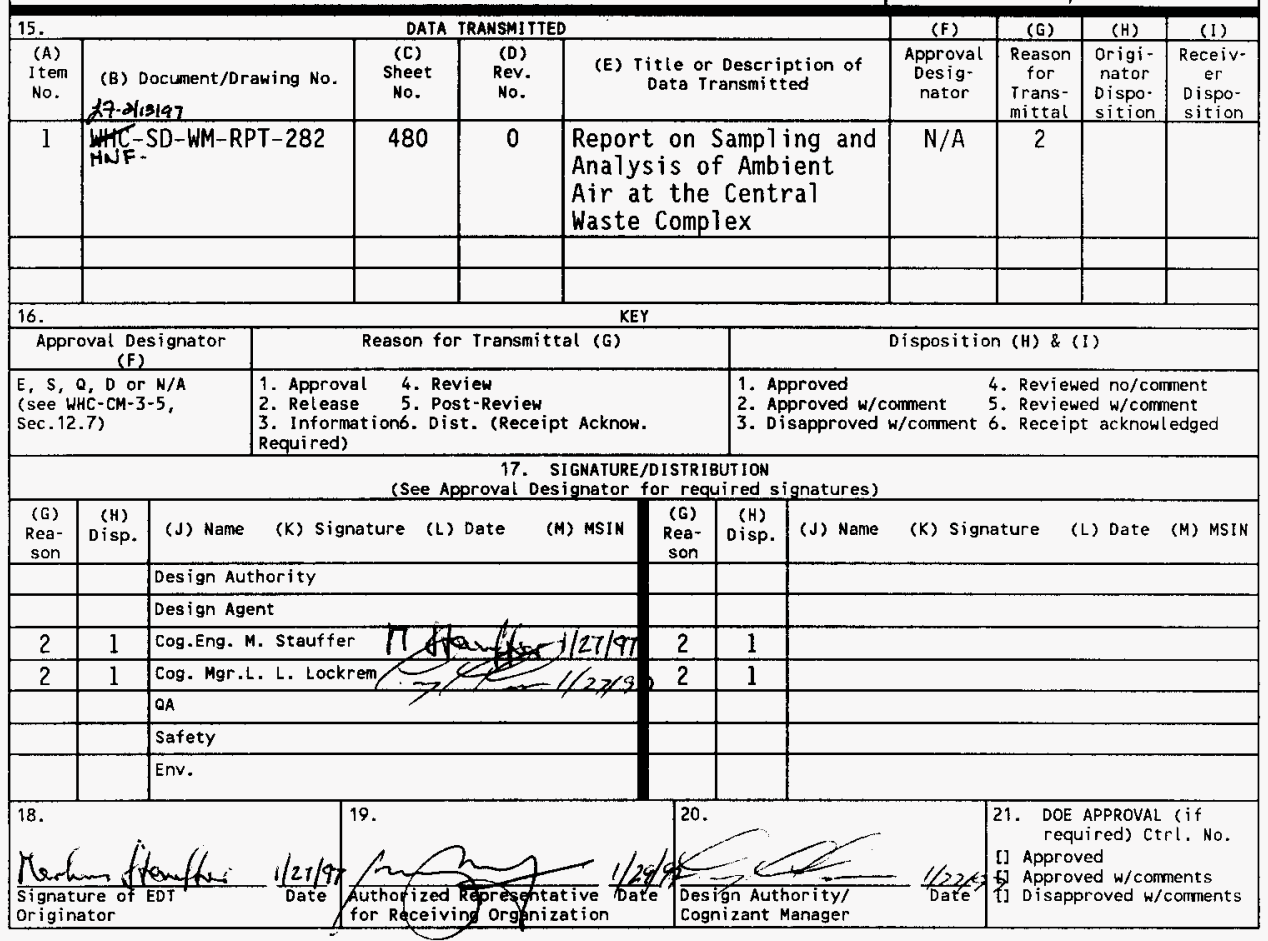

BD-7400-172-2 (05/96) GEF097 


\section{INSTRUCTIONS FOR COMPLETION OF THE ENGINEERING DATA TRANSMITTAL}

(USE BLACK INK OR TYPE)

$\frac{\text { BLOCK }}{(1)^{*}} \frac{\text { TITLE }}{\text { EDT }}$

(2) To: (Receiving Organization)

(3) From: (Orginating Organization)

(4) Releted EDT

(5) * Project/Program/Dept./Div.

(6) Cognizant/Project Engineer

(7) Purchase Order No.

(8)* Originator Remarks

(9) Equipment/Component No.

(10) System/Bldg./Facility

(11) Receiver Remarks

(12) Major Assm. Dwg. No.

(13) Permit/Permit Application No.

(14) Required Response Date

(15) * Data Transmitted
(A)* Item Number
(B) * Document/Drawing No
(C)" Sheet No.
(D)" Rev. No.
(E) Title or Description of Data Transmitted
(F) Impact Level
(G) Reason for Submittal
(H) Originator Disposition
(I) Receiver Disposition

(16) Key

(17) Signature/Distribution

(G) Reason
(H) Disposition
(J) Name
(L) Date
(M) MSIN

(18) Signature of EDT Originator

(19) Authorized Representative for Receiving Organization

(20) * Cognizant/Project Managar

(2.1) DOE Approval
- Enter the essigned EDT number.

- Enter the individual's name, title of the organization, or entity (e.g., Distribution) that the EDT is being transmitted to.

- Enter the title of the organization originating and transmitting the EDT.

- Enter EDT numbers which relate to the data being transmitted.

- Enter the Project/Program/Department/Division title or Project/Program acronym or Project Number, Work Order Number or Organization Code.

- Enter the neme of the individual identified es being responsible for coordinating disposition of the EDT.

- Enter related Purchase Order (P.O.) Number, if available.

- Enter special or additional comments concerning transmittal, or "Key" retrievel words may be entered.

- Enter equipment/component number of affected item, if appropriate.

- Enter appropriate system, building or facility number, if appropriate.

- Enter special or additional comments concerning transmittal.

- Enter epplicable drawing number of major assembly, if appropriate.

- Enter applicable permit or permit application number, if appropriate.

- Enter the date a response is required from individuals identified in Block 17 (Signature/Distribution).

- Enter sequential number, beginning with 1, of the information listed on EDT.

- Enter the unique identification number essigned to the document or drawing being transmitted.

- Enter the sheet number of the information being transmitted. If no sheot number, leave blank.

- Enter the revision number of the information being transmitted. If no revision number, leave blank.

- Enter the title of the document or drawing or a brief description of the subject if no title is identified.

- Enter the appropriate Impact Level (Block 15). Use NA for non-engineering documents.

- Enter the appropriate code to identify the purpose of the date transmittal (see Block 16).

- Enter the appropriate disposition code (see Block 16).

- Enter the appropriate disposition code (see Block 16).

- Number codes used in completion of Blocks 15 (G), (H), and (I), and 17 (G), (H) (Signature/Distribution).

- Enter the code of the reason for transmittal [Block 16 .

- Enter the code for the disposition (Block 16).

- Enter the signature of the individual completing the Disposition $17(H)$ arnd the Transmittal.

- Enter date signature is obtained.

- Enter MSIN. Note: If Distribution Sheet is used, show entire distribution lincluding that indicated on Page 1 of the EDT) on the Distribution Sheet.

- Enter the signature and date of the individual originating the EDT lentered prior to transmittal to Receiving Organization). If the EDT originator is the Cognizant/Project Engineer, sign both Blacks 17 and 18 .

- Enter the signature and date of the individual identified by the Receiving Organization as euthorized to approve disposition of the EDT and ecceptance of the data transmitted, as applicable.

- Enter the signature and date of the Cognizant/Project Engineer's mansger. (This signature is authorization for release, ]

- Enter DOE approval (if required) by letter number and indicate DOE action.

-Asterisk denote the required minimum items checked by Configuration Documentation prior to release; these are the minimum release requirements. 


\section{Report on Sampling and Analysis of Ambient Air at the Central Waste Complex}

Prepared by Marcus Stauffer, SGN Eurisys Services Corporation for Numatec Hanford Company, Richland, WA 99352

U.S. Department of Energy Contract DE-AC06-96RL13200

EDT/ECN: $\quad 158949$

UC:

Org Code: 8 C530

B\&R Code: EW3130020

Charge Code: A4X01

Total Pages: 480

Key Words: Ambient air, Central Waste Complex, volatile organic compounds

Abstract: Over 160 ambient indoor air samples were collected from warehouses at the Central Waste Complex used for the storage of lowlevel radioactive and mixed wastes. These grab (SUMMA) samples were analyzed by gas chromatography-mass spectrometry using a modified EPA T0-14 procedure. The data from this survey suggest that several buildings had elevated concentrations of volatile organic compounds.

TRADEMARK DISCLAIMER. Reference herein to any specific commercial product, process, or service by trade name, trademark, manufacturer, or otherwise, does not necessarily constitute or imply its endorsement, recommendation, or favoring by the United States Government or any agency thereof or its contractors or subcontractors.

Printed in the United States of Mmerica. To obtain copies of this docunent, contact: WHC/BCS Document Control Services, P.O. Box 1970, Mailstop H6-08, Richtand WA 99352, Phone (509) 372-2420; Fax (509) 376-4989.
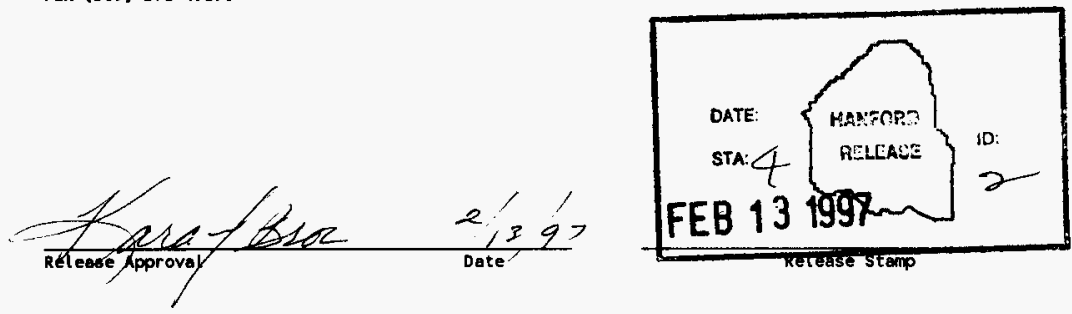

\section{Approved for Public Release}




\section{THIS PAGE LEFT BLANTIONALLY}




\title{
Report on Sampling and Analysis of Ambient Air at the Central Waste Complex
}

\author{
L. A. Pingel
}

M. Stauffer

R. S. Viswanath

SGN Eurisys Services Corporation

L. L. Lockrem

Numatec Hanford Corporation

Date Published

February 1997

Prepared for the U.S. Department of Energy

Assistant Secretany for Environmental Management 
THIS PAGE INTENTIONALLY LEFT BLANK 


\section{CONTENTS}

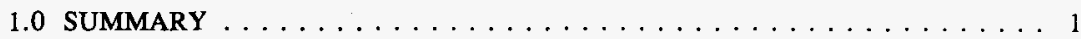

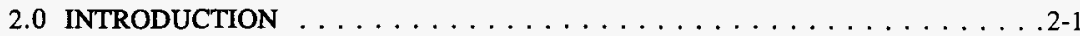

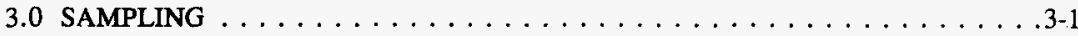

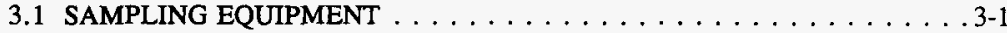

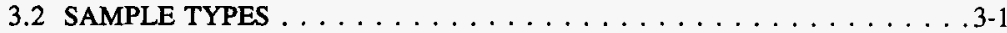

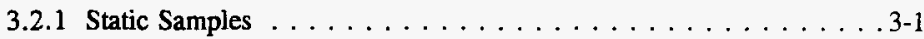

3.2 .2 Fence-line Samples . . . . . . . . . . . . . . . . . 3-1

3.2 .3 Ambient Air Blanks . . . . . . . . . . . . . . . . . . . . . . . . . . . . . . .

3.2 .4 Trip Blanks . . . . . . . . . . . . . . . . . . . . . . . . . . . . . . . . .

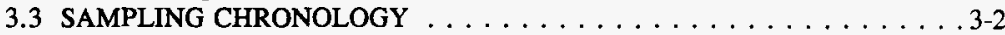

3.3.1 Low Flashpoint Modules . . . . . . . . . . . . . . . . . . . . 3-2

3.3.2 Buildings $2401 \mathrm{~W}$ and $2402 \mathrm{~W}$-Series . . . . . . . . . . 3-2

3.3 .3 Buildings $2403 \mathrm{WA}$, WB, WC . . . . . . . . . . . 3-2

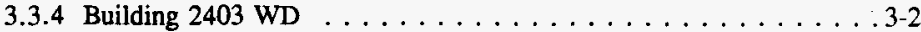

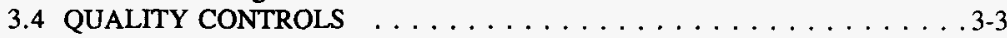

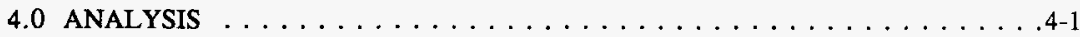

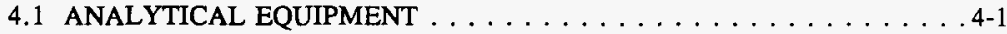

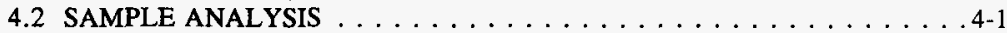

4.3 COMPOUND IDENTIFICATION AND QUANTITATION . . . . . . . . . 4-2

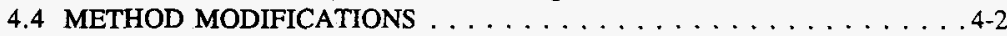

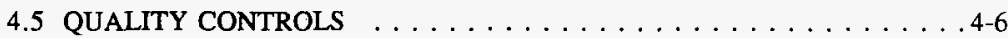

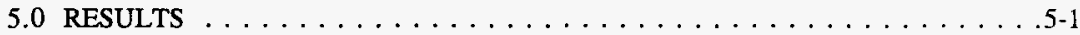

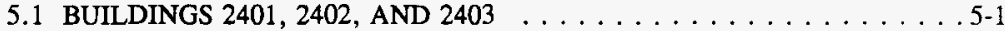

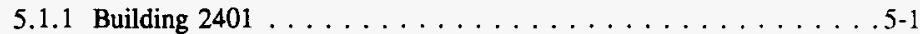

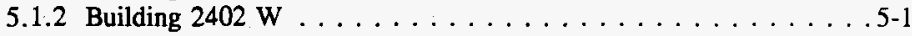

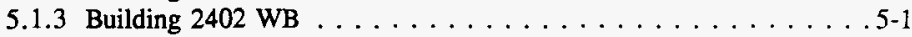

5.1 .4 Building 2402 WC . . . . . . . . . . . . . . . . 5-2

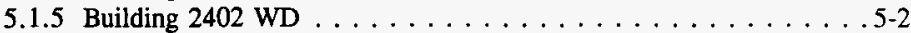

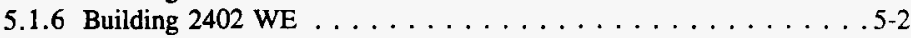

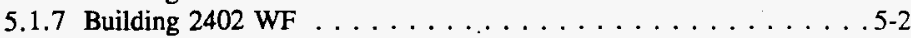

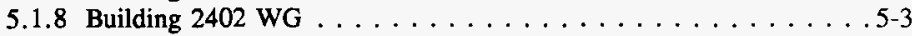

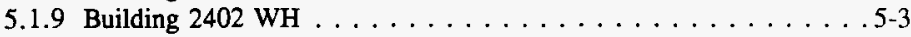

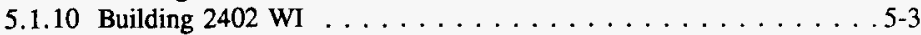

5.1 .11 Building $2402 \mathrm{WJ} \ldots \ldots \ldots \ldots \ldots \ldots \ldots$

5.1 .12 Building 2402 WK . . . . . . . . . . . . . . 5-3

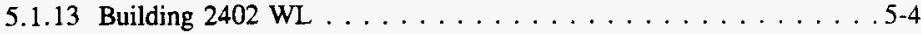

5.1 .14 Building 2403 WA . . . . . . . . . . . . . . 5-4 


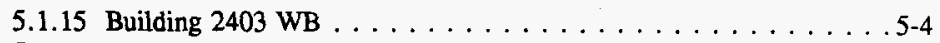

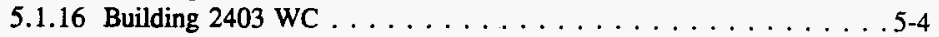

5.1 .17 Building $2403 \mathrm{WD} \ldots \ldots \ldots \ldots \ldots \ldots .5 \ldots \ldots \ldots$

5.2 LOW FLASH-POINT MIXED WASTE AND ALKALI METAL STORAGE

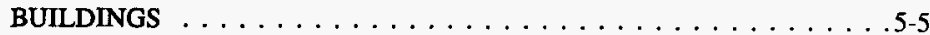

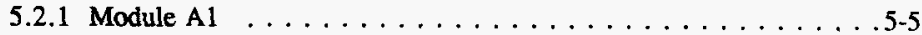

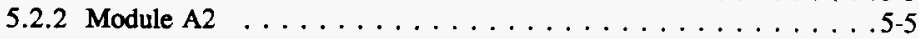

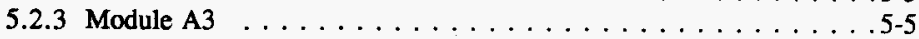

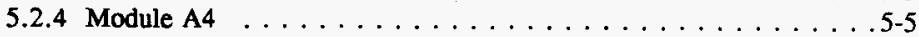

5.2 .5 Module LFM \# $1 \ldots \ldots \ldots \ldots \ldots \ldots \ldots \ldots \ldots \ldots . \ldots \ldots$. $\ldots \ldots \ldots$

5.2 .6 Module LFM \# $2 \ldots \ldots \ldots \ldots \ldots \ldots \ldots \ldots \ldots$. . . . . . . . . . 6

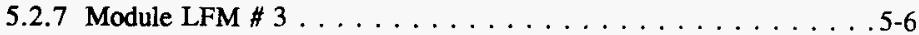

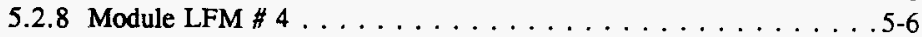

5.2 .9 Module LFM \# $5 \ldots \ldots \ldots \ldots \ldots \ldots \ldots \ldots \ldots . .6 \ldots \ldots \ldots$

5.2 .10 Module LFM \# $6 \ldots \ldots \ldots \ldots \ldots \ldots \ldots \ldots$. . . . . . . . . . .

5.2 .11 Module LFM \# $7 \ldots \ldots \ldots \ldots \ldots \ldots \ldots \ldots . .5 \ldots \ldots$

5.2 .12 Module LFM \# $8 \ldots \ldots \ldots \ldots \ldots \ldots \ldots \ldots \ldots \ldots . .5 \ldots$

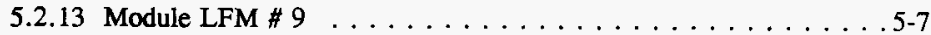

5.2 .14 Module LFM \# $10 \ldots \ldots \ldots \ldots \ldots \ldots \ldots \ldots . . \ldots \ldots$. . . . . . . . . .

5.2 .15 Module LFM \# $11 \ldots \ldots \ldots \ldots \ldots \ldots \ldots \ldots \ldots .7 .7$

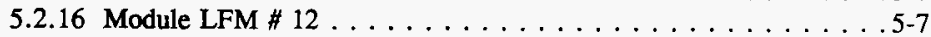

5.2 .17 Module LFM \# $13 \ldots \ldots \ldots \ldots \ldots \ldots \ldots \ldots \ldots . . .5 .6 \ldots$

5.2 .18 Module LFM \# $14 \ldots \ldots \ldots \ldots \ldots \ldots \ldots \ldots \ldots$. . . . . . . . . . . . .

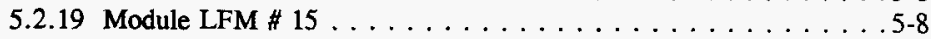

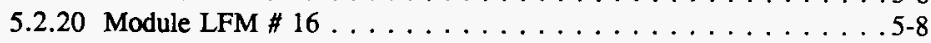

5.2 .21 Module LFM \# $17 \ldots \ldots \ldots \ldots \ldots \ldots \ldots \ldots . \ldots \ldots$

5.2 .22 Module LFM \# $18 \ldots \ldots \ldots \ldots \ldots \ldots \ldots \ldots \ldots \ldots . . \ldots \ldots \ldots$

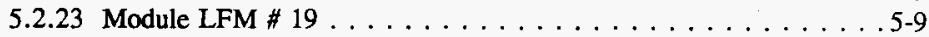

5.2 .24 Module LFM $\# 20 \ldots \ldots \ldots \ldots \ldots \ldots \ldots \ldots$. . . . . . . . . . . . . .

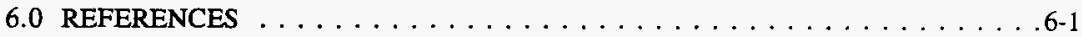

APPENDIX

A ANALYTCAL DATA FOR THE REPORT ON SAMPLING AND ANALYSIS OF AMBIENT AIR AT THE CENTRAL WASTE COMPLEX $\ldots \ldots \ldots \ldots$ A-1

A.1 CWC 2401 BUILDING $\ldots \ldots \ldots \ldots \ldots \ldots \ldots \ldots \ldots$ A-3

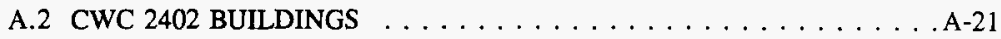

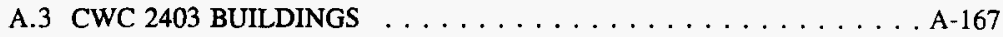

A.4 CWC LOW FLASHPOINT MODULAR UNITS $\ldots \ldots \ldots \ldots \ldots$. . . A-279 
HNF-SD-WM-RPT-282 Rev. 0

\section{LIST OF FIGURES}

1 Central Waste Complex $\ldots \ldots \ldots \ldots \ldots \ldots \ldots \ldots \ldots \ldots \ldots \ldots \ldots \ldots \ldots \ldots \ldots .2$

\section{LIST OF TABLES}

4-1 Precision and Accuracy of CWC Analyses Measured on $500 \mathrm{ppb}$

Calibration Sample . . . . . . . . . . . . . . . . . 4-3

4-2 GC-MS Data of Air Samples from the Central Waste Complex:

Concentrations in Parts Per Billion . . . . . . . . . . . . . . 4-7

5-3 GC-MS Data of indoor Air Samples From the Central Waste Complex:

Concentrations in Microgram/Meter . . . . . . . . . . . . . 5-2 
HNF-SD-WM-RPT-282 Rev. 0

\section{LIST OF TERMS}

$\begin{array}{ll}\text { CWC } & \text { Central Waste Complex } \\ \text { GC-MS } & \text { gas chromatograph-mass spectrometer } \\ \text { LOI } & \text { Letter of Instructions } \\ \text { LFM } & \text { Low Flashpoint Modules } \\ \text { MDL } & \text { Method Detection Limit } \\ \text { SAP } & \text { Sampling and Analysis Plan } \\ \text { SWD } & \text { Solid Waste Disposal } \\ \text { SAS } & \text { Special Analytical Support } \\ \text { TIC } & \text { total ion chromatogram } \\ \text { VOC } & \text { volatile organic compounds }\end{array}$




\subsection{SUMMARY}

Special Analytical Studies collected over 160 ambient indoor air samples from warehouses at the Central Waste Complex used for the storage of low-level radioactive and mixed wastes. These grab samples were analyzed by gas chromatography-mass spectrometry using a modified EPA TO-14 procedure. The data suggest that several large buildings had elevated concentrations of volatile organic compounds compared to background ambient air:

- Building 2402 WB had increased levels of chlorinated solvents, such as trichloroethene (117 ppb) and 1,1,1-trichloroethane (29 ppb).

- 2402 WE was dominated by dichloromethane (234 ppb).

- 2402 WF contained natural compounds such as pinene, carene, and limonene.

- 2402 WL had polar compounds such as 4-methyl-2-pentanone ("hexone"; range: 107 to $340 \mathrm{ppb}$ ).

- 2403 WA had predominantly dichloromethane (31 to $133 \mathrm{ppb}$ ) and 1,1,1-trichloroethane (14-70 ppb).

- 2403WC showed a buildup of dichloromethane (50 to $104 \mathrm{ppb}$ ) and tetrachloroethene (21 to $27 \mathrm{ppb}$ ).

The small low-flashpoint modules generally had high concentrations of organic compounds in the air space:

- \# 1 was dominated by toluene (239 ppb) and ethanol (83 ppb).

- \# 2 had 2-butanone ("MEK", $107 \mathrm{ppb)}$ ) and aromatic compounds such as $\mathrm{m}$ - and o-xylene (215 and $59 \mathrm{ppb}$, respectively), ethylbenzene (65 ppb), and toluene $(48 \mathrm{ppb})$.

- \# 5 contained very volatile compounds such as ethanol (137 ppb), hexane (63 ppb), 2-propanol (61 ppb), dichloromethane (37 ppb), and acetone (33 ppb).

- \#7 showed predominantly toluene (220 ppb) and ethanol (158 ppb).

- \# 11 had a very high concentration of 2-butanone ("MEK", $1465 \mathrm{ppb}$ ), followed by hexane (123 ppb), toluene (96 ppb), and others.

- \#12 contained aromatic compounds such as toluene (83 ppb) and xylenes (10 to $29 \mathrm{ppb}$ ). 


\section{HNF-SD-WM-RPT-282 Rev. 0}

- \#14 module has three separate chambers which were generally high in volatiles: 2-propanol (1,055 ppb), dichloromethane (479 ppb), carbontetrachloride (256 ppb), toluene (186 to $507 \mathrm{ppb}$ ), xylenes (273 to $329 \mathrm{ppb}$ ), Freon 113 (190 ppb), and many others.

This study represents an initial screening to establish a baseline for volatile organic compounds at the Central Waste Complex. Additional sampling and analysis cycles are needed to: a) confirm these baseline results; b) study seasonal (temperature) variations in VOCs; c) determine the concentrations of polar VOCs that were not included in the reference standard; and d) increase the number of samples from the buildings to have statistically meaningful information that can be used for emission modeling. 


\section{HNF-SD-WM-RPT-282 Rev. 0}

\subsection{INTRODUCTION}

The Central Waste Complex (CWC) at the Hanford site is a large warehousing complex for storage of containerized low-level radioactive and mixed wastes. The facility consists of four types of buildings (see Figure 1):

- Building $2401 \mathrm{~W}$, a $3000 \mathrm{ft}^{2}$ warehouse for mixed wastes.

- The $2402 \mathrm{~W}$ series consists of 12 buildings of $4000 \mathrm{ft}^{2}$ each. They are used for mixed waste storage.

- 2402 WA, WB, WC, and WD. Three of these buildings have $34,000 \mathrm{ft}^{2}$, and $2402 \mathrm{WD}$ has $55,000 \mathrm{ft}^{2}$. All are used for mixed waste storage.

- A group of 24 modules used for low flashpoint mixed waste and alkali metal waste storage. Some of these buildings are empty and presently not in use.

- A group of 8 modules for alkali metal products.

Special Analytical Support (SAS) collected ambient air at the Solid Waste Disposal (SWD) Central Waste Complex (CWC) using evacuated SUMMA ${ }^{1}$ (1) canisters. The air samples were analyzed by gas chromatography-mass spectrometry (GC-MS) for volatile organic compounds using a modified EPA protocol (TO-14). Quantitative data for 36 toxic compounds known or suspected of being present in waste managed at the CWC were generated using external standards. Furthermore, for each sample a list was compiled from the mass spectral data that gives tentative identification for all major organic compounds (>10 ppbv). These qualitative and quantitative results are an initial screening effort to estimate the baseline ambient air quality at CWC. The data from these analyses may be used to estimate the total VOC emissions from the CWC for input into the Hanford Site Air Operating Permit.

This report was requested by Work Order WHC-E35069 "Sampling and Analysis of Ambient Air at the Central Waste Complex." The objectives of the study were detailed in the Letter of Instructions (LOI), issued as an Internal Memorandum, 87600-RDP-96-005 (Pierce 1996). The strategy for collecting and analyzing the air samples was outlined in the Sampling and Analysis Plan (SAP), WHC-SD-WM-WAP-002, Rev. 0 (Lockrem 1996).

${ }^{1}$ SUMMA is a trademark of Molectrics, Inc., Cleveland, Ohio. 
HNF-SD-WM-RPT-282 Rev. 0

Figure 1. Central Waste Complex.

\section{Sompling Locotions}
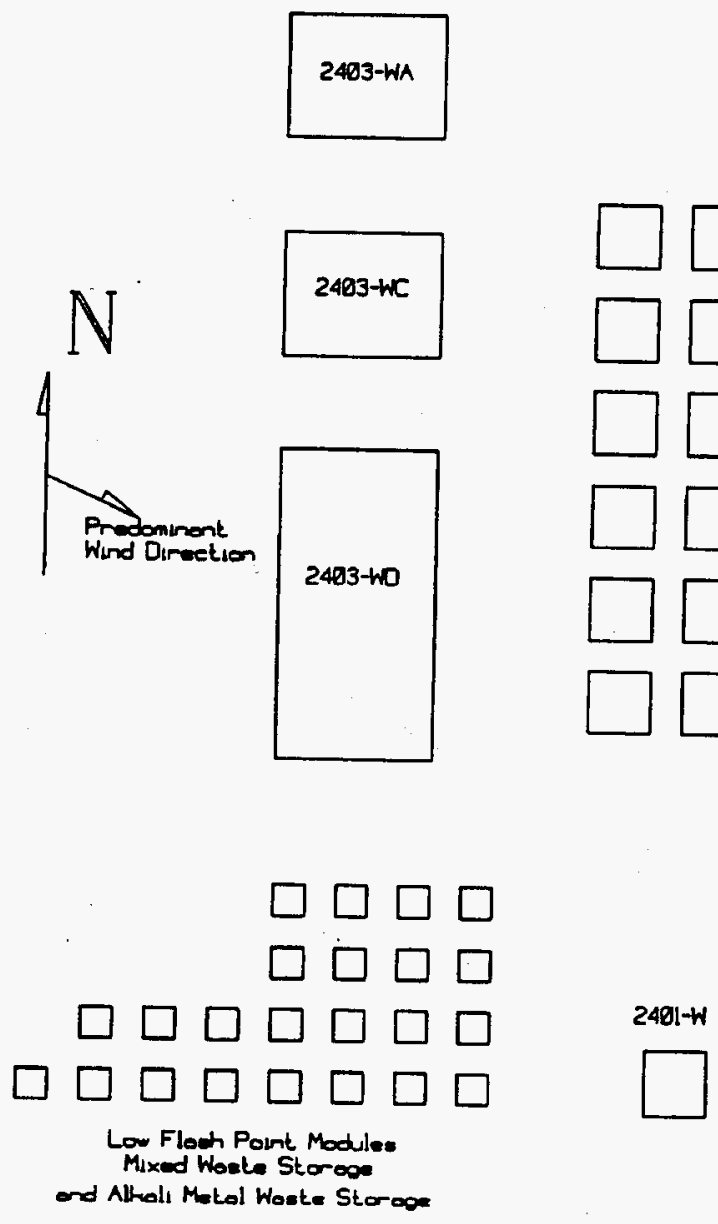

$249 !+W$
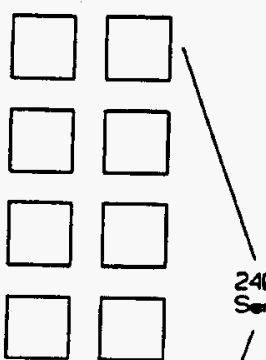

2403-60

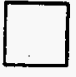

$\square$

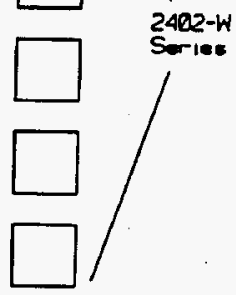

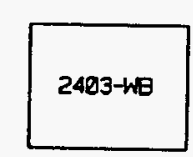


HNF-SD-WM-RPT-282 Rev. 0

\subsection{SAMPLING}

\subsection{SAMPLING EQUIPMENT}

Air sampling was done with evacuated SUMMA ${ }^{\text {TM }}$ canisters (Rasmussen, Hillsboro, OR). These spherical stainless steel containers (6.0 or 1.8 liters) have an electro-polished interior that is considered chemically inert. The canisters were cleaned by dilution with ultra zero air and evacuation. After cleaning, the canister valve was closed and capped. A new tag was attached to the SUMMA ${ }^{\mathrm{TM}}$ canister to show its status, i.e. "cleaned", "QA analyzed", "sampled", "analyzed".

\subsection{SAMPLE TYPES}

Four types of samples were collected at CWC: static samples from inside the buildings, fence-line samples, ambient air blanks, and trip blanks.

\subsubsection{Static Samples}

Air samples were taken inside the buildings by opening the valve on the evacuated SUMMA ${ }^{\mathrm{TM}}$ canister and exposing it to the ambient atmosphere over a one minute interval. Sampling locations and the number of samples collected varied for each building and are detailed in the SAP. Two different modes of valve activation were used depending on the type of the building: The large 2401, 2402, and 2403-series buildings were sampled by opening the valve about one meter above ground (waist-high). The Low Flashpoint Modules (LFM) were sampled remotely by placing the canister inside the building and after a 2-hour equilibration time, a toggle valve was actuated from outside the module.

\subsubsection{Fence-line Samples}

Two fence-line samples were collected for each day of sampling, one upwind and one downwind of CWC.

\subsubsection{Ambient Air Blanks}

Ambient air blanks were collected about one meter upwind and outside the building being sampled. The time of the sampling closely corresponded with the building samples being taken and thus represented the background for these samples. Generally, at least one ambient air blank was collected for each building, except the low flashpoint modules where one blank for every three units was collected. 


\subsubsection{Trip Blanks}

A quality assurance trip blank was used for each sampling day. This canister was carried into the field and returned to the laboratory as an evacuated canister. There it was filled with zero grade air, analyzed, and used as a background reference.

\subsection{SAMPLING CHRONOLOGY}

Sample collection was documented in a controlled logbook: WHC-N-499 \#1, pp. 117-133.

\subsubsection{Low Flashpoint Modules}

These 24 modules (1-20, A1-A4) were sampled in duplicate. Modules with multiple chambers were generally sampled only in one chamber, with the exception of \#14 where all three chambers were sampled individually. The fan on units with active ventilation (\#19 and 20) was turned off the day before sampling. One background blank was collected for every three low flashpoint modules.

\subsubsection{Buildings $2401 \mathrm{~W}$ and $2402 \mathrm{~W}$-Series}

The ventilation on these 13 buildings was stopped the night prior to sampling to allow a buildup of any volatile organic compounds (VOC). The buildings were sampled in duplicate at two locations: One was near the center of the building, and the second was near the heaviest concentration of stored material in the building. Sampling was done by manually opening the valve about one meter above ground and exposing the canister to the inside atmosphere for at least one minute. One ambient air sample was collected outside and about one meter upwind of the building.

\subsubsection{Buildings 2403 WA, WB, WC}

The ventilation on these buildings was stopped at least 16 hours before sampling. These large buildings were sampled in duplicate in each quadrant. Two ambient air blanks were collected outside and upwind of the building.

\subsubsection{Building 2403 WD}

The ventilation on this largest building was stopped 16 hours before sample collection. It was sampled by placing two SUMMA ${ }^{\mathrm{TM}}$ canisters (duplicates) in each of six equal sectors in the building. Three ambient air samples were collected about one meter upwind and equidistant from the building. 


\section{HNF-SD-WM-RPT-282 Rev. 0}

\subsection{QUALITY CONTROLS}

After cleaning of the SUMMA ${ }^{\mathrm{TM}}$ canisters, the canister valve was closed and capped. A new tag was attached to the SUMMA ${ }^{\text {TM }}$ canister at this stage to show its status, i.e., "cleaned". At least 10 percent of the cleaned cans were filled with zero grade air and analyzed to check for cleanliness. The evacuated cans were shipped to the sampling site, and after sample collection, a unique sample identifier was written on the sample tag as described in the SAP. The filled canisters were then shipped to the laboratory where the pressure was checked to assure complete filling of the can. It was pressurized to $15.0 \mathrm{psi}$ using Ultrapure ${ }^{\mathrm{TM} 2}$ air.

An upwind and one downwind fence-line sample were collected for each sampling day representing the air entering and exiting CWC. Ambient air blanks were collected about one meter upwind and outside the buildings being sampled. They were taken close to the time of the sampling of each building and thus represent the background for these samples. A quality assurance trip blank was used for each sampling event (day). This canister was carried into the field and returned to the laboratory as an evacuated canister. There it was filled with zero grade air, analyzed, and used as a background reference.

${ }^{2}$ Ultrapure is a trademark of Scott-Marrin, Riverside, Califormia. 
HNF-SD-WM-RPT-282 Rev, 0

This page intentionally left blank. 


\subsection{ANALYSIS}

The air samples were analyzed using a modified EPA procedure TO-14, "Determination of Volatile Organic Compounds in Ambient Air Using SUMMA ${ }^{\mathrm{TM}}$ Passivated Canister Sampling and Gas Chromatographic Analysis". A system with a gas chromatograph for compound separation and with mass spectrometer detection optimized for trace level organic analyses was used for qualitative and quantitative measurements.

\subsection{ANALYTICAL EQUIPMENT}

The GC-MS system consisted of the following components:

Gas Chromatograph:

A Hewlett Packard (Palo Alto, CA) gas chromatograph (GC), model 5890 series II-Plus was fitted with a 6-port sampling valve. The capillary column (DB-5; J \& W Scientific, Folsom, CA) had an internal diameter of $0.32 \mathrm{~mm}$, $60 \mathrm{~m}$ length, $1.0 \mathrm{~mm}$ film thickness, and a helium carrier with $1.5 \mathrm{ml} / \mathrm{min}$ flow rate.

Mass Spectrometer:

A Hewlett Packard mass spectrometer (MS), model 5972 MSD was used in the electron impact mode with a scan range from 29-300 amu.

Controlling Software:

HP 3365 HPChem Station for GC control

HP G1034 MSChem for data acquisition and MS control

Wiley 138 and NBS mass spectral libraries for compound identification.

\subsection{SAMPLE ANALYSIS}

An accurately measured volume of sample gas was drawn from the SUMMA ${ }^{\mathrm{TM}}$ canister (100 torr pressure differential, corresponding to about $790 \mathrm{ml}$ of air under standard conditions). The VOCs were condensed on a glass bead filled trap that was cooled to $-185^{\circ} \mathrm{C}$ (liquid argon). The GC-MS analysis was started by heating the trap to about $85^{\circ} \mathrm{C}$. The trap contents were thus transferred onto the GC column via the GC sampling valve, and refocused on the $\mathrm{GC}$ column at $-50^{\circ} \mathrm{C}$. After 1 minute, the $\mathrm{GC}$ was ramping at $6{ }^{\circ} \mathrm{C} / \mathrm{min}$ to $180{ }^{\circ} \mathrm{C}$, then heated at $25^{\circ} \mathrm{C} / \mathrm{min}$ to $250^{\circ} \mathrm{C}$, and finally held at that temperature for 2 minutes. 


\subsection{COMPOUND IDENTIFICATION AND QUANTITATION}

A three-point calibration curve was generated using $1,000 \mathrm{ppbv}, 500 \mathrm{ppbv}$ and $100 \mathrm{ppbv}$ standards (Scott-Merrin, Riverside, California). Each standard contained 40 compounds of special concern to SWD but only 36 compounds were used for quantitation of the air samples (see Table 4-1 for the compound list). No comprehensive study was done for this screening study to establish a Method Detection Limit (MDL). However, it was found that $10 \mathrm{ppbv}$ per compound could reproducibly be measured. The MDL was therefore established at $10 \mathrm{ppbv}$ or better. The following 4 compounds did not give reproducible data under the conditions used for the analyses: 2-butoxy ethanol (monobutyl ether ethylene glycol), propane, methanol, and methoxy-2-propanol. However, the presence of these 4 compounds in a sample can still be established from the qualitative mass spectral library searches.

For each sample analyzed, a total ion chromatogram (TIC) was generated which is the sum of the mass spectra over the mass range from 29 to $300 \mathrm{amu}$. This chromatogram is included in Appendix A for each air sample. The TIC data were further processed by extracting the masses characteristic for each compound used in the calibration. These extracted ion chromatograms were integrated and the area counts used to quantitate the 36 compounds used in the calibration.

In order to identify other compounds in a sample besides the 36 used for the calibration, the TIC data were searched with two library databases (Wiley 138, NBS). The mass spectrum for each compound was compared with the 190,000 spectra in the data bases. A compound was considered tentatively identified if the match quality between the unknown and the library was 70 percent or better. These library searches with tentatively identified compounds are included in Appendix A. It is important to remember that tentatively identified compounds are not necessarily indicative of the presence of these compounds in the sample. It requires at least two independent parameters to confirm the presence of a compound (positive identification). Generally this is done by analyzing a sample of the suspected compound under identical conditions. Compounds on the calibration list were identified positively when: a) the retention time matched, and b) its primary ion was present.

\subsection{METHOD MODIFICATIONS}

The TO-14 air analysis method was developed by EPA for the measurement of non-polar and slightly polar organic compounds such as halogenated hydrocarbons, aromatics, and paraffinic hydrocarbons. The compound list from CWC included many polar compounds such as 2-propanol, ethanol, methanol, 1-methoxy-propanol, and 2-butoxy ethanol. These compounds are adsorbed on many surfaces, produce poor chromatographic peaks, and are affected by humidity levels. Two modifications of the procedure were made to accommodate polar compounds: The untreated glass beads used for cryogenic trapping of the analytes were replaced with beads with a chemically modified surface (DMCS treated, Alltech, Deerfield, Illinois). Furthermore, the ultra zero air supply was fitted with a humidifier. It 
HNF-SD-WM-RPT-282 Rev. 0

Table 4-1. Precision and Accuracy of CWC Analyses Measured on 500 ppb Calibration Sample. (3 sheets)

CWC_CAL.XLS

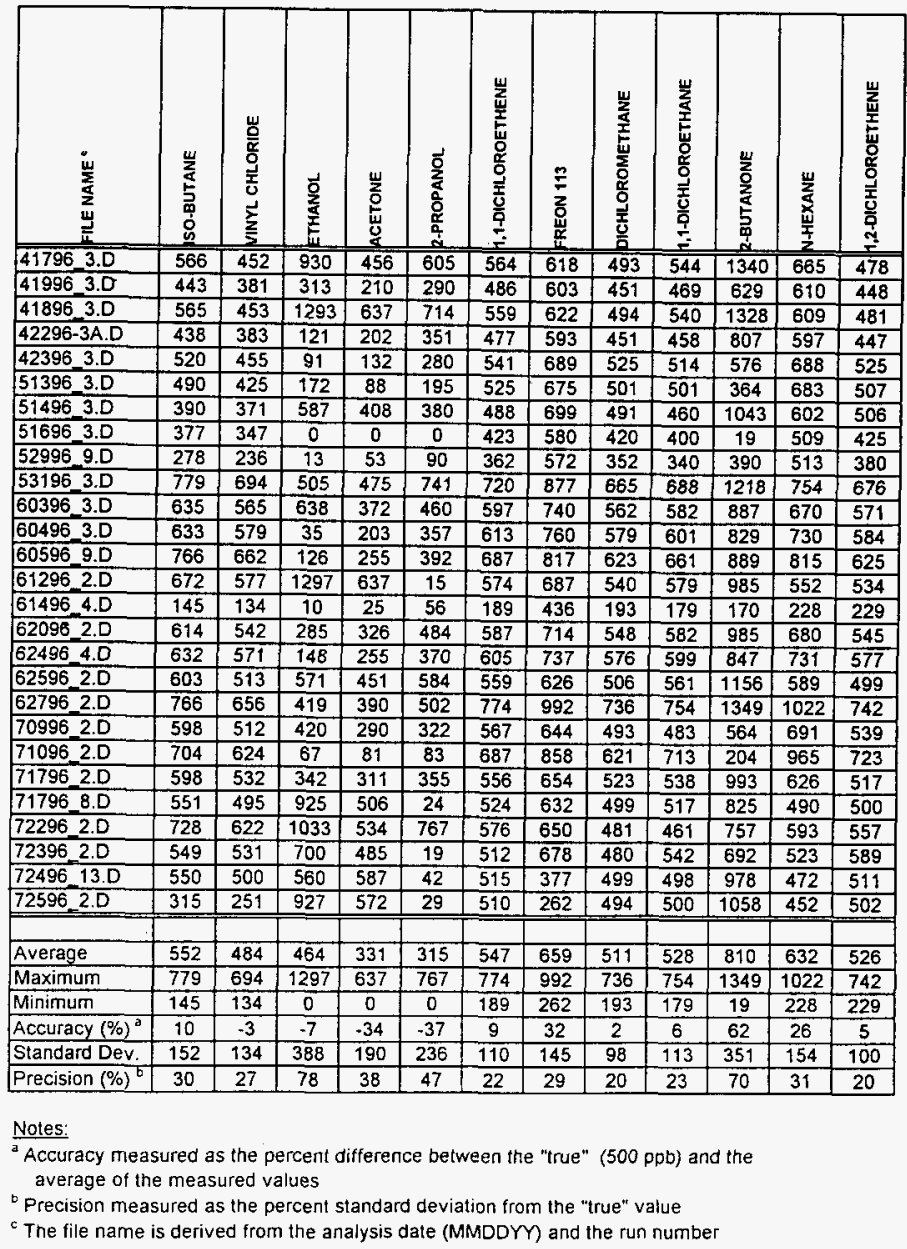

Page 1 
Table 4-1. Precision and Accuracy of CWC Analyses Measured on $500 \mathrm{ppb}$ Calibration Sample. (3 sheets)

CWC_CAL.XLS

\begin{tabular}{|c|c|c|c|c|c|c|c|c|c|c|c|c|c|}
\hline 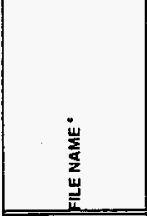 & 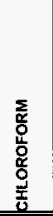 & 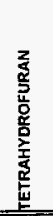 & 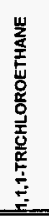 & 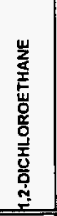 & 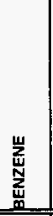 & 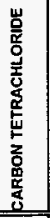 & 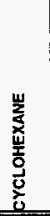 & $\begin{array}{l}1 \\
0 \\
2 \\
\vdots \\
5 \\
9 \\
9\end{array}$ & 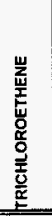 & 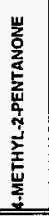 & $\begin{array}{l}\text { 岂 } \\
\text { 岁 } \\
\text { 올 }\end{array}$ & 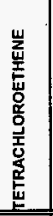 & 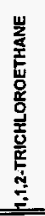 \\
\hline $41796 \quad 3 . \mathrm{D}$ & 465 & 493 & 475 & 701 & 785 & 550 & 493 & 550 & 511 & 425 & 526 & 474 & 575 \\
\hline 41996_3.D & 410 & 360 & 466 & 640 & 675 & 555 & 453 & 480 & 493 & 32 & 490 & 475 & 527 \\
\hline 41896 3.D & 463 & 586 & 493 & 718 & 758 & 561 & 492 & 362 & 516 & 270 & 525 & 478 & 574 \\
\hline 42296-3A.D & 404 & 403 & 459 & 606 & 662 & 526 & 437 & 484 & 496 & 69 & 486 & 463 & 516 \\
\hline 42396 3.D & 464 & 396 & 533 & 702 & 758 & 610 & 502 & 520 & 581 & 29 & 559 & 534 & 600 \\
\hline 51396 3.D & 441 & 305 & 501 & 660 & 790 & 580 & 493 & 550 & 554 & 43 & 542 & 513 & 602 \\
\hline 51496 3.D & 447 & 494 & 516 & 607 & 736 & 590 & 478 & 556 & 548 & 130 & 532 & 529 & 575 \\
\hline 51696_3.D & 371 & 24 & 438 & 497 & 634 & 507 & 405 & 393 & 481 & 0 & 457 & 446 & 491 \\
\hline 52996_9.D & 326 & 132 & 351 & 412 & 929 & 518 & 434 & 6 & 470 & 76 & 469 & 471 & 515 \\
\hline 5319633.0 & 635 & 685 & 698 & 1051 & 826 & 699 & 655 & 384 & 729 & 395 & 748 & 703 & 761 \\
\hline 60396_3.D & 519 & 517 & 584 & 871 & 709 & 596 & 554 & 342 & 621 & 311 & 631 & 595 & 643 \\
\hline 60496_3.D & 519 & 503 & 605 & 899 & 730 & 624 & 574 & 679 & 642 & 242 & 653 & 617 & 660 \\
\hline 60596 G.D & 554 & 498 & 644 & 1003 & 810 & 668 & 622 & 764 & 702 & 245 & 706 & 669 & 720 \\
\hline 61296 2.D & 606 & 615 & 527 & 813 & 680 & 558 & 538 & 575 & 577 & 653 & 599 & 569 & 639 \\
\hline 6149644.0 & 212 & 85 & 280 & 275 & 546 & 518 & 349 & 258 & 287 & 58 & 297 & 406 & 266 \\
\hline 62096 2.D & 499 & 533 & 550 & 819 & 726 & 588 & 544 & 196 & 603 & 305 & 608 & 594 & 631 \\
\hline $624964 . D$ & 498 & 441 & 573 & 867 & 712 & 587 & 556 & 686 & 616 & 206 & 631 & 601 & 645 \\
\hline $62596 \_2 . D$ & 465 & 529 & 492 & 797 & 650 & 514 & 499 & 549 & 537 & 374 & 556 & 515 & 586 \\
\hline $6279622 . D$ & 642 & 606 & 711 & 65 & 1155 & 850 & 810 & 853 & 844 & 364 & 862 & 831 & 914 \\
\hline $70996 \_2.0$ & 478 & 433 & 537 & 50 & 824 & 633 & 615 & 672 & 676 & 211 & 668 & 609 & 715 \\
\hline $71096 \_2 . D$ & 607 & 197 & 709 & 1062 & 1044 & 848 & 772 & 824 & 854 & 15 & 842 & 837 & 861 \\
\hline $71796 \_2 . \mathrm{D}$ & 452 & 431 & 511 & 774 & 649 & 519 & 492 & 500 & 578 & 329 & 558 & 536 & 576 \\
\hline $71796 \_8 . D$ & 556 & 547 & 499 & 769 & 605 & 518 & 485 & 500 & 533 & 566 & 545 & 520 & 567 \\
\hline $72296 \_2.0$ & 613 & 610 & 592 & 914 & 710 & 652 & 599 & 633 & 584 & 622 & 649 & 633 & 682 \\
\hline $72396 \_2 . D$ & 646 & 624 & 610 & $\overline{845}$ & 641 & 642 & 568 & 530 & 629 & 337 & 646 & 657 & 646 \\
\hline $72496 \_13.0$ & 590 & 564 & 514 & 756 & 580 & 537 & 484 & 487 & 534 & 783 & 558 & 533 & 563 \\
\hline 72596 2.D & 580 & 552 & 505 & 733 & 578 & 531 & 481 & 483 & 532 & 740 & 558 & 537 & 559 \\
\hline & & & & & & & & & & & & & \\
\hline Average & 499 & 450 & 532 & 700 & 737 & 596 & 533 & 512 & 582 & 290 & 589 & 568 & 615 \\
\hline Maximum & 646 & 685 & 711 & 1062 & 1155 & 850 & 810 & 853 & 854 & 783 & 862 & 837 & 914 \\
\hline Minimum & 212 & 24 & 280 & 50 & 546 & 507 & 349 & 6 & 287 & 0 & 297 & 406 & 266 \\
\hline Accuracy $(\%)^{\mathrm{a}}$ & 0 & -10 & 6 & 40 & 47 & 19 & 7 & 2 & 16 & .42 & 18 & 14 & 23 \\
\hline Standard Dev. & 101 & 167 & 96 & 252 & 134 & 88 & 99 & 182 & 113 & 224 & 115 & 104 & 119 \\
\hline Precision (\%) & 20 & 33 & 19 & 50 & 27 & 18 & 20 & 36 & 23 & 45 & 23 & 21 & 24 \\
\hline
\end{tabular}

Notes:

"Accuracy measured as the percent difference between the "true" (500 ppb) and the average of the measured values

"Precision measured as the percent standard deviation from the "true" value

" The file name is derived from the analysis date (MMDDY and the run number

Page 2 
Table 4-1. Precision and Accuracy of CWC Analyses Measured on $500 \mathrm{ppb}$ Calibration Sample. (3 sheets)

CWC_CAL.XLS

\begin{tabular}{|c|c|c|c|c|c|c|c|c|c|c|c|}
\hline 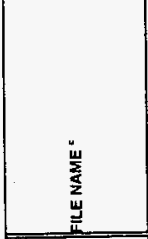 & 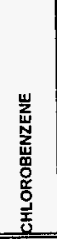 & 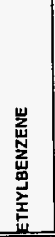 & 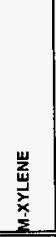 & 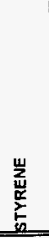 & 岸 & 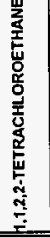 & 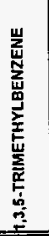 & 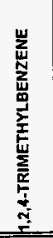 & 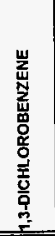 & 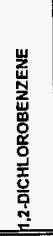 & 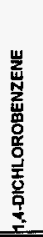 \\
\hline 41796_3.D & 469 & 506 & 474 & 460 & 505 & 521 & 466 & 458 & 454 & 461 & 445 \\
\hline 41996_3.D & 460 & 492 & 470 & 465 & 487 & 464 & 444 & 417 & 429 & 442 & 375 \\
\hline $418963 . D$ & 477 & 515 & 485 & 483 & 515 & 525 & 492 & 476 & 455 & 467 & 435 \\
\hline 42296-3A.D & 450 & 476 & 452 & 445 & 476 & 454 & 449 & 430 & 430 & 438 & 410 \\
\hline 42396 3.D & 526 & 557 & 531 & 535 & 558 & 530 & 524 & 505 & 506 & 516 & 477 \\
\hline 51396 3.D & 501 & 530 & 506 & 490 & 523 & 528 & 495 & 465 & 469 & 477 & 455 \\
\hline $51496 \quad 3 . \mathrm{D}$ & 506 & 523 & 508 & 494 & 516 & 503 & 496 & 479 & 487 & 494 & 471 \\
\hline $51696 \_3 . D$ & 438 & 456 & 435 & 413 & 443 & 405 & 392 & 326 & 407 & 408 & 351 \\
\hline 52996_9.D & 436 & 447 & 439 & 358 & 433 & 395 & 299 & 243 & 351 & 355 & 218 \\
\hline $53196 \quad 3 . D$ & 742 & 783 & 748 & 787 & 772 & 746 & 763 & 757 & 740 & 746 & 721 \\
\hline 60396 3.D & 621 & 658 & 621 & 645 & 646 & 616 & 632 & 606 & 609 & 617 & 598 \\
\hline 60496_3.D & 650 & 676 & 643 & 670 & 666 & 632 & 653 & 637 & 639 & 640 & 620 \\
\hline 60596_9.D & 694 & 730 & 689 & 716 & 719 & 682 & 698 & 674 & 682 & 684 & 658 \\
\hline $612962 . D$ & 586 & 607 & 579 & 597 & 605 & 616 & 598 & 584 & 584 & 586 & 589 \\
\hline $614964 . D$ & 300 & 306 & 301 & 274 & 291 & 247 & 280 & 249 & 303 & 304 & 240 \\
\hline 62096 2.D & 608 & 620 & 594 & 609 & 615 & 602 & 597 & 581 & 597 & 596 & 584 \\
\hline $624964 . D$ & 623 & 653 & 620 & 645 & 643 & 627 & 613 & 584 & 598 & 602 & 547 \\
\hline 62596_2.D & 549 & 575 & 541 & 559 & 563 & 570 & 545 & 524 & 523 & 530 & 488 \\
\hline $62796 \quad 2 . D$ & 828 & 852 & 841 & 840 & 864 & 844 & 827 & 784 & 809 & 820 & 747 \\
\hline 70996 2.D & 637 & 682 & 642 & 634 & 655 & 531 & 573 & 538 & 567 & 572 & 508 \\
\hline $\begin{array}{ll}71096 \_2 . D \\
\end{array}$ & 820 & 722 & 686 & 535 & 649 & 563 & 513 & 418 & 620 & 591 & 474 \\
\hline $71796 \_2 . D$ & 553 & 577 & 538 & 554 & 564 & 512 & 515 & 494 & 507 & 514 & 468 \\
\hline $71796 \div 8 . \mathrm{D}$ & 533 & 560 & 522 & 547 & 549 & 554 & 538 & 531 & 529 & 529 & 536 \\
\hline $72296 \_2 . D$ & 641 & 660 & 629 & 645 & 654 & 657 & 646 & 629 & 637 & 636 & 635 \\
\hline $72396-2 . D$ & 662 & 683 & 660 & 697 & 666 & 650 & 703 & 691 & 694 & 695 & 693 \\
\hline $72496 \_13 . D$ & 540 & 568 & 539 & 566 & 556 & 551 & 570 & 565 & 549 & 552 & 568 \\
\hline 7259622.0 & 544 & 571 & 544 & 573 & 559 & 552 & 576 & 570 & 560 & 561 & 573 \\
\hline & & & & & & & & & & & \\
\hline Average & 570 & 592 & 564 & 564 & 581 & 558 & 552 & 526 & 546 & 549 & 514 \\
\hline Maximum & 828 & 852 & 841 & 840 & 864 & 844 & 827 & 784 & 809 & 820 & 747 \\
\hline \begin{tabular}{|l} 
Minimum \\
\end{tabular} & 300 & 306 & 301 & 274 & 291 & 247 & 280 & 243 & 303 & 304 & 218 \\
\hline Accuracy $(\%)^{a}$ & 14 & 18 & 13 & 13 & 16 & 12 & 10 & 5 & 9 & 10 & 3 \\
\hline Standard Dev. & 118 & $t+3$ & 109 & 123 & 112 & 113 & 123 & 130 & 115 & 114 & 128 \\
\hline Precision $(\%)^{b}$ & 24 & 23 & 22 & 25 & 22 & 23 & 25 & 26 & 23 & 23 & 26 \\
\hline
\end{tabular}

Notes:

Accuracy measured as the percent difference between the "true" (500 ppb) and the average of the measured values

"Precision measured as the percent standard deviation from the "true" value

$c$ The file name is derived from the analysis date (MMDDYY) and the run number

Page 3 
was used to purge the sample inlet system with moist, pure air, especially after analyzing a sample rich in polar compounds, and after every calibration sample. These two modifications drastically reduced adsorption of the polars and eliminated carry-over of these compounds.

Samples analyzed before July 1996 sometimes showed polar compounds after analyzing a calibration sample. A blank (dry zero air) run immediately after the calibration sample showed no detectable organic compounds (no carryover). It was found that the humidity in "real" air samples helped to desorb these compounds from the inlet surfaces of the GC-MS. These polar analytes (ethanol, acetone, 2-propanol, 2-butanone, n-butanol, 4-methyl-2-pentanone) were therefore not quantitated before this date and are listed in Table 4-2 as "Detected" when present. The remaining non-polar and moderately polar compounds were not affected in this way and the data are reproducible.

\subsection{QUALITY CONTROLS}

\section{Sampling Equipment}

The sample volumes were determined by measuring the system pressure differential at the beginning and end of the cryogenic trapping using an absolute vacuum gauge. The gauge was calibrated by the WHC Standards Labs (ID \# 518-31-04-002); the current calibration expires $5 / 16 / 97$.

\section{Analytical Equipment}

A diagnostic check (High Sensitivity Autotune) of the GC-MS was made daily using the factory tuning standards. Daily baseline blanks using a sample of highly purified air (Ultrapure ${ }^{\mathrm{TM}}$ air) were run to verify baseline characteristics.

\section{Calibration Standards}

A three-point calibration curve was generated using a primary $1,000 \mathrm{ppbv}$ standard and secondary 500 and $100 \mathrm{ppbv}$ standards (Scott-Merrin, Riverside, California). The $500 \mathrm{ppbv}$ standard was used as an instrument check standard and was analyzed each day during the analysis cycles. The data for this standard are listed in Table 4-1 with accuracy and reproducibility information for each compound. No comprehensive study was done for this screening project to establish a Method Detection Limit (MDL). However, it was found that $10 \mathrm{ppbv}$ per compound could reproducibly be measured. The MDL was therefore established at $10 \mathrm{ppbv}$ or better.

\section{Analysis Records}

All entries were recorded into a controlled logbook: WHC-N-1236 \# 1, pp. 38-84 . 
HNF-SD-WM-RPT-282 Rev. 0

Table 4-2. GC-MS Data of Air Samples from the Central Waste Complex. (20 sheets)

CWC_LFM.XLS

1) LOW FLASH-POINT MIXED WASTE AND ALKALI METAL STORAGE BUILDINGS

\begin{tabular}{|c|c|c|c|c|c|c|c|c|c|c|}
\hline \multicolumn{4}{|c|}{ GENERAL INFORMATION } & \multicolumn{7}{|c|}{ NON-POLAR AND SLIGHTLY POLAR COMPOUNDS } \\
\hline BUILDING & 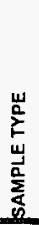 & 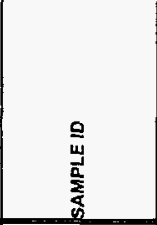 & 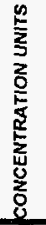 & $\begin{array}{l}\text { w } \\
\mathbf{s} \\
5 \\
\mathbf{5} \\
0 \\
0 \\
0\end{array}$ & 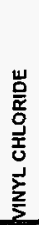 & 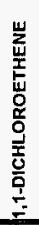 & 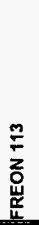 & 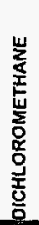 & 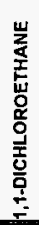 & 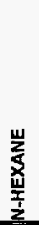 \\
\hline 1 & SR & FT6045-01.109A & PPE & NO & ND & NO & NO & ND & ND & ND \\
\hline 2 & SR & FT6045-02.108A & PPB & ND & ND & ND & ND & 6 & ND & 3 \\
\hline 3 & SR & FT $6045 \quad 03.120 \mathrm{~A}$ & PPB & ND & $\mathrm{ND}$ & ND & ND & ND & ND & ND \\
\hline 4 & SR & FT6045-04.121A & PPB & ND & ND & ND & ND & ND & $\mathrm{ND}$ & ND \\
\hline 5 & SR & FT6045-05.110A & PPB & ND & ND & 2 & ND & 37 & ND & 63 \\
\hline 6 & SR & FT $6045,06.122 \mathrm{~A}$ & PPB & ND & ND & ND & ND & ND & ND & ND \\
\hline 7 & SR & FT 6045 07.127A & PPB & ND & ND & ND & ND & 3 & ND & ND \\
\hline 8 & SR & FT6045-08.128A & PPB & ND & ND & ND & $\mathrm{ND}$ & NO & ND & ND \\
\hline 9 & SR & FT6045-09.117A & PPB & ND & ND & ND & ND & ND & ND & ND \\
\hline 10 & $\mathrm{SR}$ & FT6045-10.116A & PPB & $\mathrm{ND}$ & ND & ND & ND & ND & ND & $\mathrm{ND}$ \\
\hline$\overline{11}$ & SR & FT6045-11.115A & PPB & ND & ND & NO & 4 & 82 & ND & 123 \\
\hline 12 & SR & FT6045-12.129A & PPB & ND & ND & NO & $\overline{\mathrm{ND}}$ & ND & ND & ND \\
\hline 13 & $\mathrm{SR}$ & FT6045-13.130A & PPB & ND & $\mathrm{ND}$ & NO & ND & $\overline{N D}$ & ND & ND \\
\hline 14 (RIGHT) & SR & FT $6045,14.103 \mathrm{~A}$ & PPB & ND & NO & ND & ND & ND & ND & ND \\
\hline 14 (CENTER) & SR & FT $6045,14.104 \mathrm{~A}$ & PPB & $\overline{N D}$ & ND & ND & 190 & 479 & ND & ND \\
\hline 14 (LEFI) & SR & FT 6045 14.105 A & PPB & ND & ND & ND & ND & 87 & ND & 5 \\
\hline 15 & $\mathrm{SR}$ & FT $6045+5.102 \mathrm{~A}$ & $\mathrm{PPB}$ & ND & ND & NO & $\mathrm{ND}$ & ND & $\mathrm{ND}$ & 8 \\
\hline 16 & SR & FT6045 16.09A & PPB & ND & ND & ND & ND & ND & NO & 5 \\
\hline 17 & $\mathrm{SR}$ & FT6045 17.05A & $\mathrm{PPB}$ & ND & ND & ND & ND & ND & ND & ND \\
\hline 18 & $\overline{S R}$ & FT6045 18.03a & PPB & ND & ND & ND & ND & ND & $\overline{N D}$ & ND \\
\hline 19 & SR & FT6045 19.02A & PPB & ND & ND & ND & ND & ND & ND & ND \\
\hline 20 & SR & FT6045 20.01A & PPB & 1 & ND & ND & ND & ND & ND & ND \\
\hline A1 & SR & FT6045 A1.11 & PPB & ND & ND & ND & ND & ND & ND & ND \\
\hline $\mathrm{A} 2_{2}$ & SR & FT 6045 A2.10A & PPB & ND & NO & ND & ND & $\overline{\mathrm{ND}}$ & ND & 5 \\
\hline A3 & SR & FT6045 A3.07A & PPB & ND & ND & ND & ND & ND & ND & 10 \\
\hline A4 & SR & FT6045 A4.06A & PPB & ND & $\mathrm{ND}$ & ND & ND & NO & ND & ND \\
\hline
\end{tabular}

$\begin{array}{ll}\text { Explanations: } & \\ \text { S } & \text { SUMMA sample } \\ \text { SR } & \text { SUMMA sample collected by remole control } \\ \text { BK } & \text { Background sample } \\ \text { FL } & \text { Fenceline sample } \\ \text { UW } & \text { Upwind } \\ \text { OW } & \text { Downwind } \\ \text { ND } & \text { Not detected } \\ \text { D } & \text { Detected- not quantitated (see text) } \\ \text { Note: } & \text { Method Detection Limils are 10 ppb or better }\end{array}$

Page 1 
HNF-SD-WM-RPT-282 Rev. 0

Table 4-2. GC-MS Data of Air Samples from the Central Waste Complex. (20 sheets)

CWC_LFM.XLS

1) LOW FLASH-POINT MIXED WASTE AND ALKALI METAL STORAGE BUILDINGS

\begin{tabular}{|c|c|c|c|c|c|c|c|c|c|c|c|}
\hline NON-POLAR AND & उHTL & POLA & COMP & UNOS & & & & & & & \\
\hline BUILDING & 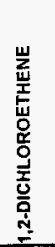 & 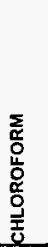 & 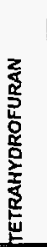 & 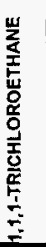 & 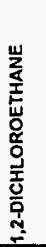 & 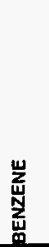 & 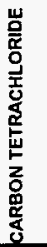 & 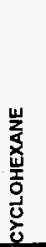 & 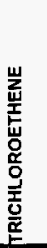 & $\begin{array}{l}\text { 岃 } \\
\text { 岃 } \\
\text { 올 }\end{array}$ & 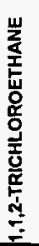 \\
\hline 1 & NO & NO & NO & NO & ND & ND & ND & ND & ND & 239 & ND \\
\hline 2 & ND & ND & $\mathrm{ND}$ & 3 & ND & ND & ND & $\theta$ & ND & 48 & ND \\
\hline 3 & ND & 4 & ND & ND & ND & ND & ND & ND & $\mathrm{ND}$ & 31 & ND \\
\hline$\frac{5}{4}$ & $\mathrm{ND}$ & ND & ND & ND & ND & ND & ND & $\mathrm{ND}$ & ND & 4 & ND \\
\hline 5 & ND & ND & 14 & ND & ND & 3 & ND & NO & ND & 19 & ND \\
\hline 6 & $\mathrm{ND}$ & ND & ND & ND & ND & $\mathrm{ND}$ & ND & ND & ND & 4 & ND \\
\hline 7 & ND & ND & ND & ND & ND & ND & ND & ND & ND & 220 & ND \\
\hline 8 & ND & ND & ND & ND & $N D$ & ND & $\mathrm{ND}$ & ND & $\mathrm{ND}$ & ND & ND \\
\hline 9 & $\mathrm{ND}$ & ND & ND & ND & ND & ND & ND & $\mathrm{ND}$ & $\mathrm{ND}$ & $\mathrm{ND}$ & ND \\
\hline 10 & ND & ND & ND & ND & ND & ND & ND & ND & ND & ND & ND \\
\hline 11 & ND & ND & 25 & ND & ND & ND & ND & ND & ND & 96 & ND \\
\hline 12 & NO & ND & $\mathrm{ND}$ & ND & NO & ND & ND & ND & ND & 83 & ND \\
\hline 13 & $\mathrm{ND}$ & NO & ND & ND & ND & ND & ND & ND & $\mathrm{ND}$ & 7 & ND \\
\hline 14 (RIGHT) & $\mathrm{ND}$ & ND & ND & ND & ND & ND & 256 & 42 & ND & 186 & NO \\
\hline 14 (CENTER) & ND & ND & ND & ND & ND & ND & 8 & ND & ND & 247 & ND \\
\hline 14 (LEFT) & ND & ND & ND & ND & ND & ND & 4 & $\mathrm{ND}$ & ND & $5 \overline{07}$ & ND \\
\hline 15 & ND & ND & ND & ND & ND & ND & 0 & $\mathrm{ND}$ & ND & 5 & ND \\
\hline 16 & ND & 1 & ND & 3 & ND & ND & $\mathrm{ND}$ & $\mathrm{ND}$ & ND & 4 & ND \\
\hline 17 & NO & ND & ND & ND & NO & ND & $\mathrm{ND}$ & ND & ND & 14 & ND \\
\hline 18 & ND & ND & ND & ND & $\mathrm{NO}$ & ND & 21 & 12 & ND & 20 & ND \\
\hline 19 & ND & ND & ND & $\mathrm{ND}$ & $\mathrm{NO}$ & ND & ND & ND & ND & ND & NO \\
\hline 20 & ND & ND & ND & $\mathrm{ND}$ & $\mathrm{ND}$ & $\mathrm{ND}$ & 5 & 21 & ND & ND & ND \\
\hline $\mathrm{A1}$ & ND & ND & ND & $\mathrm{ND}$ & ND & ND & ND & ND & ND & ND & NO \\
\hline$A 2$ & ND & ND & 3 & $\mathrm{ND}$ & 1 & ND & ND & ND & ND & ND & ND \\
\hline$A 3$ & ND & ND & 25 & ND & 3 & 1 & ND & ND & ND & 1 & $\mathrm{ND}$ \\
\hline A4 & ND & NO & NO & ND & ND & ND & ND & ND & ND & 2 & ND \\
\hline \multicolumn{12}{|l|}{ Explanations: } \\
\hline $\mathrm{s}$ & \multicolumn{11}{|c|}{ SUMMA sample } \\
\hline$S R$ & \multicolumn{11}{|c|}{ SUMMA sample collected by remote control } \\
\hline $\mathrm{BK}$ & \multicolumn{11}{|c|}{ Background sample } \\
\hline $\mathrm{FL}$ & \multicolumn{11}{|c|}{ Fenceline sample } \\
\hline UW & \multicolumn{11}{|c|}{ Upwind } \\
\hline DW & \multicolumn{11}{|c|}{ Downwind } \\
\hline ND & \multicolumn{11}{|c|}{ Not detected } \\
\hline D & \multicolumn{11}{|c|}{ Detected. not quantitated (see text) } \\
\hline Note: & \multicolumn{11}{|c|}{ Method Detection Limits are $10 \mathrm{ppb}$ or better } \\
\hline
\end{tabular}

Page 1 
HNF-SD-WM-RPT-282 Rev. 0

Table 4-2. GC-MS Data of Air Samples from the Central Waste Complex. (20 sheets)

CWC_LFM.XLS

1) LOW FLASH-POINT MIXED WASTE AND ALKALI METAL STORAGE BUILDINGS

\begin{tabular}{|c|c|c|c|c|c|c|c|c|c|c|c|c|}
\hline \multicolumn{13}{|c|}{ NON-POLAR AND SLIGHTLY POLAR COMPOUNDS } \\
\hline BUILDING & 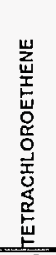 & 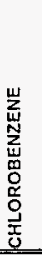 & 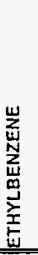 & 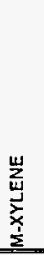 & $\frac{\frac{u}{z}}{\frac{w}{z}}$ & 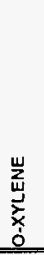 & 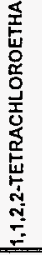 & 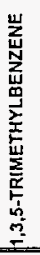 & 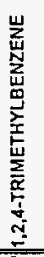 & 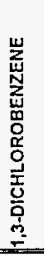 & 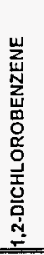 & 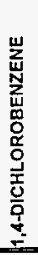 \\
\hline 1 & ND. & ND & $\overline{N D}$ & ND & NO & ND & ND & ND & 76 & ND & ND & ND \\
\hline 2 & NO & ND & 65 & 215 & 1 & 59 & ND & NO & 1 & NO & ND & ND \\
\hline 3 & ND. & ND & 2 & 16 & NO & 4 & ND & 7 & 72 & NO & ND & ND \\
\hline 4 & ND & ND & 4 & 14 & 3 & 4 & ND & 15 & 3 & ND & ND & ND \\
\hline 5 & ND & 16 & 2 & 6 & NO & 9 & NO & 9 & 2 & 30 & 31 & 30 \\
\hline 6 & NO & ND & 1 & ND & NO & $\mathrm{ND}$ & ND & ND & $\mathrm{ND}$ & $N D$ & ND & ND \\
\hline 7 & ND & ND & 1 & 10 & ND & $\mathrm{ND}$ & $\mathrm{NO}$ & ND & 9 & ND & ND & ND \\
\hline 8 & ND & ND & 9 & 11 & NO & 5 & ND & 1 & 2 & ND & ND & ND \\
\hline 9 & ND & NO & 1 & 8 & NO & 7 & ND & 1 & 2 & ND & ND & $\mathrm{NO}$ \\
\hline 10 & ND & NO & 6 & 7 & NO & 5 & ND & $\mathrm{NO}$ & ND & ND & $\mathrm{ND}$ & ND \\
\hline 11 & NO & NO & 14 & 46 & ND & 19 & ND & 3 & 8 & ND & ND & $\mathrm{ND}$ \\
\hline 12 & ND & NO & 4 & 29 & ND & 10 & $\mathrm{ND}$ & 2 & 13 & ND & ND & ND \\
\hline 13 & NO & NO & ND & $\mathrm{ND}$ & NO & NO & NO & 7 & 6 & ND & ND & ND \\
\hline 14 (RIGHT) & ND & ND & 12 & 50 & 2 & 14 & ND & 17 & 8 & ND & $\mathrm{NO}$ & NO \\
\hline 14 (CENTER) & ND & ND & 19 & 55 & 1 & 14 & $\mathrm{ND}$ & 17 & 1 & ND & $\mathrm{NO}$ & $\mathrm{ND}$ \\
\hline 14 (LEFT) & ND & ND & 42 & 329 & 6 & 273 & ND & 7 & 9 & $\mathrm{ND}$ & ND & NO \\
\hline 15 & NO & $\mathrm{ND}$ & 6 & 17 & 0 & 7 & NO & 3 & 2 & ND & $\mathrm{ND}$ & ND \\
\hline 16 & $\mathrm{ND}$ & $\mathrm{NO}$ & 3 & 5 & ND & 2 & $\mathrm{NO}$ & ND & ND & $\mathrm{ND}$ & NO & $\mathrm{ND}$ \\
\hline 17 & ND & ND & NO & ND & ND & ND & ND & 10 & 2 & $\mathrm{NO}$ & ND & ND \\
\hline 18 & ND & $\mathrm{ND}$ & 1 & 28 & ND & 23 & ND & ND & 5 & $\mathrm{ND}$ & ND & ND \\
\hline 19 & ND. & ND & ND & NO & ND & NO & $\mathrm{ND}$ & 2 & 1 & $\mathrm{ND}$ & ND & ND \\
\hline 20. & ND & NO & 6 & 7 & ND & 5 & ND & 13 & 1 & $\mathrm{NO}$ & $\mathrm{NO}$ & ND \\
\hline A1 & NO & ND & 1 & NO & ND & ND & ND & 3 & ND & $\mathrm{NO}$ & $\mathrm{ND}$ & ND \\
\hline A2 & $\mathrm{ND}$ & NO & 1 & ND & 1 & 1 & ND & 8 & 3 & 2 & 2 & 4 \\
\hline$A 3$ & NO & 1 & 2 & 2 & 3 & 2 & 1 & 3 & 5 & 2 & 3 & $\overline{4}$ \\
\hline A4 & NO & ND & 1 & 4 & NO & 2 & ND & 4 & $\mathrm{ND}$ & NO & $\mathrm{ND}$ & ND \\
\hline \multicolumn{13}{|l|}{ Explanations: } \\
\hline$s$ & \multicolumn{12}{|c|}{ SUMMA sample } \\
\hline SR & \multicolumn{12}{|c|}{ SUMMA sample collected by remote control } \\
\hline BK & \multicolumn{12}{|c|}{ Background sample } \\
\hline FL & \multicolumn{12}{|c|}{ Fenceline sample } \\
\hline UW & \multicolumn{12}{|c|}{ Upwind } \\
\hline DW & \multicolumn{12}{|c|}{ Downwind } \\
\hline NO & \multicolumn{12}{|c|}{ Not detected } \\
\hline D & \multicolumn{12}{|c|}{ Detecled. not quankitated (see text) } \\
\hline Nole: & \multicolumn{12}{|c|}{ Method Detection Limits are io pob or better } \\
\hline
\end{tabular}

Page 1 
HNF-SD-WM-RPT-282 ReV. 0

Table 4-2. GC-MS Data of Air Samples from the Central Waste Complex. (20 sheets)

CWC_LFM.XLS

1) LOW FLASH-POINT MIXED WASTE AND ALKALI METAL STORAGE BUILOINGS

\begin{tabular}{|c|c|c|c|c|c|c|c|}
\hline & \multicolumn{6}{|c|}{ POLAR COMPOUNDS } & \\
\hline BUILDING & 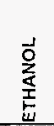 & 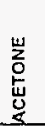 & $\begin{array}{l}\overrightarrow{1} \\
0 \\
z \\
\alpha \\
0 \\
0 \\
\alpha \\
\alpha \\
\alpha\end{array}$ & $\begin{array}{l}\text { w } \\
z \\
0 \\
z \\
5 \\
5 \\
0 \\
0 \\
0\end{array}$ & 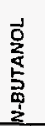 & 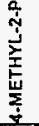 & $\begin{array}{l}\frac{n}{2} \\
\frac{w}{2} \\
0 \\
0\end{array}$ \\
\hline 1 & 83 & 3 & ND & ND & $\overline{\mathrm{ND}}$ & ND & \\
\hline 2 & $\overline{17}$ & 29 & NO & 107 & 9 & 14 & \\
\hline 3 & 10 & 5 & ND & 17 & 5 & 12 & \\
\hline 4 & $\mathrm{ND}$ & NO & NO & NO & 45 & 15 & AVERAGE OF 2 \\
\hline 5 & 137 & 33 & 61 & ND & ND & NO & AVERAGE OF $\overline{2}$ \\
\hline 6 & NO & NO & ND & ND & NO & NO & \\
\hline 7 & 158 & 6 & $\mathrm{ND}$ & 21 & 5 & ND & \\
\hline 8 & ND & ND & NO & ND & NO & NO & AVERAGE OF 2 \\
\hline 9 & ND & ND & NO & ND & NO & ND & \\
\hline 10 & ND & NO & NO & ND & ND & ND & AVERAGE OF $\overline{2}$ \\
\hline 11 & NO & 54 & $\mathrm{NO}$ & 1465 & NO & 41 & \\
\hline 12 & NO & NO & ND & ND & ND & NO & \\
\hline 13 & ND & ND & ND & NO & ND & ND & AVERAGE OF 2 \\
\hline 14 (RIGHT) & NO & 7 & 8 & 15 & NO & 105 & AVERAGE OF 2 \\
\hline 14 (CENTER) & 19 & 34 & 1055 & 20 & No & 7 & \\
\hline 14 (LEFT) & ND & 61 & 65 & 30 & 8 & 147 & \\
\hline 15 & ND & 5 & ND & ND & $\mathrm{NO}$ & ND & \\
\hline 16 & ND & D & $\mathrm{NO}$ & D & No & D & AVERAGE OF 2 \\
\hline 17 & NO & ND & NO & ND & $\bar{D}$ & ND & \\
\hline 18 & NO & $\mathrm{ND}$ & ND & ND & 10 & ND & \\
\hline 19 & $\bar{D}$ & $\bar{D}$ & ND & D & D & D & AVERAGE OF 2 \\
\hline 20 & NO & ND & NO & NO & D & ND & \\
\hline$\overline{A_{1}}$ & ND & ND & ND & ND & NO & ND & AVERAGE OF 2 \\
\hline A2 & D & D & D & D & D & D & AVERAGE OF 3 \\
\hline A3 & $\mathrm{D}$ & D & $\mathrm{D}$ & $\mathrm{D}$ & $\bar{D}$ & $\mathrm{D}$ & AVERAGE OF 3 \\
\hline A4 & ND & ND & NO & ND & ND & NO & \\
\hline
\end{tabular}

Explanations:

SR

SUMMA sample

SUMMA sample collected by remote control

BK Background sample

$\mathrm{FL} \quad$ Fenceline sample

UW Upwind

DW Downwind

NO Not detected

D

Detected- noi quantitated (see text)

Nole:

Method Detection Limits are 10 ppb or better

Page 1 
Table 4-2. GC-MS Data of Air Samples from the Central Waste Complex. (20 sheets) CWC_LFMXLS

2L BACKGROUND BLANKS FOR LOW FLASH POINT MIXER WASTE AND ALKALLIMETAL STORAGE BUHLDINGS

\begin{tabular}{|c|c|c|c|c|c|c|c|c|c|c|}
\hline \multicolumn{4}{|c|}{ GENERAL INFORMATION } & \multicolumn{7}{|c|}{ NONPOLAR AND SLIGHTLY POLAR COMPOUNDS } \\
\hline BUILDING & 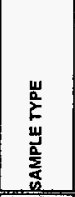 & $\begin{array}{l}\frac{0}{11} \\
\frac{1}{5} \\
\frac{1}{6}\end{array}$ & 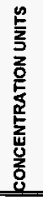 & 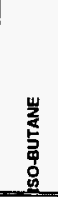 & 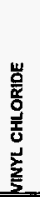 & 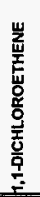 & 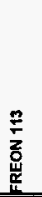 & 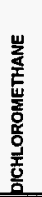 & 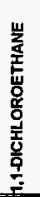 & $\begin{array}{l}\frac{w}{\mathbf{3}} \\
\mathbf{3} \\
\frac{\mathbf{m}}{7} \\
\end{array}$ \\
\hline $17,18,19,20, \mathrm{~A} 3, \mathrm{A4}$ & FL, DW & FT6045_FLCAB & $\overline{\mathrm{PPB}}$ & ND & ND & ND & ND & ND & ND & $\mathrm{ND}$ \\
\hline $17,18,19,20, \mathrm{~A}, \mathbf{3}, \mathbf{A} 4$ & $\mathrm{IR}$ & FT6045_TR08A & PPB & NA & NA & NA & NA & NA & NA & NA \\
\hline $16, \mathrm{~A} 1, \mathrm{~A} 2$ & FL, DW & FT6045_FL_13A & PPB & ND & ND & ND & ND & ND & ND & $\mathrm{ND}$ \\
\hline $16, \mathrm{~A} 1, \mathrm{~A} 2$ & FL, UW & FT6045_FL_13B & PPB & ND & ND & $\mathrm{ND}$ & ND & ND & ND & ND \\
\hline $16, \mathrm{~A} 1, \mathrm{~A} 2$ & TR & FT6045_TR14.A & $\mathrm{PPB}$ & ND & ND & ND & ND & ND & ND & ND \\
\hline $18,19,20$ & BK & FT6045 BK.15A & PPB & ND & ND & ND & ND & ND & ND & ND \\
\hline$A 1, A 2,16$ & BK & FT6015_BK.12A & PPB & ND & ND & ND & ND & ND & ND & ND \\
\hline $\mathrm{A} 3, \mathrm{~A} 4,17$ & BK & FT6015_BK.16A & PPB & ND & ND & $\mathrm{ND}$ & ND & ND & ND & 58 \\
\hline 14,15 & BK & FT6015_BK.101A & PPB & NA & NA & NA & NA & $\overline{N A}$ & NA & NA \\
\hline 14,15 & FL, DW & FT6045-DW.106A & $\mathrm{PPB}$ & ND & ND & ND & ND & ND & ND & ND \\
\hline 14,15 & $\mathrm{FL}, \mathrm{UW}$ & FT6045-UW.106A & PPB & ND & ND & ND & ND & ND & ND & ND \\
\hline 14,15 & $\mathrm{TR}$ & FT 6045_TR.106 A & PPB & ND & ND & ND & ND & ND & ND & ND \\
\hline $1,2,5$ & BK & FT6045-BK.111A & PPB & ND & ND & ND & ND & ND & ND & ND \\
\hline $9,10,11$ & $\mathrm{BK}$ & FT6045-BK.118A & $\mathrm{PPB}$ & ND & ND & ND & ND & ND & ND & ND \\
\hline $1,2,5,9,10,11$ & FL, UW & FT6045-UW.112A & PPB & ND & ND & ND & ND & ND & ND & ND \\
\hline $1,2,5,9,10,11$ & FL., DW & FT6045-DW.114A & PPB & ND & ND & ND & ND & ND & ND & ND \\
\hline $1,2,5,9,10,11$ & TR & FT6045-TR.113A & $\mathrm{PPB}$ & ND & ND & ND & ND & ND & ND & ND \\
\hline $3,4,6$ & BK & FT $6045-B K .124 A$ & PPB & ND & ND & ND & ND & ND & $\mathrm{ND}$ & ND \\
\hline $7,8,12$ & BK & FT6045-BK.131A & $\mathrm{PPB}$ & ND & ND & ND & ND & ND & $\mathrm{ND}$ & $\mathrm{ND}$ \\
\hline $3,4,6,7,8,12$ & FL, UW & FT 6045 UW.124A & PPB & ND & ND & ND & ND & ND & ND & ND \\
\hline $3,4,6,7,8,12$ & FL, DW & FT 6045_DW.125A & PPB & ND & ND & ND & ND & ND & ND & ND \\
\hline $3,4,6,7,8,12$ & $\mathrm{JR}$ & FT6045-TR.126A & $\mathrm{PPB}$ & ND & ND & ND & ND & ND & ND & ND \\
\hline \multicolumn{11}{|l|}{ Exolanations: } \\
\hline s & \multicolumn{10}{|c|}{ SUMMA sample } \\
\hline SR & \multicolumn{10}{|c|}{ SUMMA sample collected by remote control } \\
\hline BK & \multicolumn{10}{|c|}{ Background sample } \\
\hline $\mathrm{FL}$ & \multicolumn{10}{|c|}{ Fenceline sample } \\
\hline UW & \multicolumn{10}{|l|}{ Upwind } \\
\hline DW & \multicolumn{10}{|l|}{ Downwind } \\
\hline NO & \multicolumn{10}{|c|}{ Not dolected } \\
\hline D & \multicolumn{10}{|c|}{ Detected- not quantitated (see text) } \\
\hline NA & \multicolumn{10}{|c|}{ Not analyzed } \\
\hline Note. & \multicolumn{10}{|c|}{ Method Detection Limits are 10 pob or better } \\
\hline
\end{tabular}


Table 4-2. GC-MS Data of Air Samples from the Central Waste Complex. (20 sheets) CWC_LFM.XLS

2) BACKGROUND BLANKS FOR LOW FLASH-POINT MIXED WASTE AND ALKKALI METAL STORAGE BUILDINGS

\begin{tabular}{|c|c|c|c|c|c|c|c|c|c|c|c|}
\hline \multirow[b]{2}{*}{ BUILDING } & \multicolumn{11}{|c|}{ NON-POLAR AND SUIGHTLY POLAR COMPOUNOS } \\
\hline & 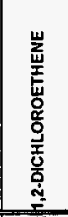 & 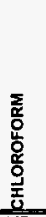 & 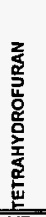 & 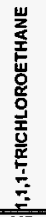 & 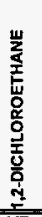 & 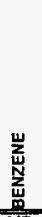 & 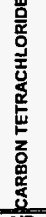 & 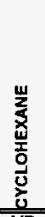 & 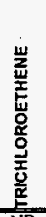 & 崫 & 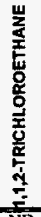 \\
\hline $17,18,19,20, \mathrm{~A} 3, \mathrm{A4}$, & ND & ND & ND & ND & ND & ND & ND & ND & ND & ND & $\mathrm{ND}$ \\
\hline $17,18,19,20, \bar{A} 3, A 4$ & NA & NA & NA & NA & NA & NA & NA & NA & NA & NA & NA \\
\hline $16, \mathrm{~A} 1, \mathrm{~A} 2$ & $\mathrm{ND}$ & ND & ND & ND & ND & ND & ND & ND & ND & ND & ND \\
\hline $16, \mathrm{~A} 1, \mathrm{~A} 2$ & ND & ND & ND & ND & ND & ND & ND & ND & ND & ND & ND \\
\hline$\overline{16, \mathrm{~A} 1, \mathrm{~A} 2}$ & ND & ND & ND & ND & ND & ND & ND & $\overline{\mathrm{ND}}$ & ND & ND & ND \\
\hline $18,19,20$ & ND & ND & ND & ND & ND & ND & ND & ND & ND & ND & $\overline{N D}$ \\
\hline $\mathrm{A} 1, \mathrm{~A} 2,16$ & ND & $\mathrm{ND}$ & ND & ND & ND & ND & ND & ND & ND & ND & ND \\
\hline $\mathrm{A} 3, \mathrm{~A} 4,17$ & ND & ND & 80 & ND & 8 & 5 & ND & ND & ND & 3 & ND \\
\hline 14,15 & NA & NA & NA & NA & NA & NA & NA & NA & NA & NA & NA \\
\hline $\mathbf{1 4 . 1 5}$ & ND & ND & $\overline{N D}$ & ND & ND & ND & $\mathrm{ND}$ & ND & ND & ND & ND \\
\hline 14,15 & ND & ND & $\mathrm{ND}$ & ND & ND & $\overline{\mathrm{ND}}$ & ND & ND & ND & ND & ND \\
\hline 14,15 & ND & ND & ND & ND & ND & ND & ND & ND & $\mathrm{ND}$ & ND & ND \\
\hline $1,2.5$ & ND & ND & ND & ND & ND & ND & ND & ND & $\mathrm{ND}$ & ND & ND \\
\hline $9,10,11$ & ND & ND & ND & ND & ND & ND & ND & ND & $\mathrm{ND}$ & $\mathrm{ND}$ & ND \\
\hline $1,2,5,9,10,11$ & ND & ND & ND & ND & ND & ND & ND & ND & ND & $\mathrm{ND}$ & ND \\
\hline $1,2,5,9,10,11$ & ND & $\mathrm{ND}$ & $\mathrm{ND}$ & ND & ND & ND & ND & ND & $\mathrm{ND}$ & ND & ND \\
\hline $1,2,5,9,10,11$ & ND & $\mathrm{ND}$ & ND & ND & ND & ND & ND & ND & $\mathrm{ND}$ & ND & ND \\
\hline $3,4,6$ & ND & ND & $\mathrm{ND}$ & ND & ND & $\mathrm{ND}$ & ND & ND & $\mathrm{ND}$ & ND & ND \\
\hline $7,8,12$ & ND & ND & ND & ND & ND & ND & ND & ND & $\mathrm{ND}$ & ND & ND \\
\hline $3,4,6,7,8,12$ & $\overline{N D}$ & ND & ND & ND & ND & ND & ND & ND & $\mathrm{ND}$ & ND & ND \\
\hline $3,4,6,7,8,12$ & ND & ND & ND & ND. & ND & ND & ND & ND & ND & ND & ND \\
\hline $3,4,6,7,8,12$ & ND & ND & ND & ND & ND & ND & ND & ND & ND & $\mathrm{ND}$ & $\mathrm{ND}$ \\
\hline \multicolumn{12}{|l|}{ Explanations: } \\
\hline $\mathbf{s}$ & \multicolumn{11}{|c|}{ SUMMA sampie } \\
\hline SR & \multicolumn{11}{|c|}{ SUMMA sample collectod by remote control } \\
\hline BK & \multicolumn{11}{|c|}{ Background sample } \\
\hline FL & \multicolumn{11}{|c|}{ Fenceline sample } \\
\hline uw & \multicolumn{11}{|c|}{ Upwind } \\
\hline DW & \multicolumn{11}{|c|}{ Downwind } \\
\hline ND & \multicolumn{11}{|c|}{ Not detected } \\
\hline D & \multicolumn{11}{|c|}{ Detected- not quantitated (see text) } \\
\hline NA & \multicolumn{11}{|c|}{ Not analyzed } \\
\hline Nol & \multicolumn{11}{|c|}{ Methad Detaction Limits are $10 \mathrm{ppb}$ or better } \\
\hline
\end{tabular}

Page 1 
HNF-SD-WM-RPT-282 Rev. 0

Table 4-2. GC-MS Data of Air Samples from the Central Waste Complex. (20 sheets) CWC_LFMXLS

21 BACKGROUND PL.ANKS FOR LOW FLASHPOINT MIXED WASTE AND ALKKALIMETAL STORAGE BUILOINGS

\begin{tabular}{|c|c|c|c|c|c|c|c|c|c|c|c|c|}
\hline \multirow[b]{2}{*}{ BUILOING } & \multicolumn{12}{|c|}{ INON POLAR AND SLIGHTLY POLAR COMPOUNOS } \\
\hline & 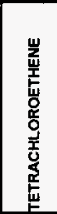 & 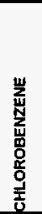 & 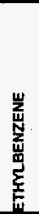 & 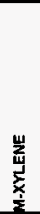 & & לֶ. & 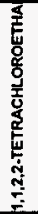 & 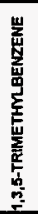 & 崖 & 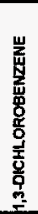 & 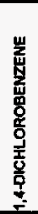 & 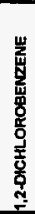 \\
\hline $17,18,19,20, \mathrm{~A}_{3}, \mathrm{A4}$ & ND & ND & ND & ND & ND & ND & ND & 2 & 4 & ND & ND & $\mathrm{ND}$ \\
\hline 17,18,19,20,A3,A4 & NA & NA & NA & NA & $\mathrm{NA}$ & NA & NA & NÁ & NA & NA & NA & NA \\
\hline $16, \mathrm{~A} 1, \mathrm{~A} 2$ & ND & ND & ND & ND & ND & ND & ND & 8 & 1 & ND & ND & $\mathrm{ND}$ \\
\hline $16, \mathrm{A1}, \mathrm{A2}$ & ND & ND & ND & ND & ND & ND & ND & 7 & ND & ND & $\mathrm{ND}$ & ND \\
\hline $16, A 1, A 2$ & ND & ND & ND & ND & ND & ND & ND. & ND & ND & ND & ND & ND \\
\hline $18,19,20$ & ND & ND & ND & ND & ND & ND & ND & 4 & ND & $\mathrm{ND}$ & $\mathrm{ND}$ & ND \\
\hline Al, A2,16 & ND & ND & ND & ND & ND & $\mathrm{ND}$ & ND & ND & $\frac{2}{2}$ & ND & ND & ND \\
\hline $\mathrm{A} 3, \mathrm{A4}, 17$ & ND & 3 & 4 & 4 & 8 & 5 & 2 & 8 & 14 & 8 & 15 & 10 \\
\hline 14,15 & NA & NA & NA & NA & NA & NA & NA & NA & NA & NA & NA & NA \\
\hline 14,15 & ND & ND & ND & ND & ND & ND & ND & ND & ND & ND & ND & ND \\
\hline 14,15 & ND & ND & ND & ND & ND & ND & ND & ND & ND & ND & ND & ND \\
\hline 14,15 & ND & ND & ND & ND & ND & ND & ND & ND & ND & ND & ND & ND \\
\hline $1,2,5$ & ND & ND & ND & ND & ND & ND & $\overline{N D}$ & ND & ND & ND & $\mathrm{ND}$ & ND \\
\hline $9,10,11$ & ND & ND & ND & ND & ND & ND & ND & ND & ND & ND & ND & ND \\
\hline $1,25,9,10,11$ & ND & ND & ND & ND & ND & ND & ND & ND & ND & ND & ND & ND \\
\hline $1,2,5,9,10,11$ & ND & ND & ND & ND & ND & ND & ND & ND & ND & ND & ND & ND \\
\hline $1,25,9,10,11$ & ND & ND & ND & ND & ND & ND & ND & ND & ND & ND & ND & ND \\
\hline$\frac{3,4,6}{3,6}$ & ND & ND & ND & ND & ND & ND & ND & ND & ND & ND & ND & ND \\
\hline $7,8,12$ & ND & ND & ND & ND & ND & ND & $\mathrm{ND}$ & ND & 2 & ND & $\mathrm{ND}$ & ND \\
\hline $3,4,6,7,8,12$ & ND & ND & ND & ND & ND & ND & ND & ND & $\mathrm{ND}$ & ND & ND & ND \\
\hline $3,4,6,7,8,12$ & ND & ND & ND & $\mathrm{ND}$ & ND & ND & ND & ND & ND & ND & ND & ND \\
\hline $3,46,7,8,12$ & ND & ND & ND & ND & ND & ND & ND & ND & ND & ND & ND & ND \\
\hline
\end{tabular}

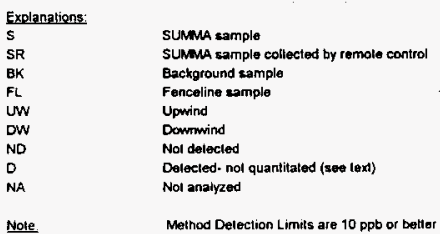

Page 1 


\section{$\nabla I-b$}

$\left[\mathrm{a} 8 \mathrm{e}_{\mathrm{d}}\right.$

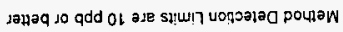

pazkieue jon

(pxaj әәs) pajejnuenb jou -pajoajad

рәроэтар jON

puipumog

- pumadn

ajdues au!|arua

әdues punos6्रेe日

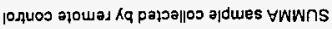

ədues $\forall W W n S$

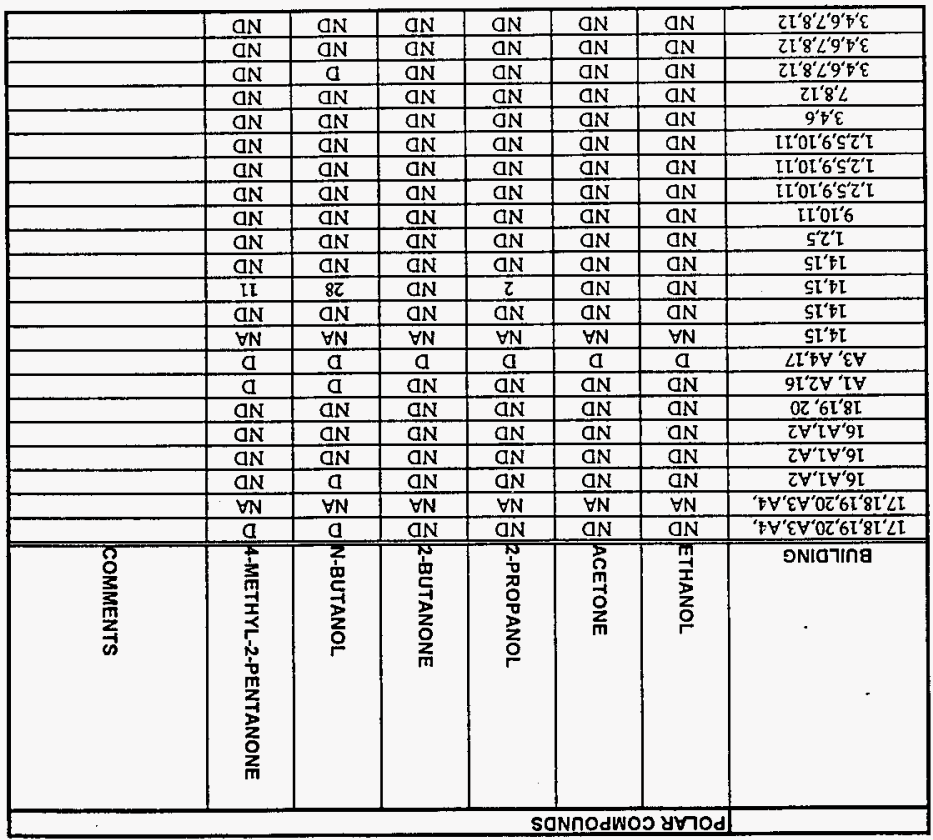

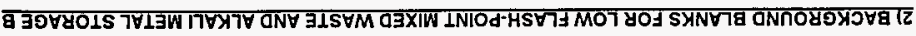

STXพ

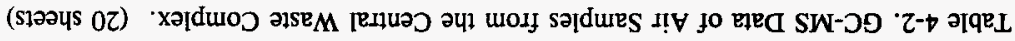


HNF-SD-WM-RPT-282 Rev. 0

Table 4-2. GC-MS Data of Air Samples from the Central Waste Complex. (20 sheets)

$2402 \times 1$ S

\section{2 aulloings}

\begin{tabular}{|c|c|c|c|c|c|c|c|c|c|c|c|}
\hline \multicolumn{4}{|c|}{ GENERAL INFORMATION } & \multicolumn{8}{|c|}{ NONFOURR ANO SLIGHTLY POLAR COMPOUNOS } \\
\hline BUILDING & 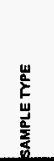 & $\begin{array}{l}\text { 은 } \\
\frac{\vec{w}}{5} \\
\frac{5}{5}\end{array}$ & 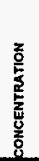 & 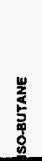 & 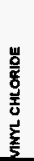 & 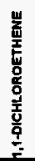 & 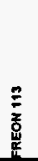 & 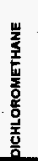 & 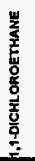 & $\begin{array}{l}\text { w } \\
\frac{5}{5} \\
\frac{7}{7} \\
7\end{array}$ & 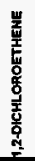 \\
\hline $2402 \mathrm{~W}$ & $\mathrm{~s}$ & FT6045_2W.20A & PPB & $\mathrm{ND}$ & ND & $\mathrm{ND}$ & ND & ND & $\mathrm{ND}$ & ND & ND \\
\hline $2402 \mathrm{~W}$ & $\mathbf{s}$ & FT6015 2W.21A & PPB & ND & ND & ND & ND & ND & No & ND & ND \\
\hline $2402 \mathrm{~W}$ & BK & FT60+5_2W.22A BK & $\mathbf{P P B}$ & ND & ND & ND & ND & ND & No & ND & ND \\
\hline $2402 \mathrm{WB}$ & $\mathrm{s}$ & FT60+5.2WB.40A & PP8 & ND & ND & ND & ND & ND & ND & ND & 3 \\
\hline $2+02 \mathrm{WB}$ & 5 & FTGO+5-2WBA3AA & PPg & ND & $\mathrm{ND}$ & ND & NO & ND & NO & ND & 3 \\
\hline $2+02 \mathrm{WB}$ & BK & FT6OHS-2WB,42ABK & PPB & ND & ND & ND & ND & No & ND & ND & ND \\
\hline $2+02 W C$ & $s$ & FToOA_2WC.23 & PPB & ND & ND & 1 & $\mathrm{ND}$ & ND & ND & 27 & ND \\
\hline $2402 \mathrm{WC}$ & 5 & FT6015 2 WC. $24 \mathrm{~A}$ & Pr8 & $\mathrm{ND}$ & ND & ND & ND & ND & $\frac{\mathrm{ND}}{\mathrm{N}}$ & $\mathrm{ND}$ & ND \\
\hline $2+02 W C$ & BK & FT6015_2WC.25 BK & Pp8 & No & $\mathrm{ND}$ & $\frac{7}{2}$ & ND & ND & 1 & 20 & 3 \\
\hline $2402 W D$ & $\mathrm{~s}$ & FT6045-2WD.43A & PP8 & NO & ND & $\mathrm{ND}$ & ND & 63 & $\mathrm{ND}$ & $\overline{\mathrm{ND}}$ & $\overline{N D}$ \\
\hline 2402 WD & 5 & FTO045 2WD +WA & Prs & NA & NA & $\mathrm{NA}$ & NA & NA & NA & NA & NA \\
\hline $2402 \mathrm{WD}$ & $B K$ & FT 6015_2WDA4SABK & PPB & ND & ND & No & ND & 104 & ND & ND & NO. \\
\hline $2+02 \mathrm{WE}$ & $\mathrm{s}$ & FT6015_2WE.26A & $P P B$ & NO & ND & ND & ND & 231 & ND & ND & ND \\
\hline $2402 \mathrm{WE}$ & s & FT6015 2WE $27 \mathrm{~A}$ & PPS & ND & ND & ND & ND & 236 & $\mathrm{ND}$ & ND & ND \\
\hline $2402 \mathrm{WE}$ & Bk & FT6015_2WE. $28 \mathrm{SA} \mathrm{BK}$ & PPPB & ND & ND & ND & ND & ND & ND & ND & ND \\
\hline $2+02 \mathrm{WF}$ & 5 & FT6015-2WF, $46 \mathrm{~A}$ & PPB & ND & ND & ND & ND & ND & NO & ND & ND \\
\hline $2402 \mathrm{WF}$ & $\mathrm{s}$ & FT6015-2WF.47A & $P P B$ & ND & ND & ND & NO & ND & ND & ND & ND \\
\hline $2402 \mathrm{WF}$ & BK & FT 60.15,2WF.48ABK & $\mathrm{PPB}$ & ND & ND & ND & $\mathrm{ND}$ & NO & ND & ND & ND \\
\hline $2402 W G$ & $\frac{\mathrm{s}}{\mathrm{S}}$ & 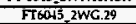 & $\mathrm{PPB}$ & ND & ND & ND & ND & ND & ND & 1 & ND \\
\hline $2+02 W C$ & 5 & FT6015,2WC,30A & $\mathrm{PPB}$ & ND & ND & NO & ND & $\mathrm{ND}$ & ND & ND & ND \\
\hline $2402 \mathrm{WC}$ & $B K$ & FT6DS_2WG_3LA BK & $\mathrm{PPB}$ & ND & ND & ND & ND & NO & NO & ND & ND \\
\hline $2402 \mathrm{WH}$ & 5 & FT6015-2WH.49A & PPB & NO & ND & ND & ND & ND & NO & $\mathrm{ND}$ & ND \\
\hline $2402 \mathrm{WH}$ & $\mathrm{s}$ & FT6045-2WH:50A & PPB & $\mathrm{ND}$ & ND & $\mathrm{ND}$ & ND & NO & NO & ND & ND \\
\hline 2402 WH & BK & FT604S2WHASIABK & PPB & ND & ND & NO & ND & ND & ND & ND & No \\
\hline $2402 \mathrm{~W}$ & 5 & Fr6045,2WL32 & PPB & ND & ND & $\mathrm{ND}$ & ND & ND & $\mathrm{ND}$ & ND & ND \\
\hline $2402 \mathrm{~W}$ & 5 & FT6045 2WT.33A & PPB & ND & ND & NO & ND & ND & ND & ND & ND \\
\hline $2402 \mathrm{~W}$ & BK & FT 6045 2WT.3A B BK & FPB & $\mathrm{ND}$ & ND & ND & NO & ND & NO & NO & ND \\
\hline $2402 \mathrm{~W}$ & 5 & FT6045-2WJ.52A & PPB & ND & ND & ND & ND & ND & ND & 14 & ND \\
\hline $2+02 \mathrm{WJ}$ & 5 & FT6015-2WT.53A & PPB & $\mathrm{ND}$ & ND & ND & ND & ND & ND & ND & ND \\
\hline$\frac{2402 \mathrm{~W}}{2402}$ & BK & FT6015-2W] $5+$ ABK & PPB & ND & ND & ND & ND & ND & NO & 1 & ND \\
\hline $2+02 \mathrm{WK}$ & 5 & FT6015 2WK 37A & $P p B$ & NO & ND & ND & $\mathrm{ND}$ & ND & NO & ND & NO \\
\hline $2402 \mathrm{WK}$ & 5 & FT6045_2WK336A & PPB & ND & ND & ND & ND & ND & ND & $\overline{N D}$ & NO \\
\hline $2+02 \mathrm{Wk}$ & BK & FT6015_2WK39A BK & PPB & ND & NO & $\mathrm{ND}$ & ND & ND & ND & NO & ND \\
\hline $2402 \mathrm{WL}$ & s & FT $60+5$ WL_55A & PFB & ND & NO & ND & $\overline{\mathrm{ND}}$ & $\mathrm{ND}$ & ND & $\overline{N D}$ & ND \\
\hline $2402 \mathrm{WL}$ & 5 & FT $60.522 \mathrm{WL}, 56 \mathrm{~A}$ & $\mathrm{PPB}$ & ND & NO & 2 & NO & $\mathrm{NO}$ & ND & 40 & ND \\
\hline $2+02 \mathrm{WL}$ & $\vec{B} \bar{K}$ & FT6015-2WL.S7A BK & PVB & ND & ND & ND & ND & ND & ND & ND & ND \\
\hline WWC.WE,WG,W. & R, UW & FT 6015_FL.35ABK & $\mathrm{PPB}$ & $\mathrm{ND}$ & NO & $\mathrm{ND}$ & ND & $\mathrm{ND}$ & $\mathrm{ND}$ & $\mathrm{ND}$ & $\mathrm{NID}$ \\
\hline WWC WEWCW. & FLDW & FT6015 FL..3SB & PPB & ND & ND & 1 & ND & ND & ND & 46 & 3 \\
\hline WWC,WE,WC,W]. & Tk & FT6045 TR.36A & Pi'b & ND & ND & ND & ND & ND & $\mathrm{ND}$ & ND & ND \\
\hline WK & FL, UW & FT $6045, \mathrm{FL} 40 \mathrm{ABK}$ & $P P B$ & ND & NO & NO & ND & ND & $\mathrm{NO}$ & ND & ND \\
\hline B.WD.WF,WH,WW,W & F.UW & TT6045-20WW5A & Pps & NO & ND & $\mathrm{ND}$ & ND & ND & $\mathrm{ND}$ & ND & ND \\
\hline R,WD,WF, WH,WT,W & K, OW & $F T 60+5.20 \mathrm{~W} .60 \mathrm{~A}$ & Pps & ND & MD & ND & NO & ND & ND & ND & ND \\
\hline B.WITF.WHWWW & $\pi R$ & FT6015-TR.59A & Pry & ND & ND & NO & NO & ND & $\mathrm{ND}$ & ND & ND \\
\hline
\end{tabular}

Exdorateons

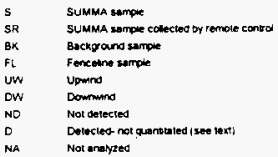

Ners:

Meroos Detection Lumb ere 10 oxe or benter 
HNF-SD-WM-RPT-282 Rev. 0

Table 4-2. GC-MS Data of Air Samples from the Central Waste Complex. (20 sheets)

2402.X2S

312402 BUILPING S Leontinued)

\begin{tabular}{|c|c|c|c|c|c|c|c|c|c|c|c|c|}
\hline \multirow[b]{2}{*}{ BULLDING } & \multirow[b]{2}{*}{ 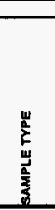 } & \multicolumn{11}{|c|}{ HONAOULA AND SLJSHTLY POLAR COMPOUUNOS } \\
\hline & & 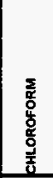 & 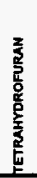 & 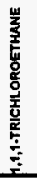 & 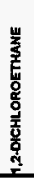 & 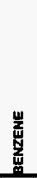 & 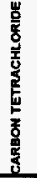 & 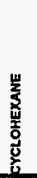 & 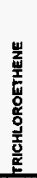 & 愛 & 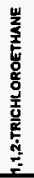 & 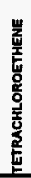 \\
\hline $2402 \mathrm{~W}$ & $\mathrm{~s}$ & ND & ND & ND & NO & No & $\mathrm{ND}$ & $\mathrm{ND}$ & ND & 3 & ND & ND \\
\hline $2402 \mathrm{~W}$ & 5 & ND & ND & ND & ND & ND & ND & NO & ND & 2 & ND & ND \\
\hline $2+02 \mathrm{w}$ & BK & ND & NO & NO & ND & ND & ND & ND & ND & ND & ND & ND \\
\hline $2402 \mathrm{WB}$ & $\mathrm{S}$ & ND & No & 24 & ND & ND & 7 & Nõ & 83 & No & ND & ND \\
\hline $2402 \mathrm{WB}$ & 5 & ND & ND & 34 & 2 & ND & 11 & $\mathrm{ND}$ & 151 & $\mathrm{ND}$ & ND & ND \\
\hline $2102 \mathrm{WB}$ & $\overline{B K}$ & ND & ND & ND & ND & ND & ND & ND & ND & ND & ND & ND \\
\hline $2+02 W C$ & $\mathrm{~s}$ & ND & 63 & 2 & 5 & 4 & ND & ND & 2 & 2 & 2 & ND \\
\hline $2402 W C$ & 5 & ND & ND & $\mathrm{ND}$ & ND & ND & ND & ND & ND & ND & ND & ND \\
\hline $2+02 W c$ & BK & 1 & 42 & ND & $\mathrm{ND}$ & 6 & $\mathrm{ND}$ & No & 3 & 1 & 2 & ND \\
\hline $2+02 \mathrm{WO}$ & 5 & ND & ND & ND & ND & $\mathrm{ND}$ & ND & No & ND & 6 & ND & ND \\
\hline $2402 \mathrm{WD}$ & 5 & NA & NA & NA & NA & NA & NA & NA & NA & NA & NA & NA \\
\hline $2+02 \mathrm{WD}$ & $\mathrm{BK}$ & $\mathrm{ND}$ & ND & $\mathrm{ND}$ & ND & ND & ND & ND & ND & 7 & $\mathbf{N D}$ & ND \\
\hline $2402 \mathrm{WE}$ & $\$$ & ND & $N D$ & 16 & ND & ND & No & ND & ND & 12 & ND & ND \\
\hline $2+02 \mathrm{WE}$ & 5 & NO & NO & 11 & ND & ND & ND & ND & ND & 12 & ND & ND \\
\hline $2+02 \mathrm{WE}$ & $B K$ & ND & ND & $\mathrm{ND}$ & ND & ND & $\mathrm{ND}$ & ND & ND & ND & ND & ND \\
\hline $2402 \mathrm{WF}$ & 5 & $\mathrm{ND}$ & ND & ND & ND & ND & $\mathrm{ND}$ & ND & NO & ND & ND & ND \\
\hline $2402 \mathrm{WF}$ & $\overline{\mathrm{S}}$ & $\mathrm{MD}$ & $\mathrm{ND}$ & $\mathrm{ND}$ & ND & ND & ND & ND & ND & ND & ND & ND \\
\hline $2402 \mathrm{WF}$ & BK & NO & NO & ND & ND & ND & ND & NO & ND & ND & $\mathrm{ND}$ & NO \\
\hline $2402 \mathrm{WC}$ & 5 & $\mathrm{ND}$ & $\mathrm{ND}$ & NO & ND & ND & $\mathrm{ND}$ & ND & ND & $\mathrm{ND}$ & ND & ND \\
\hline 2402 WG & $\mathrm{s}$ & ND & $\mathrm{ND}$ & ND & ND & ND & ND & ND & ND & ND & ND & ND \\
\hline $2402 \mathrm{WG}$ & BK & ND & ND & ND & ND & ND & ND & ND & ND & ND & ND & NO \\
\hline $2402 \mathrm{WH}$ & 5 & ND & ND & ND & ND & ND & $N D$ & ND & ND & ND & ND & ND \\
\hline $2402 \mathrm{WH}$ & 5 & ND & ND & ND & ND & ND & ND & ND & ND & ND & ND & NO \\
\hline $2+02 \mathrm{WH}$ & BK & ND & ND & ND & ND & ND & $\mathrm{ND}$ & ND & ND & ND & ND & ND \\
\hline $2402 W 1$ & 5 & ND & NO & ND & ND & $\mathrm{ND}$ & NO & ND & ND & ND & ND & ND \\
\hline $2402 \mathrm{~W}$ & 5 & ND & ND & ND & MD & ND & ND & ND & $\mathrm{ND}$ & ND & ND & ND \\
\hline $2402 W]$ & BK & ND & No & ND & ND & ND & ND & ND & ND & ND & ND & ND \\
\hline $2402 \mathrm{~W}$ & 5 & ND & 24 & ND & 6 & 3 & ND & ND & ND & 37 & ND & NO \\
\hline $2402 \mathrm{WJ}$ & 5 & ND & ND & ND & ND & ND & $\mathrm{NO}$ & ND & ND & 22 & ND & No \\
\hline 2402 W] & BK & ND & 6 & NO & ND & ND & ND & ND & $\mathrm{ND}$ & ND & ND & ND \\
\hline $2402 \mathrm{WK}$ & 5 & ND & No & $\mathrm{ND}$ & ND & ND & ND & $\mathrm{ND}$ & ND & $\mathrm{ND}$ & ND & $\mathbf{N D}$ \\
\hline $2402 \mathrm{WK}$ & $\mathrm{s}$ & ND & $\mathrm{ND}$ & $\overline{\mathrm{ND}}$ & $\overline{\mathrm{ND}}$ & ND & ND & $\overrightarrow{N D}$ & ND & ND & ND & $\mathrm{ND}$ \\
\hline $2402 W K$ & BK & ND & ND & No & ND & No & ND & ND & ND & ND & ND & $\mathrm{ND}$ \\
\hline $2402 \mathrm{WL}$ & 5 & ND & ND & ND & ND & ND & ND & ND & ND & $\mathrm{i}$ & ND & NO \\
\hline $2402 \mathrm{WL}$ & 5 & NO & 8 & ND & 4 & 7 & ND & ND & 5 & 5 & ND & ND \\
\hline $2102 \mathrm{WL}$ & BK & ND & ND & ND & ND & ND & ND & $\mathrm{ND}$ & ND & ND & ND & ND \\
\hline W,WC,WE,WG,WI, & FL, UW & ND & जाD & ND & ND & ND & ND & $\mathrm{ND}$ & ND & $\mathrm{ND}$ & NO & $\mathrm{ND}$ \\
\hline W,WC,WE,WG,WL, & FL, DW & 1 & 61 & ND & 13 & 10 & $\mathrm{ND}$ & ND & 5 & 5 & 4 & ND \\
\hline W.WC.WE.WC,WI. & IR & ND & ND & ND & ND & $\mathrm{ND}$ & ND & NO & $\mathrm{ND}$ & ND & ND & ND \\
\hline WK & FL.UW & ND & ND & ND & ND & $\mathrm{ND}$ & $\sqrt{D}$ & ND & ND & ND & ND & $\mathrm{ND}$ \\
\hline B.WD,WF,WH,W],W & Fl. UWW & ND & ND & ND & ND & NO & ND & ND & ND & ND & ND & ND \\
\hline K.WD WF,WH,W],W & F.DW & ND & ND & ND & $\mathrm{ND}$ & ND & ND & ND & ND & ND & ND & ND \\
\hline$B, W D, W F, W H, W], W$ & $T h$ & ND & ND & ND & MD & $\mathrm{ND}$ & NO & No & ND & ND & No & ND \\
\hline
\end{tabular}

Ecosmivens

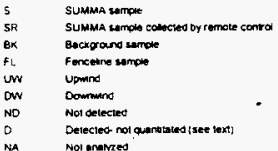


HNF-SD-WM-RPT-282 Rev. 0

Table 4-2. GC-MS Data of Air Samples from the Central Waste Complex. (20 sheets)

$2402 \times 15$

3l2402 BUILDINGS (contunued)

\begin{tabular}{|c|c|c|c|c|c|c|c|c|c|c|c|c|}
\hline \multirow[b]{2}{*}{ BUILDING } & \multirow[b]{2}{*}{ 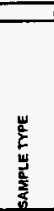 } & \multicolumn{11}{|c|}{ NON-POLAR AND SLIGHILY POLAR COMIFOUNDS } \\
\hline & & 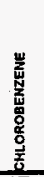 & 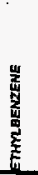 & & $\frac{\sum_{0}^{\frac{1}{2}}}{\frac{\pi}{2}}$ & 蓆 & 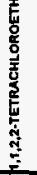 & 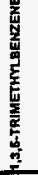 & 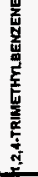 & 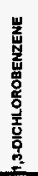 & 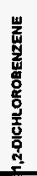 & 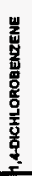 \\
\hline $2402 \mathrm{~W}$ & $\mathbf{s}$ & ND & 1 & ND & $\mathrm{ND}$ & 1 & No & ND & 1 & ND & ND & ND \\
\hline $2+02 \mathrm{~W}$ & $\mathrm{~s}$ & ND & ND & ND & ND & NO & ND & ND & ND & ND & ND & No \\
\hline $2402 W$ & BK & ND & Nī & NO & ND & ND & ND & ND & ND & $\mathrm{ND}$ & ND & ND \\
\hline $2+02 W_{8}$ & $\mathbf{s}$ & $\mathrm{ND}$ & 2 & ND & NO & 1 & ND & $\mathrm{NO}$ & ND & NO & ND & ND \\
\hline $1402 \mathrm{WB}$ & 5 & No & 1 & $\mathbf{3}$ & 1 & 2 & ND & 2 & 5 & ND & ND & ND \\
\hline $2402 \mathrm{WB}$ & BK & ND & ND & ND & ND & NO & ND & 1 & 2 & $\mathrm{ND}$ & ND & ND \\
\hline $2402 W C$ & 5 & 2 & 4 & 4 & 4 & 4 & 2 & 3 & 5 & 3 & 1 & 4 \\
\hline $2402 \mathrm{WC}$ & $s$ & $\mathrm{ND}$ & 2 & ND & ND & 1 & ND & 7 & 1 & NO & NO & ND \\
\hline $2+02 \mathrm{WC}$ & 8K & 3 & 3 & 3 & 4 & 4 & ND & 3 & 6 & 3 & 5 & 5 \\
\hline $2402 \mathrm{WD}$ & S & ND & $\overline{2}$ & 16 & ND & 3 & ND & 3 & 30 & NO & NO & ND \\
\hline 2402 WD & $\mathrm{s}$ & NA & NA & NA & NA & $\overline{N A}$ & NA & NA & NA & NA & NA & NA \\
\hline $2402 W D$ & BK & ND & 3 & 20 & 1 & 4 & ND & 1 & 36 & $\mathrm{ND}$ & ND & ND \\
\hline $2402 \mathrm{WE}$ & $\mathrm{s}$ & $\mathrm{ND}$ & 2 & 6 & $\mathrm{ND}$ & 2 & ND & ND & ND & ND & $\mathrm{ND}$ & ND \\
\hline $2402 \mathrm{WE}$ & $\mathbf{S}$ & ND & 3 & 3 & ND & 3 & ND & $\mathrm{ND}$ & $\mathrm{ND}$ & ND & ND & $\mathrm{ND}$ \\
\hline $2402 \mathrm{WE}$ & $B X$ & ND & ND & $\mathrm{ND}$ & ND & $\mathrm{ND}$ & ND & ND & $\mathrm{ND}$ & NIT & ND & ND \\
\hline $2402 \mathrm{WF}$ & 5 & ND & 2 & 3 & ND & 1 & ND & 2 & 1 & NO & NO & ND \\
\hline $2402 \mathrm{WF}$ & 5 & NO & $i$ & 3 & ND & 1 & ND & 3 & 1 & ND & ND & ND \\
\hline $2+02 \mathrm{WF}$ & $B K$ & ND & ND & ND & ND & $\overline{N D}$ & ND & 1 & 1 & ND & ND & ND \\
\hline $2402 W C$ & $\mathbf{s}$ & ND & 2 & NO & NO & 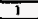 & NO & 2 & ND & ND & ND & ND \\
\hline $2402 \mathrm{WC}$ & 5 & $\overline{\mathrm{ND}}$ & ND & No & ND & ND & NO & $\mathrm{ND}$ & ND & ND & $\mathrm{ND}$ & ND \\
\hline $2402 \mathrm{WG}$ & $8 K$ & NO & $\mathrm{MD}$ & ND & No. & ND & ND & $\mathrm{ND}$ & ND & Mi & ND & ND \\
\hline $2402 \mathrm{WH}$ & $S$ & ND & 2 & $\mathrm{ND}$ & ND & ND & ND & 1 & $\mathrm{ND}$ & ND & ND & ND \\
\hline $2402 \mathrm{WH}$ & S & ND & 1 & ND & $\mathrm{ND}$ & NO & ND & ND & 1 & ND & ND & ND \\
\hline $2402 \mathrm{WH}$ & $\overline{B K}$ & ND & ND & $\mathrm{NO}$ & NO & ND & ND & $\mathrm{ND}$ & NO & NO & ND & No \\
\hline $2402 \mathrm{WI}$ & $\mathrm{s}$ & ND & ND & ND & ND & Nī & ND & 26 & 1 & ND & $\mathrm{ND}$ & $\mathrm{ND}$ \\
\hline $2402 \mathrm{WI}$ & 5 & ND & ND & ND & ND & $\mathrm{ND}$ & ND & ND & ND & NO & ND & ND \\
\hline $2402 \mathrm{WI}$ & BK & ND & ND & $\mathrm{ND}$ & ND & ND & ND & 1 & 3 & NO & No & ND \\
\hline $2402 \mathrm{WJ}$ & 5 & 2 & 5 & 8 & 7 & 6 & 3 & 5 & 14 & 4 & 6 & 6 \\
\hline 2402 WJ & 5 & ND & 1 & 3 & ND & 2 & ND & 2 & 5 & ND & ND & ND \\
\hline 2402 w & BK & ND & 2 & ND & 3 & 2 & ND & 2 & 7 & 2 & 2 & 3 \\
\hline $2+02 \mathrm{WK}$ & 5 & ND & NO & $\mathrm{ND}$ & ND & ND & $\mathrm{ND}$ & 3 & 1 & ND́ & ND & ND \\
\hline 2402 WK & 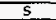 & ND & NO & $\mathrm{ND}$ & NO & No & MD & 2 & ND & ND & NO & NO \\
\hline $2402 \mathrm{Wk}$ & BK & NO & $\mathrm{ND}$ & ND & ND & $\mathrm{ND}$ & No & ND & ND & NO & $\mathrm{ND}$ & ND \\
\hline $2402 \mathrm{WL}$ & 5 & ND & 2 & 3 & 1 & 2 & ND & 1 & 6 & ND & ND & $\mathrm{ND}$ \\
\hline $2+02 \mathrm{WL}$ & 5 & 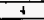 & 6 & 7 & 9 & 7 & 2 & 12 & 18 & 9 & 12. & 15 \\
\hline $2002 \mathrm{WL}$ & BK & ND & $\overline{N D}$ & ND & ND & ND & ND & ND & ND & ND & ND & ND \\
\hline W,WK,WE,WC, WI. & $F L, U W$ & NO & ND & NO & NO & $\mathrm{ND}$ & MD & ND & ND & ND & NO & ND \\
\hline WWCWE.WG.W & F. DW & 5 & 6 & 5 & 9 & $\frac{6}{6}$ & 2 & 7 & 13 & 8 & 10 & 12 \\
\hline W.WC, WE, WC, $\bar{L}$ & TR & ND & ND & ND & ND & No & Nit & ND & No & ND & ND & ND \\
\hline wK & $\bar{F}$ UW & $\mathrm{ND}$ & ND & ND & ND & ND & ND & ND & $\mathrm{ND}$ & ND & ND & ND \\
\hline B.WDWF,WHWWW & $F L, U W$ & ND & ND & ND & ND & ND & ND & ND & $\mathrm{ND}$ & NO & $\mathrm{ND}$ & ND \\
\hline$B, W U, W F, W H, W, W$ & $E L D W$ & ND & ND & $\mathrm{ND}$ & 1 & $\mathrm{ND}$ & ND & 6 & 3 & NO & ND & 2 \\
\hline B.WD,WE,WH.W.W & $T R$ & ND & ND & $\mathrm{NO}$ & ND & NO & ND & $\mathrm{ND}$ & NO & NI & ND & ND \\
\hline
\end{tabular}

Expanetons

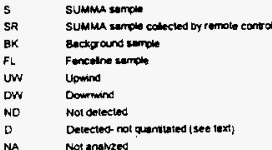

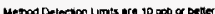


HNF-SD-WM-RPT-282 Rev. 0

Table 4-2. GC-MS Data of Air Samples from the Central Waste Complex. (20 sheets)

2402.XLS

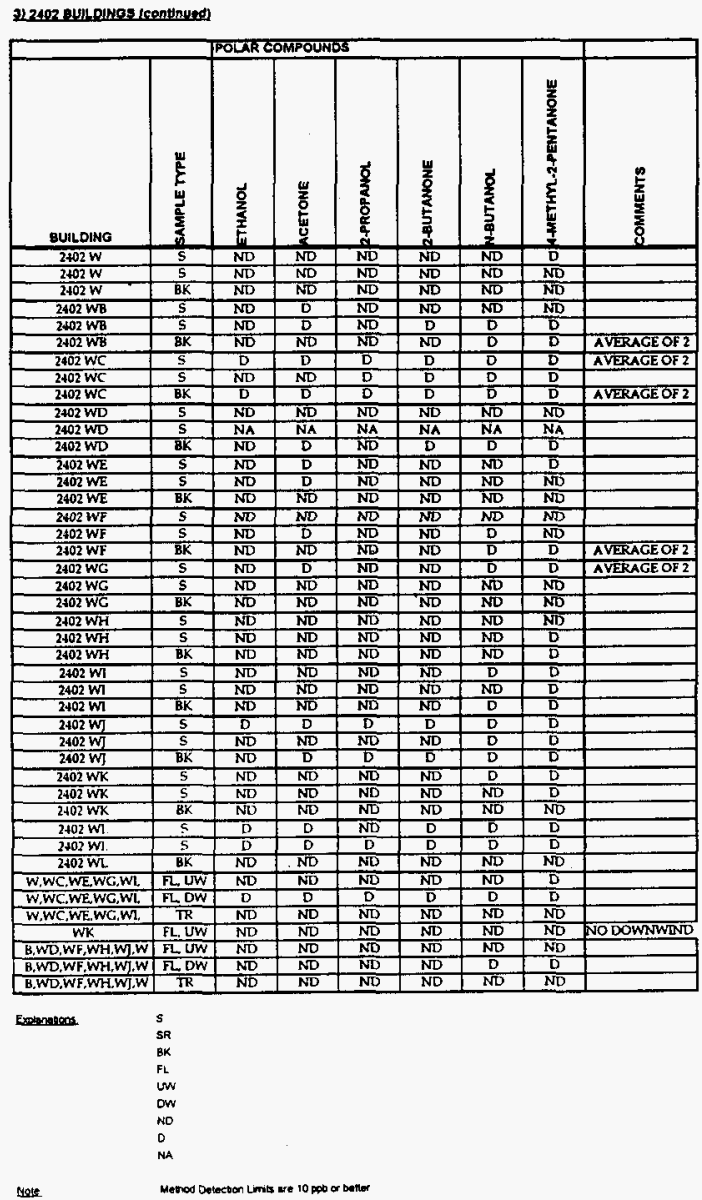

Iruge I 
HNF-SD-WM-RPT-282 Rev. 0

Table 4-2. GC-MS Data of Air Samples from the Central Waste Complex. (20 sheets)

$2403 \times 1.5$

4) 2403 BUILDINGS

\begin{tabular}{|c|c|c|c|c|c|c|c|c|c|c|c|}
\hline \multicolumn{4}{|c|}{ GENERAL INFORMATION } & \multicolumn{8}{|c|}{ NONFOLAR AND SLGHTLY POLAR COMPOUNOS } \\
\hline EUILDING & 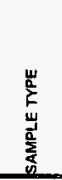 & $\begin{array}{l}\frac{0}{4} \\
\frac{a}{2} \\
\frac{a}{4} \\
n\end{array}$ & 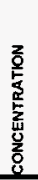 & 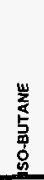 & 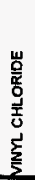 & 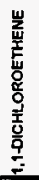 & 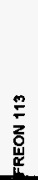 & 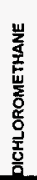 & 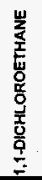 & $\begin{array}{l}\frac{w}{2} \\
\frac{3}{5} \\
\frac{10}{\frac{1}{z}}\end{array}$ & 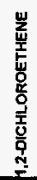 \\
\hline $2403 \mathrm{WA}$ & $S$ & FT6045_3WA.62A & PPB & ND & ND & ND & ND & 57 & ND & 6 & ND \\
\hline $2400 \mathrm{WA}$ & $\mathrm{S}$ & FT 6045 3WA.63A & PPB & ND & ND & ND & $\mathrm{ND}$ & 31 & ND & 17 & ND \\
\hline 2403 WA & 5 & FT6045_3WA.64A & PPB & ND & ND & ND & ND & 38 & ND & 4 & $\mathrm{ND}$ \\
\hline $2403 \mathrm{WA}$ & $\mathbf{S}$ & FT6045_3WA.65A & $\mathrm{PPQ}$ & ND & $\mathrm{ND}$ & ND & ND & 133 & ND & 12 & $\mathrm{ND}$ \\
\hline 2403 WA & $8 K$ & FT6045_3WA,66A & PPB & ND & ND & $\mathrm{ND}$ & ND & $\mathrm{ND}$ & ND & ND & ND \\
\hline 2403 WA & BK & FT6045_3WA.67A & PPB & ND & ND & ND & ND & ND & ND & ND & ND \\
\hline 2400 WA & TR & FT 6045-TR69A & PPB & ND & ND & $\mathrm{ND}$ & ND & ND & ND & ND & ND \\
\hline $2403 \mathrm{WA}$ & DW & FT6045-DW.72A & PPB & ND & ND & ND & ND & ND & $\mathrm{ND}$ & ND & ND \\
\hline 2403 WA & UW & FT6045-UW.73A & $\overline{\mathrm{PPB}}$ & $\mathrm{ND}$ & ND & 3 & ND & ND & 1 & 61 & $\frac{1}{2}$ \\
\hline 2403 WB & $\bar{s}$ & FT 6045_3WB 94A & PPB & ND & ND & $\mathrm{ND}$ & ND & ND & ND & ND & ND \\
\hline $2403 \mathrm{WB}$ & $\mathrm{S}$ & FT 6045_3WB.95A & PPB & ND & $\mathrm{ND}$ & ND & ND & $\mathrm{ND}$ & ND & ND & $\mathrm{ND}$ \\
\hline $2403 \mathrm{WB}$ & S & FT $6045 \_3 \mathrm{WB} .96 \mathrm{~A}$ & PPB & ND & ND & ND & ND & ND & ND & 3 & ND \\
\hline $2403 \mathrm{WB}$ & $\mathbf{S}$ & FT $60453 \mathrm{WB} .97 \mathrm{~A}$ & $\mathrm{PPB}$ & ND & ND & ND & ND & ND & ND & $\mathrm{ND}$ & ND \\
\hline 24003 WB & BK & FT6045_3WB.98A & PPB & ND & ND & ND & ND & ND & ND & ND & ND \\
\hline $2403 \mathrm{WB}$ & $8 K$ & FT 6045 3WB.99A BK & PPB & ND & ND & ND & ND & ND & $\mathrm{ND}$ & ND & ND \\
\hline $2403 \mathrm{WB}$ & TR & FT6045-TR $100 \mathrm{~A}$ & $\mathrm{PPB}$ & ND & ND & ND & ND & ND & ND & $\mathrm{ND}$ & ND \\
\hline $2403 \mathrm{WC}$ & $\mathbf{S}$ & FT6045,3WC.84A & $\mathrm{PPB}$ & ND & $\mathrm{ND}$ & $\mathrm{ND}$ & $\mathrm{ND}$ & 57 & ND & ND & ND \\
\hline $2403 W C$ & $\overline{\mathbf{s}}$ & FT $6045-3 W C .85 \mathrm{~A}$ & PPB & ND & ND & ND & $\mathrm{ND}$ & 63 & ND & ND & $\mathrm{ND}$ \\
\hline $2403 W C$ & $S$ & FT $6045-3 W C 86 A$ & PPB & ND & ND & ND & ND & 104 & ND & ND & ND \\
\hline $2403 W C$ & $s$ & FT6045-3WC.87A & PPB & ND & ND & ND & ND & 50 & ND & ND & ND \\
\hline $2403 W C$ & BK & FT 6015_3WC.B8A BK & PPB & ND & $\mathrm{ND}$ & ND & ND & $\mathrm{ND}$ & ND & ND & ND \\
\hline $2403 \mathrm{WC}$ & BK & FT6045_3WC.89A & PPB & ND & ND & ND & ND & $\mathrm{ND}$ & ND & ND & ND \\
\hline $2403 \mathrm{WD}$ & $\mathrm{s}$ & FT $6045-3 W D .75 A$ & $\mathrm{FPB}$ & ND & ND & $N D$ & ND & ND & ND & ND & ND \\
\hline $2403 W D$ & $\mathbf{S}$ & FT6045 3WD.76A & PPB & ND & ND & ND & ND & 3 & ND & 16 & ND \\
\hline $2+03 W D$ & $\overline{\mathbf{s}}$ & FT6045 3WD.77 & एPB & ND & ND & ND & ND & ND & ND & ND & ND \\
\hline $2+03 \mathrm{WD}$ & S & FT 6045 3WD.78A & PPB & ND & ND & $\mathrm{ND}$ & ND & ND & ND & ND & ND \\
\hline $2403 \mathrm{WD}$ & 5 & FT64 3W 3 WD. $79 \mathrm{~A}$ & PPB & ND & ND & ND & ND & NO & ND & ND & ND \\
\hline $2+03 \mathrm{WD}$ & $\mathrm{S}$ & FT6045 3WD.80A & $\mathrm{PPB}$ & ND & ND & ND & ND & ND & ND & $\mathrm{ND}$ & ND \\
\hline 2403 WD & BK & FT 6045 3WD. $8 \mathrm{lA} \mathrm{bk}$ & PPB & ND & ND & ND & ND & ND & ND & ND & ND \\
\hline $2403 \mathrm{WD}$ & $B K$ & FT6015_3WD.82A BK & PPB & ND & ND & ND & ND & ND & ND & ND & ND \\
\hline $2403 \mathrm{WD}$ & BK & FT 6045_3WD.83A & PPB & $\mathrm{ND}$ & ND & ND & ND & ND & ND & ND & ND \\
\hline WC,WD & FL.UW & FT6045-UW.90A & PPB & ND & ND & ND & ND & ND & ND & $\mathrm{ND}$ & ND \\
\hline WC. WD & $F L, D W$ & FT 6045-DW.91A & PPB & ND & $\mathrm{ND}$ & ND & ND & ND & ND & ND & ND \\
\hline WC, WD & TR & FT 6045 TR.91A & PPB & ND & $\mathrm{ND}$ & ND & ND & ND & ND & ND & ND \\
\hline
\end{tabular}

$\begin{array}{ll}\text { Explanations: } & \\ \text { S } & \text { SUMMA sample } \\ \text { SR } & \text { SUMMA sample coltecled ty remole control } \\ \text { BK } & \text { Background sample } \\ \text { FL } & \text { Fenceline sample } \\ \text { UW } & \text { Upwind } \\ \text { DW } & \text { Downwind } \\ \text { NO } & \text { Not delected } \\ \text { D } & \text { Detecled- not quantilated (see lexd) } \\ \text { NA } & \text { Not analyzed } \\ & \\ \text { Note } & \text { Melhod Delection Limils are 10 ppb or better }\end{array}$

Page 1 
HNF-SD-WM-RPT-282 Rev. 0

Table 4-2. GC-MS Data of Air Samples from the Central Waste Complex. (20 sheets) $2400 \times 15$

112403 BUILOINGS (continued)

\begin{tabular}{|c|c|c|c|c|c|c|c|c|c|c|c|c|}
\hline \multirow[b]{2}{*}{ BUILDING } & \multirow[b]{2}{*}{ 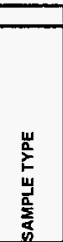 } & \multicolumn{11}{|c|}{ TNONPOLAR AND SLIGHTLY POLAR COMPOUNDS } \\
\hline & & 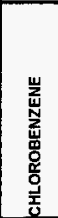 & 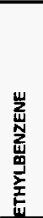 & 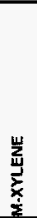 & 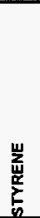 & 耑 & 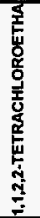 & 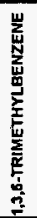 & 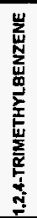 & 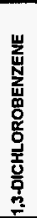 & 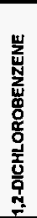 & 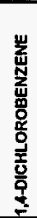 \\
\hline $2403 \mathrm{WA}$ & S & ND & 2 & 5 & 2 & 2 & 1 & 2 & 8 & 2 & 3 & 4 \\
\hline 2403 WA & $\mathbf{S}$ & 2 & 3 & 5 & 4 & 5 & 3 & $\frac{7}{8}$ & 10 & 9 & 11 & 12 \\
\hline 2403 WA & 5 & $\mathrm{ND}$ & 2 & 4 & 1 & 2 & ND & 1 & 5 & ND & ND & 2 \\
\hline 2403 WA & 5 & 2 & 6 & 14 & 3 & 5 & ND & 2 & 6 & 3 & 4 & 3 \\
\hline 2403 WA & Bर & ND & $\mathrm{ND}$ & ND & ND & $\mathrm{ND}$ & ND & $\frac{2}{4}$ & 3 & ND & ND & ND \\
\hline 2403 WA & BK & $\mathrm{ND}$ & 1 & ND & 2 & ND & ND & 5 & 2 & $\overline{2}$ & 2 & 2 \\
\hline 2403 WA & TR & ND & ND & ND & ND & ND & ND & ND & ND & ND & ND & ND \\
\hline 2403 WA & $\overline{\mathrm{DW}}$ & ND & ND & $\mathrm{ND}$ & $\mathrm{ND}$ & $\mathrm{ND}$ & ND & ND & ND & ND & ND & ND \\
\hline 2403 WA & UW & 9 & 10 & 8 & 14 & 9 & $\mathrm{ND}$ & 12 & 16 & 14 & 18 & 20 \\
\hline $2403 \mathrm{WB}$ & 5 & ND & 3 & 11 & ND & 5 & ND & 2 & 3 & ND & ND & ND \\
\hline $2403 \mathrm{WB}$ & 5 & ND & 2 & 12 & $\mathrm{ND}$ & 5 & ND & 3 & 2 & ND & ND & ND \\
\hline $2403 \mathrm{WB}$ & 5 & ND & 3 & 11 & $\mathrm{ND}$ & 5 & NO & 1 & 2 & ND & ND & $\mathrm{ND}$ \\
\hline $2403 \mathrm{WB}$ & 5 & ND & 3 & 11 & ND & 5 & ND & 1 & 3 & ND & ND & ND \\
\hline $2403 \mathrm{WB}$ & BK & $\mathrm{ND}$ & ND & ND & ND & 2 & ND & 4 & 7 & $\mathrm{ND}$ & $\mathrm{ND}$ & 2 \\
\hline $2403 \mathrm{WB}$ & BK & $\mathrm{ND}$ & 1 & ND & ND & 2 & ND & 3 & 6 & ND & ND & 2 \\
\hline $2403 \mathrm{WB}$ & TR & ND & ND & $\mathrm{ND}$ & ND & ND & ND & ND & ND & ND & $\overline{N D}$ & ND \\
\hline $2403 \mathrm{WC}$ & 5 & $\mathrm{ND}$ & 4 & 8 & 2 & 4 & ND & 2 & 6 & 2 & 2 & 3 \\
\hline $2403 \mathrm{WC}$ & 5 & ND & 2 & 7 & ND & 2 & ND & ND & 3 & ND & ND & ND \\
\hline $2403 \mathrm{WC}$ & 5 & ND & 2 & 7 & ND & 3 & $\mathrm{ND}$ & ND & 3 & ND & ND & ND \\
\hline $2403 \mathrm{WC}$ & 5 & $\mathrm{ND}$ & $\frac{2}{1}$ & 5 & ND & 2 & ND & ND & 2 & ND & $\mathrm{ND}$ & ND \\
\hline $2403 \mathrm{WC}$ & BK & ND & 1 & ND & 2 & 3 & ND & 7 & 18 & ND & ND & ND \\
\hline $2403 \mathrm{WC}$ & BK & ND & 5 & 6 & $\frac{2}{6}$ & 10 & 4 & 23 & 31 & 6 & 6 & 13 \\
\hline $2403 \mathrm{WD}$ & 5 & ND & 2 & 3 & ND & ND & ND & ND & 1 & ND & ND & ND \\
\hline $2403 \mathrm{WD}$ & $S$ & 4 & 7 & 8 & 9 & 5 & ND & 5 & 9 & 7 & 10 & 9 \\
\hline $2403 \mathrm{WD}$ & $\mathrm{s}$ & ND & 2 & 4 & ND & 1 & $\mathrm{ND}$ & 1 & I & ND & ND & ND \\
\hline $2403 \mathrm{WD}$ & 5 & ND & 1 & 3 & ND & 1 & ND & $\mathrm{ND}$ & 2 & ND & ND & ND \\
\hline $2403 \mathrm{WD}$ & 5 & ND & $\frac{1}{1}$ & 4 & $\mathrm{ND}$ & $\overline{2}$ & ND & 2 & 4 & ND & ND & ND \\
\hline $2403 \mathrm{WD}$ & $\frac{2}{5}$ & $\mathrm{ND}$ & $\frac{2}{2}$ & $\frac{7}{3}$ & 2 & $\frac{2}{2}$ & ND & $\frac{2}{1}$ & 3 & ND & ND & 2 \\
\hline $2403 \mathrm{WD}$ & $\overline{B K}$ & ND & ND & ND & ND & 1 & ND & 3 & 11 & ND & ND & 3 \\
\hline $2403 \mathrm{WD}$ & BK & ND & ND & ND & ND & ND & ND & $\mathrm{ND}$ & ND & ND & $\mathrm{ND}$ & ND \\
\hline $2403 \mathrm{WD}$ & BK & ND & ND & ND & ND & $\mathrm{ND}$ & ND & 1 & 2 & ND & ND & ND \\
\hline WC, WD & FL, UW & $\mathrm{ND}$ & ND & $\mathrm{ND}$ & $\mathrm{ND}$ & $\mathrm{ND}$ & ND & $\mathrm{ND}$ & 2 & ND & $\mathrm{ND}$ & ND \\
\hline WC, WD & FL, DW & ND & ND & $\mathrm{ND}$ & ND & $\mathrm{ND}$ & $\mathrm{ND}$ & $\mathrm{ND}$ & 2 & ND & $\mathrm{ND}$ & ND \\
\hline WC, WD & $\overline{\mathrm{TR}}$ & ND & ND & ND & ND & ND & ND & ND & 0 & ND & ND & ND \\
\hline
\end{tabular}

\begin{tabular}{ll} 
Explanations: & \\
\hline S & SUMMA sample \\
SR & SUMMR sample collected by remole conirol \\
GK & Eackground sample \\
FL & Fenceline sample \\
UW & Upwind \\
OW & Domnwind \\
ND & Nol delected \\
D & Delecleo. nol quantitaled (see texi) \\
NA & Not analyzed \\
& Mothod Delection Limits are $10 \mathrm{ppb}$ or betler \\
Note. &
\end{tabular}

Page 1 
HNF-SD-WM-RPT-282 Rev. 0

Table 4-2. GC-MS Data of Air Samples from the Central Waste Complex. (20 sheets)

$2403 \times 15$

1)2403 RUILDINGS (eontinued)

\begin{tabular}{|c|c|c|c|c|c|c|c|c|c|c|c|c|}
\hline BUILDING & $\begin{array}{l}\frac{4}{2} \\
\frac{2}{2} \\
\frac{2}{2} \\
\frac{0}{5} \\
\frac{1}{5}\end{array}$ & \multicolumn{11}{|c|}{ NONPOLAR AND SLIGHTLY POLAR COMPOUNDS } \\
\hline $2403 \mathrm{WA}$ & 5 & ND & ND & 70 & 6 & ND & ND & ND & ND & 7 & ND & ND \\
\hline $2403 \mathrm{WA}$ & 5 & ND & 23 & 14 & 4 & ND & ND & ND & 5 & 9 & ND & ND \\
\hline $2400 \mathrm{WA}$ & $\frac{2}{5}$ & ND & ND & 16 & ND & ND & ND & ND & ND & 8 & ND & ND \\
\hline 2400 WA & 5 & ND & ND & 35 & 3 & ND & ND & $\mathrm{ND}$ & $\mathrm{ND}$ & 21 & ND & 4 \\
\hline 2403 WA & BK & $\mathrm{ND}$ & ND & ND & ND & $\mathrm{ND}$ & ND & ND & $\mathrm{ND}$ & ND & ND & ND \\
\hline 2403 WA & BK & ND & ND & ND & ND & ND & ND & ND & ND & ND & ND & ND \\
\hline 2403 WA & TR & ND & ND & ND & ND & ND & $\mathrm{ND}$ & ND & ND & ND & ND & ND \\
\hline $2400 \mathrm{WA}$ & DW & ND & ND & ND & ND & ND & ND & ND & ND & ND & ND & $\mathrm{ND}$ \\
\hline $2403 \mathrm{WA}$ & UW & 1 & 90 & ND & 9 & 13 & ND & $\mathrm{NO}$ & $\frac{1}{6}$ & 8 & 4 & ND \\
\hline $2403 \mathrm{WB}$ & $S$ & $\mathrm{ND}$ & ND & ND & ND & ND & 8 & ND & ND & 1 & ND & ND \\
\hline $2403 \mathrm{WB}$ & $S$ & ND & ND & ND & ND & ND & 20 & 3 & $\mathrm{ND}$ & 2 & ND & ND \\
\hline $2403 \mathrm{WB}$ & 5 & ND & $\mathrm{ND}$ & ND & ND & 4 & 10 & 4 & ND & 2 & ND & ND \\
\hline $2403 \mathrm{WB}$ & S & ND & ND & $\mathrm{ND}$ & ND & ND & 10 & ND & ND & 2 & ND & ND \\
\hline $2403 \mathrm{WB}$ & BK & ND & ND & $\mathrm{ND}$ & ND & $\mathrm{ND}$ & ND & ND & ND & ND & ND & ND \\
\hline $2403 \mathrm{WB}$ & $\overline{B K}$ & ND & ND & ND & $\overline{\mathrm{ND}}$ & ND & ND & ND & ND & ND & ND & ND \\
\hline $2403 \mathrm{WB}$ & $\mathrm{TR}$ & ND & ND & ND & ND & ND & ND & ND & $\mathrm{ND}$ & ND & ND & $\mathrm{ND}$ \\
\hline $2403 \mathrm{WC}$ & $\mathrm{S}$ & ND & ND & 16 & ND & ND & ND & ND & ND & 6 & ND & 27 \\
\hline $2403 \mathrm{WC}$ & 5 & ND & ND & $\frac{3}{12}$ & $\mathrm{ND}$ & ND & ND & ND & $\mathrm{ND}$ & 4 & ND & 21 \\
\hline 2403 Wo & 5 & ND & ND & 15 & ND & $\mathrm{ND}$ & 5 & ND & $\mathrm{ND}$ & 3 & ND & 21 \\
\hline $2403 W C$ & $\$$ & ND & ND & 16 & ND & ND & 0 & ND & ND. & 3 & ND & 22 \\
\hline $2403 \mathrm{WC}$ & BK & ND & ND & ND & ND & ND & ND & ND & $\mathrm{ND}$ & ND & ND & ND \\
\hline $2403 W C$ & $\overline{\mathrm{BK}}$ & ND & ND & ND & ND & ND & ND & ND & ND & 1 & ND & ND \\
\hline $2403 \mathrm{WD}$ & 5 & $\mathrm{ND}$ & $\mathrm{ND}$ & $\mathrm{ND}$ & ND & $\mathrm{ND}$ & ND & $\mathrm{ND}$ & ND & 2 & ND & ND \\
\hline $2403 \mathrm{WD}$ & 5 & ND & 34 & ND & B & 6 & ND & ND & 2 & 7 & 5 & 0 \\
\hline $2403 \mathrm{WD}$ & $\overline{\mathrm{S}}$ & $\mathrm{ND}$ & ND & ND & ND & $\mathrm{ND}$ & ND & $\mathrm{ND}$ & $\mathrm{ND}$ & 3 & $\mathrm{ND}$ & $\mathrm{ND}$ \\
\hline $2403 \mathrm{wD}$ & $\mathrm{S}$ & ND & $\mathrm{ND}$ & ND & $\mathrm{ND}$ & ND & ND & ND & ND & 2 & $\mathrm{ND}$ & ND \\
\hline $2403 \mathrm{WD}$ & $\frac{5}{5}$ & ND & ND & ND & ND & ND & ND & $\mathrm{ND}$ & ND & 3 & ND & ND \\
\hline $2403 \mathrm{WD}$ & $\mathrm{S}$ & ND & ND & ND & ND & NO & ND & $\mathrm{ND}$ & $\mathrm{ND}$ & 3 & ND & ND \\
\hline 2403 WD & BK & ND & ND & ND & $\mathrm{ND}$ & ND & ND & ND & ND & ND & ND & ND \\
\hline 2403 WD & BK & ND & $\mathrm{ND}$ & ND & $\mathrm{ND}$ & ND & ND & $\mathrm{ND}$ & ND & ND & ND & ND \\
\hline $2403 \mathrm{WD}$ & BK & ND & ND & ND & ND & ND & $\mathrm{ND}$ & $\mathrm{ND}$ & ND & ND & ND & ND \\
\hline WC, WD & FL, UW & ND & ND & $\mathrm{ND}$ & ND & ND & ND & ND & ND & ND & ND & $\mathrm{ND}$ \\
\hline WC, WD & FL, DW & ND & $\mathrm{ND}$ & $\mathrm{ND}$ & ND & ND & ND & $\mathrm{ND}$ & ND & ND & ND & $\mathrm{ND}$ \\
\hline WC, WD & TR & ND & ND & ND & ND & ND & $\mathrm{ND}$ & $\mathrm{ND}$ & ND & ND & ND & $\mathrm{ND}$ \\
\hline \multicolumn{13}{|l|}{ Explanalions: } \\
\hline$s$ & \multicolumn{12}{|c|}{ SUMMA sample } \\
\hline SR & \multicolumn{12}{|c|}{ SUMMA sample collecled by remole control } \\
\hline BK & \multicolumn{12}{|c|}{ Background sample } \\
\hline $\mathrm{FL}$ & \multicolumn{12}{|c|}{ Fenceline sample } \\
\hline UW & \multicolumn{12}{|l|}{ Upwind } \\
\hline DW & \multicolumn{12}{|l|}{ Downwind } \\
\hline ND & \multicolumn{12}{|c|}{ Not detecled } \\
\hline 0 & \multicolumn{12}{|c|}{ Detected- not quantitated (see tent) } \\
\hline NA & \multicolumn{12}{|c|}{ Nol analyzed } \\
\hline Nole & \multicolumn{12}{|c|}{ Method Detection Limits are 10 ppo or betier } \\
\hline
\end{tabular}

Page 1 
$\mathrm{I}+\mathrm{B}_{\mathrm{B}} \mathrm{I}$

\begin{tabular}{|c|c|}
\hline 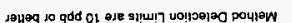 & ॠणN \\
\hline pezNeveion & $\mathbb{N}$ \\
\hline 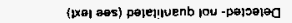 & 0 \\
\hline papojep ION & ON \\
\hline ритимиос & mo \\
\hline putwonn & $\mathrm{Mn}$ \\
\hline apdues euţasuey & 1 \\
\hline 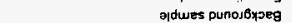 & $x \in$ \\
\hline 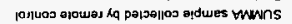 & ys \\
\hline ajdues bwwns & s \\
\hline
\end{tabular}

\begin{tabular}{|c|c|c|c|c|c|c|c|c|}
\hline & aN & aN & $\mathrm{aN}$ & aN & $a \mathrm{~N}$ & aN & 41 & $\triangle M ' O M$ \\
\hline & a & aN & $\mathrm{aN}$ & $a \mathrm{~N}$ & aN & $\mathrm{aN}$ & $M d^{7} 7 \mathrm{I}$ & $a M^{\prime} S M$ \\
\hline & a & aN & aN & aN & aN & GN & Mn'7I & $G M^{\prime} D M$ \\
\hline & a & ON & $\mathrm{aN}$ & $\mathrm{aN}$ & $\mathrm{aN}$ & $\mathrm{GN}$ & X98 & aM EO+2 \\
\hline & 6 & aN & aN. & $\mathrm{aN}$ & aN & IN & Xg & a M EORZ \\
\hline & $\mathrm{a}$ & aN & aN & aN & aN & $\mathrm{aN}$ & त्रa & OM $50 \vDash 2$ \\
\hline & $a$ & $\mathrm{a}$ & $\mathrm{aN}$ & $\mathrm{aN}$ & ON & $\mathrm{ON}$ & $s$ & aM cotz \\
\hline & $a$ & aN & aN & $a N$ & ON & ON & $s$ & OMEOF? \\
\hline & $a$ & aN & $\mathrm{aN}$ & $\mathrm{aN}$ & a & $\mathrm{aN}$ & $\mathrm{s}$ & aM \\
\hline \multirow[t]{14}{*}{ Z.JO GDYYAลY } & $a$ & a & $a N$ & $\mathrm{aN}$ & $\mathrm{aN}$ & $\mathrm{aN}$ & $S$ & GM rot? \\
\hline & $a$ & a & $\mathrm{d}$ & $d$ & $a$ & a & $s$ & $a M \cos 2$ \\
\hline & IN & $a N$ & $a N$ & $\mathrm{ON}$ & $\mathrm{ON}$ & aN & $\mathrm{s}$ & OM EOHZ \\
\hline & $\mathrm{a}$ & a & $\mathrm{ON}$ & an & 0 & GN & प्रभ & $2 M$ EOHZ \\
\hline & a & c & $\mathrm{dN}$ & ON & $\bar{a}$ & aN & प्र98 & उMEDR \\
\hline & a & aN & IN & $\mathrm{a}$ & a & ON & $\mathrm{s}$ & JM $20+2$ \\
\hline & aN & $a N$ & $\mathrm{CN}$ & $\mathrm{a}$ & aN & dN & 5 & כMEOR2 \\
\hline & aN & aN & ON & a & a & $\mathrm{d} \bar{N}$ & s & JMEOEL \\
\hline & $a$ & 0 & $\mathrm{~d}$ & a & a & $a$ & $\mathbf{s}$ & DM EOFZ \\
\hline & IN & $a N$ & ON & $\mathrm{ON}$ & $\mathrm{dN}$ & QN & $y, t$ & gM cotz \\
\hline & $\mathrm{dN}$ & $a N$ & $\mathrm{dN}$ & ON & ON & $a \mathrm{~N}$ & 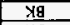 & gM COH2 \\
\hline & $\mathrm{GN}$ & aN & $\mathrm{dN}$ & aN & $\mathrm{GN}$ & aN & X्रg & gM COF \\
\hline & IN & aN & $\mathrm{dN}$ & $\mathrm{aN}$ & $L$ & ON & $\mathrm{s}$ & gM E0t? \\
\hline & $\mathrm{ON}$ & 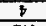 & aN & $\triangle \mathrm{N}$ & $p$ & aN & $\mathrm{s}$ & gM cont \\
\hline \multirow[t]{10}{*}{ ZJO JDV } & aN & GN & GN & an & $i$ & 5 & 5 & gM EOFZ \\
\hline & aN & GN & $\mathrm{ON}$ & aN & $s$ & IN & $\mathrm{s}$ & gM EOF \\
\hline & a & $\mathrm{a}$ & $\mathrm{a}$ & a & a & a & $\mathrm{Mn}$ & $\forall M$ EOEZ \\
\hline & aN & $\mathrm{aN}$ & aN & ON & $\mathrm{dN}$ & aN & $\mathrm{MO}$ & $\forall M E O B Z$ \\
\hline & $\mathrm{aN}$ & $\mathrm{aN}$ & ON & aN & ON & ON & प.1. & YMSOFZ \\
\hline & a & a & $\mathrm{aN}$ & $a N$ & $\mathrm{ON}$ & $\mathrm{dN}$ & 818 & YMEDEZ \\
\hline & $\mathrm{aN}$ & $\mathrm{CN}$ & aN & aN & aN & $\mathrm{aN}$ & प्रg & YM EDEZ \\
\hline & 0 & $\mathrm{a}$ & $a$ & d & a & a & $\mathrm{s}$ & $\forall M$ EDEZ \\
\hline & aN & aN & aN & aN & a & aN & $\mathrm{S}$ & $\forall M E 0 R 2$ \\
\hline & $\mathrm{a}$ & a & a & $a$ & $a$ & a & $\mathrm{s}$ & $\forall M \cos 2$ \\
\hline 2 J0 3DVRAАY & $\mathbf{Q}$ & a & a & an & $a$ & aN & $\mathrm{s}$ & $\forall M$ EOLZ \\
\hline 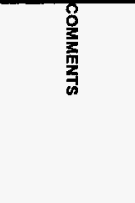 & 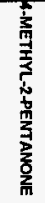 & 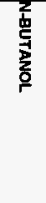 & 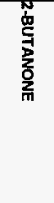 & 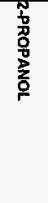 & $\begin{array}{l}\text { 学 } \\
\text { 晋 } \\
\text { 紊 }\end{array}$ & $\begin{array}{l}\text { 览 } \\
\text { 章 } \\
\text { o }\end{array}$ & 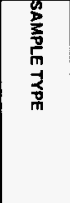 & ontding \\
\hline & & & & & inodw & 8470 & & \\
\hline
\end{tabular}

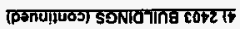

5าx $\times 902$

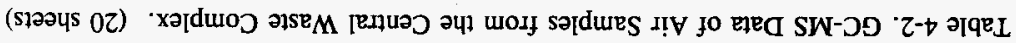


HNF-SD-WM-RPT-282 Rev. 0

Table 4-2. GC-MS Data of Air Samples from the Central Waste Complex. (20 sheets)

2401.xis

6.2401 BUILOANG

\begin{tabular}{|c|c|c|c|c|c|c|c|c|c|c|c|}
\hline \multicolumn{4}{|c|}{ GENERAL INFORMATION } & \multicolumn{8}{|c|}{ NON-POLAR AND SLIGHTLY POLAR COMPOUNDS } \\
\hline BUILDING & 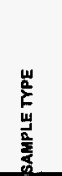 & 号 & 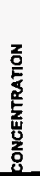 & 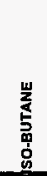 & 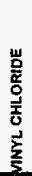 & 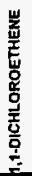 & $\frac{2}{\underline{z}}$ & 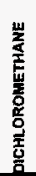 & 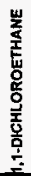 & 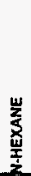 & 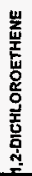 \\
\hline $2401 \mathrm{~W}$ & 5 & FT6045-1W.6BA & $\mathrm{PPB}$ & NO & ND & ND & NO & ND & $\mathrm{ND}$ & ND & ND \\
\hline $2401 \mathrm{~W}$ & $\frac{5}{5}$ & FTB045-1W.7OA & $P P B$ & ND & NO & ND & ND & ND & ND & 8 & ND \\
\hline $2401 \mathrm{w}$ & BK & FT6045-1W.71A & $\mathrm{PPB}$ & ND & ND & ND & ND & ND & ND & NO & ND \\
\hline $2401 \mathrm{~W}$ & $F L, D W$ & FT6045-DW.72A & PPB & NO & $\mathrm{ND}$ & $\mathrm{ND}$ & $\mathrm{ND}$ & $\mathrm{NO}$ & $\mathrm{ND}$ & ND & ND \\
\hline$\frac{6101 \mathrm{~W}}{2401}$ & $F L, U W$ & FT6045-UW:73A & $P P B$ & ND & ND & ND & NO & ND & $\frac{\pi}{1}$ & $\frac{\pi 1}{61}$ & $\frac{12}{2}$ \\
\hline $2401 \mathrm{~W}$ & $T R$ & FT 6045-TR.69A & PPB & ND & ND & ND & ND & ND & ND & ND & ND \\
\hline
\end{tabular}

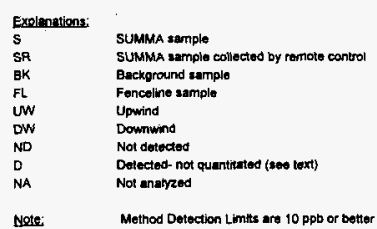


HNF-SD-WM-RPT-282 Rev. 0

Table 4-2. GC-MS Data of Air Samples from the Central Waste Complex. (20 sheets) $2401 . x 15$

5) 2401 auiLDiNe.(continuad)

\begin{tabular}{|c|c|c|c|c|c|c|c|c|c|c|c|c|}
\hline \multirow[b]{2}{*}{ BUILDING } & \multirow[b]{2}{*}{ 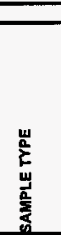 } & \multicolumn{11}{|c|}{ NON-POLAR AND SUGHTLY POLAR COMPOUNDS } \\
\hline & & 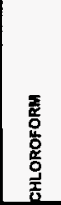 & 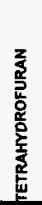 & 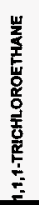 & 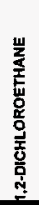 & 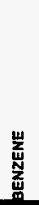 & 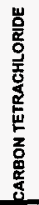 & 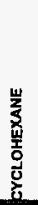 & 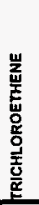 & 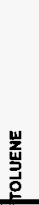 & 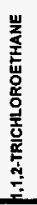 & 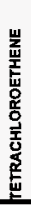 \\
\hline $2401 \mathrm{~W}$ & 's & ND & ND & ND & $\mathrm{ND}$ & $\mathrm{ND}$ & ND & ND & ND & 2 & $\mathrm{ND}$ & ND \\
\hline $2401 \mathrm{~W}$ & 5 & ND & 35 & NO & 1 & $\mathrm{ND}$ & ND & ND & ND & 1 & ND & ND \\
\hline $2401 \mathrm{~W}$ & $B K$ & ND & ND & ND & ND & $\mathrm{ND}$ & NO & $N D$ & ND & ND & ND & ND \\
\hline $2401 \mathrm{~W}$ & $\mathrm{FL}, \mathrm{DW}$ & ND & ND & NO & ND & ND & ND & ND & ND & ND & ND & ND \\
\hline $2401 \mathrm{~W}$ & $F L, U W$ & 1 & 80 & NO & $\frac{1}{9}$ & 13 & NO & $\mathrm{ND}$ & 6 & B & 4 & ND \\
\hline $2401 \mathrm{~W}$ & TR & ND & ND & ND & ND & $\mathrm{ND}$ & NO & ND & ND & ND & ND & ND \\
\hline
\end{tabular}

\begin{tabular}{|c|c|}
\hline \\
\hline \multicolumn{2}{|c|}{$\begin{array}{l}\text { Explanationa: } \\
S \text { SUMMA sarnphe }\end{array}$} \\
\hline $\mathbf{S R}$ & SUMMA sample colloctod by remote control \\
\hline BK & Background sumple \\
\hline FL & Fencoline sample \\
\hline uw & Upwing \\
\hline DW & Downmind \\
\hline ND & Not detectiod \\
\hline D & Detected- not quimititated (soe text) \\
\hline NA & Nat analyzed \\
\hline Note: & Method Detection Limits aro 10 of \\
\hline
\end{tabular}


Table 4-2. GC-MS Data of Air Samples from the Central Waste Complex. (20 sheets) $2401 \times 1 s$

5)2401 BUiLDiwe (continued)

\begin{tabular}{|c|c|c|c|c|c|c|c|c|c|c|c|c|}
\hline \multirow[b]{2}{*}{ BUILDING } & \multirow[b]{2}{*}{ 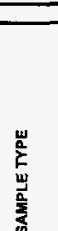 } & \multicolumn{11}{|c|}{ NON-POLAR AND SLIGHTLY POLAR COMPOUNDS } \\
\hline & & 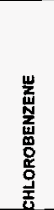 & 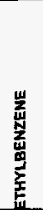 & 岩 & 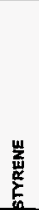 & 㟶 & 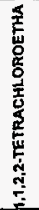 & 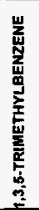 & 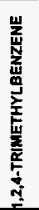 & 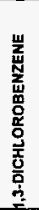 & 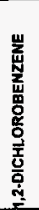 & 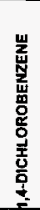 \\
\hline $2401 \mathrm{~W}$ & s & ND & 2 & NO & 2 & $\frac{1}{2}$ & ND & 18 & 2 & 2 & 2 & 2 \\
\hline $2401 \mathrm{~W}$ & s & 9 & 3 & 3 & 8 & 3 & 5 & 8 & 16 & 6 & $\frac{2}{10}$ & 10 \\
\hline$\frac{4001 \mathrm{~W}}{2401 \mathrm{~W}}$ & 5 & $\frac{1}{N D}$ & ND & ND & ND & ND & No & ND & ND & No & NO & NO \\
\hline $2401 \mathrm{~W}$ & $F L, D W$ & ND & ND & ND & ND & $\mathrm{ND}$ & $\mathrm{ND}$ & $\mathrm{ND}$ & ND & NO & $\mathrm{ND}$ & ND \\
\hline$\frac{2401 W}{2401 w}$ & $\frac{r L, U W}{F L, U W}$ & $\frac{100}{9}$ & $\frac{100}{10}$ & $\frac{\pi}{8}$ & 14 & $\frac{1}{9}$ & $\frac{N D}{N D}$ & $\frac{\sqrt{12}}{12}$ & $\frac{14}{16}$ & $\frac{114}{14}$ & 18 & $\frac{12}{20}$ \\
\hline$\frac{2401 W}{2401 W}$ & $\frac{1}{T R}$ & $\frac{9}{N D}$ & ND & ND & NO & $\mathrm{ND}$ & NO & ND & $\mathrm{ND}$ & ND & ND & ND \\
\hline
\end{tabular}

\begin{tabular}{|c|c|}
\hline \multirow{2}{*}{\multicolumn{2}{|c|}{ Explanations: }} \\
\hline & SUMMa somple \\
\hline & SUMMA remple collectod by remoto contiol \\
\hline BK & Beckground sample \\
\hline FL & Fencaline semple \\
\hline IW & Upwind \\
\hline DW & Downwind \\
\hline NO & Not detected \\
\hline D & Deterted- not quantinted (soe text) \\
\hline NA & Not enalyzed \\
\hline Mot: & Dellection \\
\hline
\end{tabular}


HNF-SD-WM-RPT-282 Rev. 0

Table 4-2. GC-MS Data of Air Samples from the Central Waste Complex. (20 sheets) 2401.xls

\section{5) 2401 BUILDING (continued)}

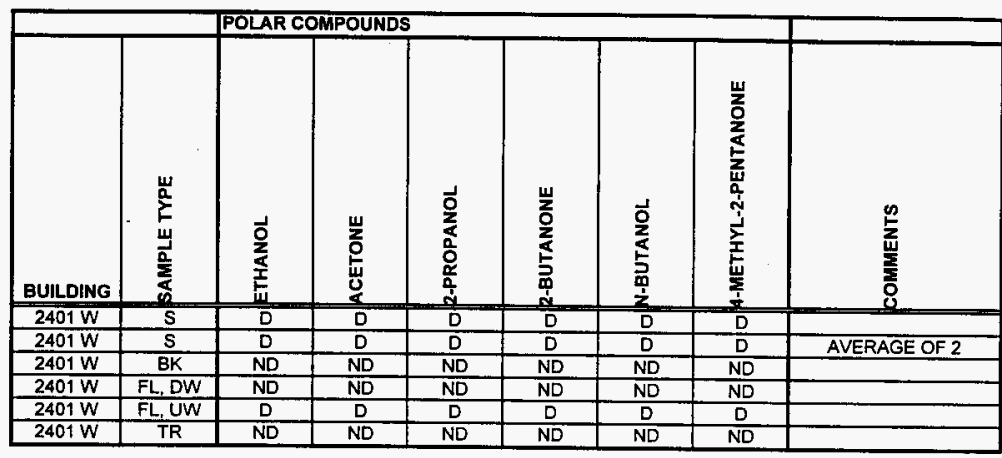

$\begin{array}{ll}\text { Explanations: } & \\ \text { S } & \text { SUMMA sample } \\ \text { SR } & \text { SUMMA sample collected by remote control } \\ \text { BK } & \text { Background sample } \\ \text { FL } & \text { Fenceline sample } \\ \text { UW } & \text { Upwind } \\ \text { DW } & \text { Downwind } \\ \text { ND } & \text { Not detected } \\ \text { D } & \text { Detected- not quantitated (see text) } \\ \text { NA } & \text { Not analyzed }\end{array}$

Note: Method Detection Limits are $10 \mathrm{ppb}$ or better 


\subsection{RESULTS}

The following is a compilation of the analysis results from each building based on the quantitative data using external standards (see Tables 2 and 3) and the qualitative library searches of the GC-MS data (see Appendix A) from the SUMMA ${ }^{\mathrm{TM}}$ grab samples taken at the CWC facility.

\subsection{BUILDINGS 2401, 2402, AND 2403}

\subsubsection{Building 2401}

Three samples from two different locations from inside the building were analyzed. The results of the duplicate samples (1W_70 A and B) were averaged in Table 2. The data suggest that only low levels of non-polar and slightly polar organic compounds are present. Some of the grab samples contained tetrahydrofuran ( $35 \mathrm{ppb})$ and trimethyl and dichlorobenzenes ( 2 to $18 \mathrm{ppb}$ ). The upwind samples had similar levels of these compounds and they resemble the indoor ambient air. Significant concentrations of polar compounds such as 2-butoxyethanol, 4-methyl-2-pentanone, 1-butanol, and 2-methoxyethanol were detected but the amounts vary between the two sampling locations. The analysis system was at that time not optimized for such polar compounds and the data should be verified with additional sampling in the future.

\subsubsection{Building $2402 \mathrm{~W}$}

The two samples have very low levels of organic compounds, mostly toluene ( 2 and $3 \mathrm{ppb}$ ). The mass spectral library searches also showed the presence of: 4-methyl-2-pentanone which also was in the background sample; 2-butoxyethanol; and of pinene and 3-carene, similar to compounds from turpentine solvent.

\subsubsection{Building 2402 WB}

The three samples from two different locations (one duplicate) consistently showed the presence of chlorinated solvents: trichloroethene was dominant (117 ppb average), followed by 1,1,1- trichloroethane (29 ppb), carbon tetrachloride ( $9 \mathrm{ppb}$ ) and 1,2-dichloroethene (3 ppb). The following polar compounds were detected but not quantitated: $n$-butanol and 4-methyl-2-pentanone (both of which also appeared in the background sample), and 2-butanone. The library searches also indicated that 2 -heptanone, benzaldehyde, and 2-butoxyethanol were present in these samples. 
Table 5-3. GC-MS Data of Indoor Air Samples From the Central Waste Complex: Concentrations in Microgram/Meter. ${ }^{3}$ (20 sheets)

2401.x1s

5) 2401 BUILDING

\begin{tabular}{|c|c|c|c|c|c|c|c|c|c|c|c|}
\hline \multicolumn{4}{|c|}{ GENERALINFORMATION } & \multicolumn{8}{|c|}{ NON-POLAR AND SLIGHTLY POLAR COMPOUNDS } \\
\hline BULLDING & 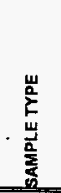 & 包 & 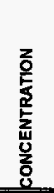 & 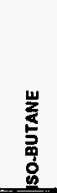 & 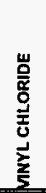 & 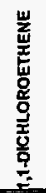 & 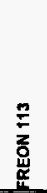 & 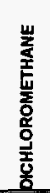 & 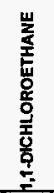 & 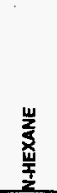 & 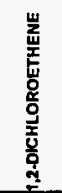 \\
\hline $2401 \mathrm{~W}$ & 5 & FT6045-1W.68A & $\mu a / m^{3}$ & ND & ND & ND & ND & ND & ND & ND & ND \\
\hline $2401 W$ & $\mathrm{~s}$ & FT6045-1W.70A & $\mu \mathrm{g} / \mathrm{m}^{3}$ & ND & ND & ND & ND & ND & ND & 31 & ND \\
\hline $2401 \mathrm{~W}$ & BK & FT6045-1W.71A & $\mu g / m^{3}$ & ND & ND & ND & ND & ND & ND & ND & ND \\
\hline $2401 \mathrm{~W}$ & FL. DW & FT6045-DW.72A & $\mu g / m^{3}$ & ND & $\mathrm{ND}$ & ND & ND & ND & ND & ND & ND \\
\hline $2401 \mathrm{~W}$ & FL.UW & FT6045-UW.73A & $\mu g / m^{3}$ & ND & ND & $\overline{\mathbf{N D}}$ & ND & ND & 4 & 234 & 10 \\
\hline $2401 w$ & $\mathrm{TR}$ & FT 6045-TR $69 \mathrm{~A}$ & $\mu g / m^{3}$ & ND & $\mathrm{ND}$ & ND & ND & ND & ND & ND & ND \\
\hline \multicolumn{4}{|c|}{$\begin{array}{l}\text { Molecular weight of analyte } \\
\text { CAS \# }\end{array}$} & $\begin{array}{c}58.1 \\
75285\end{array}$ & $\begin{array}{l}620 \\
73014\end{array}$ & $\begin{array}{r}96.0 \\
75354\end{array}$ & $\begin{array}{r}187.4 \\
75131\end{array}$ & $\begin{array}{l}84.0 \\
75092\end{array}$ & $\begin{array}{c}98.0 \\
75343\end{array}$ & $\begin{array}{c}86.1 \\
110543\end{array}$ & $\begin{array}{c}96.0 \\
156592\end{array}$ \\
\hline
\end{tabular}

Explanations:

$S$ SUMMA sample

SR SUMMA sample collectiod by remote control

BK Background sample

FL Fenceline sample

UW Upwind

DW Downwind

ND Not detectod

D Detected- not quantitatod (sop toxt)

NA Not analyzed

Noles: $\quad$ 1) Mathod Detection Limits are $10 \mathrm{ppb}$ or better

2) The quantitative analysis rosulns for the target compounds were calculated from the calibration curve which was gernerated from primary standards with concentrations in ppDV. The conversion from ppbv to $\mu / \mathrm{m}^{3}$ assumes standard temperature and pressure (STP) conditions of 760 tort and $273 \mathrm{~K}$ and was calculated from the following oquation:

$\mu \mathrm{g}^{\prime} \mathrm{m}^{3}=$ conc. in opby $\times$ mol wh. of compound (o)

$22.4(\mathrm{Hmol})$

Page 1 
Table 5-3. GC-MS Data of Indoor Air Samples From the Central Waste Complex:

Concentrations in Microgram/Meter. ${ }^{3}$ (20 sheets)

2401.xls

\section{5) 2401 BUILDING (continued)}

\begin{tabular}{|c|c|c|c|c|c|c|c|c|c|c|c|c|}
\hline \multirow[b]{2}{*}{ BUILING } & \multirow[b]{2}{*}{ 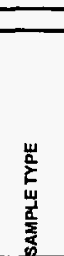 } & \multicolumn{11}{|c|}{ NON.POLAR AND SLIGKTLY POLAR COMPOUNDS } \\
\hline & & 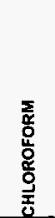 & 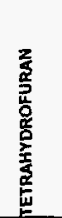 & 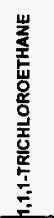 & 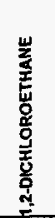 & 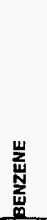 & 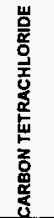 & 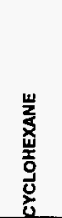 & 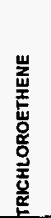 & 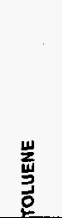 & 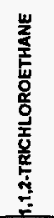 & 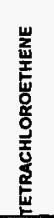 \\
\hline $2401 \bar{W}$ & $\frac{10}{s}$ & $\overline{\mathrm{ND}}$ & $\overline{\mathrm{ND}}$ & ND & ND & ND & ND & $=\frac{2}{N D}$ & ND & $\frac{1}{7}$ & ND & ND \\
\hline $2401 \mathrm{~W}$ & S & ND & 113 & ND & 4 & ND & ND & ND & ND & 4 & ND & ND \\
\hline $2801 \mathrm{~W}$ & BK & ND & ND & ND & ND & ND & ND & ND & ND & ND & ND & ND \\
\hline $2401 \mathrm{~W}$ & FL DW & ND & ND & ND & ND & ND & ND & ND & ND & ND & ND & ND \\
\hline $2401 \mathrm{~W}$ & FL. UW & $\frac{7 L}{7}$ & 288 & NO & 40 & 44 & ND & ND & 37 & 34 & 22 & ND \\
\hline $2401 \mathrm{~W}$ & TR & ND & ND & ND & ND & ND & ND & $\mathrm{ND}$ & ND & ND & ND & $\mathrm{ND}$ \\
\hline Molecular & in & 117.9 & 72.1 & 131.9 & 98.0 & 78.0 & 151.9 & 84.1 & 129.9 & 92.1 & 131.9 & 163.9 \\
\hline
\end{tabular}

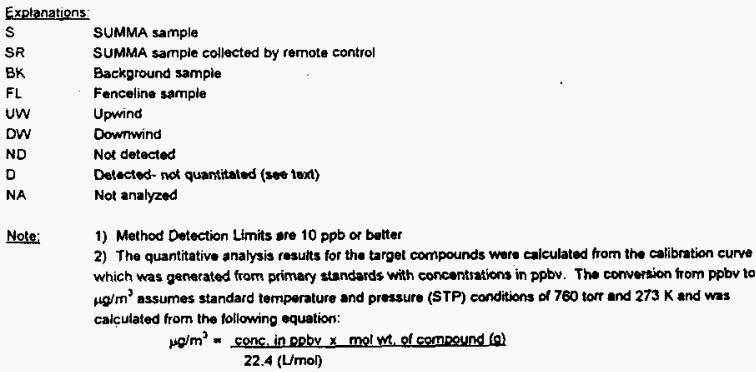


Table 5-3. GC-MS Data of Indoor Air Samples From the Central Waste Complex: Concentrations in Microgram/Meter. ${ }^{3}$ (20 sheets)

2401.xls

5) 2401 BUILDING (continued)

\begin{tabular}{|c|c|c|c|c|c|c|c|c|c|c|c|c|}
\hline & & ONFPC & AND & GHrL & SUAR & TIPOU & & & & & & \\
\hline BUILDING & 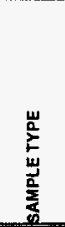 & 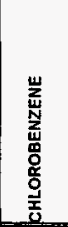 & 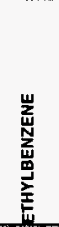 & 崖 & 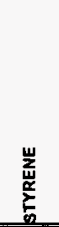 & $\frac{w}{\frac{w}{4}}$ & 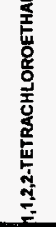 & 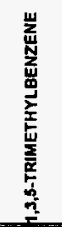 & 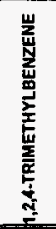 & 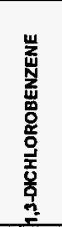 & 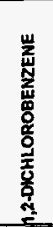 & 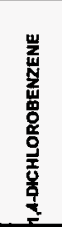 \\
\hline $2401 \mathrm{~W}$ & S & ND & 8 & ND & 10 & 8 & ND & 97 & 12 & 15 & 15 & 13 \\
\hline $2401 W$ & S & 5 & 14 & 14 & 37 & 14 & 37 & 43 & 86 & 39 & 65 & 65 \\
\hline $2401 \mathrm{~W}$ & BK & ND & ND & ND & ND & ND & ND & ND & ND & ND & $N D$ & ND \\
\hline $2401 \mathrm{~W}$ & FL, DW & ND & ND & ND & ND & ND & ND & ND & ND & ND & ND & ND \\
\hline $2401 \mathrm{~W}$ & FL, UW & 43 & 45 & 38 & 66 & 41 & ND & 64 & 88 & 91 & 116 & 130 \\
\hline $2401 \mathrm{~W}$ & TR & ND & ND & ND & $\overrightarrow{N D}$ & ND & ND & ND & ND & ND & ND & ND \\
\hline $\begin{array}{l}\text { Molecular } \\
\text { CAS \# }\end{array}$ & ight & $\begin{array}{c}112.0 \\
108907\end{array}$ & $\begin{array}{c}106.1 \\
100+114\end{array}$ & $\begin{array}{c}106.1 \\
108383\end{array}$ & $\begin{array}{c}104.1 \\
100425\end{array}$ & $\begin{array}{l}106.1 \\
95.476\end{array}$ & $\begin{array}{l}165.9 \\
79345\end{array}$ & $\begin{array}{c}120.1 \\
108678\end{array}$ & $\begin{array}{l}120.1 \\
95636\end{array}$ & $\begin{array}{c}146.0 \\
541731\end{array}$ & $\begin{array}{l}146.0 \\
95501\end{array}$ & $\begin{array}{c}146.0 \\
106467\end{array}$ \\
\hline
\end{tabular}

Explanations:

SUMMA sample

SR SUMMA sample collected by remote control

BK Background sample

FL Fenceline sample

UW Upwind

DW Dowmind

NO Not detectod

D Detected- not quantitated (see text)

NA Not analyzed

Note: 1) Method Detection Limits are $10 \mathrm{ppb}$ or belter

2) The quantitative analysis results for the larget eompounds were calculated from the calibration curve which was generated trom primary standards whth concentrations in ppbv. The conversion from ppby to

$\mu \mathrm{g} / \mathrm{m}^{3}$ assumes stendard tomparature and pressure (STP) conditions of 760 torr and $273 \mathrm{~K}$ and wes calculated from the tollowing equation:

$\mu \mathrm{g} / \mathrm{m}^{3}=$ conc, in poby $\times$ mol wh of compound (o) 22.4 (Umol) 
HNF-SD-WM-RPT-282 Rev. 0

Table 5-3. GC-MS Data of Indoor Air Samples From the Central Waste Complex: Concentrations in Microgram/Meter. ${ }^{3}$ (20 sheets)

$2401 . \times 1 s$

\section{5) 2401 BUILDING (continued)}

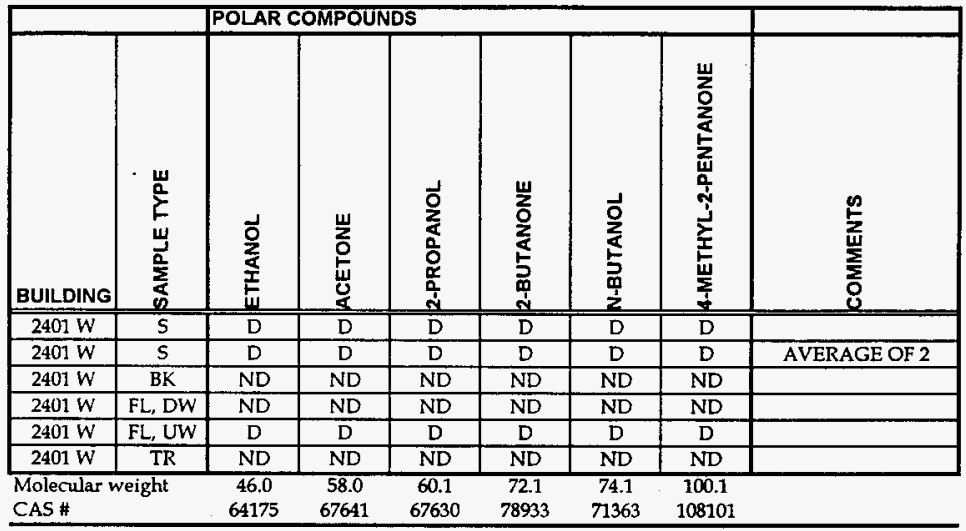

Explanations:

$\begin{array}{ll}\text { S } & \text { SUMMA sample } \\ \text { SR } & \text { SUMMA sample collected by remote control } \\ \text { BK } & \text { Background sample } \\ \text { FL } & \text { Fenceline sample } \\ \text { UW } & \text { Upwind } \\ \text { OW } & \text { Downwind } \\ \text { ND } & \text { Not detected } \\ \text { D } & \text { Detected- not quantitated (see text) } \\ \text { NA } & \text { Not analyzed }\end{array}$

Note. 1) Method Detection Limits are $10 \mathrm{ppb}$ or better

2) The quantitative analysis results for the target compounds were calculated from the calibration curve which was generated from primary standards with concentrations in ppbv. The conversion from ppbv to $\mu \mathrm{g} / \mathrm{m}^{3}$ assumes standard temperature and pressure (STP) conditions of 760 torr and $273 \mathrm{~K}$ and was calculated from the following equation:

$\mu \mathrm{g} / \mathrm{m}^{3}=$ conc in ppbr $\times$ mol wh of compound (a)

22.4 (Lmol) 
Table 5-3. GC-MS Data of Indoor Air Samples From the Central Waste Complex: Concentrations in Microgram/Meter. ${ }^{3}$ (20 sheets)

$2400 \times 5$

II R4P BบIIPINGS

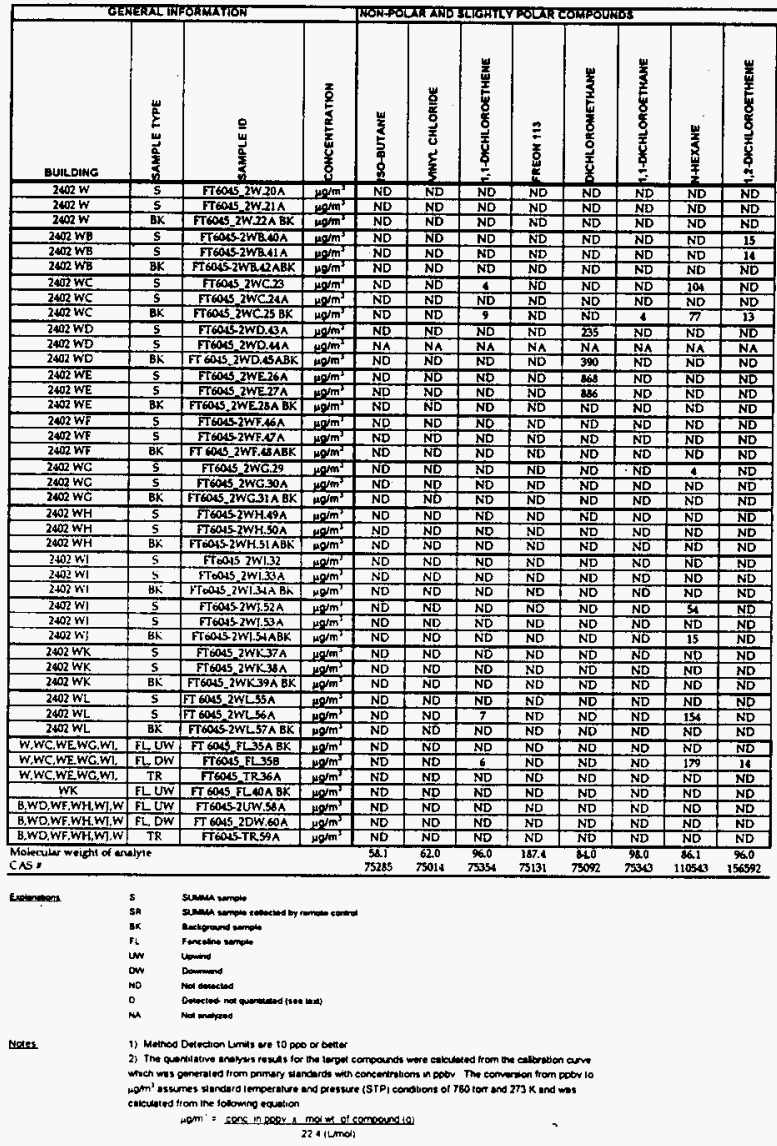


Table 5-3. GC-MS Data of Indoor Air Samples From the Central Waste Complex: Concentrations in Microgram/Meter. ${ }^{3}$ (20 sheets) 
HNF-SD-WM-RPT-282 Rev. 0

Table 5-3. GC-MS Data of Indoor Air Samples From the Central Waste Complex: Concentrations in Microgram/Meter. ${ }^{3}$ (20 sheets)

zoores

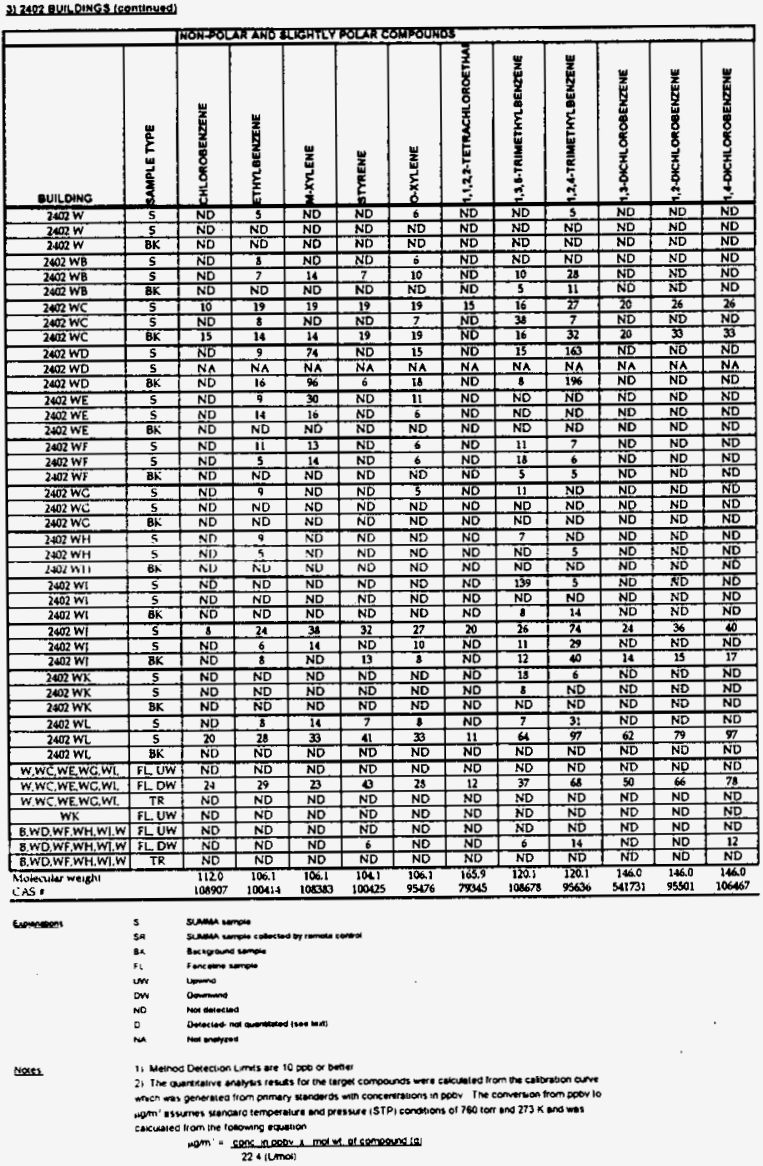


Table 5-3. GC-MS Data of Indoor Air Samples From the Central Waste Complex: Concentrations in Microgram/Meter. ${ }^{3}$ (20 sheets)

$2402 \times 15$

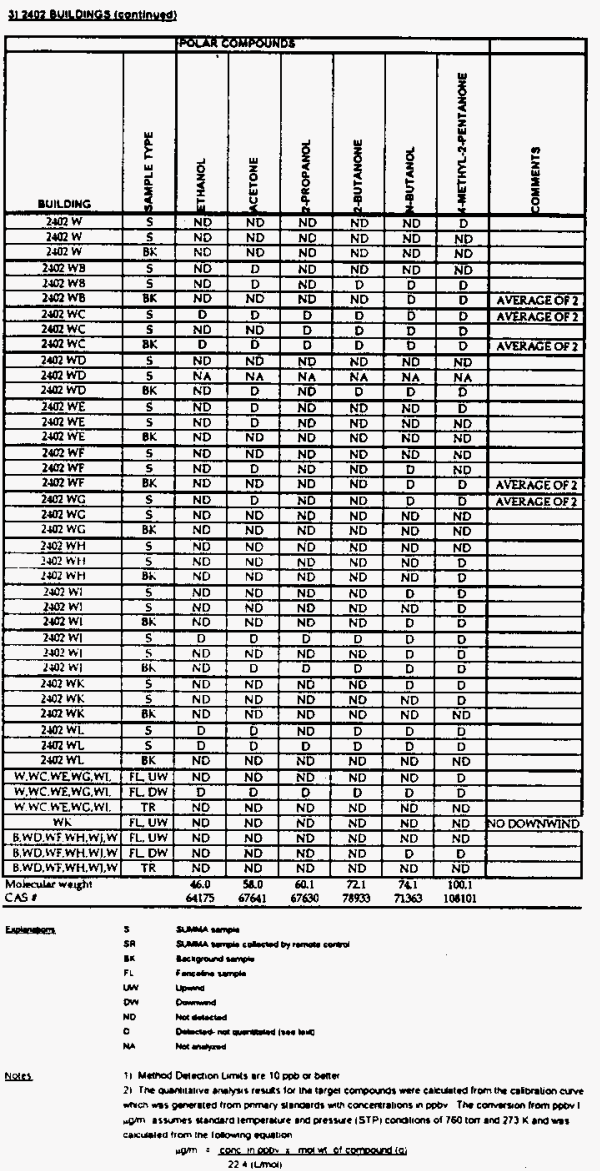


HNF-SD-WM-RPT-282 Rev. 0

Table 5-3. GC-MS Data of Indoor Air Samples From the Central Waste Complex: Concentrations in Microgram/Meter. ${ }^{3}$ (20 sheets)

2403.XLS

4)2403 gullomos

\begin{tabular}{|c|c|c|c|c|c|c|c|c|c|c|c|}
\hline \multicolumn{4}{|c|}{ GENEERALITFOCRMATION } & \multicolumn{8}{|c|}{ 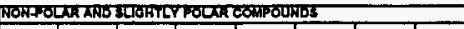 } \\
\hline BUILDING & 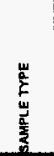 & 문 & 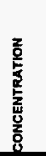 & 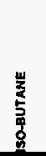 & 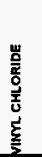 & 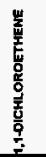 & 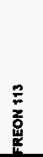 & 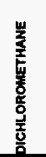 & 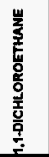 & 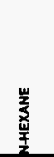 & 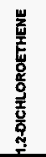 \\
\hline$\frac{2403 W A}{2400 W A}$ & $\frac{5}{5}$ & FT6015.3WA.62A & 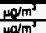 & $\frac{N D}{N D}$ & $\frac{N D}{N D}$ & $\frac{\mathrm{ND}}{\mathrm{NO}}$ & $\frac{N D}{N D D}$ & 214 & ND & 24 & ND \\
\hline 2400 WA & 5 & FT 6045,3WA.63A & $\frac{\mu \mathrm{N} / \mathrm{m}^{2}}{2}$ & $\mathrm{NP}$ & & No & ND & 115 & ND & 64 & ND \\
\hline 2403 WA & $\mathrm{s}$ & FT6045_3WA.6tA & $\mathrm{N} / \mathrm{m}^{3}$ & ND & ND & ND & ND & 142 & ND & 16 & NO \\
\hline $2403 \mathrm{WA}$ & $\mathrm{s}$ & FT60045 3WA 3 WASA & mom & ND & ND & ND & ND & 500 & ND & 48 & $\mathrm{ND}$ \\
\hline 2403 WA & BK & FT6045 3WA.66A & $\mu m^{2}$ & ND & ND & ND & ND & ND & $\mathrm{ND}$ & ND & NO \\
\hline $2400 \mathrm{WA}$ & BK & FT6015 3WA.67A & $=0 \sqrt{m^{2}}$ & $\mathrm{ND}$ & ND & ND & ND & ND & ND & ND & ND \\
\hline 2400 WA & $\mathrm{TR}$ & FI 6045TR .69A & $\mathrm{g}^{2} \mathrm{~m}^{2}$ & ND & NO & ND & ND & NO & ND & ND & ND \\
\hline 2400 WA & DW & FT6045DW.72A & $-\infty=1 m^{2}$ & ND & $\mathrm{ND}$ & $\mathrm{ND}$ & $\mathrm{ND}$ & NO & $\mathrm{ND}$ & $\mathrm{ND}$ & ND \\
\hline 2403 WA & UW & FT6045-UW.73A & $\omega m^{2}$ & $\mathrm{ND}$ & ND & 12 & ND & ND & 6 & 234 & 10 \\
\hline $2403 \mathrm{WB}$ & $\mathrm{s}$ & FT 6045 3WB,94A & $20 m^{3}$ & NO & ND & $\mathrm{ND}$ & $\mathrm{ND}$ & $\mathrm{NO}$ & ND & ND & ND \\
\hline $2400 \mathrm{WB}$ & $\frac{5}{5}$ & FT $60+5,3 W B .95 A$ & $\frac{m i n}{20}$ & ND & ND & ND & $\mathrm{ND}$ & ND & ND & ND & ND \\
\hline 2403 WB & s & FP $6045.3 W B .96 A$ & intin & NO & NO & ND & NO & NO & $\mathrm{ND}$ & 13 & ND \\
\hline 24003 WB & 5 & FT $60453 \mathrm{WB} 97 \mathrm{~A}$ & moim? & ND & ND & ND & $\mathrm{ND}$ & ND & $\mathrm{ND}$ & ND & No \\
\hline $2403 \mathrm{WB}$ & $B K$ & FT6045 3 WBB.98A & nom & ND & NO & ND & $\mathrm{ND}$ & NO & ND & ND & ND \\
\hline 2403 WB & BK & FT 6045,3 WB.99A BK & $\log ^{3}$ & ND & ND & NO & ND & ND & ND & ND & ND \\
\hline $2400 \mathrm{WB}$ & TR & FT604L-TR IOOA & $\mathrm{m} / \mathrm{m}$ & ND & ND & NO & ND & NO & ND & ND & ND \\
\hline 2403 WC & s & FT6045_3WC..4A & $\mathrm{\mu} 0 \mathrm{dm^{3 }}$ & ND & ND & ND & ND & 214 & ND & NO & ND \\
\hline 2400 WC & s & FT 6045-3WC. $85 A$ & $\mu \mathrm{m}^{3}$ & ND & ND & ND & ND & 237 & ND & ND & ND \\
\hline $2403 \mathrm{WC}$ & s & FT $6045-3 W C$ CB $6 A A$ & $\mu \nu m^{2}$ & ND & ND & ND & ND & 389 & ND & ND & NO \\
\hline 2403 WC & s & FT6045-3WC.87A & ing & ND & ND & ND & ND & 189 & ND & ND & ND \\
\hline $2403 \mathrm{WC}$ & BK & FT 6015 3WC.BEA BK & $\mu \mu^{2}{ }^{2}$ & ND & ND & ND & ND & ND & ND & ND & ND \\
\hline $2403 \mathrm{WC}$ & BK & FT6015_3WC.89A & $\mu \mathrm{g} \mathrm{m}^{3}$ & ND & ND & ND & ND & ND & NO & $\mathrm{ND}$ & ND \\
\hline 2403 WD & $s$ & FT 6045-3WDT3A & poim & $\mathrm{ND}$ & ND & ND & ND & ND & ND & ND & ND \\
\hline 2403 WD & $\mathrm{s}$ & FT6045_3WD.76A & 䧃 & ND & ND & ND & ND & 10 & ND & 62 & ND \\
\hline 2403 WD & 5 & FT6045 3WO 77 & nim & NO & $\mathrm{ND}$ & ND & ND & ND & ND & NO & ND \\
\hline $2403 \mathrm{WD}$ & s & FT6045_3WD.78A & $\mu / m^{\prime}$ & NO & $\mathrm{ND}$ & ND & $\mathrm{ND}$ & NO & ND & ND & ND \\
\hline 2403 WO & 5 & FT6015 3WD.79A & im & ND & ND & ND & ND & ND & ND & ND & ND \\
\hline 2403 wo & $s$ & FT'O045_3WD.80A & $\mathrm{spl}^{3}$ & NO & ND & ND & ND & ND & ND & ND & $\mathrm{ND}$ \\
\hline $2403 \mathrm{WL}$ & $B K$ & FTo045 3WD, BIA bk & sim & ND & $M D$ & $N D$ & ND & ND & ND & NO & ND \\
\hline $2+02$ WD & BK & FT6045_3WD.82A BK & $\alpha / m^{2}$ & ND & $\mathrm{NO}$ & ND & ND & ND & $\mathrm{ND}$ & ND & NO \\
\hline $2+100 \mathrm{WD}$ & $B K$ & 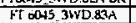 & 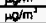 & ND & NO & ND & ND & ND & ND & ND & $\frac{1}{N D}$ \\
\hline WC.WD & FLUW & FT6015-15W.90A & $2 / \mathrm{m}^{2}$ & ND & NO & ND & ND & ND & $\mathrm{ND}$ & ND & ND \\
\hline WC, WD & F. DW & FT 6015-DW.91A & $2 / m^{3}$ & ND & ND & ND & ND & ND & ND & ND & ND \\
\hline WC, WD & TR & FT $60+5-7 R .91 \mathrm{~A}$ & $4 / m^{3}$ & ND & ND & ND & ND & ND & ND & $\mathrm{ND}$ & $\mathrm{ND}$ \\
\hline Seculer wei & 1 & & & $\begin{array}{c}36.1 \\
73285\end{array}$ & $\begin{array}{c}62.0 \\
75014\end{array}$ & $\begin{array}{l}96.0 \\
73354\end{array}$ & $\begin{array}{l}187.4 \\
75131\end{array}$ & $\begin{array}{l}840 \\
75092\end{array}$ & $\begin{array}{l}98.0 \\
73343\end{array}$ & $\begin{array}{c}86.1 \\
110543 \\
\end{array}$ & $\begin{array}{c}96.0 \\
156592 \\
\end{array}$ \\
\hline
\end{tabular}

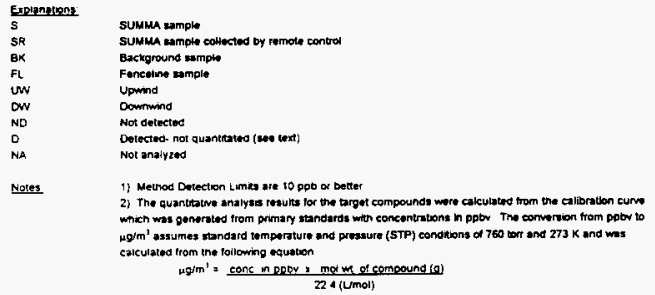


HNF-SD-WM-RPT-282 Rev. 0

Table 5-3. GC-MS Data of Indoor Air Samples From the Central Waste Complex: Concentrations in Microgram/Meter. ${ }^{3}$ (20 sheets)

$2403 \times 1.5$

412 Lo3 Bullowos (continused)

\begin{tabular}{|c|c|c|c|c|c|c|c|c|c|c|c|c|}
\hline \multirow[b]{2}{*}{ BUILDMO } & \multirow[b]{2}{*}{$\sum_{\frac{1}{2}}^{\frac{u}{2}}$} & \multicolumn{11}{|c|}{ 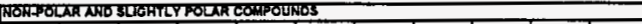 } \\
\hline & & $\begin{array}{l}\frac{5}{5} \\
\frac{5}{0} \\
\frac{1}{8} \\
\frac{5}{0} \\
\frac{7}{7}\end{array}$ & 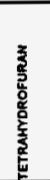 & 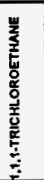 & 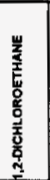 & 宸 & 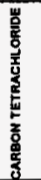 & $\frac{\frac{w}{2}}{\mathbf{s}}$ & 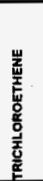 & $\begin{array}{l}\text { 삘 } \\
\text { 홀 }\end{array}$ & 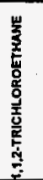 & 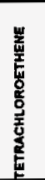 \\
\hline 2403 WA & 5 & ND & ND & 412 & 26 & ND & ND & ND & ND & 29 & ND & ND \\
\hline 2400 WA & $\cdot 5$ & ND & $\frac{10}{73}$ & 80 & 16 & ND & ND & ND & $\frac{107}{27}$ & 36 & $\mathrm{NO}$ & ND \\
\hline 2403 WA & 5 & ND & ND & 9 & $\mathrm{ND}$ & ND & $\frac{\mathrm{ND}}{\mathrm{ND}}$ & ND & $\frac{R D}{N D}$ & 32 & ND & ND \\
\hline 2403 WA & S & $\mathrm{ND}$ & ND & 208 & 12 & $\mathrm{ND}$ & ND & ND & ND & 85 & ND & 29 \\
\hline $2403 \mathrm{WA}$ & EK & ND & ND & 140 & NO & $\mathrm{ND}$ & ND & ND & $\mathrm{ND}$ & ND & ND & ND \\
\hline $2403 W \mathrm{AA}$ & $\mathrm{BK}$ & ND & ND & ND & $\mathrm{ND}$ & ND & $\mathrm{ND}$ & $\mathrm{ND}$ & ND & ND & ND & ND \\
\hline 2403 WA & TR & ND & ND & $\mathrm{ND}$ & ND & $\mathrm{ND}$ & ND & ND & ND & $\mathrm{ND}$ & ND & $\mathrm{ND}$ \\
\hline $2403 \mathrm{WA}$ & DW & ND & $\mathrm{ND}$ & ND & ND & $\mathrm{ND}$ & ND & ND & ND & $\sqrt{\mathrm{NO}}$ & ND & ND \\
\hline $2400 \mathrm{WA}$ & Uw & 7 & 288 & ND & 40 & 4 & ND & ND & 37 & 34 & $\frac{2}{22}$ & ND \\
\hline $2+03 \mathrm{WB}$ & 5 & ND & ND & ND & $\mathrm{No}$ & ND & 56 & ND & ND & 6 & $\sqrt{\mathrm{N}}$ & ND \\
\hline 2403 WB & s & $\sqrt{0}$ & NDD & $\mathrm{ND}$ & ND & ND & 136 & 11 & NO & 8 & ND & ND \\
\hline 2403 wo & 5 & ND & ND & 150 & ND & 14 & 7 & 14 & NU & 9 & ND & ND \\
\hline $2403 \mathrm{WB}$ & $s$ & NO & ND & ND & ND & ND & $\pi 0$ & $\mathrm{ND}$ & ND & 7 & ND & ND \\
\hline 2403 WB & BK & ND & ND & ND & ND & $\mathrm{ND}$ & ND & $\mathrm{ND}$ & ND & ND & ND & $\mathrm{ND}$ \\
\hline $2403 \mathrm{WB}$ & BK & ND & ND & No & NO & $\mathrm{ND}$ & $\mathrm{ND}$ & ND & $\mathrm{ND}$ & ND & ND & ND \\
\hline 2403 wB & TR & No & ND & ND & ND & ND & ND & $\mathrm{ND}$ & ND & ND & ND & ND \\
\hline $240 \mathrm{WC}$ & s & ND & ND & 9 & No & $\mathrm{ND}$ & ND & $\mathrm{ND}$ & ND & 24 & ND & 195 \\
\hline $2403 \mathrm{WC}$ & $S$ & $\sqrt{D}$ & ND & 73 & ND & ND & $\mathrm{ND}$ & ND & ND & 16 & ND & 151 \\
\hline 2403 WC & 5 & $N D$ & ND & 89 & ND & ND & 34 & ND & $\mathrm{ND}$ & 14 & ND & 154 \\
\hline $2403 W C$ & $\mathrm{~s}$ & ND & ND & 94 & ND & ND & $\mathrm{ND}$ & ND & ND & 11 & NO & 158 \\
\hline $2403 W C$ & BK & $\mathrm{ND}$ & ND & ND & ND & ND & ND & ND & $\mathrm{NO}$ & NO & $\mathrm{ND}$ & ND \\
\hline $2400 W C$ & BK & ND & ND & ND & ND & NO & ND & ND & ND & 5 & ND & ND \\
\hline 2403 WD & $\mathrm{s}$ & $\mathrm{ND}$ & ND & $\mathrm{ND}$ & ND & NO & ND & ND & ND & 8 & ND & ND \\
\hline 2403 WD & s & ND & 109 & ND & 35 & $\frac{\pi v}{2 t}$ & ND & ND & 12 & 29 & 29 & ND \\
\hline $2409 \mathrm{WD}$ & s & $N D$ & ND & $\mathrm{ND}$ & $\mathrm{ND}$ & $\mathrm{ND}$ & ND & ND & ND & $\frac{5}{12}$ & ND & ND \\
\hline $2+03 \mathrm{WD}$ & 5 & ND & $\overline{N D}$ & ND & ND & ND & ND & ND & ND & 10 & ND & $\mathrm{ND}$ \\
\hline 2403 WD & $s$ & ND & No & ND & $\mathrm{ND}$ & ND & $\mathrm{ND}$ & ND & ND & 12 & ND & ND \\
\hline $2403 \mathrm{WD}$ & 5 & $N$ & $\mathrm{NC}$ & ND & ND & ND & NO & $\mathrm{ND}$ & ND & 11 & $\mathrm{ND}$ & ND \\
\hline $2+c / 2 w 0$ & BK & ND & ND & ND & ND & ND & ND & ND & $\mathrm{ND}$ & ND & ND & ND \\
\hline $2+03 \mathrm{WD}$ & $B K$ & $\mathrm{ND}$ & ND & ND & ND & ND & $\mathrm{ND}$ & ND & $\mathrm{ND}$ & ND & ND & ND \\
\hline $2403 \mathrm{WD}$ & $\overline{\mathrm{BK}}$ & ND & ND & ND & ND & ND & ND & ND & ND & ND & ND & ND \\
\hline wC, wD & Fiv & ND & ND & ND & ND & ND & ND & NO & NO & ND & ND & ND \\
\hline Wc wo & FLDW & ND & ND & ND & ND & ND & ND & NO & ND & ND & ND & ND \\
\hline WC, WD & IR & ND & ND & ND & NO & ND & No & ND & ND & $\overline{M D}$ & $\mathrm{ND}$ & NT \\
\hline $\begin{array}{l}\text { Modeculint weig } \\
\text { CAS* }\end{array}$ & & $\begin{array}{l}117.9 \\
67663\end{array}$ & $\begin{array}{l}72.1 \\
1009 \%\end{array}$ & $\begin{array}{l}131.9 \\
71566\end{array}$ & $\begin{array}{c}900 \\
107062\end{array}$ & $\begin{array}{l}78.0 \\
n 432\end{array}$ & $\begin{array}{l}151.9 \\
56225\end{array}$ & $\begin{array}{c}84.1 \\
110827\end{array}$ & $\begin{array}{l}129.9 \\
79016\end{array}$ & $\begin{array}{c}12.1 \\
108839\end{array}$ & $\begin{array}{r}131.9 \\
79005\end{array}$ & $\begin{array}{r}103.9 \\
12784\end{array}$ \\
\hline
\end{tabular}

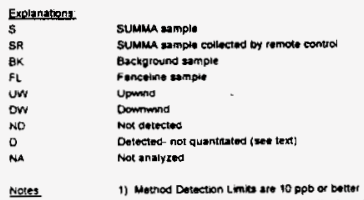

1) Wethod Detectian Limita are 10 p pb or befttor

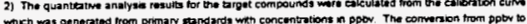

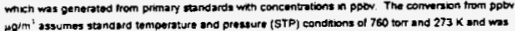

caculated trom the followng equation

nom' = conc $n$ notor $x$ mol me of compound (a)

22 A (UTrol) 
HNF-SD-WM-RPT-282 Rev, 0

Table 5-3. GC-MS Data of Indoor Air Samples From the Central Waste Complex: Concentrations in Microgram/Meter. ${ }^{3}$ (20 sheets)

$2 \cos x \operatorname{sis}$

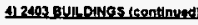

\begin{tabular}{|c|c|c|c|c|c|c|c|c|c|c|c|c|}
\hline \multirow[b]{2}{*}{ BUILDINO } & \multirow[b]{2}{*}{ 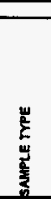 } & \multicolumn{11}{|c|}{ 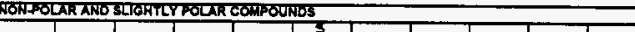 } \\
\hline & & 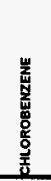 & 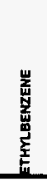 & 崖 & $\frac{w}{\frac{m}{2}}$ & $\frac{\text { 㟽 }}{\frac{3}{3}}$ & 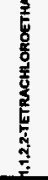 & 崖 & 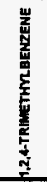 & 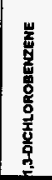 & 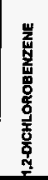 & 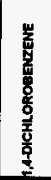 \\
\hline $2403 \mathrm{WA}$ & 5 & $\mathrm{ND}$ & 9 & 24 & 9 & 9 & 7 & 11 & 3 & 13 & 20 & 26 \\
\hline 2400 WA & $\mathrm{s}$ & 8 & 16 & 26 & 20 & 22 & 20 & 41 & 53 & 61 & 69 & 75 \\
\hline $2400 \mathrm{WA}$ & $\mathrm{s}$ & ND & $\mathbf{g}$ & 18 & 6 & 9 & ND & 7 & 29 & No & ND & 16 \\
\hline $2+100 \mathrm{WA}$ & $\mathbf{S}$ & 8 & 30 & 68 & 15 & 25 & $\mathrm{ND}$ & 8 & 30 & 16 & 23 & 22 \\
\hline 2403 WA & BK & NO & NO & NO & ND & ND & ND & 23 & 15 & $\mathrm{ND}$ & ND & $\mathrm{ND}$ \\
\hline $2400 \mathrm{WA}$ & BK & ND & 7 & $\mathrm{NO}$ & 9 & ND & ND & 28 & 12 & 14 & 15 & 14 \\
\hline $2403 \mathrm{WA}$ & TR & $\mathrm{ND}$ & $\mathrm{ND}$ & $\mathrm{ND}$ & $\mathrm{ND}$ & ND & ND & ND & ND & ND & ND & ND \\
\hline 2403 WA & DW & $\mathrm{NL}$ & $\mathrm{ND}$ & ND & ND & ND & ND & ND & ND & ND & ND & ND \\
\hline $2403 \mathrm{WA}$ & UW & 43 & 45 & 38 & 66 & 41 & NO & 64 & 88 & 91 & 116 & 130 \\
\hline $2400 \mathrm{WB}$ & $s$ & ND & 14 & 34 & ND & 23 & ND & 11 & 14 & ND & ND & No \\
\hline $2403 \mathrm{WB}$ & 5 & $\mathrm{ND}$ & 12 & 57 & ND & 22 & ND & 16 & 10 & ND & $\mathrm{ND}$ & ND \\
\hline $2400 \mathrm{WB}$ & $\mathrm{s}$ & ND & 13 & 53 & ND & 23 & $\mathrm{ND}$ & 7 & 13 & ND & $\mathrm{MD}$ & No \\
\hline $2403 \mathrm{WB}$ & 5 & ND & 15 & 54 & ND & 23 & ND & 6 & 16 & ND & ND & ND \\
\hline 2403 WB & $\mathrm{BK}$ & $\mathrm{ND}$ & ND & NO & NO & 9 & ND & 22 & 36 & ND & ND & 16 \\
\hline $2403 \mathrm{WB}$ & BK & ND & 5 & ND & ND & 11 & ND & 17 & 31 & ND & NO & 13 \\
\hline 24003 WB & $\mathrm{TR}$ & ND & $\mathrm{ND}$ & $\mathrm{ND}$ & ND & ND & ND & ND & $\sqrt{0}$ & ND & $\mathrm{ND}$ & ND \\
\hline $2+\sqrt{3}$ WC & 5 & ND & 18 & 40 & 12 & $1 \overline{9}$ & ND & 9 & 33 & 13 & 16 & 21 \\
\hline $2403 \mathrm{WC}$ & $s$ & ND & 9 & 33 & ND & 11 & NO & ND & 18 & ND & NO & ND \\
\hline $2400 \mathrm{WC}$ & 5 & $\mathrm{ND}$ & 8 & 34 & $\mathrm{ND}$ & 13 & ND & ND & 19 & ND & ND & ND \\
\hline $2403 w C$ & $\mathbf{s}$ & NO & 7 & 24 & ND & 10 & ND & ND & 73 & $\mathrm{ND}$ & ND & NO \\
\hline 2403 WC & $B \overline{B K}$ & ND & 7 & NO & 7 & 15 & ND & 39 & 95 & ND & ND & ND \\
\hline $2400 W C$ & BK & NO & 23 & 30 & 28 & 46 & 30 & 125 & 168 & 41 & 37 & है \\
\hline $2403 \mathrm{WD}$ & 5 & $\mathrm{ND}$ & 12 & 14 & ND & ND & ND & NO & 7 & $\mathrm{ND}$ & ND & ND \\
\hline 2403 WD & 5 & 20 & 33 & 38 & 42 & 24 & ND & 27 & 48 & 46 & 65 & 3 \\
\hline $2403 \mathrm{WD}$ & 5 & $\mathrm{NO}$ & 8 & 19 & $\mathrm{ND}$ & 5 & ND & 5 & 5 & ND & ND & ND \\
\hline $2400 \mathrm{WD}$ & $\mathrm{S}$ & $\mathrm{ND}$ & 6 & 16 & ND & 6 & $\mathrm{ND}$ & $\mathrm{ND}$ & 9 & ND & ND & ND \\
\hline $2403 \mathrm{WD}$ & 5 & ND & 7 & 20 & $\mathrm{ND}$ & 8 & ND & 8 & 20 & ND & ND & ND \\
\hline $2400 \mathrm{WD}$ & 5 & ND & 9 & 15 & 8 & 9 & ND & 7 & 16 & ND & ND & 13 \\
\hline $2403 \mathrm{WD}$ & BK & ND & NO & ND & ND & 6 & ND & 16 & 57 & ND & ND & 18 \\
\hline 2403 WD & $\overline{\mathrm{BK}}$ & ND & $\mathrm{ND}$ & ND & $\mathrm{ND}$ & ND & ND & ND & ND & ND & ND & $\mathrm{ND}$ \\
\hline $2+00 \mathrm{WD}$ & BK & ND & ND & No & ND & No & ND & 7 & 13 & ND & ND & ND \\
\hline WC, WD & FL UW & ND & ND & $\mathrm{ND}$ & ND & $\mathrm{NO}$ & No & ND & 8 & ND & ND & ND \\
\hline WC. WD & FL. DW & NO & $\mathrm{ND}$ & ND & NO & ND & ND & ND & 9 & ND & $\mathrm{ND}$ & $\mathrm{ND}$ \\
\hline WC. Wo & $T R$ & ND & ND & ND & ND & ND & ND & ND & ND & ND & ND & NO \\
\hline $\begin{array}{l}\text { Molecular weigh } \\
\text { CAS }\end{array}$ & & $\begin{array}{c}112.0 \\
108907\end{array}$ & $\begin{array}{c}106.1 \\
100414\end{array}$ & $\begin{array}{c}106.1 \\
100383\end{array}$ & $\begin{array}{c}104.1 \\
100425\end{array}$ & $\begin{array}{l}106 . \mathrm{I} \\
95476\end{array}$ & $\begin{array}{l}165.9 \\
79345\end{array}$ & $\begin{array}{c}120.1 \\
108678\end{array}$ & $\begin{array}{l}120.1 \\
95636\end{array}$ & $\begin{array}{r}146.0 \\
541731\end{array}$ & $\begin{array}{l}146.0 \\
95501\end{array}$ & $\begin{array}{r}146.0 \\
106467\end{array}$ \\
\hline
\end{tabular}

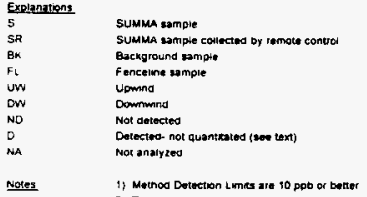

Notes 1) Mathod Detertion Lamis are 10 poto or better

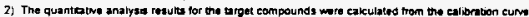

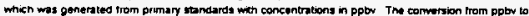

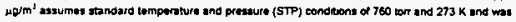

calculated from the tollowing equation.

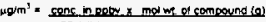
2.4 (Umol) 
Table 5-3. GC-MS Data of Indoor Air Samples From the Central Waste Complex: Concentrations in Microgram/Meter. ${ }^{3}$ (20 sheets)

2403.XIS

142403 BUILOINas icontinuad

\begin{tabular}{|c|c|c|c|c|c|c|c|c|}
\hline \multirow[b]{2}{*}{ EUILOINO } & \multirow[b]{2}{*}{$\frac{w}{4}$} & \multicolumn{6}{|c|}{ 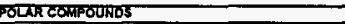 } & \\
\hline & & $\begin{array}{l}\frac{\mathbf{0}}{2} \\
\frac{5}{5} \\
\frac{5}{51}\end{array}$ & 訔 & $\begin{array}{l}\frac{1}{0} \\
\frac{0}{2} \\
\frac{\alpha}{\alpha} \\
\frac{\alpha}{4} \\
\frac{\alpha}{4} \\
\alpha\end{array}$ & $\begin{array}{l}\frac{1}{2} \\
\frac{7}{9} \\
\frac{3}{5} \\
\frac{3}{7}\end{array}$ & $\begin{array}{l}\frac{6}{0} \\
\frac{3}{2} \\
\frac{5}{9} \\
\frac{1}{2}\end{array}$ & 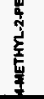 & 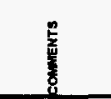 \\
\hline $2400 \mathrm{WA}$ & 5 & ND & D & ND & D & D & D & AVERAGE OF? \\
\hline $2403 \mathrm{WA}$ & s & $\mathrm{D}$ & D & D & D & $\bar{D}$ & D & \\
\hline 2403 WA & S & ND & $\mathrm{D}$ & ND & ND & ND & ND & \\
\hline 2400 WA & 5 & $\bar{D}$ & $\bar{D}$ & D & $D$ & D & $D$ & \\
\hline $2400 \mathrm{WA}$ & $B K$ & ND & ND & ND & $\mathrm{ND}$ & ND & ND & \\
\hline $2400 \mathrm{WA}$ & BK & $\mathrm{ND}$ & ND & $\mathrm{ND}$ & ND & $\mathrm{D}$ & $\frac{D}{D}$ & \\
\hline 2400 WA & $\sqrt{\mathrm{K}}$ & ND & ND & ND & ND & ND & ND & \\
\hline $2400 \mathrm{WA}$ & $\overline{\mathrm{DW}}$ & ND & ND & ND & ND & ND & ND & \\
\hline $2400 \mathrm{WA}$ & $\mathrm{uw}$ & D & $\overline{\mathrm{D}}$ & $\overline{\mathrm{D}}$ & $\bar{D}$ & $\bar{D}$ & D & \\
\hline $2+00 \mathrm{WB}$ & $\mathrm{s}$ & $\mathrm{ND}$ & 13 & ND & ND & ND & ND & \\
\hline $2+003 \mathrm{WB}^{\circ}$ & 5 & 10 & 9 & ND & ND & ND & ND & AVERAGE OF 2 . \\
\hline $2400 w 6$ & $\mathrm{~S}$ & ND & 17 & ND & NO & 13 & ND & \\
\hline $2403 \mathrm{WB}$ & s & NO & 19 & ND & $\mathrm{ND}$ & ND & ND & \\
\hline 2403 WB & BK & NO & ND & ND & ND & ND & ND & \\
\hline $2+003$ WB & BK & $\mathrm{ND}$ & ND & $\mathrm{NO}$ & ND & ND & ND & \\
\hline $2+00$ WB & Tर & ND & ND & ND & ND & ND & ND & \\
\hline $2+03 W C$ & 5 & $\bar{D}$ & $\mathrm{D}$ & $\bar{D}$ & $\overline{\mathrm{D}}$ & D & $\overline{\mathbf{D}}$ & \\
\hline $2403 W C$ & 5 & ND & D & $\bar{D}$ & NO & ND & NO & \\
\hline 2403 WC & $\mathrm{s}$ & ND & $\mathrm{NO}$ & D & NO & No & NO & \\
\hline $2+106 \mathrm{WC}$ & s & NO & D & D & $\sqrt{\mathrm{N}}$ & $\mathrm{ND}$ & $\mathrm{D}$ & \\
\hline $2400 \mathrm{WC}$ & BK & ND & $\mathrm{D}$ & $\mathrm{ND}$ & ND & D & $D$ & \\
\hline $2400 \mathrm{WC}$ & BK & $\sqrt{D}$ & D & $\mathrm{ND}$ & ND & $D$ & D & \\
\hline $2400 \mathrm{WD}$ & 5 & ND & ND & NO & ND & ND & ND & \\
\hline $2+03$ wO & 5 & $\bar{D}$ & D & $\mathrm{D}$ & $\mathrm{D}$ & D & D & \\
\hline 2400 WD & 5 & ND & ND & ND & ND & D & D & AVERAGE OF 2 \\
\hline $2+103 \mathrm{WD}$ & s & ND & D & ND & NO & NO & D & \\
\hline $2403 \mathrm{WD}$ & $\mathrm{s}$ & ND & NO & ND & ND & ND & D & \\
\hline $2403 \mathrm{WD}$ & $\mathbf{s}$ & ND & ND & ND & ND & $\bar{D}$ & D & \\
\hline 2403 WO & BK & ND & $\overline{N D}$ & ND & NO & $\mathrm{ND}$ & $\bar{D}$ & \\
\hline $2403 \mathrm{WV}$ & BK & $\sqrt{\mathrm{D}}$ & ND & ND & ND & ND & 40 & \\
\hline $2403 \mathrm{WD}$ & $\overline{B K}$ & ND & ND & NO & ND & ND & D & \\
\hline WC.WD & FLUW & NO & ND & NO & ND & No & D & \\
\hline WC, WD & $\mathrm{FL} D W$ & ND & ND & ND & ND & NO & D & \\
\hline WC, WD & TR & ND & ND & $\overline{\mathrm{ND}}$ & NO & ND & ND & \\
\hline $\begin{array}{l}\text { Molecular weig } \\
\text { AS " }\end{array}$ & & $\begin{array}{l}46.0 \\
64175\end{array}$ & $\begin{array}{l}58.0 \\
67641\end{array}$ & $\begin{array}{l}67.1 \\
67630\end{array}$ & $\begin{array}{c}721 \\
78933\end{array}$ & $\begin{array}{l}74.1 \\
77363\end{array}$ & $\begin{array}{c}100.1 \\
108101\end{array}$ & \\
\hline
\end{tabular}

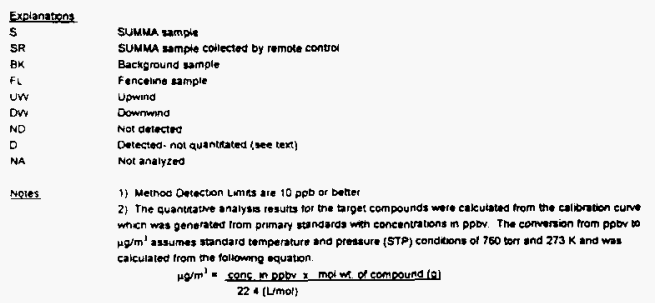


Table 5-3. GC-MS Data of Indoor Air Samples From the Central Waste Complex: Concentrations in Microgram/Meter. ${ }^{3}$ (20 sheets)

Ifm background

21 BACKGROUND BLANKS FOR LOW FLASH-POINT MIXED WASTE ANO ALKALIMETAL STORACE BUILDINGS

\begin{tabular}{|c|c|c|c|c|c|c|c|c|c|c|}
\hline \multicolumn{4}{|c|}{ GENERAL TNFORMATION } & \multicolumn{7}{|c|}{ NONPOLAR AND SLIOHTLY POLAR COMPOUNDS } \\
\hline BUILDING & 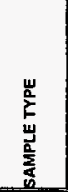 & $\frac{0}{\frac{1}{5}}$ & 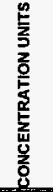 & 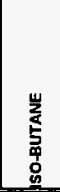 & 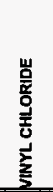 & 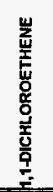 & 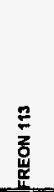 & 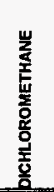 & 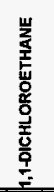 & $\begin{array}{l}\frac{w}{3} \\
\frac{3}{3} \\
\frac{w}{7} \\
\frac{7}{2}\end{array}$ \\
\hline $17,18,19,20, \mathrm{~A} 3, \mathrm{~A} 4$ & FL. DW & FT $6045, \mathrm{FL}, 04 \mathrm{~B}$ & $\mu \mathrm{g} / \mathrm{m}^{\mathrm{s}}$ & ND & ND & ND & ND & ND & $\mathrm{ND}$ & ND \\
\hline $17,18,19,20, \mathrm{~A} 3, \mathrm{A4}$ & $\mathrm{TR}$ & FT6045_TR08A & $\mu \mathrm{g} \mathrm{m}^{3}$ & NA & NA & NA & NA & NA & NA & NA \\
\hline $16, \mathrm{~A} 1, \mathrm{~A} 2$ & FL, DW & FT6045_FL13A & $\mu \mathrm{g} / \mathrm{m}^{3}$ & ND & ND & ND & ND & ND & ND & ND \\
\hline$\overline{16, \mathrm{~A} 1, \mathrm{~A} 2}$ & $\mathrm{FL}, \mathrm{UW}$ & FT6045FL.13B & $\mu g / \mathrm{m}^{3}$ & ND & ND & ND & ND & ND & ND & ND \\
\hline $16, \mathrm{~A} 1, \mathrm{~A} 2$ & $T R$ & FT6045_TRI4.A & $\mu \mathrm{g} / \mathrm{m}^{3}$ & ND & ND & ND & ND & ND & ND & ND \\
\hline $18,19,20$ & BK & FT6045_BK.15A & $\mu g / m^{3}$ & ND & ND & ND & ND & ND & ND & ND \\
\hline $\mathrm{Al}, \mathrm{A} 2,16$ & BK & FT6045_BK.12A & $\mu \mathrm{gm}^{3}$ & ND & ND & ND & ND & ND & ND & ND \\
\hline$A 3, A 4,17$ & BK & FT6045_BK.16A & $\mu \mathrm{g} / \mathrm{m}^{3}$ & ND & ND & ND & ND & ND & ND & 233 \\
\hline 14,15 & BK & FT6045BK.101A & $\mu g / m^{3}$ & NA & NA & NA & NA & NA & $\overline{\mathrm{NA}}$ & NA \\
\hline 14,15 & FL, DW & FT6045-DW.106A & $\mu g / m^{3}$ & ND & $\overline{\mathrm{ND}}$ & ND & ND & ND & ND & ND \\
\hline 14,15 & $\overline{F L}, \mathrm{UW}$ & FT6045-UW.106A & $\mu \mathrm{g} / \mathrm{m}^{3}$ & ND & ND & ND & ND & ND & ND & ND \\
\hline 14,15 & TR & FT 6045_TR.106 A & $\mu \mathrm{g} / \mathrm{m}^{3}$ & ND & ND & ND & ND & ND & ND & ND \\
\hline $1,2,5$ & BK & FT6045-BK.111A & $\mu g / m^{3}$ & ND & ND & ND & ND & ND & ND & ND \\
\hline $9,10,11$ & BK & FT6045-BK.118A & $\mu \mathrm{g} \mathrm{m}^{3}$ & ND & ND & ND & ND & ND & ND & ND \\
\hline $1,2,5,9,10,11$ & $\mathrm{FL}, \mathrm{UW}$ & FT6045-UW.112A & $\mu \mathrm{g} / \mathrm{m}^{3}$ & ND & ND & ND & ND & ND & ND & ND \\
\hline $1,2,5,9,10,11$ & $\overline{F L}, D W$ & FT6045-DW.114A & $\mu \mathrm{g} / \mathrm{m}^{3}$ & ND & ND & ND & $\overline{N D}$ & ND & ND & ND \\
\hline $1,2,5,9,10,11$ & TR & FT6045-TR.113A & $\mu g / m^{\top}$ & ND & ND & $\mathrm{ND}$ & ND & ND & $\overline{\mathrm{ND}}$ & ND \\
\hline $3,4,6$ & BK & FT6045-BK.124A & $\mu g / m^{3}$ & ND & ND & ND & ND & ND & ND & ND \\
\hline $7,8,12$ & BK & FT6045-BK.131A & $\mu \mathrm{g} / \mathrm{m}^{3}$ & ND & ND & ND & ND & ND & ND & ND \\
\hline $3,4,6,7,8,12$ & FL, UW & FT 6045 UW.124A & $\mu \mathrm{g} / \mathrm{m}^{3}$ & ND & ND & ND & ND & ND & ND & ND \\
\hline $3,4,6,7,8,12$ & FL, DW & FT 6045_DW.125A & $\mu \mathrm{g} / \mathrm{m}^{3}$ & ND & ND & ND & ND & ND & ND. & ND \\
\hline $3,4,6,7,8,12$ & $\mathrm{TR}$ & FT6045-TR $126 \mathrm{~A}$ & $\mu \mathrm{g} / \mathrm{m}^{3}$ & ND & ND & ND & ND & ND & ND & ND \\
\hline \multirow{2}{*}{\multicolumn{4}{|c|}{$\begin{array}{l}\text { Molecular weight of analyte } \\
\text { CAS " }\end{array}$}} & 58.1 & 62.0 & 96.0 & 187.4 & 84.0 & 98.0 & 86.1 \\
\hline & & & & 75285 & 75014 & 75354 & 75131 & 75092 & $753+13$ & 110543 \\
\hline
\end{tabular}

Explanations: $\mathrm{S}$

SR

BK

FL

UW

DW

ND

D

NA

Notes:
SUMMA sample

SUMMA sample collected by remote control

Background sample

Fenceline sample

Upunind

Downwind

Not delected

Detected- not quantiated (see text)

Not analyzed

1) Method Detection Limits are 10 ppb or better

2) The quantitative analygis results for the barget compeunds were ealeulated from the calibration eurve

which was generated from primary standarts with concentrations in ppbv. The conversion from ppbv to

$\mu g / m^{3}$ assumes standard temperature and pressure (STP) conditions of 760 torr and $273 \mathrm{~K}$ and was

calculated from the following equation:

$\mu \mathrm{g}^{\prime} \mathrm{m}^{3}=\frac{\text { conc, in poby } \times \text { mol me of compound (o) }}{22.4}$ 
Table 5-3. GC-MS Data of Indoor Air Samples From the Central Waste Complex: Concentrations in Microgram/Meter ${ }^{3}$ (20 sheets)

Ifm background

3) BACKGROUNO BLANKS FOR LOWFLASHPOINT MIXEO WASTE AND ALKALUMETALSTORAGE BUILDINGS

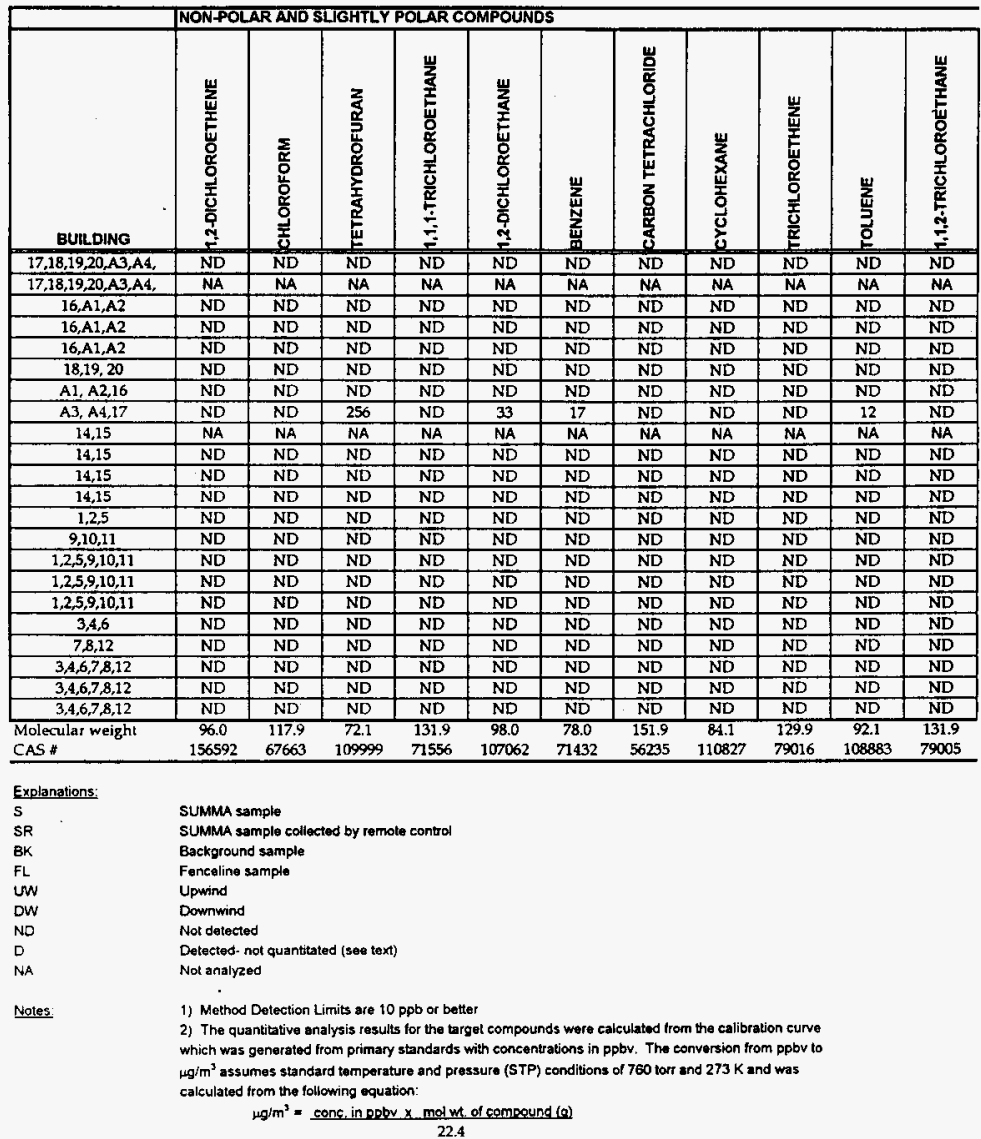


Table 5-3. GC-MS Data of Indoor Air Samples From the Central Waste Complex: Concentrations in Microgram/Meter. ${ }^{3}$ (20 sheets)

Ifm background

2) BACKGROUND BLANKS FOR LOW ELASH POIMT MIXED WASTE AND ALKALI METALSSTORAGE BUILDIHCS

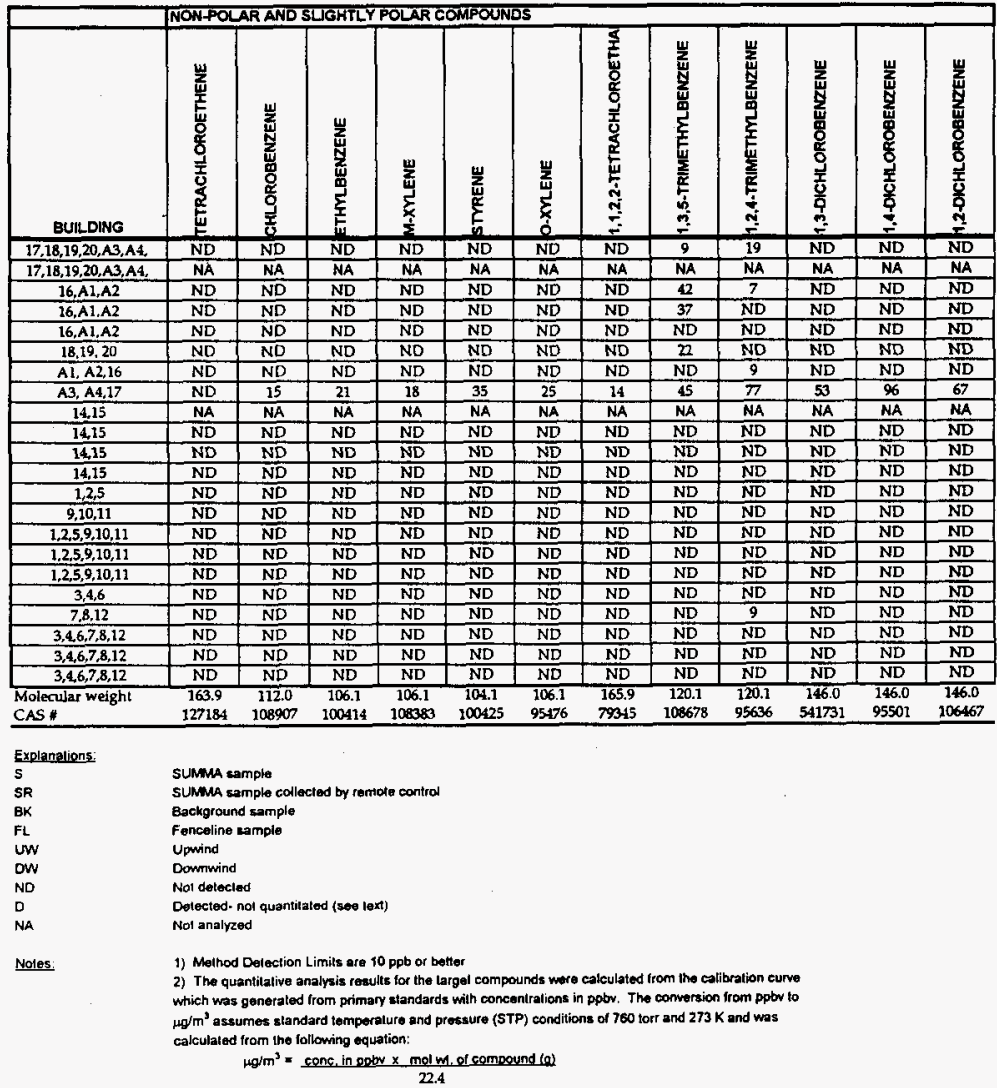


Table 5-3. GC-MS Data of Indoor Air Samples From the Central Waste Complex: Concentrations in Microgram/Meter. ${ }^{3}$ (20 sheets)

Ifm background

2LBACKGRQUND BLANKS FOR LOW FLASH POINI MIXEO WIASIE ANDALKALU MEIAL STORAGE BUHLDINGS

\begin{tabular}{|c|c|c|c|c|c|c|c|}
\hline \multirow[b]{2}{*}{ EULLDING } & \multicolumn{7}{|c|}{ POLAR COMPOUNDS } \\
\hline & 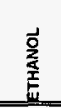 & 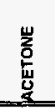 & 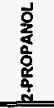 & 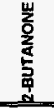 & $\begin{array}{l}\frac{1}{2} \\
\frac{2}{5} \\
\frac{9}{2} \\
\end{array}$ & 站 & 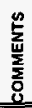 \\
\hline$\overline{17,18,19,20, A 3, A 4}$ & ND & ND & ND & $\overline{\mathrm{ND}}$ & D & D & \\
\hline $17,18,19,20, A 3, A 4$ & NA & NA & NA & NA & NA & NA & \\
\hline $16, \mathrm{~A} 1, \mathrm{~A} 2$ & $\mathrm{ND}$ & ND & ND & $\mathrm{ND}$ & $\bar{D}$ & ND & \\
\hline $16, \mathrm{Al}, \mathrm{A} 2$ & ND & ND & ND & ND & ND & ND & \\
\hline $16, \mathrm{~A} 1, \mathrm{~A} 2$ & $\mathrm{ND}$ & ND & $\mathrm{ND}$ & NO & ND & ND & \\
\hline $18,19,20$ & ND & ND & ND & ND & ND & ND & \\
\hline$A 1, A 2,16$ & ND & ND & ND & ND & $\bar{D}$ & $\bar{D}$ & \\
\hline $\mathrm{A}^{3}, \mathrm{~A} 4,17$ & $\bar{D}$ & $D$ & $\bar{D}$ & D & D & $\mathrm{D}$ & \\
\hline 14,15 & NA & NA & NA & NA & NA & NA & \\
\hline 14,15 & ND & ND & ND & ND & ND & ND & \\
\hline 14,15 & ND & ND & 5 & ND & 93 & 51 & \\
\hline 14,15 & ND & ND & ND. & ND & ND & ND & \\
\hline $1,2,5$ & ND & ND & ND & ND & ND & $\mathrm{ND}$ & \\
\hline $9,10,11$ & $\overline{\mathrm{ND}}$ & ND & ND & ND & ND & ND & \\
\hline $1,2,5,9,10,11$ & ND & ND & ND & ND & ND & ND & \\
\hline $1,2,5,9,10,11$ & ND & ND & $\mathrm{ND}$ & ND & ND & ND & \\
\hline $1,2,5,9,10,11$ & ND & ND & ND & ND & ND & ND & \\
\hline $3,4,6$ & ND & ND & ND & ND & ND & ND & \\
\hline $7,8,12$ & ND & $\mathrm{ND}$ & ND & ND & ND & ND & \\
\hline $3,4,6,7,8,12$ & ND & ND & ND & ND & $\bar{D}$ & ND & \\
\hline $3,4,6,7,8,12$ & ND & ND & ND & ND & ND & ND & \\
\hline $3,4,6,7,8,12$ & ND & ND & ND & ND & ND & ND & \\
\hline Molecular weight & 46.0 & 58.0 & 60.1 & 72.1 & 74.1 & 100.1 & \\
\hline CAS \# & 64175 & 67641 & 67630 & 78933 & 71363 & 108101 & \\
\hline
\end{tabular}

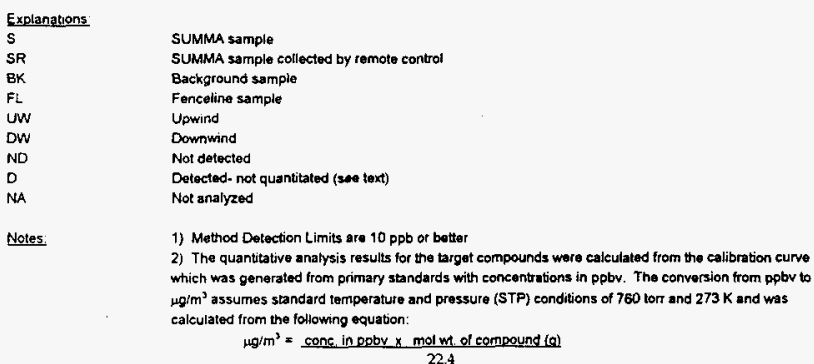




\section{$8 \mathrm{I}-\varsigma$}

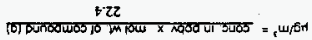

uolienbe Gumoing an! woll pajelnales

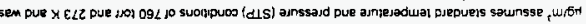

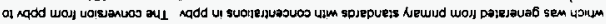

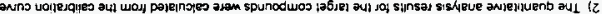

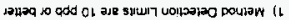

\$9ा0

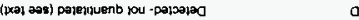

pejajep lon

purmunoa

puindn

adues autiasuad

oduce Dunojbyee

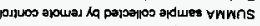

aqdues vwinns

\begin{tabular}{|c|c|c|c|c|c|c|c|c|c|c|}
\hline $\begin{array}{l}\text { EFoll } \\
1.96\end{array}$ & $\begin{array}{c}\text { ErES } \\
0.86\end{array}$ & $\begin{array}{c}260 E L \\
\text { Ot8 }\end{array}$ & $\begin{array}{l}\text { IEISL } \\
\text { V } \angle 8 t\end{array}$ & $\begin{array}{l}\log 24 \\
096\end{array}$ & $\begin{array}{c}\text { b1052 } \\
029\end{array}$ & $\begin{array}{l}58252 \\
189\end{array}$ & & & \multicolumn{2}{|c|}{ 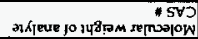 } \\
\hline$a \mathbf{N}$ & aN & $\overline{a N}$ & $\overline{a N}$ & $a N$ & aN & aN & Шबन्त & $\forall 90$ bV stogld & ds & $5 \mathrm{~V}$ \\
\hline $8 E$ & ON & $a N$ & $\mathrm{GN}$ & $\mathrm{dN}$ & aN & aN & $\omega / \bar{n}$ & YLOEV St09L1 & dS & $\varepsilon \forall$ \\
\hline $6 \mathrm{~L}$ & aN & aN & dN & aN & aN & an & 내유 & V0I'Z S509 IL & ds & $z V$ \\
\hline IN & aN & aN & aN & GN & aN & $\mathrm{aN}$ & एणन & II'IY'S6OSII & dS & IV \\
\hline aN & IN & an & aN & $\mathrm{aN}$ & aN & $E$ & Wfon & $\forall$ LOOR' SHO9LA & ys & $0 z$ \\
\hline aN & aN & बN & aN & $\mathrm{GN}$ & aN & aN & एकाष्त & V20'61'5r09LA & עS & $6 \mathrm{~L}$ \\
\hline IN & $a N$ & $\square \mathrm{N}$ & aN & aN & aN & aN & एकाषणत & "Co:8I StogLI & is & $8 \mathrm{I}$ \\
\hline ầ & $\mathrm{dN}$ & aN & $a N$ & aN & $d N$ & aN & ए। & $\forall 50<\angle T$ S509LU & $\frac{25}{d S}$ & $\frac{\text { L }}{\mathrm{LI}}$ \\
\hline $6 \mathrm{t}$ & aN & $\mathrm{aN}$ & $a N$ & aN & aN & an & एकाज्त & $\forall 60^{\circ} 91^{-} \mathrm{S} 50912$ & d5 & 9I \\
\hline tE & GN & an & aN & CN & aN & $\mathrm{aN}$ & WW & $\forall$ zoI'st $^{-} s t 09 \perp \mathrm{J}$ & is & $\leqq \mathfrak{l}$ \\
\hline $6 \mathrm{~L}$ & GN & 9ZE & aN & $\mathrm{CN}$ & GN & aN & LW/Fה & $\forall$ sol'ts $5+09 . \mathrm{LI}$ & ys & $(4-197) \mathrm{El}$ \\
\hline aN & aN & 9642 & $\angle 891$ & $\mathrm{ON}$ & aN & aN & W/ & 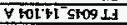 & yS & 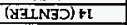 \\
\hline an & $\mathrm{aN}$ & $\mathrm{aN}$ & aN & $\mathrm{aN}$ & aN & $\mathrm{dN}$ & $\omega \beta$ & $\forall$ got $t^{-}$sto9 Lt & ys & $(\mathrm{LH}$ DId $) \mathrm{bl}$ \\
\hline aN & aN & $\mathrm{aN}$ & $a_{N}$ & $\mathrm{aN}$ & aN & aN & W/ & VOET EI SFO9LI & لys & EI \\
\hline aN & aN & aN & aN & $\overline{\mathrm{CN}}$ & aN & aN & W/ & V6RI'ZI-9609]1 & ys & 21 \\
\hline ELt & aN & $80 \varepsilon$ & $9 \varepsilon$ & aN & aN & aN & +W/ 제 & VSIT'LI-STOPLI & ys & it \\
\hline IN & aN & aN & GN & aN & aN & aN & WW & V9lt ố-shogld & ys & OL \\
\hline aN & aN & GN & aN & aN & aN & aN & шक्न & $\checkmark \angle I I^{\prime} 60-9509 L I$ & ys & 6 \\
\hline aN & aN & aN & aN & $\mathrm{dN}$ & aN & aN & समन्त & V8ZI'g0-Sh09LI & $\Delta S$ & 8 \\
\hline an & aN & EI & $\mathrm{aN}$ & aN & GN & an & कात्त & $\forall \angle 2 L^{2} \angle 0^{-} S 609$ LI & $\Delta S$ & $\div$ \\
\hline aN & GN & dN & aN & aN & an & aN & प्यमित्र & $\forall Z 2{ }^{\circ} 90^{\circ}$ S609 LA & dS & 9 \\
\hline 272 & aN & 6EL & $\overrightarrow{\mathrm{dN}}$ & 6 & $\mathrm{aN}$ & $a_{N}$ & एकत & FoLI'so-shos!-I & dS & 5 \\
\hline aN & aN & $\mathrm{CN}$ & $\mathrm{dN}$ & aN & $\mathrm{dN}$ & aN & एकान्त & 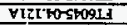 & ds & $b$ \\
\hline aN & an & GN & aN & $a N$ & aN & an & W/ & $\forall 0 z L^{\prime} \mid 00^{-} 5109$ LS & $\frac{5}{d S}$ & $\varepsilon$ \\
\hline $\mathbf{z I}$ & aN & $\pi$ & GN & aN & an & aN & Wस & $\forall 80{ }^{2} 20-96091 \mathrm{I}$ & ys & $z$ \\
\hline GN & an & aN & aN & GN & aN & aN & समझक्त & V60I'LO-GE09.LI & पS & L \\
\hline$\frac{2}{\frac{1}{\mathbf{m}}}$ & 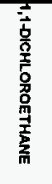 & 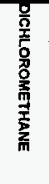 & 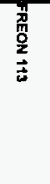 & 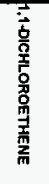 & $\begin{array}{l}\frac{5}{3} \\
3 \\
\mathbf{3} \\
9 \\
\mathbf{9} \\
\mathbf{9} \\
\frac{9}{7}\end{array}$ & 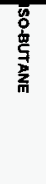 & 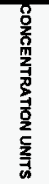 & $\begin{array}{l}\frac{a}{2} \\
\frac{5}{7} \\
\text { m } \\
\overline{0}\end{array}$ & 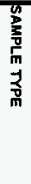 & ONIOTII) \\
\hline & Q: & yn & $\pm H$ & & & & & & 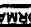 & VEANFP \\
\hline
\end{tabular}

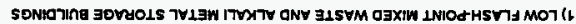

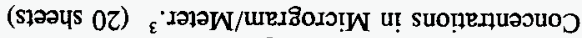

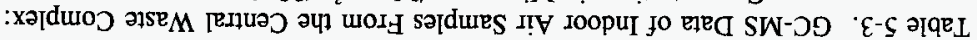


Table 5-3. GC-MS Data of Indoor Air Samples From the Central Waste Complex: Concentrations in Microgram/Meter. ${ }^{3}$ (20 sheets)

CWC_LFM,XLS

1) LOW FLASHPOINT MIXED WASTE AND ALKALI METAL STORAGE BUILDINOS

\begin{tabular}{|c|c|c|c|c|c|c|c|c|c|c|c|}
\hline \multicolumn{12}{|c|}{ NON POLAR AND SLGHTLY POLAR COMPOUNDS } \\
\hline BUILDINO - & 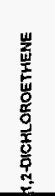 & 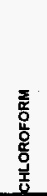 & 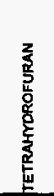 & 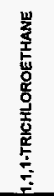 & 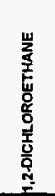 & 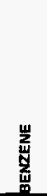 & 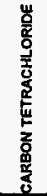 & 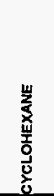 & 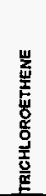 & $\begin{array}{l}\text { 崖 } \\
\text { 岇 } \\
\text { 号 }\end{array}$ & 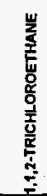 \\
\hline 1 & ND & ND & ND & $\mathrm{ND}$ & ND & ND & ND & ND & ND & 984 & ND \\
\hline 2 & ND & ND & ND & 20 & ND & ND & ND & 34 & ND & 198 & ND \\
\hline 3 & $\mathrm{ND}$ & 19 & ND & ND & $\mathrm{ND}$ & ND & No & ND & ND & 127 & ND \\
\hline 4 & $\mathrm{ND}$ & ND & ND & ND & ND & ND & ND & NO & ND & 16 & ND \\
\hline 5 & $\mathrm{ND}$ & ND & 45 & ND & ND & 10 & ND & $\mathrm{ND}$ & ND & 78 & $\overline{\mathrm{ND}}$ \\
\hline 6 & NO & ND & ND & ND & ND & ND & ND & ND & ND & 15 & ND \\
\hline 7 & ND & ND & ND & ND & ND & ND & ND & ND & ND & 904 & ND \\
\hline 8 & ND & ND & ND & ND & ND & ND & ND & ND & ND & ND & ND \\
\hline 9 & $\overline{\mathrm{ND}}$ & ND & ND & $\overline{N D}$ & ND & ND & ND & ND & ND & ND & ND \\
\hline 10 & NO & ND & ND & ND & ND & ND & ND & NO & ND & ND & ND \\
\hline 11 & $\mathrm{ND}$ & ND & 79 & ND & ND & ND & ND & ND & ND & 395 & ND \\
\hline 12 & ND & ND. & ND & $\overline{\mathrm{ND}}$ & $\mathrm{ND}$ & ND & ND & ND & ND & 341 & ND \\
\hline 13 & ND & ND & ND & ND & ND & ND & ND & ND & ND & 29 & ND \\
\hline $14(\overline{R G G H T})$ & ND & ND & ND & ND & ND & ND & 1736 & 158 & ND & 765 & ND \\
\hline 14 (CENTER) & ND & ND & ND & ND & ND & ND & 57 & NO & ND & 1014 & ND \\
\hline $1+$ (LEFT) & $\mathrm{ND}$ & ND & ND & ND & ND & ND & 29 & ND & ND & 2084 & ND \\
\hline 15 & $N \bar{D}$ & ND & ND & ND & ND & ND & ND & $\overline{N D}$ & ND & 19 & ND \\
\hline 16 & ND & 5 & ND & 28 & ND & ND & ND & ND & ND & 17 & ND \\
\hline 17 & ND & ND & ND & $\mathrm{ND}$ & ND & $\mathrm{ND}$ & ND & $\overline{N D}$ & ND & 57 & ND \\
\hline 18 & ND & ND & ND & ND & ND & ND & 145 & 44 & ND & 82 & ND \\
\hline 19 & $\overline{\mathrm{MO}}$ & ND & ND & ND & NDD & ND & ND & ND & ND & ND & ND \\
\hline 20 & $\overline{\mathrm{ND}}$ & ND & ND & $\overline{N D}$ & ND & ND & 32 & 79 & ND & ND & ND \\
\hline$\overline{A 1}$ & $\mathrm{ND}$ & ND & ND & ND & $\mathrm{ND}$ & $\mathrm{ND}$ & ND & ND & ND & ND & ND \\
\hline$A 2$ & ND & ND & 10 & $\mathrm{ND}$ & 4 & ND & ND & ND & ND & ND & ND \\
\hline $\mathrm{A}^{\overline{3}}$ & $\overline{\mathrm{ND}}$ & ND & 80 & $\overline{N D}$ & 13 & 3 & $\mathrm{ND}$ & ND & ND & 4 & ND \\
\hline A4 & $\mathrm{ND}$ & ND & ND & ND & $\mathrm{ND}$ & ND & ND & ND & ND & 7 & ND \\
\hline $\begin{array}{l}\text { Molecular weight } \\
\text { CAS n }\end{array}$ & $\begin{array}{c}96.0 \\
156592\end{array}$ & $\begin{array}{l}117.9 \\
67663\end{array}$ & $\begin{array}{c}72.1 \\
109999\end{array}$ & $\begin{array}{l}731.9 \\
71556\end{array}$ & $\begin{array}{c}98.0 \\
107062\end{array}$ & $\begin{array}{c}78.0 \\
71432\end{array}$ & $\begin{array}{l}151.9 \\
56235\end{array}$ & $\begin{array}{c}84.1 \\
110827\end{array}$ & $\begin{array}{l}129.9 \\
79016\end{array}$ & $\begin{array}{r}92.1 \\
103883\end{array}$ & $\begin{array}{l}731.9 \\
79006\end{array}$ \\
\hline
\end{tabular}

$\begin{array}{ll}\text { Explapations } & \\ \text { S } & \text { SUMMA sample } \\ \text { SR } & \text { SUMSA sompie coliected Dy remote contro } \\ \text { BK } & \text { Background sample } \\ \text { FL } & \text { Fenceline sample } \\ \text { UW } & \text { Upwind } \\ \text { OW } & \text { Downinind } \\ \text { ND } & \text { Not detected } \\ \text { D } & \text { Detected- not Quantitated (see text) }\end{array}$

Noles 1) Method Detection Limits are 10 spob or better

2) The quanttative anahysis resuns tor the arget compounds were calculated hom the calibration curve which was generated from priman standards with concentrations in pobv. The comversion from pptv to Hgim' assumes standard temperature and pressure (STP) canditions of 760 torr and $273 \mathrm{~K}$ and was calculated from the following equation:

$\mu \mathrm{g}^{2} \mathrm{~m}^{2}=$ conc in opto $\times \mathrm{mol}$ w of compeuns (c) 


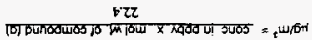

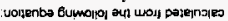

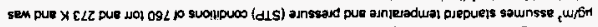

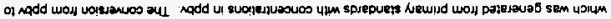

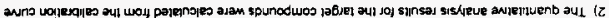

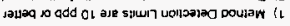

(prat a.s) paleupuenb you - paljala

dejceiad ion

Dulmumar

pliundn

adwes punojoxioga

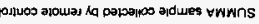

adwes vwwns

\begin{tabular}{|c|c|c|c|c|c|c|c|c|c|c|c|c|}
\hline $\begin{array}{l}\angle 9690 \mathrm{I} \\
0.97 \mathrm{I}\end{array}$ & $\begin{array}{l}\text { L0956 } \\
0.9+1\end{array}$ & $\begin{array}{l}\text { IE } L \text { LtF } \\
\text { 09t I }\end{array}$ & $\begin{array}{l}98956 \\
1021\end{array}$ & $\begin{array}{l}\text { 8L980L } \\
\text { I0ZL }\end{array}$ & $\begin{array}{l}\text { SFESL } \\
6991\end{array}$ & $\begin{array}{l}9 \angle F-56 \\
t=0 \mathrm{t}\end{array}$ & $\begin{array}{l}\text { SZtDOL } \\
\text { ['tDI }\end{array}$ & $\begin{array}{l}\text { C85SOI } \\
\text { I'90I }\end{array}$ & $\begin{array}{c}\text { TLCOL } \\
\text { ['90L }\end{array}$ & $\begin{array}{l}\angle 0680 \mathrm{I} \\
0 \mathrm{ZII}\end{array}$ & $\begin{array}{l}\text { FLLZL } \\
6 \mathrm{gl}\end{array}$ & 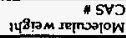 \\
\hline $9 z$ & $\overline{0 z}$ & EL & $\angle 2$ & 91 & $L$ & 6 & FI & 6 & 6 & $s$ & $\overline{Q N}$ & $\mathrm{EV}$ \\
\hline$a \mathrm{~N}$ & aN & $a N$ & aN & 91 & $\mathrm{aN}$ & GN & $\overrightarrow{O N}$ & aN & $L$ & aN & $a N$ & IV \\
\hline$\overline{a N}$ & IN & aN & 9 & $u$ & $\mathrm{aN}$ & $z z$ & aN & $E$ & $O E$ & $\mathrm{aN}$ & $\mathrm{gN}$ & $\overline{0 z}$ \\
\hline aN & IN & aN & $s$ & $\mathrm{tl}$ & $\mathrm{dN}$ & aN & an & aN & aN & GN & aN & 6I \\
\hline$a N$ & GN & $a N$ & $\mathrm{aN}$ & $\mathrm{aN}$ & $\mathrm{aN}$ & 6 & aN & 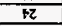 & st & aN & CN & 9t \\
\hline aN & $\mathrm{aN}$ & aN & 6 & 81 & $\mathrm{GN}$ & $\overline{z E}$ & $\mathrm{aN}$ & 28 & 62 & aN & $\mathrm{dN}$ & SI \\
\hline $\mathrm{aN}$ & aN & aN & 8 & $\angle E$ & $\mathbf{a N}$ & S6ZL & 82 & OSSI & $00 z$ & aN & aN & $(4 . \sqrt{3} 7)+\mathrm{L}$ \\
\hline aN & GN & $\mathrm{dN}$ & 9 & 06 & aN & 89 & $L$ & 85 & 06 & IN & ON & (YZINAD) $+\mathrm{I}$ \\
\hline $\mathrm{aN}$ & an & $\mathrm{aN}$ & a & I6 & IN & 99 & 4 & $4 \mathrm{Z}$ & $4 S$ & $\mathrm{aN}$ & $\mathrm{dN}$ & (1HON) \\
\hline $\mathrm{aN}$ & â & $\mathrm{aN}$ & $z \varepsilon$ & 8 & aN & aN & $a N$ & aN & $\mathrm{aN}$ & $\mathrm{aN}$ & ON & EI \\
\hline GN & aN & aN & II & $\mathbf{S}$ & GN & +2 & aN & 25 & $\varepsilon$ & aN & $\mathrm{GN}$ & 8 \\
\hline aN & $\mathrm{aN}$ & aN & 90 & $\mathrm{aN}$ & aN & GN & $\mathrm{CN}$ & $\Delta t$ & 6 & $\mathrm{ON}$ & $a N$ & 6 \\
\hline$a N$ & aN & $\frac{a}{a}$ & $\mathrm{aN}$ & $\frac{d N}{d N}$ & $\mathrm{GN}$ & $\mathrm{ON}$ & $\mathrm{GN}$ & $\mathrm{GN}$ & $\frac{5}{5}$ & on & ON & $\frac{6}{9}$ \\
\hline \%I & 202 & 961 & 6 & 84 & $\mathrm{aN}$ & 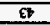 & 【N & 82 & 6 & 08 & $\mathrm{GN}$ & 5 \\
\hline $\mathrm{aN}$ & aN & $\mathrm{aN}$ & 9I & 08 & $\frac{\mathrm{N}}{\mathrm{N}}$ & $6 \mathrm{t}$ & $\mathrm{fI}$ & 99 & $6 \mathrm{II}$ & aN & $\mathrm{aN}$ & 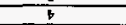 \\
\hline GN & aN & aN & $\angle B E$ & $6 \varepsilon$ & $G N$ & IZ & an & $t L$ & $\mathrm{ot}$ & aN & $\mathrm{ON}$ & $\bar{\varepsilon}$ \\
\hline $\mathrm{aN}$ & aN & aN & $L$ & aN & aN & $\overline{\mathrm{ZBZ}}$ & $L$ & 6IOL & $80 \varepsilon$ & aN & $\mathrm{dN}$ & $z$ \\
\hline aN & GN & aN & $\angle 00$ & aN & aN & GN & aN & aN & $G N$ & IN & $\mathrm{aN}$ & I \\
\hline 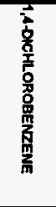 & 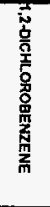 & 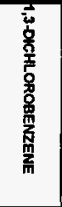 & 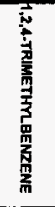 & 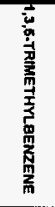 & 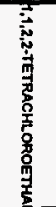 & 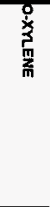 & 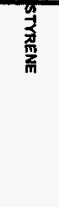 & $\begin{array}{l}\frac{5}{3} \\
\frac{3}{3} \\
\frac{m}{m}\end{array}$ & 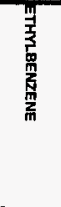 & 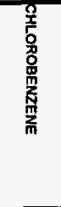 & 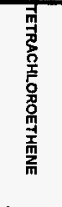 & oxigning \\
\hline
\end{tabular}

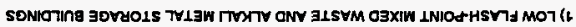

\author{
S1XNIT TMD
}

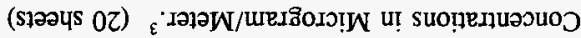

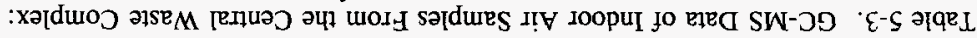


Table 5-3. GC-MS Data of Indoor Air Samples From the Central Waste Complex: Concentrations in Microgram/Meter. ${ }^{3}$ (20 sheets)

CWC.LFM.XLS

1) LOW FLASHPOINT MIXED WASTE AND ALKALI METAL STORAGE BUILDINGS

\begin{tabular}{|c|c|c|c|c|c|c|c|}
\hline \multirow[b]{2}{*}{ BUILDING } & \multicolumn{6}{|c|}{ POLAFCOMPOUNDS } & \multirow[b]{2}{*}{$\frac{5}{\frac{5}{5}}$} \\
\hline & $\begin{array}{l}\text { ㅁ } \\
\frac{3}{5} \\
\frac{1}{111}\end{array}$ & $\frac{\text { w }}{\frac{1}{0}}$ & 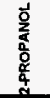 & $\begin{array}{l}\mathbf{u} \\
\mathbf{2} \\
\mathbf{2} \\
\mathbf{5} \\
\mathbf{5} \\
\mathbf{9}\end{array}$ & $\begin{array}{l}\frac{1}{0} \\
2 \\
5 \\
5 \\
0\end{array}$ & 站 & \\
\hline 1 & 170 & 7 & ND & ND & ND & ND & \\
\hline 2 & 35 & 76. & ND & 344 & 28 & 61 & \\
\hline 3 & 21 & 14 & ND & 56 & 16 & 56 & \\
\hline 4 & ND & ND & ND & ND & 149 & 67 & AVERAGEOF 2 \\
\hline 5 & 281 & 85 & 61 & ND & ND & ND & AVERAGE OF 2 \\
\hline 6 & ND & ND & ND & ND & ND & ND & \\
\hline 7 & 324 & 17 & ND & 66 & 18 & ND & \\
\hline 8 & ND & ND & ND & ND & ND & ND & AVERAGE OF 2 \\
\hline 9 & ND & ND & ND & ND & ND & ND & \\
\hline 10 & ND & ND & ND & ND & ND & ND & AVERAGE OF 2 \\
\hline 11 & ND & 139 & ND & 475 & ND & 182 & \\
\hline 12 & $\mathrm{ND}$ & ND & ND & ND & ND & ND & \\
\hline 13 & ND & $\mathrm{ND}$ & ND & ND & ND & ND & AVERAGE OF 2 \\
\hline 14 (RIGHT) & ND & 18 & 8 & 48 & ND & 469 & AVERAGE OF 2 \\
\hline 14 (CENTER) & 39 & 88 & 1055 & 66 & ND & 30 & \\
\hline 14 (LEFT) & ND & 157 & 65 & 97 & 26 & 655 & \\
\hline 15 & ND & $1 \overline{4}$ & ND & ND & ND & ND & \\
\hline 16 & ND & $\mathrm{D}$ & ND & $\mathrm{D}$ & ND & $\bar{D}$ & AVERAGEOF 2 \\
\hline 17 & ND & ND & $\mathrm{ND}$ & ND & $\mathrm{D}$ & ND & \\
\hline 18 & ND & ND & ND & ND & 34 & ND & \\
\hline 19 & D & $\mathrm{D}$ & ND & $\bar{D}$ & D & $\overline{\mathrm{D}}$ & AVERAGE OF 2 \\
\hline 20 & ND & ND & ND & ND & D & ND & \\
\hline $\mathrm{Al}$ & ND & ND & ND & ND & ND & ND & AVERAGEOE 2 \\
\hline A2 & D & D & D & $\mathrm{D}$ & $\mathrm{D}$ & D & AVERAGE OF 3 \\
\hline A3 & D & $D$ & $\mathrm{D}$ & D & $\mathrm{D}$ & $\bar{D}$ & AVERAGE OF 3 \\
\hline A4 & ND & ND & ND & ND & ND & ND & \\
\hline Molecular weight & 46.0 & 58.0 & 60.1 & 72.1 & 74.1 & 100.1 & \\
\hline CAS * & 64175 & 67641 & 67630 & 78933 & 71363 & 108101 & \\
\hline
\end{tabular}

Explanations:

$S \quad$ SUMMA sample

SR SUMMA sample coltected by remote control

EK Background sample

$\mathrm{FL} \quad$ Fenceline sample

UW Uownes

$\begin{array}{ll}\text { OW } & \text { Dowriwnd } \\ \text { ND } & \text { Not detected }\end{array}$

D Detected-not quantitated (see text)

Noles $\quad$ 1) Method Detection Limits are $10 \mathrm{ppb}$ or better

2) The quantitative analysis results for the target comoounds were calculated from the calibralion tume which was generated from primary standards with concentrations in ppbv. The comversion from pobu to $\mu \mathrm{gm} \mathrm{m}^{2}$ assumes slancard temperature and pressure (STP) conditions of 760 tor and $273 \mathrm{~K}$ and was calculated from the followng equation.

$\mu \mathrm{g} / \mathrm{m}^{3}=$ senc in opov $\times$ mol it of compound (o) 


\subsubsection{Building 2402 WC}

This building had low levels of non-polar and moderately polar compounds. Hexane and tetrahydrofuran showed up in one sample but they were also present at similar concentrations in one background sample. Similarly, polar compounds such as ethanol, acetone, 2-propanol, 2-butanone, n-butanol, and 4-methyl-2-pentanone were present at similar concentration levels in both the indoor and the background sample. In addition to these compounds, the library searches found 1,4-dioxane, benzaldehyde, 1-phenylethanone, and a,a-dimethylbenzene methanol at slightly elevated levels ( 1 to 5 percent of total area counts). The data for polar compounds differ between the samples. It is suggested to re-sample this building if these types of compounds are of special concern.

\subsubsection{Building 2402 WD}

Although only one indoor and one background sample were analyzed, the data are consistent. They show that the compounds found were present at similar concentrations both inside and outside the building: dichloromethane ( $63 \mathrm{ppb}$ inside, $104 \mathrm{ppb}$ outside); toluene ( 6 and $7 \mathrm{ppb}$ ); m-xylene (16 and $20 \mathrm{ppb}$ ); and 1,2,4- trimethylbenzene ( 30 and $36 \mathrm{ppb}$ ). Of the polar compounds, 2-butanone was present both inside and outside (26 and $31 \mathrm{ppb}$ ) but 4-methyl-2-pentanone (67 ppb) and n-butanol (10 ppb) was only in the background sample. The upwind and downwind samples collected on this day did not show significant levels of any of these compounds. Additional sampling is required to find the sources of these compounds .

\subsubsection{Building 2402 WE}

The two samples from different location inside this building gave very good agreement showing elevated concentrations of dichloromethane $(234 \mathrm{ppb})$. Furthermore, minor levels of 1,1,1-trichloroethane (14 ppb), Toluene (12 ppb), and xylenes (about $5 \mathrm{ppb}$ ) were measured. None of these compounds were found in the background sample. No additional compounds were found at significant levels with library matching.

\subsubsection{Building 2402 WF}

The analytical results showed consistently low levels $(<10 \mathrm{ppb})$ of the priority analytes. 2-butoxyethanol and 4-methyl-2-pentanone were present in both the indoor samples and in the background and could have an external source. The major components of the air samples were predominantly pinene, with smaller amounts of 3-carene, limonene, phellandrene, sabinene, and camphene. Because these natural essential oils did not appear in the background samples, they most likely were emitted by the waste stored in this building. The probable origin was natural turpentine oil which is 58 to 65 percent a-pinene and 30 percent b-pinene. 


\subsubsection{Building 2402 WG}

There is very little buildup of volatile organic compounds in this building.

4-methyl-2-pentanone is present in both the indoor and the background samples. The non-polars are all below $10 \mathrm{ppb}$ and the polar compounds consist predominantly of 2-butoxyethanol. In addition to the compounds used in the calibration standard, the library search found small concentration of benzaldehyde.

\subsubsection{Building 2402 WH}

The limited data of this baseline study indicate that this building has low levels of VOCs. 4-methyl-2-pentanone is present in the building samples and in the background. One sample showed that 2-butoxyethanol was the dominant polar constituent. The analysis of a duplicate sample (data not included in this report) showed elevated levels of polar compounds such as 2-butanone, ethanol, acetone, 4-methyl-2-pentanone. It is felt that this is an artifact of the analysis conditions at that time (which were later corrected) but it is recommended to re-sample this building to confirm these conclusions.

\subsubsection{Building 2402 WI}

This building has low concentrations of organic compounds. One sample showed the presence of 1,3,5-trimethylbenzene but this compound did not appear in the second sample. 4-methyl-2-pentanone appears in all indoor and in the background samples. The library searches identified benzaldehyde as the major compound outside the quantitation list.

\subsubsection{Building $2402 \mathrm{WJ}$}

Both samples from this location showed low levels of toluene ( $30 \mathrm{ppb}$ average). Polar compounds such as 2-butanone, n-butanol, 4-methyl-2-pentanone, and 2-butoxyethanol are present in both the background and the building samples. 1-methoxy-2-propanol and benzaldehyde are the major compounds identified by library search.

\subsubsection{Building 2402 WK}

The concentrations for non-polar and moderately polar compounds are below $10 \mathrm{ppb}$. Polars such as 2-butoxyethanol, n-butanol, 4-methyl-2-pentanone are present indoors but not in the background sample. Benzaldehyde was found in the indoors sample with the mass spectral library. 


\subsubsection{Building 2402 WL}

The two samples taken at different locations in the building differ somewhat in their compositions: Both have elevated levels of 4-methyl-2-pentanone (107 and $340 \mathrm{ppb}$ ) and 2-butoxyethanol (not quantified). The second sample has higher levels of polar compounds such as 2-butanone (773 ppb), n-butanol (545 ppb), ethanol (168 ppb), acetone ( $97 \mathrm{ppb})$, and moderate polars such as tetrahydrofuran $(84 \mathrm{ppb})$. This discrepancy needs to be resolved with an additional sampling event. Other compounds identified from their mass spectra include: benzaldehyde, 1-phenylethanone, and 1,4-dioxane.

\subsubsection{Building 2403 WA}

This building was sampled in four different locations. The analyses from the quadrants gave consistent results showing elevated levels of dichloromethane ( 31 to $133 \mathrm{ppb}$ ) and 1,1,1-trichloroethane (14 to $70 \mathrm{ppb}$ ) compared to the background samples. Lower concentrations of toluene (7 to $21 \mathrm{ppb})$ and $n$-hexane ( 4 to $17 \mathrm{ppb}$ ) were measured but the upwind sample had similar concentrations of these compounds. Some samples had higher concentrations of polars (4-methyl-2-pentanone, n-butanol, 2-butanone, ) but these compounds were also in the upwind sample. Additional compounds found include 2-butoxyethanol, benzaldehyde, and natural oils such as pinene, carene, and limonene.

\subsubsection{Building 2403 WB}

The results from the four sampled sections are in very good agreement showing that this building has very low levels of VOCs. Carbontetrachloride is present at 8 to $20 \mathrm{ppb}$, and smaller quantities of xylenes, acetone, ethyl- and trimethylbenzenes were measured. Other compounds in these samples included pinene and trichlorofluoromethane.

\subsubsection{Building 2403 WC}

The results of the 4 analyses are very reproducible and show that this building has elevated concentrations of dichloromethane ( 50 to $104 \mathrm{ppb}$ ) compared to the background sample. Lower concentrations of tetrachloroethene ( 21 to $27 \mathrm{ppb}$ ), 1,1,1-trichloroethane (12 to $16 \mathrm{ppb}$ ), and toluene ( 3 to $6 \mathrm{ppb}$ ) were measured. Polars are generally low and consist of n-butanol, 4-methyl-2-pentanone, and acetone all of which show up in the background samples. Additional compounds that were found inside the building but not in the background samples include 2-butoxyethanol, 1-methoxy-2-propanol, hexanal, pinene, and carene. 


\subsubsection{Building $2403 \mathrm{WD}$}

Seven indoor (one is a duplicate) and three outside background samples were analyzed from this large building. The data show that this building has very low concentrations of non-polar and moderately polar volatile organic: Ethylbenzene, xylenes, and trimethylbenzenes were present in all samples at concentrations below $10 \mathrm{ppb}$. One sample also had small amounts of hexane $(16 \mathrm{ppb})$ and tetrahydrofuran ( $34 \mathrm{ppb})$. Polars such as 4-methyl-2-pentanone and 2-butoxyethanol occur in most samples. One sample also contained other polar compounds like n-butanol, 2-butanone, acetone, ethanol, and 2-propanol. The library searches tentatively identified pinene, 3-carene, 1-methoxy-2-propanol, and benzaldehyde as significant components of the indoor air.

\subsection{LOW FLASH-POINT MIXED WASTE AND ALKALI METAL STORAGE BUILDINGS}

\subsubsection{Module A1}

The two samples taken from this module showed very low levels of volatile organics. Aromatic compounds such as ethyl- and trimethylbenzene were present in concentrations below the Method Detection Limit of $10 \mathrm{ppb}$. Additional compounds found in this unit include 2-butoxyethanol and benzaldehyde.

\subsubsection{Module A2}

The three samples from this module contained non-polar and moderately polar VOC below $10 \mathrm{ppb}$. Polar compounds such as methanol, ethanol, acetone, 2-butanone, n-butanol, and 2-butoxyethanol were detected but their concentration needs to be determined.

\subsubsection{Module A3}

Hexane and tetrahydrofuran are above the MDL at 10 and $25 \mathrm{ppb}$, respectively. However, the background sample does also contain similar levels of these compounds. Polar compounds such as Ethanol, acetone, 2-propanol, 2-butanone, n-butanol, 4-methyl-2-pentanone, and 2-butoxyethanol were detected in both the module and the background.

\subsubsection{Module A4}

This unit has no VOCs above the MDL of $10 \mathrm{ppb}$. 2-butoxyethanol was detected with library searching. 


\subsubsection{Module LFM \# 1}

One sample collected from this unit showed increased concentrations of toluene (239 ppb), ethanol ( $83 \mathrm{ppb})$, and 1,2,4-trimethylbenzene (76 ppb). None of these compounds were found in the background sample. The library matching found 3-heptanone in the indoor sample.

\subsubsection{Module LFM \# 2}

Aromatic compounds such as $\mathrm{m}$-xylene (215 ppb), o-xylene ( $59 \mathrm{ppb}$ ), ethylbenzene (65 ppb), and toluene ( $48 \mathrm{ppb}$ ) were prominent in this sample. It also had higher concentrations of 2-butanone (MEK, $107 \mathrm{ppb}$ ), acetone (29 ppb), and ethanol (17 ppb). None of these compounds were in the background.

\subsubsection{Module LFM \# 3}

1,2,4-trimethylbenzene ( $72 \mathrm{ppb})$ and toluene $(31 \mathrm{ppb})$ were somewhat increased inside the module but did not show up in the background sample. Benzaldehyde was also detected.

\subsubsection{Module LFM \# 4}

The two samples analyzed from this unit detected low concentrations of 4-methyl-2-pentanone (15 ppb) and $\mathrm{m}$-xylene (14 ppb). One sample also contained $\mathrm{n}$-butanol and 1,3,5-trimethylbenzene. Additionally, a considerable amount of benzaldehyde was found in both samples. The concentration needs to be determined with standards.

\subsubsection{Module LFM \# 5}

Analyses of two grab samples determined that this module is dominated by very volatile compounds: Ethanol (137 ppb), hexane (63 ppb), 2-propanol (61 ppb), acetone (33 ppb) and dichloromethane ( $37 \mathrm{ppb}$ ). Curiously, one sample had about equal concentrations of the three dichlorobenzene isomers (about $60 \mathrm{ppb}$ each) but these compounds were not detected in the second sample. The presence of these higher boiling VOC needs to be confirmed. Benzaldehyde, 3-heptanone, and 2-hexanone were detected with spectral library searches.

\subsubsection{Module LFM \# 6}

This unit did not show any significant levels of volatile organic compounds. 


\subsubsection{Module LFM \# 7}

The one sample analyzed from this module contained toluene $(220 \mathrm{ppb})$ and ethanol (158 ppb). Furthermore, lower concentrations of 2-butanone (21 ppb) and m-xylene (10 ppb) were detected. 3-heptanone was also present in this sample.

\subsubsection{Module LFM \# 8}

Both samples from this unit showed that low concentrations of aromatic hydrocarbons were present: $\mathrm{m}$-xylene (11 ppb), ethylbenzene (9 ppb), and o-xylene (5 ppb). Additionally, 3-heptanone was identified from its mass spectrum.

\subsubsection{Module LFM \# 9}

Analysis of one sample shows that the VOCs in this module are very low in concentration, all below $10 \mathrm{ppb}$. The library searches indicate that 1-phenylethanone and 3-heptanone are also present.

\subsubsection{Module LFM \# 10}

This unit has low levels of aromatic VOCs: xylenes and ethylbenzene are all below $10 \mathrm{ppb}$. The major components in this sample were identified as 2-heptanone and 3-heptanone. However, they were not quantitated with an external standard.

\subsubsection{Module LFM \# 11}

This sample has significant amounts of 2-butanone (also known as methyl ethyl ketone or MEK) with $1465 \mathrm{ppb}$. Other major compounds include hexane (123 ppb), toluene (96 ppb), dichloromethane (82 ppb), acetone (54 ppb), m-xylene (46 ppb), 4-metyl-2-pentanone or "hexone" (41 ppb), and tetrahydrofuran (25 ppb). The additional compounds identified with library searches are relatively minor.

\subsubsection{Module LFM \# 12}

The compounds in this unit are all aromatic VOCs: toluene (83 ppb), m-xylene (29 ppb), 1,2,4-trimethylbenzene (13 ppb), and o-xylene (10 ppb). 3-heptanone and benzaldehyde were also identified. 


\subsubsection{Module LFM \# 13}

Minor quantities of toluene (7 ppb), 1,3,5-trimethylbenzene ( $7 \mathrm{ppb}$ ) and $1,2,4$-trimethylbenzene ( $5 \mathrm{ppb}$ ) were measured. Benzaldehyde is also in this sample at similar concentration levels.

\subsubsection{Module LFM \# 14}

This module contains three separate compartments that were sampled individually. The first compartment had elevated levels of carbontetrachloride (256 ppb), toluene (186 ppb), 4-methyl-2-pentanone (105 ppb), m-xylene (50 ppb), and cyclohexane (42 ppb).

The center unit had high concentrations of 2-propanol $(1,055 \mathrm{ppb})$, dichloromethane (479 ppb), toluene (247 ppb), Freon 113 (190 ppb), m-xylene (55 ppb), and acetone (34 ppb).

The third compartment was dominated by toluene (507 ppb), m-xylene (329 ppb), and $\mathrm{p}$-xylene (273 ppb), followed by 4-methyl-2-pentanone (147 ppb), dichloromethane (87 ppb), 2-propanol (65 ppb), acetone (61 ppb), ethylbenzene (42 ppb), and 2-butanone (30 ppb). This sample contains a relatively high concentration of a compound that has been tentatively identified as acetonitrile. The identity and concentration of this compound have to be confirmed with a neat standard.

\subsubsection{Module LFM \# 15}

This module contains low concentrations of $\mathrm{m}$-xylene (17 ppb); $\mathrm{n}$-hexane, o-xylene, ethylbenzene and others are below the $10 \mathrm{ppb}$ MDL. Octanal was also identified in this sample as a significant component.

\subsubsection{Module LFM \# 16}

Less than $10 \mathrm{ppb}$ were measured for $\mathrm{n}$-hexane, m-xylene, and toluene in the two samples. Of the polar compounds, 2-butanone was present in both analyses ( $35 \mathrm{ppb}$ average), but 2-butoxyethanol was only detected in one sample.

\subsubsection{Module LFM \# 17}

Low concentrations of toluene $(14 \mathrm{ppb})$ and $1,3,5$-trimethylbenzene $(10 \mathrm{ppb})$ were present in this module. 2-butoxyethanol was the major polar compound in this sample. However, this compound also appeared in the background sample and its presence needs to be confirmed by analyzing with the new, modified procedure. 


\subsubsection{Module LFM \# 18}

Several VOCs were detected in this unit: $\mathrm{m}$ - and p-xylene ( 28 and $23 \mathrm{ppb}$, respectively), carbon tetrachloride $(21 \mathrm{ppb})$, toluene $(20 \mathrm{ppb})$, cyclohexane $(12 \mathrm{ppb})$, and $\mathrm{n}$-butanol $(10 \mathrm{ppb})$. The library search also indicated the presence of small quantities of 2-butoxyethanol.

\subsubsection{Module LFM \# 19}

No non-polar or moderately polar compounds were detected above the MDL of $10 \mathrm{ppb}$. The two samples analyzed from this module also had polar compounds such as $n$-butanol and 2-butoxyethanol but the concentrations have not been determined. Small quantities of 1-phenyl ethanone and benzaldehyde are also present.

\subsubsection{Module LFM \# 20}

One sample analyzed from this unit contained cyclohexane (21 ppb), n-butanol (18 ppb), and 1,3,5-trimethylbenzene (13 ppb). The major peak in the chromatogram corresponds to 2-butoxy ethanol. This compound also shows up in the background sample and its concentration has to be measured by analyzing a new sample with the modified procedure. The mass spectral data indicate that benzaldehyde also appears in this sample. 
HNF-SD-WM-RPT-282 Rev, 0

This page intentionally left blank. 
HNF-SD-WM-RPT-282 Rev. 0

\subsection{REFERENCES}

Lockrem, L. L., 1996, Sampling and Analysis Plan, WHC-SD-WM-WAP-002, Rev. 0, Westinghouse Hanford Company, Richland, Washington.

Pierce, R. D., 1996, Internal Memorandum, 87600-RDP-96-005, Westinghouse Hanford Company, Richland, Washington. 
HNF-SD-WM-RPT-282 Rev. 0

This page intentionally left blank. 
HNF-SD-WM-RPT-282 Rev. 0

\author{
APPENDIX A \\ ANALYTICAL DATA FOR THE REPORT ON \\ SAMPLING AND ANALYSIS OF AMBIENT AIR \\ AT THE CENTRAL WASTE COMPLEX
}

A-1 
HNF-SD-WM-RPT-282 Rev. 0

This page intentionally left blank. 
HNF-SD-WM-RPT-282 Rev. 0

A.1 CWC 2401 BUILDING

A-3 
HNF-SD-WM-RPT-282 Rev. 0

This page intentionally left blank. 
File

Operator

: C: $\backslash$ HPCHEM $\backslash 2 \backslash D A T A \backslash 61396 \_4 . D$

Acquired

: LAP

Instrument

: 13 Jun 96 10:19 am using AcqMethod CWC

Misc Info : DELTA $\mathrm{P}=100$ torx

Vial Number: 1

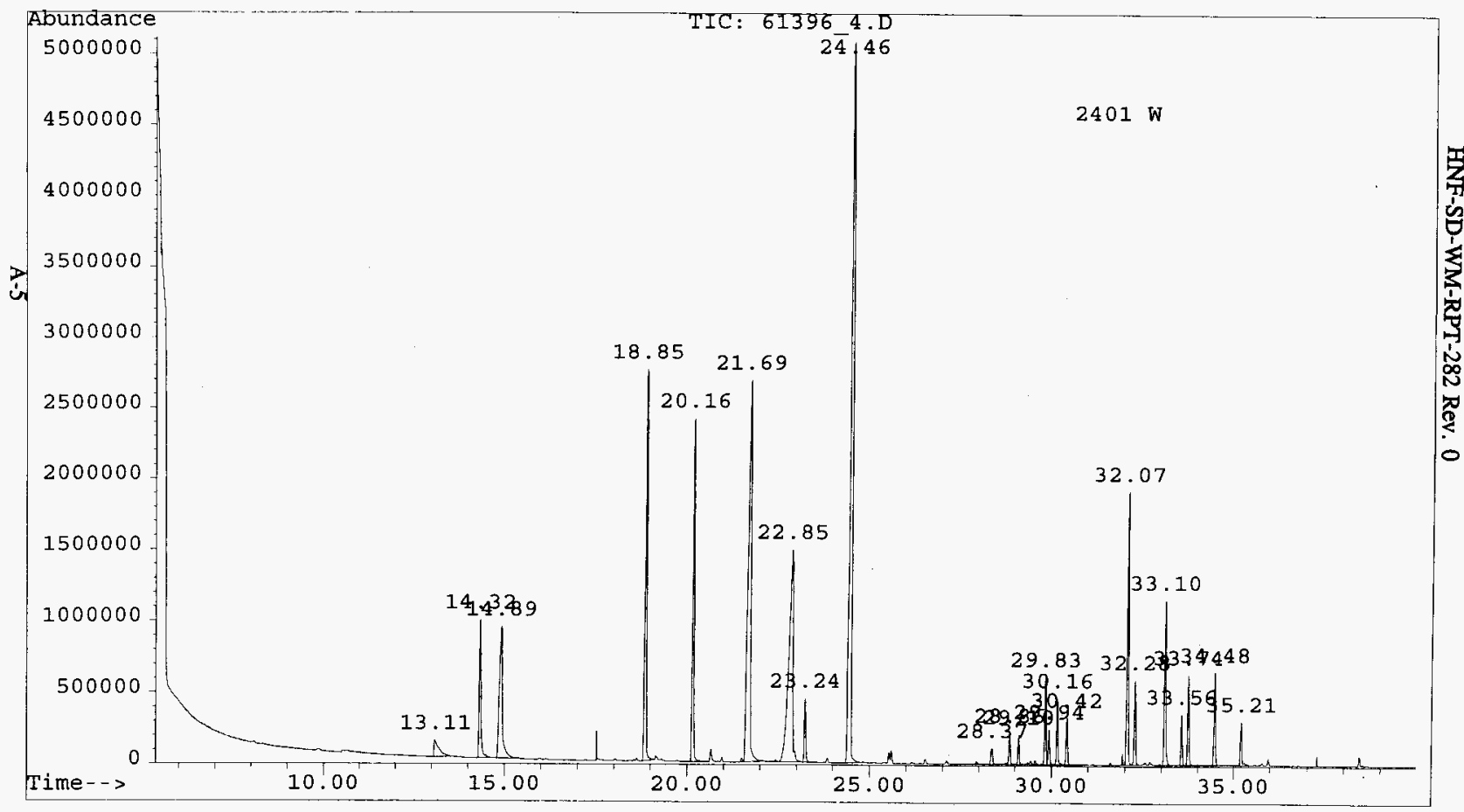


HNF-SD-WM-RPT-282 Rev. 0

CWC_REP.XLS

\section{ANALYSIS REPORT FOR CWC AIR SAMPLES}

File Name:

Analyst:

Analysis Date:

Method:

Sample Name:
61396_4.D

LAP

06/13/96

CWC

FT6045-1W.70A

\begin{tabular}{|c|c|c|c|c|}
\hline COMPOUND NAME & CAS \# & Concentration & Units & Comments \\
\hline ISO-BUTANE & $75-28-5$ & 0 & $\overline{P P B}$ & \\
\hline VINYL CHLORIDE & 75-01-4 & 0 & $\overline{\mathrm{PPB}}$ & \\
\hline ETHANOL & 64-17-5 & 25 & PPB & \\
\hline ACETONE & $67-64-1$ & 26 & PPB & \\
\hline 2-PROPANOL & $67-63-0$ & 60 & PPB & \\
\hline 1,1-DICHLOROETHENE & $75-35-4$ & 0 & PPB & \\
\hline FREON 113 & $76-13-1$ & 0 & PPB & \\
\hline DICHLOROMETHANE & $75-09-2$ & $\overline{0}$ & PPB & . \\
\hline 1,1-DICHLOROETHANE & $75-34-3$ & 0 & PPB & \\
\hline 2-BUTANONE & $78-93-3$ & 338 & PPB & \\
\hline N-HEXANE & $110-54-3$ & 16 & PPB & \\
\hline 1,2-DICHLOROETHENE & $156-59-2$ & 0 & PPB & \\
\hline CHLOROFORM & $67-66-3$ & 0 & PPB & \\
\hline TETRAHYDROFURAN & $109-99-9$ & 69 & PPB & \\
\hline 1,1,1-TRICHLOROETHANE & $71-55-6$ & 0 & PPB & \\
\hline 1,2-DICHLOROETHANE & $107-06-2$ & 2 & $\overline{\mathrm{PPB}}$ & \\
\hline BENZENE & $71-\overline{43-2}$ & 0 & PPB & \\
\hline CARBON TETRACHLORIDE & $56-23-5$ & 0 & PPB & \\
\hline CYCLOHEXANE & $110-82-7$ & 0 & $\overline{\mathrm{PPB}}$ & \\
\hline N-BUTANOL & $71-36-3$ & 216 & PPB & \\
\hline TRICHLOROETHENE & $79-01-6$ & 0 & PPB & \\
\hline 4-METHYL-2-PENTANONE & $108-10-1$ & 195 & $\overline{\mathrm{PPB}}$ & \\
\hline TOLUENE & $108-88-3$ & 2 & PPB & \\
\hline 1,1,2-TRICHLOROETHANE & $79-00-5$ & 0 & PPB & \\
\hline TETRACHLOROETHENE & $127-18-4$ & 0 & PPB & \\
\hline CHLOROBENZENE & $108-90-7$ & 2 & $\overline{P P B}$ & \\
\hline ETHYLBENZENE & $100-41-4$ & 6 & PPB & \\
\hline M-XYLENE & $108-38-3$ & 5 & PPB & \\
\hline STYRENE & $100-42-5$ & 15 & PPB & \\
\hline O-XYLENE & $95-47-6$ & 6 & $\overline{\mathrm{PPB}}$ & \\
\hline 1,1,2,2-TETRACHLORETHANE & $79-34-5$ & 10 & PPB & \\
\hline 1,3,5-TRIMETHYLBENZENE & $108-67-8$ & 16 & PPB & \\
\hline 1,2,4-TRIMETHYLBENZENE & $95-63-6$ & 31 & PPB & \\
\hline 1,3-DICHLOROBENZENE & $541-73-1$ & 11 & PPB & \\
\hline 1,2-DICHLOROBENZENE & $95-50-1$ & 20 & PPB & \\
\hline 1,4-DICHLOROBENZENE & $106-46-7$ & 20 & PPB & \\
\hline
\end{tabular}


HNF-SD-WM-RPT-282 Rev. 0

\section{LIST OF COMPOUNDS TENTATIVELY IDENTIFIED WITH MASS SPECTRAL LIBRARY}

Sample: $\quad 2401 \mathrm{~W}$

File:

ID:

C:UHPCHEMU2LATA161396_4,D

FT6045-1W.70A

Compound

CAS\#

Ethanol

000064-17-5

2-Propanone

000067-64-1

2-Propanol

000067-63-0

2-Butanone

000078-93-3

Furan, tetrahydro-

000109-99-9

2-Butanol, 2-methyl-

000075-85-4

Ethane, 1,2-dichloro-

000107-06-2

Benzene

$000071-43-2$

1-Butanol

000071-36-3

Ethanol, 2-methoxy-

000109-86-4

1,4-Dioxane

000123-91-1

Formic acid, butyl ester

000592-84-7

2-Pentanone, 4-methyl-

000108-10-1

Benzene, methyl-

000108-88-3

Ethane, 1,1,2-trichloro-

000079-00-5

Hexanal

000066-25-1

Ethene, tetrachloro-

000127-18-4 


\section{LIST OF COMPOUNDS TENTATIVELY IDENTIFIED WITH MASS SPECTRAL LIBRARY}

Sample: $\quad 2401 \mathrm{~W}$ (CONTINUED)

File: $\quad$ C:IHPCHEMLLDATA161396_4.D

ID: $\quad$ FT6045-1W.70A

Compound

CAS\#

Benzene, chloro-

000108-90-7

Benzene, ethyl-

$000100-41-4$

Benzene, 1,3-dimethyl-

000108-38-3

3-Heptanone

000106-35-4

2-Heptanone

000110-43-0

Styrene

$000100-42-5$

Benzene, 1,4-dimethyl-

000106-42-3

Ethanol, 2-butoxy-

000111-76-2

Ethane, 1,1,2,2-tetrachloro-

000079-34-5

Benzaldehyde

000100-52-7

Benzene, 1,3,5-trimethyl-

000108-67-8

Benzene, 1,2,4-trimethyl-

000095-63-6

Benzene, 1,3-dichloro-

000541-73-1

Benzene, 1,2-dichloro-

000095-50-1

Ethanone, 1-phenyl-

000098-86-2

Benzaldehyde, 2-methyl-

000529-20-4 
HNF-SD-WM-RPT-282 Rev. 0

Sample: $\quad 2401 \mathrm{~W}$ (CONTINUED)

File: $\quad$ C:LHPCHEMIDATAI61396_4.D

ID: FT6045-1W.70A

Compound

CAS\#

Nonanal

000124-19-6

Dodecane

000112-40-3 
File

Operator

Acquired

Instrument

Sample Nar

FT6045-1W.68A

Misc Info : DELTA $\mathrm{P}=100$ torr

Vial Number: 1

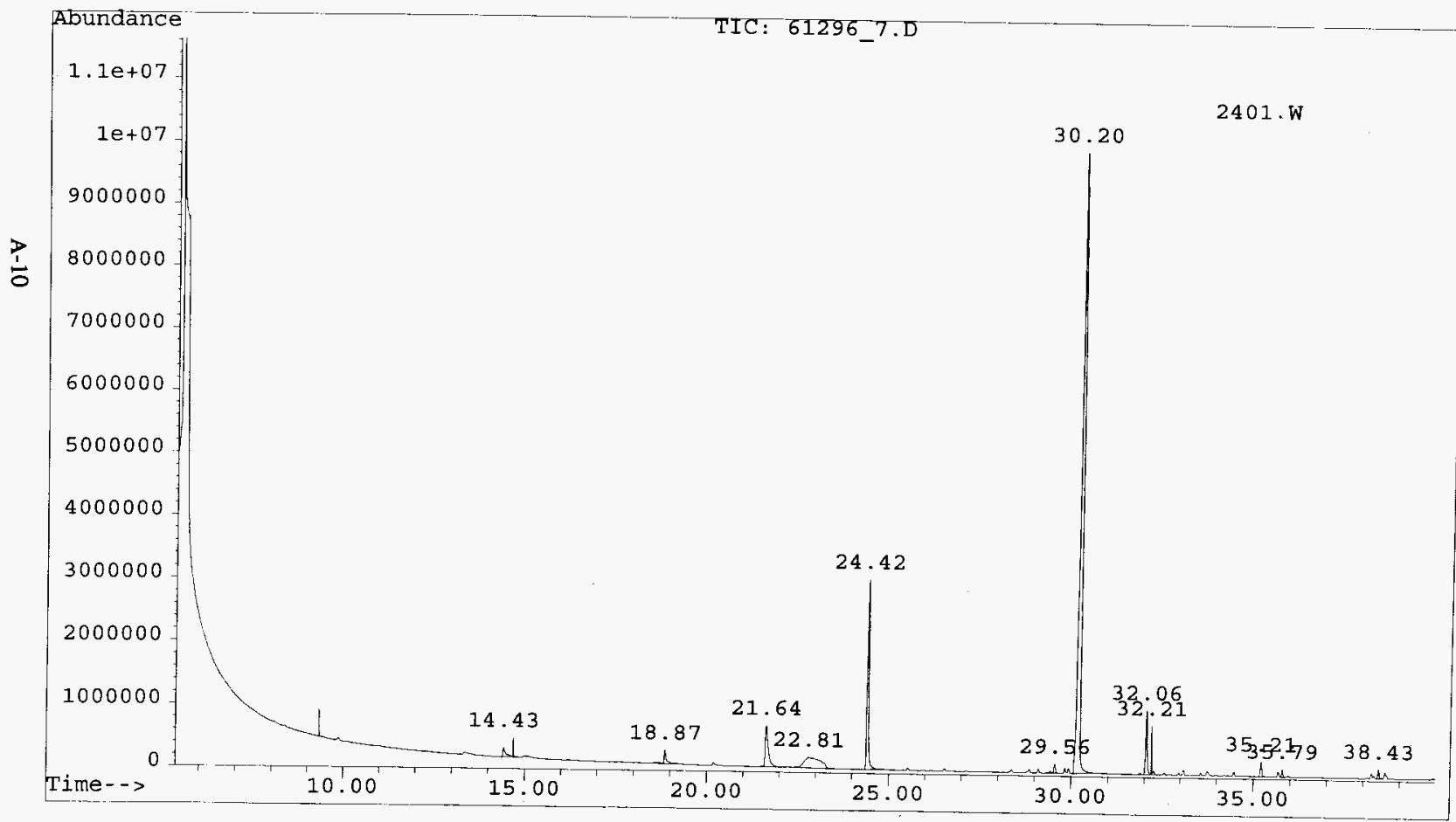


HNF-SD-WM-RPT-282 Rev. 0

CWC_REP.XLS

\section{ANALYSIS REPORT FOR CWC AIR SAMPLES}

File Name:

Analyst:

Analysis Date:

Method:

Sample Name:
61296_7.D

LAP

06/12/96

CWC

FT6045-1W.68A

\begin{tabular}{|c|c|c|c|c|}
\hline COMPOUND NAME & CAS \# & Concentration & Units & Comments \\
\hline ISO-BUTANE & $75-28-5$ & 0 & PPB & \\
\hline VINYL CHLORIDE & $75-01-4$ & 0 & PPB & \\
\hline ETHANOL & $64-17-5$ & 11 & PPB & \\
\hline ACETONE & $67-64-1$ & 6 & PPB & \\
\hline 2-PROPANOL & $67-63-0$ & 4 & PPB & \\
\hline 1,1-DICHLOROETHENE & $75-35-4$ & 0 & PPB & \\
\hline FREON 113 & $76-13-1$ & 0 & PPB & \\
\hline DICHLOROMETHANE & $75-09-2$ & 0 & PPB & \\
\hline 1,1-DICHLOROETHANE & $75-34-3$ & 0 & PPB & \\
\hline 2-BUTANONE & $78-93-3$ & 25 & PPB & \\
\hline N-HEXANE & $110-54-3$ & 0 & $\overline{\mathrm{PPB}}$ & \\
\hline 1,2-DICHLOROETHENE & $156-59-2$ & 0 & PPB & \\
\hline CHLOROFORM & $67-66-3$ & 0 & PPB & \\
\hline TETRAHYDROFURAN & $109-99-9$ & 0 & $\mathrm{PPB}$ & \\
\hline 1,1,1-TRICHLOROETHANE & $71-55-6$ & 0 & PPB & \\
\hline 1,2-DICHLOROETHANE & $107-06-2$ & 0 & PPB & \\
\hline BENZENE & $71-43-2$ & 0 & PPB & \\
\hline CARBON TETRACHLORIDE & $56-23-5$ & 0 & PPB & \\
\hline CYCLOHEXANE & $110-82-7$ & 0 & PPB & \\
\hline N-BUTANOL & $71-36-3$ & 59 & PPB & \\
\hline TRICHLOROETHENE & $79-01-6$ & 0 & PPB & \\
\hline 4-METHYL-2-PENTANONE & $108-10-1$ & 56 & PPB & \\
\hline TOLUENE & $108-88-3$ & 2 & PPB & \\
\hline 1,1,2-TRICHLOROETHANE & $79-00-5$ & 0 & PPB & \\
\hline TETRACHLOROETHENE & $127-18-4$ & 0 & PPB & \\
\hline CHLOROBENZENE & $108-90-7$ & 0 & PPB & \\
\hline ETHYLBENZENE & $100-41-4$ & 2 & PPB & \\
\hline M-XYLENE & $108-38-3$ & 0 & PPB & \\
\hline STYRENE & $100-42-5$ & 2 & PPB & \\
\hline O-XYLENE & $95-47-6$ & 2 & PPB & \\
\hline 1,1,2,2-TETRACHLORETHANE & $79-34-5$ & 0 & PPB & \\
\hline 1,3,5-TRIMETHYLBENZENE & $108-67-8$ & 18 & PPB & \\
\hline 1,2,4-TRIMETHYLBENZENE & $95-63-6$ & 2 & PPB & \\
\hline 1,3-DICHLOROBENZENE & $541-73-1$ & 2 & PPB & \\
\hline 1,2-DICHLOROBENZENE & $95-50-1$ & 2 & PPB & \\
\hline 1,4-DICHLOROBENZENE & $106-46-7$ & 2 & PPB & \\
\hline
\end{tabular}


HNF-SD-WM-RPT-282 Rev. 0

\section{LIST OF COMPOUNDS TENTATIVELY IDENTIFIED WITH MASS SPECTRAL LIBRARY}

Sample: $\quad 2401 \mathrm{~W}$

File: $\quad$ C:LHPCHEML2LATA161296_7.D

ID: $\quad$ FT6045-1W.68A

Compound

2-Butanone

1,3-Benzenediol, 2-methyl-

1-Butanol

2-Propanol, 1-methoxy-

2-Pentanone, 4-methyl-

Benzene, methyl-

Benzene, chloro-

Benzene, ethyl-

Benzene, 1,3-dimethyl-

2-Heptanone

Styrene

Benzene, 1,2-dimethyl-

Ethanol, 2-butoxy-

Benzaldehyde

Phenol

Decane

Benzene, 1,3,5-trimethyl-
CAS\#

000078-93-3

000608-25-3

000071-36-3

000107-98-2

000108-10-1

000108-88-3

000108-90-7

$000100-41-4$

000108-38-3

000110-43-0

$000100-42-5$

$000095-47-6$

$000111-76-2$

000100-52-7

000108-95-2

000124-18-5

000108-67-8 
HNF-SD-WM-RPT-282 Rev. 0

\section{LIST OF COMPOUNDS TENTATIVELY IDENTIFIED WITH MASS SPECTRAL LIBRARY}

Sample: $\quad 2401 \mathrm{~W}$ (CONTINUED)

File: $\quad$ C:LHPCHEM 2LDATAl61296_7.D

ID: FT6045-1W.68A

Compound CAS\#

Benzene, 1,4-dichloro- $\quad$ 000106-46-7

Benzene, 1,4-dichloro- $\quad$ 000106-46-7

Benzene, 1,4-dichloro- $\quad$ 000106-46-7

Ethanone, 1-phenyl- $\quad 000098-86-2$

Decane 000124-18-5

Naphthalene, 1,2,3,4-tetrahydro- 000119-64-2

Benzaldehyde, 3,4-dimethyl- 005973-71-7

2,3-DIMETHYLBENZALDEHYDE 005779-93-1

Dodecane 000112-40-3

Benzene, 1,2,4-trichloro- $\quad$ 000120-82-1

Benzaldehyde, ethyl- $\quad$ 053951-50-1 
File

Operator

Acquired

Instrument

Sample Name:

Misc Info

Vial Number:

C: \HPCHEM $\backslash 2 \backslash D A T A \backslash 61396 \quad 9 . D$

: MS

: 13 Jun 96 5:33 pm using AcqMethod CWC

5972 - In

FT6045-1W. 70B

DELTA $P=100$ torr

1

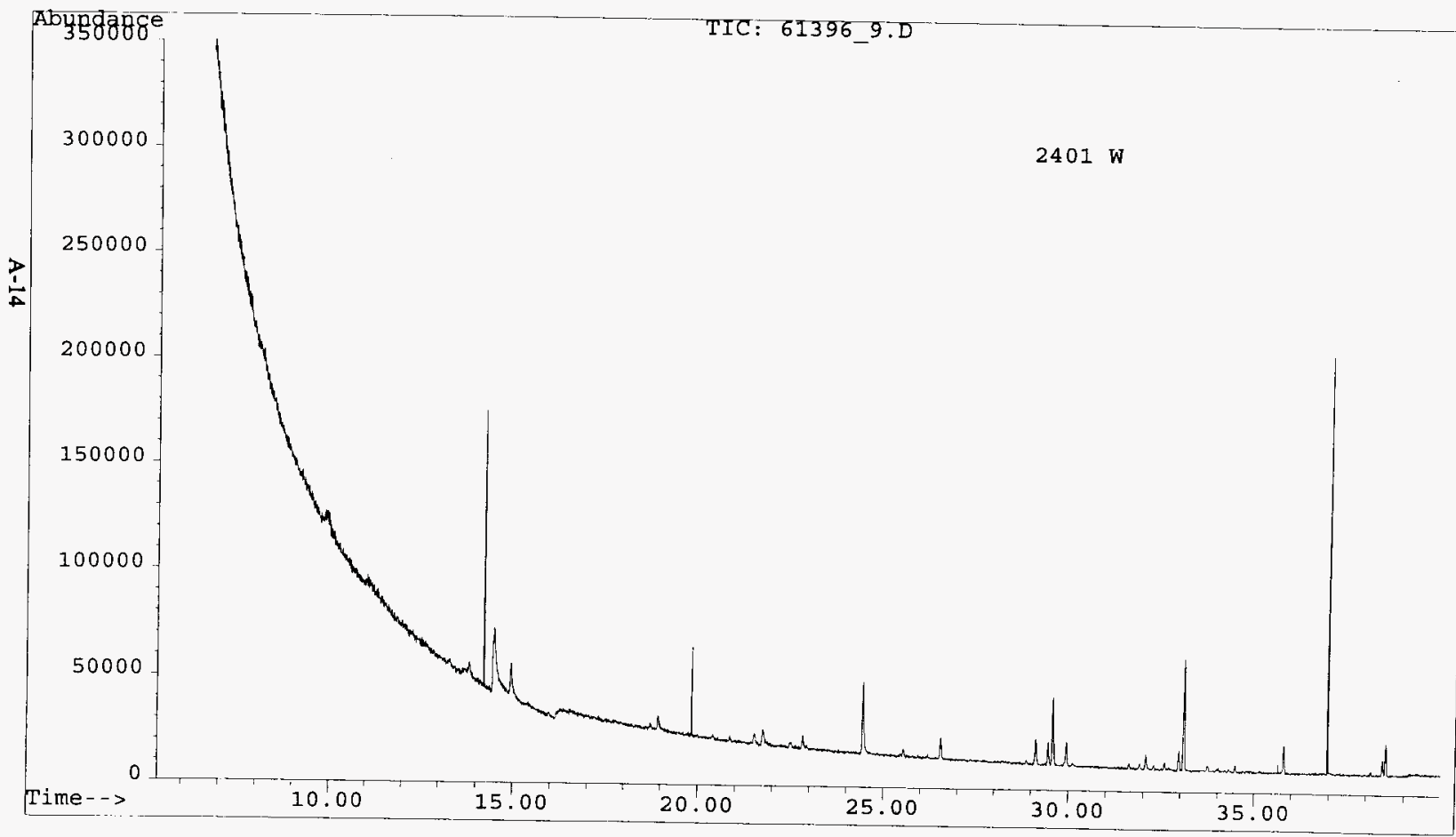


HNF-SD-WM-RPT-282 Rev. 0

CWC_REP.XLS

\section{ANALYSIS REPORT FOR CWC AIR SAMPLES}

File Name:

Analyst:

Analysis Date:

Method:

Sample Name:
61396_9.D

MS

06/13/96

CWC

FT6045-1W.70B

\begin{tabular}{|c|c|c|c|c|}
\hline COMPOUND NAME & CAS\# & Concentration & Units & Comments \\
\hline ISO-BUTANE & $75-28-5$ & 0 & PPB & \\
\hline VINYL CHLORIDE & $75-01-4$ & 0 & PPB & \\
\hline ETHANOL & $64-17-5$ & 0 & PPB & \\
\hline ACETONE & $67-64-1$ & 0 & PPB & \\
\hline 2-PROPANOL & $67-63-0$ & 0 & PPB & \\
\hline 1,1-DICHLOROETHENE & $75-35-4$ & 0 & PPB & \\
\hline FREON 113 & $76-13-1$ & 0 & PPB & \\
\hline DICHLOROMETHANE & $75-09-2$ & 0 & $\overline{\mathrm{PPB}}$ & \\
\hline 1,1-DICHLOROETHANE & $75-34-3$ & 0 & $\overline{P P B}$ & \\
\hline 2-BUTANONE & $78-93-3$ & 0 & $\overline{P P B}$ & \\
\hline N-HEXANE & $110-54-3$ & 0 & PPB & \\
\hline 1,2-DICHLOROETHENE & $156-59-2$ & 0 & PPB & \\
\hline CHLOROFORM & $67-66-3$ & 0 & PPB & \\
\hline TETRAHYDROFURAN & $109-99-9$ & 0 & PPB & \\
\hline 1,1,1-TRICHLOROETHANE & $71-55-6$ & 0 & PPB & \\
\hline 1,2-DICHLOROETHANE & $107-06-2$ & 0 & PPB & \\
\hline BENZENE & $71-43-2$ & 0 & PPB & \\
\hline CARBON TETRACHLORIDE & $56-23-5$ & 0 & PPB & \\
\hline CYCLOHEXANE & $110-82-7$ & 0 & PPB & \\
\hline N-BUTANOL & $71-36-3$ & 0 & PPB & \\
\hline TRICHLOROETHENE & $79-01-6$ & 0 & PPB & \\
\hline 4-METHYL-2-PENTANONE & $108-10-1$ & $\overline{0}$ & PPB & \\
\hline TOLUENE & $108-88-3$ & 0 & PPB & \\
\hline 1,1,2-TRICHLOROETHANE & $79-00-5$ & 0 & PPB & \\
\hline TETRACHLOROETHENE & $127-18-4$ & 0 & PPB & \\
\hline CHLOROBENZENE & $108-90-7$ & 0 & PPB & \\
\hline ETHYLBENZENE & $100-41-4$ & 0 & $\overline{\mathrm{PPB}}$ & \\
\hline M-XYLENE & $108-38-3$ & 0 & PPB & \\
\hline STYRENE & $100-42-5$ & 0 & PPB & \\
\hline O-XYLENE & $95-47-6$ & 0 & PPB & \\
\hline 1,1,2,2-TETRACHLORETHANE & $79-34-5$ & 0 & PPB & \\
\hline 1,3,5-TRIMETHYLBENZENE & $108-67-8$ & 0 & $\overline{\mathrm{PPB}}$ & \\
\hline 1,2,4-TRIMETHYLBENZENE & $95-63-6$ & 1 & PPB & \\
\hline 1,3-DICHLOROBENZENE & $541-73-1$ & 0 & PPB & \\
\hline 1,2-DICHLOROBENZENE & $95-50-1$ & 0 & PPB & \\
\hline 1,4-DICHLOROBENZENE & $106-46-7$ & 0 & PPB & \\
\hline
\end{tabular}


HNF-SD-WM-RPT-282 Rev. 0

LIST OF COMPOUNDS TENTATIVELY IDENTIFIED WITH MASS SPECTRAL

LIBRARY

Sample: $\quad 2401 \mathrm{~W}$

File: $\quad$ C:LHPCHEMI2LATA161396_9.D

ID: FT6045-1W.70B

Compound

CAS\#

2-Heptanone

000110-43-0

Benzene, 1,2,3-trimethyl-

000526-73-8

Benzene, 1,2,3-trichloro-

$000087-61-6$ 
File

: C: \HPCHEM $\backslash 2 \backslash D A T A \backslash 61396 \_11 . D$

Operator

Acquired : 13 Jun $96 \quad 7: 57$ pm using AcqMethod CWC

Instrument : 5972 - In

Sample Name: FT6045-1W.71A

Misc Info : DELTA $\mathrm{P}=100$ torr

Vial Number: 1

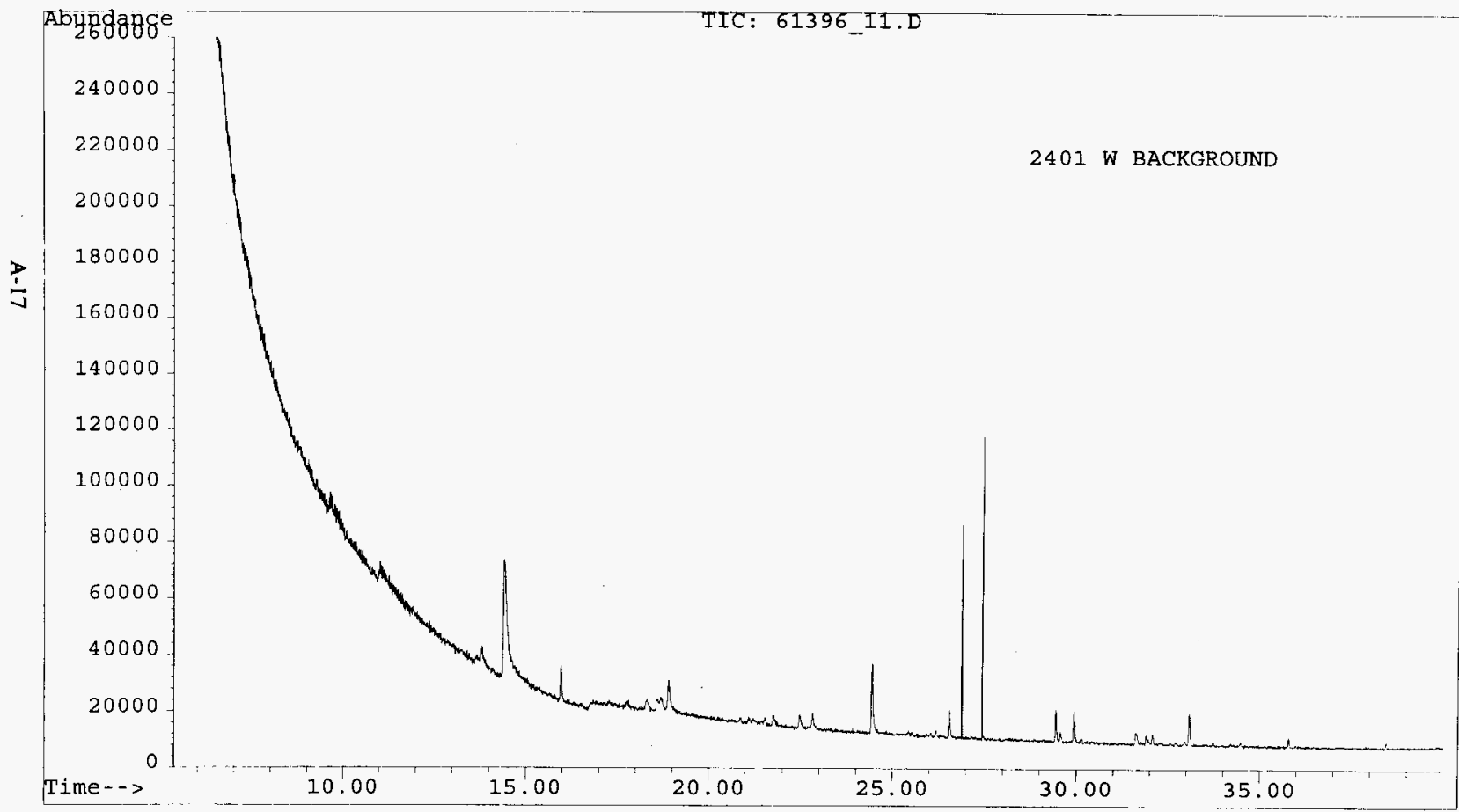


HNF-SD-WM-RPT-282 Rev. 0

CWC_REP.XLS

\section{ANALYSIS REPORT FOR CWC AIR SAMPLES}

File Name:

Analyst:

Analysis Date:

61396 11.D

Method:

MS

Sample Name:

06/13/96

CWC

FT6045-1W.71A

\begin{tabular}{|c|c|c|c|c|}
\hline COMPOUND NAME & CAS\# & Concentration & Units & Comments \\
\hline ISO-BUTANE & $75-28-5$ & $\overline{0}$ & PPB & \\
\hline VINYL CHLORIDE & $75-01-4$ & 0 & PPB & \\
\hline ETHANOL & $64-17-5$ & 0 & PPB & \\
\hline ACETONE & $67-64-1$ & 0 & PPB & \\
\hline 2-PROPANOL & $67-63-0$ & 0 & PPB & \\
\hline 1,1-DICHLOROETHENE & $75-35-4$ & 0 & PPB & \\
\hline FREON 113 & 76-13-1 & 0 & $\overline{P P B}$ & \\
\hline DICHLOROMETHANE & $75-09-2$ & 0 & PPB & \\
\hline 1,1-DICHLOROETHANE & $75-34-3$ & 0 & PPB & \\
\hline 2-BUTANONE & 78-93-3 & $\overline{0}$ & PPB & \\
\hline N-HEXANE & $110-54-3$ & 0 & PPB & \\
\hline 1,2-DICHLOROETHENE & $156-59-2$ & 0 & PPB & \\
\hline CHLOROFORM & $67-66-3$ & $\overline{0}$ & PPB & \\
\hline TETRAHYDROFURAN & $109-99-9$ & 0 & PPB & \\
\hline 1,1,1-TRICHLOROETHANE & $71.55-6$ & $\overline{0}$ & PPB & \\
\hline 1,2-DICHLOROETHANE & $107-06-2$ & 0 & PPB & $\therefore$ \\
\hline BENZENE & $71-43-2$ & 0 & PPB & \\
\hline CARBON TETRACHLORIDE & $56-23-5$ & 0 & PPB & \\
\hline CYCLOHEXANE & $110-82-7$ & 0 & PPB & \\
\hline N-BUTANOL & $71-36-3$ & 0 & PPB & \\
\hline TRICHLOROETHENE & $79-01-6$ & 0 & PPB & \\
\hline 4-METHYL-2-PENTANONE & $108-10-1$ & 0 & PPB & \\
\hline TOLUENE & $108-88-3$ & 0 & PPB & \\
\hline 1,1,2-TRICHLOROETHANE & $79-00-5$ & 0 & PPB & \\
\hline TETRACHLOROETHENE & $127-18-4$ & 0 & PPB & \\
\hline CHLOROBENZENE & $108-90-7$ & 0 & PPB & \\
\hline ETHYLBENZENE & $100-41-4$ & 0 & PPB & \\
\hline M-XYLENE & $108-38-3$ & 0 & PPB & \\
\hline STYRENE & $100-42-5$ & 0 & PPB & \\
\hline O-XYLENE & $95-47-6$ & 0 & PPB & \\
\hline 1,1,2,2-TETRACHLORETHANE & $79-34-5$ & 0 & PPB & \\
\hline 1,3,5-TRIMETHYLBENZENE & $108-67-8$ & 0 & PPB & \\
\hline 1,2,4-TRIMETHYLBENZENE & $95-63-6$ & 0 & PPB & \\
\hline 1,3-DICHLOROBENZENE & $541-73-1$ & 0 & PPB & \\
\hline 1,2-DICHLOROBENZENE & $95-50-1$ & 0 & PPB & \\
\hline 1,4-DICHLOROBENZENE & $106-46-7$ & 0 & PPB & \\
\hline
\end{tabular}


HNF-SD-WM-RPT-282 Rev. 0

\section{LIST OF COMPOUNDS TENTATIVELY IDENTIFIED WITH MASS SPECTRAL} LIBRARY

Sample: $\quad 2401$ W BACKGROUND

File:

ID: C:LHPCHEM2LDATAI61396 11.D

FT6045-1W.71A

Compound

CAS\#

None 
HNF-SD-WM-RPT-282 Rev. 0

This page intentionally left blank. 
HNF-SD-WM-RPT-282 Rev. 0

\section{A.2 CWC 2402 BUILDINGS}


HNF-SD-WM-RPT-282 Rev. 0

This page intentionally left blank. 
File

$C: \backslash H P C H E M \backslash 2 \backslash D A T A \backslash 51496-9 . D$

Operator : lap

Acquired : 14 May $96 \quad 12: 25$ pm using AcqMethod CWC

Instrument : 5972 - In

Sample Name: FT6045-2W.20A

Misc Info : DELTA $P=100$ torr

Vial Number: 1

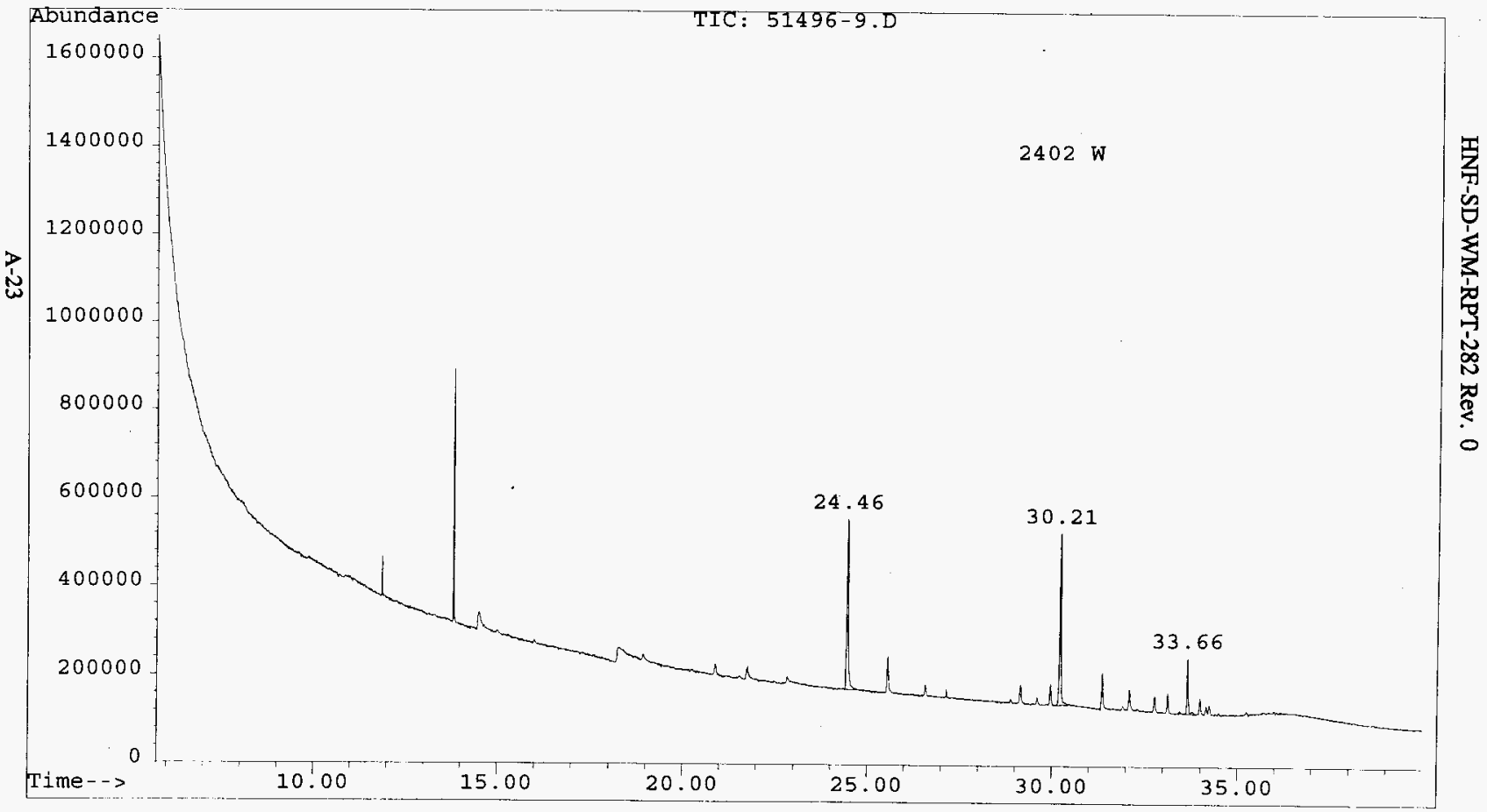


HNF-SD-WM-RPT-282 Rev. 0

CWC_REP.XLS

\section{ANALYSIS REPORT FOR CWC AIR SAMPLES}

File Name:

Analyst:

Analysis Date:

Method:

Sample Name:
51496-9.D

lap

05/14/96

CWC

FT6045-2W.20A

\begin{tabular}{|c|c|c|c|c|}
\hline COMPOUND NAME & CAS \# & Concentration & Units & Comments \\
\hline ISO-BUTANE & $75-28-5$ & 0 & $\overline{\mathrm{PPB}}$ & \\
\hline VINYL CHLORIDE & $75-01-4$ & 0 & PPB & \\
\hline ETHANOL & $64-17-5$ & 0 & PPB & \\
\hline ACETONE & $67-64-1$ & 0 & PPB & \\
\hline 2-PROPANOL & $67-63-0$ & 0 & PPB & \\
\hline 1,1-DICHLOROETHENE & $75-35-4$ & 0 & PPB & \\
\hline FREON 113 & $76-13-1$ & 0 & PPB & \\
\hline DICHLOROMETHANE & $75-09-2$ & $\overline{0}$ & PPB & \\
\hline 1,1-DICHLOROETHANE & $75-34-3$ & 0 & PPB & \\
\hline 2-BUTANONE & $78-93-3$ & 0 & PPB & \\
\hline N-HEXANE & $110-54-3$ & 0 & PPB & \\
\hline 1,2-DICHLOROETHENE & $156-59-2$ & 0 & PPB & \\
\hline CHLOROFORM & $67-66-3$ & 0 & PPB & \\
\hline TETRAHYDROFURAN & $109-99-9$ & 0 & PPB & \\
\hline 1,1,1-TRICHLOROETHANE & $71-55-6$ & 0 & PPB & \\
\hline 1,2-DICHLOROETHANE & $107-06-2$ & 0 & PPB & \\
\hline BENZENE & $71-43-2$ & 0 & $\overline{\mathrm{PPB}}$ & \\
\hline CARBON TETRACHLORIDE & $56-23-5$ & 0 & PPB & \\
\hline CYCLOHEXANE & $110-82-7$ & 0 & PPB & \\
\hline N-BUTANOL & $71-36-3$ & 0 & PPB & \\
\hline TRICHLOROETHENE & $79-01-6$ & 0 & PPB & \\
\hline 4-METHYL-2-PENTANONE & $108-10-1$ & 8 & PPB & \\
\hline TOLUENE & $108-88-3$ & 3 & PPB & \\
\hline 1,1,2-TRICHLOROETHANE & $79-00-5$ & 0 & PPB & \\
\hline TETRACHLOROETHENE & $127-18-4$ & 0 & PPB & \\
\hline CHLOROBENZENE & $108-90-7$ & 0 & PPB & \\
\hline ETHYLBENZENE & $100-41-4$ & 1 & PPB & \\
\hline M-XYLENE & $108-38-3$ & 0 & PPB & \\
\hline STYRENE & $100-42-5$ & 0 & $\overline{\mathrm{PPB}}$ & \\
\hline O-XYLENE & $95-47-6$ & 1 & PPB & \\
\hline 1,1,2,2-TETRACHLORETHANE & $79-34-5$ & 0 & PPB & \\
\hline 1,3,5-TRIMETHYLBENZENE & $108-67-8$ & 0 & PPB & \\
\hline 1,2,4-TRIMETHYLBENZENE & $95-63-6$ & 1 & PPB & \\
\hline 1,3-DICHLOROBENZENE & $541-73-1$ & 0 & PPB & \\
\hline 1,2-DICHLOROBENZENE & $95-50-1$ & 0 & PPB & \\
\hline 1,4-DICHLOROBENZENE & $106-46-7$ & 0 & PPB & \\
\hline
\end{tabular}


HNF-SD-WM-RPT-282 Rev. 0

\section{LIST OF COMPOUNDS TENTATIVELY IDENTIFIED WITH MASS SPECTRAL} LIBRARY

$\begin{array}{ll}\text { Sample: } & \text { 2402 W20 } \\ \text { File } & \text { C. }: \text { HPCHEM 2LDATAI51496-9.D } \\ \text { ID } & \text { FT6045-2W.20A }\end{array}$

Compound

CAS\#

Ethane, 1,1,1-trichloro-

$000071-55-6$

2-Pentanone, 4-methyl-

000108-10-1

Benzene, methyl-

000108-88-3

Benzene, 1,3-dimethyl-

000108-38-3

Benzene, 1,2-dimethyl-

$000095-47-6$

Ethanol, 2-butoxy-

$000111-76-2$

ALPHA -PINENE, (-)-

000080-56-8

Benzaldehyde

000100-52-7

2-BETA.-PINENE

000127-91-3

Benzene, 1,2,4-trimethyl-

000095-63-6

DELTA.3-Carene

13466-78-9 95

Benzene, 1-methyl-3-(1-methylethyl)-

000535-77-3 93 

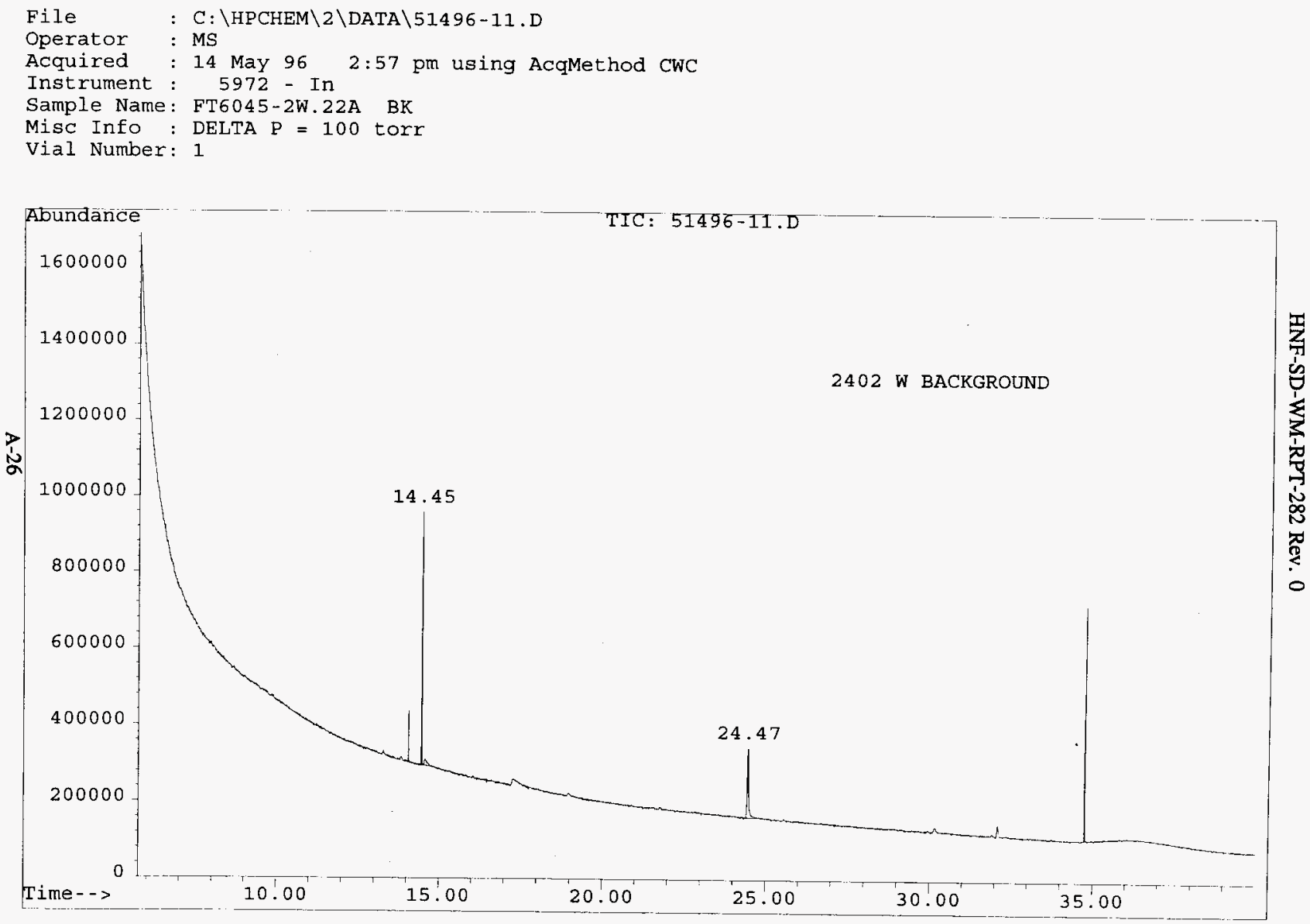
HNF-SD-WM-RPT-282 Rev. 0

CWC_REP.XLS

\section{ANALYSIS REPORT FOR CWC AIR SAMPLES}

File Name:

Analyst:

Analysis Date:

Method:

Sample Name:
51496-11.D

MS

05/14/96

CWC

FT6045-2W.22A BK

\begin{tabular}{|c|c|c|c|c|}
\hline COMPOUND NAME & CAS \# & Concentration & Units & Comments \\
\hline ISO-BUTANE & $75-28-5$ & 0 & PPB & \\
\hline VINYL CHLORIDE & $75-01-4$ & 0 & PPB & \\
\hline ETHANOL & $64-17-5$ & 0 & PPB & \\
\hline ACETONE & $67-64-1$ & 0 & PPB & \\
\hline 2-PROPANOL & $67-63-0$ & 0 & PPB & \\
\hline 1,1-DICHLOROETHENE & $75-35-4$ & 0 & PPB & \\
\hline FREON 113 & $76-13-1$ & $\overline{0}$ & $\overline{P P B}$ & \\
\hline DICHLOROMETHANE & $75-09-2$ & 0 & $\mathrm{PPB}$ & \\
\hline 1,1-DICHLOROETHANE & $75-34-3$ & 0 & PPB & \\
\hline 2-BUTANONE & $78-93-3$ & 0 & PPB & \\
\hline N-HEXANE & $110-54-3$ & 0 & PPB & \\
\hline 1,2-DICHLOROETHENE & $156-59-2$ & 0 & PPB & \\
\hline CHLOROFORM & $67-66-3$ & 0 & PPB & \\
\hline TETRAHYDROFURAN & $109-99-9$ & 0 & PPB & \\
\hline 1,1,1-TRICHLOROETHANE & $71-55-6$ & 0 & PPB & \\
\hline 1,2-DICHLOROETHANE & 107-06-2 & 0 & PPB & \\
\hline BENZENE & $71-43-2$ & 0 & PPB & \\
\hline CARBON TETRACHLORIDE & $56-23-5$ & 0 & PPB & \\
\hline CYCLOHEXANE & $110-82-7$ & 0 & PPB & \\
\hline N-BUTANOL & $71-36-3$ & 0 & PPB & \\
\hline TRICHLOROETHENE & $79-01-6$ & 0 & PPB & \\
\hline 4-METHYL-2-PENTANONE & $108-10-1$ & 0 & PPB & \\
\hline TOLUENE & $108-88-3$ & 0 & PPB & \\
\hline 1,1,2-TRICHLOROETHANE & $79-00-5$ & 0 & PPB & \\
\hline TETRACHLOROETHENE & $127-18-4$ & 0 & PPB & \\
\hline CHLOROBENZENE & $108-90-7$ & 0 & PPB & \\
\hline ETHYLBENZENE & $100-41-4$ & 0 & $\overline{\mathrm{PPB}}$ & \\
\hline M-XYLENE & $108-38-3$ & 0 & $\overline{P P B}$ & \\
\hline STYRENE & $100-42-5$ & 0 & PPB & \\
\hline O-XYLENE & $95-47-6$ & 0 & PPB & \\
\hline 1,1,2,2-TETRACHLORETHANE & $79-34-5$ & 0 & PPB & \\
\hline 1,3,5-TRIMETHYLBENZENE & $108-67-8$ & 0 & $\mathrm{PPB}$ & \\
\hline 1,2,4-TRIMETHYLBENZENE & $95-63-6$ & 0 & $\overline{P P B}$ & \\
\hline 1,3-DICHLOROBENZENE & $541-73-1$ & 0 & PPB & \\
\hline 1,2-DICHLOROBENZENE & $95-50-1$ & 0 & PPB & \\
\hline 1,4-DICHLOROBENZENE & $106-46-7$ & 0 & PPB & \\
\hline
\end{tabular}


Sample: $\quad 2402 \mathrm{~W}$ Background

Sample Name: FT6045-2W.22A BK

Misc Info: DELTA $P=100$ torr

Search Libraries: C:IDATABASEIWILEY138.L Minimum Quality: 70

Unknown Spectrum: Apex

Integration Params: VOA.E

Pk\# RT Area\% Library/ID Ref\# CAS\# Qual

524.4627 .77 C:IDATABASEIWILEY138.L

2-Pentanone, 4-methyl-

732.105 .22 C:DATABASEIWILEY138.L

Benzaldehyde
$118019000108-10-187$

$118546000100-52-796$ 
File

Operator

Acquired

Instrument

Sample Name:

Misc Info : DELTA $\overline{\mathrm{P}}=100 \mathrm{TORR}$

Vial Number: 1

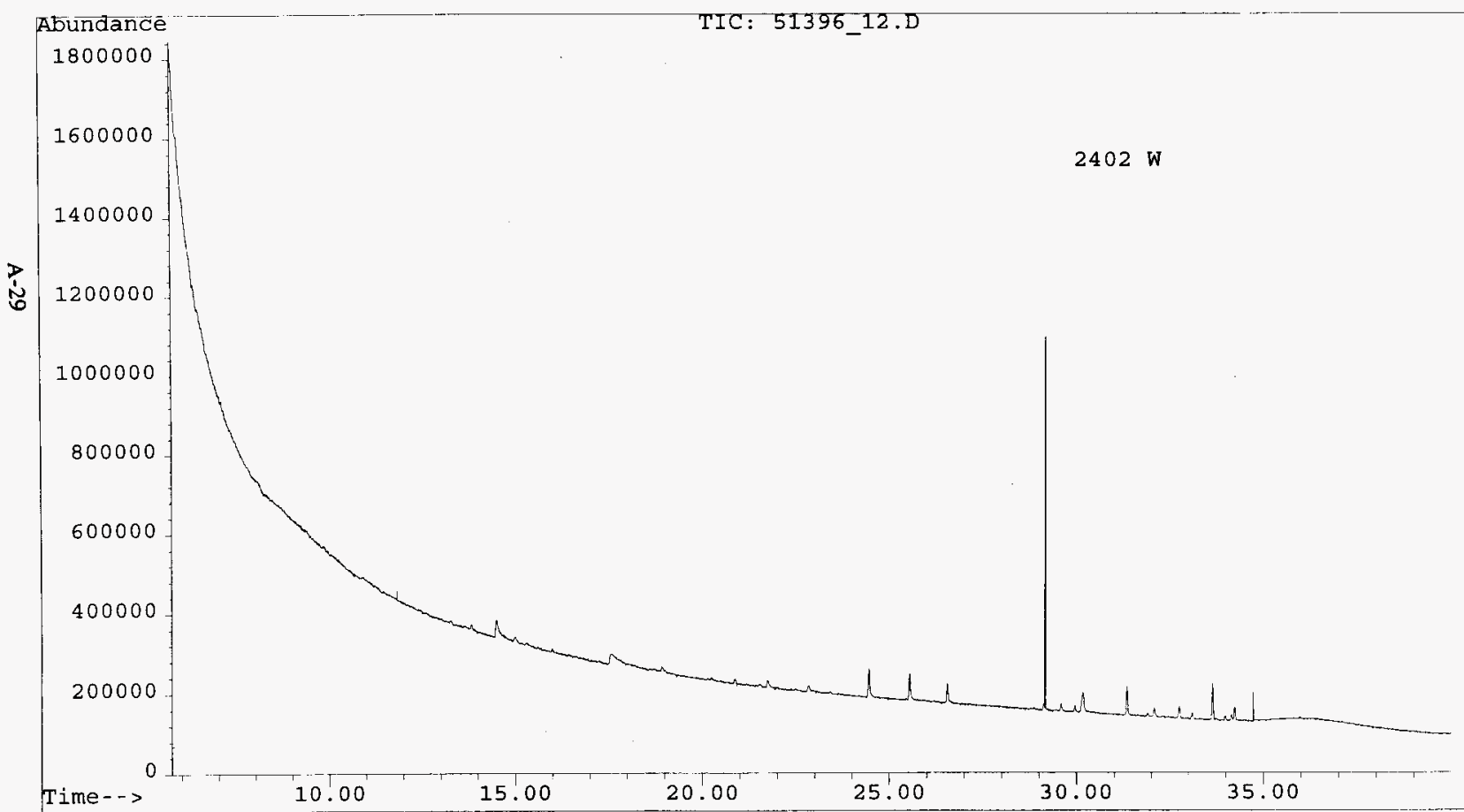


HNF-SD-WM-RPT-282 Rev. 0

CWC_REP.XLS

\section{ANALYSIS REPORT FOR CWC AIR SAMPLES}

File Name:

Analyst:

Analysis Date:

Method:

Sample Name:
51396_12.D

MS

05/13/96

CWC

FT6045_2W.21A

\begin{tabular}{|c|c|c|c|c|}
\hline COMPOUND NAME & CAS\# & Concentration & Units & Comments \\
\hline ISO-BUTANE & $75-28-5$ & 0 & $\overline{\mathrm{PPB}}$ & \\
\hline VINYL CHLORIDE & $75-01-4$ & 0 & PPB & \\
\hline ETHANOL & $64-17-5$ & 0 & $\mathrm{PPB}$ & \\
\hline ACETONE & $67-64-1$ & 0 & PPB & \\
\hline 2-PROPANOL & $67-63-0$ & 0 & PPB & \\
\hline 1,1-DICHLOROETHENE & $75-35-4$ & 0 & PPB & \\
\hline FREON 113 & $76-13-1$ & 0 & PPB & \\
\hline DICHLOROMETHANE & $75-09-2$ & $\overline{0}$ & $\overline{P P B}$ & \\
\hline 1,1-DICHLOROETHANE & $75-34-3$ & 0 & PPB & \\
\hline 2-BUTANONE & $78-93-3$ & 0 & PPB & \\
\hline N-HEXANE & $110-54-3$ & 0 & PPB & \\
\hline 1,2-DICHLOROETHENE & $156-59-2$ & 0 & $\mathrm{PPB}$ & \\
\hline CHLOROFORM & $67-66-3$ & 0 & PPB & \\
\hline TETRAHYDROFURAN & $109-99-9$ & 0 & PPB & \\
\hline 1,1,1-TRICHLOROETHANE & $71-55-6$ & 0 & PPB & \\
\hline 1,2-DICHLOROETHANE & $107-06-2$ & 0 & PPB & \\
\hline BENZENE & $71-43-2$ & 0 & PPB & \\
\hline CARBON TETRACHLORIDE & $56-23-5$ & 0 & PPB & \\
\hline CYCLOHEXANE & $110-82-7$ & 0 & $\mathrm{PPB}$ & \\
\hline N-BUTANOL & $71-36-3$ & 0 & $\mathrm{PPB}$ & \\
\hline TRICHLOROETHENE & $79-01-6$ & 0 & PPB & \\
\hline 4-METHYL-2-PENTANONE & $108-10-1$ & 0 & PPB & \\
\hline TOLUENE & $108-88-3$ & 2 & $\mathrm{PPB}$ & \\
\hline 1,1,2-TRICHLOROETHANE & $79-00-5$ & 0 & PPB & \\
\hline TETRACHLOROETHENE & $127-18-4$ & 0 & PPB & \\
\hline CHLOROBENZENE & $108-90-7$ & $\overline{0}$ & PPB & \\
\hline ETHYLBENZENE & $100-41-4$ & 0 & PPB & \\
\hline M-XYLENE & $108-38-3$ & 0 & PPB & \\
\hline STYRENE & $100-42-5$ & 0 & PPB & \\
\hline O-XYLENE & $95-47-6$ & $\overline{0}$ & PPB & \\
\hline 1,1,2,2-TETRACHLORETHANE & $79-34-5$ & 0 & PPB & \\
\hline 1,3,5-TRIMETHYLBENZENE & $108-67-8$ & 0 & PPB & \\
\hline 1,2,4-TRIMETHYLBENZENE & $95-63-6$ & 0 & PPB & \\
\hline 1,3-DICHLOROBENZENE & $5 4 \longdiv { 1 - 7 3 - 1 }$ & 0 & PPB & \\
\hline 1,2-DICHLOROBENZENE & $95-50-1$ & 0 & PPB & \\
\hline 1,4-DICHLOROBENZENE & $106-46-7$ & 0 & PPB & \\
\hline
\end{tabular}


Sample: $\quad 2402 \mathrm{~W}$

Sample Name: FT6045_2W.21A

Search Libraries: C:DATABASEIWILEY138.L Minimum Quality: 70

Unknown Spectrum: Apex

Integration Params: VOA.E

$\begin{array}{lllll}\text { Pk\# RT Area\% } & \text { Library/ID } & \text { Ref\# } & \text { CAS\# } & \text { Qual }\end{array}$

624.46 6.97 C:IDATABASEIWILEY138.L

2-Pentanone, 4-methyl-

$118017000108-10-176$

725.55 5.90 C:DATABASEIWILEY138.L

Benzene, methyl-

$117430000108-88-394$

1331.34 6.24 C:DATABASEIWILEY138.L ALPHA.-PINENE, (-)- $\quad 122065$ 000080-56-8 87

$1532.752 .63 \mathrm{C}:$ :DATABASEIWILEY138.L 2-BETA.-PINENE

$8163000127-91-3 \quad 95$

$1733.65 \quad 7.02$ C: DATABASEIWILEY138.L DELTA.3-Carene

$122092013466-78-996$

$1934.242 .98 \mathrm{C} \cdot$ :DATABASEIWILEY138.L

beta.-Phellandrene

Sabinene

beta.-Thujene
$8118000555-10-2 \quad 93$

$122063003387-41-590$

8155 028634-89-1 81 
File

Operator

Acquired

Instrument

Sample Name: FT6045-2WB.41A

Misc Info : DELTA $P=100$ torr

Vial Number: 1

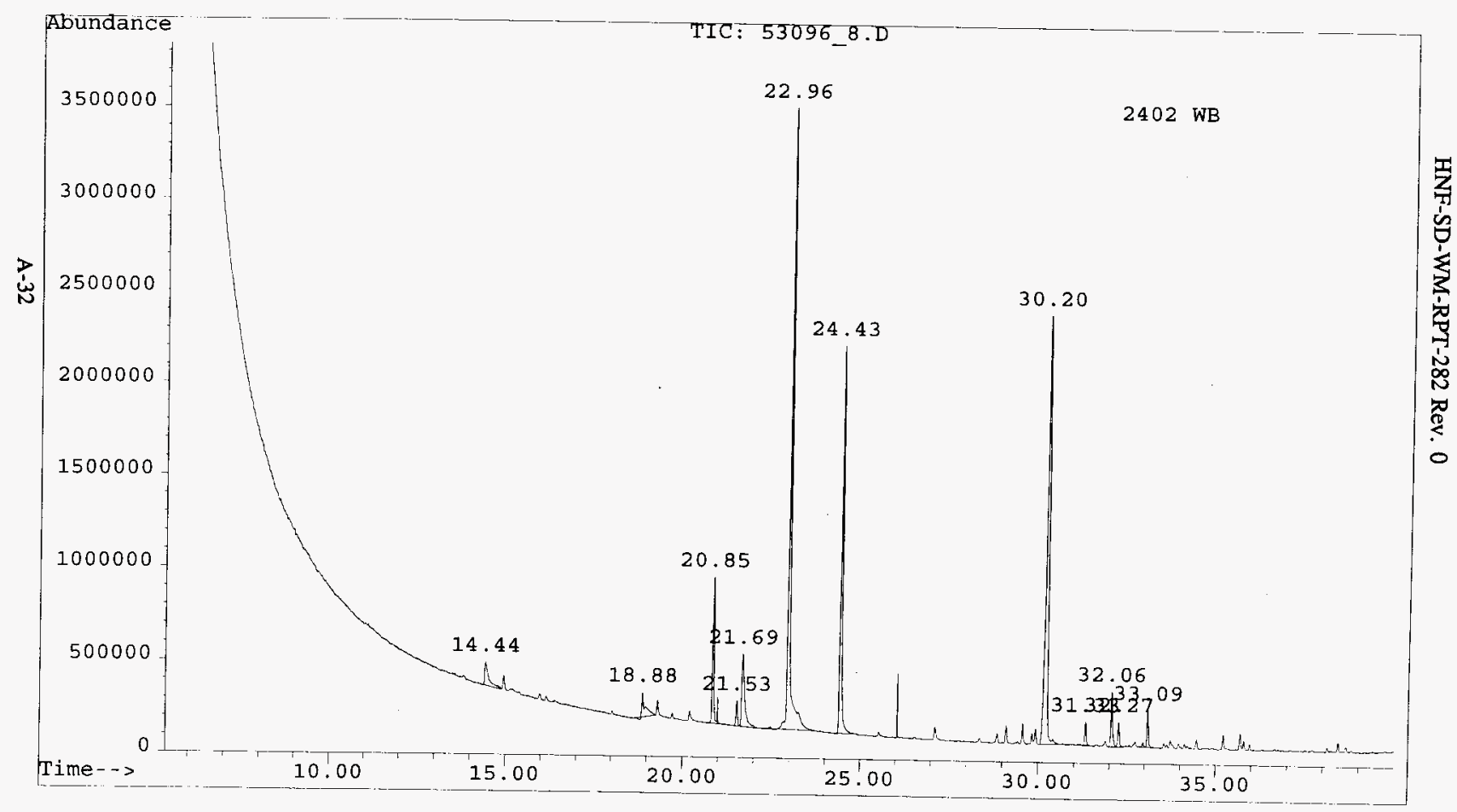


HNF-SD-WM-RPT-282 Rev. 0

CWC_REP.XLS

ANALYSIS REPORT FOR CWC AIR SAMPLES

File Name:

Analyst:

Analysis Date:

Method:

Sample Name:

53096_8.D

LAP

05/30/96

CWC

FT6045-2WB.41A

\begin{tabular}{|c|c|c|c|c|}
\hline COMPOUND NAME & CAS \# & Concentration & Units & Comments \\
\hline ISO-BUTANE & $75-28-5$ & 0 & PPB & \\
\hline VINYL CHLORIDE & $75-01-4$ & 0 & PPB & \\
\hline ETHANOL & $64-17-5$ & 0 & PPB & \\
\hline ACETONE & $67-64-1$ & 7 & PPB & \\
\hline 2-PROPANOL & $67-63-0$ & 0 & PPB & \\
\hline 1,1-DICHLOROETHENE & $75-35-4$ & 0 & PPB & \\
\hline FREON 113 & $76-13-1$ & 0 & PPB & \\
\hline DICHLOROMETHANE & $75-09-2$ & 0 & PPB & \\
\hline 1,1-DICHLOROETHANE & $75-34-3$ & $\overline{0}$ & PPB & \\
\hline 2-BUTANONE & $78-93-3$ & 19 & PPB & \\
\hline N-HEXANE & $110-54-3$ & $\overline{0}$ & PPB & \\
\hline 1,2-DICHLOROETHENE & $156-59-2$ & 3 & PPB & \\
\hline CHLOROFORM & $67-66-3$ & 0 & PPB & \\
\hline TETRAHYDROFURAN & $109-99-9$ & 0 & PPB & \\
\hline 1,1,1-TRICHLOROETHANE & $71-55-6$ & 34 & PPB & \\
\hline 1,2-DICHLOROETHANE & $107-06-2$ & 2 & PPB & \\
\hline BENZENE & $71-43-2$ & 0 & PPB & \\
\hline CARBON TETRACHLORIDE & $56-23-5$ & 11 & PPB & \\
\hline CYCLOHEXANE & $110-82-7$ & 0 & PPB & \\
\hline N-BUTANOL & $71-36-3$ & 34 & PPB & \\
\hline TRICHLOROETHENE & $79-01-6$ & 151 & PPB & \\
\hline 4-METHYL-2-PENTANONE & $108-10-1$ & 45 & PPB & \\
\hline TOLUENE & $108-88-3$ & 0 & $\overline{\mathrm{PPB}}$ & \\
\hline 1,1,2-TRICHLOROETHANE & $79-00-5$ & 0 & PPB & \\
\hline TETRACHLOROETHENE & $127-18-4$ & 0 & PPB & \\
\hline CHLOROBENZENE & $108-90-7$ & 0 & PPB & \\
\hline ETHYLBENZENE & $100-41-4$ & 1 & PPB & \\
\hline M-XYLENE & $108-38-3$ & 3 & PPB & \\
\hline STYRENE & $100-42-5$ & 1 & PPB & \\
\hline O-XYLENE & $95-47-6$ & 2 & PPB & \\
\hline 1,1,2,2-TETRACHLORETHANE & $79-34-5$ & 0 & PPB & \\
\hline 1,3,5-TRIMETHYLBENZENE & $108-67-8$ & 2 & PPB & \\
\hline 1,2,4-TRIMETHYLBENZENE & $95-63-6$ & 5 & PPB & \\
\hline 1,3-DICHLOROBENZENE & $541-73-1$ & 0 & PPB & \\
\hline 1,2-DICHLOROBENZENE & $95-50-1$ & 0 & PPB & \\
\hline 1,4-DICHLOROBENZENE & $106-46-7$ & 0 & PPB & \\
\hline
\end{tabular}


HNF-SD-WM-RPT-282 Rev. 0

\section{LIST OF COMPOUNDS TENTATIVELY IDENTIFIED WITH MASS SPECTRAL LIBRARY}

Sample: $\quad 2402 \mathrm{WB}$

File: $\quad$ C:LHPCHEM2LDATA153096_8.D

ID $\quad$ FT6045-2WB.41A

Compound CAS\#

Ethane, 1,1,1-trichloro-

$000071-55-6$

1-Butanol

$000071-36-3$

Ethene, trichloro-

000079-01-6

2-Pentanone, 4-methyl-

000108-10-1

Benzene, 1,3-dimethyl-

000108-38-3

Ethanol, 2-butoxy-

$000111-76-2$

ALPHA.-PINENE, (-)-

000080-56-8

Benzaldehyde

$000100-52-7$

Benzene, 1,3,5-trimethyl-

$000108-67-8$

Ethanone, 1-phenyl-

000098-86-2 
File

: C: $\backslash$ HPCHEM $\backslash 2 \backslash D A T A \backslash 62096 \_5 . D$

Operator : MS

Acquired : 20 Jun 96 10:54 am using AcqMethod CWC

Instrument : 5972 - In

Sample Name: FT 6045 2WB. $40 B$

Misc Info : 100 TORR; HOLDING TIME EXPIRED

Vial Number: 1

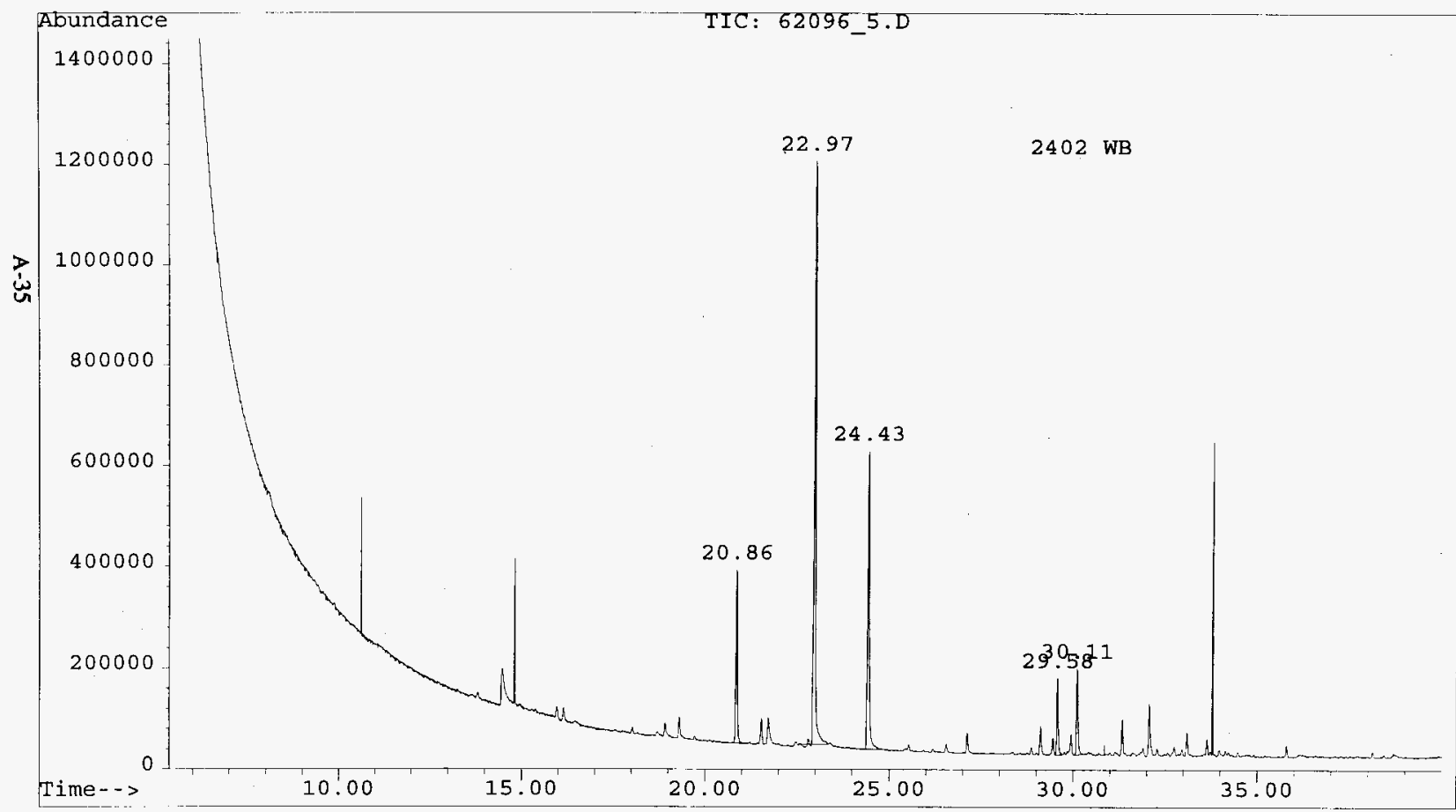


HNF-SD-WM-RPT-282 Rev. 0

CWC_REP.XLS

\section{ANALYSIS REPORT FOR CWC AIR SAMPLES}

File Name:

Analyst:

Analysis Date:

Method:

Sample Name:
62096_5.D

MS

06/20/96

CWC

FT 6045_2WB.40B

\begin{tabular}{|c|c|c|c|c|}
\hline COMPOUND NAME & CAS \# & Concentration & Units & Comments \\
\hline ISO-BUTANE & $75-28-5$ & 0 & PPB & \\
\hline VINYL CHLORIDE & $75-01-4$ & 0 & PPB & \\
\hline ETHANOL & $64-17-5$ & 0 & PPB & \\
\hline ACETONE & $67-64-1$ & 4 & PPB & \\
\hline 2-PROPANOL & $67-63-0$ & 0 & PPB & \\
\hline 1,1-DICHLOROETHENE & $75-35-4$ & 0 & PPB & \\
\hline FREON 113 & $76-13-1$ & 0 & PPB & \\
\hline DICHLOROMETHANE & $75-09-2$ & 0 & PPB & \\
\hline 1,1-DICHLOROETHANE & $75-34-3$ & 0 & PPB & \\
\hline 2-BUTANONE & $78-93-3$ & 0 & PPB & \\
\hline N-HEXANE & $+10-54-3$ & 0 & PPB & \\
\hline 1,2-DICHLOROETHENE & $156-59-2$ & 0 & $\mathrm{PPB}$ & \\
\hline CHLOROFORM & $67-66-3$ & 0 & PPB & \\
\hline TETRAHYDROFURAN & $109-99-9$ & 0 & PPB & \\
\hline 1,1,1-TRICHLOROETHANE & $71-55-6$ & 14 & PPB & \\
\hline 1,2-DICHLOROETHANE & $107-06-2$ & 0 & $\overline{\mathrm{PPB}}$ & \\
\hline BENZENE & $71-43-2$ & 0 & PPB & \\
\hline CARBON TETRACHLORIDE & $56-23-5$ & 0 & PPB & \\
\hline CYCLOHEXANE & $110-82-7$ & 0 & PPB & \\
\hline N-BUTANOL & $71-36-3$ & 0 & PPB & \\
\hline TRICHLOROETHENE & $79-01-6$ & 51 & PPB & \\
\hline 4-METHYL-2-PENTANONE & $108-10-1$ & 12 & PPB & \\
\hline TOLUENE & $108-88-3$ & 0 & PPB & \\
\hline 1,1,2-TRICHLOROETHANE & $79-00-5$ & 0 & $\mathrm{PPB}$ & \\
\hline TETRACHLOROETHENE & $127-18-4$ & 0 & PPB & \\
\hline CHLOROBENZENE & $108-90-7$ & 0 & PPB & \\
\hline ETHYLBENZENE & $100-41-4$ & 2 & PPB & \\
\hline M-XYLENE & $108-38-3$ & 0 & PPB & \\
\hline STYRENE & $100-42-5$ & 0 & PPB & \\
\hline O-XYLENE & $95-47-6$ & 0 & PPB & \\
\hline 1,1,2,2-TETRACHLORETHANE & $79-34-5$ & 0 & PPB & \\
\hline 1,3,5-TRIMETHYLBENZENE & $108-67-8$ & 2 & PPB & \\
\hline 1,2,4-TRIMETHYLBENZENE & $95-63-6$ & 1 & PPB & \\
\hline 1,3-DICHLOROBENZENE & $541-73-1$ & 0 & PPB & \\
\hline 1,2-DICHLOROBENZENE & $95-50-1$ & 0 & PPB & \\
\hline 1.4-DICHLOROBENZENE & $106-46-7$ & 0 & PPB & \\
\hline
\end{tabular}


HNF-SD-WM-RPT-282 Rev. 0

\section{LIST OF COMPOUNDS TENTATIVELY IDENTIFIED WITH MASS SPECTRAL LIBRARY}

Sample: $\quad 2402 \mathrm{WB}$

File: $\quad$ C:LHPCHEML2LDATA162096_5.D

ID: $\quad$ FT6045 2WB.40B

Compound

CAS\#

Methane, dichloro-

000075-09-2

Ethene, 1,2-dichloro-, (Z)-

000156-59-2

Ethane, 1,1,1-trichloro-

000071-55-6

Methane, tetrachloro-

000056-23-5

l-Butanol

000071-36-3

Ethene, trichloro-

000079-01-6

2-Pentanone, 4-methyl-

000108-10-1

Ethene, tetrachloro-

000127-18-4

Benzene, ethyl-

000100-41-4

Benzene, 1,3-dimethyl-

000108-38-3

3-Heptanone

000106-35-4

2-Heptanone

000110-43-0

Benzene, 1,2-dimethyl-

000095-47-6

Ethanol, 2-butoxy-

000111-76-2

ALPHA-PINENE, (-)-

000080-56-8

Benzaldehyde

000100-52-7

Benzene, 1,3,5-trimethyl-

$000108-67-8$ 
HNF-SD-WM-RPT-282 Rev. 0

LIST OF COMPOUNDS TENTATIVELY IDENTIFIED WITH MASS SPECTRAL LIBRARY

Sample: $\quad 2402$ WB (CONTINUED)

File: $\quad$ C:LHPCHEMLLDATAl62096_5.D

ID: $\quad$ FT6045_2WB.40B

Compound

CAS\#

Benzene, 1,3,5-trimethyl-

000108-67-8

DELTA.3-Carene

013466-78-9 
File

Operator

Acquired

Instrument

Sample Name

Misc Info

Vial Number:

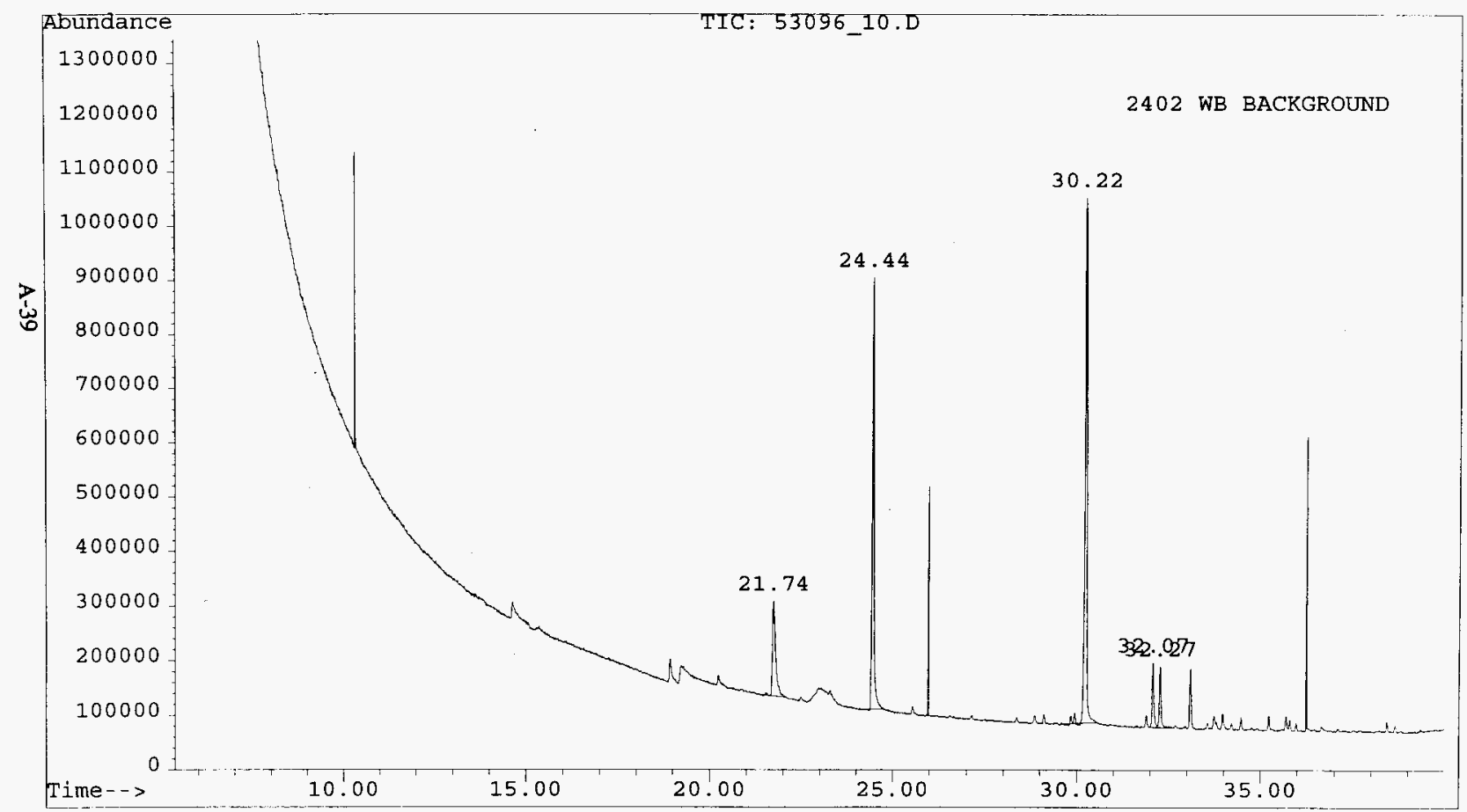

LAP

30 May $96 \quad 12: 10$ pm using AcqMethod CWC 5972 - In

FT6045-2WB . 42A

DELTA $P=100$ torr

1
C: $\backslash$ HPCHEM $\backslash 2 \backslash D A T A \backslash 53096 \quad 10 . D$ 
HNF-SD-WM-RPT-282 Rev. 0

CWC_REP.XLS

\section{ANALYSIS REPORT FOR CWC AIR SAMPLES}

File Name:

Analyst:

Analysis Date:

Method:

Sample Name:
53096_10.D

LAP

05/30/96

CWC

FT6045-2WB.42A

\begin{tabular}{|c|c|c|c|c|}
\hline COMPOUND NAME & CAS\# & Concentration & Units & Comments \\
\hline ISO-BUTANE & $75-28-5$ & 0 & PPB & \\
\hline VINYL CHLORIDE & $75-01-4$ & 0 & PPB & \\
\hline ETHANOL & $64-17-5$ & 0 & PPB & \\
\hline ACETONE & $67-64-1$ & 0 & PPB & \\
\hline 2-PROPANOL & $67-63-0$ & 0 & PPB & \\
\hline 1,1-DICHLOROETHENE & $75-35-4$ & 0 & PPB & \\
\hline FREON 113 & $76-13-1$ & 0 & PPB & \\
\hline DICHLOROMETHANE & 75-09-2 & 0 & PPB & \\
\hline 1,1-DICHLOROETHANE & 75-34-3 & 0 & PPB & \\
\hline 2-BUTANONE & $78-93-3$ & 0 & PPB & \\
\hline N-HEXANE & $110-54-3$ & 0 & $\overline{\mathrm{PPB}}$ & \\
\hline 1,2-DICHLOROETHENE & $156-59-2$ & 0 & PPB & \\
\hline CHLOROFORM & $67-66-3$ & 0 & PPB & \\
\hline TETRAHYDROFURAN & $109-99-9$ & 0 & PPB & \\
\hline 1,1,1-TRICHLOROETHANE & $71-55-6$ & 0 & PPB & \\
\hline 1,2-DICHLOROETHANE & $107-06-2$ & 0 & PPB & \\
\hline BENZENE & $71-43-2$ & 0 & $\mathrm{PPB}$ & \\
\hline CARBON TETRACHLORIDE & $56-23-5$ & 0 & $\mathrm{PPB}$ & \\
\hline CYCLOHEXANE & $110-82-7$ & 0 & $\mathrm{PPB}$ & \\
\hline N-BUTANOL & $71-36-3$ & 14 & PPB & \\
\hline TRICHLOROETHENE & $79-01-6$ & 0 & PPB & \\
\hline 4-METHYL-2-PENTANONE & $108-10-1$ & 18 & PPB & \\
\hline TOLUENE & $108-88-3$ & 0 & PPB & \\
\hline 1,1,2-TRICHLOROETHANE & $79-00-5$ & 0 & PPB & \\
\hline TETRACHLOROETHENE & $127-18-4$ & 0 & PPB & \\
\hline CHLOROBENZENE & $108-90-7$ & 0 & PPB & \\
\hline ETHYLBENZENE & $100-41-4$ & 0 & PPB & \\
\hline M-XYLENE & $108-38-3$ & 0 & PPB & \\
\hline STYRENE & $100-42-5$ & 0 & PPB & \\
\hline O-XYLENE & $95-47-6$ & 0 & PPB & \\
\hline $1,1,2,2$-TETRACHLORETHANE & $79-34-5$ & 0 & PPB & \\
\hline 1,3,5-TRIMETHYLBENZENE & $108-67-8$ & 2 & PPB & \\
\hline 1,2,4-TRIMETHYLBENZENE & $95-63-6$ & 3 & PPB & \\
\hline 1,3-DICHLOROBENZENE & $541-73-1$ & 0 & PPB & \\
\hline 1,2-DICHLOROBENZENE & $95-50-1$ & 0 & PPB & \\
\hline 1,4-DICHLOROBENZENE & $106-46-7$ & 0 & PPB & \\
\hline
\end{tabular}


HNF-SD-WM-RPT-282 Rev. 0.

LIST OF COMPOUNDS TENTATIVELY IDENTIFIED WITH MASS SPECTRAL

\section{LIBRARY}

Sample: 2402 WB BACKGROUND

File: $\quad$ C:LHPCHEMLLDATA153096_10.D

ID: FT6045-2WB.42A

Compound

CAS\#

2-Pentanone, 4-methyl-

000108-10-1

Ethanol, 2-butoxy-

$000111-76-2$

Benzaldehyde

$000100-52-7$

Phenol

000108-95-2

Benzene, 1,2,3-trimethyl-

000526-73-8 


\section{File}

Operator

Acquired

Instrument

Sample Name:

Misc Info

Vial Number: 1

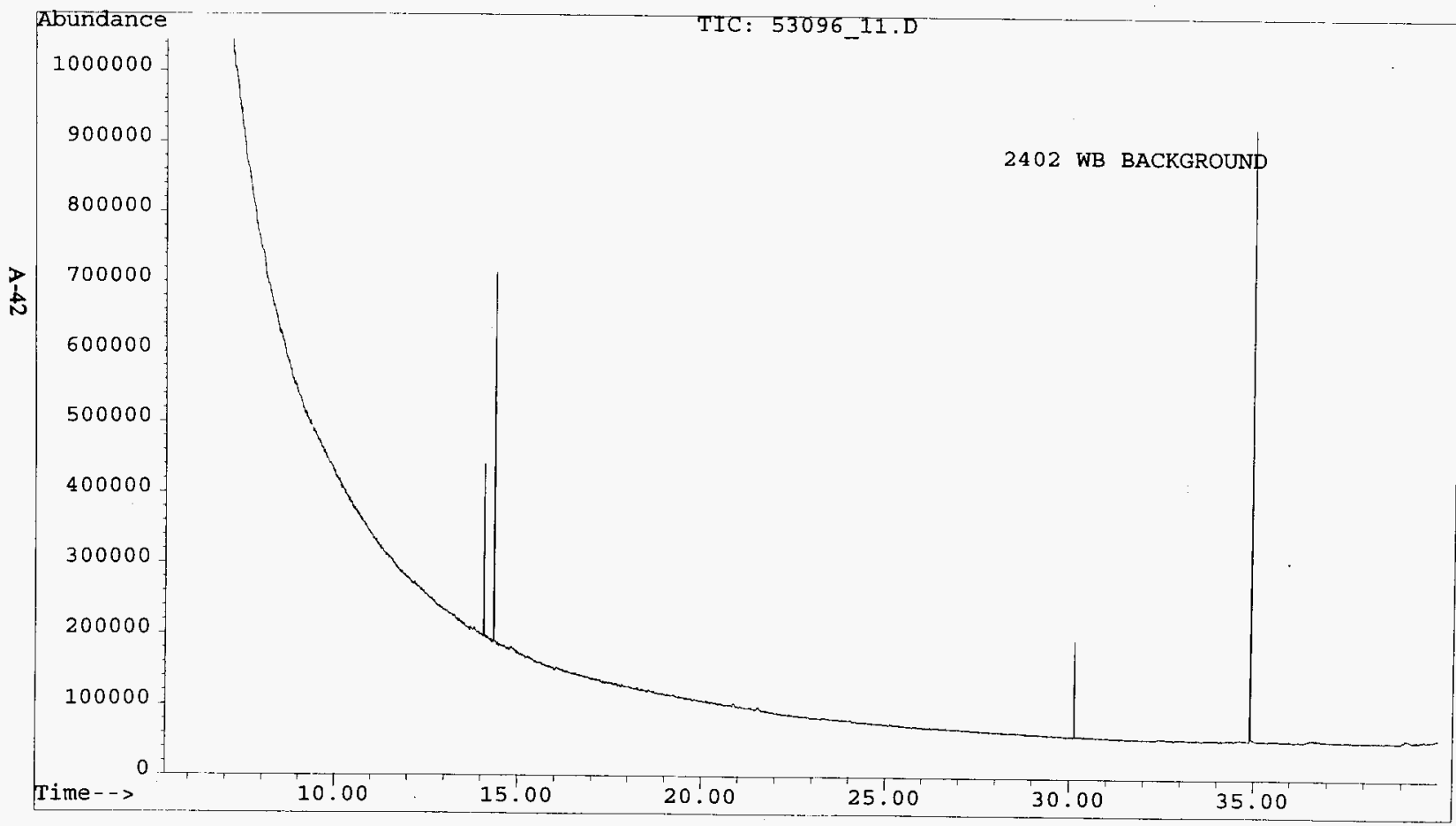


HNF-SD-WM-RPT-282 Rev. 0

CWC_REP.XLS

\section{ANALYSIS REPORT FOR CWC AIR SAMPLES}

File Name:

Analyst:

Analysis Date:

Method:

Sample Name:
53096_11.D

LAP

05/30/96

CWC

FT6045-2WB.42A

\begin{tabular}{|c|c|c|c|c|}
\hline COMPOUND NAME & CAS \# & Concentration & Units & Comments \\
\hline ISO-BUTANE & $75-28-5$ & $\overline{0}$ & PPB & \\
\hline VINYL CHLORIDE & $75-01-4$ & 0 & PPB & \\
\hline ETHANOL & $64-17-5$ & 0 & PPB & \\
\hline ACETONE & $67-64-1$ & 0 & PPB & \\
\hline 2-PROPANOL & $67-63-0$ & 0 & PPB & \\
\hline 1,1-DICHLOROETHENE & $75-35-4$ & 0 & PPB & \\
\hline FREON 113 & $76-13-1$ & 0 & PPB & \\
\hline DICHLOROMETHANE & $75-09-2$ & 0 & PPB & \\
\hline 1,1-DICHLOROETHANE & $75-34-3$ & 0 & PPB & \\
\hline 2-BUTANONE & $78-93-3$ & 0 & PPB & \\
\hline N-HEXANE & $110-54-3$ & 0 & PPB & \\
\hline 1,2-DICHLOROETHENE & $156-59-2$ & 0 & $\overline{\text { PPB }}$ & \\
\hline CHLOROFORM & $67-66-3$ & 0 & PPB & \\
\hline TETRAHYDROFURAN & $109-99-9$ & 0 & PPB & \\
\hline 1,1,1-TRICHLOROETHANE & $71-55-6$ & 0 & PPB & \\
\hline 1,2-DICHLOROETHANE & $107-06-2$ & 0 & PPB & \\
\hline BENZENE & $71-43-2$ & 0 & $\mathrm{PPB}$ & \\
\hline CARBON TETRACHLORIDE & $56-23-5$ & 0 & PPB & \\
\hline CYCLOHEXANE & $110-82-7$ & 0 & PPB & \\
\hline N-BUTANOL & $71-36-3$ & 0 & PPB & \\
\hline TRICHLOROETHENE & $79-01-6$ & 0 & PPB & \\
\hline 4-METHYL-2-PENTANONE & $108-10-1$ & 0 & PPB & \\
\hline TOLUENE & $108-88-3$ & 0 & PPB & \\
\hline 1,1,2-TRICHLOROETHANE & $79-00-5$ & 0 & PPB & \\
\hline TETRACHLOROETHENE & $127-18-4$ & 0 & PPB & \\
\hline CHLOROBENZENE & $108-90-7$ & 0 & PPB & \\
\hline ETHYLBENZENE & $100-41-4$ & 0 & PPB & \\
\hline M-XYLENE & $108-38-3$ & 0 & PPB & \\
\hline STYRENE & $100-42-5$ & 0 & PPB & \\
\hline O-XYLENE & $95-47-6$ & 0 & PPB & \\
\hline 1,1,2,2-TETRACHLORETHANE & $79-34-5$ & 0 & PPB & \\
\hline 1,3,5-TRIMETHYLBENZENE & $108-67-8$ & 0 & PPB & \\
\hline 1,2,4-TRIMETHYLBENZENE & $95-63-6$ & 0 & PPB & \\
\hline 1,3-DICHLOROBENZENE & $541-73-1$ & 0 & PPB & \\
\hline 1,2-DICHLOROBENZENE & $95-50-1$ & 0 & PPB & \\
\hline 1,4-DICHLOROBENZENE & $106-46-7$ & 0 & PPB & \\
\hline
\end{tabular}


HNF-SD-WM-RPT-282 Rev. 0

LIST OF COMPOUNDS TENTATIVELY IDENTIFIED WITH MASS SPECTRAL LIBRARY

Sample: $\quad 2402$ WB

File: $\quad$ C:UHPCHEML2LDATAI53096_11.D

ID: $\quad$ FT6045-2WB.42A

Compound

CAS\#

None 
File

Operator

Acquired

Instrument

Sample Name:

Misc Info

Vial Number:

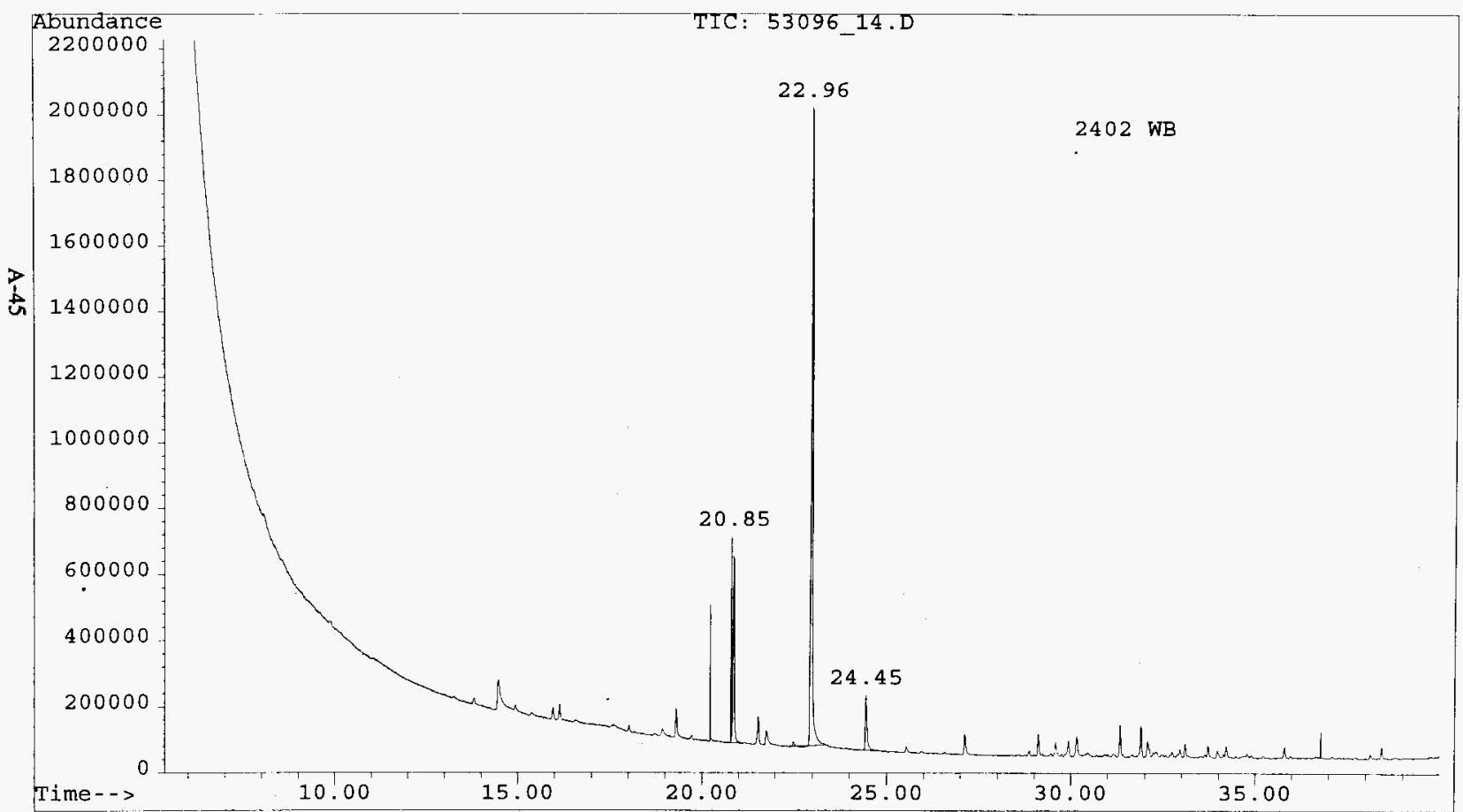

C: $\backslash H P C H E M \backslash 2 \backslash D A T A \backslash 53096 \quad 14 . D$

MS

30 May $96 \quad 4: 24$ pm using AcqMethod CWC $5972-$ In

FT6045-2WB , 40A

1
DELTA $P=100$ torr 
HNF-SD-WM-RPT-282 Rev. 0

CWC_REP.XLS

\section{ANALYSIS REPORT FOR CWC AIR SAMPLES}

File Name:

Analyst:

Analysis Date:

Method:

Sample Name:
53096_14.D

MS

05/30/96

CWC

FT6045-2WB.40A

\begin{tabular}{|c|c|c|c|c|}
\hline COMPOUND NAME & CAS \# & Concentration & Units & Comments \\
\hline ISO-BUTANE & $75-28-5$ & 0 & PPB & \\
\hline VINYL CHLORIDE & $75-01-4$ & 0 & PPB & \\
\hline ETHANOL & $64-17-5$ & 0 & PPB & \\
\hline ACETONE & $67-64-1$ & 5 & PPB & \\
\hline 2-PROPANOL & $67-63-0$ & 0 & PPB & \\
\hline 1,1-DICHLOROETHENE & $75-35-4$ & 0 & PPB & \\
\hline FREON 113 & $76-13-1$ & 0 & PPB & \\
\hline DICHLOROMETHANE & $75-09-2$ & 0 & PPB & \\
\hline 1,1-DICHLOROETHANE & $75-34-3$ & 0 & PPB & \\
\hline 2-BUTANONE & $78-93-3$ & 0 & PPB & \\
\hline N-HEXANE & $110-54-3$ & 0 & PPB & \\
\hline 1,2-DICHLOROETHENE & $156-59-2$ & 3 & PPB & \\
\hline CHLOROFORM & $67-66-3$ & 0 & PPB & \\
\hline TETRAHYDROFURAN & $109-99-9$ & 0 & PPB & \\
\hline 1,1,1-TRICHLOROETHANE & $71-55-6$ & 24 & PPB & \\
\hline 1,2-DICHLOROETHANE & $107-06-2$ & 0 & PPB & \\
\hline BENZENE & $71-43-2$ & 0 & PPB & \\
\hline CARBON TETRACHLORIDE & $56-23-5$ & 7 & PPB & \\
\hline CYCLOHEXANE & $110-82-7$ & 0 & PPB & \\
\hline N-BUTANOL & $71-36-3$ & 0 & PPB & \\
\hline TRICHLOROETHENE & $79-01-6$ & 83 & PPB & \\
\hline 4-METHYL-2-PENTANONE & $108-10-1$ & 0 & PPB & \\
\hline TOLUENE & 108-88-3 & 0 & PPB & \\
\hline 1,1,2-TRICHLOROETHANE & $79-00-5$ & 0 & PPB & \\
\hline TETRACHLOROETHENE & $127-18-4$ & 0 & PPB & \\
\hline CHLOROBENZENE & $108-90-7$ & 0 & PPB & \\
\hline ETHYLBENZENE & $100-41-4$ & 2 & PPB & \\
\hline M-XYLENE & $108-38-3$ & 0 & PPB & \\
\hline STYRENE & $100-42-5$ & 0 & PPB & \\
\hline O-XYLENE & $95-47-6$ & 1 & PPB & \\
\hline 1,1,2,2-TETRACHLORETHANE & $79-34-5$ & 0 & PPB & \\
\hline 1,3,5-TRIMETHYLBENZENE & $108-67-8$ & 0 & PPB & \\
\hline 1,2,4-TRIMETHYLBENZENE & $95-63-6$ & 0 & PPB & \\
\hline 1,3-DICHLOROBENZENE & $541-73-1$ & 0 & PPB & \\
\hline 1,2-DICHLOROBENZENE & $95-50-1$ & 0 & PPB & \\
\hline 1,4-DICHLOROBENZENE & $106-46-7$ & 0 & PPB & \\
\hline
\end{tabular}


HNF-SD-WM-RPT-282 Rev. 0

\section{LIST OF COMPOUNDS TENTATIVELY IDENTIFIED WITH MASS SPECTRAL LIBRARY}

Sample: $\quad 2402$ WB

File: $\quad$ C.LHPCHEMLLDATA153096_14.D

ID $\quad$ FT6045-2WB .40A

Compound.

CAS\#

Methane, dichloro-

$000075-09-2$

Ethene, 1,2-dichloro-, (Z)-

$000156-59-2$

Ethane, 1,1,1-trichloro-

$000071-55-6$

1-Bromobicyclo[4.3.1]decan-8-one

070576-35-1

Ethene, trichloro-

000079-01-6

2-Pentanone, 4-methyl-

000108-10-1

Ethene, tetrachloro-

000127-18-4

Benzene, 1,2-dimethyl-

$000095-47-6$

Benzene, 1,4-dimethyl-

$000106-42-3$

Benzene, 1,2-dimethyl-

000095-47-6

ALPHA.-PINENE, (-)-

000080-56-8

Benzene, 1,2,4-trimethyl-

000095-63-6 
File

Operator

: C: $\backslash$ HPCHEM $\backslash 2 \backslash D A T A \backslash 51596 \_5 . D$

Acquired

: 15 May $96 \quad 1: 28$ pm using AcqMethod CWC

Instrument : 5972 - In

Sample Name: FT 6045 2WC.25A BK

MisC Info : DELTA $\mathrm{P}^{-}=100$ torr

Vial Number: 1

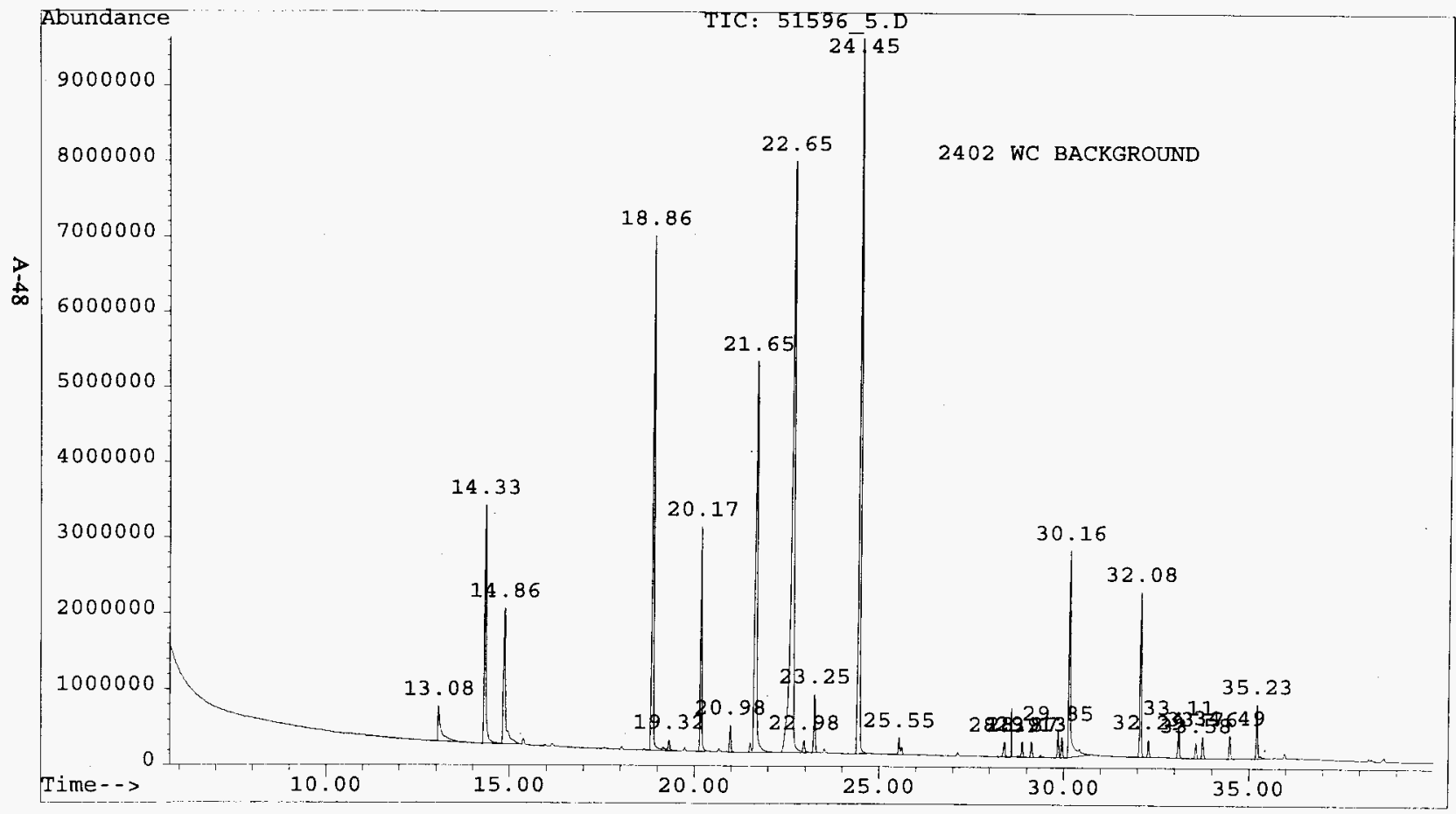


HNF-SD-WM-RPT-282 Rev. 0

CWC_REP.XLS

\section{ANALYSIS REPORT FOR CWC AIR SAMPLES}

File Name:

Analyst:

Analysis Date:

Method:

Sample Name:
51596_5.D

MS

05/15/96

CWC

FT 6045_2WC.25A BK

\begin{tabular}{|c|c|c|c|c|}
\hline COMPOUND NAME & CAS \# & Concentration & Units & Comments \\
\hline ISO-BUTANE & $75-28-5$ & 0 & $\overline{\mathrm{PPB}}$ & \\
\hline VINYL CHLORIDE & $75-01-4$ & D & PPB & \\
\hline ETHANOL & $64-17-5$ & 58 & PPB & \\
\hline ACETONE & $67-64-1$ & 96 & PPB & \\
\hline 2-PROPANOL & $67-63-0$ & 90 & PPB & \\
\hline 1,1-DICHLOROETHENE & $75-35-4$ & 3 & PPB & \\
\hline FREON 113 & $76-13-1$ & 0 & PPB & \\
\hline DICHLOROMETHANE & $75-09-2$ & 0 & PPB & \\
\hline 1,1-DICHLOROETHANE & $75-34-3$ & 1 & PPB & \\
\hline 2-BUTANONE & $78-93-3$ & 794 & $\overline{\mathrm{PPB}}$ & \\
\hline N-HEXANE & $110-54-3$ & 40 & PPB & \\
\hline 1,2-DICHLOROETHENE & $156-59-2$ & 5 & PPB & \\
\hline CHLOROFORM & $67-66-3$ & 1 & $\overline{\mathrm{PPB}}$ & \\
\hline TETRAHYDROFURAN & $109-99-9$ & 83 & PPB & \\
\hline 1,1,1-TRICHLOROETHANE & $71-55-6$ & 0 & $\mathrm{PPB}$ & \\
\hline 1,2-DICHLOROETHANE & $107-06-2$ & 21 & PPB & \\
\hline BENZENE & $71-43-2$ & 12 & PPB & \\
\hline CARBON TETRACHLORIDE & $56-23-5$ & 0 & PPB & \\
\hline CYCLOHEXANE & $110-82-7$ & 0 & $\mathrm{PPB}$ & \\
\hline N-BUTANOL & $71-36-3$ & 313 & PPB & \\
\hline TRICHLOROETHENE & $79-01-6$ & 5 & PPB & \\
\hline 4-METHYL-2-PENTANONE & $108-10-1$ & 246 & PPB & \\
\hline TOLUENE & $108-88-3$ & 7 & PPB & \\
\hline 1,1,2-TRICHLOROETHANE & $79-00-5$ & 4 & PPB & \\
\hline TETRACHLOROETHENE & $127-18-4$ & 0 & PPB & \\
\hline CHLOROBENZENE & $108-90-7$ & 5 & PPB & \\
\hline ETHYLBENZENE & $100-41-4$ & 5 & PPB & \\
\hline $\mathrm{M}-\mathrm{XYLENE}$ & $108-38-3$ & 6 & PPB & \\
\hline STYRENE & $100-42-5$ & 8 & PPB & \\
\hline O-XYLENE & $95-47-6$ & 7 & PPB & \\
\hline 1,1,2,2-TETRACHLORETHANE & $79-34-5$ & 0 & PPB & \\
\hline 1,3,5-TRIMETHYLBENZENE & $108-67-8$ & 6 & PPB & \\
\hline 1,2,4-TRIMETHYLBENZENE & $95-63-6$ & 11 & PPB & \\
\hline 1,3-DICHLOROBENZENE & $541-73-1$ & 6 & PPB & \\
\hline 1,2-DICHLOROBENZENE & $95-50-1$ & 9 & PPB & \\
\hline 1,4-DICHLOROBENZENE & $106-46-7$ & 10 & PPB & \\
\hline
\end{tabular}


HNF-SD-WM-RPT-282 Rev. 0

\section{LIST OF COMPOUNDS TENTATIVELY IDENTIFIED WITH MASS SPECTRAL LIBRARY}

Sample: $\quad$ W402 Background ${ }^{1}$

Sample Name: FT 6045_2WC.25A BK

Search Libraries: C:LDATABASEIWILEY138.L Minimum Quality: 70

C:DATABASELNBS54K.L Minimum Quality: 70

Pk\# RT Area\% Library/ID $\quad$ Ref\# CAS\# Qual

113.07 1.66 C:DATABASEIWILEY138.L Ethanol

$115963000064-17-590$

$214.33 \quad 5.68 \mathrm{C}:$ DATABASEIWILEY138.L

2-Propanone

$116051000067-64-172$

$314.864 .75 \mathrm{C}:$ :DATABASEIWILEY138.L 2-Propanol

$116152000067-63-090$

$4 \quad 15.390 .21 \mathrm{C}:$ DATABASEIWLEY138.L

Ethene, 1, 1-dichloro- $\quad 535$ 000075-35-4 96

$5 \quad 16.160 .13$ C:IDATABASE|WILEY138.L Methane, dichloro- $\quad 116807$ 000075-09-2 95

$7 \quad 18.04 \quad 0.08$ C:DATABASEIWLEY138.L

Ethane, 1,1-dichloro- $\quad 712$ 000075-34-3 90

$918.8611 .81 \mathrm{C}:$ DATABASEIWILEY138.L 2-Butanone

$116393000078-93-386$

$11 \quad 19.320 .28 \mathrm{C}:$ :DATABASEIWILEY138.L Ethene, 1,2-dichloro-, (Z)-

$117549000156-59-296$

$12 \quad 19.74 \quad 0.09$ C:DATABASEIWILEY138.L Chloroform $119703000067-66-395$

1320.174 .60 C:DATABASEIWILEY 138.L Furan, tetrahydro$116446000109-99-991$

\footnotetext{
${ }^{1}$ Note comments in report regarding possible carryover of polar analytes
} 
HNF-SD-WM-RPT-282 Rev. 0

Sample: 2402 Background $^{2}$ (continued)

Pk\# RT Area\% Library/ID Ref\# CAS\# Qual

$1620.98 \quad 0.56$ C:IDATABASEIWILEY138.L

Ethane, 1,2-dichloro-

$117658000107-06-295$

$\begin{array}{lll}17 & 21.51 & 0.21 \mathrm{C}: \text { DATABASEIWILEY138.L }\end{array}$ Benzene

$116687000071-43-295$

1821.6611 .87 C:DATABASEIWILEY138.L 1-Butanol

$116549000071-36-391$

$2022.98 \quad 0.38$ C:LDATABASEIWILEY138.L Ethene, trichloro-

$121019000079-01-698$

2123.251 .26 C:DATABASEIWILEY138.L 1,4-Dioxane

$117225000123-91-195$

2424.46 17.53 C:DATABASEIWILEY138.L 2-Pentanone, 4-methyl-

118018 000108-10-191

$2525.55 \quad 0.31$ C:IDATABASEIWILEY138.L Benzene, methyl-

117430 000108-88-395

$2625.610 .15 \mathrm{C}:$ :DATABASEIWILEY138.L Ethane, 1,1,2-trichloro-

$121295000079-00-599$

2727.130 .09 C:IDATABASEIWILEY138.L Ethene, tetrachloro-

$125230000127-18-497$

2828.390 .36 C:IDATABASEIWILEY138.L Benzene, chloro-

118974 000108-90-7 96

$3128.870 .30 \mathrm{C}:$ :DATABASEIWILEY138.L Benzene, ethyl-

$118560000100-41-495$

$32 \quad 29.13 \quad 0.34$ C:IDATABASEIWILEY138.L

Benzene, 1,3-dimethyl-

$118575000108-38-397$

$3429.850 .50 \mathrm{C}:$ :DATABASEIWILEY138.L Styrene $1662000100-42-597$

${ }^{2}$ Note comments in report regarding possible carryover of polar analytes 
Sample: 2402 Background $^{3}$ (continued)

Pk\# RT Area\% Library/ID Ref\# CAS\# Qual

$3529.960 .41 \mathrm{C}:$ DATABASEIWILEY138.L XYLENE

118580 001330-20-7 97

3630.164 .90 C:LDATABASE|WILEY138.L Ethanol, 2-butoxy-

$119786000111-76-290$

$3730.43 \quad 0.87$ C:IDATABASEIWLEY138.L Ethane, 1,1,2,2-tetrachloro-

$125455000079-34-584$

$3832.083 .34 \mathrm{C}:$ IDATABASEIWILEY138.L Benzaldehyde

$118546000100-52-796$

3932.290 .34 C:IDATABASEIWILEY138.L Benzene, 1,3,5-trimethyl-

$4147000108-67-895$

4033.110 .67 C:LDATABASEIWLEY138.L Benzene, 1,3,5-trimethyl120037 000108-67-8 95

4133.580 .31 C:LDATABASEIWILEY138.L Benzene, 1,3-dichloro-

$123079000541-73-198$

$4233.760 .49 \mathrm{C}:$ :DATABASEIWILEY138.L Benzene, 1,3-dichloro$123079000541-73-198$

4334.490 .48 C:DATABASEIWILEY138.L

Benzene, 1,3-dichloro-

$123079000541-73-198$

$4435.231 .06 \mathrm{C}$ :IDATABASEIWILEY138.L Ethanone, 1-phenyl-

$119985000098-86-295$

$45 \quad 35.320 .10 \mathrm{C}$ :IDATABASEIWILEY138.L

Benzaldehyde, 2-methyl-

$119973000529-20-490$

$4738.250 .06 \mathrm{C}:$ DATABASEIWILEY138.L

Benzaldehyde, ethyl-

$121568053951-50-181$

$4838.33 \quad 0.05$ C:IDATABASEIWILEY138.L

Benzaldehyde, 3,4-dimethyl-

$7381005973-71-793$

${ }^{3}$ Note comments in report regarding possible carryover of polar analytes 
File

Operator

Acquired

Instrument

NT6045 2WC. 23B

Misc Info : NOT PRESS. TO 15 PSI; DELTA $\mathrm{P}=100$ torr

Vial Number: 1

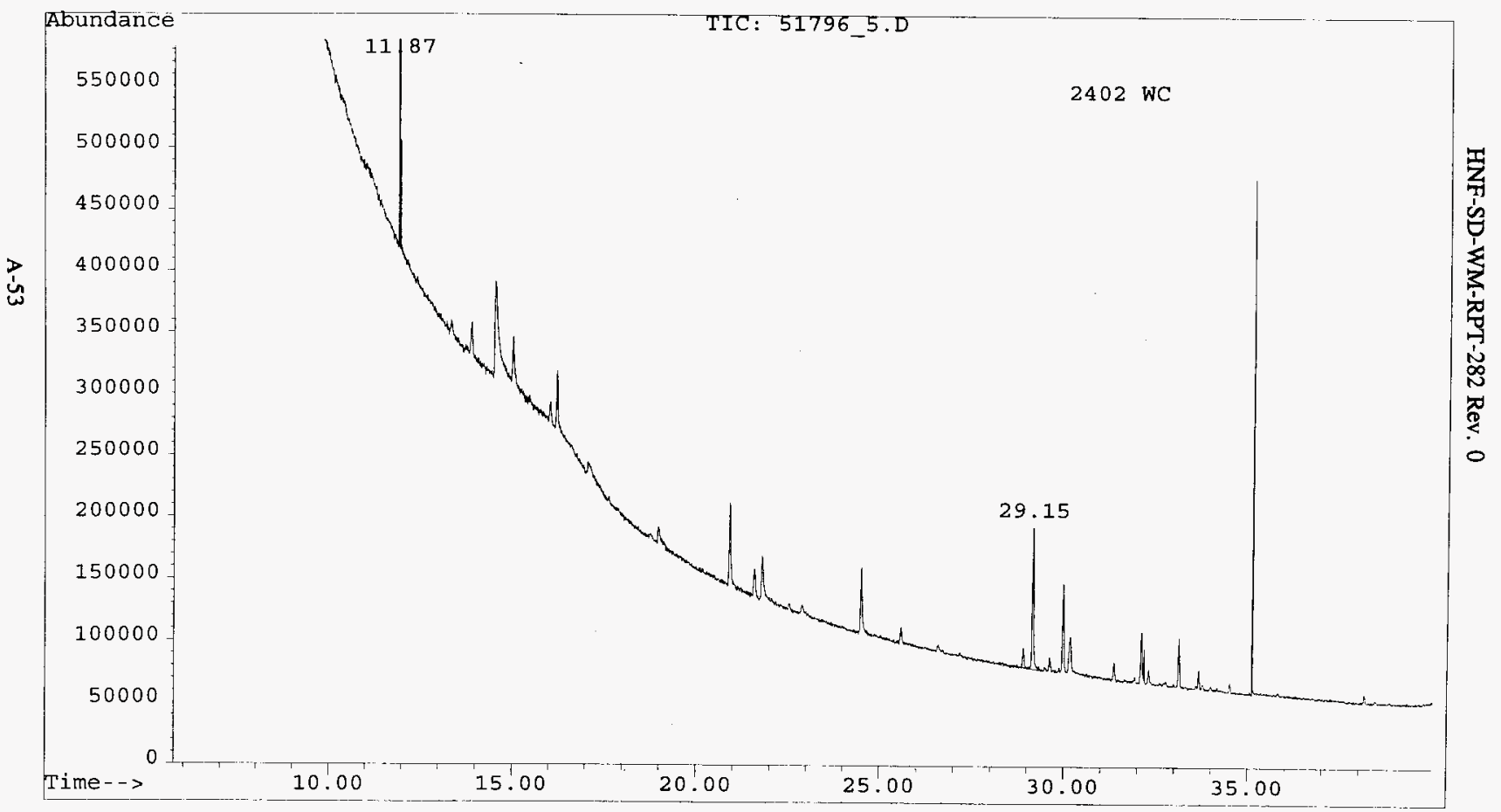


HNF-SD-WM-RPT-282 Rev. 0

CWC_REP.XLS

\section{ANALYSIS REPORT FOR CWC AIR SAMPLES}

File Name:

Analyst:

Analysis Date:

Method:

Sample Name:
51796_5.D

$\mathrm{ms}$

05/17/96

CWC

FT6045_2WC.23B

\begin{tabular}{|c|c|c|c|c|}
\hline COMPOUND NAME & CAS \# & Concentration & Units & Comments \\
\hline ISO-BUTANE & $75-28-5$ & 0 & PPB & \\
\hline VINYL CHLORIDE & $75-01-4$ & 0 & PPB & \\
\hline ETHANOL & $64-17-5$ & 0 & PPB & \\
\hline ACETONE & $67-64-1$ & 4 & PPB & \\
\hline 2-PROPANOL & $67-63-0$ & 0 & PPB & \\
\hline 1,1-DICHLOROETHENE & $75-35-4$ & 0 & $\overline{\mathrm{PPB}}$ & \\
\hline FREON 113 & $76-13-1$ & 0 & PPB & \\
\hline DICHLOROMETHANE & $75-09-2$ & 0 & PPB & \\
\hline 1,1-DICHLOROETHANE & $75-34-3$ & 0 & PPB & \\
\hline 2-BUTANONE & 78-93-3 & 0 & PPB & \\
\hline N-HEXANE & $110-54-3$ & 0 & PPB & \\
\hline 1,2-DICHLOROETHENE & $156-59-2$ & 0 & PPB & \\
\hline CHLOROFORM & $67-66-3$ & 0 & PPB & \\
\hline TETRAHYDROFURAN & $109-99-9$ & 0 & PPB & \\
\hline 1,1,1-TRICHLOROETHANE & $71-55-6$ & 3 & PPB & \\
\hline 1,2-DICHLOROETHANE & $107-06-2$ & 0 & PPB & \\
\hline BENZENE & $71-43-2$ & 0 & PPB & \\
\hline CARBON TETRACHLORIDE & $56-23-5$ & 0 & PPB & \\
\hline CYCLOHEXANE & $110-82-7$ & 0 & PPB & \\
\hline N-BUTANOL & $71-36-3$ & 0 & PPB & \\
\hline TRICHLOROETHENE & $79-01-6$ & 0 & $\overline{\mathrm{PPB}}$ & \\
\hline 4-METHYL-2-PENTANONE & $108-10-1$ & 0 & PPB & \\
\hline TOLUENE & 108-88-3 & 0 & $\overline{\mathrm{PPB}}$ & \\
\hline 1,1,2-TRICHLOROETHANE & $79-00-5$ & 0 & PPB & \\
\hline TETRACHLOROETHENE & $127-18-4$ & 0 & PPB & \\
\hline CHLOROBENZENE & $108-90-7$ & 0 & PPB & \\
\hline ETHYLBENZENE & $100-41-4$ & 3 & PPB & \\
\hline M-XYLENE & $108-38-3$ & 3 & PPB & \\
\hline STYRENE & $100-42-5$ & 0 & PPB & \\
\hline O-XYLENE & $95-47-6$ & 2 & PPB & \\
\hline 1,1,2,2-TETRACHLORETHANE & $79-34-5$ & 0 & PPB & \\
\hline 1,3,5-TRIMETHYLBENZENE & $108-67-8$ & 0 & PPB & \\
\hline 1,2,4-TRIMETHYLBENZENE & $95-63-6$ & 0 & PPB & \\
\hline 1,3-DICHLOROBENZENE & $541-73-1$ & 0 & PPB & \\
\hline 1,2-DICHLOROBENZENE & $95-50-1$ & 0 & PPB & \\
\hline 1,4-DICHLOROBENZENE & $106-46-7$ & 0 & PPB & \\
\hline
\end{tabular}


HNF-SD-WM-RPT-282 Rev. 0

\section{LIST OF COMPOUNDS TENTATIVELY IDENTIFIED WITH MASS SPECTRAL LIBRARY}

Sample: $\quad 2402$ WC

File: $\quad$ C:LHPCHEMLDLTAL151796_5.D

ID: $\quad$ FT6045_2WC.23B

Compound

CAS \#

Benzene, 1,4-dimethyl-

$000106-42-3$

Benzene, 1,2-dimethyl-

$000095-47-6$

Benzene, 1,2,4-trimethyl-

000095-63-6 
File

$: C: \backslash H P C H E M \backslash 2 \backslash D A T A \backslash 51396 \quad 6 . D$

Operator : MS

Acquired : 13 May 96 11:09 am using AcqMethod CWC

Instrument : 5972 - In

Sample Name: FT6045 2WC.23A

Misc Info: DELTA $\overline{\mathrm{P}}=100$ TORR

Vial Number: 1

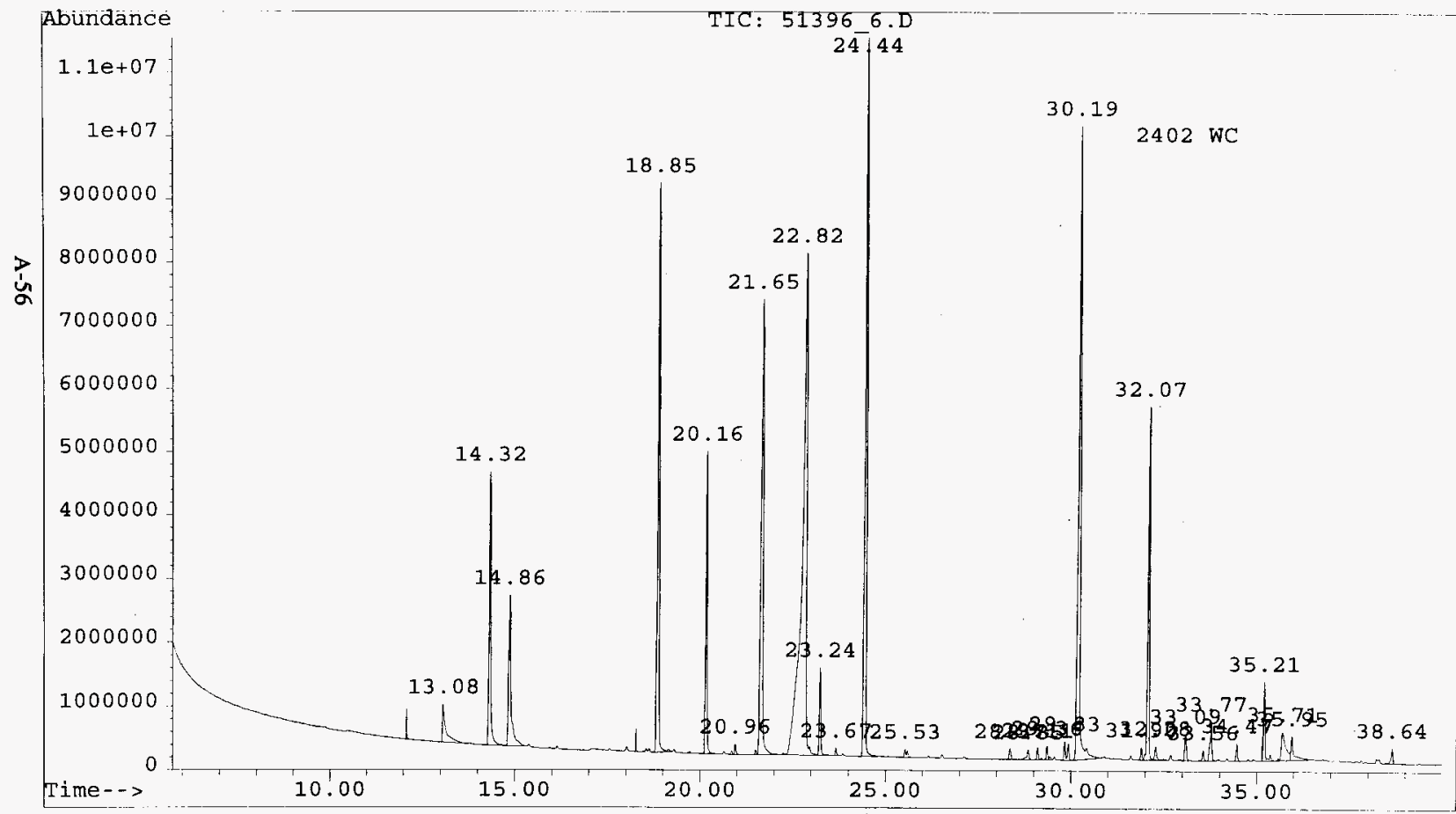


HNF-SD-WM-RPT-282 Rev. 0

CWC_REP.XLS

\section{ANALYSIS REPORT FOR CWC AIR SAMPLES}

File Name:

Analyst:

Analysis Date:

Method:

Sample Name:
51396_6.D

MS

05/13/96

CWC

FT6045_2WC.23A

\begin{tabular}{|c|c|c|c|c|}
\hline COMPOUND NAME & CAS \# & Concentration & Units & Comments \\
\hline ISO-BUTANE & $75-28-5$ & 0 & PPB & \\
\hline VINYL CHLORIDE & $75-01-4$ & 0 & PPB & \\
\hline ETHANOL & $64-17-5$ & 82 & PPB & \\
\hline ACETONE & 67-64-1 & 129 & PPB & \\
\hline 2-PROPANOL & $67-63-0$ & 131 & PPB & \\
\hline 1,1-DICHLOROETHENE & $75-35-4$ & 2 & PPB & \\
\hline FREON 113 & $76-13-1$ & 0 & PPB & \\
\hline DICHLOROMETHANE & $75-09-2$ & 0 & PPB & \\
\hline 1,1-DICHLOROETHANE & $75-34-3$ & 0 & PPB & \\
\hline 2-BUTANONE & 78-93-3 & 1051 & PPB & \\
\hline N-HEXANE & $110-54-3$ & 54 & PPB & \\
\hline 1,2-DICHLOROETHENE & $156-59-2$ & 0 & PPB & \\
\hline CHLOROFORM & $67-66-3$ & 0 & PPB & \\
\hline TETRAHYDROFURAN & $109-99-9$ & 126 & PPB & \\
\hline 1,1,1-TRICHLOROETHANE & $71-55-6$ & 2 & PPB & \\
\hline 1,2-DICHLOROETHANE & 107-06-2 & 9 & PPB & \\
\hline BENZENE & $71-43-2$ & 7 & PPB & \\
\hline CARBON TETRACHLORIDE & $56-23-5$ & 0 & PPB & \\
\hline CYCLOHEXANE & $110-82-7$ & 0 & PPB & \\
\hline N-BUTANOL & $71-36-3$ & 446 & PPB & \\
\hline TRICHLOROETHENE & $79-01-6$ & 3 & PPB & \\
\hline 4-METHYL-2-PENTANONE & $108-10-1$ & 254 & PPB & \\
\hline TOLUENE & $108-88-3$ & 4 & PPB & \\
\hline 1,1,2-TRICHLOROETHANE & $79-00-5$ & 4 & PPB & \\
\hline TETRACHLOROETHENE & $127-18-4$ & 0 & PPB & \\
\hline CHLOROBENZENE & $108-90-7$ & 3 & PPB & \\
\hline ETHYLBENZENE & $100-41-4$ & 4 & PPB & \\
\hline M-XYLENE & 108-38-3 & 5 & PPB & \\
\hline STYRENE & $100-42-5$ & 7 & PPB & \\
\hline O-XYLENE & $95-47-6$ & 5 & PPB & \\
\hline $1,1,2,2$-TETRACHLORETHANE & $79-34-5$ & 3 & PPB & \\
\hline 1,3,5-TRIMETHYLBENZENE & $108-67-8$ & 6 & PPB & \\
\hline 1,2,4-TRIMETHYLBENZENE & $95-63-6$ & 10 & PPB & \\
\hline 1,3-DICHLOROBENZENE & $541-73-1$ & 5 & $\mathrm{PPB}$ & \\
\hline 1,2-DICHLOROBENZENE & $95-50-1$ & 8 & PPB & \\
\hline 1,4-DICHLOROBENZENE & $106-46-7$ & 8 & PPB & \\
\hline
\end{tabular}


HNF-SD-WM-RPT-282 Rev. 0

\section{LIST OF COMPOUNDS TENTATIVELY IDENTIFIED WITH MASS SPECTRAL \\ LIBRARY}

Sample: $\quad 2402 \mathrm{WC}$

Sample Name: FT6045_2WC.23A

Misc Info : $\quad$ DELTA $\bar{P}=100$ TORR

Search Libraries: C:DATABASEIWILEY138.L Minimum Quality: 70

Unknown Spectrum: Apex

Integration Params: VOA.E

Pk\# RT Area\% Library/ID Ref\# CAS\# $\quad$ Qual

$3 \quad 13.07 \quad 1.39 \mathrm{C}:$ DATABASEIWLEY138.L Ethanol

4 14.32 4.67 C:DATABASEIWILEY138.L 2-Propanone

$5 \quad 14.86 \quad 4.07$ C:DATABASE $I W I L E Y 138 . L$ 2-Propanol

$\begin{array}{lll}6 & 15.37 & 0.10 \mathrm{C}: \text { DATABASEIWILEY138.L }\end{array}$ Ethene, 1,1-dichloro-

$8 \quad 16.14 \quad 0.05$ C:DATABASEIWILEY138.L Methane, dichloro-

$15 \quad 18.869 .81 \mathrm{C}:$ DATABASEIWILEY138.L 2-Butanone

$17 \quad 19.30 \quad 0.07$ C:IDATABASEIWILEY138.L Ethene, 1,2-dichloro-, (E)-

1920.164 .41 C:DATABASEIWILEY138.L Furan, tetrahydro-

$2120.860 .06 \mathrm{C}:$ DATABASEIWLEY138.L Ethane, 1,1,1-trichloro-

2220.960 .16 C:IDATABASEIWILEY138.L Ethane, 1,2-dichloro$116807000075-09-294$

$115963000064-17-590$

$116050000067-64-180$

$116152000067-63-090$

$535000075-35-4 \quad 96$

$116393000078-93-3 \quad 86$

$117551000156-60-596$

116445 000109-99-9 91

$121290000071-55-6 \quad 74$

$117659000107-06-296$ 
Sample: $\quad 2402$ WC continued

2321.500 .08 C:IDATABASEIWILEY138.L

Benzene

$116687000071-43-295$

2421.66 10.23 C:IDATABASEIWILEY138.L

1-Butanol

$116556000071-36-391$

$2723.24 \quad 1.35$ C: IDATABASEIWILEY138.L 1,4-Dioxane

$117225000123-91-195$

$2823.670 .13 \mathrm{C}:$ :DATABASEIWILEY138.L 1-Propanol, 2-methoxy-

$345001589-47-5 \quad 78$

3024.4412 .45 C:IDATABASEIWILEY138.L 2-Pentanone, 4-methyl-

118019000108-10-191

$3125.530 .11 \mathrm{C}:$ :DATABASEIWILEY138.L Benzene, methyl- $\quad 117433$ 000108-88-3 93 BICYCLO-(2,2,1)-HEPTADIENE-(2,5) 117446 000121-46-0 87

$32 \quad 25.60 \quad 0.10 \mathrm{C}:$ IDATABASEIWILEY138.L

Ethane, 1,1,2-trichloro- $\quad 121295 \quad 000079-00-5 \quad 99$

$3728.850 .21 \mathrm{C}:$ DATABASEIWILEY138.L

Benzene, ethyl-

$118560000100-41-495$

3829.110 .17 C:LDATABASEIWILEY138.L Benzene, 1,3-dimethyl$118578000108-38-397$ Benzene, 1,4-dimethyl$118586000106-42-397$

$42 \quad 29.55 \quad 0.05$ C:IDATABASEIWILEY138.L 2-Heptanone $119298000110-43-081$

$4329.83 \quad 0.25$ C:LATABASEIWILEY138.L 1,3,5,7-Cyclooctatetraene Styrene

$118487000629-20-997$ $118481000100-42-596$

$4429.93 \quad 0.26 \mathrm{C}:$ DATABASEIWLEY138.L Benzene, 1,2-dimethyl$118564000095-47-695$ XYLENE $118580001330-20-794$

Benzene, 1,3-dimethyl$118576000108-38-394$

$45 \quad 30.1912 .63 \mathrm{C}:$ LDATABASEIWILEY138.L Ethanol, 2-butoxy$119793000111-76-286$ 
Sample: $\quad 2402$ WC continued

4630.420 .65 C:IDATABASEIWILEY138.L

Ethane, 1,1,2,2-tetrachloro- $\quad 125454$ 000079-34-5 87

$50 \quad 31.90 \quad 0.18 \mathrm{C}:$ DATABASEIWILEY138.L

Decane, 2,2,8-trimethyl-

27775 062238-01-1 78

Octane, 2,2,6-trimethyl15635 062016-28-8 78

Heptane, 4-ethyl-2,2,6,6-tetramethyl27801 062108-31-0 78

51 $32.075 .56 \mathrm{C}:$ DATABASEIWILEY138.L Benzaldehyde

$118546000100-52-797$

$52 \quad 32.27 \quad 0.27$ C:IDATABASEIWILEY138.L

Benzene, 1,3,5-trimethyl-

4147000108-67-8 94

Benzene, 1,2,3-trimethyl-

4145 000526-73-8 93

$55 \quad 33.09 \quad 0.49$ C:IDATABASEIWILEY138.L

Benzene, 1,3,5-trimethyl-

Benzene, 1,2,3-trimethyl-

$120037000108-67-895$

$4145000526-73-8 \quad 94$

Benzene, 1,2,4-trimethyl-

120028 000095-63-694

$56 \quad 33.56 \quad 0.17$ C:IDATABASEIWILEY 138.L

Benzene, 1,4-dichloro-

$123085000106-46-798$

5733.770 .69 C:IDATABASEIWHLEY138.L 1-Hexanol, 2-ethyl-

121201 000104-76-7 78

$6034.47 \quad 0.27$ C:LATABASEIWILEY138.L

Benzene, 1,2-dichloro-

$11151000095-50-1 \quad 98$

Benzene, 1,3-dichloro-

123079000541-73-198

$6335.21 \quad 1.23$ C:LATABASEIWILEY138.L

Ethanone, 1-phenyl-

$4126000098-86-2 \quad 95$

$6435.380 .1]$ C.DATABASEIWILEY 138.L

Benzenamine, N,N,2-trimethyl-

$7629000609-72-3 \quad 89$ 
HNF-SD-WM-RPT-282 Rev. 0

Sample: 2402 WC continued

$65 \quad 35.590 .04$ C:LDATABASEIWILEY138.L 2-Nonanone $122692000821-55-670$

$663570 \quad 1.26 \mathrm{C}:$ DATABASEIWILEY138.L

Benzenemethanol, alpha., alpha.-dime 121924 000617-94-7 90

$6735.960 .86 \mathrm{C}:$ DATABASEIWILEY138.L Nonanal

$122682000124-19-6 \quad 74$

6837.810 .03 C:LATABASEIWILEY138.L Benzenepropanal $121562000104-53-0 \quad 89$

6938.240 .06 C:IDATABASEIWILEY138.L

2,3-DIMETHYLBENZALDEHYDE 7378 005779-93-1 90

Benzaldehyde, ethyl$7377053951-50-1 \quad 83$

Benzaldehyde, 3,4-dimethyl$7381005973-71-7 \quad 80$

$70 \quad 38.29 \quad 0.07$ C:IDATABASEIWILEY138.L

2,3-DIMETHYLBENZALDEHYDE $\quad 7378$ 005779-93-1 74

7138.640 .24 C:IDATABASEIWILEY138.L

Decanal

$15512000112-31-2 \quad 72$ 
File

Operator

Acquired

Instrument

Sample Name: FT6045 2WC.25B BK

Misc Info: DELTA $\overline{\mathrm{P}}=100$ torr

Vial Number: 1

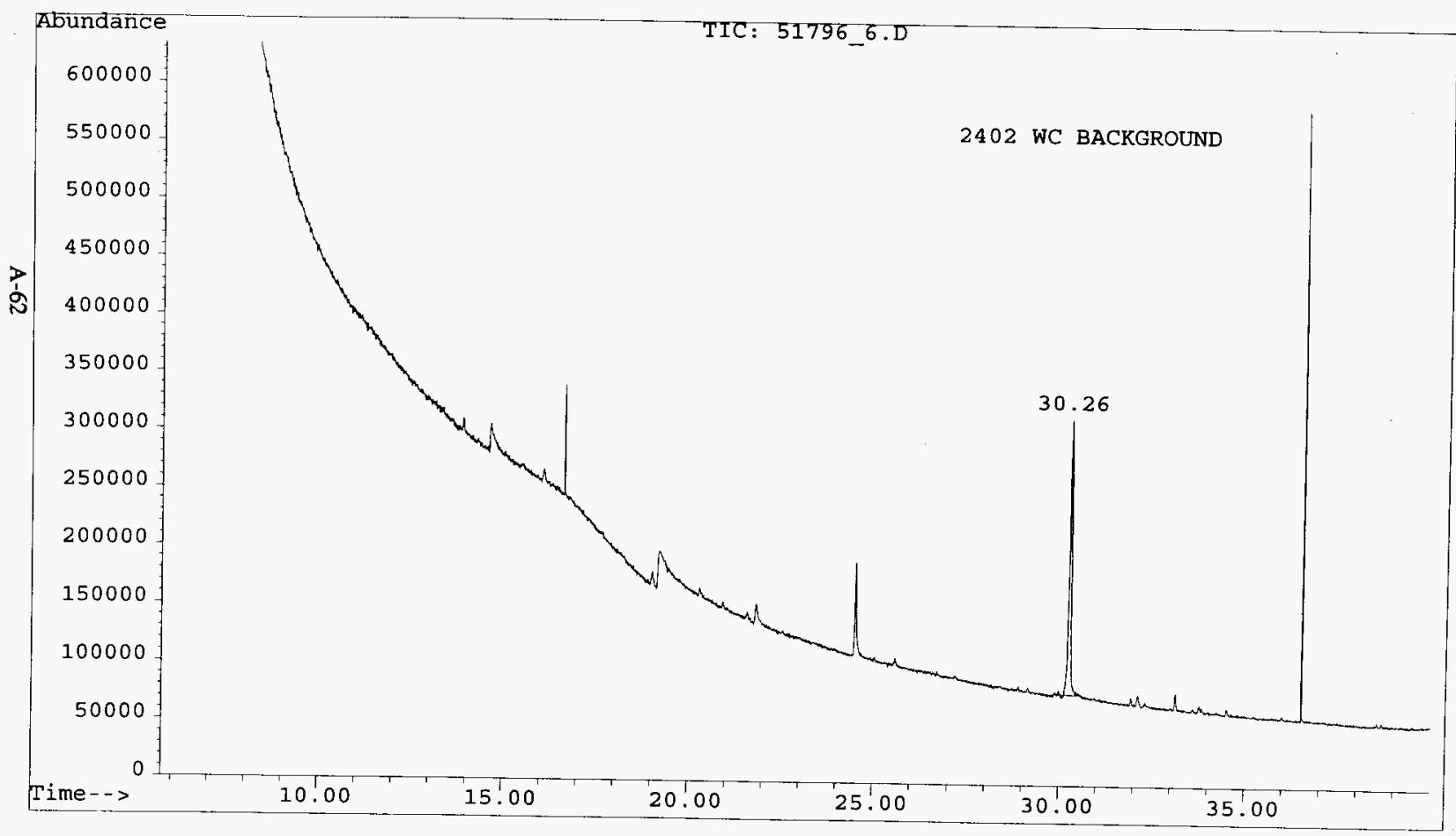


HNF-SD-WM-RPT-282 Rev. 0

CWC_REP.XLS

\section{ANALYSIS REPORT FOR CWC AIR SAMPLES}

File Name:

Analyst:

Analysis Date:

Method:

Sample Name:
51796_6.D

ms

05/17/96

CWC

FT6045_2WC.25B BK

\begin{tabular}{|c|c|c|c|c|}
\hline COMPOUND NAME & CAS \# & Concentration & Units & Comments \\
\hline ISO-BUTANE & $75-28-5$ & 0 & $\overline{\mathrm{PPB}}$ & \\
\hline VINYL CHLORIDE & $75-01-4$ & 0 & PPB & \\
\hline ETHANOL & $64-17-5$ & 0 & PPB & \\
\hline ACETONE & $67-64-1$ & 0 & $\overline{\mathrm{PPB}}$ & \\
\hline 2-PROPANOL & $67-63-0$ & 0 & PPB & \\
\hline 1,1-DICHLOROETHENE & $75-35-4$ & 0 & $\mathrm{PPB}$ & \\
\hline FREON 113 & $76-13-1$ & 0 & PPB & \\
\hline DICHLOROMETHANE & 75-09-2 & 0 & PPB & \\
\hline 1,1-DICHLOROETHANE & $75-34-3$ & 0 & PPB & \\
\hline 2-BUTANONE & $78-93-3$ & 0 & PPB & \\
\hline N-HEXANE & 110-54-3 & 0 & PPB & \\
\hline 1,2-DICHLOROETHENE & $156-59-2$ & 0 & PPB & \\
\hline CHLOROFORM & $67-66-3$ & 0 & PPB & \\
\hline TETRAHYDROFURAN & 109-99-9 & 0 & PPB & \\
\hline 1,1,1-TRICHLOROETHANE & $71-55-6$ & 0 & PPB & \\
\hline 1,2-DICHLOROETHANE & $107-06-2$ & 0 & PPB & \\
\hline BENZENE & $71-43-2$ & 0 & PPB & \\
\hline CARBON TETRACHLORIDE & $56-23-5$ & 0 & PPB & \\
\hline CYCLOHEXANE & $110-82-7$ & 0 & PPB & \\
\hline N-BUTANOL & $71-36-3$ & 0 & PPB & \\
\hline TRICHLOROETHENE & $79-01-6$ & 0 & PPB & \\
\hline 4-METHYL-2-PENTANONE & $108-10-1$ & 0 & PPB & \\
\hline TOLUENE & $108-88-3$ & 0 & PPB & \\
\hline 1,1,2-TRICHLOROETHANE & $79-00-5$ & 0 & PPB & \\
\hline TETRACHLOROETHENE & $127-18-4$ & 0 & $\mathrm{PPB}$ & \\
\hline CHLOROBENZENE & $108-90-7$ & 0 & $\overline{P P B}$ & \\
\hline ETHYLBENZENE & $100-41-4$ & 0 & PPB & \\
\hline M-XYLENE & $108-38-3$ & 0 & $\overline{\mathrm{PPB}}$ & \\
\hline STYRENE & $100-42-5$ & 0 & PPB & \\
\hline O-XYLENE & $95-47-6$ & 0 & $\overline{P P B}$ & \\
\hline $1,1,2,2$-TETRACHLORETHANE & $79-34-5$ & 0 & PPB & \\
\hline 1,3,5-TRIMETHYLBENZENE & $108-67-8$ & 0 & PPB & \\
\hline 1,2,4-TRIMETHYLBENZENE & $95-63-6$ & 0 & PPB & \\
\hline 1,3-DICHLOROBENZENE & $541-73-1$ & 0 & PPB & \\
\hline 1,2-DICHLOROBENZENE & $95-50-1$ & 0 & PPB & \\
\hline 1,4-DICHLOROBENZENE & $106-46-7$ & 0 & PPB & \\
\hline
\end{tabular}


HNF-SD-WM-RPT-282 Rev. 0

\section{LIST OF COMPOUNDS TENTATIVELY IDENTTFIED WITH MASS SPECTRAL} LIBRARY

Sample: $\quad 2402$ WC Background

File: $\quad$ C:LHPCHEMLIDATA151796_6.D

ID: $\quad$ FT6045_2WC.25B BK

Compound CAS \#

Ethanol, 2-butoxy- 000111-76-2 


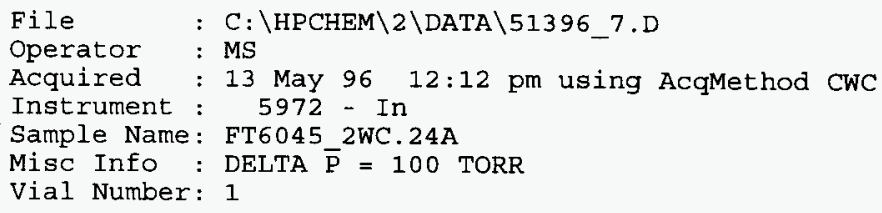

Sample Name: FT6045_2WC.24A

Misc Info: DELTA $\overline{\mathrm{P}}=100$ TORR

Vial Number: 1

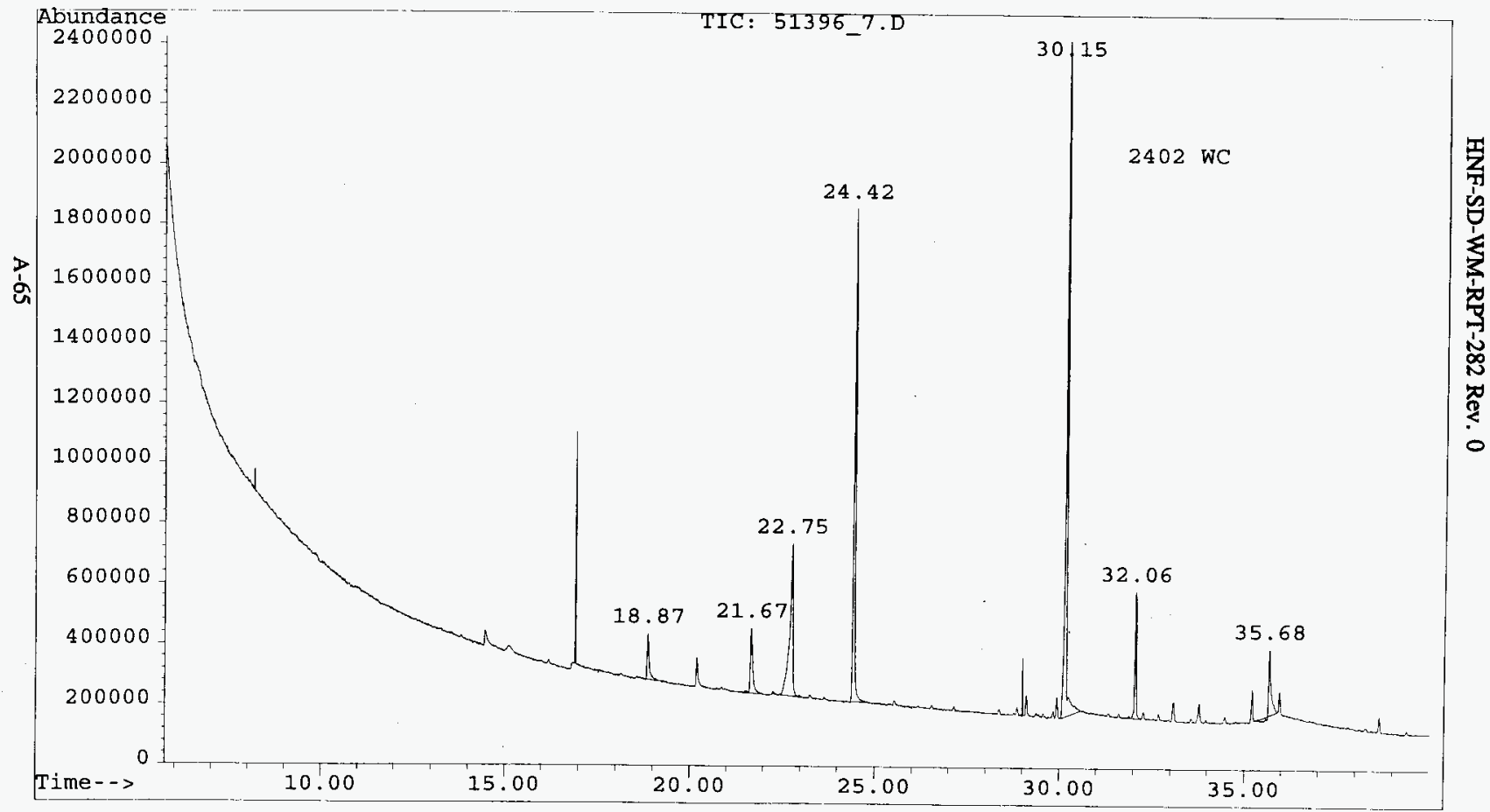


HNF-SD-WM-RPT-282 Rev. 0

CWC_REP.XLS

\section{ANALYSIS REPORT FOR CWC AIR SAMPLES}

File Name:

Analyst:

Analysis Date:

Method:

Sample Name:
51396_7.D

MS

05/13/96

CWC

FT6045_2WC.24A

\begin{tabular}{|c|c|c|c|c|}
\hline COMPOUND NAME & CAS \# & Concentration & Units & Comments \\
\hline ISO-BUTANE & $75-28-5$ & 0 & $\overline{\mathrm{PPB}}$ & \\
\hline VINYL CHLORIDE & $75-01-4$ & 0 & PPB & \\
\hline ETHANOL & $64-17-5$ & 0 & PPB & \\
\hline ACETONE & $67-64-1$ & 0 & PPB & \\
\hline 2-PROPANOL & $67-63-0$ & 2 & PPB & \\
\hline 1,1-DICHLOROETHENE & $75-35-4$ & 0 & PPB & \\
\hline FREON 113 & $76-13-1$ & 0 & PPB & \\
\hline DICHLOROMETHANE & $75-09-2$ & 0 & PPB & \\
\hline 1,1-DICHLOROETHANE & $75-34-3$ & 0 & PPB & \\
\hline 2-BUTANONE & $78-93-3$ & 17 & PPB & \\
\hline N-HEXANE & $110-54-3$ & 0 & PPB & \\
\hline 1,2-DICHLOROETHENE & $156-59-2$ & 0 & PPB & \\
\hline CHLOROFORM & $67-66-3$ & 0 & PPB & \\
\hline TETRAHYDROFURAN & $109-99-9$ & 0 & PPB & \\
\hline 1,1,1-TRICHLOROETHANE & $71-55-6$ & 0 & PPB & \\
\hline 1,2-DICHLOROETHANE & $107-06-2$ & 0 & PPB & \\
\hline BENZENE & $71-43-2$ & 0 & $\overline{\mathrm{PPB}}$ & \\
\hline CARBON TETRACHLORIDE & $56-23-5$ & 0 & PPB & \\
\hline CYCLOHEXANE & $110-82-7$ & 0 & PPB & \\
\hline N-BUTANOL & $71-36-3$ & 14 & $\overline{\mathrm{PPB}}$ & \\
\hline TRICHLOROETHENE & $79-01-6$ & 0 & PPB & \\
\hline 4-METHYL-2-PENTANONE & $108-10-1$ & 30 & PPB & \\
\hline TOLUENE & $108-88-3$ & 0 & PPB & \\
\hline 1,1,2-TRICHLOROETHANE & $79-00-5$ & 0 & PPB & \\
\hline TETRACHLOROETHENE & $127-18-4$ & 0 & PPB & \\
\hline CHLOROBENZENE & $108-90-7$ & 0 & PPB & \\
\hline ETHYLBENZENE & $100-41-4$ & 2 & PPB & \\
\hline M-XYLENE & $108-38-3$ & 0 & PPB & \\
\hline STYRENE & $100-42-5$ & 0 & PPB & \\
\hline O-XYLENE & $95-47-6$ & 1 & PPB & \\
\hline 1,1,2,2-TETRACHLORETHANE & $79-34-5$ & 0 & PPB & \\
\hline 1,3,5-TRIMETHYLBENZENE & $108-67-8$ & 7 & PPB & \\
\hline 1,2,4-TRIMETHYLBENZENE & $95-63-6$ & 1 & PPB & \\
\hline 1,3-DICHLOROBENZENE & $541-73-1$ & 0 & PPB & \\
\hline 1,2-DICHLOROBENZENE & $95-50-1$ & 0 & PPB & \\
\hline 1,4-DICHLOROBENZENE & $106-46-7$ & 0 & PPB & \\
\hline
\end{tabular}


HNF-SD-WM-RPT-282 Rev. 0

\section{LIST OF COMPOUNDS TENTATIVELY IDENTIFIED WITH MASS SPECTRAL LIBRARY}

Sample: $\quad 2402 \mathrm{WC}$

File: $\quad$ FT6045_2WC.24A

Search Libraries: C:DATABASEIWILEY138.L Minimum Quality: 70

Unknown Spectrum: Apex

Integration Params: VOA.E

Pk\# RT Area\% Library/ID Ref\# CAS\# Qual

$520.20 \quad 1.60$ C:DATABASEIWILEY138.L Furan, tetrahydro-

$116445000109-99-972$

621.664 .35 C:IDATABASEIWILEY138.L 1-Butanol

$116554000071-36-387$

722.7613 .49 C:IDATABASEIWILEY138.L 2-Propanol, 1-methoxy-

347 000107-98-2 83

824.4220 .02 C:IDATABASEIWILEY138.L 2-Pentanone, 4-methyl118019 000108-10-1 91

$1128.86 \quad 0.42$ C:DATABASEIWILEY138.L Benzene, ethylBenzene, 1,2-dimethyl$118571000095-47-672$

1329.111 .04 C:DATABASEIWILEY138.L Benzene, 1,4-dimethylXYLENE

Benzene, 1,3-dimethyl118587 000106-42-3 95 $118580001330-20-795$ 118578 000108-38-3 95

1429.840 .34 C:IDATABASEIWILEY138.L Styrene

$118484000100-42-594$

1529.940 .81 C:DATABASEIWILEY138.L Benzene, 1,2-dimethyl$118564000095-47-692$ Benzene, 1,3-dimethyl$118576000108-38-391$

1630.1530 .70 C:DATABASEIWILEY138.L Ethanol, 2-butoxy$119793000111-76-290$ 
HNF-SD-WM-RPT-282 Rev. 0

Sample: $\quad 2402$ WC (continued)

Pk\# RT Area\% Library/ID Ref\# CAS\# Qual

832.06 5.01 C:DATABASEIWLEY138.L

Benzaldehyde

$118546000100-52-797$

$1932.270 .32 \mathrm{C}:$ LDATABASEIWILEY138.L

Benzene, 1,3,5-trimethyl-

$120037000108-67-874$

Benzene, 1,2,3-trimethyl-

4145 000526-73-8 70

$2133.08 \quad 0.95 \mathrm{C}:$ DATABASEIWILEY138.L

Benzene, 1,3,5-trimethyl-

$120037000108-67-895$

Benzene, 1,2,4-trimethyl-

$120030000095-63-693$

$2233.560 .17 \mathrm{C}:$ :DATABASEIWILEY138.L

Benzene, 1,4-dichloro-

123085 000106-46-7 97

Benzene, 1,3-dichloro-

123079 000541-73-1 91

$2434.48 \quad 0.35$ C:IDATABASEIWLEY138.L

Benzene, 1,3-dichloro-

$123081000541-73-196$

Benzene, 1,4-dichloro-

123085 000106-46-7 96

Benzene, 1,2-dichloro-

$123077000095-50-196$

$\begin{array}{lll}25 & 35.21 & 1.09 \\ \text { C:LATABASEIWILEY138.L }\end{array}$ Ethanone, 1-phenyl-

$119989000098-86-297$

$2635.685 .52 \mathrm{C}:$ :DATABASEIWILEY138.L

Benzenemethanol, alpha., alpha.-dime 121925 000617-94-7 76 
File

Operator

: C: $\backslash$ HPCHEM $\backslash 2 \backslash D A T A \backslash 60496 \quad 7 . D$

$\begin{array}{ll}\text { Acquired : } & 4 \text { Jun } 96 \text { 2:49 pm using AcqMethod CWC } \\ \text { Instrument : } & 5972-\text { In }\end{array}$

Sample Name: FT 6045 2WD.45A

Misc Info : 100 TOR $\bar{R}$

Vial Number: 1

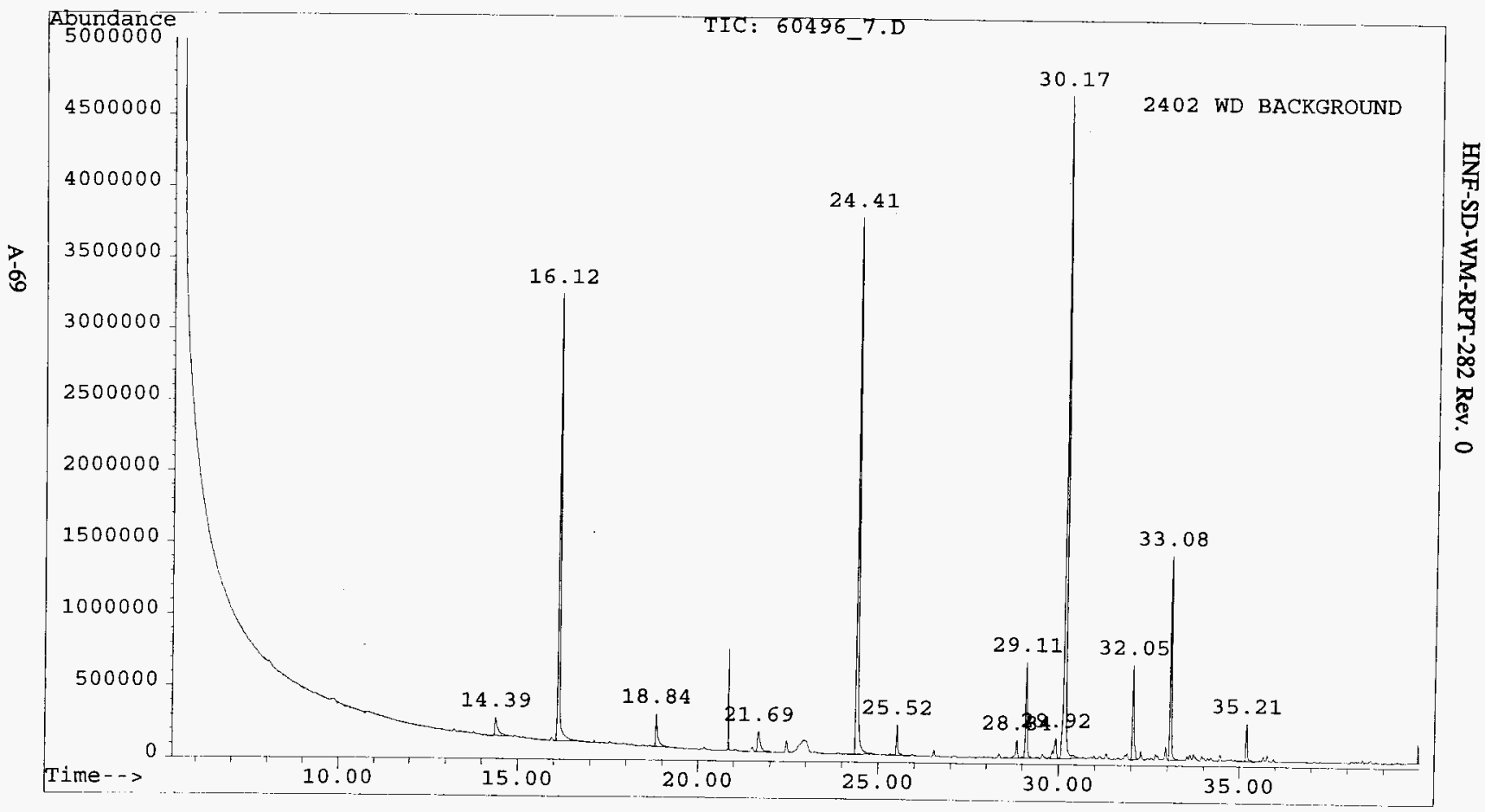


HNF-SD-WM-RPT-282 Rev. 0

CWC_REP.XLS

\section{ANALYSIS REPORT FOR CWC AIR SAMPLES}

File Name:

Analyst:

Analysis Date:

Method:

Sample Name:
60496_7.D

MS

06/04/96

CWC

FT 6045_2WD.45A

\begin{tabular}{|c|c|c|c|c|}
\hline COMPOUND NAME & CAS\# & Concentration & Units & Comments \\
\hline ISO-BUTANE & $75-28-5$ & 0 & PPB & \\
\hline VINYL CHLORIDE & $75-01-4$ & 0 & PPB & \\
\hline ETHANOL & $64-17-5$ & 0 & PPB & \\
\hline ACETONE & $67-64-1$ & 5 & PPB & \\
\hline 2-PROPANOL & $67-63-0$ & 0 & $\mathrm{PPB}$ & \\
\hline 1,1-DICHLOROETHENE & $75-35-4$ & 0 & PPB & \\
\hline FREON 113 & $76-13-1$ & 0 & PPB & \\
\hline DICHLOROMETHANE & $75-09-2$ & 104 & $\mathrm{PPB}$ & \\
\hline 1,1-DICHLOROETHANE & $75-34-3$ & 0 & PPB & \\
\hline 2-BUTANONE & $78-93-3$ & 26 & PPB & \\
\hline N-HEXANE & $110-54-3$ & 0 & PPB & \\
\hline 1,2-DICHLOROETHENE & $156-59-2$ & 0 & PPB & \\
\hline CHLOROFORM & $67-66-3$ & 0 & PPB & \\
\hline TETRAHYDROFURAN & $109-99-9$ & 0 & PPB & \\
\hline 1,1,1-TRICHLOROETHANE & $71-55-6$ & 0 & $\overline{\text { PPB }}$ & \\
\hline 1,2-DICHLOROETHANE & $107-06-2$ & 0 & PPB & \\
\hline BENZENE & $71-43-2$ & 0 & PPB & \\
\hline CARBON TETRACHLORIDE & $56-23-5$ & 0 & PPB & \\
\hline CYCLOHEXANE & $110-82-7$ & 0 & PPB & \\
\hline N-BUTANOL & $71-36-3$ & 10 & PPB & \\
\hline TRICHLOROETHENE & $79-01-6$ & 0 & $\overline{\mathrm{PPB}}$ & \\
\hline 4-METHYL-2-PENTANONE & $108-10-1$ & 67 & $\overline{\mathrm{PPB}}$ & \\
\hline TOLUENE & $108-88-3$ & 7 & PPB & \\
\hline 1,1,2-TRICHLOROETHANE & $79-00-5$ & 0 & $\mathrm{PPB}$ & \\
\hline TETRACHLOROETHENE & $127-18-4$ & 0 & PPB & \\
\hline CHLOROBENZENE & $108-90-7$ & 0 & PPB & \\
\hline ETHYLBENZENE & $100-41-4$ & 3 & PPB & \\
\hline M-XYLENE & $108-38-3$ & 20 & $\mathrm{PPB}$ & \\
\hline STYRENE & $100-42-5$ & 1 & PPB & \\
\hline O-XYLENE & $95-47-6$ & 4 & PPB & \\
\hline 1,1,2,2-TETRACHLORETHANE & $79-34-5$ & 0 & PPB & \\
\hline 1,3,5-TRIMETHYLBENZENE & $108-67-8$ & 1 & PPB & \\
\hline 1,2,4-TRIMETHYLBENZENE & $95-63-6$ & 36 & PPB & \\
\hline 1,3-DICHLOROBENZENE & $541-73-1$ & 0 & PPB & \\
\hline 1,2-DICHLOROBENZENE & $95-50-1$ & 0 & PPB & \\
\hline 1,4-DICHLOROBENZENE & $106-46-7$ & 0 & PPB & \\
\hline
\end{tabular}




\section{LIST OF COMPOUNDS TENTATIVELY IDENTIFIED WITH MASS SPECTRAL LIBRARY}

$\begin{array}{ll}\text { Sample: } & \text { 2402 WD BACKGROUND } \\ \text { File: } & \text { C:IHPCHEM2IDATAI60496_7.D } \\ \text { ID: } & \text { FT6045_2WD.45A }\end{array}$

Compound

CAS\#

Methane, dichloro-

000075-09-2

2-Butanone

000078-93-3

l-Butanol

$000071-36-3$

2-Pentanone, 4-methyl-

000108-10-1

Benzene, methyl-

000108-88-3

Benzene, ethyl-

$000100-41-4$

XYLENE

001330-20-7

Ethanol, 2-butoxy-

$000111-76-2$

ALPHA.-PINENE, (-)-

$000080-56-8$

Benzaldehyde

$000100-52-7$

Benzene, 1,3,5-trimethyl-

000108-67-8

Decane

000124-18-5

Benzene, 1,2,4-trimethyl-

000095-63-6

Benzene, 1,4-dichloro-

000106-46-7

Benzene, 1-methyl-2-(1-methylethyl)-

$000527-84-4$

Ethanone, 1-phenyl-

000098-86-2

Naphthalene, 1,2,3,4-tetrahydro-

000119-64-2 
File

Operator

Acquired

Instrument

FT6045-2WD.43A

Misc Info : DELTA $P=100$ torr

Vial Number: 1

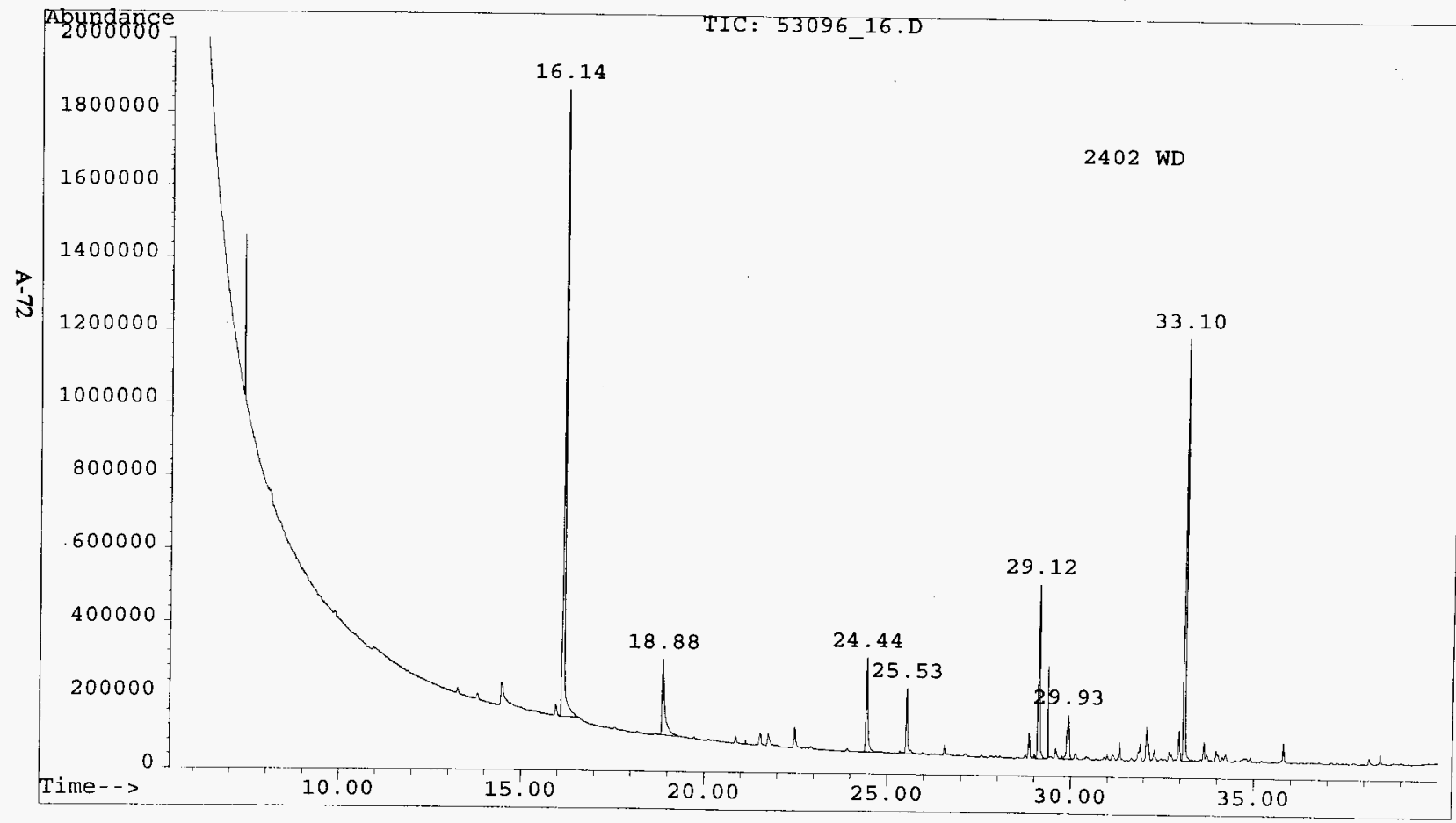


HNF-SD-WM-RPT-282 Rev. 0

CWC_REP.XLS

\section{ANALYSIS REPORT FOR CWC AIR SAMPLES}

File Name:

Analyst:

Analysis Date:

Method:

Sample Name:
53096_16.D

MS

05/30/96

CWC

FT6045-2WD.43A

\begin{tabular}{|c|c|c|c|c|}
\hline COMPOUND NAME & CAS \# & Concentration & Units & Comments \\
\hline ISO-BUTANE & $75-28-5$ & 0 & PPB & \\
\hline VINYL CHLORIDE & $75-01-4$ & 0 & PPB & \\
\hline ETHANOL & $64-17-5$ & 0 & PPB & \\
\hline ACETONE & $67-64-1$ & 0 & PPB & \\
\hline 2-PROPANOL & $67-63-0$ & 0 & PPB & \\
\hline 1,1-DICHLOROETHENE & $75-35-4$ & 0 & PPB & \\
\hline FREON 113 & $76-13-1$ & 0 & PPB & \\
\hline DICHLOROMETHANE & $75-09-2$ & 63 & PPB & \\
\hline 1,1-DICHLOROETHANE & $75-34-3$ & 0 & PPB & \\
\hline 2-BUTANONE & $78-93-3$ & 31 & PPB & \\
\hline N-HEXANE & $110-54-3$ & 0 & PPB & \\
\hline 1,2-DICHLOROETHENE & $156-59-2$ & 0 & $\overline{\text { PPB }}$ & \\
\hline CHLOROFORM & $67-66-3$ & 0 & PPB & \\
\hline TETRAHYDROFURAN & $109-99-9$ & 0 & PPB & \\
\hline 1,1,1-TRICHLOROETHANE & $71-55-6$ & 0 & PPB & \\
\hline 1,2-DICHLOROETHANE & $107-06-2$ & 0 & PPB & \\
\hline BENZENE & $71-43-2$ & 0 & PPB & \\
\hline CARBON TETRACHLORIDE & $56-23-5$ & 0 & PPB & \\
\hline CYCLOHEXANE & $110-82-7$ & 0 & PPB & \\
\hline N-BUTANOL & $71-36-3$ & 0 & $\overline{P P B}$ & \\
\hline TRICHLOROETHENE & 79-01-6 & 0 & PPB & \\
\hline 4-METHYL-2-PENTANONE & $108-10-1$ & 0 & PPB & \\
\hline TOLUENE & $108-88-3$ & 6 & PPB & \\
\hline 1,1,2-TRICHLOROETHANE & $79-00-5$ & 0 & PPB & \\
\hline TETRACHLOROETHENE & $127-18-4$ & 0 & PPB & \\
\hline CHLOROBENZENE & $108-90-7$ & 0 & PPB & \\
\hline ETHYLBENZENE & $100-41-4$ & 2 & PPB & \\
\hline M-XYLENE & $108-38-3$ & 16 & PPB & \\
\hline STYRENE & $100-42-5$ & 0 & PPB & \\
\hline O-XYLENE & $95-47-6$ & 3 & PPB & \\
\hline 1,1,2,2-TETRACHLORETHANE & $79-34-5$ & 0 & PPB & \\
\hline १,3,5-TRIMETHYLBENZENE & $108-67-8$ & 3 & PPB & \\
\hline 1,2,4-TRIMETHYLBENZENE & $95-63-6$ & 30 & PPB & \\
\hline 1,3-DICHLOROBENZENE & $541-73-1$ & 0 & PPB & \\
\hline 1,2-DICHLOROBENZENE & $95-50-1$ & 0 & PPB & \\
\hline 1,4-DICHLOROBENZENE & $106-46-7$ & 0 & PPB & \\
\hline
\end{tabular}


HNF-SD-WM-RPT-282 Rev. 0

\section{LIST OF COMPOUNDS TENTATIVELY IDENTIFIED WITH MASS SPECTRAL LIBRARY}

Sample: $\quad 2402$ WD

File $\quad$ C:LHPCHEMLLDATA153096_16.D

ID: $\quad$ FT6045-2WD.43A

Compound

CAS\#

Methane, dichloro-

$000075-09-2$

2-Pentanone, 4-methyl-

$000108-10-1$

Benzene, methyl-

000108-88-3

Benzene, ethyl-

$000100-41-4$

Benzene, 1,3-dimethyl-

000108-38-3

Benzene, 1,3-dimethyl-

000108-38-3

ALPHA.-PINENE, (-)-

$000080-56-8$

Benzene, 1,3,5-trimethyl

000108-67-8

Decane

000124-18-5

Benzene, 1,3,5-trimethyl-

000108-67-8

Benzene, 1-methyl-4-(1-methylethyl)-

000099-87-6

Undecane

001120-21-4

Naphthalene, 1,2,3,4-tetrahydro-

000119-64-2 
File

Operator

Acquired

Instrum

Instrument

Misc Info : DELTA $\overline{\mathrm{P}}=100$ TORR

Vial Number: 1

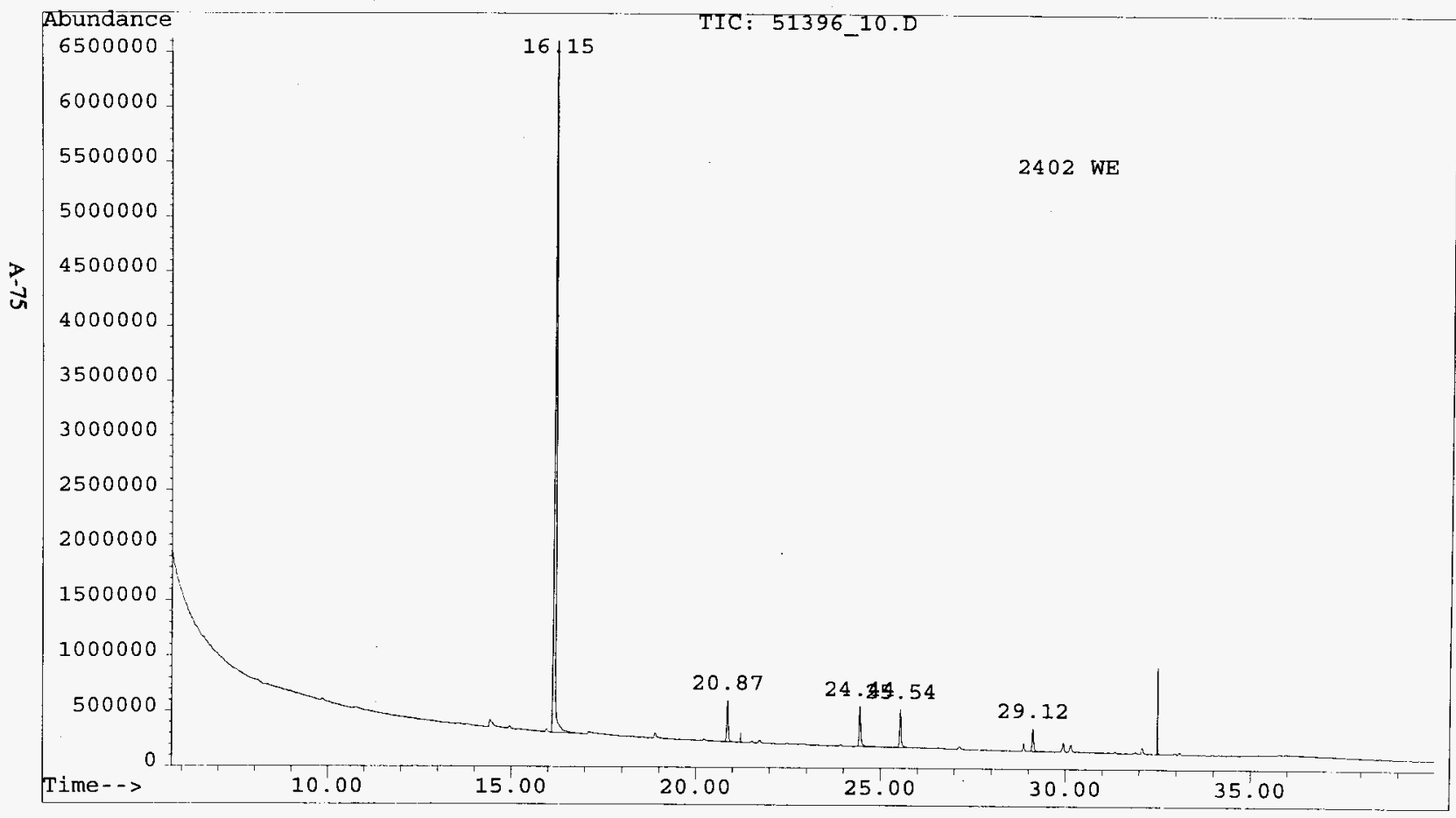


HNF-SD-WM-RPT-282 Rev. 0

CWC_REP.XLS

\section{ANALYSIS REPORT FOR CWC AIR SAMPLES}

File Name:

Analyst:

Analysis Date:

Method:

Sample Name:

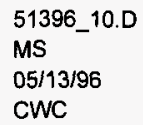

05/13/96

CWC

FT6045_2WE.26A

\begin{tabular}{|c|c|c|c|c|}
\hline COMPOUND NAME & CAS \# & Concentration & Units & Comments \\
\hline ISO-BUTANE & $75-28-5$ & 0 & PPB & \\
\hline VINYL CHLORIDE & $75-01-4$ & 0 & PPB & \\
\hline ETHANOL & $64-17-5$ & 0 & PPB & \\
\hline ACETONE & $67-64-1$ & 3 & PPB & \\
\hline 2-PROPANOL & $67-63-0$ & 0 & $\overline{\mathrm{PPB}}$ & \\
\hline 1,1-DICHLOROETHENE & $75-35-4$ & 0 & PPB & \\
\hline FREON 113 & $76-13-1$ & 0 & PPB & \\
\hline DICHLOROMETHANE & $75-09-2$ & 231 & PPB & \\
\hline 1,1-DICHLOROETHANE & $75-34-3$ & 0 & PPB & \\
\hline 2-BUTANONE & $78-93-3$ & 0 & $\mathrm{PPB}$ & \\
\hline N-HEXANE & $110-54-3$ & 0 & PPB & \\
\hline 1,2-DICHLOROETHENE & $156-59-2$ & 0 & PPB & \\
\hline CHLOROFORM & $67-66-3$ & 0 & PPB & \\
\hline TETRAHYDROFURAN & $109-99-9$ & 0 & PPB & \\
\hline 1,1,1-TRICHLOROETHANE & $71-55-6$ & 16 & PPB & \\
\hline 1,2-DICHLOROETHANE & $107-06-2$ & 0 & PPB & \\
\hline BENZENE & $71-43-2$ & 0 & PPB & \\
\hline CARBON TETRACHLORIDE & $56-23-5$ & 0 & PPB & \\
\hline CYCLOHEXANE & $110-82-7$ & 0 & PPB & \\
\hline N-BUTANOL & $71-36-3$ & 0 & PPB & \\
\hline TRICHLOROETHENE & $79-01-6$ & 0 & PPB & \\
\hline 4-METHYL-2-PENTANONE & $108-10-1$ & 7 & PPB & \\
\hline TOLUENE & $108-88-3$ & 12 & $\mathrm{PPB}$ & \\
\hline 1,1,2-TRICHLOROETHANE & $79-00-5$ & 0 & PPB & \\
\hline TETRACHLOROETHENE & $127-18-4$ & 0 & PPB & \\
\hline CHLOROBENZENE & $108-90-7$ & $\overline{0}$ & PPB & \\
\hline ETHYLBENZENE & $100-41-4$ & 2 & PPB & \\
\hline M-XYLENE & $108-38-3$ & 6 & PPB & \\
\hline STYRENE & $100-42-5$ & 0 & PPB & \\
\hline O-XYLENE & $95-47-6$ & 2 & PPB & \\
\hline $1,1,2,2$-TETRACHLORETHANE & $79-34-5$ & 0 & PPB & \\
\hline 1,3,5-TRIMETHYLBENZENE & $108-67-8$ & 0 & PिB & \\
\hline 1,2,4-TRIMETHYLBENZENE & $95-63-6$ & 0 & PPB & \\
\hline 1,3-DICHLOROBENZENE & $541-73-1$ & 0 & PPB & \\
\hline 1,2-DICHLOROBENZENE & $95-50-1$ & 0 & PPB & \\
\hline 1,4-DICHLOROBENZENE & $106-46-7$ & 0 & PPB & \\
\hline
\end{tabular}




\section{LIST OF COMPOUNDS TENTATIVELY IDENTIFIED WITH MASS SPECTRAL LIBRARY}

Sample: $\quad 2402$ WE

Sample Name: FT6045_2WE.26A

Search Libraries: C:DATABASEIWILEY138.L Minimum Quality: 70

Unknown Spectrum: Apex

Integration Params: VOA.E

Pk\# RT Area\% Library/ID Ref\# CAS\# Qual

$5 \quad 16.1671 .34$ C:LDATABASEIWLEY138.L Methane, dichloro-

116807 000075-09-2 97

$920.873 .88 \mathrm{C}:$ DATABASEIWILEY138.L Ethane, 1,1,1-trichloro- $\quad 121290000071-55-691$

$1324.44 \quad 4.29$ C:IDATABASEIWILEY138.L 2-Pentanone, 4-methyl118021 000108-10-190

$1425.543 .89 \mathrm{C}:$ DATABASEIWILEY138.L Benzene, methyl-

$117430000108-88-395$

1628.870 .74 C:DATABASEIWILEY138.L Benzene, ethyl-

$118560000100-41-493$

1729.122 .38 C:IDATABASEIWILEY138.L Benzene, 1,4-dimethyl-

$118587000106-42-397$

Benzene, 1,3-dimethyl-

$118578000108-38-397$

$1829.95 \quad 1.25$ C:IDATABASEIWILEY138.L

Benzene, 1,2-dimethyl-

118564 000095-47-6 95

Benzene, 1,3-dimethyl-

$118579000108-38-395$

Benzene, 1,4-dimethyl-

$118586000106-42-394$

$1930.151 .17 \mathrm{C}:$ DATABASEIWILEY138.L Ethanol, 2-butoxy-

$119789000111-76-272$

$2232.08 \quad 0.79 \mathrm{C}:$ :DATABASEIWILEY 138.L Benzaldehyde

$118552000100-52-795$ 
HNF-SD-WM-RPT-282 Rev. 0

\section{LIST OF COMPOUNDS TENTATIVELY IDENTIFIED WITH MASS SPECTRAL} LIBRARY

Sample: $\quad 2402$ WE (continued)

Pk\# RT Area\% Library/ID Ref\# CAS\# Qual

$2533.10 \quad 0.28$ C:DATABASEIWILEY138.L

Benzene, 1,3,5-trimethyl-

Benzene, 1,2,3-trimethyl-

Benzene, 1,2,4-trimethyl-

$120037000108-67-893$

$120024000526-73-870$

$120030000095-63-670$ 
File

: C $\backslash \backslash$ HPCHEM $\backslash 2 \backslash D A T A \backslash 51496-12 . \mathrm{D}$

Operator : MS

Acquired : 14 May $96 \quad 4: 04$ pm using AcqMethod CWC

Instrument : 5972 - In

Sample Name: FT6045-2WR.28A BK

Misc Info : DELTA $P=100$ torr

Vial Number: 1

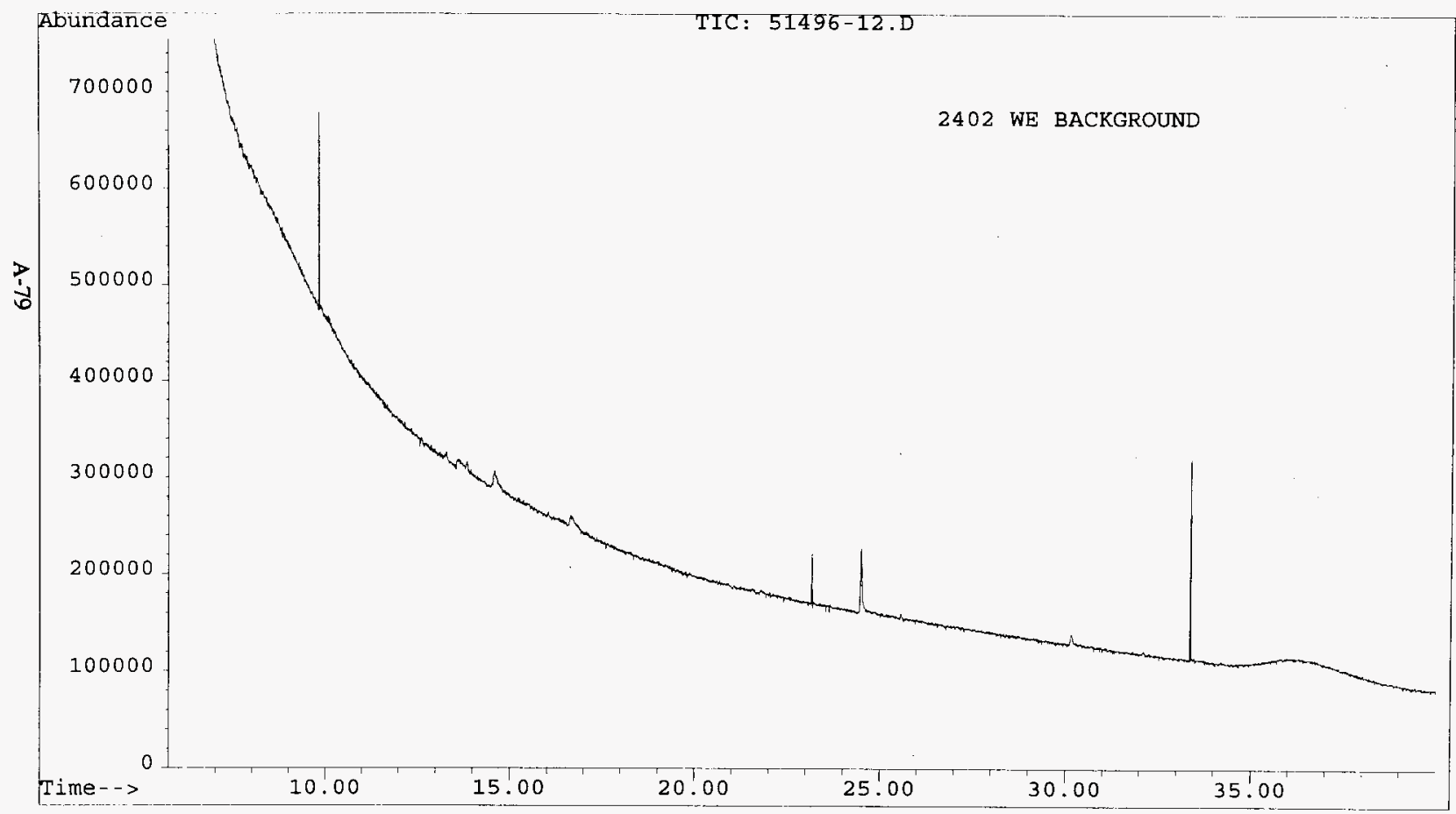


HNF-SD-WM-RPT-282 Rev. 0

CWC_REP.XLS

\section{ANALYSIS REPORT FOR CWC AIR SAMPLES}

File Name:

Analyst:

Analysis Date:

Method:

Sample Name:
51496-12.D

MS

05/14/96

CWC

FT6045-2WA.28A BK

\begin{tabular}{|c|c|c|c|c|}
\hline COMPOUND NAME & CAS \# & Concentration & Units & Comments \\
\hline ISO-BUTANE & $75-28-5$ & 0 & $\overline{\text { PPB }}$ & \\
\hline VINYL CHLORIDE & $75-01-4$ & 0 & PPB & \\
\hline ETHANOL & $64-17-5$ & 0 & PPB & \\
\hline ACETONE & $67-64-1$ & 0 & PPB & \\
\hline 2-PROPANOL & $67-63-0$ & 0 & PPB & \\
\hline 1,1-DICHLOROETHENE & $75-35-4$ & 0 & PPB & \\
\hline FREON 113 & $76-13-1$ & 0 & PPB & \\
\hline DICHLOROMETHANE & $75-09-2$ & 0 & PPB & \\
\hline 1,1-DICHLOROETHANE & $75-34-3$ & 0 & PPB & \\
\hline 2-BUTANONE & $78-93-3$ & 0 & PPB & \\
\hline N-HEXANE & $110-54-3$ & 0 & PPB & \\
\hline 1,2-DICHLOROETHENE & $156-59-2$ & 0 & PPB & \\
\hline CHLOROFORM & $67-66-3$ & $\underline{0}$ & PPB & \\
\hline TETRAHYDROFURAN & $109-99-9$ & 0 & PPB & \\
\hline 1,1,1-TRICHLOROETHANE & $71-55-6$ & 0 & PPB & \\
\hline 1,2-DICHLOROETHANE & $107-06-2$ & 0 & PPB & \\
\hline BENZENE & $71-43-2$ & 0 & PPB & \\
\hline CARBON TETRACHLORIDE & $56-23-5$ & 0 & PPB & \\
\hline CYCLOHEXANE & $110-82-7$ & 0 & PPB & \\
\hline N-BUTANOL & $71-36-3$ & 0 & PPB & \\
\hline TRICHLOROETHENE & $79-01-6$ & 0 & PPB & \\
\hline 4-METHYL-2-PENTANONE & $108-10-1$ & 0 & PPB & \\
\hline TOLUENE & $108-88-3$ & 0 & PPB & \\
\hline 1,1,2-TRICHLOROETHANE & $79-00-5$ & 0 & PPB & \\
\hline TETRACHLOROETHENE & $127-18-4$ & 0 & PPB & \\
\hline CHLOROBENZENE & $108-90-7$ & 0 & PPB & \\
\hline ETHYLBENZENE & $100-41-4$ & 0 & PPB & \\
\hline M-XYLENE & $108-38-3$ & 0 & PPB & \\
\hline STYRENE & $100-42-5$ & 0 & PPB & \\
\hline O-XYLENE & $95-47-6$ & 0 & PPB & \\
\hline 1,1,2,2-TETRACHLORETHANE & $79-34-5$ & 0 & PPB & \\
\hline 1,3,5-TRIMETHYLBENZENE & $108-67-8$ & 0 & PPB & \\
\hline 1,2,4-TRIMETHYLBENZENE & $95-63-6$ & 0 & PPB & \\
\hline 1,3-DICHLOROBENZENE & $541-73-1$ & 0 & PPB & \\
\hline 1,2-DICHLOROBENZENE & $95-50-1$ & 0 & PPB & \\
\hline 1,4-DICHLOROBENZENE & $106-46-7$ & 0 & PPB & \\
\hline
\end{tabular}


HNF-SD-WM-RPT-282 Rev. 0

LIST OF COMPOUNDS TENTATIVELY IDENTIFIED WITH MASS SPECTRAL LIBRARY

Sample: $\quad 2402$ WE Background

Sample Name: FT6045-2WA.28A BK

Search Libraries: C:LDATABASEIWILEY138.L $\quad$ Minimum Quality: 70

Unknown Spectrum: Apex

Integration Params: VOA.E

Pk\# RT Area\% Library/ID $\quad$ Ref\# CAS\# Qual

224.4840 .16 C:IDATABASEIWILEY138.L

2-Pentanone, 4-methyl-

$118017000108-10-176$ 
File

: C: \HPCHEM $\backslash 2 \backslash \mathrm{DATA} \backslash 51396 \_13 . \mathrm{D}$

Operator

: MS

Acquired

Instrument :

Sample Name: FT6045 2WE.27A

Misc Info : DELTA $\overline{\mathrm{P}}=100$ TORR

Vial Number: 1

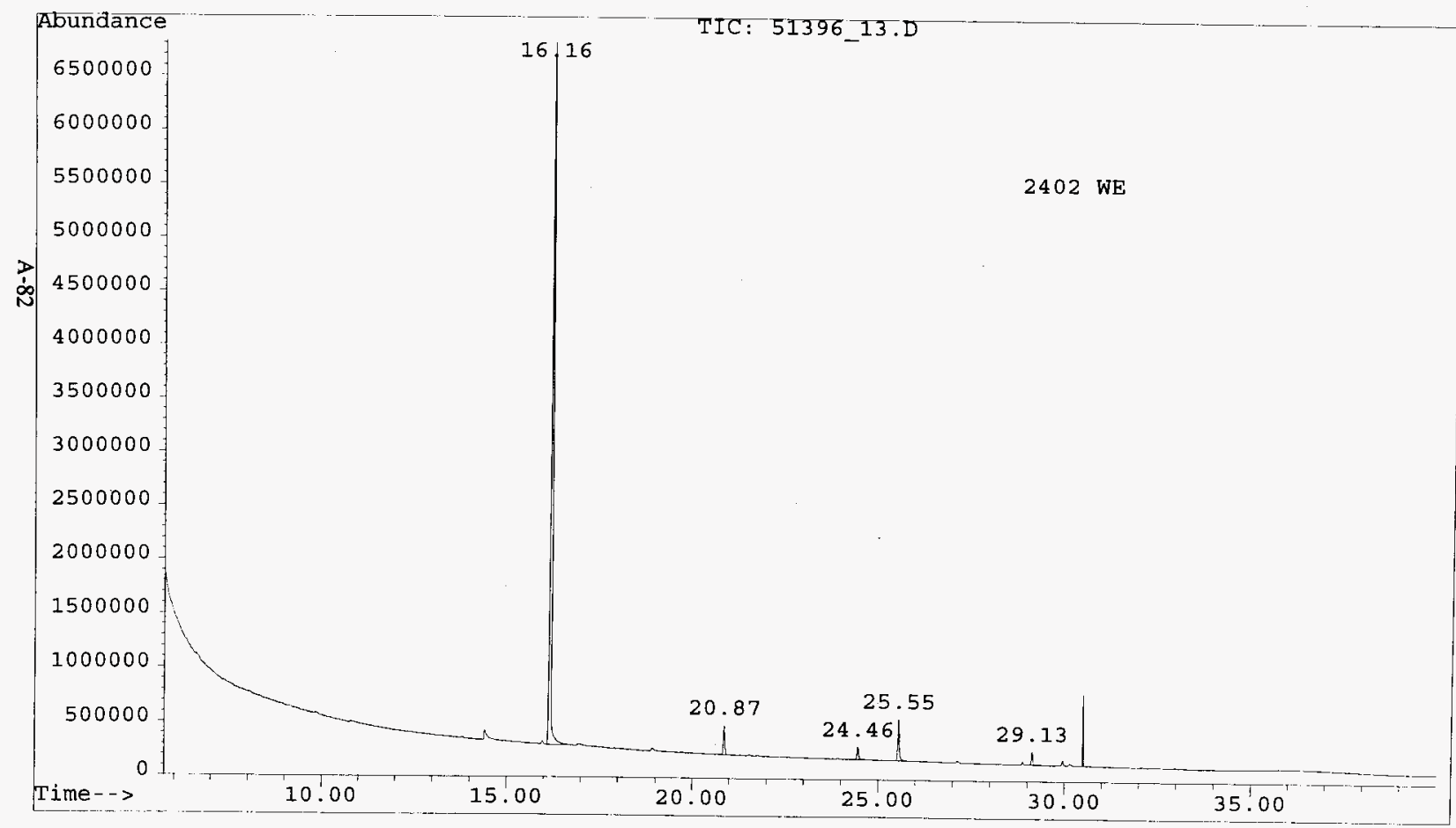


HNF-SD-WM-RPT-282 Rev. 0

CWC_REP.XLS

\section{ANALYSIS REPORT FOR CWC AIR SAMPLES}

File Name:

Analyst:

Analysis Date:

Method:

Sample Name:
51396_13.D

MS

05/13/96

CWC

FT6045_2WE.27A

\begin{tabular}{|c|c|c|c|c|}
\hline COMPOUND NAME & CAS\# & Concentration & Units & Comments \\
\hline ISO-BUTANE & $75-28-5$ & 0 & PPB & \\
\hline VINYL CHLORIDE & $75-01-4$ & 0 & PPB & \\
\hline ETHANOL & $64-17-5$ & 0 & $\mathrm{PPB}$ & \\
\hline ACETONE & $67-64-1$ & 4 & PPB & \\
\hline 2-PROPANOL & $67-63-0$ & 0 & PPB & \\
\hline 1,1-DICHLOROETHENE & $75-35-4$ & 0 & PPB & \\
\hline FREON 113 & $76-13-1$ & 0 & PPB & \\
\hline DICHLOROMETHANE & $75-09-2$ & 236 & PPB & \\
\hline 1,1-DICHLOROETHANE & $75-34-3$ & 0 & PPB & \\
\hline 2-BUTANONE & $78-93-3$ & 0 & PPB & \\
\hline N-HEXANE & $110-54-3$ & 0 & PPB & \\
\hline 1,2-DICHLOROETHENE & $156-59-2$ & 0 & $\overline{\mathrm{PPB}}$ & \\
\hline CHLOROFORM & $67-66-3$ & 0 & $\overline{\mathrm{PPB}}$ & \\
\hline TETRAHYDROFURAN & $109-99-9$ & 0 & PPB & \\
\hline 1,1,1-TRICHLOROETHANE & $71-55-6$ & 11 & PPB & \\
\hline 1,2-DICHLOROETHANE & $107-06-2$ & 0 & PPB & \\
\hline BENZENE & $71-43-2$ & 0 & PPB & \\
\hline CARBON TETRACHLORIDE & $56-23-5$ & 0 & PPB & \\
\hline CYCLOHEXANE & $110-82-7$ & 0 & PPB & \\
\hline N-BUTANOL & $71-36-3$ & 0 & PPB & \\
\hline TRICHLOROETHENE & $79-01-6$ & 0 & $\overline{\mathrm{PPB}}$ & \\
\hline 4-METHYL-2-PENTANONE & $108-10-1$ & 0 & PPB & \\
\hline TOLUENE & $108-88-3$ & 12 & PPB & \\
\hline 1,1,2-TRICHLOROETHANE & $79-00-5$ & 0 & PPB & \\
\hline TETRACHLOROETHENE & $127-18-4$ & 0 & PPB & \\
\hline CHLOROBENZENE & $108-90-7$ & 0 & $\overline{\mathrm{PPB}}$ & \\
\hline ETHYLBENZENE & $100-41-4$ & 3 & $\overline{\mathrm{PPB}}$ & \\
\hline M-XYLENE & $108-38-3$ & 3 & PPB & \\
\hline STYRENE & $100-42-5$ & 0 & PPB & \\
\hline O-XYLENE & $95-47-6$ & 1 & PPB & \\
\hline 1,1,2,2-TETRACHLORETHANE & $79-34-5$ & 0 & PPB & \\
\hline 1,3,5-TRIMETHYLBENZENE & $108-67-8$ & 0 & PPB & \\
\hline 1,2,4-TRIMETHYLBENZENE & $95-63-6$ & 0 & PPB & \\
\hline 1,3-DICHLOROBENZENE & $541-73-1$ & 0 & PPB & \\
\hline 1,2-DICHLOROBENZENE & $95-50-1$ & 0 & PPB & \\
\hline 1,4-DICHLOROBENZENE & $106-46-7$ & 0 & PPB & \\
\hline
\end{tabular}




\section{LIST OF COMPOUNDS TENTATIVELY IDENTIFIED WITH MASS SPECTRAL LIBRARY}

Sample: 2402 WE

Sample Name: FT6045_2WE.27A

Search Libraries: C:DATABASEIWILEY138.L Minimum Quality: 70

Unknown Spectrum: Apex

Integration Params: VOA.E

Pk\# RT Area\% Library/ID $\quad$ Ref\# CAS\# Qual

316.1680 .93 C:IDATABASEIWLEY138.L Methane, dichloro-

$116807000075-09-297$

620.873 .15 C:DATABASElWILEY138.L

Ethane, 1,1,1-trichloro-

$121290000071-55-691$

$724.461 .98 \mathrm{C}:$ DATABASEIWILEY138.L

2-Pentanone, 4-methyl-

$118019000108-10-187$

825.55 4.59 C:IDATABASE $\backslash$ WILEY138.L

Benzene, methyl-

$117430000108-88-395$

1028.870 .33 C:IDATABASEIWILEY138.L

Benzene, ethyl-

Benzene, 1,2-dimethyl-

$1129.131 .51 \mathrm{C}:$ DATABASE\WLEY138.L Benzene, 1,3-dimethyl-

Benzene, 1,4-dimethyl-

$118586000106-42-395$

$1229.960 .68 \mathrm{C}:$ DATABASEIWILEY138.L

Benzene, 1,2-dimethyl-

$118564000095-47-695$

Benzene, 1,3-dimethyl-

$118578000108-38-395$

Benzene, 1,4-dimethyl-

118586 000106-42-395 
File

Operator

: C: \HPCHEM \2\DATA \60596_4.D

Acquired

Instrument : 5972 - In

Sample Name: FT6045-2WF.47A re-run

Misc Info : 100 torr

Vial Number: 1

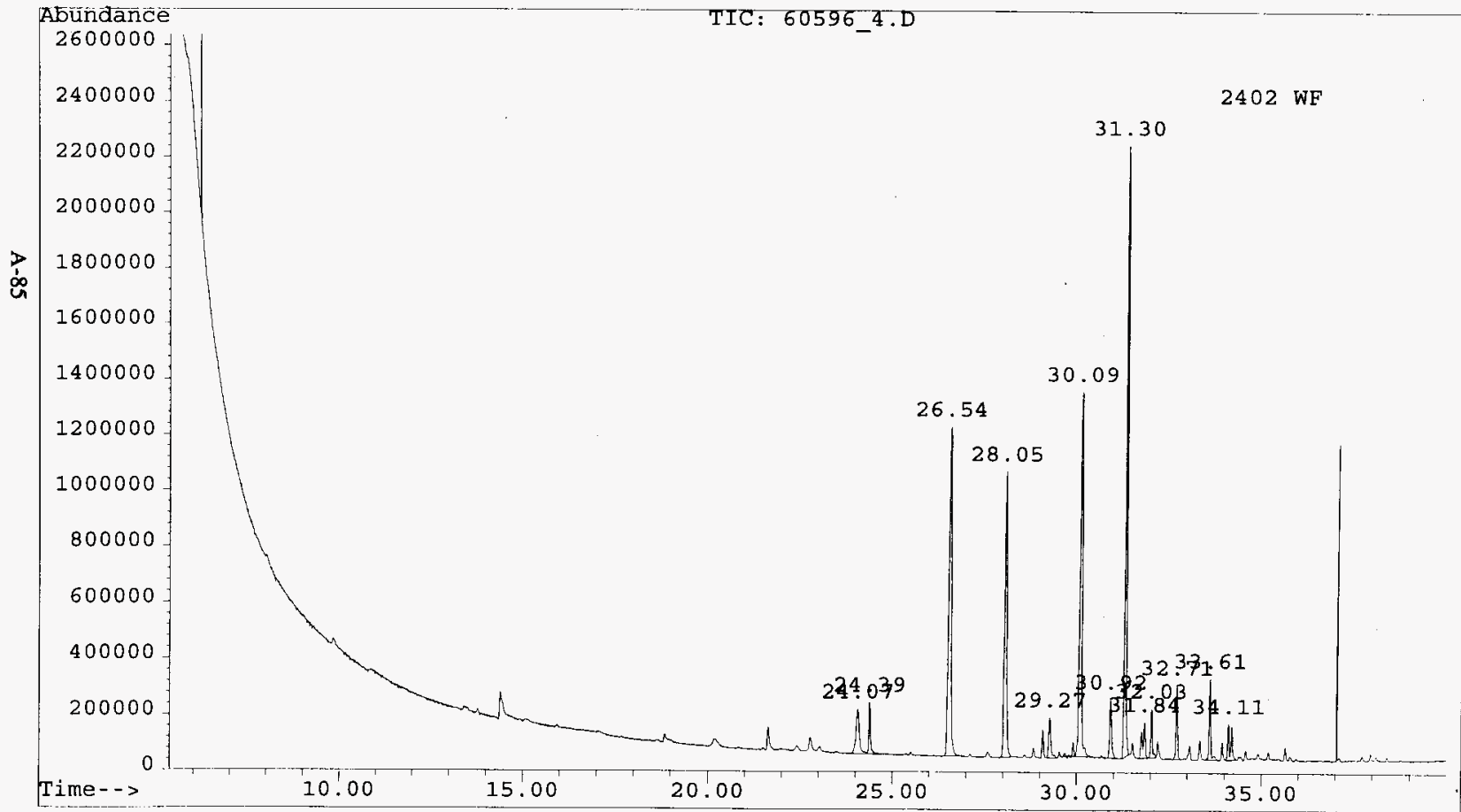


HNF-SD-WM-RPT-282 Rev. 0

CWC_REP.XLS

\section{ANALYSIS REPORT FOR CWC AIR SAMPLES}

File Name:

Analyst:

Analysis Date:

Method:

Sample Name:
60596_4.D

lap

06/05/96

CWC

FT6045-2WF.47A re-run

\begin{tabular}{|c|c|c|c|c|}
\hline COMPOUND NAME & CAS\# & Concentration & Units & Comments \\
\hline ISO-BUTANE & $75-28-5$ & 0 & PPB & \\
\hline VINYL CHLORIDE & $75-01-4$ & 0 & PPB & \\
\hline ETHANOL & $64-17-5$ & $\overline{0}$ & PPB & \\
\hline ACETONE & $67-64-1$ & 4 & $\mathrm{PPB}$ & \\
\hline 2-PROPANOL & $67-63-0$ & 0 & PPB & \\
\hline 1,1-DICHLOROETHENE & $75-35-4$ & $\underline{0}$ & PPB & \\
\hline FREON 113 & $76-13-1$ & 0 & PPB & \\
\hline DICHLOROMETHANE & $75-09-2$ & 0 & PPB & \\
\hline 1,1-DICHLOROETHANE & $75-34-3$ & 0 & PPB & \\
\hline 2-BUTANONE & $78-93-3$ & 0 & $\overline{\mathrm{PPB}}$ & \\
\hline N-HEXANE & $110-54-3$ & 0 & PPB & \\
\hline 1,2-DICHLOROETHENE & $156-59-2$ & 0 & PPB & \\
\hline CHLOROFORM & $67-66-3$ & 0 & PPB & \\
\hline TETRAHYDROFURAN & $109-99-9$ & 0 & PPB & \\
\hline 1,1,1-TRICHLOROETHANE & $71-55-6$ & 0 & PPB & \\
\hline 1,2-DICHLOROETHANE & $107-06-2$ & 0 & PPB & \\
\hline BENZENE & $71-43-2$ & 0 & PPB & \\
\hline CARBON TETRACHLORIDE & $56-23-5$ & 0 & PPB & \\
\hline CYCLOHEXANE & $110-82-7$ & 0 & PPB & \\
\hline N-BUTANOL & $71-36-3$ & 4 & PPB & \\
\hline TRICHLOROETHENE & $79-01-6$ & 0 & PPB & \\
\hline 4-METHYL-2-PENTANONE & $108-10-1$ & 0 & PPB & \\
\hline TOLUENE & $108-88-3$ & 0 & PPB & \\
\hline 1,1,2-TRICHLOROETHANE & $79-00-5$ & 0 & $\overline{\mathrm{PPB}}$ & \\
\hline TETRACHLOROETHENE & $127-18-4$ & 0 & PPB & \\
\hline CHLOROBENZENE & $108-90-7$ & 0 & PPB & \\
\hline ETHYLBENZENE & $100-41-4$ & 1 & PPB & \\
\hline M-XYLENE & $108-38-3$ & 3 & PPB & \\
\hline STYRENE & $100-42-5$ & 0 & PPB & \\
\hline O-XYLENE & $95-47-6$ & 1 & PPB & \\
\hline 1,1,2,2-TETRACHLORETHANE & $79-34-5$ & 0 & PPB & \\
\hline 1,3,5-TRIMETHYLBENZENE & $108-67-8$ & 3 & PPB & \\
\hline 1,2,4-TRIMETHYLBENZENE & $95-63-6$ & 1 & PPB & \\
\hline 1,3-DICHLOROBENZENE & $541-73-1$ & 0 & PPB & \\
\hline 1,2-DICHLOROBENZENE & $95-50-1$ & 0 & PPB & \\
\hline 1,4-DICHLOROBENZENE & $106-46-7$ & 0 & PPB & \\
\hline
\end{tabular}


HNF-SD-WM-RPT-282 Rev. 0

\section{LIST OF COMPOUNDS TENTATIVELY IDENTIFIED WITH MASS SPECTRAL LIBRARY}

Sample: $\quad 2402$ WF

File: $\quad$ C:IHPCHEMLIDATAl60596_4.D

ID: $\quad$ FT6045-2WF.47A re-run

Compound CAS\#

2-Pentanone, 4-methyl-

000108-10-1

ALPHA.-PINENE, (-)-

000080-56-8

Benzaldehyde

000100-52-7

Benzene, ethyl-

$000100-41-4$

Benzene, 1,3-dimethyl-

000108-38-3

Sabinene

$003387-41-5$

Benzene, 1,2-dimethyl-

$000095-47-6$

Ethanol, 2-butoxy-

$000111-76-2$

DELTA.3-Carene

013466-78-9

ALPHA.-PINENE, (-)-

$000080-56-8$

Benzene, 1-methyl-4-(1-methylethyl)-

000099-87-6

dl-Limonene

000138-86-3

2-BETA.-PINENE

000127-91-3

Benzaldehyde

000100-52-7

Phenol

000108-95-2

2-BETA-PINENE

000127-91-3

Benzene, 1,2,4-trimethyl-

000095-63-6 


\section{LIST OF COMPOUNDS TENTATIVELY IDENTIFIED WITH MASS SPECTRAL LIBRARY}

Sample: $\quad 2402$ WF (CONTINUED)

File: $\quad$ C:LHPCHEMIDLDATAI60596_4.D

ID: $\quad$ FT6045-2WF.47A re-run

Compound

CAS\#

Ethanone, 1-phenyl-

000098-86-2

DELTA.3-Carene

013466-78-9

Benzene, 1-ethyl-2,3-dimethyl-

000933-98-2

Cyclohexene, 1-methyl-4-(1-methylethe

005989-27-5

beta.-Phellandrene

$000555-10-2$

Peroxide, bis(dichlorobenzoyl)

$028604-90-2$

Ethanone, 1-phenyl-

000098-86-2 
File

Operator

: C: \HPCHEM \2\DATA \60596_5.D

: 1 ap

Acquired

5 Jun 96 5972 - In

Sample Name: FT6045-2WF.46A re-run

Misc Info : 100 torr

Vial Number: 1

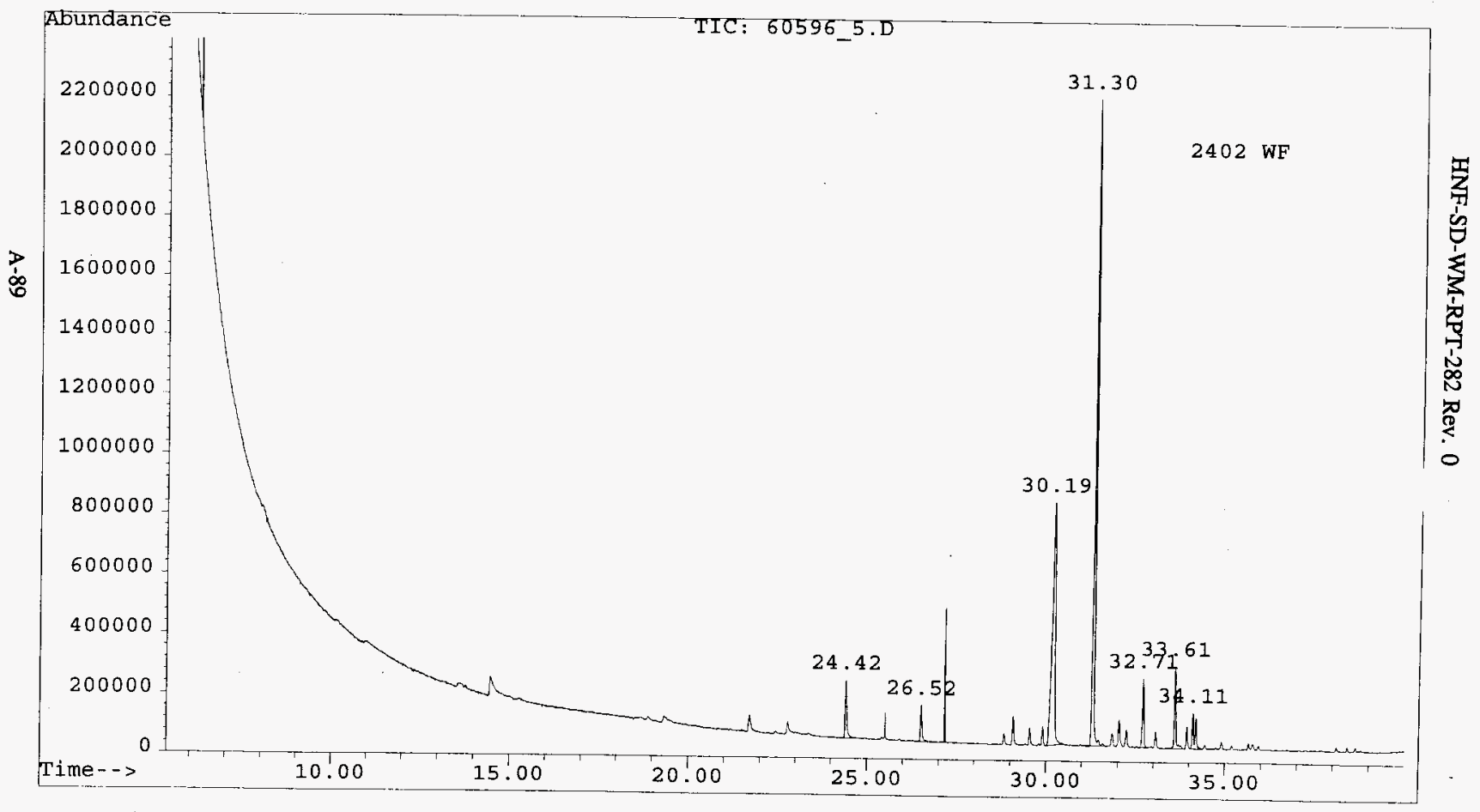


HNF-SD-WM-RPT-282 Rev. 0

CWC_REP.XLS

\section{ANALYSIS REPORT FOR CWC AIR SAMPLES}

File Name:

Analyst:

Analysis Date:

Method:

Sample Name:
60596_5.D

lap

06/05/96

CWC

FT6045-2WF.46A re-run

\begin{tabular}{|c|c|c|c|c|}
\hline COMPOUND NAME & CAS \# & Concentration & Units & Comments \\
\hline ISO-BUTANE & $75-28-5$ & 0 & PPB & \\
\hline VINYL CHLORIDE & $75-01-4$ & 0 & PPB & \\
\hline ETHANOL & $64-17-5$ & 0 & PPB & \\
\hline ACETONE & $67-64-1$ & 0 & PPB & \\
\hline 2-PROPANOL & $67-63-0$ & 0 & PPB & \\
\hline 1,1-DICHLOROETHENE & $75-35-4$ & 0 & PPB & \\
\hline FREON 113 & $76-13-1$ & 0 & PPB & \\
\hline DICHLOROMETHANE & $75-09-2$ & $\underline{0}$ & PPB & \\
\hline 1,1-DICHLOROETHANE & $75-34-3$ & 0 & PPB & \\
\hline 2-BUTANONE & 78-93-3 & 0 & PPB & \\
\hline N-HEXANE & $110-54-3$ & 0 & PPB & \\
\hline 1,2-DICHLOROETHENE & $156-59-2$ & 0 & PPB & \\
\hline CHLOROFORM & $67-66-3$ & 0 & PPB & \\
\hline TETRAHYDROFURAN & $109-99-9$ & 0 & PPB & \\
\hline 1,1,1-TRICHLOROETHANE & $71-55-6$ & 0 & PPB & \\
\hline 1,2-DICHLOROETHANE & $107-06-2$ & 0 & PPB & \\
\hline BENZENE & $71-43-2$ & 0 & PPB & \\
\hline CARBON TETRACHLORIDE & $56-23-5$ & 0 & PPB & \\
\hline CYCLOHEXANE & $110-82-7$ & 0 & PPB & \\
\hline N-BUTANOL & $71-36-3$ & 0 & PPB & \\
\hline TRICHLOROETHENE & $79-01-6$ & 0 & PPB & \\
\hline 4-METHYL-2-PENTANONE & $108-10-1$ & 0 & PPB & \\
\hline TOLUENE & $108-88-3$ & 0 & PPB & \\
\hline 1,1,2-TRICHLOROETHANE & $79-00-5$ & 0 & PPB & \\
\hline TETRACHLOROETHENE & $127-18-4$ & 0 & PPB & \\
\hline CHLOROBENZENE & $108-90-7$ & 0 & PPB & \\
\hline ETHYLBENZENE & $100-41-4$ & 2 & PPB & \\
\hline M-XYLENE & $108-38-3$ & 3 & PPB & \\
\hline STYRENE & $100-42-5$ & 0 & PPB & \\
\hline O-XYLENE & $95-47-6$ & 1 & PPB & \\
\hline 1,1,2,2-TETRACHLORETHANE & $79-34-5$ & 0 & PPB & \\
\hline 1,3,5-TRIMETHYLBENZENE & $108-67-8$ & 2 & PPB & \\
\hline 1,2,4-TRIMETHYLBENZENE & $95-63-6$ & 1 & PPB & \\
\hline 1,3-DICHLOROBENZENE & $541-73-1$ & 0 & PPB & \\
\hline 1,2-DICHLOROBENZENE & $95-50-1$ & 0 & PPB & \\
\hline 1,4-DICHLOROBENZENE & $106-46-7$ & 0 & PPB & \\
\hline
\end{tabular}


HNF-SD-WM-RPT-282 Rev. 0

LIST OF COMPOUNDS TENTATIVELY IDENTIFIED WITH MASS SPECTRAL LIBRARY

$\begin{array}{ll}\text { Sample: } & \text { 2402 WF } \\ \text { File: } & \text { C:LHPCHEM)2LDATAl60596_5.D } \\ \text { ID: } & \text { FT6045-2WF.46A re-run }\end{array}$

Compound

CAS\#

2-Pentanone, 4-methyl-

000108-10-1

Benzene, ethyl-

$000100-41-4$

XYLENE

001330-20-7

Benzene, 1,2-dimethyl-

000095-47-6

Ethanol, 2-butoxy-

$000111-76-2$

ALPHA.-PINENE, (-)-

000080-56-8

Camphene

$000079-92-5$

Benzaldehyde

000100-52-7

Phenol

000108-95-2

2-BETA-PINENE

$000127-91-3$

Benzene, 1,2,4-trimethyl-

000095-63-6

DELTA.3-Carene

013466-78-9

Benzene, 1-methyl-3-(1-methylethyl)-

$000535-77-3$

dl-Limonene

000138-86-3

beta.-Phellandrene

$000555-10-2$ 
File

Operator

Acquired

Instrument

Sample Name: 5972 - In

Misc Info : 100 TORR; HOLDING TIME EXPIRED

Vial Number: 1

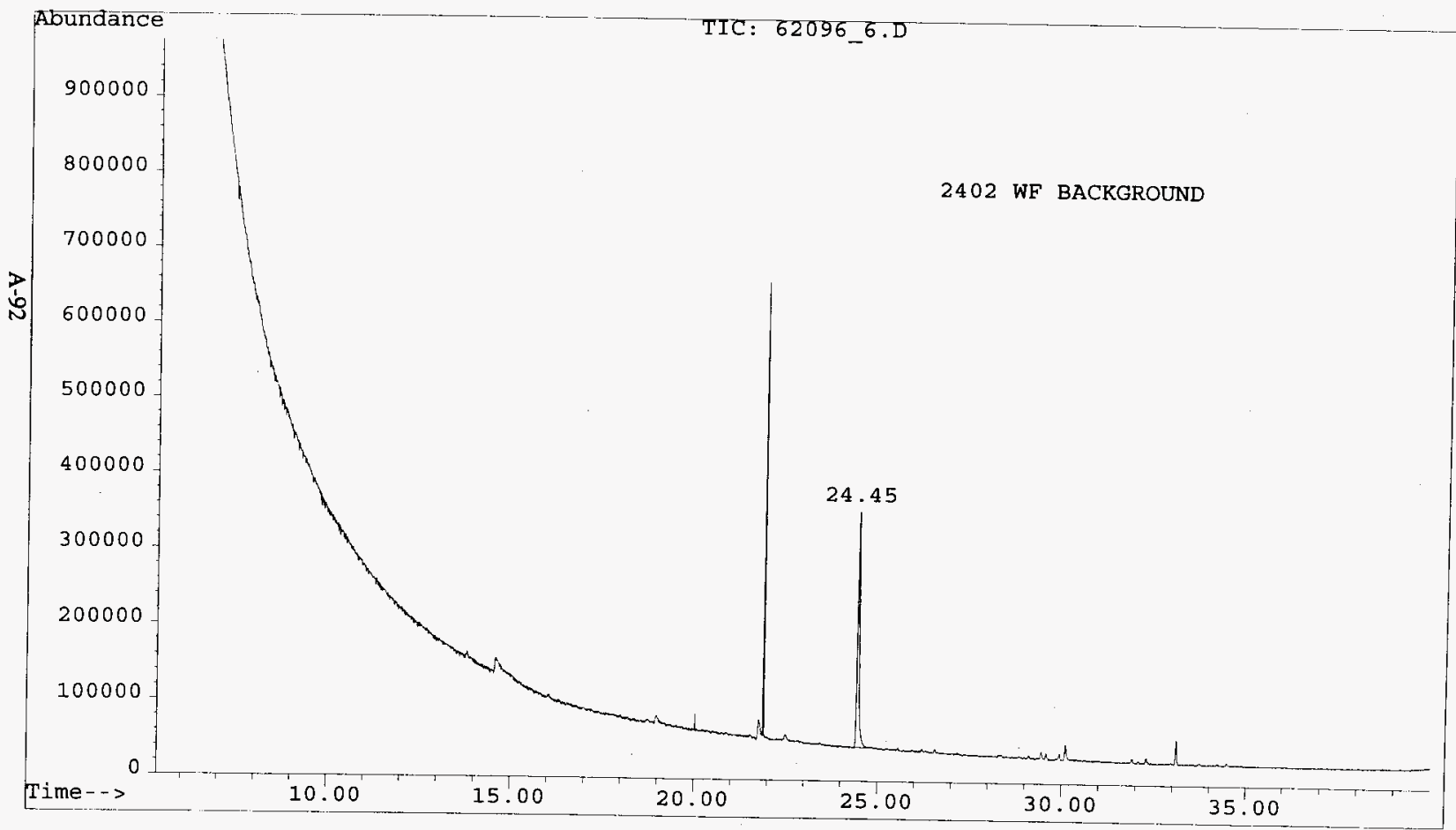




\begin{tabular}{|c|c|c|c|c|}
\hline & 8dd & 0 & $L-9 t-90 L$ & 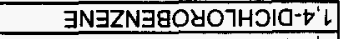 \\
\hline & 8dd & 0 & $1-0 \mathrm{~g}-\mathrm{S} 6$ & 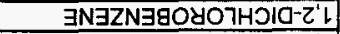 \\
\hline & gdd & 0 & $1-\varepsilon L-1, G$ & 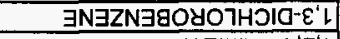 \\
\hline & gdd & 0 & $9-\varepsilon 9-96$ & 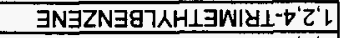 \\
\hline & 8dd & 0 & $8-\angle 9-801$ & 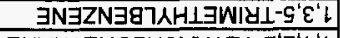 \\
\hline & 8dd & 0 & $S-t \varepsilon-6 L$ & $\exists N \forall H \perp \exists y O 7 H \supset \forall Y \perp \exists \perp-Z^{\prime} Z^{\prime} L^{\prime} L$ \\
\hline & Add & 0 & $9-15-96$ & $\exists \mathrm{N} \exists 7 \mathrm{~A} \times \mathrm{X}-\mathrm{O}$ \\
\hline & Add & 0 & s-Zt-00l & $\exists N \exists y \lambda \Lambda S$ \\
\hline & add & 0 & $\varepsilon-8 \varepsilon-80 \mathrm{~L}$ & $\exists N \exists 7 \lambda X-W$ \\
\hline & 8dd & 0 & bL & 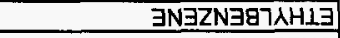 \\
\hline & gdd & 0 & $\angle-06-801$ & $\exists \mathrm{N} \exists \mathrm{ZN} \exists \mathrm{gO} \mathrm{OO} \mathrm{OHO}$ \\
\hline & 8dd & 0 & $t-8 l-\angle Z l$ & $\exists N \exists H \perp \exists 0807 H O \forall \triangle \perp \exists \perp$ \\
\hline & Edd & 0 & $S-00-6 L$ & $\exists N \forall H \perp \exists O Y O T H O 181-Z^{\prime} \downarrow ' \downarrow$ \\
\hline & 8dd & 0 & $\varepsilon-88-801$ & $\exists \mathrm{N} \exists \cap 7 \mathrm{OL}$ \\
\hline & Add & 0 & $1-01-801$ & $\exists N O N \forall \perp N \exists d-Z-7 \wedge H \perp \exists W-t$ \\
\hline & Edd & 0 & $9-10-6 L$ & 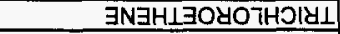 \\
\hline & gdd & 0 & $\varepsilon-9 \varepsilon-1 L$ & 7ON $\forall \perp \cap A-N$ \\
\hline & add & 0 & $\angle-28-011$ & $\exists N \forall x \exists H O 70 \wedge 0$ \\
\hline & 8dd & 0 & G- $\varepsilon \tau-9 g$ & $\exists$ ᄏalyo $7 \mathrm{HO \forall} \forall \perp \exists \perp$ Nogy $\forall 0$ \\
\hline & 8dd & 0 & $z-\varepsilon b-L L$ & $\exists \mathrm{N} \exists Z \mathrm{~N} \exists \mathrm{g}$ \\
\hline & 9dd & 0 & $2-90-\angle 0 l$ & $\exists N \forall H \perp \exists O Y O 7 H O I a-Z^{\prime} \mathrm{L}$ \\
\hline & idd & $\underline{0}$ & $9-99-L L$ & $\exists N \forall H \perp \exists O Y O=7 H O I Y \perp-L ' L^{\prime} l$ \\
\hline & Add & $\underline{0}$ & 6-66-60l & 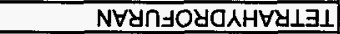 \\
\hline & gdd & 0 & $\varepsilon-99<9$ & wyOJOYOาHว \\
\hline & Edd & 0 & $z-69-99 l$ & $\exists N \exists H \perp \exists O У O 7 H O I a-Z^{\prime} l$ \\
\hline & 8dd & 0 & $\varepsilon-b 9-01 L$ & $\exists \mathrm{N} \forall \times \exists \mathrm{H}-\mathrm{N}$ \\
\hline & gdd & $\underline{0}$ & $\varepsilon-\varepsilon 6-8 L$ & $\exists N O N \forall \perp \cap A-Z$ \\
\hline & Edd & 0 & $\varepsilon-\nabla \varepsilon-G L$ & $\exists N \forall H \perp \exists O Y 07 H \supset I 0-\perp ' L$ \\
\hline & gdd & 0 & $2-60-9 L$ & $\exists N \forall H \perp \exists W O Y O 7 \mathrm{HOIO}$ \\
\hline & 8dd & 0 & $L-\varepsilon L-9 L$ & ELレ NOتYy \\
\hline & 8dd & 0 & $t-G \varepsilon-G L$ & 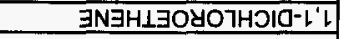 \\
\hline & add & 0 & $0-\varepsilon 9-\angle 9$ & 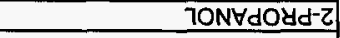 \\
\hline & gdd & 0 & $1-b 9<9$ & $\exists N O \perp \exists O \forall$ \\
\hline & gdd & 0 & $s-\angle 1-69$ & $70 N \forall H \perp \exists$ \\
\hline & Edd & 0 & $D-10-G L$ & 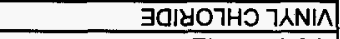 \\
\hline & gdd & 0 & $9-82-9 L$ & $\exists N \forall \perp \cap 8-O S I$ \\
\hline squeutuos & si!un & uolpedquesuos & \# SVO & BW \\
\hline
\end{tabular}

\begin{tabular}{|c|c|}
\hline 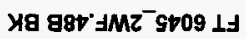 & :ameN ə|dues \\
\hline OMD & :poupaw \\
\hline $96 / 0 z / 90$ & :əjeg s!sא|eur \\
\hline $\begin{array}{r}\text { SW } \\
0 \cdot 9^{-} 96029\end{array}$ & $\begin{array}{l}\text { is גieur } \\
\text { :aweN 키- }\end{array}$ \\
\hline
\end{tabular}

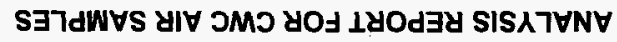

\author{
S7X $\cdot d \exists y^{-} J M O$
}

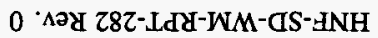


HNF-SD-WM-RPT-282 Rev. 0

LIST OF COMPOUNDS TENTATIVELY IDENTIFIED WITH MASS SPECTRAL LIBRARY

Sample: $\quad 2402$ WF BACKGROUND

File: $\quad$ C:UHPCHEMLLDATA162096_6.D

ID: FT6045_2WF.48B BK

Compound CAS\#

2-Pentanone, 4-methyl- 000108-10-1

Benzene, 1,3,5-trimethyl- 000108-67-8 
File

C : \HPCHEM \2\DATA \53196_13.D

operator

MS

Acquired

31 May 96 6:15 pm using AcqMethod CWC

Instrument : 5972 - In

Sample Name: FT 6045 2WF.48A BK

Misc Info : DELTA $\mathrm{P}^{-}=100$ torr

Vial Number: 1

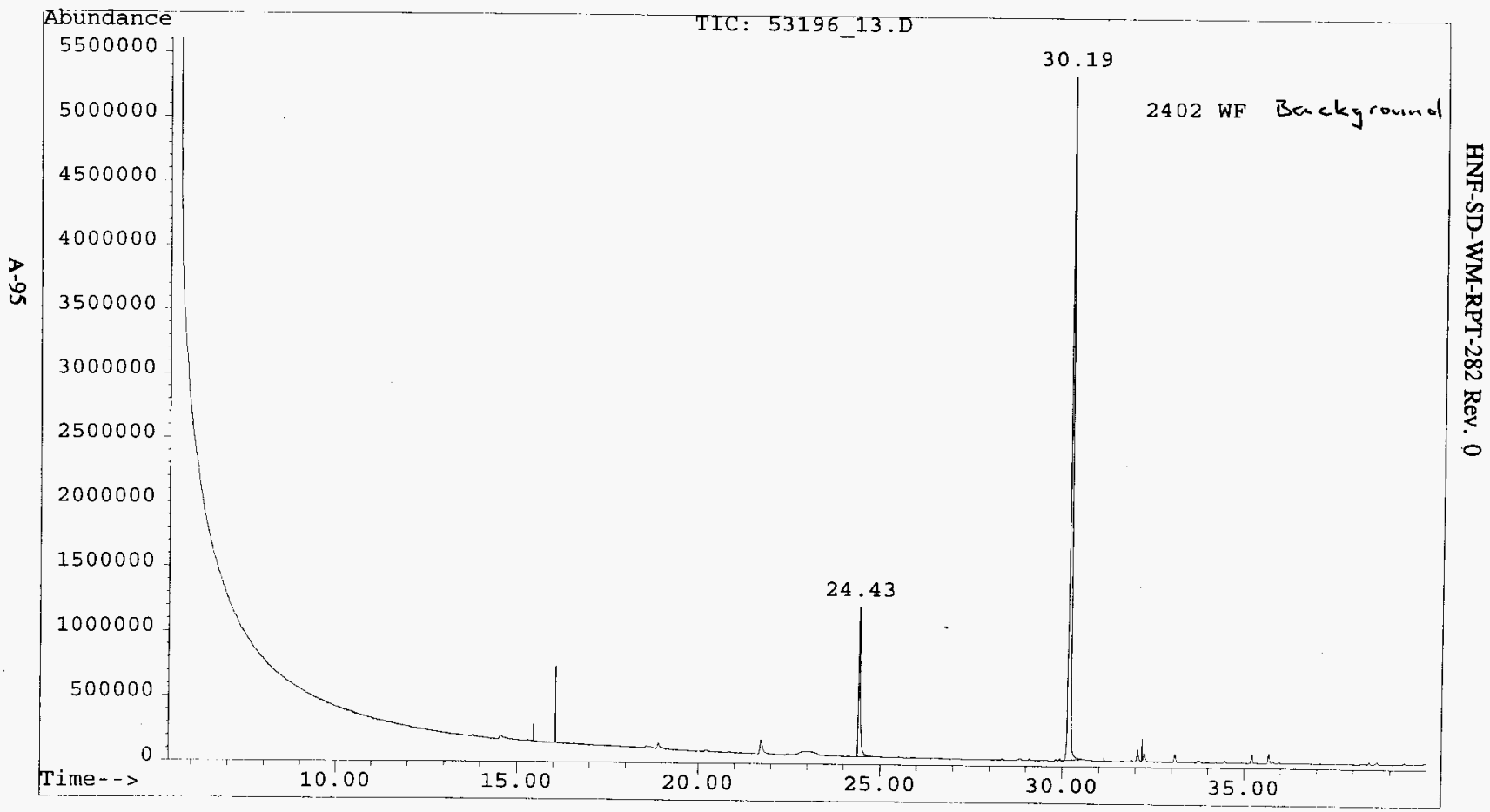


HNF-SD-WM-RPT-282 Rev. 0

CWC_REP.XLS

\section{ANALYSIS REPORT FOR CWC AIR SAMPLES}

File Name:

Analyst:

Analysis Date:

Method:

Sample Name:

53196_13.D

MS

05/31/96

CWC

FT 6045_2WF.48A BK

\begin{tabular}{|c|c|c|c|c|}
\hline COMPOUND NAME & CAS \# & Concentration & Units & Comments \\
\hline ISO-BUTANE & $75-28-5$ & $\overline{0}$ & PPB & \\
\hline VINYL CHLORIDE & $75-01-4$ & 0 & PिB & \\
\hline ETHANOL & $64-17-5$ & 0 & PPB & \\
\hline ACETONE & $67-64-1$ & 0 & PPB & \\
\hline 2-PROPANOL & $67-63-0$ & 0 & PPB & \\
\hline 1,1-DICHLOROETHENE & $75-35-4$ & 0 & PPB & \\
\hline FREON 113 & $76-13-1$ & 0 & PPB & \\
\hline DICHLOROMETHANE & $75-09-2$ & 0 & PPB & \\
\hline 1,1-DICHLOROETHANE & $75-34-3$ & 0 & PPB & \\
\hline 2-BUTANONE & $78-93-3$ & 0 & PPB & \\
\hline N-HEXANE & $110-54-3$ & 0 & PPB & \\
\hline 1,2-DICHLOROETHENE & $156-59-2$ & 0 & PPB & \\
\hline CHLOROFORM & $67-66-3$ & 0 & $\mathrm{PPB}$ & \\
\hline TETRAHYDROFURAN & $109-99-9$ & 0 & PPB & \\
\hline 1,1,1-TRICHLOROETHANE & $71-55-6$ & 0 & PPB & \\
\hline 1,2-DICHLOROETHANE & $107-06-2$ & 0 & PPB & \\
\hline BENZENE & $71-43-2$ & 0 & PPB & \\
\hline CARBON TETRACHLORIDE & $56-23-5$ & 0 & PPB & \\
\hline CYCLOHEXANE & $110-82-7$ & 0 & PPB & \\
\hline N-BUTANOL & $71-36-3$ & 7 & PPB & \\
\hline TRICHLOROETHENE & $79-01-6$ & 0 & PPB & \\
\hline 4-METHYL-2-PENTANONE & $108-10-1$ & 27 & PPB & \\
\hline TOLUENE & $108-88-3$ & 0 & PPB & \\
\hline 1,1,2-TRICHLOROETHANE & $79-00-5$ & 0 & PPB & \\
\hline TETRACHLOROETTHENE & $127-18-4$ & 0 & PPB & \\
\hline CHLOROBENZENE & $108-90-7$ & 0 & PPB & \\
\hline ETHYLBENZENE & $100-41-4$ & 0 & PPB & \\
\hline M-XYLENE & $108-38-3$ & 0 & PPB & \\
\hline STYRENE & $100-42-5$ & 0 & PPB & \\
\hline O-XYLENE & $95-47-6$ & 0 & PPB & \\
\hline 1,1,2,2-TETRACHLORETHANE & $79-34-5$ & 0 & PPB & \\
\hline 1,3,5-TRIMETHYLBENZENE & $108-67-8$ & 2 & PPB & \\
\hline 1,2,4-TRIMETHYLBENZENE & $95-63-6$ & 2 & PPB & \\
\hline 1,3-DICHLOROBENZENE & $541-73-1$ & 0 & PPB & \\
\hline 1,2-DICHLOROBENZENE & $95-50-1$ & 0 & PPB & \\
\hline 1,4-DICHLOROBENZENE & $106-46-7$ & 0 & PPB & \\
\hline
\end{tabular}


HNF-SD-WM-RPT-282 Rev. 0

\section{LIST OF COMPOUNDS TENTATIVELY IDENTIFIED WITH MASS SPECTRAL LIBRARY}

Sample: $\quad 2402$ WF BACKGROUND

File: $\quad$ C:LHPCHEMLLDATAIS3196 13.D

ID: $\quad$ FT6045_2WF.48A BK

Compound

CAS\#

1-Butanol

000071-36-3

2-Pentanone, 4-methyl-

000108-10-1

Ethanol, 2-butoxy-

$000111-76-2$

Benzaldehyde

000100-52-7

Benzene, 1,2,4-trimethyl-

000095-63-6

Benzene, 1,4-dichloro-

000106-46-7

Benzene, 1,4-dichloro-

$000106-46-7$

Ethanone, 1-phenyl-

000098-86-2

Benzene, (1-methylethenyl)-

000098-83-9 
File

Operator

Acquired

Instrument

MT6045 2WG.30A

Misc Info : DELTA $\overline{\mathrm{P}}=100$ TORR

Vial Number: 1

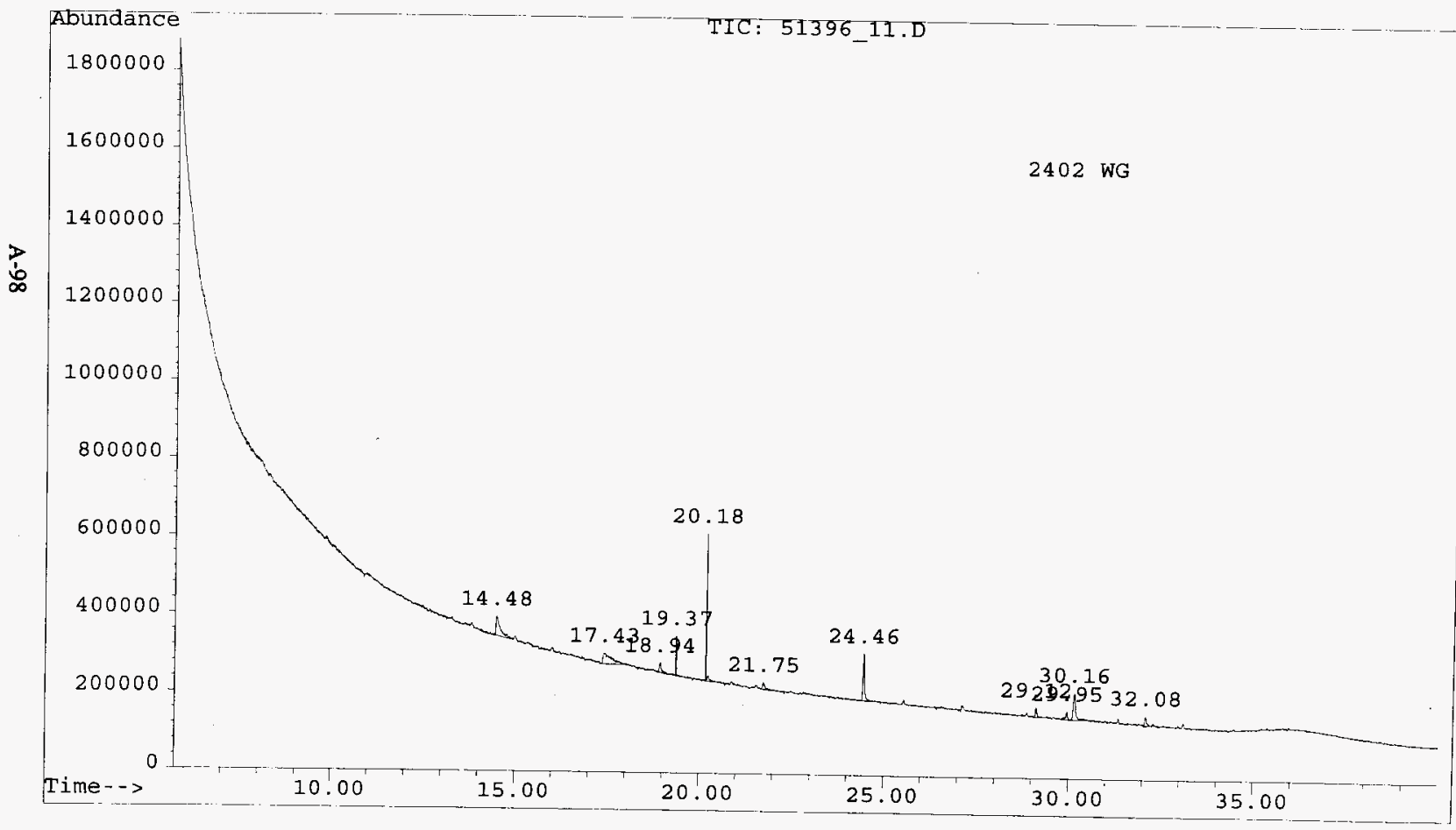




\section{LIST OF COMPOUNDS TENTATIVELY WENTIFIED WITH MASS SPECTRAL}

\section{LIBRARY}

Sample: $\quad 2402$ WG

Sample Name: FT6045_2WG.30A

Search Libraries: C:DATABASEIWILEY138.L Minimum Quality: 70

Unknown Spectrum: Apex

Integration Params: VOA.E

Pk\# RT Area\% Library/ID Re\# CAS\# Qual

724.46 16.44 C:DATABASEIWILEY138.L

2-Pentanone, 4-methyl-

$118019000108-10-187$

829.123 .06 C:DATABASEIWLEY138.L

Benzene, 1,2-dimethyl-

$118571000095-47-694$

Benzene, 1,3-dimethyl-

$118575000108-38-393$

929.953 .48 C:DATABASEIWILEY138.L

Benzene, 1,2-dimethyl- $\quad 118571000095-47-690$

$1030.1614 .41 \mathrm{C}:$ DATABASEIWILEY138.L

Ethanol, 2-butoxy-

$119788000111-76-278$

1132.083 .96 C:DATABASEIWILEY138.L

Benzaldehyde

$118546000100-52-795$ 
HNF-SD-WM-RPT-282 Rev. 0

CWC_REP.XLS

\section{ANALYSIS REPORT FOR CWC AIR SAMPLES}

File Name:

Analyst:

Analysis Date:

Method:

Sample Name:
51396_11.D

MS

05/13/96

CWC

FT6045 2WG.30A

\begin{tabular}{|c|c|c|c|c|}
\hline COMPOUND NAME & CAS\# & Concentration & Units & Comments \\
\hline ISO-BUTANE & $75-28-5$ & 0 & PPB & \\
\hline VINYL CHLORIDE & $75-01-4$ & 0 & PPB & \\
\hline ETHANOL & $64-17-5$ & 0 & $\overline{\mathrm{PPB}}$ & \\
\hline ACETONE & $67-64-1$ & 0 & PPB & \\
\hline 2-PROPANOL & $67-63-0$ & 0 & PPB & \\
\hline 1,1-DICHLOROETHENE & $75-35-4$ & 0 & PPB & \\
\hline FREON 113 & $76-13-1$ & 0 & PPB & \\
\hline DICHLOROMETHANE & $75-09-2$ & 0 & PPB & \\
\hline 1,1-DICHLOROETHANE & $75-34-3$ & 0 & $\mathrm{PPB}$ & \\
\hline 2-BUTANONE & $78-93-3$ & 0 & PPB & \\
\hline N-HEXANE & $110-54-3$ & 0 & PPB & \\
\hline 1,2-DICHLOROETHENE & $156-59-2$ & 0 & PPB & \\
\hline CHLOROFORM & $67-66-3$ & 0 & PPB & \\
\hline TETRAHYDROFURAN & 109-99-9 & $D$ & PPB & \\
\hline 1,1,1-TRICHLOROETHANE & $71-55-6$ & 0 & PPB & \\
\hline 1,2-DICHLOROETHANE & $107-06-2$ & 0 & PPB & \\
\hline BENZENE & $71-43-2$ & 0 & PPB & \\
\hline CARBON TETRACHLORIDE & $56-23-5$ & 0 & PPB & \\
\hline CYCLOHEXANE & $110-82-7$ & 0 & PPB & \\
\hline N-BUTANOL & $71-36-3$ & 0 & PPB & \\
\hline TRICHLOROETHENE & $79-01-6$ & 0 & PPB & \\
\hline 4-METHYL-2-PENTANONE & $108-10-1$ & 0 & $\overline{P P B}$ & \\
\hline TOLUENE & $108-88-3$ & $\underline{0}$ & $\mathrm{PPB}$ & \\
\hline 1,1,2-TRICHLOROETHANE & $79-00-5$ & 0 & $\mathrm{PPB}$ & \\
\hline TETRACHLOROETHENE & $127-18-4$ & 0 & $\mathrm{PPB}$ & \\
\hline CHLOROBENZENE & $108-90-7$ & 0 & $\mathrm{PPB}$ & \\
\hline ETHYLBENZENE & $100-41-4$ & 0 & $\mathrm{PPB}$ & \\
\hline M-XYLENE & $108-38-3$ & 0 & $\mathrm{PPB}$ & \\
\hline STYRENE & $100-42-5$ & 0 & PPB & \\
\hline O-XYLENE & $95-47-6$ & 0 & PPB & \\
\hline 1,1,2,2-TETRACHLORETHANE & $79-34-5$ & 0 & PPB & \\
\hline 1,3,5-TRIMETHYLBENZENE & $108-67-8$ & 0 & PPB & \\
\hline 1,2,4-TRIMETHYLBENZENE & $95-63-6$ & 0 & PPB & \\
\hline 1,3-DICHLOROBENZENE & $541-73-1$ & 0 & PPB & \\
\hline 1,2-DICHLOROBENZENE & $95-50-1$ & 0 & PPB & \\
\hline 1,4-DICHLOROBENZENE & $106-46-7$ & 0 & PPB & \\
\hline
\end{tabular}




\section{File}

Operator

C: \HPCHEM \2\DATA \51796_4.D

Acquired

: ms

Instrument

17 May 96 10:06 am using AcqMethod CWC

5972 - In

Sample Name: FT6045_2WG.29B

Misc Info : NOT PRESS. TO 15 PSI; DELTA P = 100 torr

Vial Number: 1

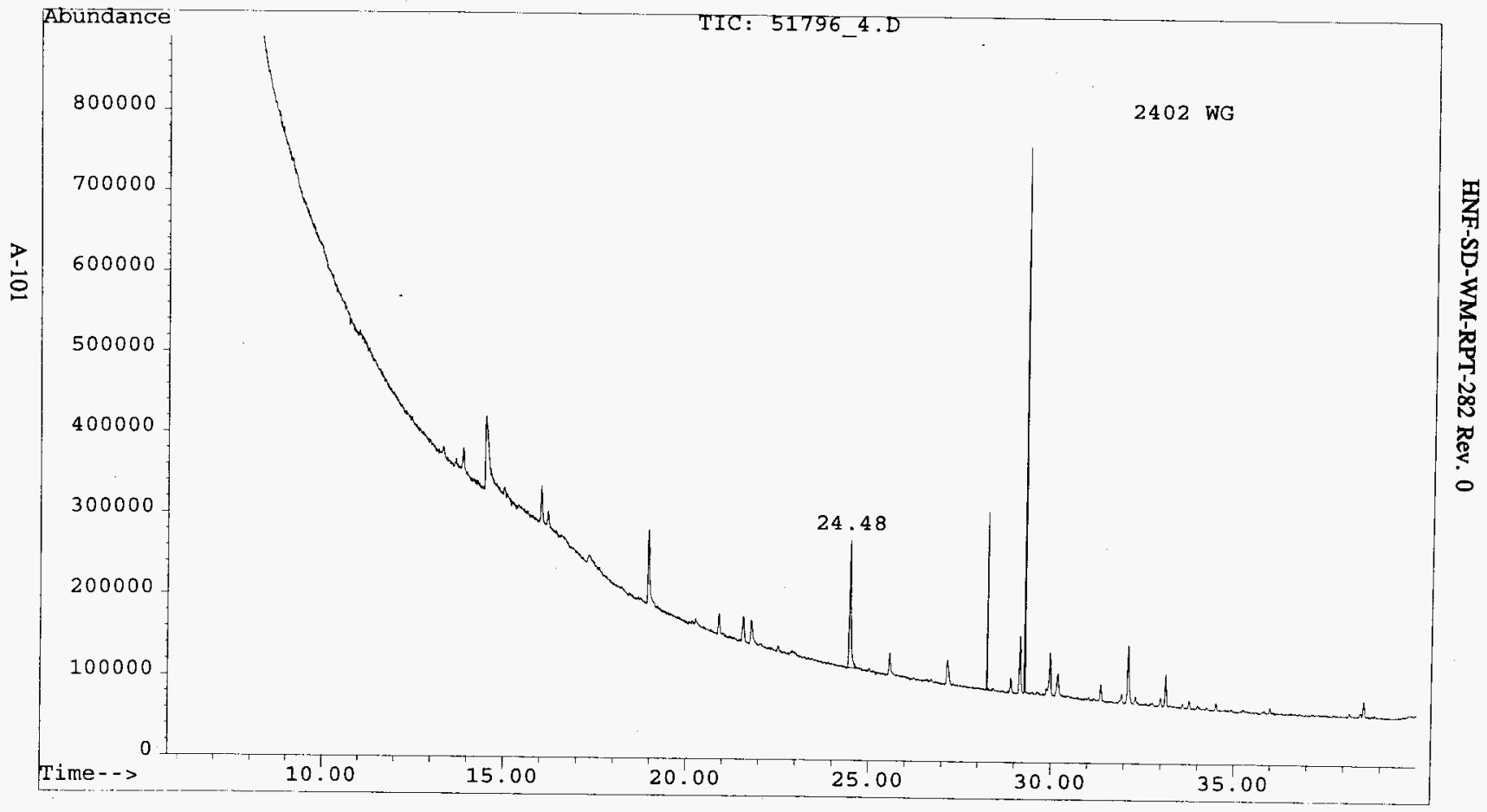


HNF-SD-WM-RPT-282 Rev. 0

CWC_REP.XIS

\section{ANALYSIS REPORT FOR CWC AIR SAMPLES}

File Name:

Analyst:

Analysis Date:

Method:

Sample Name:
51796_4.D

$\mathrm{ms}$

05/17/96

CWC

FT6045_2WG.29B

\begin{tabular}{|c|c|c|c|c|}
\hline COMPOUND NAME & CAS \# & Concentration & Units & Comments \\
\hline ISO-BUTANE & $75-28-5$ & 0 & $\overline{\mathrm{PPB}}$ & \\
\hline VINYL CHLORIDE & $75-01-4$ & 0 & PPB & \\
\hline ETHANOL & $64-17-5$ & 0 & PPB & \\
\hline ACETONE & $67-64-1$ & 5 & PPB & \\
\hline 2-PROPANOL & $67-63-0$ & 0 & PPB & \\
\hline 1,1-DICHLOROETHENE & $75-35-4$ & 0 & PPB & \\
\hline FREON 113 & $76-13-1$ & 0 & PPB & \\
\hline DICHLOROMETHANE & $75-09-2$ & 0 & PPB & \\
\hline 1,1-DICHLOROETHANE & $75-34-3$ & 0 & PPB & \\
\hline 2-BUTANONE & $78-93-3$ & 0 & PPB & \\
\hline N-HEXANE & $110-54-3$ & 2 & PPB & \\
\hline 1,2-DICHLOROETHENE & $156-59-2$ & 0 & PPB & \\
\hline CHLOROFORM & $67-66-3$ & 0 & $\overline{P P B}$ & \\
\hline TETRAHYDROFURAN & $109-99-9$ & 0 & $\mathrm{PPB}$ & \\
\hline 1,1,1-TRICHLOROETHANE & $71-55-6$ & 0 & PPB & \\
\hline 1,2-DICHLOROETHANE & $107-06-2$ & 0 & PPB & \\
\hline BENZENE & $71-43-2$ & 0 & PPB & \\
\hline CARBON TETRACHLORIDE & $56-23-5$ & 0 & PPB & \\
\hline CYCLOHEXANE & $110-82-7$ & 0 & PPB & \\
\hline N-BUTANOL & $71-36-3$ & 0 & PPB & \\
\hline TRICHLOROETHENE & $79-01-6$ & 0 & PPB & \\
\hline 4-METHYL-2-PENTANONE & $108-10-1$ & 0 & PPB & \\
\hline TOLUENE & $108-88-3$ & 0 & PPB & \\
\hline 1,1,2-TRICHLOROETHANE & $79-00-5$ & 0 & PPB & \\
\hline TETRACHLOROETHENE & $127-18-4$ & 0 & PPB & \\
\hline CHLOROBENZENE & $108-90-7$ & 0 & PPB & \\
\hline ETHYLBENZENE & $100-41-4$ & 2 & PPB & \\
\hline M-XYLENE & $108-38-3$ & 0 & PPB & \\
\hline STYRENE & $100-42-5$ & 0 & PPB & \\
\hline O-XYLENE & $95-47-6$ & 1 & PPB & \\
\hline 1,1,2,2-TETRACHLORETHANE & $79-34-5$ & 0 & PPB & \\
\hline 1,3,5-TRIMETHYLBENZENE & $108-67-8$ & 2 & PPB & \\
\hline 1,2,4-TRIMETHYLBENZENE & $95-63-6$ & 0 & $\overline{P P B}$ & \\
\hline 1,3-DICHLOROBENZENE & $541-73-1$ & 0 & PPB & \\
\hline 1,2-DICHLOROBENZENE & $95-50-1$ & 0 & PPB & \\
\hline 1,4-DICHLOROBENZENE & $106-46-7$ & 0 & PPB & \\
\hline
\end{tabular}


HNF-SD-WM-RPT-282 Rev. 0

\section{LIST OF COMPOUNDS TENTATIVELY IDENTIFIED WITH MASS SPECTRAL}

\section{LIBRARY}

\section{Sample: $\quad 2402$ WG}

File $\quad$ C:LHPCHEM2LDATAL51796_4.D

ID: $\quad$ FT6045_2WG.29B

Compound

CAS \#

Benzene, 1,2-dimethyl-

$000095-47-6$

Benzene, 1,2-dimethyl-

$000095-47-6$

Benzaldehyde

000100-52-7

Benzene, 1,2,3-trimethyl-

000526-73-8 
File

Operator

Acquired

Instrument

Sample 5972 - In

Misc Info : DELTA $\mathrm{P}^{-}=100$ torr

Vial Number: 1

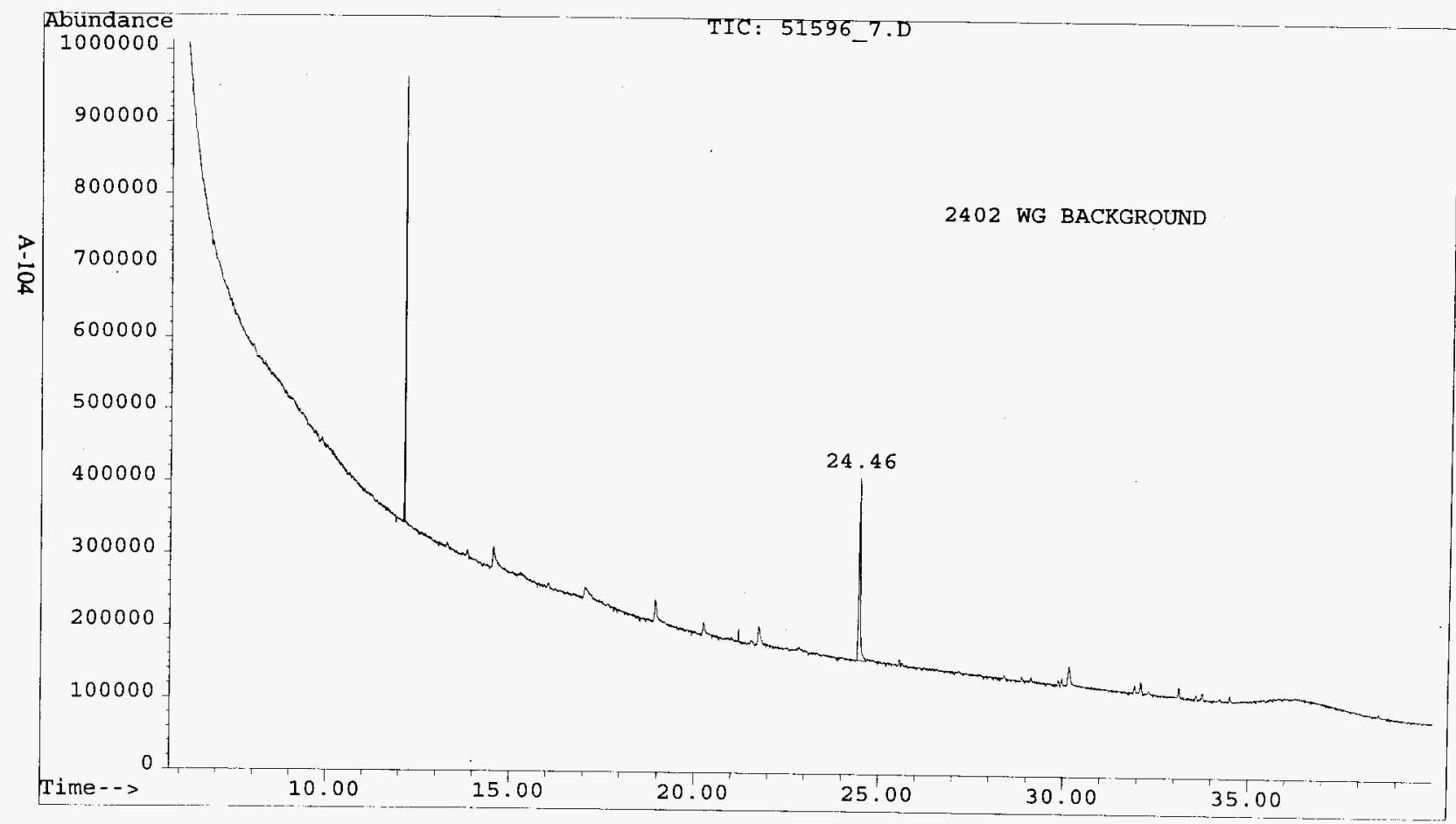


HNF-SD-WM-RPT-282 Rev. 0

CWC_REP.XLS

\section{ANALYSIS REPORT FOR CWC AIR SAMPLES}

File Name:

Analyst:

Analysis Date:

Method:

Sample Name:
51596_7.D

MS

05/15/96

CWC

FT 6045_2WG.31A BK

\begin{tabular}{|c|c|c|c|c|}
\hline COMPOUND NAME & CAS \# & Concentration & Units & Comments \\
\hline ISO-BUTANE & $75-28-5$ & 0 & PPB & \\
\hline VINYL CHLORIDE & $75-01-4$ & 0 & PPB & \\
\hline ETHANOL & $64-17-5$ & 0 & PPB & \\
\hline ACETONE & $67-64-1$ & 0 & PPB & \\
\hline 2-PROPANOL & $67-63-0$ & $\overline{0}$ & PPB & \\
\hline 1,1-DICHLOROETHENE & $75-35-4$ & 0 & PPB & \\
\hline FREON 113 & $76-13-1$ & 0 & PPB & \\
\hline DICHLOROMETHANE & $75-09-2$ & 0 & PPB & \\
\hline 1,1-DICHLOROETHANE & $75-34-3$ & 0 & PPB & \\
\hline 2-BUTANONE & $78-93-3$ & 0 & PPB & \\
\hline N-HEXANE & $110-54-3$ & 0 & PPB & \\
\hline 1,2-DICHLOROETHENE & $156-59-2$ & 0 & PPB & \\
\hline CHLOROFORM & $67-66-3$ & 0 & PPB & \\
\hline TETRAHYDROFURAN & $109-99-9$ & 0 & PPB & \\
\hline 1,1,1-TRICHLOROETHANE & $71-55-6$ & 0 & PPB & \\
\hline 1,2-DICHLOROETHANE & $107-06-2$ & 0 & PPB & \\
\hline BENZENE & $71-43-2$ & 0 & PPB & \\
\hline CARBON TETRACHLORIDE & $56-23-5$ & 0 & PPB & \\
\hline CYCLOHEXANE & $110-82-7$ & 0 & PPB & \\
\hline N-BUTANOL & $71-36-3$ & 0 & PPB & \\
\hline TRICHLOROETHENE & $79-01-6$ & 0 & PPB & \\
\hline 4-METHYL-2-PENTANONE & $108-10-1$ & 0 & PPB & \\
\hline TOLUENE & $108-88-3$ & 0 & PPB & \\
\hline 1,1,2-TRICHLOROETHANE & $79-00-5$ & 0 & PPB & \\
\hline TETRACHLOROETHENE & $127-18-4$ & 0 & PPB & \\
\hline CHLOROBENZENE & $108-90-7$ & 0 & PPB & \\
\hline ETHYLBENZENE & $100-41-4$ & 0 & PPB & \\
\hline M-XYLENE & $108-38-3$ & 0 & PPB & \\
\hline STYRENE & $100-42-5$ & 0 & PPB & \\
\hline O-XYLENE & $95-47-6$ & 0 & PPB & \\
\hline 1,1,2,2-TETRACHLORETHANE & $79-34-5$ & 0 & PPB & \\
\hline 1,3,5-TRIMETHYLBENZENE & $108-67-8$ & 0 & PPB & \\
\hline 1,2,4-TRIMETHYLBENZENE & $95-63-6$ & 0 & $\overline{P P B}$ & \\
\hline 1,3-DICHLOROBENZENE & $541-73-1$ & 0 & PPB & \\
\hline 1,2-DICHLOROBENZENE & $95-50-1$ & 0 & PPB & \\
\hline 1,4-DICHLOROBENZENE & $106-46-7$ & 0 & PPB & \\
\hline
\end{tabular}


HNF-SD-WM-RPT-282 Rev. 0

LIST OF COMPOUNDS TENTATIVELY IDENTIFIED WITH MASS SPECTRAL LIBRARY

Sample: 2402 WG Background

File: $\quad$ FT 6045_2WG.31A BK

Libraries: C:LatabaselWiley 138.L

C:IDATABASEINBS54K.L Minimum Quality: 70

Unknown Spectrum: Apex

Integration Params: VOA.E

Pk\# RT Area\% Library/ID $\quad$ Ref\# CAS\# Qual

724.4635 .13 C:LDATABASEIWILEY138.L

2-Pentanone, 4-methyl-

118023 000108-10-1 91

$830.16 \quad 6.87$ C:LDATABASEIWILEY138.L

Ethanol, 2-butoxy- $\quad 119789000111-76-272$

$932.092 .48 \mathrm{C}:$ DATABASEIWILEY138.L

Benzaldehyde $118546000100-52-793$

1033.122 .72 C:DATABASEIWILEY138.L

Benzene, 1,3,5-trimethyl- $\quad 120037$ 000108-67-8 92 
File

Operator

: C $: \backslash H P C H E M \backslash 2 \backslash D A T A \backslash 51396 \_8 . D$

Acquired

: 13 May 96

Sample Name: FT6045 2WG.29A

Misc Info : DELTA $\overline{\mathrm{P}}=100$ TORR

Vial Number: 1

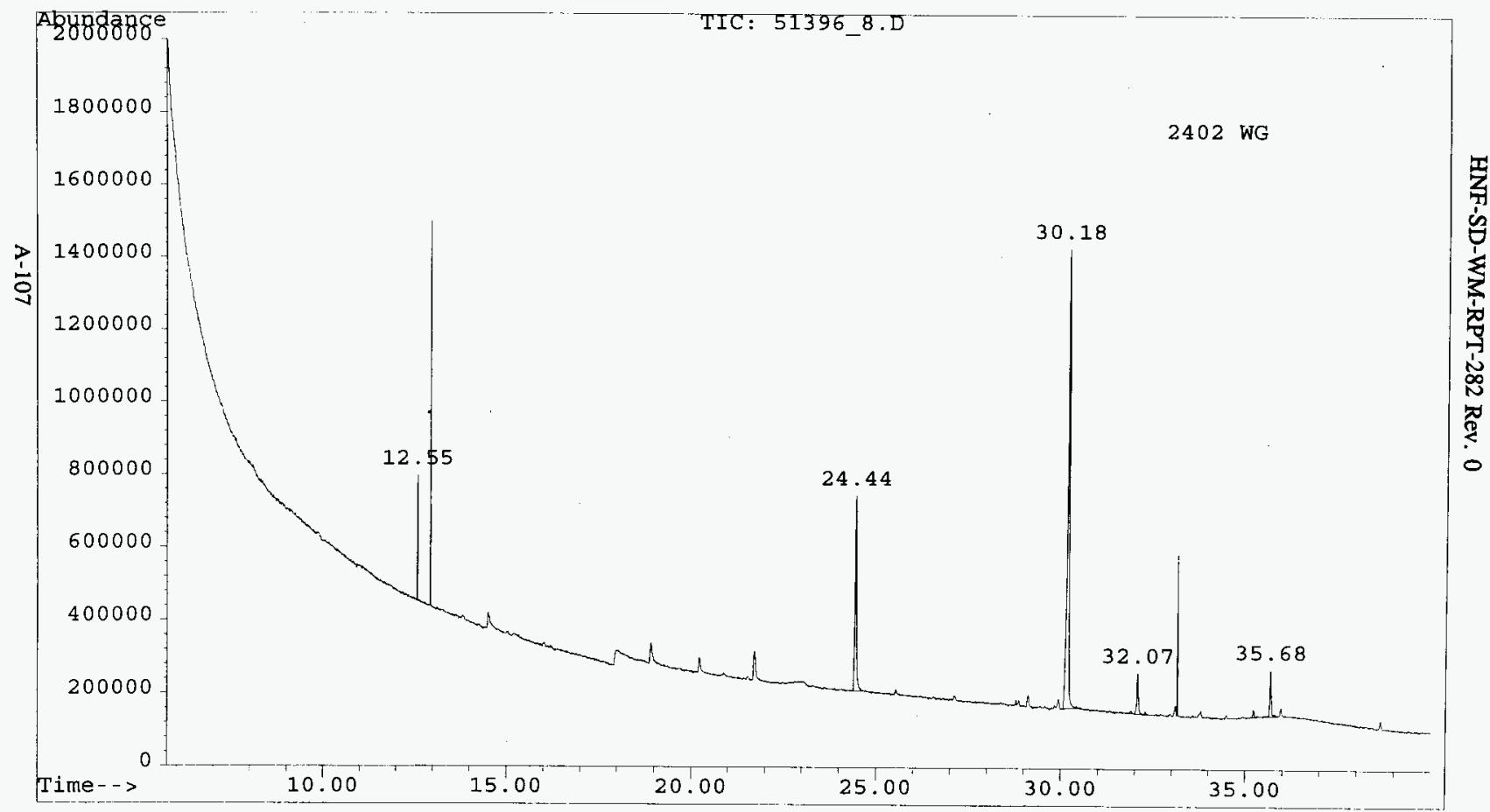


HNF-SD-WM-RPT-282 Rev. 0

\section{LIST OF COMPOUNDS TENTATIVELY IENTIFIED WITH MASS SPECTRAL LIBRARY}

Sample: $\quad 2402$ WG

Sample Name: FT6045_2WG.29A

Search Libraries: C:DATABASEIWLEY138.L Minimum Quality: 70

Unknown Spectrum: Apex

Integration Params: VOA.E

Pk\# RT Area\% Library/ID Ref\# CAS\# Qual

721.713 .85 C:DATABASEIWILEY138.L

1-Butanol

$116551000071-36-372$

824.44 16.54 C:LDATABASEIWILEY138.L

2-Pentanone, 4-methyl-

$118023000108-10-191$

$1028.860 .91 \mathrm{C}:$ IDATABASEIWILEY138.L

Benzene, ethyl-

$118561000100-41-486$

1129.12 1.31 C:DATABASEIWILEY138.L

Benzene, 1,4-dimethyl-

$118587000106-42-393$

Benzene, 1,3-dimethyl-

$118578000108-38-393$

1229.951 .39 C:DATABASEIWILEY138.L XYLENE

$118580001330-20-793$

Benzene, 1,4-dimethyl-

$118587000106-42-392$

Benzene, 1,3-dimethyl-

$118578000108-38-392$

1432.073 .49 C:IDATABASEIWILEY138.L

Benzaldehyde

$118552000100-52-796$

$1533.090 .88 \mathrm{C}:$ DATABASEIWILEY138.L

Benzene, 1,3,5-trimethyl-

$120037000108-67-889$ 
HNF-SD-WM-RPT-282 Rev. 0

CWC_REP.XLS

\section{ANALYSIS REPORT FOR CWC AIR SAMPLES}

File Name:

Analyst:

Analysis Date:

Method:

Sample Name:
51396_8.D

MS

05/13/96

CWC

FT6045_2WG.29A

\begin{tabular}{|c|c|c|c|c|}
\hline COMPOUND NAME & CAS \# & Concentration & Units & Comments \\
\hline ISO-BUTANE & $75-28-5$ & 0 & PPB & \\
\hline VINYL CHLORIDE & $75-01-4$ & 0 & PPB & \\
\hline ETHANOL & $64-17-5$ & 0 & PPB & \\
\hline ACETONE & $67-64-1$ & 0 & PPB & \\
\hline 2-PROPANOL & $67-63-0$ & 0 & PPB & \\
\hline 1,1-DICHLOROETHENE & $75-35-4$ & 0 & PPB & \\
\hline FREON 113 & $76-13-1$ & 0 & PPB & \\
\hline DICHLOROMETHANE & $75-09-2$ & 0 & PPB & \\
\hline 1,1-DICHLOROETHANE & $75-34-3$ & 0 & PPB & \\
\hline 2-BUTANONE & $78-93-3$ & 0 & PPB & \\
\hline N-HEXANE & $110-54-3$ & 0 & PPB & \\
\hline 1,2-DICHLOROETHENE & $156-59-2$ & 0 & PPB & \\
\hline CHLOROFORM & $67-66-3$ & 0 & PPB & \\
\hline TETRAHYDROFURAN & $109-99-9$ & 0 & PPB & \\
\hline 1,1,1-TRICHLOROETHANE & $71-55-6$ & 0 & PPB & \\
\hline 1,2-DICHLOROETHANE & $107-06-2$ & 0 & PPB & \\
\hline BENZENE & $71-43-2$ & 0 & PPB & \\
\hline CARBON TETRACHLORIDE & $56-23-5$ & 0 & PPB & \\
\hline CYCLOHEXANE & $110-82-7$ & 0 & PPB & \\
\hline N-BUTANOL & $71-36-3$ & 4 & PPB & \\
\hline TRICHLOROETHENE & $79-01-6$ & 0 & PPB & \\
\hline 4-METHYL-2-PENTANONE & $108-10-1$ & 10 & PPB & \\
\hline TOLUENE & $108-88-3$ & 0 & PPB & \\
\hline 1,1,2-TRICHLOROETHANE & $79-00-5$ & 0 & PPB & \\
\hline TETRACHLOROETHENE & $127-18-4$ & 0 & PPB & \\
\hline CHLOROBENZENE & $108-90-7$ & 0 & PPB & \\
\hline ETHYLBENZENE & $100-41-4$ & 0 & PPB & \\
\hline M-XYLENE & 108-38-3 & 0 & PPB & \\
\hline STYRENE & $100-42-5$ & 0 & PPB & \\
\hline O-XYLENE & $95-47-6$ & 0 & PPB & \\
\hline $1,1,2,2-$ TETRACHLORETHANE & $79-34-5$ & 0 & PPB & \\
\hline 1,3,5-TRIMETHYLBENZENE & $108-67-8$ & 2 & PPB & \\
\hline 1,2,4-TRIMETHYLBENZENE & $95-63-6$ & 0 & PPB & \\
\hline 1,3-DICHLOROBENZENE & $541.73-1$ & 0 & PPB & \\
\hline 1,2-DICHLOROBENZENE & $95-50-1$ & 0 & PPB & \\
\hline 1,4-DICHLOROBENZENE & $106-46-7$ & 0 & PPB & \\
\hline
\end{tabular}




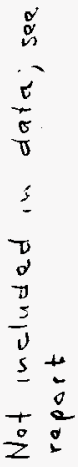

HNF-SD-WM-RPT-282 Rev. 0
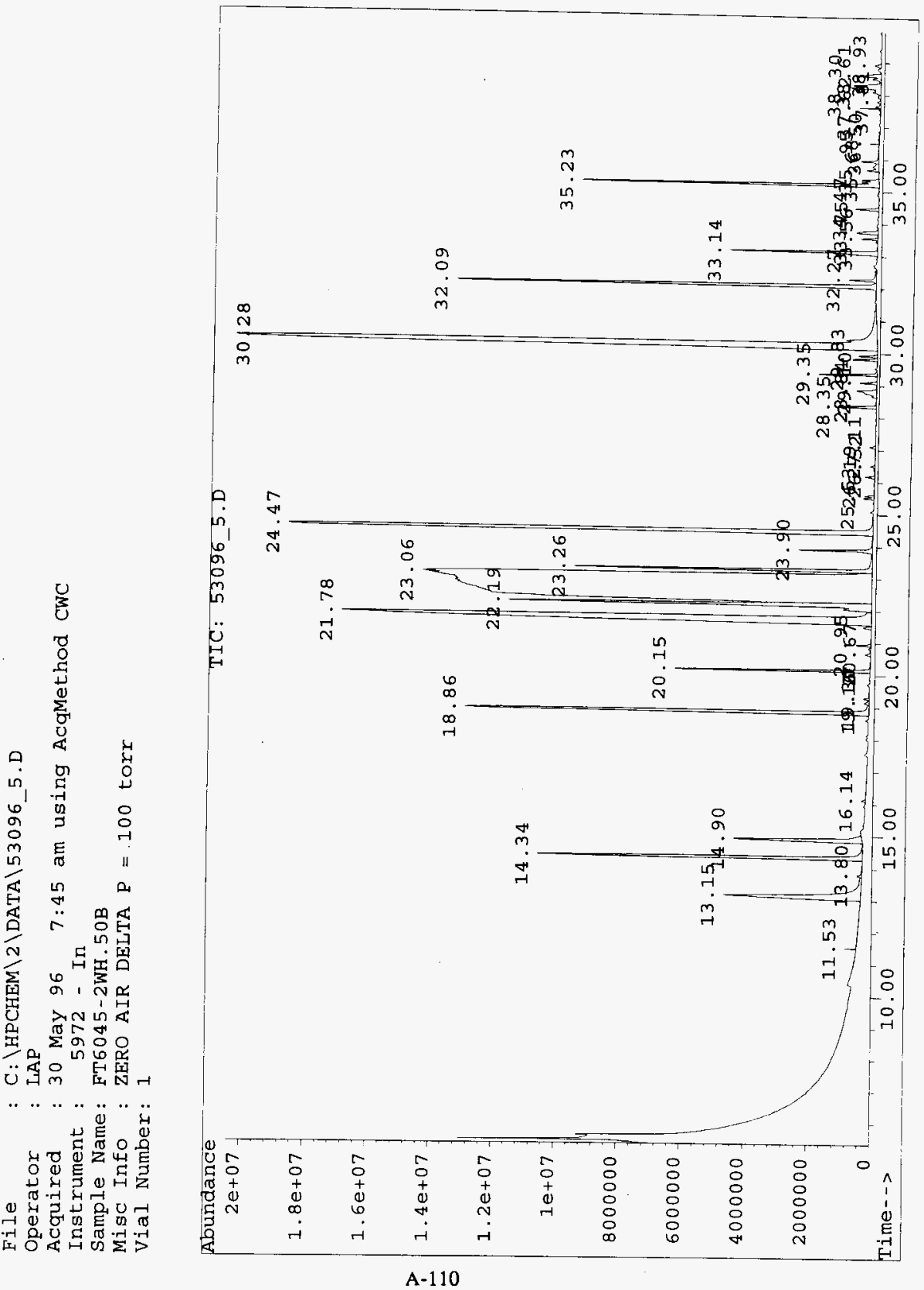
HNF-SD-WM-RPT-282 Rev. 0

CWC_REP.XLS

ANALYSIS REPORT FOR CWC AIR SAMPLES

File Name:

Analyst:

53096_5.D

Analysis Date:

Method:

Sample Name:

LAP

05/30/96

CWC

FT6045-2WH.50B

\begin{tabular}{|c|c|c|c|c|}
\hline COMPOUND NAME & CAS \# & Concentration & Units & Comments \\
\hline ISO-BUTANE & $75-28-5$ & $\overline{0}$ & PPB & \\
\hline VINYL CHLORIDE & $75-01-4$ & 0 & $\overline{P P B}$ & \\
\hline ETHANOL & $64-17-5$ & 727 & PPB & \\
\hline ACETONE & $67-64-1$ & 463 & PPB & \\
\hline 2-PROPANOL & $67-63-0$ & 241 & PPB & \\
\hline 1,1-DICHLOROETHENE & $75-35-4$ & 0 & PPB & \\
\hline FREON 113 & $76-13-1$ & 0 & PPB & \\
\hline DICHLOROMETHANE & $75-09-2$ & 4 & PPB & \\
\hline 1,1-DICHLOROETHANE & $75-34-3$ & 1 & PPB & \\
\hline 2-BUTANONE & $78-93-3$ & 2470 & PPB & \\
\hline N-HEXANE & $110-54-3$ & 129 & PPB & \\
\hline 1,2-DICHLOROETHENE & $156-59-2$ & 5 & PPB & \\
\hline CHLOROFORM & $67-66-3$ & 2 & PPB & \\
\hline TETRAHYDROFURAN & $109-99-9$ & 165 & PPB & \\
\hline 1,1,1-TRICHLOROETHANE & $71-55-6$ & 4 & PPB & \\
\hline 1,2-DICHLOROETHANE & $107-06-2$ & 26 & PPB & \\
\hline BENZENE & $71-43-2$ & 17 & $\overline{\mathrm{PPB}}$ & \\
\hline CARBON TETRACHLORIDE & $56-23-5$ & 8 & PPB & \\
\hline CYCLOHEXANE & $110-82-7$ & 5 & PPB & \\
\hline N-BUTANOL & $71-36-3$ & 4 & PPB & \\
\hline TRICHLOROETHENE & $79-01-6$ & 6 & PPB & \\
\hline 4-METHYL-2-PENTANONE & $108-10-1$ & 790 & PPB & \\
\hline TOLUENE & $108-88-3$ & 11 & PPB & \\
\hline 1,1,2-TRICHLOROETHANE & $79-00-5$ & 12 & PPB & \\
\hline TETRACHLOROETHENE & $127-18-4$ & 7 & PPB & \\
\hline CHLOROBENZENE & $108-90-7$ & 9 & PPB & \\
\hline ETHYLBENZENE & $100-41-4$ & 14 & PPB & \\
\hline M-XYLENE & $108-38-3$ & 15 & PPB & \\
\hline STYRENE & $100-42-5$ & 19 & PPB & \\
\hline O-XYLENE & $95-47-6$ & 14 & PPB & \\
\hline 1,1,2,2-TETRACHLORETHANE & $79-34-5$ & 15 & PPB & \\
\hline 1,3,5-TRIMETHYLBENZENE & $108-67-8$ & 21 & PPB & \\
\hline 1,2,4-TRIMETHYLBENZENE & $95-63-6$ & 26 & PPB & \\
\hline 1,3-DICHLOROBENZENE & $541-73-1$ & 17 & PPB & \\
\hline 1,2-DICHLOROBENZENE & $95-50-1$ & 21 & PPB & \\
\hline 1.4-DICHLOROBENZENE & $106-46-7$ & 24 & PPB & \\
\hline
\end{tabular}


HNF-SD-WM-RPT-282 Rev. 0

\section{LIST OF COMPOUNDS TENTATIVELY IDENTIFIED WITH MASS SPECTRAL LIBRARY}

$\begin{array}{ll}\text { Sample: } & \text { 2402 WH } \\ \text { File: } & \text { C:UPCHEM2LDATA153096_5.D } \\ \text { ID } & \text { FT6045-2WH.50B }\end{array}$

Compound

CAS\#

Ethanol

$000064-17-5$

2-Propanone

000067-64-1

2-Propanol

000067-63-0

2-Butanone

000078-93-3

Ethene, 1,2-dichloro-, (Z)-

000156-59-2

Acetic acid, trichloro-

000076-03-9

Furan, tetrahydro-

000109-99-9

Ethane, 1.2-dichloro-

000107-06-2

I-Butanol

000071-36-3

1,4-Dioxane

000123-9I-1

I-Propanol, 2-methoxy-

$001589-47-5$

2-Pentanone, 4-methyl-

000108-10-1

Benzene, methyl-

$000108-88-3$

Ethane, 1,1,2-trichloro-

000079-00-5

Ethene, tetrachloro-

$000127-18-4$

Benzene, ethyl-

$000100-41-4$

Benzene, 1,3-dimethyl-

000108-38-3 
Sample: $\quad 2402$ WH (CONTINUED)

File C.UHPCHEM 2\DATA153096_5.D

ID $\quad$ FT6045-2WH.50B

Compound

Styrene

XYLENE

Ethanol, 2-butoxy-

Benzaldehyde

Benzene, 1,3,5-trimethyl-

Benzene, 1,3-dichloro-

Benzene, 1,2-dichloro-

Benzene, 1,2-dichloro-

Ethanone, 1-phenyl-

Benzenamine, N,N,2-trimethyl-

Benzenemethanol, alpha, alpha-dime

Benzaldehyde, ethyl-
CAS\#

$000100-42-5$

001330-20-7

$000111-76-2$

000100-52-7

000108-67-8

$000541-73-1$

000095-50-1

000095-50-1

000098-86-2

000609-72-3

000617-94-7

053951-50-1 
File

Operator

Acquired

Instrument

Sample Name:

Misc Info

vial Number:

$\mathrm{C}: \backslash$ HPCHEM $\backslash 2 \backslash \mathrm{DATA} \backslash 53096 \_13 . \mathrm{D}$

MS

30 May $96 \quad 3: 22$ pm using AcqMethod CWC

5972 - In

FT6045-2WH. 49A

DELTA $\mathrm{P}=100$ torr

1

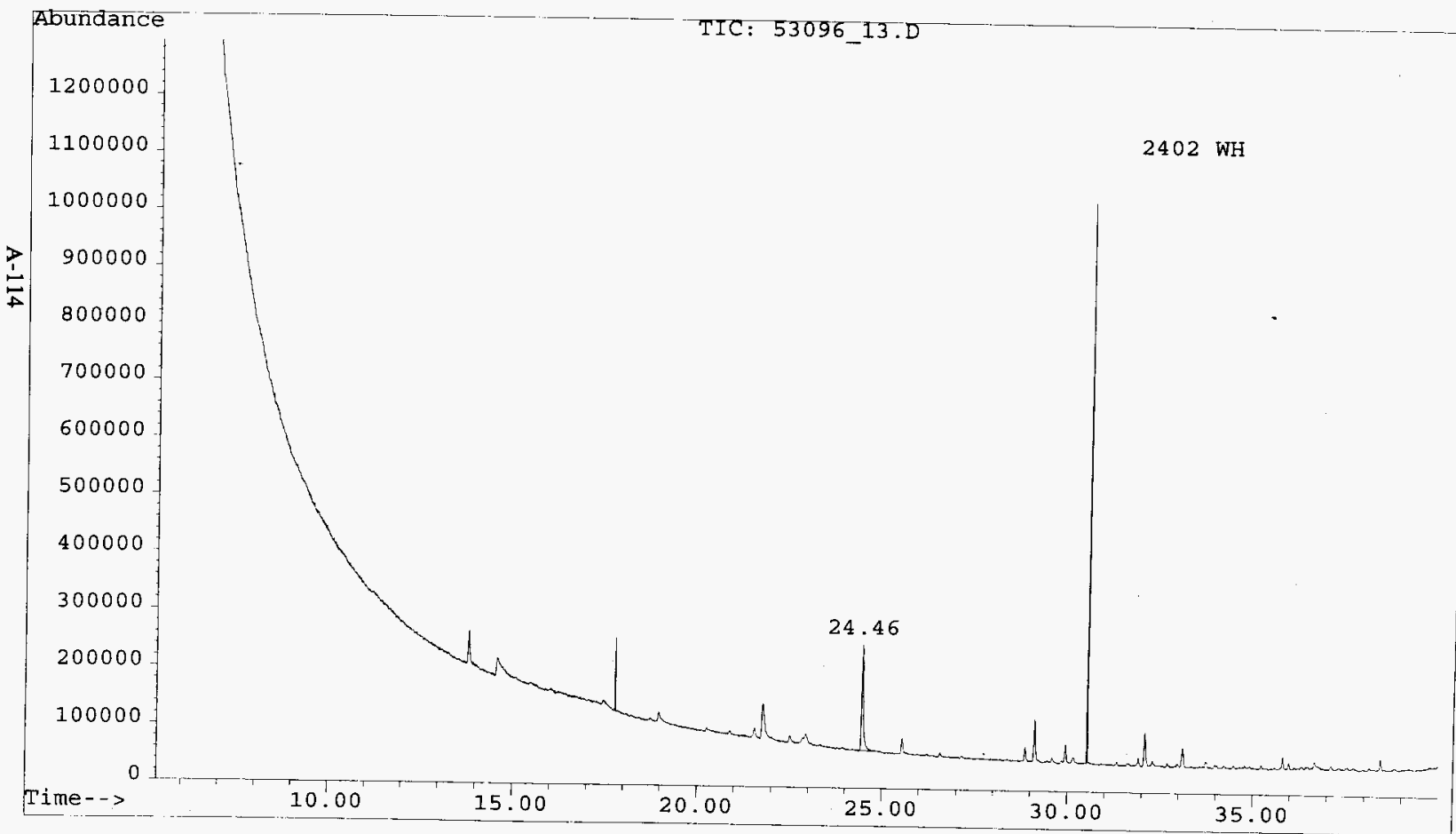


HNF-SD-WM-RPT-282 Rev. 0

CWC_REP.XLS

\section{ANALYSIS REPORT FOR CWC AIR SAMPLES}

File Name:

Analyst:

Analysis Date:

Method:

Sample Name:
53096_13.D

MS

05/30/96

CWC

FT6045-2WH.49A

\begin{tabular}{|c|c|c|c|c|}
\hline COMPOUND NAME & CAS \# & Concentration & Units & Comments \\
\hline ISO-BUTANE & $75-28-5$ & $\overline{0}$ & $\overline{P P B}$ & \\
\hline VINYL CHLORIDE & $75-01-4$ & 0 & PPB & \\
\hline ETHANOL & $64-17-5$ & 0 & PPB & \\
\hline ACETONE & $67-64-1$ & 0 & PPB & \\
\hline 2-PROPANOL & $67-63-0$ & 0 & PPB & \\
\hline 1,1-DICHLOROETHENE & $75-35-4$ & 0 & PPB & \\
\hline FREON 113 & $76-13-1$ & 0 & PPB & \\
\hline DICHLOROMETHANE & $75-09-2$ & 0 & PPB & \\
\hline 1,1-DICHLOROETHANE & $75-34-3$ & 0 & PPB & \\
\hline 2-BUTANONE & $78-93-3$ & 0 & PPB & \\
\hline N-HEXANE & $110-54-3$ & 0 & PPB & \\
\hline 1,2-DICHLOROETHENE & $156-59-2$ & 0 & $\overline{\mathrm{PPB}}$ & \\
\hline CHLOROFORM & $67-66-3$ & 0 & PPB & \\
\hline TETRAHYDROFURAN & $109-99-9$ & 0 & PPB & \\
\hline 1,1,1-TRICHLOROETHANE & $71-55-6$ & 0 & PPB & \\
\hline 1,2-DICHLOROETHANE & $107-06-2$ & 0 & PPB & \\
\hline BENZENE & $71-43-2$ & 0 & PPB & \\
\hline CARBON TETRACHLORIDE & $56-23-5$ & 0 & PPB & \\
\hline CYCLOHEXANE & $110-82-7$ & 0 & PPB & \\
\hline N-BUTANOL & $71-36-3$ & 0 & PPB & \\
\hline TRICHLOROETHENE & $79-01-6$ & 0 & $\overline{\mathbf{P P B}}$ & \\
\hline 4-METHYL-2-PENTANONE & $108-10-1$ & 0 & PPB & \\
\hline TOLUENE & $108-88-3$ & 0 & PPB & \\
\hline 1,1,2-TRICHLOROETHANE & $79-00-5$ & 0 & PPB & \\
\hline TETRACHLOROETHENE & $127-18-4$ & 0 & PPB & \\
\hline CHLOROBENZENE & $108-90-7$ & 0 & PPB & \\
\hline ETHYLBENZENE & $100-41-4$ & 2 & PPB & \\
\hline M-XYLENE & $108-38-3$ & 0 & PPB & \\
\hline STYRENE & $100-42-5$ & 0 & PPB & \\
\hline O-XYLENE & $95-47-6$ & 0 & PPB & \\
\hline 1,1,2,2-TETRACHLORETHANE & $79-34-5$ & 0 & PPB & \\
\hline 1,3,5-TRIMETHYLBENZENE & $108-67-8$ & 1 & PPB & \\
\hline 1,2,4-TRIMETHYLBENZENE & $95-63-6$ & 0 & PPB & \\
\hline 1,3-DICHLOROBENZENE & $541-73-1$ & 0 & PPB & \\
\hline 1,2-DICHLOROBENZENE & $95-50-1$ & 0 & PPB & \\
\hline 1,4-DICHLOROBENZENE & $106-46-7$ & 0 & PPB & \\
\hline
\end{tabular}


HNF-SD-WM-RPT-282 Rev. 0

\section{LIST OF COMPOUNDS TENTATIVELY IDENTIFIED WITH MASS SPECTRAL LIBRARY}

Sample: $\quad 2402$ WH

File: $\quad$ C:UHPCHEMI2LATA153096_13.D

D: $\quad$ FT6045-2WH.49A

Compound CAS\#

2-Pentanone, 4-methyl-

000108-10-1

Benzene, methyl-

000108-88-3

Benzene, ethyl-

000100-41-4

Benzene, 1,3-dimethyl-

000108-38-3

XYLENE

001330-20-7

Benzaldehyde

$000100-52-7$

Benzene, 1,2,3-trimethyl-

000526-73-8

Decane

000124-18-5 
File

Operator

: C: \HPCHEM \2\DATA \53196 14.D

Acquired : 31 May $96 \quad 7: 16$ pm using AcqMethod CWC

Instrument : 5972 - In

Sample Name: FT 6045 2WH.50A RERUN

Misc Info : DELTA $\mathrm{P}^{-}=100$ torr

Vial Number: 1

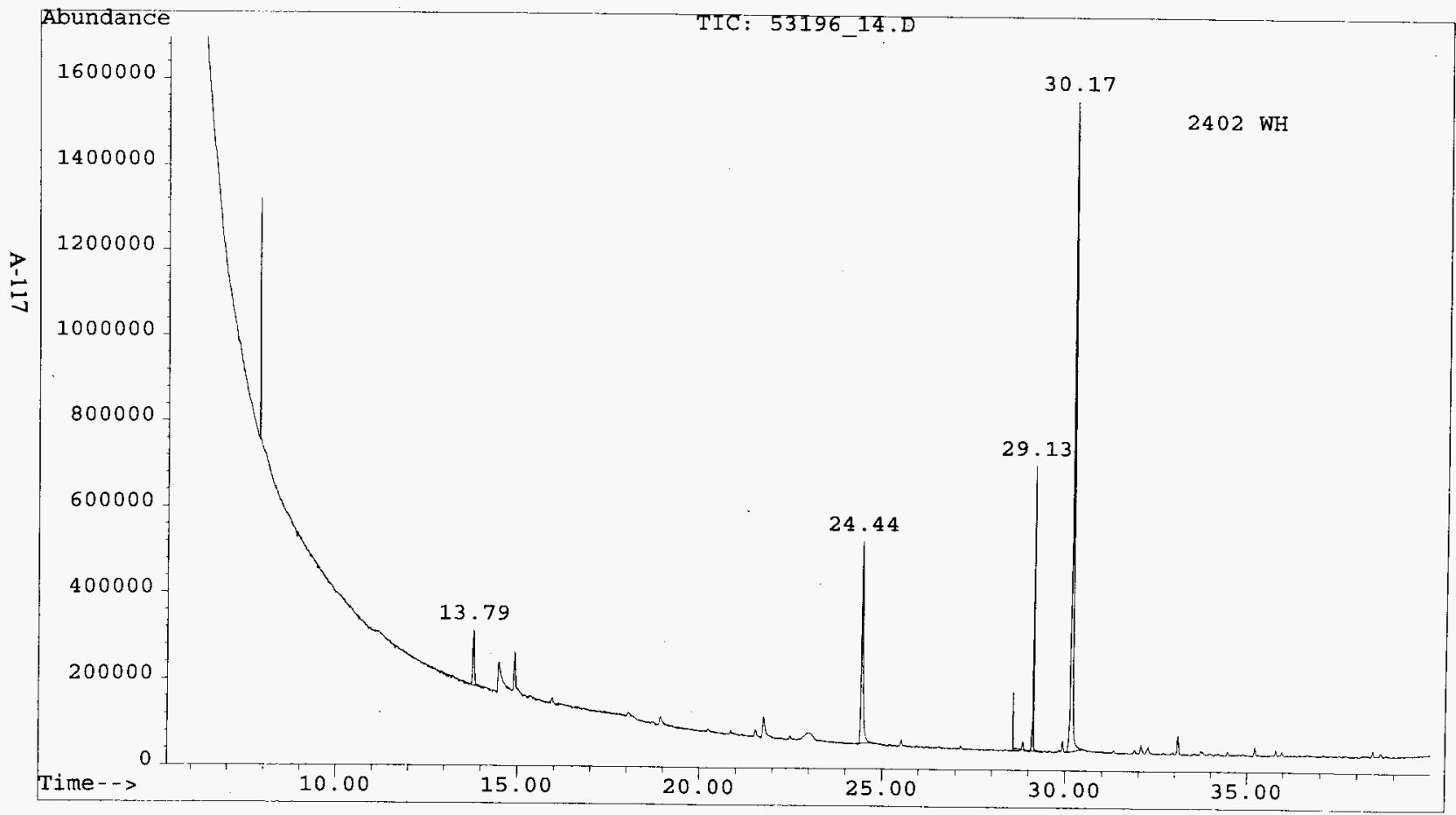




\begin{tabular}{|c|c|c|c|c|}
\hline & gdd & 0 & $L-9 b-90 L$ & 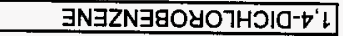 \\
\hline & gdd & 0 & $\downarrow-0 S-96$ & $\exists \mathrm{N} \exists \mathrm{ZN} \exists g 0807 \mathrm{HOI0-乙^{ \prime } \mathrm { L }}$ \\
\hline & gdd & 0 & $L-\varepsilon L-L \downarrow G$ & 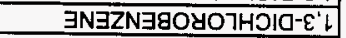 \\
\hline & 8dd & 1 & $9-\varepsilon 9-96$ & $\exists N \exists Z N \exists g 7 \lambda H \perp \exists W \mid{ }^{\prime} \perp-\nabla^{\prime} Z^{\prime}$ \\
\hline & Gdd & 0 & $8-\angle 9-80 L$ & $\exists N \exists Z N \exists g 7 \wedge H \perp \exists W 1 Y \perp-G^{\prime} \varepsilon^{\prime} L$ \\
\hline & gdd & 0 & $S-b \varepsilon-6 L$ & $\exists N \forall H \perp \exists y O\urcorner H O \forall Y \perp \exists \perp-Z^{\prime} Z^{\prime} L^{\prime} \downarrow$ \\
\hline & gdd & 0 & $9-\angle t S 6$ & $\exists N \exists 7 \lambda \times-0$ \\
\hline & gdd & 0 & $s-2 t-00 t$ & $\exists N \exists Y \wedge \perp S$ \\
\hline & gdd & 0 & $\varepsilon-8 \varepsilon-801$ & $\exists N \exists า \wedge x-1$ \\
\hline & gdd & 1 & $516-001$ & $\exists N \exists Z N \exists g 7 \wedge H \perp \exists$ \\
\hline & gdd & 0 & $\angle-06-80 L$ & $\exists N \exists Z N \exists g O Y O 7 \mathrm{HO}$ \\
\hline & gdd & 0 & $b-8 L-\angle 2 l$ & $\exists N \exists H \perp \exists O У O 7 H O \forall \& \perp \exists \perp$ \\
\hline & 8dd & 0 & $S=00-6 L$ & $\exists N \forall H \perp \exists O Y O T H O I Y \perp-Z^{\prime} \iota^{\prime} l$ \\
\hline & gdd & 0 & $\varepsilon-88-801$ & $\exists N \exists \cap 701$ \\
\hline & gdd & IL & $1-0 l-80 L$ & $\exists N O N \forall \perp N \exists d-\tau-7 \lambda H \perp \exists W-t$ \\
\hline & gdd & 0 & $9-10-6 L$ & $\exists N \exists H \perp \exists O Y 07 \mathrm{HOI} \perp$ \\
\hline & gdd & 0 & $\varepsilon-9 \varepsilon-\downarrow L$ & 10N $\forall \perp n g-N$ \\
\hline & add & 0 & $\angle-28-016$ & $\exists N \vee \times \exists H O T \supset \lambda \supset$ \\
\hline & gdd & 0 & $9-\varepsilon z-99$ & 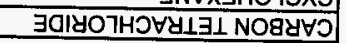 \\
\hline & gdd & 0 & $z-\varepsilon t-1 L$ & $\exists N \exists Z N \exists G$ \\
\hline & gdd & 0 & $2-90-\angle 0 l$ & $\exists N \forall H \perp \exists O$ УОา \\
\hline & gdd & 0 & $9-9 g-1 L$ & 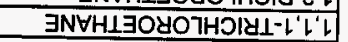 \\
\hline & gdd & 0 & $6-66-601$ & 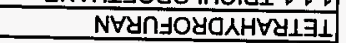 \\
\hline & 8dd & 0 & $\varepsilon-99-\angle 9$ & WyOJOYO7Hว \\
\hline & gdd & 0 & $\overline{z-69-9 G l}$ & $\exists N \exists H \perp \exists O У 07 H 0 \mid a-Z^{\prime}$ \\
\hline & gdd & 0 & $\varepsilon-t G-01 L$ & $\exists N \forall \times \exists H-N$ \\
\hline & gdd & 0 & $\varepsilon-\varepsilon 6-8 L$ & BNON $\forall \perp \cap Q-Z$ \\
\hline & gdd & 0 & $\varepsilon-t \varepsilon-\varsigma L$ & 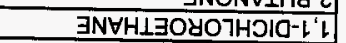 \\
\hline & 8dd & 0 & $z-60-S L$ & $\exists N \forall H \perp \exists$ WOУOרHगI \\
\hline & 8dd & 0 & $1-\varepsilon l-9 L$ & ELL NOتYy \\
\hline & 8dd & 0 & $t-G \varepsilon-S L$ & $\exists N \exists H \perp \exists O 807 \mathrm{HOI0-เ'L}$ \\
\hline & gdd & 0 & $0-89-\angle 9$ & 10N $\forall$ dOYd-Z \\
\hline & gdd & 0 & $1-69-\angle 9$ & $\exists N O \perp \exists O \forall$ \\
\hline & gdd & 0 & $s-\angle L-79$ & 7ON \\
\hline$\div$ & gdd & 0 & VLO-SL & 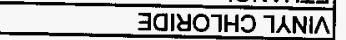 \\
\hline & 8dd & 0 & $G-8 Z-G L$ & $\exists N \forall \perp \cap 8-O S \mid$ \\
\hline squeumuos & sł!un & uopenuesuos & \# SVo & IWVN ONNOdWOS \\
\hline & & Nกyヨy $\forall 0 s$ & 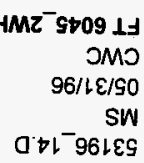 & 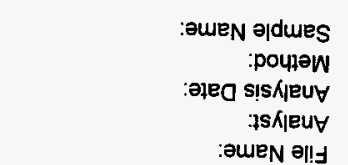 \\
\hline
\end{tabular}


HNF-SD-WM-RPT-282 Rev. 0

\section{LIST OF COMPOUNDS TENTATIVELY IDENTIFIED WITH MASS SPECTRAL LIBRARY}

Sample: $\quad 2402$ WH

File: $\quad$ C:UHPCHEMLLDATAI53196_14.D

ID: FT6045_2WH.50A RERUN

Compound

CAS \#

1,3-Butadiene, 2-methyl-

000078-79-5

2-Pentanone, 4-methyl-

000108-10-1

Benzene, 1,2-dimethyl-

000095-47-6

Ethanol, 2-butoxy-

000111-76-2

Benzene, 1,3,5-trimethyl-

000108-67-8 
File

Operator

Acquired

Instrument

5972 - In

Misc Info : DELTA $P=100$ torr

Vial Number: 1

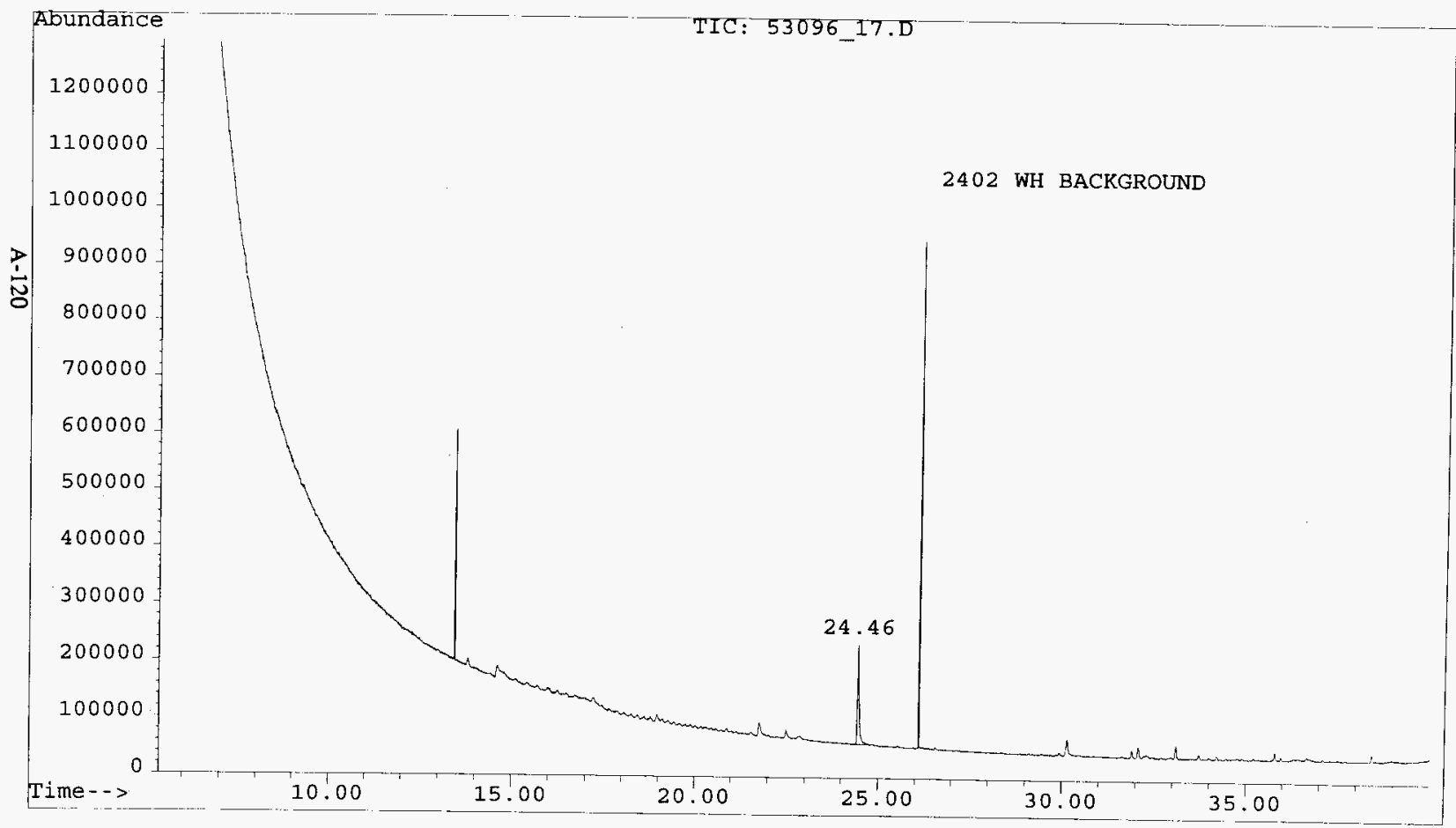


HNF-SD-WM-RPT-282 Rev. 0

CWC_REP.XLS

\section{ANALYSIS REPORT FOR CWC AIR SAMPLES}

File Name:

Analyst:

Analysis Date

Method:

Sample Name:
53096_17.D

MS

05/30/96

CWC

FT6045-2WH.51A

\begin{tabular}{|c|c|c|c|c|}
\hline COMPOUND NAME & CAS \# & Concentration & Units & Comments \\
\hline ISO-BUTANE & $75-28-5$ & 0 & PPB & \\
\hline VINYL CHLORIDE & $75-01-4$ & 0 & PPB & \\
\hline ETHANOL & $64-17-5$ & 0 & PPB & \\
\hline ACETONE & $67-64-1$ & 0 & PPB & \\
\hline 2-PROPANOL & $67-63-0$ & 0 & PPB & \\
\hline 1,1-DICHLOROETHENE & $75-35-4$ & 0 & PPB & \\
\hline FREON 113 & $76-13-1$ & 0 & PPB & \\
\hline DICHLOROMETHANE & $75-09-2$ & 0 & PPB & \\
\hline 1,1-DICHLOROETHANE & $75-34-3$ & 0 & PPB & \\
\hline 2-BUTANONE & $78-93-3$ & 0 & PPB & \\
\hline N-HEXANE & $110-54-3$ & 0 & PPB & \\
\hline 1,2-DICHLOROETHENE & $156-59-2$ & 0 & PPB & \\
\hline CHLOROFORM & $67-66-3$ & 0 & PPB & \\
\hline TETRAHYDROFURAN & $109-99-9$ & 0 & $\overline{\mathrm{PPB}}$ & \\
\hline 1,1,1-TRICHLOROETHANE & $71-55-6$ & 0 & PPB & \\
\hline 1,2-DICHLOROETHANE & $107-06-2$ & 0 & PPB & \\
\hline BENZENE & $71-43-2$ & 0 & PPB & \\
\hline CARBON TETRACHLORIDE & $56-23-5$ & 0 & PPB & \\
\hline CYCLOHEXANE & $110-82-7$ & 0 & PPB & \\
\hline N-BUTANOL & $71-36-3$ & 0 & PPB & \\
\hline TRICHLOROETHENE & $79-01-6$ & 0 & PPB & \\
\hline 4-METHYL-2-PENTANONE & $108-10-1$ & 0 & PPB & \\
\hline TOLUENE & $108-88-3$ & 0 & PPB & \\
\hline 1,1,2-TRICHLOROETHANE & $79-00-5$ & $\overline{0}$ & PPB & \\
\hline TETRACHLOROETHENE & $127-18-4$ & 0 & PPB & \\
\hline CHLOROBENZENE & $108-90-7$ & 0 & PPB & \\
\hline ETHYLBENZENE & $100-41-4$ & 0 & PPB & \\
\hline M-XYLENE & $108-38-3$ & 0 & PPB & \\
\hline STYRENE & $100-42-5$ & 0 & PPB & \\
\hline O-XYLENE & $95-47-6$ & 0 & $\overline{P P B}$ & \\
\hline 1,1,2,2-TETRACHLORETHANE & $79-34-5$ & 0 & $\overline{\mathrm{PPB}}$ & \\
\hline 1,3,5-TRIMETHYLBENZENE & $108-67-8$ & 0 & PPB & \\
\hline 1,2,4-TRIMETHYLBENZENE & $95-63-6$ & 0 & PPB & \\
\hline 1,3-DICHLOROBENZENE & $541-73-1$ & 0 & PPB & \\
\hline 1,2-DICHLOROBENZENE & $95-50-1$ & 0 & PPB & \\
\hline 1,4-DICHLOROBENZENE & $106-46-7$ & 0 & PPB & \\
\hline
\end{tabular}


HNF-SD-WM-RPT-282 Rev. 0

LIST OF COMPOUNDS TENTATIVELY IDENTIFIED WITH MASS SPECTRAL LIBRARY

Sample: $\quad$ 2402WH

File: $\quad$ C:IHPCHEML2LDATAL53096_17.D

ID: $\quad$ FT6045-2WH.51A

Compound CAS\#

2-Pentanone, 4-methyl-

000108-10-1

Benzaldehyde

$000100-52-7$

Benzene, 1,3,5-trimethyl-

$000108-67-8$ 
HNF-SD-WM-RPT-282 Rev. 0

\section{LIST OF COMPOUNDS TENTATIVELY IDENTIFIED WITH MASS SPECTRAL LIBRARY}

Sample: $\quad$ 2402WH

File: $\quad$ C:UHPCHEM2LDATA153096 17.D

ID: $\quad$ FT6045-2WH.51A

Compound

CAS\#

2-Pentanone, 4-methyl-

000108-10-1

Benzaldehyde

000100-52-7

Benzene, 1,3,5-trimethyl-

000108-67-8 
File

Operator

Acquired

Instrument

Sample Name: 5972 - In

$20452 \mathrm{WI} 32 \mathrm{~B}$

Misc Info : DELTA $\overline{\mathrm{P}}=100$ torr

Vial Number: 1

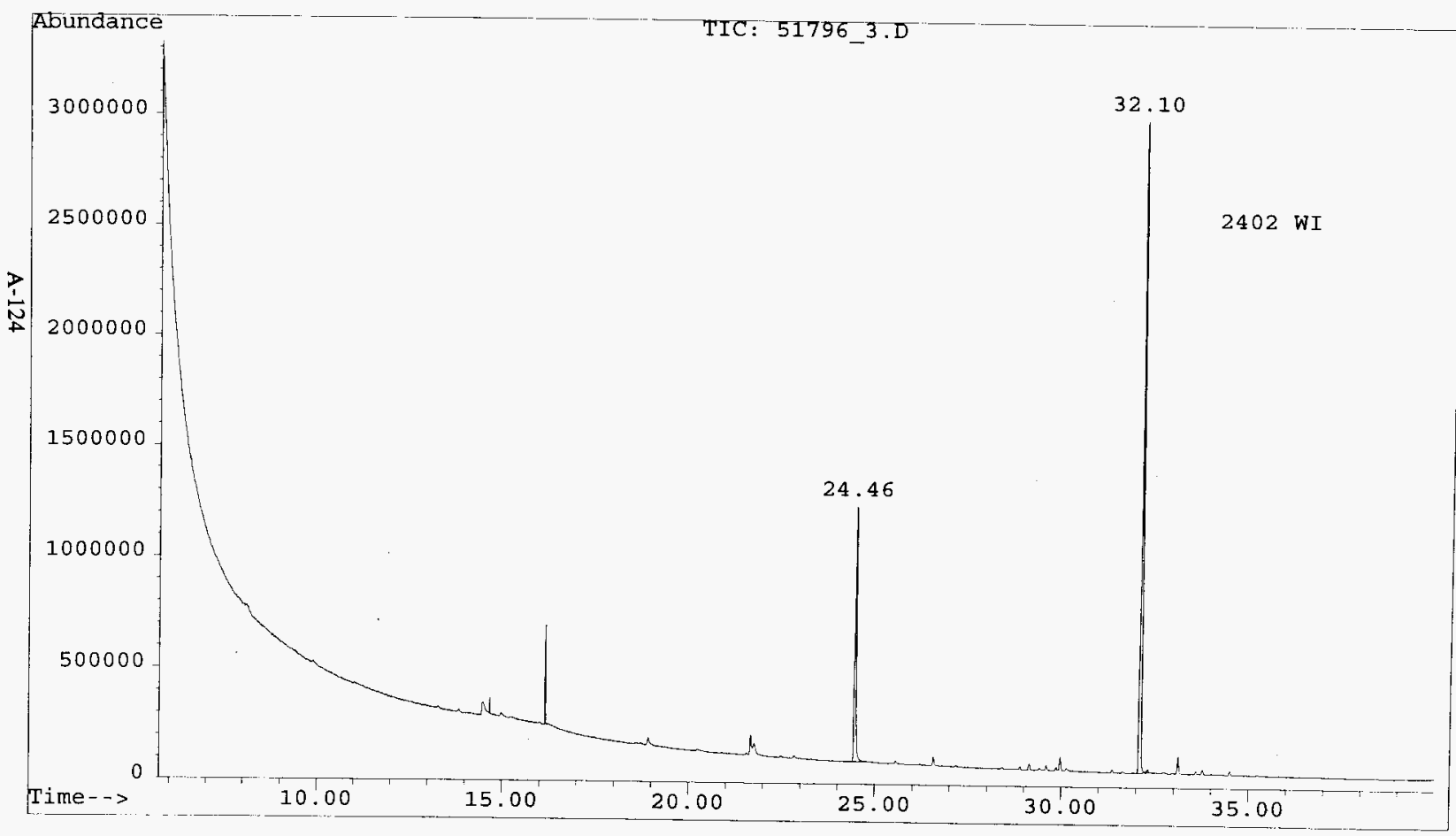


HNF-SD-WM-RPT-282 Rev. 0

CWC_REP.XLS

\section{ANALYSIS REPORT FOR CWC AIR SAMPLES}

File Name:

Analyst:

51796_3.D

Analysis Date:

Method:

$\mathrm{ms}$

05/17/96

Sample Name:

CWC

FT6045_2WI.32B

\begin{tabular}{|c|c|c|c|c|}
\hline COMPOUND NAME & CAS \# & Concentration & Units & Comments \\
\hline ISO-BUTANE & $\overline{75-28-5}$ & $\overline{0}$ & $\overline{P P B}$ & \\
\hline VINYL CHLORIDE & $75-01-4$ & 0 & PPB & \\
\hline ETHANOL & $64-17-5$ & 0 & PPB & \\
\hline ACETONE & $67-64-1$ & 0 & PPB & \\
\hline 2-PROPANOL & $67-63-0$ & 0 & PPB & \\
\hline 1,1-DICHLOROETHENE & $75-35-4$ & 0 & PPB & \\
\hline FREON 113 & $76-13-1$ & 0 & PPB & \\
\hline DICHLOROMETHANE & $75-09-2$ & 0 & PPB & \\
\hline 1,1-DICHLOROETHANE & $75-34-3$ & 0 & PPB & \\
\hline 2-BUTANONE & $78-93-3$ & 0 & PPB & \\
\hline N-HEXANE & $110-54-3$ & 0 & $\overline{\mathrm{PPB}}$ & \\
\hline 1,2-DICHLOROETHENE & $156-59-2$ & 0 & PPB & \\
\hline CHLOROFORM & $67-66-3$ & 0 & PPB & \\
\hline TETRAHYDROFURAN & $109-99-9$ & 0 & PPB & \\
\hline 1,1,1-TRICHLOROETHANE & $71-55-6$ & 0 & PPB & \\
\hline 1,2-DICHLOROETHANE & $107-06-2$ & 0 & PPB & \\
\hline BENZENE & $71-43-2$ & 0 & PPB & \\
\hline CARBON TETRACHLORIDE & $56-23-5$ & 0 & PPB & \\
\hline CYCLOHEXANE & $110-82-7$ & 0 & PPB & \\
\hline N-BUTANOL & $71-36-3$ & 2 & PPB & \\
\hline TRICHLOROETHENE & $79-01-6$ & 0 & PPB & \\
\hline 4-METHYL-2-PENTANONE & $108-10-1$ & 24 & PPB & \\
\hline TOLUENE & $108-88-3$ & 0 & PPB & \\
\hline 1,1,2-TRICHLOROETHANE & $79-00-5$ & 0 & PPB & \\
\hline TETRACHLOROETHENE & $127-18-4$ & 0 & PPB & \\
\hline CHLOROBENZENE & $108-90-7$ & 0 & PPB & \\
\hline ETHYLBENZENE & $100-41-4$ & 0 & PPB & \\
\hline M-XYLENE & $108-38-3$ & 0 & PPB & \\
\hline STYRENE & $100-42-5$ & 0 & PPB & \\
\hline O-XYLENE & $95-47-6$ & 0 & PPB & \\
\hline 1,1,2,2-TETRACHLORETHANE & $79-34-5$ & 0 & PPB & \\
\hline 1,3,5-TRIMETHYLBENZENE & $108-67-8$ & 51 & PPB & \\
\hline 1,2,4-TRIMETHYLBENZENE & $95-63-6$ & 2 & PPB & \\
\hline 1,3-DICHLOROBENZENE & $541-73-1$ & 0 & PPB & \\
\hline 1,2-DICHLOROBENZENE & $95-50-1$ & 0 & PPB & \\
\hline 1,4-DICHLOROBENZENE & $106-46-7$ & 0 & PPB & \\
\hline
\end{tabular}


HNF-SD-WM-RPT-282 Rev. 0

\section{LIST OF COMPOUNDS TENTATIVELY IDENTIFIED WITH MASS SPECTRAL LIBRARY}

Sample: $\quad 2402$ WI

File: C:UHPCHEM 2LDATAI51796_3.D

ID: FT6045_2WI.32B

Compound

CAS \#

1-Butanol

$000071-36-3$

2-Pentanone, 4-methyl-

000108-10-1

Benzene, ethyl-

$000100-41-4$

XYLENE

001330-20-7

Styrene

$000100-42-5$

Benzene, 1,2-dimethyl-

$000095-47-6$

Benzaldehyde

000100-52-7

Benzene, 1,3,5-trimethyl-

000108-67-8

Benzene, 1,4-dichloro-

000106-46-7

Benzene, 1,2-dichloro-

000095-50-1 
File

Operator

Acquired

Instrument

Sample Name:

Misc Info

Vial Number:

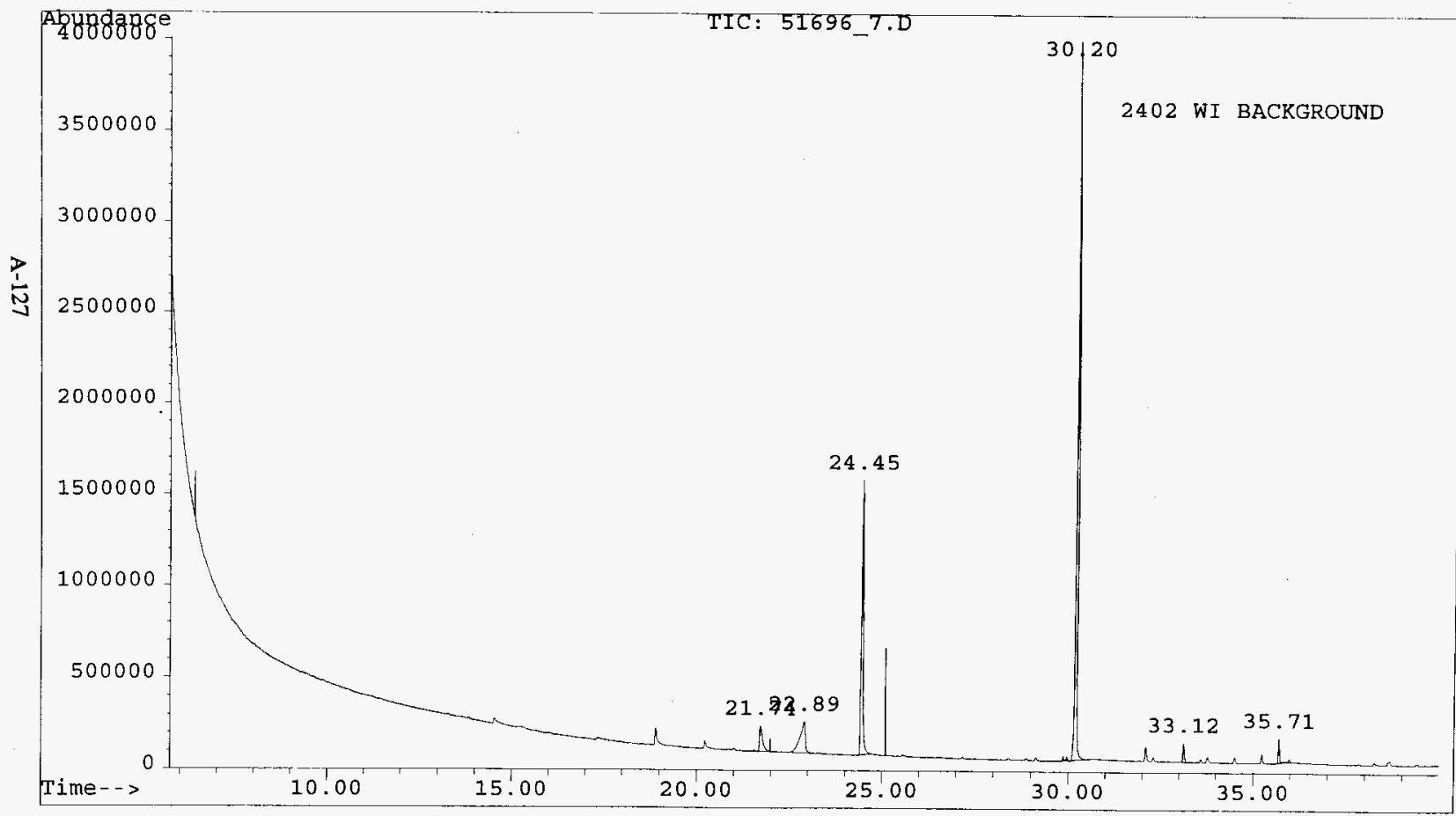

ms

16 May 96 4:31 pm using AcqMethod CWC

$5972-$ In

FT6045 2WI.34A BK

RERUN; 100 TORR
$\mathrm{C}: \backslash \mathrm{HPCHEM} \backslash 2 \backslash \mathrm{DATA} \backslash 51696 \_7 . \mathrm{D}$ 
HNF-SD-WM-RPT-282 Rev. 0

CWC_REP.XLS

\section{ANALYSIS REPORT FOR CWC AIR SAMPLES}

File Name:

Anaiyst:

Analysis Date:

Method:

Sample Name:
51696_7.D

$\mathrm{ms}$

05/16/96

CWC

FT6045_2W.34A BK

\begin{tabular}{|c|c|c|c|c|}
\hline COMPOUND NAME & CAS \# & Concentration & Units & Comments \\
\hline ISO-BUTANE & $75-28-5$ & $\overline{0}$ & PPB & \\
\hline VINYL CHLORIDE & $75-01-4$ & 0 & PPB & \\
\hline ETHANOL & $64-17-5$ & 0 & $\mathrm{PPB}$ & \\
\hline ACETONE & $67-\overline{64-1}$ & 0 & PPB & \\
\hline 2-PROPANOL & $67-63-0$ & $\overline{0}$ & PPB & \\
\hline 1,1-DICHLOROETHENE & $75-35-4$ & 0 & PPB & \\
\hline FREON 113 & $76-13-1$ & 0 & PPB & \\
\hline DICHLOROMETHANE & $75-09-2$ & 0 & PPB & \\
\hline 1,1-DICHLOROETHANE & $75-34-3$ & $\overline{0}$ & PPB & \\
\hline 2-BUTANONE & $78-93-3$ & 0 & PPB & \\
\hline N-HEXANE & $110-54-3$ & 0 & PPB & \\
\hline 1,2-DICHLOROETHENE & $156-59-2$ & 0 & PPB & \\
\hline CHLOROFORM & $67-66-3$ & 0 & PPB & \\
\hline TETRAHYDROFURAN & $109-99-9$ & 0 & PPB & \\
\hline 1,1,1-TRICHLOROETHANE & $71-55-6$ & 0 & PPB & \\
\hline 1,2-DICHLOROETHANE & $107-06-2$ & 0 & PPB & \\
\hline BENZENE & $71-43-2$ & 0 & PPB & \\
\hline CARBON TETRACHLORIDE & $56-23-5$ & $\overline{0}$ & PPB & \\
\hline CYCLOHEXANE & $110-82-7$ & 0 & PPB & \\
\hline N-BUTANOL & $71-36-3$ & 11 & PPB & \\
\hline TRICHLOROETHENE & $79-01-6$ & 0 & PPB & \\
\hline 4-METHYL-2-PENTANONE & $108-10-1$ & 35 & PPB & \\
\hline TOLUENE & $108-88-3$ & 0 & PPB & \\
\hline 1,1,2-TRICHLOROETHANE & $79-00-5$ & 0 & PPB & \\
\hline TETRACHLOROETHENE & $127-18-4$ & 0 & PPB & \\
\hline CHLOROBENZENE & $108-90-7$ & 0 & PPB & \\
\hline ETHYLBENZENE & $100-41-4$ & 0 & $\mathrm{PPB}$ & \\
\hline M-XYLENE & $108-38-3$ & 0 & PPB & \\
\hline STYRENE & $100-42-5$ & $\overline{0}$ & PPB & \\
\hline O-XYLENE & $95-47-6$ & 0 & PPB & \\
\hline $1,1,2,2$-TETRACHLORETHANE & $79-34-5$ & 0 & PPB & \\
\hline 1,3,5-TRIMETHYLBENZENE & $108-67-8$ & 1 & PPB & \\
\hline 1,2,4-TRIMETHYLBENZENE & $95-63-6$ & 3 & PPB & \\
\hline 1,3-DICHLOROBENZENE & $541-73-1$ & 0 & PPB & \\
\hline 1,2-DICHLOROBENZENE & $95-50-1$ & 0 & PPB & \\
\hline 1,4-DICHLOROBENZENE & $106-46-7$ & 0 & PPB & \\
\hline
\end{tabular}


HNF-SD-WM-RPT-282 Rev. 0

\section{LIST OF COMPOUNDS TENTATIVELY IDENTIFIED WITH MASS SPECTRAL LIBRARY}

Sample: 2402 WI Background

File: C:UHPCHEML2LATA151696_7.D

ID: FT6045_2WI.34A BK

Compound CAS \#

1-Butanol

$000071-36-3$

2-Propanol, 1-methoxy-

000107-98-2

2-Pentanone, 4-methyl-

000108-10-1

Benzene, 1,4-dimethyl-

000106-42-3

Styrene

$000100-42-5$

XYLENE

01330-20-7 95

Ethanol, 2-butoxy-

$000111-76-2$

Benzaldehyde

$000100-52-7$

Benzene, 1,3,5-trimethyl-

$000108-67-8$

Benzene, 1,2,4-trimethyl-

$000095-63-6$

Benzene, 1,4-dichloro-

000106-46-7

Ethanone, 1-phenyl-

000098-86-2 


\section{File}

Operator

Acquired

Instrument

Sample Na

Misc Info

Vial Number:

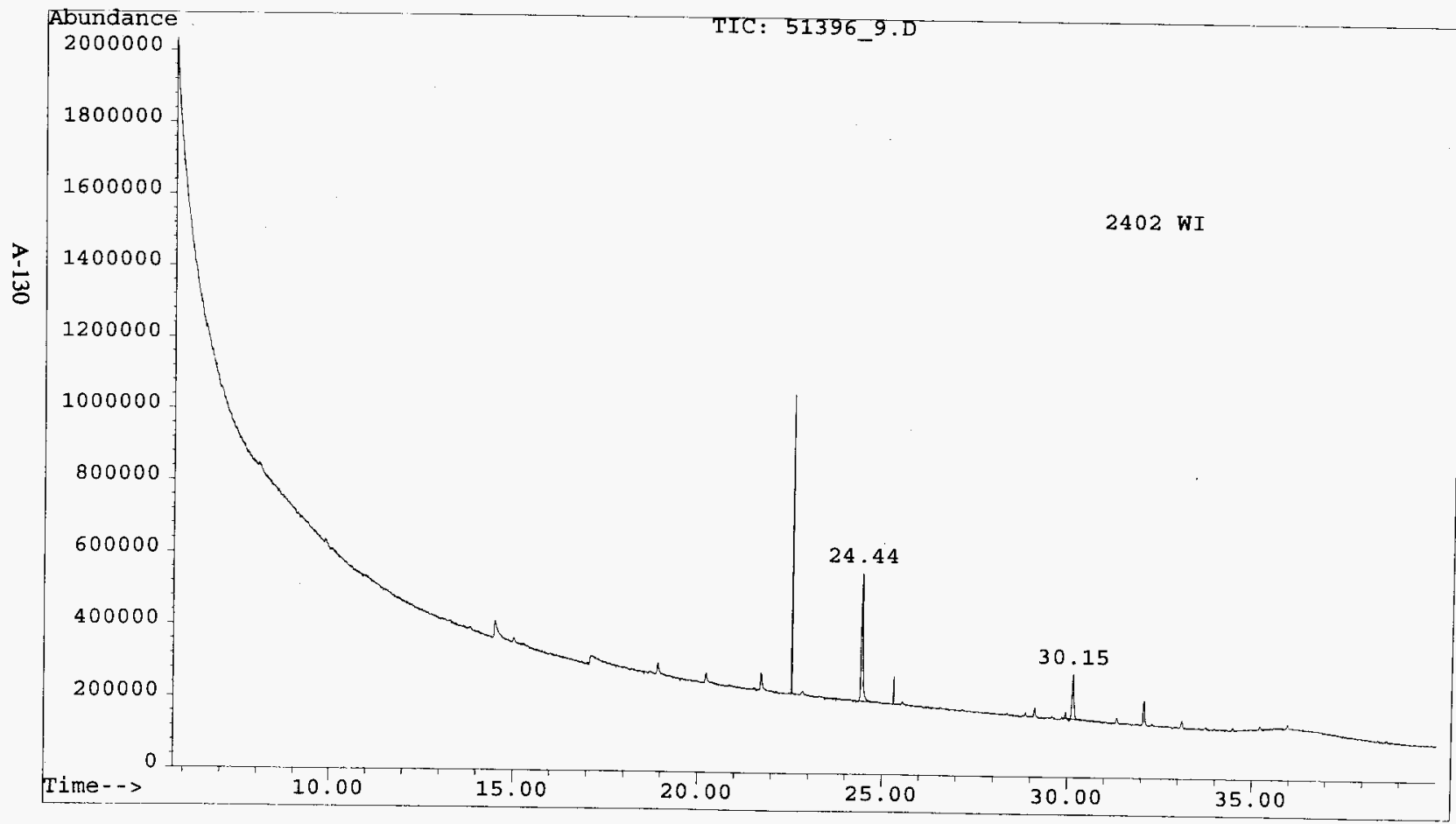

$\mathrm{C}:$ 5972 - In

FT6045 2WI.32A

DELTA $\overline{\mathrm{P}}=100$ TORR
: 13 May 96 2:14 pm using AcqMethod CWC 


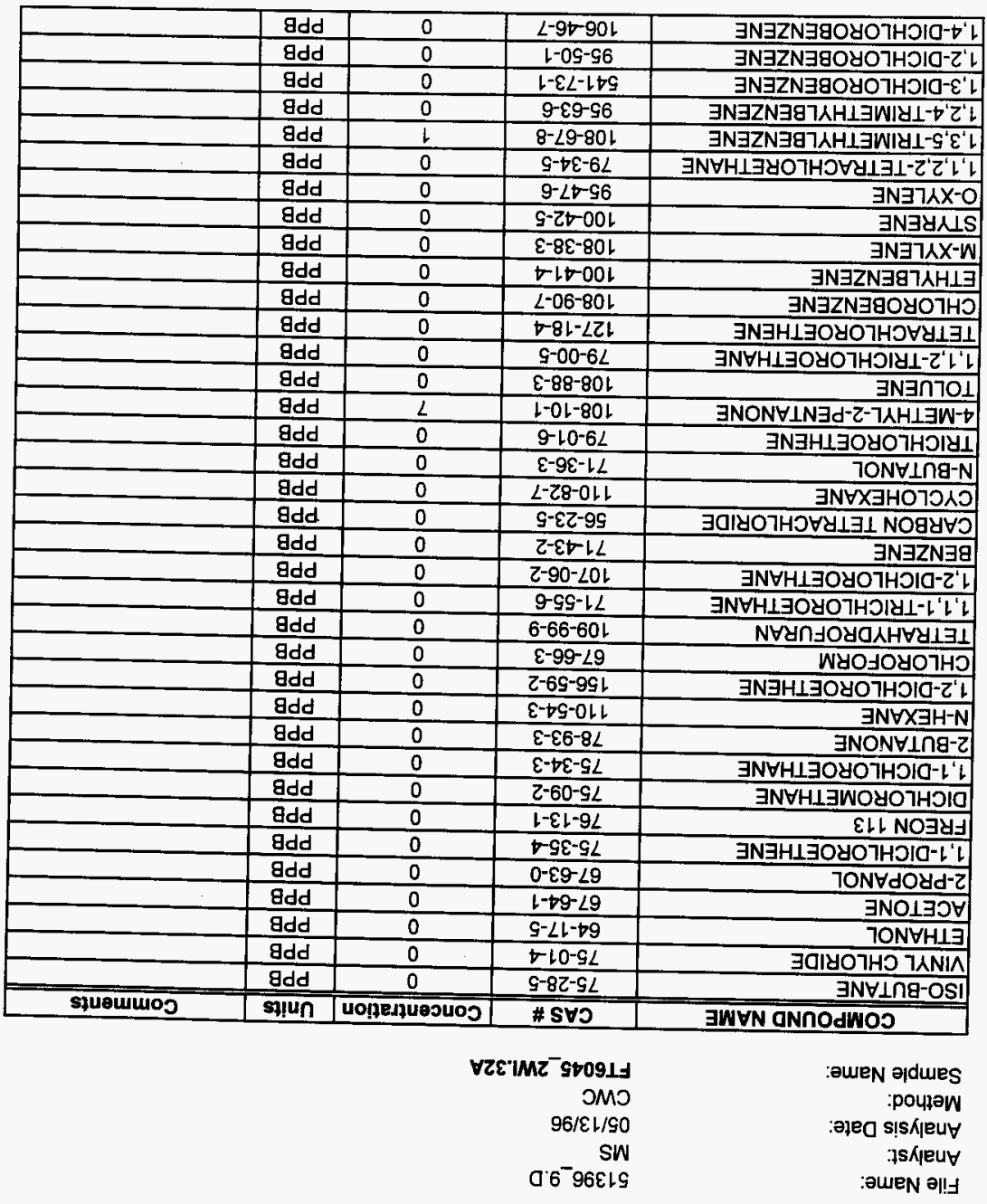




\section{LIST OF COMPOUNDS TENTATIVELY IDENTIFIED WITH MASS SPECTRAL LIBRARY}

Sample: $\quad 2402$ WI

Sample Name: FT6045_2WI.32A

Search Libraries: C:DATABASEIWILEY138.L Minimum Quality: 70

Unknown Spectrum: Apex

Integration Params: VOA.E

Pk\# RT Area\% Library/mD Ref\# CAS\# Qual

621.72 5.40 C:IDATABASEIWILEY138.L 1-Butanol

$116551000071-36-372$

824.4427 .86 C:DATABASEIWILEY138.L

2-Pentanone, 4-methyl-

$118019000108-10-190$

1029.112 .34 C:IDATABASEIWILEY138.L Benzene, 1,3-dimethyl-

$118578000108-38-392$

1129.941 .97 C:DATABASEIWLEY138.L Benzene, 1,2-dimethyl$118571000095-47-691$

1432.07 5.15 C:IDATABASEIWILEY138.L Benzaldehyde

$118546000100-52-796$

1533.09 1.68 C:DATABASEIWLEY138.L Benzene, 1,3,5-trimethyl-

$120037000108-67-890$ 
File

Operator

Acquired

Instrument

FTle Name: FT645-2WI.33A

Misc Info : DELTA P $=100$ torr

Vial Number: 1

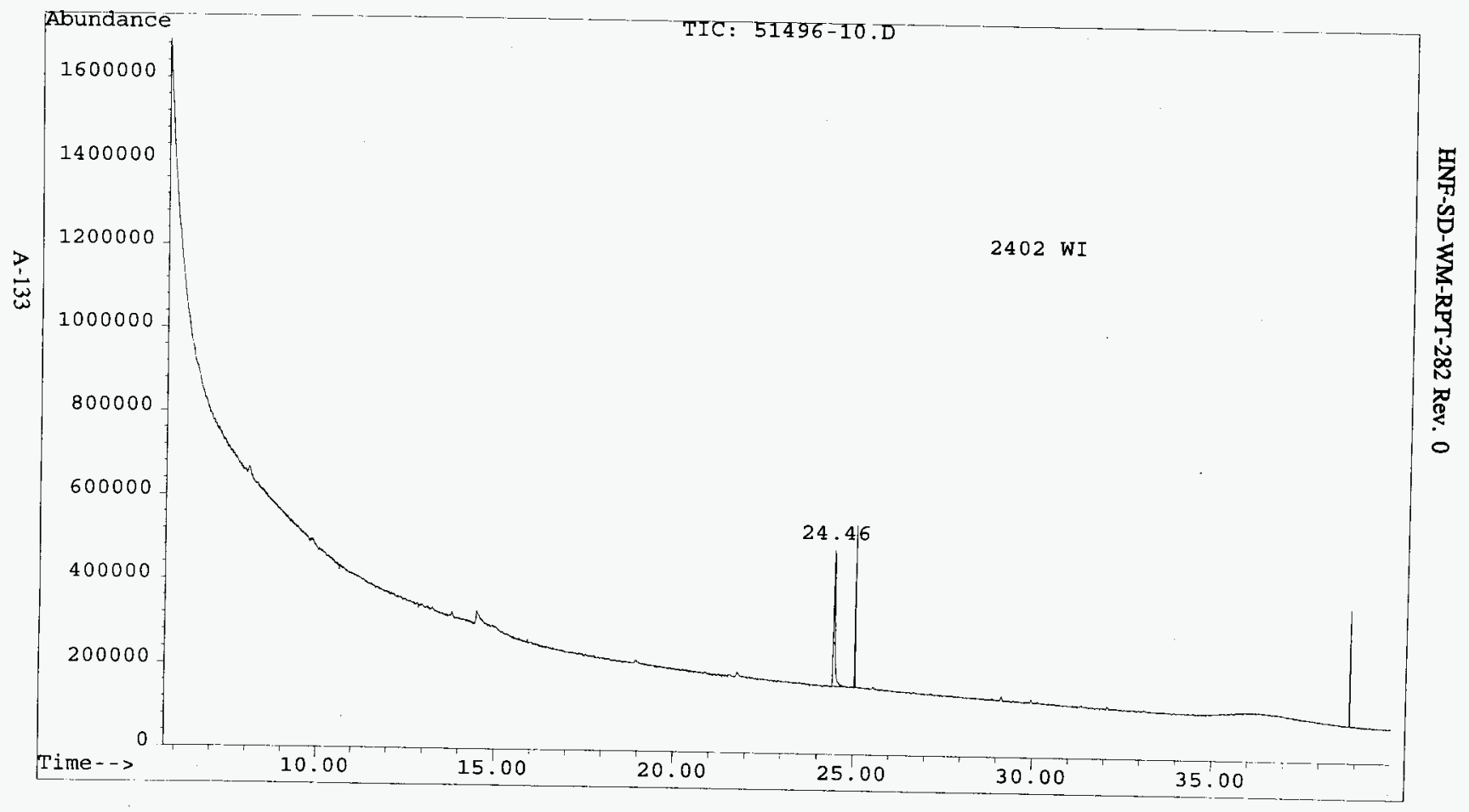


HNF-SD-WM-RPT-282 Rev. 0

CWC_REP.XLS

\section{ANALYSIS REPORT FOR CWC AIR SAMPLES}

File Name:

Analyst:

Analysis Date:

Method:

Sample Name:
51496-10.D

MS

05/14/96

CWC

FT6045-2WI.33A

\begin{tabular}{|c|c|c|c|c|}
\hline COMPOUND NAME & CAS \# & Concentration & Units & Comments \\
\hline ISO-BUTANE & $75-28-5$ & 0 & PPB & \\
\hline VINYL CHLORIDE & $75-01-4$ & 0 & PPB & \\
\hline ETHANOL & $64-17-5$ & 0 & PPB & \\
\hline ACETONE & $67-64-1$ & 0 & PPB & \\
\hline 2-PROPANOL & $67-63-0$ & 0 & PPB & \\
\hline 1,1-DICHLOROETHENE & $75-35-4$ & 0 & PPB & \\
\hline FREON 113 & $76-13-1$ & 0 & PPB & \\
\hline DICHLOROMETHANE & $75-\overline{09-2}$ & 0 & PPB & + \\
\hline 1,1-DICHLOROETHANE & $75-34-3$ & 0 & PPB & \\
\hline 2-BUTANONE & $78-93-3$ & 0 & PPB & \\
\hline N-HEXANE & $110-54-3$ & 0 & PPB & \\
\hline 1,2-DICHLOROETHENE & $156-59-2$ & 0 & PPB & \\
\hline CHLOROFORM & $67-66-3$ & 0 & PPB & \\
\hline TETRAHYDROFURAN & $109-99-9$ & 0 & PPB & \\
\hline 1,1,1-TRICHLOROETHANE & $71-55-6$ & 0 & PPB & \\
\hline 1,2-DICHLOROETHANE & $107-06-2$ & 0 & PPB & \\
\hline BENZENE & $71-43-2$ & 0 & $\overline{\mathrm{PPB}}$ & \\
\hline CARBON TETRACHLORIDE & $56-23-5$ & 0 & PPB & \\
\hline CYCLOHEXANE & $110-82-7$ & 0 & PPB & \\
\hline N-BUTANOL & $71-36-3$ & 0 & PPB & \\
\hline TRICHLOROETHENE & $79-01-6$ & 0 & PPB & \\
\hline 4-METHYL-2-PENTANONE & $108-10-1$ & 7 & PPB & \\
\hline TOLUENE & $108-88-3$ & 0 & PPB & \\
\hline 1,1,2-TRICHLOROETHANE & $79-00-5$ & 0 & PPB & \\
\hline TETRACHLOROETHENE & $127-18-4$ & 0 & PPB & \\
\hline CHLOROBENZENE & $108-90-7$ & 0 & PPB & \\
\hline ETHYLBENZENE & $100-41-4$ & 0 & PPB & \\
\hline M-XYLENE & $108-38-3$ & 0 & PPB & \\
\hline STYRENE & $100-42-5$ & 0 & PPB & \\
\hline O-XYLENE & $95-47-6$ & 0 & PPB & \\
\hline 1,1,2,2-TETRACHLORETHANE & $79-34-5$ & 0 & PPB & \\
\hline 1,3,5-TRIMETHYLBENZENE & $108-67-8$ & 0 & PPB & \\
\hline 1,2,4-TRIMETHYLBENZENE & $95-63-6$ & 0 & PPB & \\
\hline 1,3-DICHLOROBENZENE & $541-73-1$ & 0 & PPB & \\
\hline 1,2-DICHLOROBENZENE & $95-50-1$ & 0 & PPB & \\
\hline 1,4-DICHLOROBENZENE & $106-46-7$ & 0 & PPB & \\
\hline
\end{tabular}


HNF-SD-WM-RPT-282 Rev. 0

LIST OF COMPOUNDS TENTATIVELY IDENTIFIED WITH MASS SPECTRAL LIBRARY

Sample: $\quad 2402$ WI

Sample Name: FT6045-2WI.33A

Search Libraries: C:DATABASEIWLEY138.L Minimum Quality: 70

Unknown Spectrum: Apex

Integration Prams: VOA.E

Pk\# RT Area\% Library/ID Ref\# CAS\# Qua

224.4555 .72 C:DATABASEIWILEY138.L

2-Pentanone, 4-methyl-

$118019000108-10-191$

A-135 
File

Operator

Acquired

Inst rument

Sample Name: FT6045-2WJ

Vial Number: 1

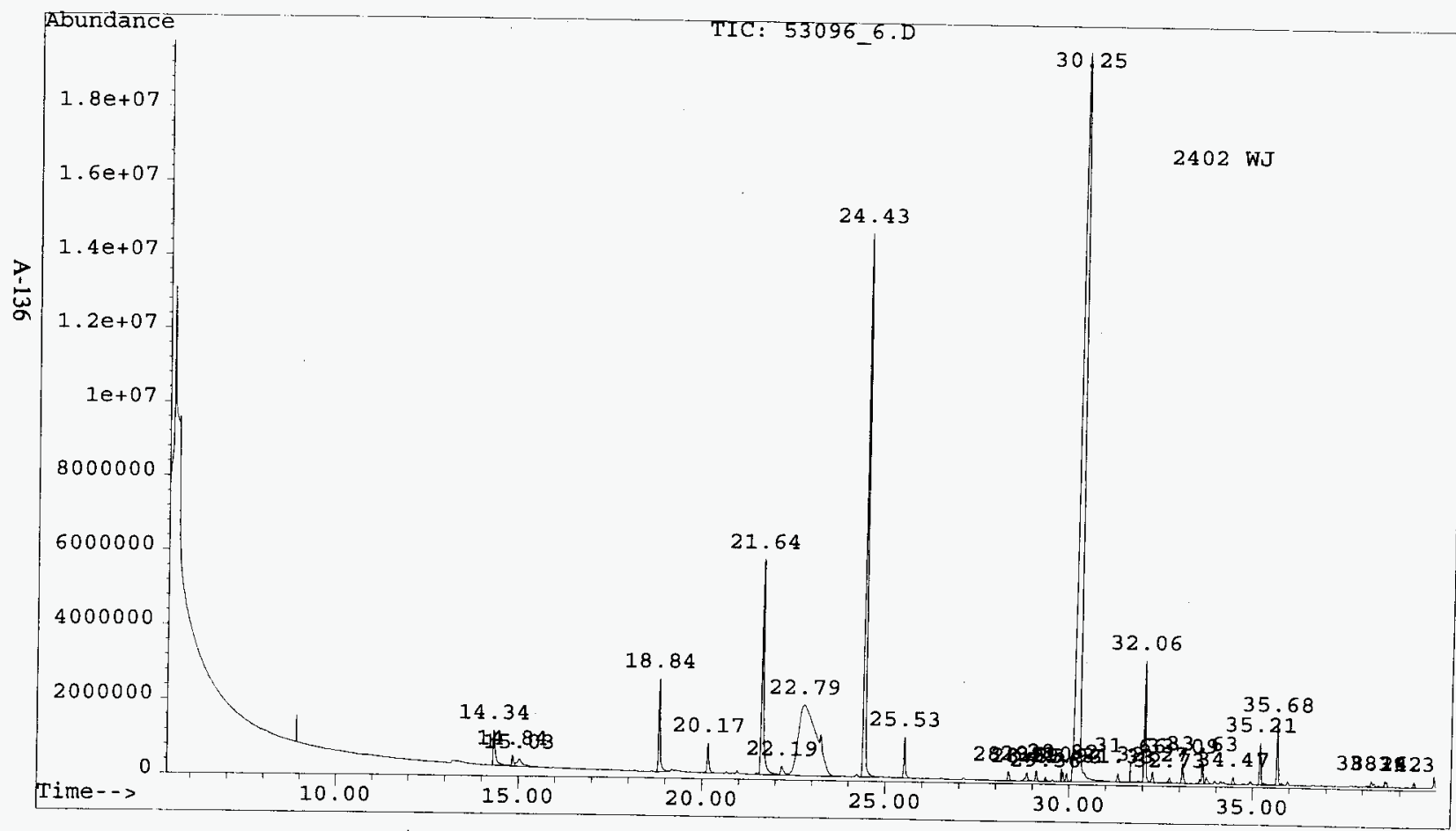


HNF-SD-WM-RPT-282 Rev. 0

CWC_REP.XLS

\section{ANALYSIS REPORT FOR CWC AIR SAMPLES}

File Name:

Analyst:

Analysis Date:

Method:

Sample Name:
53096_6.D

LAP

05/30/96

CWC

FT6045-2WJ.52A

\begin{tabular}{|c|c|c|c|c|}
\hline COMPOUND NAME & CAS \# & Concentration & Units & Comments \\
\hline ISO-BUTANE & $75-28-5$ & 0 & PPB & \\
\hline VINYL CHLORIDE & $75-01-4$ & 0 & PPB & \\
\hline ETHANOL & $64-17-5$ & 6 & PPB & \\
\hline ACETONE & $67-64-1$ & 39 & PPB & \\
\hline 2-PROPANOL & $67-63-0$ & 22 & PPB & \\
\hline 1,1-DICHLOROETHENE & $75-35-4$ & 0 & PPB & \\
\hline FREON 113 & $76-13-1$ & 0 & PPB & \\
\hline DICHLOROMETHANE & $75-09-2$ & 0 & PPB & \\
\hline 1,1-DICHLOROETHANE & $75-34-3$ & 0 & $\overline{\mathrm{PPB}}$ & \\
\hline 2-BUTANONE & 78-93-3 & 245 & $\mathrm{PPB}$ & \\
\hline N-HEXANE & $110-54-3$ & 14 & PPB & \\
\hline 1,2-DICHLOROETHENE & $156-59-2$ & 0 & PPB & \\
\hline CHLOROFORM & $67-66-3$ & 0 & PPB & \\
\hline TETRAHYDROFURAN & $109-99-9$ & 24 & PPB & \\
\hline 1,1,1-TRICHLOROETHANE & $71-55-6$ & 0 & PPB & \\
\hline 1,2-DICHLOROETHANE & 107-06-2 & 6 & PPB & \\
\hline BENZENE & $71-43-2$ & 3 & PPB & \\
\hline CARBON TETRACHLORIDE & $56-23-5$ & 0 & PPB & \\
\hline CYCLOHEXANE & $110-82-7$ & 0 & PPB & \\
\hline N-BUTANOL & $71-36-3$ & 336 & PPB & \\
\hline TRICHLOROETHENE & $79-01-6$ & 0 & PPB & \\
\hline 4-METHYL-2-PENTANONE & $108-10-1$ & 345 & PPB & \\
\hline TOLUENE & $108-88-3$ & 37 & PPB & \\
\hline 1,1,2-TRICHLOROETHANE & $79-00-5$ & 0 & PPB & \\
\hline TETRACHLOROETHENE & $127-18-4$ & 0 & PPB & \\
\hline CHLOROBENZENE & $108-90-7$ & 2 & $\overline{\mathrm{PPB}}$ & \\
\hline ETHYLBENZENE & $100-41-4$ & 5 & PPB & \\
\hline M-XYLENE & $108-38-3$ & 8 & PPB & \\
\hline STYRENE & $100-42-5$ & 7 & PPB & \\
\hline O-XYLENE & $95-47-6$ & 6 & PPB & \\
\hline 1,1,2,2-TETRACHLORETHANE & $79-34-5$ & 3 & PPB & \\
\hline 1,3,5-TRIMETHYLBENZENE & $108-67-8$ & 5 & PPB & \\
\hline 1,2,4-TRIMETHYLBENZENE & $95-63-6$ & 14 & PPB & \\
\hline 1,3-DICHLOROBENZENE & $54 \uparrow-73-1$ & 4 & PPB & \\
\hline 1,2-DICHLOROBENZENE & $95-50-1$ & 6 & PPB & \\
\hline 1,4-DICHLOROBENZENE & $106-46-7$ & 6 & PPB & \\
\hline
\end{tabular}




\section{LIST OF COMPOUNDS TENTATIVELY IDENTIFIED WITH MASS SPECTRAL LIBRARY}

$\begin{array}{ll}\text { Sample: } & \text { 2402 WJ } \\ \text { File: } & \text { C:LHPCHEMI2LATA153096_6.D } \\ \text { ID: } & \text { FT6045-2WJ.52A }\end{array}$

Compound

CAS\#

2-Butanone

$000078-93-3$

Furan, tetrahydro-

000109-99-9

Ethane, 1,2-dichloro

000107-06-2

l-Butanol

000071-36-3

2-Propanol, 1-methoxy-

000107-98-2

2-Pentanone, 4-methyl-

000108-10-1

Benzene, methyl-

000108-88-3

Benzene, ethyl-

$000100-41-4$

Benzene, 1,3-dimethyl-

000108-38-3

Styrene

$000100-42-5$

XYLENE

001330-20-7

Ethanol, 2-butoxy-

000111-76-2

ALPHA -PINENE, (-)-

000080-56-8

Benzaldehyde

000100-52-7

Benzene, 1,2,4-trimethyl-

000095-63-6

2-BETA.-PINENE

000127-91-3

Benzene, 1,3,5-trimethyl-

000108-67-8 
HNF-SD-WM-RPT-282 Rev. 0

\section{LIST OF COMPOUNDS TENTATIVELY IDENTIFIED WITH MASS SPECTRAL LIBRARY}

$\begin{array}{ll}\text { Sample: } & \text { 2402 WJ (CONTINUED) } \\ \text { File: } & \text { C: } 1 \text { HPCHEM2LATA } 53096 \_6 . D \\ \text { ID: } & \text { FT6045-2WJ.52A }\end{array}$

Compound

CAS\#

DELTA.3-Carene

$013466-78-9$

Benzene, 1,3-dichloro-

$000541-73-1$

Benzene, 1-methyl-4-(1-methylethyl)-

$000099-87-6$

Cyclohexene, 1-methyl-4-(1-methylethe

005989-27-5

Benzene, 1,2-dichloro-

0095-50-1

Ethanone, 1-phenyl-

0098-86-2

Benzenemethanol, .alpha.,,alpha.-dime

000617-94-7

2,3-DIMETHYLBENZALDEHYDE

005779-93-1

Benzaldehyde, 3,4-dimethyl-

005973-71-7 
File

Operator

Acquired

Instrument

Sample Name:

Misc Info

Vial Number: 1

C: $\backslash$ HPCHEM $\backslash 2 \backslash D A T A \backslash 53096 \_7 . D$

LAP

: 30 May $96 \quad 9: 37$ am using AcqMethod CWC

5972 - In

FT6045-2WJ. 54A

DELTA $\mathrm{P}=100$ torr

1

\section{Abundañè}

?

A000000

7000000

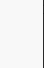

$\overrightarrow{1}$
$\stackrel{1}{0}$

5000000

4

4000000

.

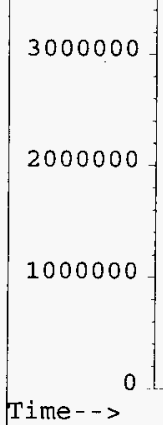

Time-- >

0

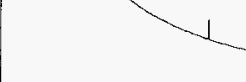

TIC: $53096 \_7 . D$

24.41

2402 WJ BACKGROUND

201. 4604

$18.84 \quad 22.85$

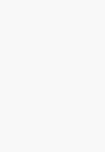

|

30.16

32.06

33.0935 .21 
HNF-SD-WM-RPT-282 Rev. 0

CWC_REP.XLS

\section{ANALYSIS REPORT FOR CWC AIR SAMPLES}

File Name:

Analyst:

Analysis Date:

Method:

Sample Name:
53096_7.D

LAP

05/30/96

CWC

FT6045-2WJ.54A

\begin{tabular}{|c|c|c|c|c|}
\hline COMPOUND NAME & CAS\# & Concentration & Units & Comments \\
\hline ISO-BUTANE & $75-28-5$ & 0 & PPB & \\
\hline VINYL CHLORIDE & $75-01-4$ & 0 & PPB & \\
\hline ETHANOL & $64-17-5$ & 0 & PPB & \\
\hline ACETONE & $67-64-1$ & 5 & PPB & \\
\hline 2-PROPANOL & $67-63-0$ & 2 & PPB & \\
\hline 1,1-DICHLOROETHENE & $75-35-4$ & 0 & PPB & \\
\hline FREON 113 & $76-13-1$ & 0 & PPB & \\
\hline DICHLOROMETHANE & $75-09-2$ & 0 & $\overline{\mathrm{PPB}}$ & \\
\hline 1,1-DICHLOROETHANE & $75-34-3$ & 0 & $\overline{\text { PPB }}$ & \\
\hline 2-BUTANONE & $78-93-3$ & 85 & PPB & \\
\hline N-HEXANE & $110-54-3$ & 4 & PPB & \\
\hline 1,2-DICHLOROETHENE & $156-59-2$ & 0 & PPB & \\
\hline CHLOROFORM & $67-66-3$ & 0 & PPB & \\
\hline TETRAHYDROFURAN & $109-99-9$ & 6 & PPB & \\
\hline 1,1,1-TRICHLOROETHANE & $71-55-6$ & 0 & PPB & \\
\hline 1,2-DICHLOROETHANE & $107-06-2$ & 0 & PPB & \\
\hline BENZENE & $71-43-2$ & 0 & PPB & \\
\hline CARBON TETRACHLORIDE & $56-23-5$ & 0 & PPB & \\
\hline CYCLOHEXANE & $110-82-7$ & 0 & $\overline{\mathrm{PPB}}$ & \\
\hline N-BUTTANOL & $71-36-3$ & 100 & PPB & \\
\hline TRICHLOROETHENE & $79-01-6$ & 0 & $\overline{P P B}$ & \\
\hline 4-METHYL-2-PENTANONE & $108-10-1$ & 142 & PPB & \\
\hline TOLUENE & $108-88-3$ & 0 & PPB & \\
\hline 1,1,2-TRICHLOROETHANE & $79-00-5$ & 0 & PPB & \\
\hline TETRACHLOROETHENE & $127-18-4$ & 0 & PPB & \\
\hline CHLOROBENZENE & $108-90-7$ & 0 & PPB & \\
\hline ETHYLBENZENE & $100-41-4$ & 2 & PPB & \\
\hline M-XYLENE & $108-38-3$ & 0 & PPB & \\
\hline STYRENE & $100-42-5$ & 3 & PPB & \\
\hline O-XYLENE & $95-47-6$ & 2 & PPB & \\
\hline $1,1,2,2$-TETRACHLORETHANE & $79-34-5$ & 0 & PPB & \\
\hline 1,3,5-TRIMETHYLBENZENE & $108-67-8$ & 2 & PPB & \\
\hline 1,2,4-TRIMETHYLBENZENE & $95-63-6$ & 7 & PPB & \\
\hline 1,3-DICHLOROBENZENE & $541-73-1$ & 2 & PPB & \\
\hline 1,2-DICHLOROBENZENE & $95-50-1$ & 2 & PPB & \\
\hline 1,4-DICHLOROBENZENE & $106-46-7$ & 3 & PPB & \\
\hline
\end{tabular}


HNF-SD-WM-RPT-282 Rev. 0

\section{LIST OF COMPOUNDS TENTATIVELY IDENTIFIED WITH MASS SPECTRAL LIBRARY}

$\begin{array}{ll}\text { Sample: } & \text { 2402 WJ BACKGROUND } \\ \text { File: } & \text { C:IHPCHEM2LDATA153096_7.D } \\ \text { D: } & \text { FT6045-2WJ.54A }\end{array}$

Compound

CAS\#

1-Butanol

$000071-36-3$

2-Propanol, 1-methoxy-

000107-98-2

2-Pentanone, 4-methyl-

000108-10-1

Benzene, ethyl-

$000100-41-4$

Benzene, 1,4-dimethyl-

$000106-42-3$

Styrene

$000100-42-5$

Benzene, 1,2-dimethyl-

000095-47-6

Ethanol, 2-butoxy-

$000111-76-2$

Benzaldehyde

000100-52-7

Benzene, 1,2,3-trimethyl-

000526-73-8

Benzene, 1,2,3-trimethyl-

000526-73-8

Benzene, 1,4-dichloro-

000106-46-7

Benzene, 1,4-dichloro-

000106-46-7

Benzene, 1,4-dichloro-

000106-46-7

Ethanone, 1-phenyl-

000098-86-2 
File

Operator

Acquired

Instrument :

Sample Name: FT6045-2WJ 53A

Misc Info : DELTA $P=100$ torr

Vial Number: 1

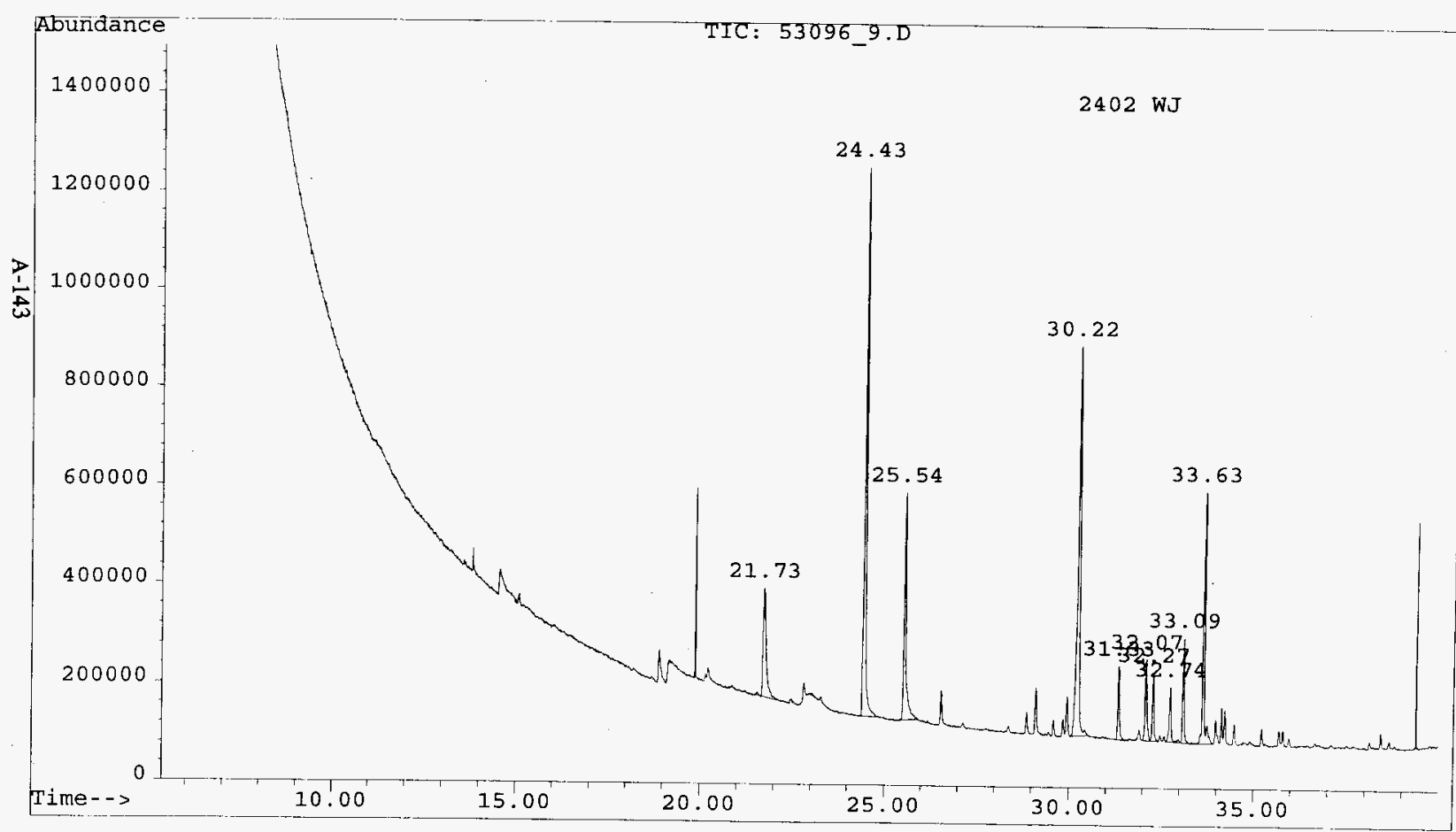


HNF-SD-WM-RPT-282 Rev. 0

CWC_REP.XLS

\section{ANALYSIS REPORT FOR CWC AIR SAMPLES}

File Name:

Analyst

Analysis Date:

Method:

Sample Name:
53096_9.D

LAP

05/30/96

CWC

FT6045-2WJ.53A

\begin{tabular}{|c|c|c|c|c|}
\hline COMPOUND NAME & CAS \# & Concentration & Units & Comments \\
\hline ISO-BUTANE & $75-28-5$ & 0 & $\overline{\mathrm{PPB}}$ & \\
\hline VINYL CHLORIDE & $75-01-4$ & 0 & PPB & \\
\hline ETHANOL & 64-17-5 & 0 & PPB & \\
\hline ACETONE & $67-64-1$ & 0 & PPB & \\
\hline 2-PROPANOL & $67-63-0$ & 0 & PPB & \\
\hline 1,1-DICHLOROETHENE & $75-35-4$ & 0 & PPB & \\
\hline FREON 113 & 76-13-1 & 0 & PPB & \\
\hline DICHLOROMETHANE & $75-09-2$ & 0 & PPB & \\
\hline 1,1-DICHLOROETHANE & $75-34-3$ & 0 & PPB & \\
\hline 2-BUTANONE & $78-93-3$ & 0 & PPB & \\
\hline N-HEXANE & $110-54-3$ & 0 & PPB & \\
\hline 1,2-DICHLOROETHENE & $156-59-2$ & 0 & PPB & \\
\hline CHLOROFORM & $67-66-3$ & 0 & PPB & \\
\hline TETRAHYDROFURAN & $109-99-9$ & 0 & PPB & \\
\hline 1,1,1-TRICHLOROETHANE & $71-55-6$ & 0 & PPB & \\
\hline 1,2-DICHLOROETHANE & $107-06-2$ & 0 & PPB & \\
\hline BENZENE & $71-43-2$ & 0 & PPB & \\
\hline CARBON TETRACHLORIDE & $56-23-5$ & 0 & PPB & \\
\hline CYCLOHEXANE & $110-82-7$ & 0 & PPB & \\
\hline N-BUTANOL & $71-36-3$ & 18 & PPB & \\
\hline TRICHLOROETHENE & $79-01-6$ & 0 & PPB & \\
\hline 4-METHYL-2-PENTANONE & $108-10-1$ & 25 & PPB & \\
\hline TOLUENE & $108-88-3$ & 22 & PPB & \\
\hline 1,1,2-TRICHLOROETHANE & $79-00-5$ & 0 & PPB & \\
\hline TETRACHLOROETHENE & $127-18-4$ & 0 & PPB & \\
\hline CHLOROBENZENE & $108-90-7$ & 0 & PPB & \\
\hline ETHYLBENZENE & $100-41-4$ & 1 & PPB & \\
\hline M-XYLENE & $108-38-3$ & 3 & PPB & \\
\hline STYRENE & $100-42-5$ & 0 & PPB & \\
\hline O-XYLENE & $95-47-6$ & 2 & PPB & \\
\hline 1,1,2,2-TETRACHLORETHANE & $79-34-5$ & 0 & PPB & \\
\hline 1,3,5-TRIMETHYLBENZENE & $108-67-8$ & 2 & PPB & \\
\hline $1,2,4$-TRIMETHYLBENZENE & $95-63-6$ & 5 & PPB & \\
\hline 1,3-DICHLOROBENZENE & $541-73-1$ & 0 & PPB & \\
\hline 1,2-DICHLOROBENZENE & $95-50-1$ & 0 & PPB & \\
\hline 1,4-DICHLOROBENZENE & $106-46-7$ & 0 & PPB & \\
\hline
\end{tabular}


HNF-SD-WM-RPT-282 Rev. 0

LIST OF COMPOUNDS TENTATIVELY IDENTIFIED WITH MASS SPECTRAL LIBRARY

Sample: $\quad 2402 \mathrm{WJ}$

File: C:LHPCHEML2LDATA153096_9.D

ID: $\quad$ FT6045-2WJ.53A

Compound

CAS\#

2-Pentanone, 4-methyl-

000108-10-1

Benzene, methyl-

$000108-88-3$

Benzene, 1,3-dimethyl-

$000108-38-3$

alpha.-Pinene

$000080-56-8$

Benzaldehyde

000100-52-7

Benzene, 1,2,3-trimethyl-

$000526-73-8$

DELTA.3-Carene

013466-78-9

Peroxide, bis(dichlorobenzoyl)

028604-90-2 
File

: D : \CWC \SAMPLES $\backslash 51496-7 . D$

Operator

lap

Acquired

14 May $96 \quad 10: 30$ am using AcqMethod CWC

Instrument : 5972 - In

Sample Name: FT6045-2WK.37A

Misc Info : DELTA P $=100$ torr

Vial Number: 1

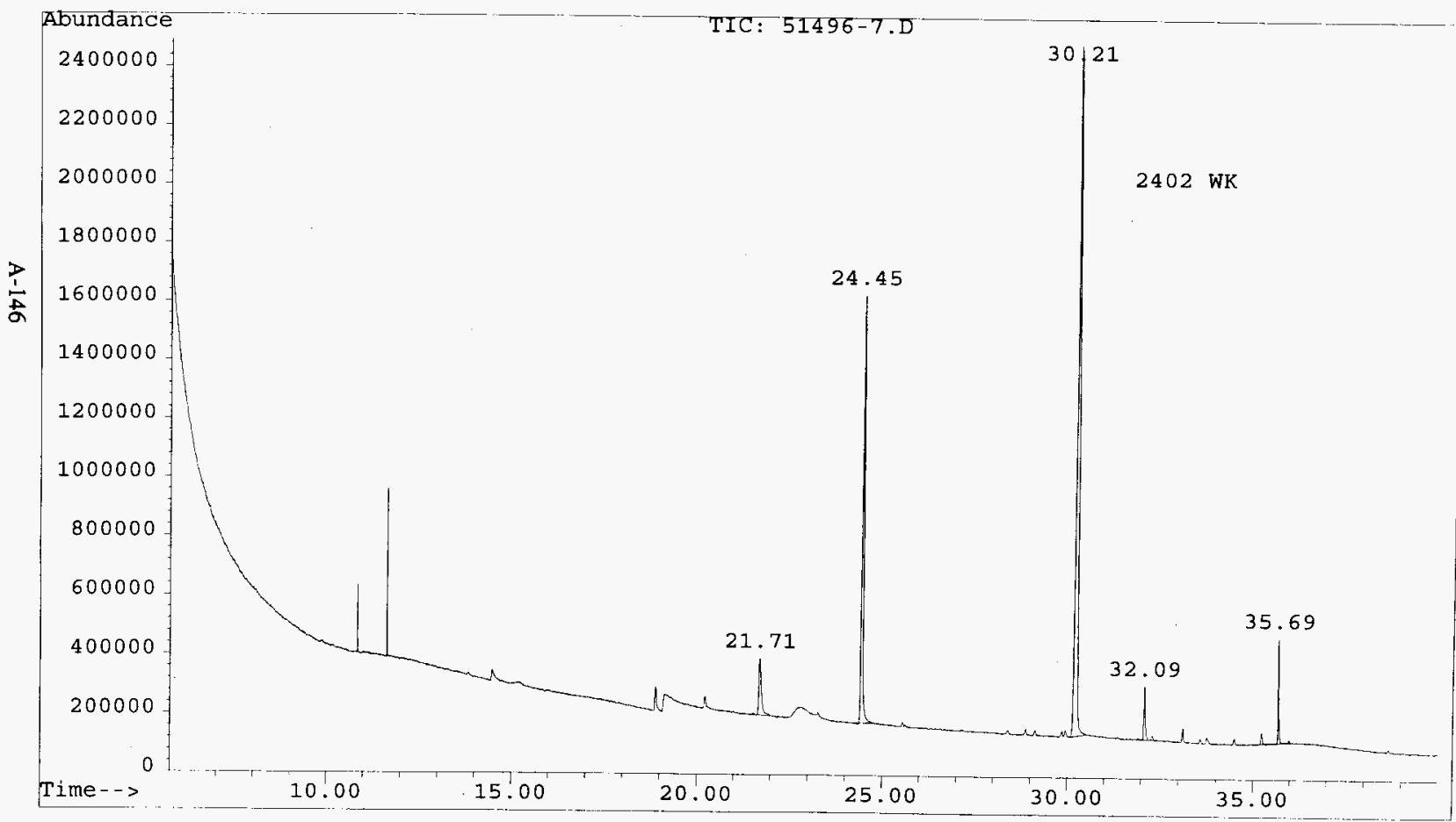


HNF-SD-WM-RPT-282 Rev. 0

CWC_REP.XLS

\section{ANALYSIS REPORT FOR CWC AIR SAMPLES}

File Name:

Analyst:

Analysis Date:

Method:

Sample Name:
51496-7.D

lap

05/14/96

CWC

FT6045-2WK.37A

\begin{tabular}{|c|c|c|c|c|}
\hline COMPOUND NAME & CAS \# & Concentration & Units & Comments \\
\hline ISO-BUTANE & $75-28-5$ & $\overline{0}$ & PPB & \\
\hline VINYL CHLORIDE & $75-01-4$ & 0 & PPB & \\
\hline ETHANOL & $64-17-5$ & $\overline{0}$ & PPB & \\
\hline ACETONE & $67-64-1$ & 0 & PPB & \\
\hline 2-PROPANOL & $67-63-0$ & 0 & $\overline{\mathrm{PPB}}$ & \\
\hline 1,1-DICHLOROETHENE & $75-35-4$ & 0 & PPB & \\
\hline FREON 113 & $76-1 \overline{3-1}$ & $\overline{0}$ & PPB & \\
\hline DICHLOROMETHANE & $75-09-2$ & 0 & $\overline{\mathrm{PPB}}$ & \\
\hline 1,1-DICHLOROETHANE & $75-34-3$ & 0 & PPB & \\
\hline 2-BUTANONE & $78-93-3$ & 0 & PPB & \\
\hline N-HEXANE & $110-54-3$ & $\overline{0}$ & PPB & \\
\hline 1,2-DICHLOROETHENE & $156-59-2$ & $\overline{0}$ & PPB & \\
\hline CHLOROFORM & $67-66-3$ & 0 & PPB & \\
\hline TETRAHYDROFURAN & $109-99-9$ & 0 & PPB & \\
\hline 1,1,1-TRICHLOROETHANE & $71-55-6$ & $\overline{0}$ & PPB & \\
\hline 1,2-DICHLOROETHANE & $107-06-2$ & D & PPB & \\
\hline BENZENE & $71-43-2$ & 0 & PPB & \\
\hline CARBON TETRACHLORIDE & $56-23-5$ & 0 & PPB & \\
\hline CYCLOHEXANE & $110-82-7$ & 0 & PPB & \\
\hline N-BUTANOL & $71-36-3$ & 13 & PPB & \\
\hline TRICHLOROETHENE & $79-01-6$ & 0 & PPB & \\
\hline 4-METHYL-2-PENTANONE & $108-10-1$ & 32 & PPB & \\
\hline TOLUENE & $108-88-3$ & 0 & $\mathrm{PPB}$ & \\
\hline 1,1,2-TRICHLOROETHANE & $79-00-5$ & 0 & PPB & \\
\hline TETRACHLOROETHENE & $127-18-4$ & 0 & PPB & \\
\hline CHLOROBENZENE & $108-90-7$ & 0 & PPB & \\
\hline ETHYLBENZENE & $100-41-4$ & 0 & PPB & \\
\hline M-XYLENE & $108-38-3$ & 0 & PPB & \\
\hline STYRENE & $100-42-5$ & 0 & PPB & \\
\hline O-XYLENE & $95-47-6$ & 0 & PPB & \\
\hline 1,1,2,2-TETRACHLORETHANE & $79-34-5$ & 0 & PPB & \\
\hline 1,3,5-TRIMETHYLBENZENE & $108-67-8$ & 3 & PPB & \\
\hline 1,2,4-TRIMETHYLBENZENE & $95-63-6$ & 1 & PPB & \\
\hline 1,3-DICHLOROBENZENE & $541-73-1$ & 0 & PPB & \\
\hline 1,2-DICHLOROBENZENE & $95-50-1$ & 0 & PPB & \\
\hline 1,4-DICHLOROBENZENE & $106-46-7$ & 0 & PPB & \\
\hline
\end{tabular}




\section{LIST OF COMPOUNDS TENTATIVELY IDENTIFIED WITH MASS SPECTRAL LIBRARY}

Sample: $\quad 2402$ WK

Sample Name: FT6045-2WK.37A

Search Libraries: C:LATABASEIWLEY138.L Minimum Quality: 70

Unknown Spectrum: Apex

Integration Params: VOA.E

Pk\# RT Area\% Library/ID $\quad$ Ref\# CAS\# $\quad$ Qual

721.704 .84 C:LATABASEIWILEY138.L 1-Butanol

$116554000071-36-390$

$1024.4520 .11 \mathrm{C}:$ DATABASEIWILEY138.L 2-Pentanone, 4-methyl-

$118019000108-10-191$

1228.890 .32 C:DATABASEIWILEY138.L Benzene, 1,2-dimethyl-

Benzene, ethyl-

$118571000095-47-683$

$118561000100-41-480$

1329.140 .30 C:IDATABASEIWILEY138.L

Benzene, 1,2-dimethyl-

XYLENE

Benzene, 1,3-dimethyl-

$118571000095-47-691$

$118580001330-20-790$

$118578000108-38-387$

1429.860 .26 C: $\backslash$ DATABASEIWILEY138.L

Styrene

1,3,5,7-Cyclooctatetraene

$118481000100-42-592$

118487 000629-20-9 91

$1529.960 .34 \mathrm{C}:$ :DATABASEIWILEY138.L

Benzene, 1,2-dimethyl-

$118571000095-47-691$

$1630.2146 .06 \mathrm{C}:$ DATABASEIWILEY138.L Ethanol, 2-butoxy-

$119786000111-76-290$

$1732.082 .44 \mathrm{C}:$ :DATABASEIWILEY138.L

Benzaldehyde

$118545000100-52-797$ 
Sample: $\quad 2402$ WK (continued)

Pk\# RT Area\% $\quad$ Library/ID Ref\# CAS\# Qual

$1832.290 .24 \mathrm{C}:$ :DATABASEIWILEY138.L

Benzene, 1,3,5-trimethyl- $\quad 120037000108-67-886$

$1933.110 .58 \mathrm{C}:$ WATABASEIWILEY138.L

Benzene, 1,2,3-trimethyl- $\quad 4145$ 000526-73-8 95

Benzene, 1,3,5-trimethyl- $\quad 4147$ 000108-67-8 95

2033.760 .39 C:LDATABASE\WILEY I38.L

Benzene, 1,2-dichloro- $\quad 11151000095-50-197$

Benzene, 1,4-dichloro- $\quad 11154$ 000106-46-7 96

2134.490 .28 C:IDATABASEIWILEY138.L

Benzene, 1,4-dichloro-

$123085000106-46-796$

Benzene, 1,3-dichloro-

$123079000541-73-196$

$2235.23 \quad 0.69$ C:IDATABASEIWILEY138.L Ethanone, 1-phenyl-

119988 000098-86-2 95 
HNF-SD-WM-RPT-282 Rev. 0

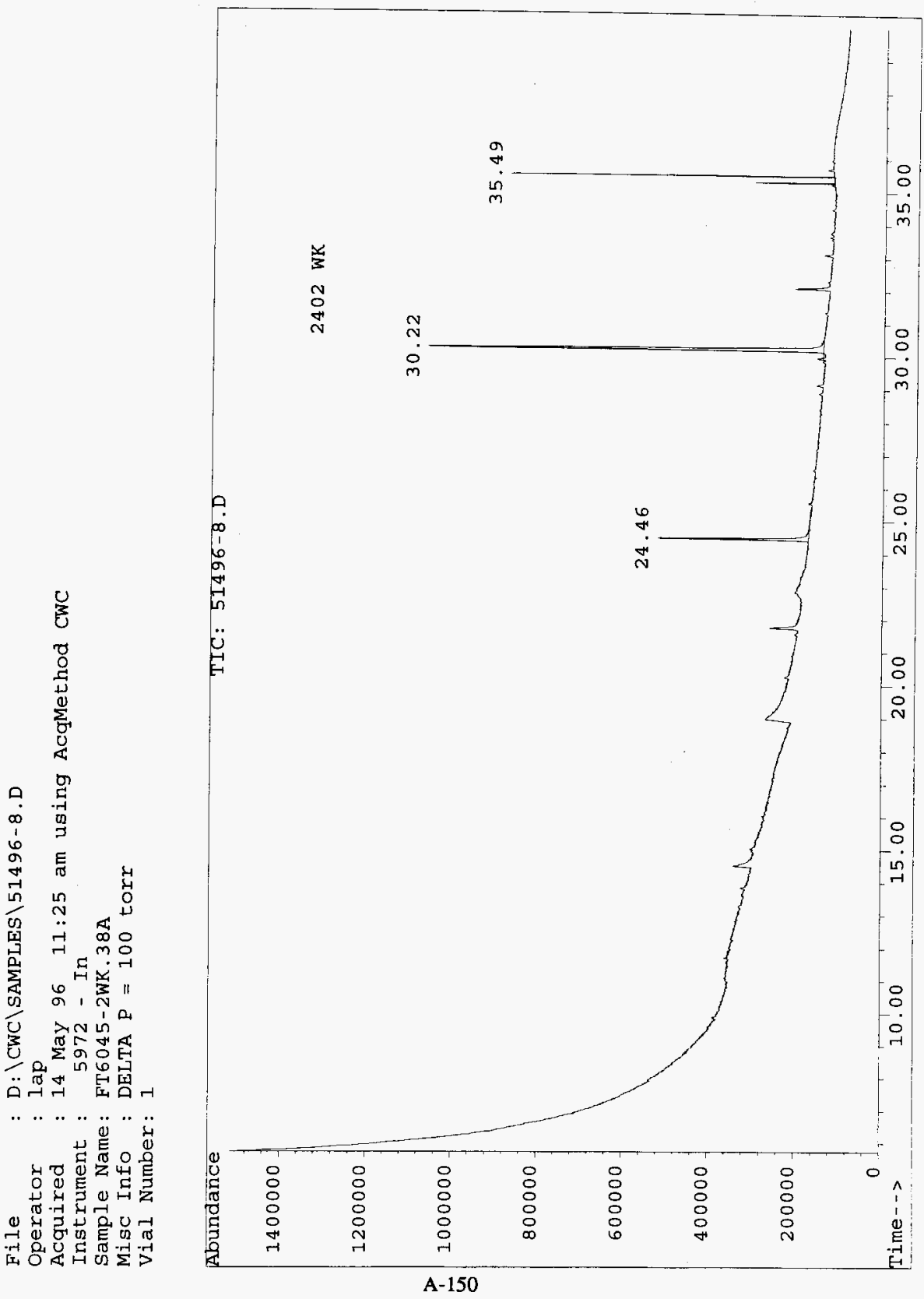


HNF-SD-WM-RPT-282 Rev. 0

CWC_REP.XLS

\section{ANALYSIS REPORT FOR CWC AIR SAMPLES}

File Name:

Analyst:

Analysis Date:

Method:

Sample Name:
51496-8.D

lap

05/14/96

CWC

FT6045-2WK.38A

\begin{tabular}{|c|c|c|c|c|}
\hline COMPOUND NAME & CAS \# & Concentration & Units & Comments \\
\hline ISO-BUTANE & $75-28-5$ & 0 & PPB & \\
\hline VINYL CHLORIDE & $75-01-4$ & 0 & PPB & \\
\hline ETHANOL & $64-17-5$ & 0 & PPB & \\
\hline ACETONE & $67-64-1$ & 0 & PPB & \\
\hline 2-PROPANOL & $67-63-0$ & 0 & PPB & \\
\hline 1,1-DICHLOROETHENE & $75-35-4$ & 0 & PPB & \\
\hline FREON 113 & $76-13-1$ & 0 & PPB & \\
\hline DICHLOROMETHANE & $75-09-2$ & 0 & PPB & \\
\hline 1,1-DICHLOROETHANE & $75-34-3$ & 0 & PPB & \\
\hline 2-BUTANONE & $78-93-3$ & $\overline{0}$ & PPB & \\
\hline N-HEXANE & $110-54-3$ & 0 & PPB & \\
\hline 1,2-DICHLOROETHENE & $156-59-2$ & 0 & PPB & \\
\hline CHLOROFORM & $67-66-3$ & 0 & PPB & \\
\hline TETRAHYDROFURAN & $109-99-9$ & 0 & PPB & \\
\hline 1,1,1-TRICHLOROETHANE & $71-55-6$ & 0 & PPB & \\
\hline 1,2-DICHLOROETHANE & $107-06-2$ & 0 & PPB & \\
\hline BENZENE & $71-43-2$ & 0 & PPB & \\
\hline CARBON TETRACHLORIDE & $56-23-5$ & 0 & PPB & \\
\hline CYCLOHEXANE & $110-82-7$ & 0 & PPB & \\
\hline N-BUTANOL & $71-36-3$ & 0 & $\overline{\mathrm{PPB}}$ & \\
\hline TRICHLOROETHENE & $79-01-6$ & 0 & PPB & \\
\hline 4-METHYL-2-PENTANONE & $108-10-1$ & 8 & PPB & \\
\hline TOLUENE & $108-88-3$ & 0 & PPB & \\
\hline 1,1,2-TRICHLOROETHANE & $79-00-5$ & 0 & PPB & \\
\hline TETRACHLOROETHENE & $127-18-4$ & 0 & $\overline{\mathrm{PPB}}$ & \\
\hline CHLOROBENZENE & $108-90-7$ & 0 & PPB & \\
\hline ETHYLBENZENE & $100-41-4$ & 0 & PPB & \\
\hline M-XYLENE & $108-38-3$ & 0 & PPB & \\
\hline STYRENE & $100-42-5$ & 0 & PPB & \\
\hline O-XYLENE & $95-47-6$ & 0 & PPB & \\
\hline 1,1,2,2-TETRACHLORETHANE & $79-34-5$ & 0 & PPB & \\
\hline 1,3,5-TRIMETHYLBENZENE & $108-67-8$ & 2 & PPB & \\
\hline 1,2,4-TRIMETHYLBENZENE & $95-63-6$ & 0 & PPB & \\
\hline 1,3-DICHLOROBENZENE & $541-73-1$ & 0 & PPB & \\
\hline 1,2-DICHLOROBENZENE & $95-50-1$ & 0 & PPB & \\
\hline 1,4-DICHLOROBENZENE & $106-46-7$ & 0 & PPB & \\
\hline
\end{tabular}


HNF-SD-WM-RPT-282 Rev. 0

\section{LIST OF COMPOUNDS TENTATIVELY IDENTIFIED WITH MASS SPECTRAL LIBRARY}

Sample: $\quad 2402$ WK

Sample Name: FT6045-2WK.38A

Search Libraries: C:DATABASEIWILEY138.L Minimum Quality: 70

Unknown Spectrum: Apex

Integration Params: VOA.E

Pk\# RT Area\% Library/ID Ref\# CAS\# Qual

524.4614 .44 C:LDATABASEIWILEY138.L

2-Pentanone, 4-methyl-

$118021000108-10-191$

629.140 .73 C:LDATABASEIWILEY138.L

Benzene, 1,2-dimethyl- $\quad 118571000095-47-691$

XYLENE

$118580001330-20-783$

$729.960 .71 \mathrm{C}:$ LATABASEIWILEY138.L

Benzene, 1,2-dimethyl-

XYLENE

$118571000095-47-691$

$118580001330-20-789$

830.2248 .68 C:LDATABASEIWILEY138.L

Ethanol, 2-butoxy-

$119786000111-76-290$

$932.093 .18 \mathrm{C}:$ LATABASEIWILEYI38.L

Benzaldehyde

$1777000100-52-794$

1033.110 .93 C:LDATABASEIWILEY138.L

Benzene, 1,3,5-trimethyl-

$120037000108-67-887$ 


\section{File}

Operator

: D: \CWC \SAMPLES $\backslash 51496-13 . \mathrm{D}$

Acquired

Instrument

Sample Name: FT6045-2WK.39A BK

Misc Info : DELTA $P=100$ torr

Vial Number: 1

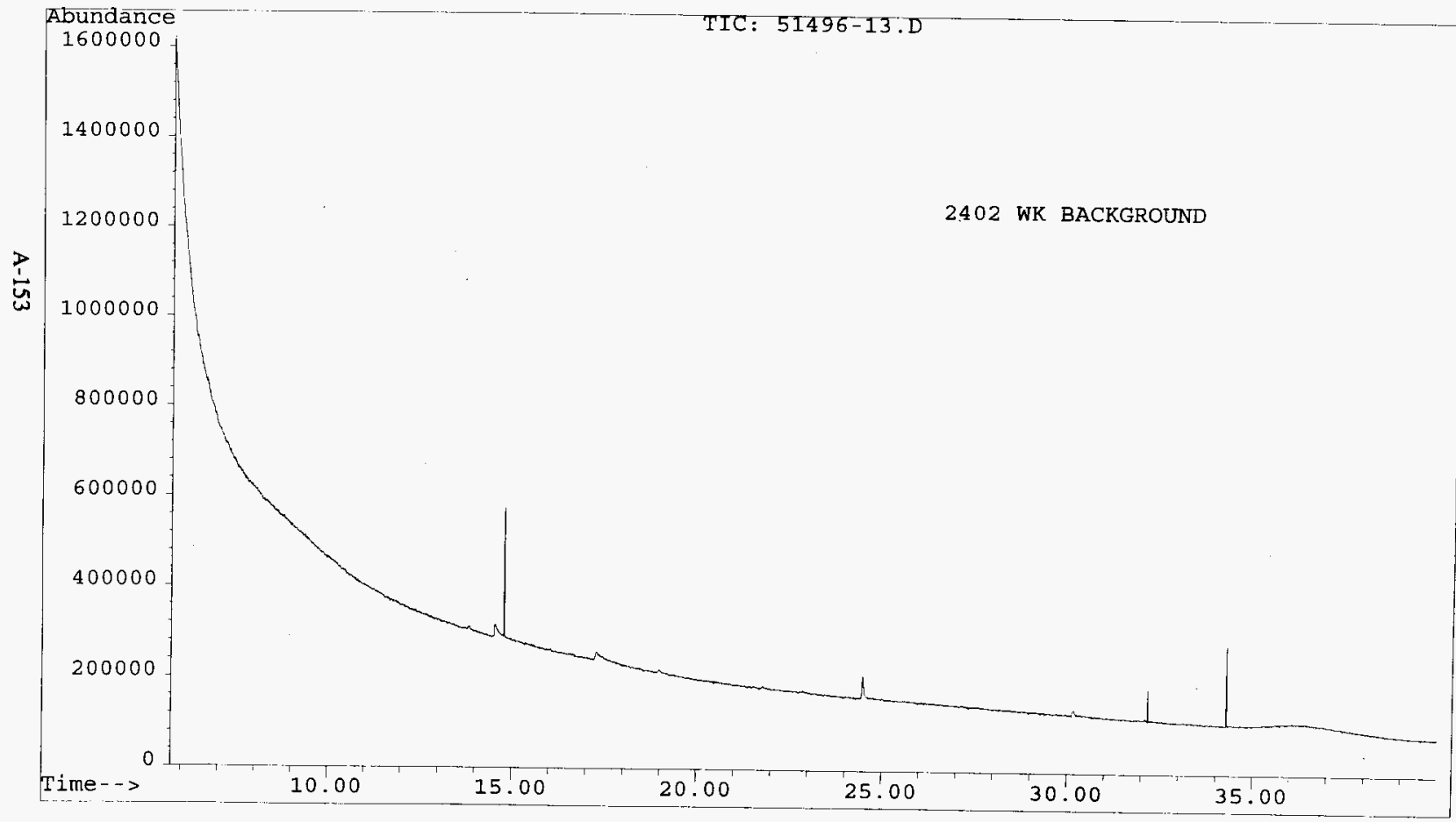


HNF-SD-WM-RPT-282 Rev. 0

CWC_REP.XLS

\section{ANALYSIS REPORT FOR CWC AIR SAMPLES}

File Name:

Analyst:

Analysis Date:

Method:

Sample Name:
51496-13.D

MS

05/14/96

CWC

FT6045-2WK.39A BK

\begin{tabular}{|c|c|c|c|c|}
\hline COMPOUND NAME & CAS \# & Concentration & Units & Comments \\
\hline ISO-BUTANE & $75-28-5$ & 0 & PPB & \\
\hline VINYL CHLORIDE & $75-01-4$ & $\overline{0}$ & PPB & \\
\hline ETHANOL & $64-17-5$ & 0 & PPB & \\
\hline ACETONE & $67-64-1$ & 0 & $\overline{\mathrm{PPB}}$ & \\
\hline 2-PROPANOL & $67-63-0$ & $\overline{0}$ & $\overline{\mathrm{PPB}}$ & \\
\hline 1,1-DICHLOROETHENE & $75-35-4$ & 0 & PPB & \\
\hline FREON 113 & $76-13-1$ & $\overline{0}$ & PPB & \\
\hline DICHLOROMETHANE & $75-\overline{09-2}$ & 0 & PPB & \\
\hline 1,1-DICHLOROETHANE & $75-34-3$ & 0 & PPB & \\
\hline 2-BUTANONE & $78-93-3$ & 0 & PPB & \\
\hline N-HEXANE & $110-54-3$ & 0 & PPB & \\
\hline 1,2-DICHLOROETHENE & $156-59-2$ & 0 & $\overline{\text { PPB }}$ & \\
\hline CHLOROFORM & $67-66-3$ & 0 & PPB & \\
\hline TETRAHYDROFURAN & $109-99-9$ & 0 & PPB & \\
\hline 1,1,1-TRICHLOROETHANE & $71-55-6$ & 0 & PPB & \\
\hline 1,2-DICHLOROETHANE & $107-06-2$ & 0 & PPB & \\
\hline BENZENE & $71-43-2$ & 0 & PPB & \\
\hline CARBON TETRACHLORIDE & $56-23-5$ & 0 & $\mathrm{PPB}$ & \\
\hline CYCLOHEXANE & $110-82-7$ & 0 & PPB & \\
\hline N-BUTANOL & $71-36-3$ & 0 & $\overline{\mathrm{PPB}}$ & \\
\hline TRICHLOROETHENE & $79-01-6$ & 0 & $\overline{P P B}$ & \\
\hline 4-METHYL-2-PENTANONE & $108-10-1$ & 0 & PPB & \\
\hline TOLUENE & $108-88-3$ & 0 & PPB & \\
\hline 1,1,2-TRICHLOROETHANE & $79-00-5$ & 0 & PPB & \\
\hline TETRACHLOROETHENE & $127-18-4$ & 0 & PPB & \\
\hline CHLOROBENZENE & $108-90-7$ & 0 & PPB & \\
\hline ETHYLBENZENE & $100-41-4$ & 0 & PPB & \\
\hline M-XYLENE & $108-38-3$ & 0 & $\overline{\mathrm{PPB}}$ & \\
\hline STYRENE & $100-42-5$ & 0 & PPB & \\
\hline O-XYLENE & $95-47-6$ & 0 & PPB & \\
\hline 1,1,2,2-TETRACHLORETHANE & $79-34-5$ & 0 & PPB & \\
\hline 1,3,5-TRIMETHYLBENZENE & $108-67-8$ & 0 & PPB & \\
\hline 1,2,4-TRIMETHYLBENZENE & $95-63-6$ & 0 & PPB & \\
\hline 1,3-DICHLOROBENZENE & $541-73-1$ & 0 & PPB & \\
\hline 1,2-DICHLOROBENZENE & $95-50-1$ & 0 & PPB & \\
\hline 1,4-DICHLOROBENZENE & $106-46-7$ & 0 & PPB & \\
\hline
\end{tabular}


HNF-SD-WM-RPT-282 Rev. 0

\section{LIST OF COMPOUNDS TENTATIVELY IDENTIFIED WITH MASS SPECTRAL LIBRARY}

Sample: $\quad 2402$ WK Background

Sample Name: FT6045-2WK.39A BK

Search Libraries: C:DATABASEIWILEY138.L Minimum Quality: 70

Unknown Spectrum: Apex

Integration Params: VOA.E

Pk\# RT Area\% Library/ID Ref\# CAS\#

$424.4820 .95 \mathrm{C}:$ :DATABASEIWILEY138.L

2-Pentanone, 4-methyl-

$118017000108-10-176$ 
HNF-SD-WM-RPT-282 Rev. 0
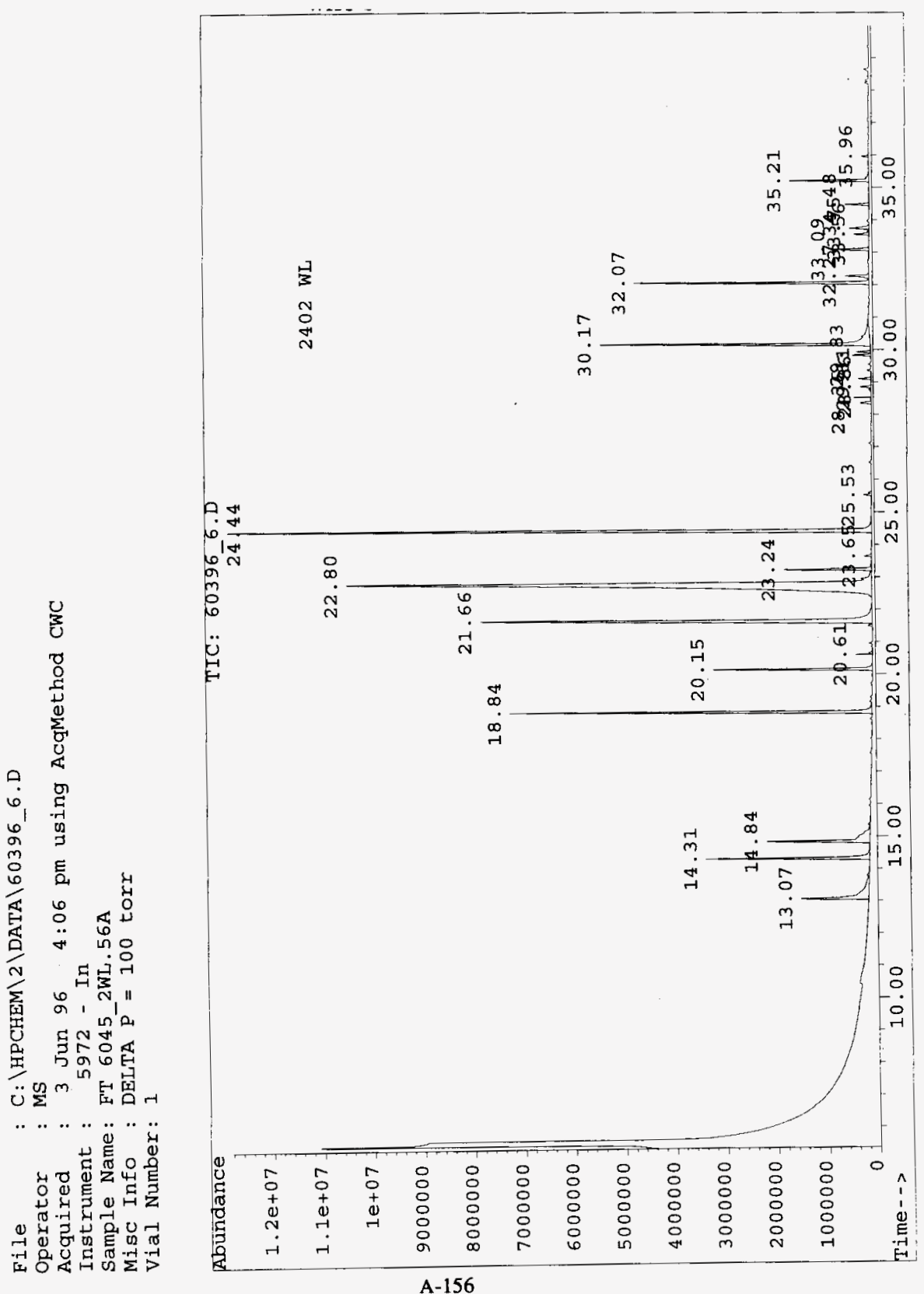
HNF-SD-WM-RPT-282 Rev. 0

CWC_REP.XLS

\section{ANALYSIS REPORT FOR CWC AIR SAMPLES}

File Name:

Analyst:

60396_6.D

Analysis Date:

Method:

MS

Sample Name:

06/03/96

CWC

FT 6045_2WL.56A

\begin{tabular}{|c|c|c|c|c|}
\hline COMPOUND NAME & CAS \# & Concentration & Units & Comments \\
\hline ISO-BUTANE & $75-28-5$ & 0 & PPB & \\
\hline VINYL CHLORIDE & $75-01-4$ & 0 & $\mathrm{PPB}$ & \\
\hline ETHANOL & $64-17-5$ & 168 & PPB & \\
\hline ACETONE & $67-64-1$ & 97 & PPB & \\
\hline 2-PROPANOL & $67-63-0$ & 26 & PPB & \\
\hline 1,1-DICHLOROETHENE & $75-35-4$ & 2 & PPB & \\
\hline FREON 113 & $76-13-1$ & 0 & PPB & \\
\hline DICHLOROMETHANE & $75-09-2$ & 0 & PPB & \\
\hline 1,1-DICHLOROETHANE & $75-34-3$ & 0 & PPB & \\
\hline 2-BUTANONE & $78-93-3$ & 773 & PPB & \\
\hline N-HEXANE & $110-54-3$ & 40 & PPB & \\
\hline 1,2-DICHLOROETHENE & $156-59-2$ & 0 & PPB & \\
\hline CHLOROFORM & $67-66-3$ & 0 & PPB & \\
\hline TETRAHYDROFURAN & $109-99-9$ & 84 & PPB & \\
\hline 1,1,1-TRICHLOROETHANE & $71-55-6$ & 0 & PPB & \\
\hline 1,2-DICHLOROETHANE & $107-06-2$ & 4 & PPB & \\
\hline BENZENE & $71-43-2$ & 7 & PPB & \\
\hline CARBON TETRACHLORIDE & $56-23-5$ & 0 & PPB & \\
\hline CYCLOHEXANE & $110-82-7$ & 0 & PPB & \\
\hline N-BUTANOL & $71-36-3$ & 545 & PPB & \\
\hline TRICHLOROETHENE & $79-01-6$ & 5 & PPB & \\
\hline 4-METHYL-2-PENTANONE & $108-10-1$ & 340 & PPB & \\
\hline TOLUENE & $108-88-3$ & 5 & PPB & \\
\hline 1,1,2-TRICHLOROETHANE & $79-00-5$ & 0 & PPB & \\
\hline TETRACHLOROETHENE & $127-18-4$ & 0 & PPB & \\
\hline CHLOROBENZENE & $108-90-7$ & 4 & PPB & \\
\hline ETHYLBENZENE & $100-41-4$ & 6 & PPB & \\
\hline M-XYLENE & $108-38-3$ & 7 & PPB & \\
\hline STYRENE & $100-42-5$ & 9 & PPB & \\
\hline O-XYLENE & $95-47-6$ & 7 & PPB & \\
\hline 1,1,2,2-TETRACHLORETHANE & $79-34-5$ & 2 & PPB & \\
\hline 1,3,5-TRIMETHYLBENZENE & $108-67-8$ & 12 & PPB & \\
\hline 1,2,4-TRIMETHYLBENZENE & $95-63-6$ & 18 & PPB & \\
\hline 1,3-DICHLOROBENZENE & $541-73-1$ & 9 & PPB & \\
\hline 1,2-DICHLOROBENZENE & $95-50-1$ & 12 & $\overline{\mathrm{PPB}}$ & \\
\hline 1,4-DICHLOROBENZENE & $106-46-7$ & 15 & PPB & \\
\hline
\end{tabular}




\section{LIST OF COMPOUNDS TENTATIVELY IDENTIFIED WITH MASS SPECTRAL LIBRARY}

Sample: $\quad 2402 \mathrm{WL}$

File: $\quad$ C:LHPCHEM2LDATAl60396_6.D

ID: FT6045_2WL.56A

Compound CAS\#

Glycidol 000556-52-5

$\begin{array}{ll}\text { Ethanol } & 000064-17-5\end{array}$

2-Propanone 000067-64-1

2-Propanol 000067-63-0

Ethene, 1,1-dichloro- 000075-35-4

Ethane, 1,1-dichloro- $\quad 000075-34-3$

2-Butanone 000078-93-3

Ethene, 1,2-dichloro-, (Z)- 000156-59-2

Chloroform $\quad 000067-66-3$

Furan, tetrahydro- $\quad$ 000109-99-9

Ethane, 1,2-dichloro- 000107-06-2

$\begin{array}{ll}\text { Benzene } & 000071-43-2\end{array}$

1-Butanol $\quad 000071-36-3$

Ethene, trichloro- $\quad$ 000079-01-6

1,4-Dioxane 000123-91-1

Propanoic acid, 2-hydroxy-2-methyl-, $\quad$ 002110-78-3

2-Pentanone, 4-methyl- 000108-10-1 
HNF-SD-WM-RPT-282 Rev. 0

\section{LIST OF COMPOUNDS TENTATIVELY IDENTIFIED WITH MASS SPECTRAL LIBRARY}

Sample: $\quad 2402$ WL (CONTINUED)

File: $\quad$ C:LHPCHEM2LDATAl60396_6.D

ID: $\quad$ FT6045_2WL.56A

Compound

CAS\#

Benzene, methyl-

000108-88-3

Ethane, 1,1,2-trichloro-

000079-00-5

Hexanal

000066-25-1

Ethene, tetrachloro-

000127-18-4

Benzene, ethyl-

$000100-41-4$

Benzene, 1,4-dimethyl-

$000106-42-3$

2-Heptanone

$000110-43-0$

Styrene

$000100-42-5$

XYLENE

001330-20-7

Ethanol, 2-butoxy-

000111-76-2

Benzaldehyde

000100-52-7

Benzene, 1,3,5-trimethyl-

000108-67-8

Benzene, 1,3-dichloro-

000541-73-1

Benzene, 1-methyl-3-(1-methylethyl)-

$000535-77-3$

Benzene, 1,2-dichloro-

000095-50-1

Nonanal

000124-19-6

Benzaldehyde, 3,4-dimethyl-

005973-71-7

2,3-DIMETHYLBENZALDEHYDE

005779-93-1 
HNF-SD-WM-RPT-282 Rev. 0

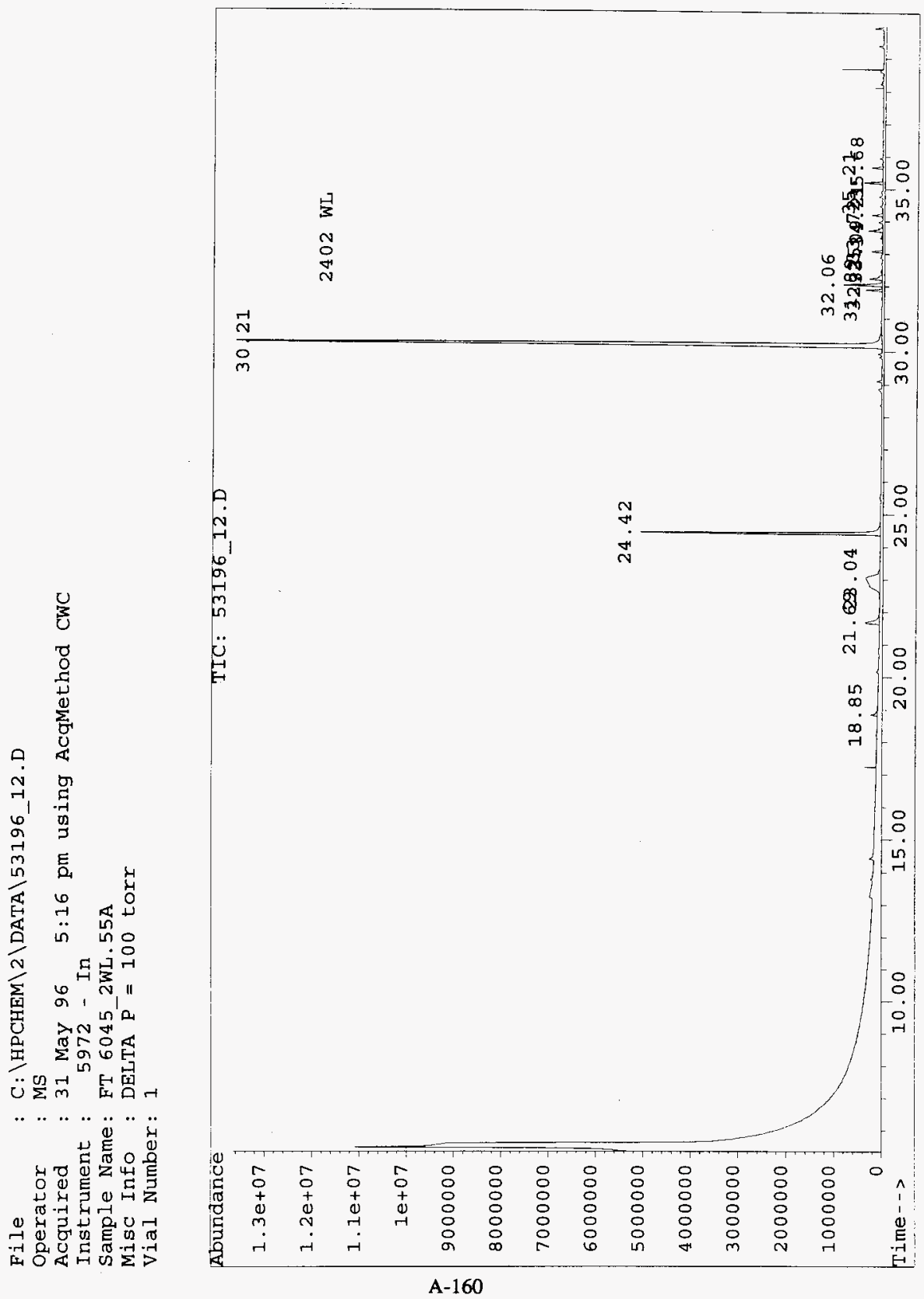


HNF-SD-WM-RPT-282 Rev. 0

CWC_REP.XLS

\section{ANALYSIS REPORT FOR CWC AIR SAMPLES}

File Name:

Analyst:

Analysis Date:

Method:

Sample Name:
53196_12.D

MS

05/31/96

CWC

FT 6045_2WL.55A

\begin{tabular}{|c|c|c|c|c|}
\hline COMPOUND NAME & CAS \# & Concentration & Units & Comments \\
\hline ISO-BUTANE & $75-28-5$ & 0 & PPB & \\
\hline VINYL CHLORIDE & $75-01-4$ & 0 & PPB & \\
\hline ETHANOL & $64-17-5$ & 15 & PPB & \\
\hline ACETONE & $67-64-1$ & 3 & PPB & \\
\hline 2-PROPANOL & $67-63-0$ & 0 & PPB & \\
\hline 1,1-DICHLOROETHENE & $75-35-4$ & 0 & PPB & \\
\hline FREON 113 & $76-13-1$ & 0 & PPB & \\
\hline DICHLOROMETHANE & $75-09-2$ & 0 & $\overline{\mathrm{PPB}}$ & \\
\hline 1,1-DICHLOROETHANE & $75-34-3$ & 0 & PPB & \\
\hline 2-BUTANONE & 78-93-3 & 16 & PPB & \\
\hline N-HEXANE & $110-54-3$ & 0 & PPB & \\
\hline 1,2-DICHLOROETHENE & $156-59-2$ & 0 & $\overline{\mathrm{PPB}}$ & \\
\hline CHLOROFORM & $67-66-3$ & 0 & PPB & \\
\hline TETRAHYDROFURAN & $109-99-9$ & 0 & PPB & \\
\hline 1,1,1-TRICHLOROETHANE & $71-55-6$ & 0 & PPB & \\
\hline 1,2-DICHLOROETHANE & $107-06-2$ & 0 & PPB & \\
\hline BENZENE & $71-43-2$ & 0 & PPB & \\
\hline CARBON TETRACHLORIDE & $56-23-5$ & 0 & PPB & \\
\hline CYCLOHEXANE & $110-82-7$ & 0 & $\mathrm{PPB}$ & \\
\hline N-BUTANOL & $71-36-3$ & 25 & PPB & \\
\hline TRICHLOROETHENE & $79-01-6$ & 0 & PPB & \\
\hline 4-METHYL-2-PENTANONE & $108-10-1$ & 107 & PPB & \\
\hline TOLUENE & $108-88-3$ & 1 & $\overline{\mathrm{PPB}}$ & \\
\hline 1,1,2-TRICHLOROETHANE & $79-00-5$ & 0 & PPB & \\
\hline TETRACHLOROETHENE & $127-18-4$ & 0 & $\overline{\mathrm{PPB}}$ & \\
\hline CHLOROBENZENE & $108-90-7$ & 0 & PPB & \\
\hline ETHYLBENZENE & $100-41-4$ & 2 & PPB & \\
\hline M-XYLENE & $108-38-3$ & 3 & PPB & \\
\hline STYRENE & $100-42-5$ & 1 & PPB & \\
\hline O-XYLENE & $95-47-6$ & 2 & PPB & \\
\hline $1,1,2,2$-TETRACHLORETHANE & $79-34-5$ & 0 & PPB & \\
\hline 1,3,5-TRIMETHYLBENZENE & $108-67-8$ & 1 & PPB & \\
\hline 1,2,4-TRIMETHYLBENZENE & $95-63-6$ & 6 & PPB & \\
\hline 1,3-DICHLOROBENZENE & $541-73-1$ & 0 & PPB & \\
\hline 1,2-DICHLOROBENZENE & $95-50-1$ & 0 & PPB & \\
\hline 1,4-DICHLOROBENZENE & $106-46-7$ & 0 & PPB & \\
\hline
\end{tabular}


HNF-SD-WM-RPT-282 Rev. 0

\section{LIST OF COMPOUNDS TENTATIVELY IDENTIFIED WITH MASS SPECTRAL LIBRARY}

$\begin{array}{ll}\text { Sample: } & \text { 2402 WL } \\ \text { File: } & \text { C:UHPCHEM 2LDATA153196_12.D } \\ \text { ID: } & \text { FT6045_2WL.55A }\end{array}$

Compound

CAS\#

1-Butanol

$000071-36-3$

2-Pentanone, 4-methyl-

000108-10-1

Benzene, methyl-

000108-88-3

Benzene, ethyl-

00010v-41-4

Benzene, 1,3-dimethyl-

000108-38-3

Styrene

$000100-42-5$

XYLENE

001330-20-7

Ethanol, 2-butoxy-

$000111-76-2$

Decane, 2,2,8-trimethyl-

062238-01-1

Benzaldehyde

000100-52-7

Phenol

000108-95-2

Benzene, 1,2,4-trimethyl-

000095-63-6

Benzene, 1,3-dichloro-

000541-73-1

Hexadecane

000544-76-3

Benzene, 1,4-dichloro-

000106-46-7

Ethanone, 1-phenyl-

000098-86-2

Benzene, (1-methyletheriyl)-

000098-83-9 
HNF-SD-WM-RPT-282 Rev. 0

\section{LIST OF COMPOUNDS TENTATIVELY IDENTIFIED WITH MASS SPECTRAL} LIBRARY

Sample: $\quad 2402$ WL (CONTINUED)

File: $\quad$ C:LHPCHEM2LDATA153196_12.D

ID: $\quad$ FT6045_2WL.55A

Compound

CAS\#

Nonanal

000124-19-6

Benzaldehyde, 3,4-dimethyl-

005973-71-7

2,3-DIMETHYLBENZALDEHYDE

005779-93-1

Benzaldehyde, ethyl-

053951-50-1 
File

C $: \backslash H P C H E M \backslash 2 \backslash D A T A \backslash 53096 \_18 . D$

Operator : MS

Acquired

: 30 May 96

5972 - In

Sample Name: FT6045-2WL.57A BK

Misc Info : DELTA $P=100$ torr

Vial Number: 1

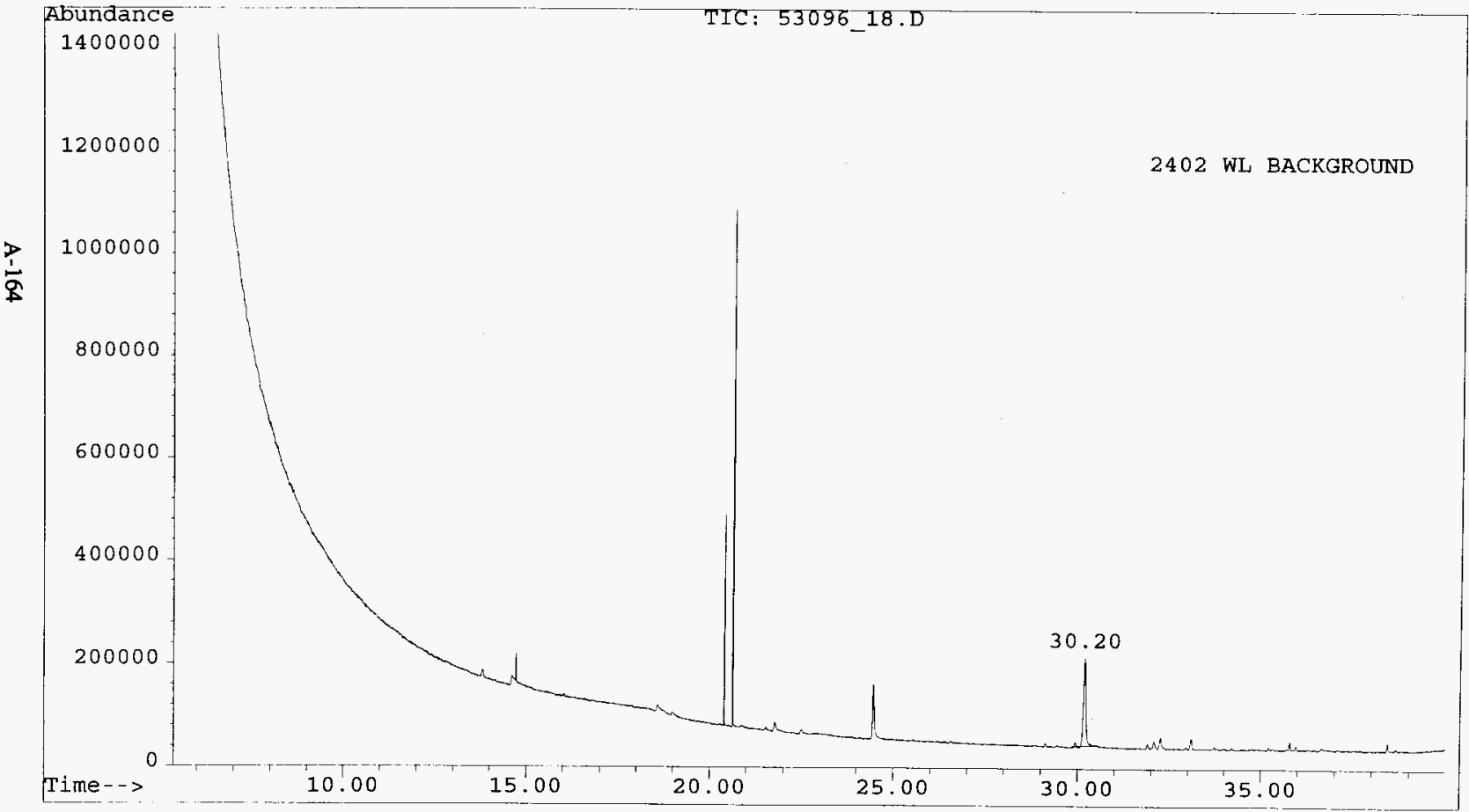


HNF-SD-WM-RPT-282 Rev. 0

CWC_REP.XLS

\section{ANALYSIS REPORT FOR CWC AIR SAMPLES}

File Name:

Analyst:

Analysis Date:

Method:

Sample Name:

\author{
53096_18.D \\ MS \\ 05/30/96 \\ CWC \\ FT6045-2WL.57A BK
}

\begin{tabular}{|c|c|c|c|c|}
\hline COMPOUND NAME & CAS \# & Concentration & Units & Comments \\
\hline ISO-BUTANE & $75-28-5$ & 0 & PPB & \\
\hline VINYL CHLORIDE & $75-01-4$ & 0 & PPB & \\
\hline ETHANOL & $64-17-5$ & 0 & PPB & \\
\hline ACETONE & $67-64-1$ & 0 & PPB & \\
\hline 2-PROPANOL & $67-63-0$ & 0 & PPB & \\
\hline 1,1-DICHLOROETHENE & $75-35-4$ & 0 & PPB & \\
\hline FREON 113 & $76-13-1$ & 0 & PPB & \\
\hline DICHLOROMETHANE & $75-09-2$ & 0 & $\overline{\mathrm{PPB}}$ & \\
\hline 1,1-DICHLOROETHANE & $75-34-3$ & 0 & PPB & \\
\hline 2-BUTANONE & $78-93-3$ & 0 & PPB & \\
\hline N-HEXANE & $110-54-3$ & 0 & PPB & \\
\hline 1,2-DICHLOROETHENE & $156-59-2$ & 0 & PPB & \\
\hline CHLOROFORM & $67-66-3$ & 0 & PPB & \\
\hline TETRAHYDROFURAN & $109-99-9$ & 0 & PPB & \\
\hline 1,1,1-TRICHLOROETHANE & $71-55-6$ & 0 & PPB & \\
\hline 1,2-DICHLOROETHANE & $107-06-2$ & 0 & PPB & \\
\hline BENZENE & $71-43-2$ & 0 & PPB & \\
\hline CARBON TETRACHLORIDE & $56-23-5$ & 0 & PPB & \\
\hline CYCLOHEXANE & $110-82-7$ & 0 & PPB & \\
\hline N-BUTANOL & $71-36-3$ & 0 & PPB & \\
\hline TRICHLOROETHENE & $79-01-6$ & 0 & $\mathrm{PPB}$ & \\
\hline 4-METHYL-2-PENTANONE & $108-10-1$ & 0 & PPB & \\
\hline TOLUENE & $108-88-3$ & 0 & PPB & \\
\hline 1,1,2-TRICHLOROETHANE & $79-00-5$ & 0 & PPB & \\
\hline TETRACHLOROETHENE & $127-18-4$ & 0 & PPB & \\
\hline CHLOROBENZENE & $108-90-7$ & 0 & PPB & \\
\hline ETHYLBENZENE & $100-41-4$ & 0 & PPB & \\
\hline M-XYLENE & $108-38-3$ & 0 & $\overline{\mathrm{PPB}}$ & \\
\hline STYRENE & $100-42-5$ & 0 & PPB & \\
\hline O-XYLENE & $95-47-6$ & 0 & PPB & \\
\hline $1,1,2,2$-TETRACHLORETHANE & $79-34-5$ & 0 & PPB & \\
\hline 1,3,5-TRIMETHYLBENZENE & $108-67-8$ & 0 & PPB & \\
\hline 1,2,4-TRIMETHYLBENZENE & $95-63-6$ & 0 & PPB & \\
\hline 1,3-DICHLOROBENZENE & $541-73-1$ & 0 & PPB & \\
\hline 1,2-DICHLOROBENZENE & $95-50-1$ & 0 & PPB & \\
\hline 1,4-DICHLOROBENZENE & $106-46-7$ & 0 & PPB & \\
\hline
\end{tabular}


HNF-SD-WM-RPT-282 Rev. 0

\section{LIST OF COMPOUNDS TENTATIVELY DENTIFIED WITH MASS SPECTRAL LIBRARY}

$\begin{array}{ll}\text { Sample: } & \text { 2402 WL BACKGROUND } \\ \text { File: } & \text { C:UHPCHEM } \backslash 2 \text { DATA } 53096 \_18 . D \\ \text { ID: } & \text { FT6045-2WL.57A BK }\end{array}$

Compound

CAS\#

2-Pentanone, 4-methyl-

000108-10-1 87

Ethanol, 2-butoxy-

$000111-76-280$

Benzaldehyde

000100-52-791

Phenol

$000108-95-281$

Benzene, 1,3,5-trimethyl-

000108-67-8 90 
HNF-SD-WM-RPT-282 Rev. 0

\section{A.3 CWC 2403 BUILDINGS}




\section{HNF-SD-WM-RPT-282 Rev. 0}

This page intentionally left blank. 
File

: C $: \backslash H P C H E M \backslash 2 \backslash D A T A \backslash 61396 \_5 . D$

Operator

: LAP

Acquired

: 13 Jun 96 11:23 am using AcqMethod CWC

Instrument : 5972 - In

Sample Name: FT6045-3WA.62A

Misc Info : DELTA $\mathrm{P}=100$ torr

Vial Number:

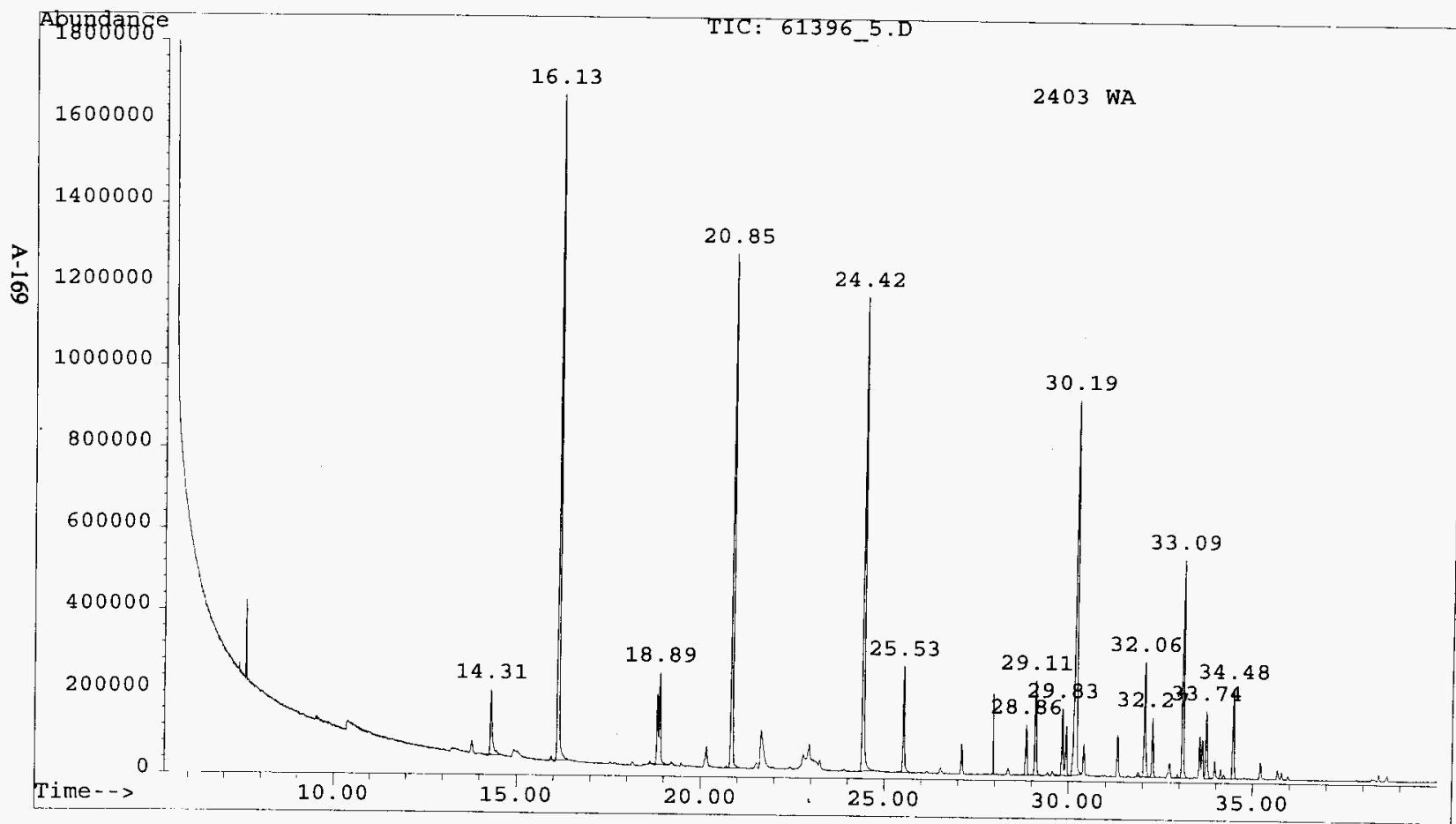


HNF-SD-WM-RPT-282 Rev. 0

CWC_REP.XLS

\section{ANALYSIS REPORT FOR CWC AIR SAMPLES}

File Name:

Analyst:

Analysis Date:

Method:

Sample Name:
61396_5.D

LAP

O6/13/96

CWC

FT6045-3WA.62A

\begin{tabular}{|c|c|c|c|c|}
\hline COMPOUND NAME & CAS \# & Concentration & Units & Comments \\
\hline ISO-BUTANE & $75-28-5$ & 0 & PPB & \\
\hline VINYL CHLORIDE & $75-01-4$ & 0 & PPB & \\
\hline ETHANOL & $64-17-5$ & 0 & PPB & \\
\hline ACETONE & $67-64-1$ & 5 & PPB & \\
\hline 2-PROPANOL & $67-63-0$ & 0 & PPB & \\
\hline 1,1-DICHLOROETHENE & $75-35-4$ & 0 & PPB & \\
\hline FREON 113 & $76-13-1$ & 0 & PPB & \\
\hline DICHLOROMETHANE & $75-09-2$ & 60 & PPB & \\
\hline 1,1-DICHLOROETHANE & $75-34-3$ & 0 & PPB & \\
\hline 2-BUTANONE & $78-93-3$ & 18 & PPB & \\
\hline N-HEXANE & $110-54-3$ & 6 & PPB & \\
\hline 1,2-DICHLOROETHENE & $156-59-2$ & 0 & PPB & \\
\hline CHLOROFORM & $67-66-3$ & 0 & PPB & \\
\hline TETRAHYDROFURAN & $109-99-9$ & 0 & PPB & \\
\hline $1,1,1$-TRICHLOROETHANE & $71-55-6$ & 56 & PPB & \\
\hline 1,2-DICHLOROETHANE & $107-06-2$ & 4 & PPB & \\
\hline BENZENE & $71-43-2$ & 0 & PPB & \\
\hline CARBON TETRACHLORIDE & $56-23-5$ & 0 & PPB & \\
\hline CYCLOHEXANE & $110-82-7$ & 0 & PPB & \\
\hline N-BUTANOL & $71-36-3$ & 8 & PPB & \\
\hline TRICHLOROETHENE & $79-01-6$ & 0 & PPB & \\
\hline 4-METHYL-2-PENTANONE & $108-10-1$ & 24 & PPB & \\
\hline TOLUENE & $108-88-3$ & 8 & PPB & \\
\hline 1,1,2-TRICHLOROETHANE & $79-00-5$ & 0 & PPB & \\
\hline TETRACHLOROETHENE & $127-18-4$ & 0 & PPB & \\
\hline CHLOROBENZENE & $108-90-7$ & 0 & PPB & \\
\hline ETHYLBENZENE & $100-41-4$ & 3 & PPB & \\
\hline $\mathrm{M}-\mathrm{XYLENE}$ & $108-38-3$ & 6 & PPB & \\
\hline STYRENE & $100-42-5$ & 4 & PPB & \\
\hline O-XYLENE & $95-47-6$ & 3 & PPB & \\
\hline 1,1,2,2-TETRACHLORETHANE & $79-34-5$ & 2 & PPB & \\
\hline 1,3,5-TRIMETHYLBENZENE & $108-67-8$ & 4 & PPB & \\
\hline 1,2,4-TRIMETHYLBENZENE & $95-63-6$ & 14 & PPB & \\
\hline 1,3-DICHLOROBENZENE & $541-73-1$ & 3 & PPB & \\
\hline 1,2-DICHLOROBENZENE & $95-50-1$ & 5 & PPB & \\
\hline 1,4-DICHLOROBENZENE & $106-46-7$ & 7 & PPB & \\
\hline
\end{tabular}


HNF-SD-WM-RPT-282 Rev. 0

\section{LIST OF COMPOUNDS TENTATIVELY IDENTIFIED WITH MASS SPECTRAL LIBRARY}

Sample: $\quad 2403$ WA

File: $\quad$ C:LHPCHEM2LDATAl61396_5.D

ID: $\quad$ FT6045-3WA.62A

Compound

CAS\#

Methane, dichloro-

000075-09-2

2-Butanone

000078-93-3

Hexane

000110-54-3

Furan, tetrahydro-

000109-99-9 .

Ethane, 1,1,1-trichloro-

$0000071-55-6$

1-Butanol

000071-36-3

2-Pentanone, 4-methyl-

000108-10-1

Benzene, methyl-

000108-88-3

Ethene, tetrachloro-

000127-18-4

Benzene, chloro-

000108-90-7

Benzene, ethyl-

$000100-41-4$

Benzene, 1,3-dimethyl-

000108-38-3

Styrene

$000100-42-5$

Benzene, 1,2-dimethyl-

000095-47-6

Ethanol, 2-butoxy-

000111-76-2

Ethane, 1,1,2,2-tetrachloro-

000079-34-5

ALPHA.-PINENE, (-)-

000080-56-8

Benzaldehyde

000100-52-7 


\section{LIST OF COMPOUNDS TENTATIVELY IDENTIFIED WITH MASS SPECTRAL} LIBRARY

Sample: $\quad 2403$ WA (CONTINUED)

File: $\quad$ C:LHPCHEMLLDATAl61396_5.D

ID: $\quad$ FT6045-3WA.62A

Compound

CAS\#

Benzene, 1,3,5-trimethyl-

$000108-67-8$

2-.BETA.-PINENE

000127-91-3

Benzene, 1,2,4-trimethyl-

000095-63-6

Benzene, 1,4-dichloro-

000106-46-7

DELTA.3-Carene

013466-78-9

Benzene, 1,4-dichloro-

000106-46-7

Benzene, 1-methyl-2-(1-methylethyl)-

000527-84-4

dl-Limonene

000138-86-3

Benzene, 1,2-dichloro-

000095-50-1

Ethanone, 1-phenyl-

000098-86-2

Benzenemethanol, .alpha.,,alpha.-dime

000617-94-7 
File

Operator

: C: $\backslash$ HPCHEM $\backslash 2 \backslash D A T A \backslash 61396 \_6 . D$

Acquired

: LAP

Instrument

: 13 Jun $96 \quad 1: 18$ pm using AcqMethod CWC

Sample Name: FT6045-3WA.64A

Misc Info : DELTA $\mathrm{P}=100$ torr

Vial Number: 1

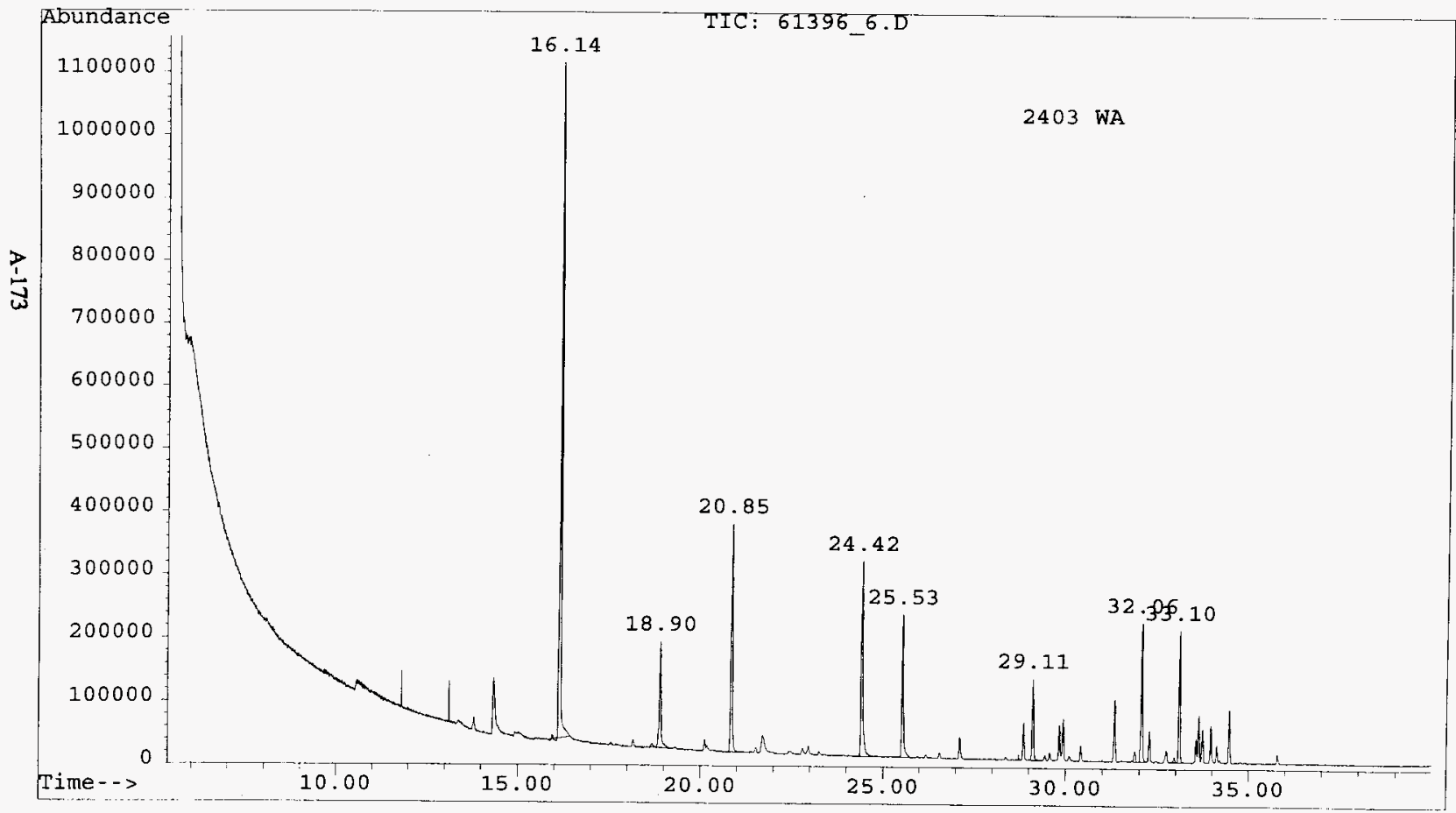


HNF-SD-WM-RPT-282 Rev. 0

CWC_REP.XLS

\section{ANALYSIS REPORT FOR CWC AIR SAMPLES}

File Name:

Analyst:

Analysis Date:

Method:

Sample Name:
61396_6.D

LAP

06/13/96

CWC

FT6045-3WA.64A

\begin{tabular}{|c|c|c|c|c|}
\hline COMPOUND NAME & CAS\# & Concentration & Units & Comments \\
\hline ISO-BUTANE & $75-28-5$ & 0 & PPB & \\
\hline VINYL CHLORIDE & $75-01-4$ & 0 & PPB & \\
\hline ETHANOL & $64-17-5$ & 0 & PPB & \\
\hline ACETONE & $67-64-1$ & 3 & PPB & \\
\hline 2-PROPANOL & $67-63-0$ & 0 & PPB & \\
\hline 1,1-DICHLOROETHENE & $75-35-4$ & 0 & PPB & \\
\hline FREON 113 & $76-13-1$ & 0 & PPB & \\
\hline DICHLOROMETHANE & $75-09-2$ & 38 & PPB & \\
\hline 1,1-DICHLOROETHANE & $75-34-3$ & 0 & PPB & \\
\hline 2-BUTANONE & $78-93-3$ & 0 & PPB & \\
\hline N-HEXANE & $110-54-3$ & 4 & PPB & \\
\hline 1,2-DICHLOROETHENE & $156-59-2$ & 0 & PPB & \\
\hline CHLOROFORM & $67-66-3$ & 0 & PPB & \\
\hline TETRAHYDROFURAN & $109-99-9$ & 0 & PPB & \\
\hline 1,1,1-TRICHLOROETHANE & $71-55-6$ & 16 & PPB & \\
\hline 1,2-DICHLOROETHANE & $107-06-2$ & 0 & PPB & \\
\hline BENZENE & $71-43-2$ & 0 & PPB & \\
\hline CARBON TETRACHLORIDE & $56-23-5$ & 0 & PPB & \\
\hline CYCLOHEXANE & $110-82-7$ & 0 & PPB & \\
\hline N-BUTANOL & $71-36-3$ & 0 & PPB & \\
\hline TRICHLOROETHENE & $79-01-6$ & 0 & PPB & \\
\hline 4-METHYL-2-PENTANONE & $108-10-1$ & 0 & PPB & \\
\hline TOLUENE & $108-88-3$ & 8 & PPB & \\
\hline 1,1,2-TRICHLOROETHANE & $79-00-5$ & 0 & PPB & \\
\hline TETRACHLOROETHENE & $127-18-4$ & 0 & PPB & \\
\hline CHLOROBENZENE & $108-90-7$ & 0 & PPB & \\
\hline ETHYLBENZENE & $100-41-4$ & 2 & PPB & \\
\hline M-XYLENE & $108-38-3$ & 4 & PPB & \\
\hline STYRENE & $100-42-5$ & 1 & PPB & \\
\hline O-XYLENE & $95-47-6$ & 2 & PPB & \\
\hline 1,1,2,2-TETRACHLORETHANE & $79-34-5$ & 0 & PPB & \\
\hline 1,3,5-TRIMETHYLBENZENE & $108-67-8$ & 1 & PPB & \\
\hline 1,2,4-TRIMETHYLBENZENE & $95-63-6$ & 5 & PPB & \\
\hline 1,3-DICHLOROBENZENE & $541-73-1$ & 0 & PPB & \\
\hline 1,2-DICHLOROBENZENE & $95-50-1$ & 0 & PPB & \\
\hline 1,4-DICHLOROBENZENE & $106-46-7$ & 2 & PPB & \\
\hline
\end{tabular}




\section{LIST OF COMPOUNDS TENTATIVELY IDENTIFIED WITH MASS SPECTRAL LIBRARY}

Sample: 2403 WA

File C.UPCHEMLLDATA161396_6.D

ID FT6045-3WA.64A

Compound

CAS\#

Methane, dichloro-

000075-09-2

Hexane

$000110-54-3$

Ethane, 1,1,1-trichloro-

$000071-55-6$

2-Pentanone, 4-methyl-

000108-10-1

Benzene, methyl-

$000108-88-3$

Ethene, tetrachioro-

$000127-18-4$

Benzene, ethyl-

$000100-41-4$

Benzene, 1,3-dimethyl-

000108-38-3

Styrene

$000100-42-5$

XYLENE

001330-20-7

Ethane, 1,1,2,2-tetrachloro-

000079-34-5

ALPHA.-PINENE, (-)-

000080-56-8

Benzaldehyde

000100-52-7

Benzene, 1,3,5-trimethyl-

000108-67-8

2-.BETA.PINENE

$000127-91-3$

Benzene, 1,2,3-trimethyl-

000526-73-8

Benzene, 1,4-dichloro-

000106-46-7

DELTA -4-CARENE

000554-61-0 


\section{LIST OF COMPOUNDS TENTATIVELY IDENTIFIED WITH MASS SPECTRAL LIBRARY}

Sample: $\quad 2403$ WA (CONTINUED)

File: $\quad$ C:LHPCHEM2LDATAl61396_6.D

ID $\quad$ FT6045-3WA.64A

Compound $\quad$ CAS\#

Benzene, 1,4-dichloro- $\quad 000106-46-7$

Benzene, 1-methyl-4-(1-methylethyl)- $\quad$ 000099-87-6

l-Limonene 005989-54-8 
File

Operator

C: $\backslash$ HPCHEM $\backslash 2 \backslash D A T A \backslash 61296 \_8 . D$

Acquired

: LAP

Instrument

12 Jun $96 \quad 2: 48$ pm using AcqMethod CWC

Sample Name: FT6045-3WA.65A

Misc Info : DELTA $\mathrm{P}=100$ torr

Vial Number: 1

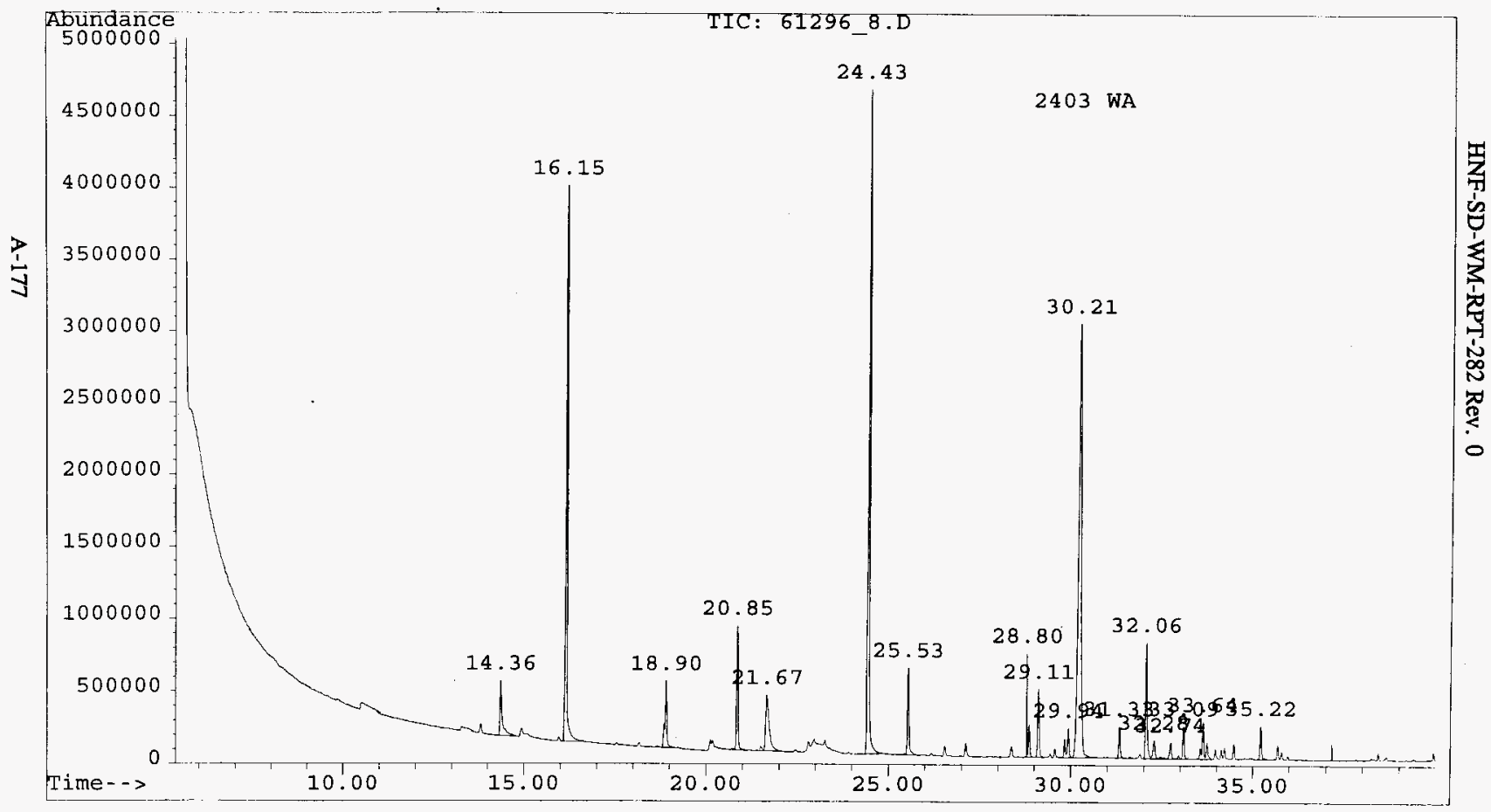


HNF-SD-WM-RPT-282 Rev. 0

CWC_REP.XLS

\section{ANALYSIS REPORT FOR CWC AIR SAMPLES}

File Name:

Analyst:

Analysis Date:

Method:

Sample Name:
61296_8.D

LAP

06/12/96

CWC

FT6045-3WA.65A

\begin{tabular}{|c|c|c|c|c|}
\hline COMPOUND NAME & CAS \# & Concentration & Units & Comments \\
\hline ISO-BUTANE & $75-28-5$ & 0 & $\overline{\mathrm{PPB}}$ & \\
\hline VINYL CHLORIDE & $75-01-4$ & 0 & PPB & \\
\hline ETHANOL & $64-17-5$ & 10 & PPB & \\
\hline ACETONE & $67-64-1$ & 15 & PPB & \\
\hline 2-PROPANOL & $67-63-0$ & 3 & PPB & \\
\hline 1,1-DICHLOROETHENE & $75-35-4$ & 0 & PPB & \\
\hline FREON 113 & $76-13-1$ & 0 & PPB & \\
\hline DICHLOROMETHANE & $75-09-2$ & 133 & PPB & \\
\hline 1,1-DICHLOROETHANE & $75-34-3$ & 0 & PPB & \\
\hline 2-BUTANONE & $78-93-3$ & 19 & PPB & \\
\hline N-HEXANE & $110-54-3$ & 12 & PPB & \\
\hline 1,2-DICHLOROETHENE & $156-59-2$ & 0 & PPB & \\
\hline CHLOROFORM & $67-66-3$ & 0 & PPB & \\
\hline TETRAHYDROFURAN & $109-99-9$ & 0 & PPB & \\
\hline 1,1,1-TRICHLOROETHANE & $71-55-6$ & 35 & PPB & \\
\hline 1,2-DICHLOROETHANE & $107-06-2$ & 3 & PPB & \\
\hline BENZENE & $71-43-2$ & 0 & PPB & \\
\hline CARBON TETRACHLORIDE & $56-23-5$ & 0 & PPB & \\
\hline CYCLOHEXANE & $110-82-7$ & 0 & PPB & \\
\hline N-BUTANOL & $71-36-3$ & 40 & PPB & \\
\hline TRICHLOROETHENE & $79-01-6$ & 0 & PPB & \\
\hline 4-METHYL-2-PENTANONE & $108-10-1$ & 87 & PPB & \\
\hline TOLUENE & $108-88-3$ & 21 & PPB & \\
\hline 1,1,2-TRICHLOROETHANE & $79-00-5$ & 0 & PPB & \\
\hline TETRACHLOROETHENE & $127-18-4$ & 4 & PPB & \\
\hline CHLOROBENZENE & $108-90-7$ & 2 & PPB & \\
\hline ETHYLBENZENE & $100-41-4$ & 6 & PPB & \\
\hline M-XYLENE & $108-38-3$ & 14 & PPB & \\
\hline STYRENE & $100-42-5$ & 3 & PPB & \\
\hline O-XYLENE & $95-47-6$ & 5 & PPB & \\
\hline 1,1,2,2-TETRACHLORETHANE & $79-34-5$ & 0 & PPB & \\
\hline 1,3,5-TRIMETHYLBENZENE & $108-67-8$ & 2 & PPB & \\
\hline 1,2,4-TRIMETHYLBENZENE & $95-63-6$ & 6 & PPB & \\
\hline 1,3-DICHLOROBENZENE & $541-73-1$ & 3 & PPB & \\
\hline 1,2-DICHLOROBENZENE & $95-50-1$ & 4 & PPB & \\
\hline 1,4-DICHLOROBENZENE & $106-46-7$ & 3 & PPB & \\
\hline
\end{tabular}




\section{LIST OF COMPOUNDS TENTATIVELY IDENTIFIED WITH MASS SPECTRAL LIBRARY}

$\begin{array}{ll}\text { Sample: } & \text { 2403 WA } \\ \text { File: } & \text { C:LPCHEML2WATAl61296_8.D } \\ \text { ID: } & \text { FT6045-3WA.65A }\end{array}$

Compound CAS\#

Methane, dichloro-

000075-09-2

Ethane, 1,1,1-trichloro-

$000071-55-6$

1-Butanol

000071-36-3

2-Pentanone, 4-methyl-

000108-10-1

Benzene, methyl-

000108-88-3

Hexanal

000066-25-1

Ethene, tetrachloro-

000127-18-4

Benzene, chloro-

000108-90-7

Benzene, 1,2-dimethyl-

000095-47-6

2-Heptanone

000110-43-0

Styrene

000100-42-5

Benzene, 1,3-dimethyl-

000108-38-3

Ethanol, 2-butoxy-

000111-76-2

ALPHA.-PINENE, (-)-

000080-56-8

Benzaldehyde

000100-52-7

Phenol

000108-95-2

2-BETA-PINENE

000127-91-3 


\section{LIST OF COMPOUNDS TENTATTVELY IDENTIFIED WITH MASS SPECTRAL LIBRARY}

Sample: $\quad 2403$ WA (CONTINUED)

File: $\quad$ C:LHPCHEMVLDATAl61296_8.D

ID: $\quad$ FT6045-3WA.65A

Compound

CAS\#

Decane

000124-18-5

Benzene, 1,2,3-trimethyl-

000526-73-8

Benzene, 1,4-dichloro-

000106-46-7

DELTA.3-Carene

013466-78-9

Benzene, 1,4-dichloro-

$000106-46-7$

Benzene, 1-methyl-2-(1-methylethyl)-

$000527-84-4$

dl-Limonene

000138-86-3

Beta.-Phellandrene

000555-10-2

Benzene, 1,3-dichloro-

000541-73-1

Ethanone, 1-phenyl-

000098-86-2

Undecane

001120-21-4 
File

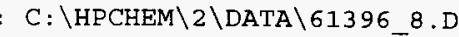

Operator

MS

Acquired

13 Jun 96 $3: 38$ pm using AcqMethod CWC

Instrument : 5972 - In

Sample Name: FT6045-3WA.62B

Misc Info : DELTA $\mathrm{P}=100$ torr

Vial Number: 1

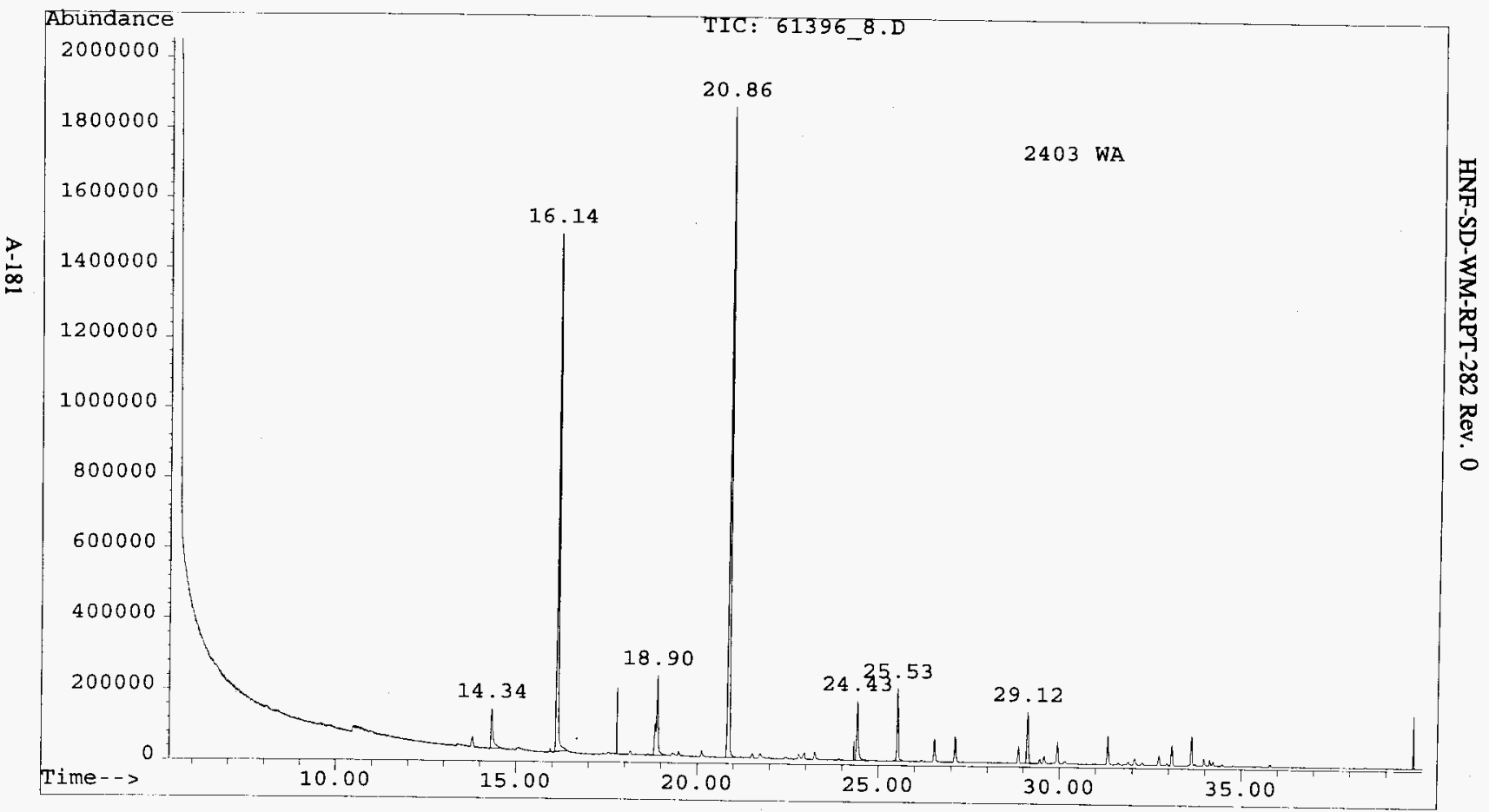


HNF-SD-WM-RPT-282 Rev. 0

CWC_REP.XLS

\section{ANALYSIS REPORT FOR CWC AIR SAMPLES}

File Name:

Analyst:

Analysis Date:

Method:

Sample Name:
61396_8.D

MS

06/13/96

CWC

FT6045-3WA.62B

\begin{tabular}{|c|c|c|c|c|}
\hline COMPOUND NAME & CAS\# & Concentration & Units & Comments \\
\hline ISO-BUTANE & $75-28-5$ & 0 & $\overline{\mathrm{PPB}}$ & \\
\hline VINYL CHLORIDE & $75-01-4$ & 0 & PPB & \\
\hline ETHANOL & $64-17-5$ & 0 & $\mathrm{PPB}$ & \\
\hline ACETONE & $67-64-1$ & 3 & PPB & \\
\hline 2-PROPANOL & $67-63-0$ & 0 & PPB & \\
\hline 1,1-DICHLOROETHENE & $75-35-4$ & 0 & PPB & \\
\hline FREON 113 & $76-13-1$ & $\overline{0}$ & PPB & \\
\hline DICHLOROMETHANE & $75-09-2$ & 53 & PPB & \\
\hline 1,1-DICHLOROETHANE & $75-34-3$ & 0 & PPB & \\
\hline 2-BUTANONE & $78-93-3$ & 0 & PPB & \\
\hline N-HEXANE & $110-54-3$ & 6 & PPB & \\
\hline 1,2-DICHLOROETHENE & $156-59-2$ & 0 & PPB & \\
\hline CHLOROFORM & $67-66-3$ & 0 & PPB & \\
\hline TETRAHYDROFURAN & $109-99-9$ & 0 & PPB & \\
\hline 1,1,1-TRICHLOROETHANE & $71-55-6$ & 83 & PPB & \\
\hline 1,2-DICHLOROETHANE & $107-06-2$ & 7 & PPB & \\
\hline BENZENE & $71-43-2$ & 0 & $\mathrm{PPB}$ & \\
\hline CARBON TETRACHLORIDE & $56-23-5$ & 0 & PPB & \\
\hline CYCLOHEXANE & 110-82-7 & 0 & PPB & \\
\hline N-BUTANOL & $71-36-3$ & 0 & PPB & \\
\hline TRICHLOROETHENE & $79-04-6$ & 0 & PPB & \\
\hline 4-METHYL-2-PENTANONE & 108-10-1 & 0 & PPB & \\
\hline TOLUENE & 108-88-3 & 6 & PPB & \\
\hline 1,1,2-TRICHLOROETHANE & $79-00-5$ & 0 & PPB & \\
\hline TETRACHLOROETHENE & $127-18-4$ & 0 & PPB & \\
\hline CHLOROBENZENE & 108-90-7 & 0 & PPB & \\
\hline ETHYLBENZENE & $100-41-4$ & 1 & PPB & \\
\hline M-XYLENE & 108-38-3 & 4 & PPB & \\
\hline STYRENE & $100-42-5$ & 0 & PPB & \\
\hline O-XYLENE & $95-47-6$ & 1 & PPB & \\
\hline 1,1,2,2-TETRACHLORETHANE & $79-34-5$ & 0 & PPB & \\
\hline 1,3,5-TRIMETHYLBENZENE & $108-67-8$ & 0 & PPB & \\
\hline 1,2,4-TRIMETHYLBENZENE & $95-63-6$ & 1 & PPB & \\
\hline 1,3-DICHLOROBENZENE & 541-73-1 & 0 & PPB & \\
\hline 1,2-DICHLOROBENZENE & $95-50-1$ & $\overline{0}$ & $\overline{P P B}$ & \\
\hline 1,4-DICHLOROBENZENE & $106-46-7$ & 0 & PPB & \\
\hline
\end{tabular}


HNF-SD-WM-RPT-282 Rev. 0

LIST OF COMPOUNDS TENTATIVELY IDENTIFIED WITH MASS SPECTRAL

\section{LIBRARY}

Sample: $\quad 2403$ WA

File: $\quad$ C:UHPCHEMULDATAll61396_8.D

ID: $\quad$ FT6045-3WA.62B

Compound CAS\#

Methane, trichlorofluoro- $\quad$ 000075-69-4

Methane, dichloro- $\quad$ 000075-09-2

Hexane 000110-54-3

$\begin{array}{ll}\text { Cyclopentane, methyl- } & \text { 000096-37-7 }\end{array}$

Ethane, 1,1,1-trichloro- 000071-55-6

$\begin{array}{ll}\text { Pentanal 000110-62-3 } & \end{array}$

Ethene, trichloro- $\quad$ 000079-01-6

1,4-Dioxane 000123-91-1

2-Pentanone, 4-methyl- 000108-10-1

Benzene, methyl- 000108-88-3

$\begin{array}{ll}\text { Hexanal 00006-25-1 } & \end{array}$

Ethene, tetrachloro- $\quad$ 000127-18-4

Benzene, ethyl- $\quad 000100-41-4$

Benzene, 1,3-dimethyl- 000108-38-3

2-Heptanone 000110-43-0

Benzene, 1,3-dimethyl- 000108-38-3

ALPHA.-PINENE, (-)- 000080-56-8 
HNF-SD-WM-RPT-282 Rev, 0

\title{
LIST OF COMPOUNDS TENTATIVELY IDENTIFIED WITH MASS SPECTRAL LIBRARY
}

\author{
Sample: $\quad 2403$ WA (CONTINUED) \\ File: $\quad$ C:LHPCHEMLLDATAII61396 8.D \\ ID: FT6045-3WA.62B
}

Compound

CAS\#

Benzaldehyde

$000100-52-7$

2-.BETA.-PINENE

$000127-91-3$

Benzene, 1,2,4-trimethyl-

000095-63-6

DELTA.3-Carene

013466-78-9

Benzene, 1-methyl-4-(1-methylethyl)-

000099-87-6

dl-Limonene

000138-86-3 
File

Operator

: C: \HPCHEM $\backslash 2 \backslash D A T A \backslash 61396 \_7 . D$

$\therefore$ LAP

Instrument : $\quad 5972$ Jun 96

Sample Name: FT6045-3WA.66A

Misc Info : DELTA $\mathrm{P}=100$ torr

Vial Number: 1

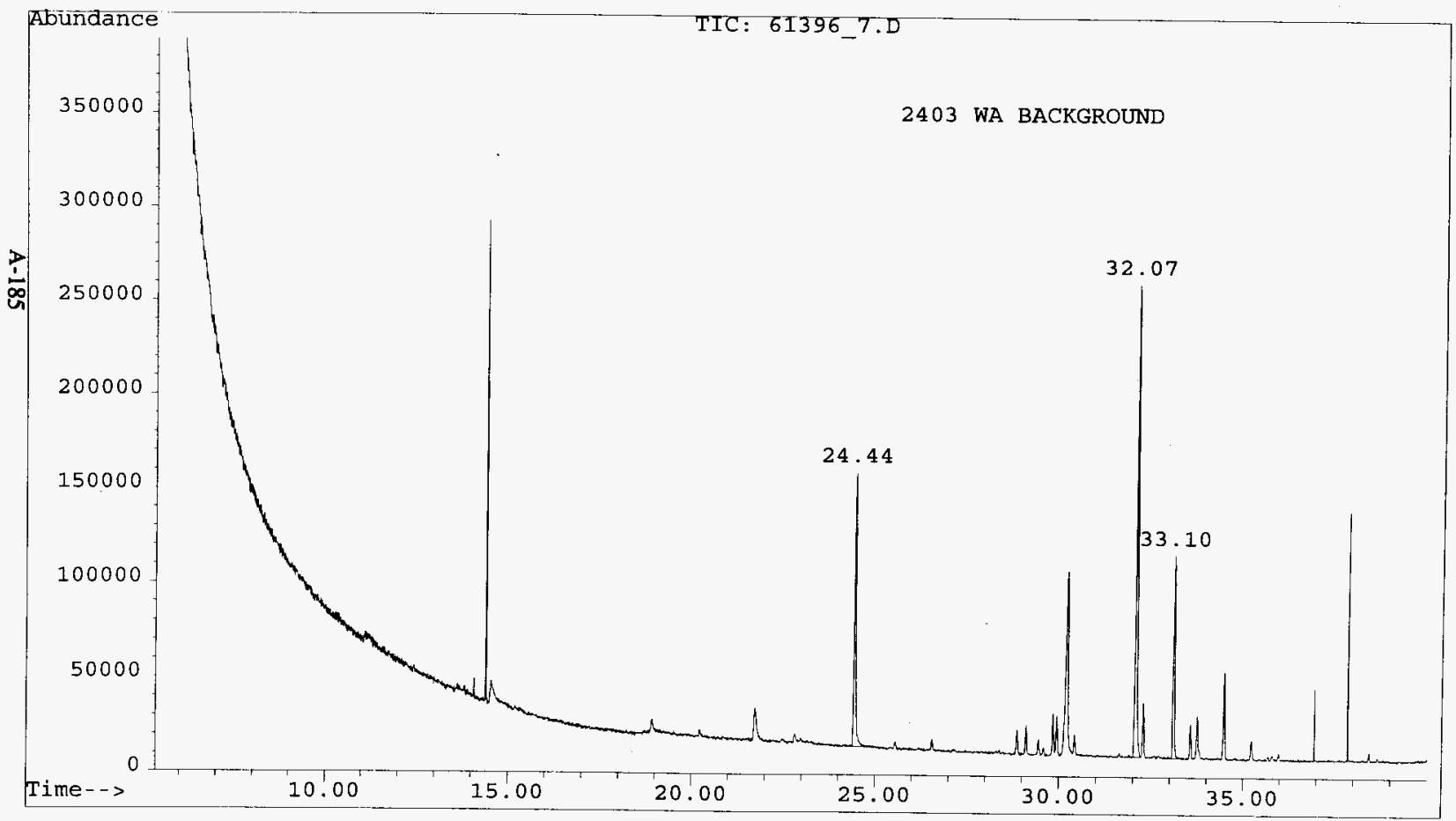


HNF-SD-WM-RPT-282 Rev. 0

CWC_REP.XLS

\section{ANALYSIS REPORT FOR CWC AIR SAMPLES}

File Name:

Analyst:

Analysis Date:

Method:

Sample Name:
61396_7.D

LAP

06/13/96

CWC

FT6045-3WA.66A

\begin{tabular}{|c|c|c|c|c|}
\hline COMPOUND NAME & CAS \# & Concentration & Units & Comments \\
\hline ISO-BUTANE & $75-28-5$ & 0 & PPB & \\
\hline VINYL CHLORIDE & $75-01-4$ & 0 & PPB & \\
\hline ETHANOL & $64-17-5$ & 0 & PPB & \\
\hline ACETONE & $67-64-1$ & 0 & $\overline{\mathrm{PPB}}$ & \\
\hline 2-PROPANOL & $67-63-0$ & 0 & PPB & \\
\hline 1,1-DICHLOROETHENE & $75-35-4$ & 0 & PPB & \\
\hline FREON 113 & $76-13-1$ & 0 & PPB & \\
\hline DICHLOROMETHANE & $75-09-2$ & 0 & PPB & \\
\hline 1,1-DICHLOROETHANE & $75-34-3$ & 0 & PPB & \\
\hline 2-BUTANONE & $78-93-3$ & 0 & PPB & \\
\hline N-HEXANE & $110-54-3$ & 0 & PPB & \\
\hline 1,2-DICHLOROETHENE & $156-59-2$ & 0 & PPB & \\
\hline CHLOROFORM & $67-66-3$ & 0 & PPB & \\
\hline TETRAHYDROFURAN & $109-99-9$ & 0 & PPB & \\
\hline 1,1,1-TRICHLOROETHANE & $71-55-6$ & 0 & PPB & \\
\hline 1,2-DICHLOROETHANE & $107-06-2$ & 0 & PPB & \\
\hline BENZENE & $71-43-2$ & 0 & PPB & \\
\hline CARBON TETRACHLORIDE & $56-23-5$ & 0 & PPB & \\
\hline CYCLOHEXANE & $110-82-7$ & 0 & PPB & \\
\hline N-BUTANOL & $71-36-3$ & 0 & PPB & \\
\hline TRICHLOROETHENE & $79-01-6$ & 0 & PPB & \\
\hline 4-METHYL-2-PENTANONE & $108-10-1$ & 0 & PPB & \\
\hline TOLUENE & $108-8 \overline{8-3}$ & 0 & PPB & \\
\hline 1,1,2-TRICHLOROETHANE & $79-00-5$ & 0 & PPB & \\
\hline TETRACHLOROETHENE & $127-18-4$ & 0 & PPB & \\
\hline CHLOROBENZENE & $108-90-7$ & 0 & PPB & \\
\hline ETHYLBENZENE & $100-41-4$ & 0 & PPB & \\
\hline M-XYLENE & $108-38-3$ & 0 & PPB & \\
\hline STYRENE & $100-42-5$ & 0 & PPB & \\
\hline O-XYLENE & $95-47-6$ & 0 & PPB & \\
\hline 1,1,2,2-TETRACHLORETHANE & $79-34-5$ & 0 & PPB & \\
\hline 1,3,5-TRIMETHYLBENZENE & $108-67-8$ & 4 & PPB & \\
\hline 1,2,4-TRIMETHYLBENZENE & $95-63-6$ & 3 & PPB & \\
\hline 1,3-DICHLOROBENZENE & $541-73-1$ & 0 & PPB & \\
\hline 1,2-DICHLOROBENZENE & $95-50-1$ & 0 & PPB & \\
\hline 1,4-DICHLOROBENZENE & $106-46-7$ & 0 & PPB & \\
\hline
\end{tabular}




\section{LIST OF COMPOUNDS TENTATIVELY IDENTIFIED WITH MASS SPECTRAL LIBRARY}

Sample: 2403WA BACKGROUND

File: C:LHPCHEMLLDATAl61396_7.D

ID: FT6045-3WA.66A

Compound CAS\#

2-Pentanone, 4-methyl-

Benzene, 1,2-dimethyl-

Styrene

Benzene, 1,2-dimethyl-

Ethanol, 2-butoxy-

Benzaldehyde

Benzene, 1,3,5-trimethyl-

Benzene, 1,2,3-trimethyl-

Benzene, 1,2-dichloro-

Benzene, 1,3-dichloro-

Benzene, 1,2-dichloro-
000108-10-1

000095-47-6

$000100-42-5$

000095-47-6

$000111-76-2$

$000100-52-7$

000108-67-8

000526-73-8

000095-50-1

000541-73-1

000095-50-1 
File

$\mathrm{C}: \backslash H P C H E M \backslash 2 \backslash D A T A \backslash 61296 \_9 . \mathrm{D}$

Operator

IAP

Acquired

: 12 Jun 96 3:41 pm using AcqMethod CWC

Instrument : 5972 - In

Sample Name: FT6045-3WA.67A

Misc Info : DELTA $\mathrm{P}=100$ torr

Vial Number: I

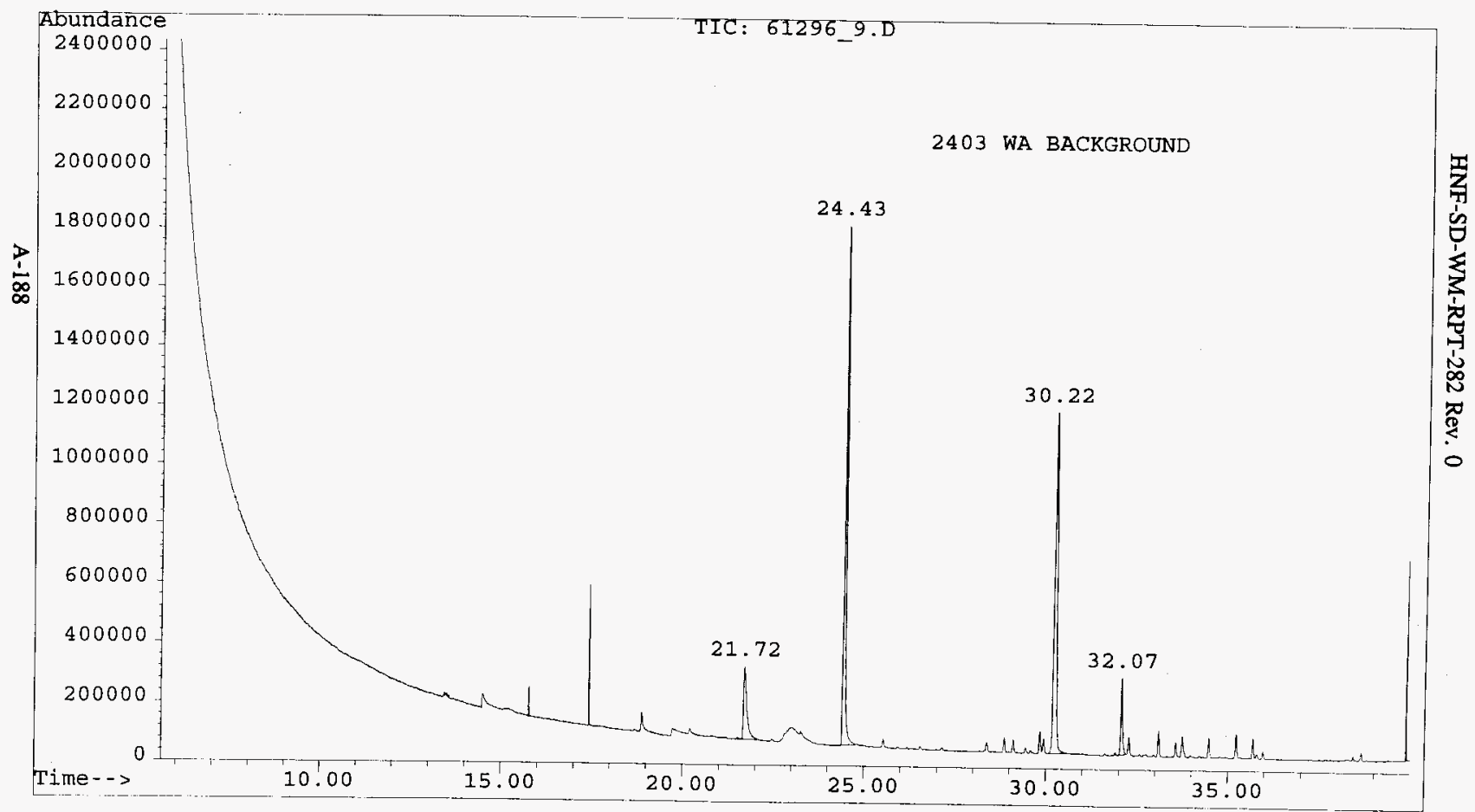




\begin{tabular}{|c|c|c|c|c|}
\hline & 8dd & $\bar{Z}$ & $L-9 b-90 L$ & 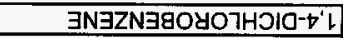 \\
\hline & gdd & $z$ & l-OS-S6 & $\exists N \exists Z N \exists B O Y 07 \mathrm{H}$ \\
\hline & gdd & $z$ & $L-\varepsilon L-L b S$ & 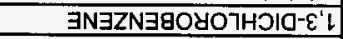 \\
\hline & 8dd & $z$ & $9-89-96$ & $\exists N \exists Z N \exists B 7 \lambda H \perp \exists W I Y \perp^{-1} \nabla^{\prime} Z^{\prime} L$ \\
\hline & gdd & $\underline{9}$ & $8-\angle 9-801$ & $\exists N \exists Z N \exists g 7 \lambda \mathrm{H} \perp \exists W I Y \perp-\varsigma^{\prime} \varepsilon^{\prime} \downarrow$ \\
\hline & gdd & 0 & $S-b \varepsilon-6 L$ & $\exists N \forall H \perp \exists У O 7 H O \forall y \perp \exists \perp-Z^{\prime} Z^{\prime} \downarrow\llcorner$ \\
\hline & gdd & 0 & $9-\angle b-96$ & $\exists \mathrm{N} \exists 7 \times \mathrm{X}-\mathrm{O}$ \\
\hline & gdd & $z$ & $\mathrm{~s}-2 \mathrm{~b}-00 \mathrm{~L}$ & $\exists N \exists y \lambda \perp S$ \\
\hline & gdd & 0 & $\varepsilon-8 \varepsilon-801$ & $\exists N \exists 7 \lambda X-W$ \\
\hline & gdd & 1 & $\forall-t \sigma 00 t$ & $\exists N \exists Z N \exists g 7 \lambda H \perp \exists$ \\
\hline & gdd & 0 & $\angle-06-80 \mathrm{~L}$ & 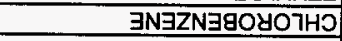 \\
\hline & gdd & 0 & $t-8 L-\angle Z L$ & $\exists N \exists H \perp \exists O Y O T H O \forall Y \perp \exists \perp$ \\
\hline & gdd & 0 & S-00-6L & $\exists N \forall H \perp \exists O \forall O\urcorner H O I+Y \perp-Z^{\prime} L^{\prime} \vdash$ \\
\hline & gdd & 0 & $\varepsilon-88-80 \mathrm{~L}$ & $\exists N \exists \cap 70 \perp$ \\
\hline & gdd & $9 \varepsilon$ & $1-0 L-801$ & $\exists N O N \forall \perp N \exists d-\tau-7 \lambda H \perp \exists W-t$ \\
\hline & 8dd & 0 & $9-10-6 L$ & 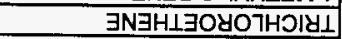 \\
\hline & 8dd & $\varepsilon z$ & $\varepsilon-9 \varepsilon-1 L$ & רON $\perp \cap \cap 9-N$ \\
\hline & gdd & 0 & $\angle-28-011$ & $\exists N \forall \times \exists H O 7 \supset \wedge ว$ \\
\hline & gdd & 0 & $s-\varepsilon z-9 s$ & $\exists 0 l y 07 H \supset \forall y \perp \exists \perp$ NOgy $\forall 0$ \\
\hline & gdd & 0 & $z-\varepsilon t-1 L$ & ヨN $\mathrm{ZNN} \exists \mathrm{G}$ \\
\hline & gdd & 0 & $2-90-\angle 01$ & $\exists N \forall H \perp \exists O Y O 7 H O I O-Z^{2} L$ \\
\hline & gdd & 0 & $9-9 g-1 L$ & $\exists N \forall H \perp \exists O Y O 7 H O I Y \perp-l^{\prime} l^{\prime} l$ \\
\hline & gdd & 0 & $6-66-601$ & 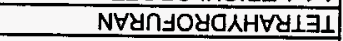 \\
\hline & gdd & 0 & $\varepsilon-99-\angle 9$ & WyOzOYO7Hว \\
\hline & gdd & 0 & $2-69-991$ & 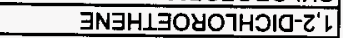 \\
\hline & gdd & 0 & $\varepsilon-+\varepsilon-0\llcorner l$ & $\exists N \forall X \exists H-N$ \\
\hline & gdd & 0 & $\varepsilon-\varepsilon 6-8 L$ & $\exists N O N \forall \perp \cap 9-Z$ \\
\hline & gdd & 0 & $\varepsilon-\nabla \varepsilon-g L$ & 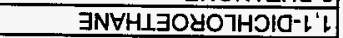 \\
\hline & 8dd & 0 & $2-60-9 L$ & $\exists N \forall H \perp \exists W O Y O 7 H D I 0$ \\
\hline & 8dd & 0 & $L-\varepsilon l-9 L$ & ELLNOJYA \\
\hline & gdd & 0 & $\nabla-G \varepsilon-9 L$ & $\exists N \exists H \perp \exists O Y 07 H O I 0-L^{\prime \prime}$ \\
\hline & gdd & 0 & $0-\varepsilon 9-\angle 9$ & $70 N \forall d O d d-Z$ \\
\hline & gdd & 0 & $1-t 9-29$ & $\exists N O \perp \exists O V$ \\
\hline & gdd & 0 & $G-\angle 1-t 9$ & 7ON $\forall H \perp \exists$ \\
\hline & gdd & 0 & $D-10-G L$ & 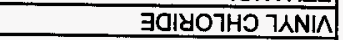 \\
\hline & gdd & 0 & $G-8 Z-G L$ & $\exists N \forall \perp \cap Q-O S \mid$ \\
\hline squouuros & sq!un & uonp? & \# S*O & IWVN aNnOdWOS \\
\hline
\end{tabular}

$\left.\forall \angle 9^{\circ} \forall M E-S \$ 09 \perp\right\rfloor$

OMO

96/ZL/90

dV7

व.6 66 เட9
:ameN әdwes

:pouław

:әjeg s!sরןeur

ìsfieur

:əueN 리! 
HNF-SD-WM-RPT-282 Rev, 0

\section{LIST OF COMPOUNDS TENTATIVELY IDENTIFIED WITH MASS SPECTRAL LIBRARY}

$\begin{array}{ll}\text { Sample: } & \text { 2403 WA } \\ \text { File: } & \text { C:LHPCHEML2LDATAl61296_9.D } \\ \text { ID: } & \text { FT6045-3WA.67A }\end{array}$

Compound

CAS\#

1-Butanol

$000071-36-3$

2-Pentanone, 4-methyl-

000108-10-1

Benzene, methyl-

000108-88-3

Benzene, chloro-

000108-90-7

Benzene, ethyl-

$000100-41-4$

Benzene, 1,3-dimethyl-

000108-38-3

Styrene

$00100-42-5$

Benzene, 1,2-dimethyl-

$000095-47-6$

Ethanol, 2-butoxy-

000111-76-2

Benzaldehyde

000100-52-7

Phenol

000108-95-2

Benzene, 1,3,5-trimethyl-

000108-67-8

Benzene, 1,3-dichloro-

000541-73-1

Benzene, 1,4-dichloro-

000106-46-7

Ethanone, 1-phenyl-

000098-86-2

Benzene, (1-methylethenyl)-

000098-83-9 
File

Operator

C: \HPCHEM $\backslash 2 \backslash D A T A \backslash 61496$ 9.D

Acquired

: MS

Instrument : $5972-$ In

Sample Name: FT 6045-3WA.63A

Misc Info : DELTA $\mathrm{P}=100$ TORR

Vial Number: 1

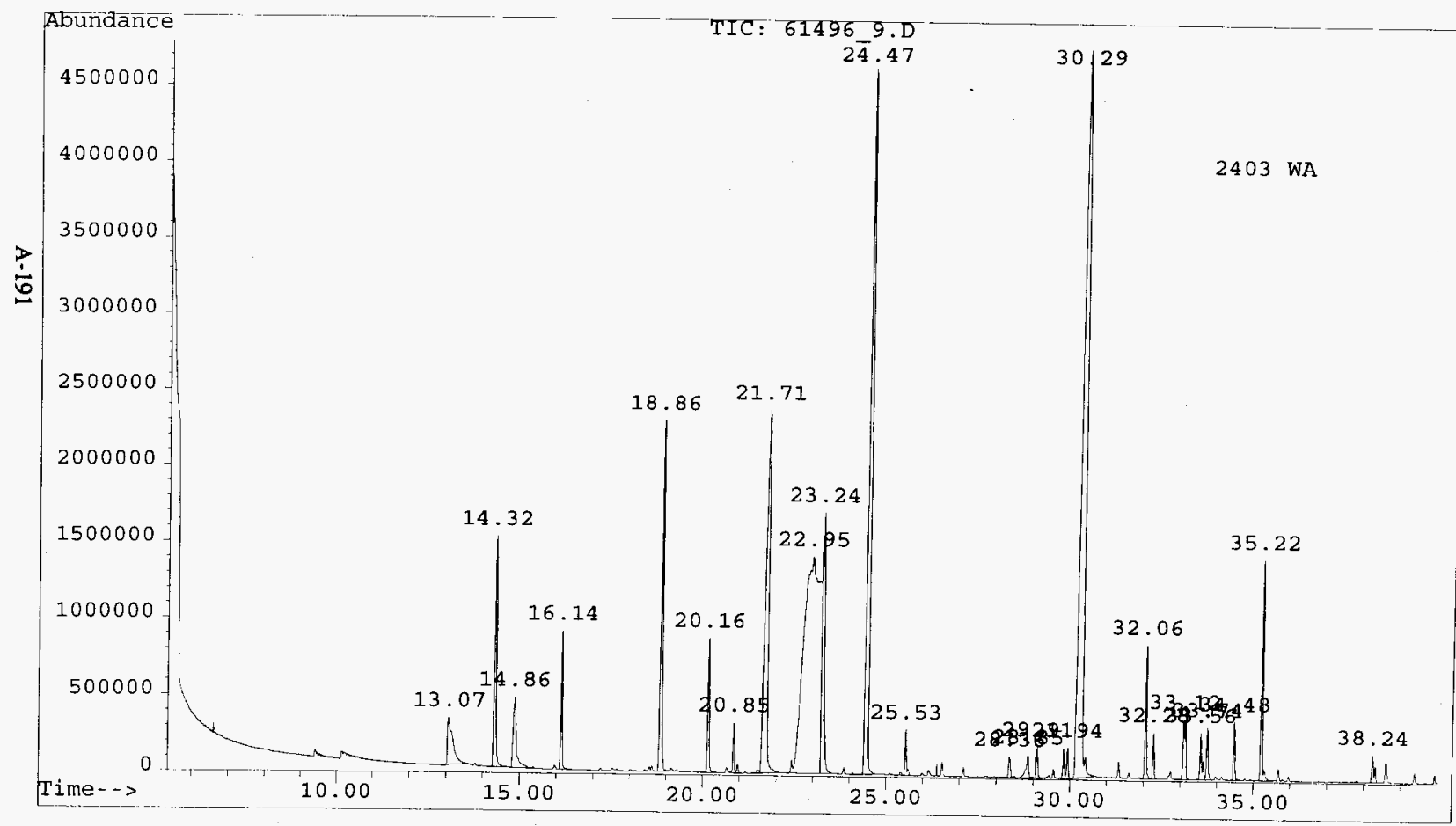


HNF-SD-WM-RPT-282 Rev. 0

CWC_REP.XLS

\section{ANALYSIS REPORT FOR CWC AIR SAMPLES}

File Name:

Analyst:

Analysis Date:

Method:

Sample Name:
61496_9.D

MS

06/14/96

CWC

FT 6045-3WA.63A

\begin{tabular}{|c|c|c|c|c|}
\hline COMPOUND NAME & CAS \# & Concentration & Units & Comments \\
\hline ISO-BUTANE & $75-28-5$ & 0 & $\overline{\mathrm{PPB}}$ & \\
\hline VINYL CHLORIDE & $75-01-4$ & 0 & PPB & \\
\hline ETHANOL & $64-17-5$ & 73 & PPB & \\
\hline ACETONE & $67-64-1$ & 42 & PPB & \\
\hline 2-PROPANOL & $67-63-0$ & 29 & PPB & \\
\hline 1,1-DICHLOROETHENE & $75-35-4$ & 0 & PPB & \\
\hline FREON 113 & $76-13-1$ & 0 & PPB & \\
\hline DICHLOROMETHANE & $75-09-2$ & 31 & PPB & \\
\hline 1,1-DICHLOROETHANE & $75-34-3$ & 0 & PPB & \\
\hline 2-BUTANONE & $78-93-3$ & 276 & PPB & \\
\hline N-HEXANE & $110-54-3$ & 17 & $\overline{\mathrm{PPB}}$ & \\
\hline 1,2-DICHLOROETHENE & $156-59-2$ & 0 & $\overline{\mathrm{PPB}}$ & \\
\hline CHLOROFORM & $67-66-3$ & 0 & PPB & \\
\hline TETRAHYDROFURAN & $109-99-9$ & 23 & PPB & \\
\hline 1,1,1-TRICHLOROETHANE & $71-55-6$ & 14 & PPB & \\
\hline 1,2-DICHLOROETHANE & $107-06-2$ & 4 & PPB & \\
\hline BENZENE & $71-43-2$ & 0 & PPB & \\
\hline CARBON TETRACHLORIDE & $56-23-5$ & 0 & PPB & \\
\hline CYCLOHEXANE & $110-82-7$ & 0 & PPB & \\
\hline N-BUTANOL & $71-36-3$ & 203 & PPB & \\
\hline TRICHLOROETHENE & $79-01-6$ & 5 & PPB & \\
\hline 4-METHYL-2-PENTANONE & $108-10-1$ & 189 & PPB & \\
\hline TOLUENE & $108-88-3$ & 9 & PPB & \\
\hline 1,1,2-TRICHLOROETHANE & $79-00-5$ & 0 & PPB & \\
\hline TETRACHLOROETHENE & $127-18-4$ & $\overline{0}$ & PPB & \\
\hline CHLOROBENZENE & $108-90-7$ & 2 & PPB & \\
\hline ETHYLBENZENE & $100-41-4$ & 3 & PPB & \\
\hline M-XYLENE & $108-38-3$ & 5 & PPB & \\
\hline STYRENE & $100-42-5$ & 4 & PPB & \\
\hline O-XYLENE & $95-47-6$ & 5 & PPB & \\
\hline 1,1,2,2-TETRACHLORETHANE & $79-34-5$ & 3 & PPB & \\
\hline 1,3,5-TRIMETHYLBENZENE & $108-67-8$ & 8 & PPB & \\
\hline 1,2,4-TRIMETHYLBENZENE & $95-63-6$ & 10 & PPB & \\
\hline 1,3-DICHLOROBENZENE & $541-73-1$ & 9 & PPB & \\
\hline 1,2-DICHLOROBENZENE & $95-50-1$ & 11 & PPB & \\
\hline 1,4-DICHLOROBENZENE & $106-46-7$ & 12 & PPB & \\
\hline
\end{tabular}


HNF-SD-WM-RPT-282 Rev. 0

\section{LIST OF COMPOUNDS TENTATIVELY IDENTIFIED WITH MASS SPECTRAL LIBRARY}

Sample: $\quad 2403$ WA

File: C:LHPCHEML2LDATAl61496_9.D

ID: FT6045-3WA.63A

Compound CAS\#

Ethanol

2-Propanone

2-Propanol

Methane, dichloro-

Butanal

1-Hexyn-3-ol

2-Butanone

Ethene, 1,2-dichloro-, (E)-

Furan, tetrahydro-

2-Butanol, 2-methyl-

Ethane, 1,1,1-trichloro-

Ethane, 1,2-dichloro-

1-Butanol

2-Pentanone

2-Pentanone, 4-methyl-

Benzene, methyl-
000064-17-5

000067-64-1

000067-63-0

000075-09-2

000123-72-8

000105-31-7

000078-93-3

000156-60-5

000109-99-9

000075-85-4

000071-55-6

000107-06-2

000071-36-3

000107-87-9

000108-10-1

000108-88-3 


\section{LIST OF COMPOUNDS TENTATIVELY IDENTIFIED WITH MASS SPECTRAL LIBRARY}

Sample: 2403 WA (Continued)

File: $\quad$ C:LHPCHEMI2LDATA161496_9.D

ID: $\quad$ FT6045-3WA.63A

Compound

CAS\#

2-Hexanone

000591-78-6

Hexanal

000066-25-1

Ethene, tetrachloro-

000127-18-4

Benzene, ethyl-

$000100-41-4$

Benzene, 1,3-dimethyl-

000108-38-3

3-Heptanone

000106-35-4

2-Heptanone

000110-43-0

Styrene

$000100-42-5$

Benzene, 1,3-dimethyl-

000108-38-3

Ethanol, 2-butoxy-

000111-76-2

Ethane, 1,1,2,2-tetrachloro-

000079-34-5

ALPHA.-PINENE, (-)-

000080-56-8

Benzaldehyde

000100-52-7

Benzene, 1,3,5-trimethyl-

000108-67-8

2-.BETA.-PINENE

000127-91-3

Benzene, 1,2,4-trimethyl-

000095-63-6 


\section{LIST OF COMPOUNDS TENTATIVELY IDENTIFIED WITH MASS SPECTRAL LIBRARY}

Sample: $\quad 2403$ WA (CONTINUED)

File: $\quad$ C:LHPCHEMLLDATAl61496_9.D

ID: FT6045-3WA.63A

Compound

CAS\#

Benzene, 1,4-dichloro-

000106-46-7

DELTA.3-Carene

013466-78-9

Benzene, 1,2-dichloro-

000095-50-1

Benzene, 1-ethyl-2,3-dimethyl-

00933-98-2

dl-Limonene

000138-86-3

Benzene, 1,2-dichloro-

000095-50-1

Ethanone, 1-phenyl-

000098-86-2

Nonanal

$000124-19-6$

Benzaldehyde, ethyl-

053951-50-1

Benzaldehyde, 3,4-dimethyl-

005973-71-7

Benzaldehyde, ethyl-

053951-50-1

Benzaldehyde, ethyl-

053951-50-1 
HNF-SD-WM-RPT-282 Rev. 0

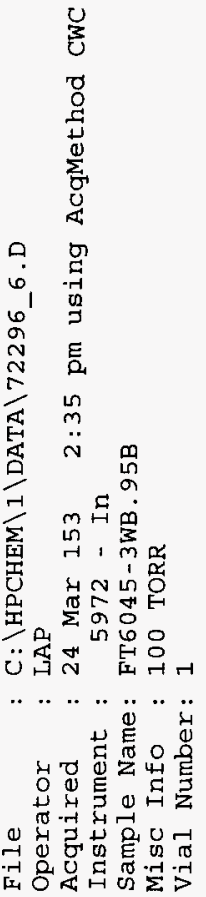

$M$
3
$m$
$o$
$\stackrel{\sim}{*}$
v

n

6

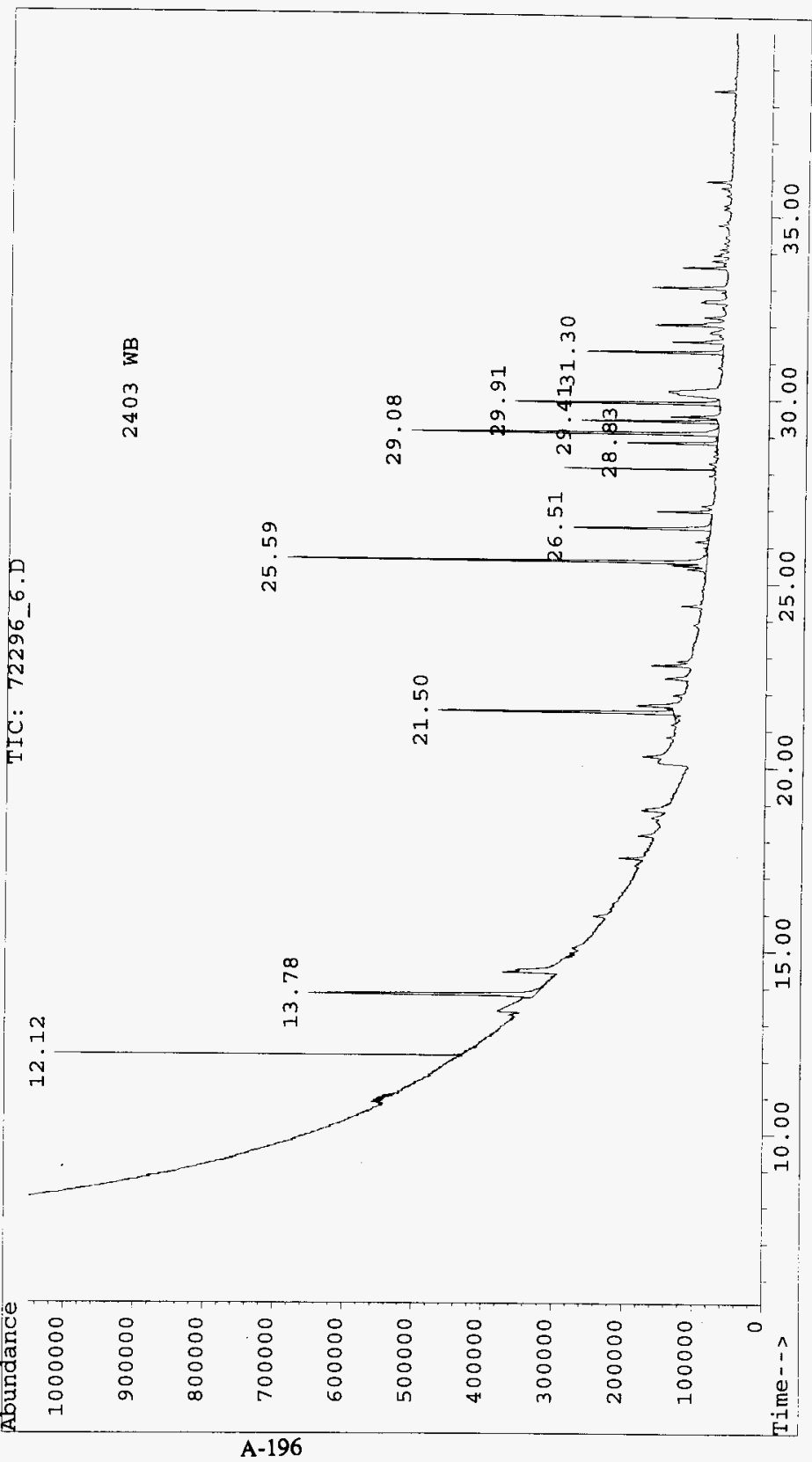


HNF-SD-WM-RPT-282 Rev. 0

CWC_REP.XLS

\section{ANALYSIS REPORT FOR CWC AIR SAMPLES}

File Name:

Analyst:

$72296 \_6.0$

Analysis Date:

LAP

Method:

\#VALUE!

Sample Name:

CWC

FT6045-3WB.95B

\begin{tabular}{|c|c|c|c|c|}
\hline COMPOUND NAME & CAS\# & Concentration & Units & Comments \\
\hline ISO-BUTANE & $75-28-5$ & 0 & PPB & \\
\hline VINYL CHLORIDE & $75-01-4$ & 0 & PPB & \\
\hline ETHANOL & $64-17-5$ & 9 & PPB & \\
\hline ACETONE & $67-64-1$ & 4 & PPB & \\
\hline 2-PROPANOL & $67-63-0$ & 0 & PPB & \\
\hline 1,1-DICHLOROETHENE & $75-35-4$ & 0 & PPB & \\
\hline FREON 113 & $76-13-1$ & 0 & PPB & \\
\hline DICHLOROMETHANE & $75-09-2$ & 0 & PPB & \\
\hline 1,1-DICHLOROETHANE & $75-34-3$ & 0 & PPB & \\
\hline 2-BUTANONE & $78-93-\overline{3}$ & 0 & PPB & \\
\hline N-HEXANE & $110-54-3$ & 0 & PPB & \\
\hline 1,2-DICHLOROETHENE & $156-59-2$ & $\overline{0}$ & PPB & \\
\hline CHLOROFORM & $67-66-3$ & 0 & PPB & \\
\hline TETRAHYDROFURAN & $109-99-9$ & 0 & PPB & \\
\hline 1,1,1-TRICHLOROETHANE & $71-55-6$ & 0 & PPB & \\
\hline 1,2-DICHLOROETHANE & $107-06-2$ & 0 & PPB & \\
\hline BENZENE & $71-43-2$ & 0 & PPB & \\
\hline CARBON TETRACHLORIDE & $56-23-5$ & 26 & PPB & \\
\hline CYCLOHEXANE & $110-82-7$ & 5 & PPB & \\
\hline N-BUTANOL & $71-36-3$ & 0 & PPB & \\
\hline TRICHLOROETHENE & $79-01-6$ & $\overline{0}$ & PPB & \\
\hline 4-METHYL-2-PENTANONE & $108-10-1$ & 0 & $\mathrm{PPB}$ & \\
\hline TOLUENE & $108-88-3$ & 2 & PPB & \\
\hline 1,1,2-TRICHLOROETHANE & $79-00-5$ & 0 & PPB & \\
\hline TETRACHLOROETHENE & $127-18-4$ & 0 & PPB & \\
\hline CHLOROBENZENE & $108-90-7$ & 0 & PPB & \\
\hline ETHYLBENZENE & $100-41-4$ & 3 & PPB & \\
\hline M-XYLENE & $108-38-3$ & 13 & PPB & \\
\hline STYRENE & $100-42-5$ & 0 & PPB & \\
\hline O-XYLENE & $95-47-6$ & 6 & PPB & \\
\hline 1,1,2,2-TETRACHLORETHANE & $79-34-5$ & 0 & PPB & \\
\hline 1,3,5-TRIMETHYLBENZENE & $108-67-8$ & 3 & PPB & \\
\hline 1,2,4-TRIMETHYLBENZENE & $95-63-6$ & 2 & PPB & \\
\hline 1,3-DICHLOROBENZENE & $541-73-1$ & 0 & PPB & \\
\hline 1,2-DICHLOROBENZENE & $95-50-1$ & 0 & PPB & \\
\hline 1,4-DICHLOROBENZENE & $106-46-7$ & 0 & PPB & \\
\hline
\end{tabular}


HNF-SD-WM-RPT-282 Rev. 0

\section{LIST OF COMPOUNDS TENTATIVELY IDENTIFIED WITH MASS SPECTRAL} LIBRARY

Sample: $\quad 2403$ WB

File: $\quad$ C:LHPCHEML2LDATA172296_6.D

ID: $\quad$ FT6045-3WB.95B

Compound

CAS\#

Benzene, ethyl-

$000100-41-4$

XYLENE

001330-20-7

ALPHA.-PINENE, (-)-

000080-56-8 
File

Operator

: C: \HPCHEM \I\DATA \70996_6.D

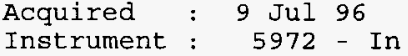

Sample Name: FT 6045 3WB.97A

Misc Info : Delta $\mathrm{P}^{-}=100$ TORR

Vial Number: 1

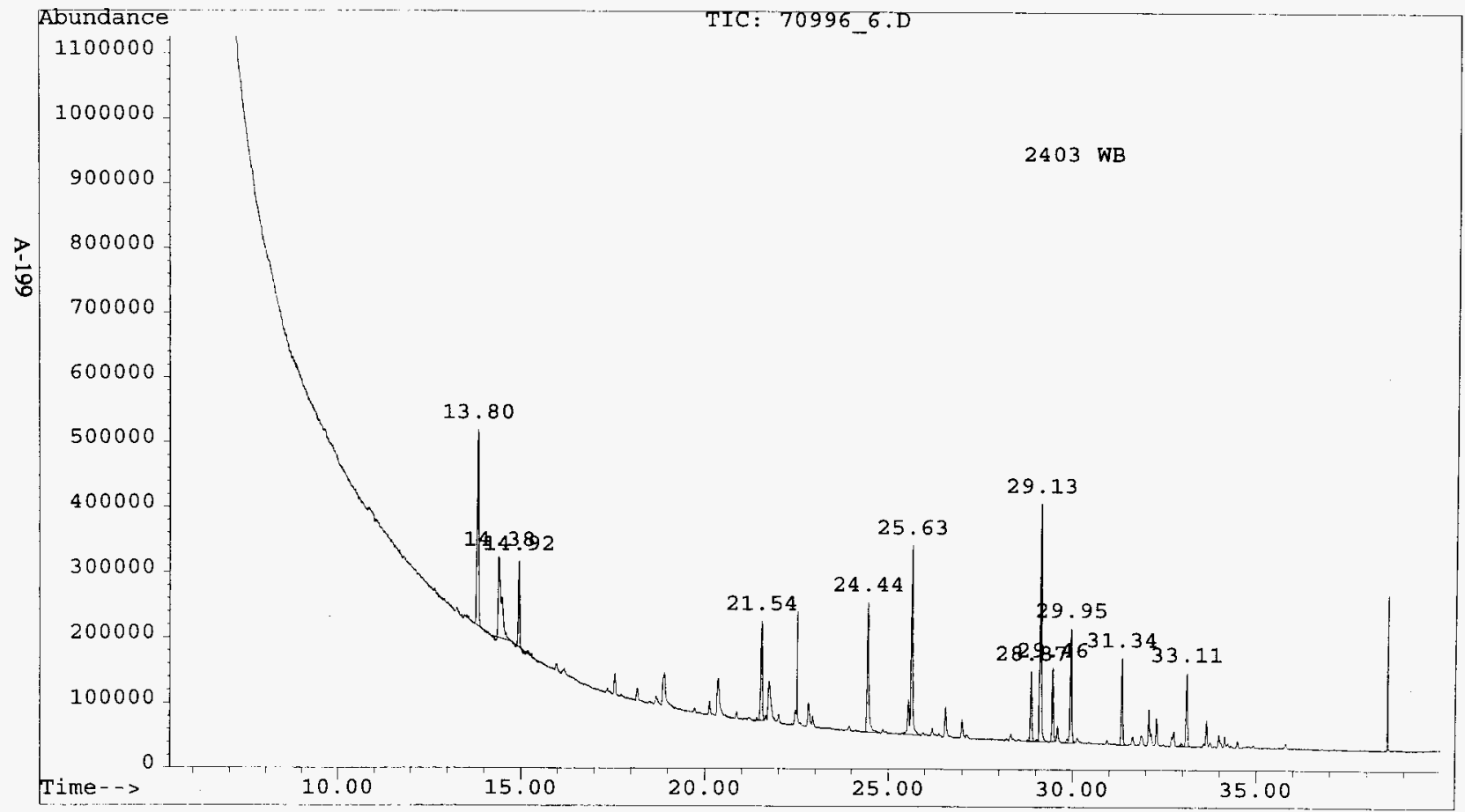


HNF-SD-WM-RPT-282 Rev. 0

CWC_REP.XLS

\section{ANALYSIS REPORT FOR CWC AIR SAMPLES}

File Name:

Analyst:

Analysis Date:

Method:

Sample Name:
70996_6.D

MS

07/09/96

CWC

FT 6045_3WB.97A

\begin{tabular}{|c|c|c|c|c|}
\hline COMPOUND NAME & CAS \# & Concentration & Units & Comments \\
\hline ISO-BUTANE & $75-28-5$ & 0 & PPB & \\
\hline VINYL CHLORIDE & $75-01-4$ & 0 & PPB & \\
\hline ETHANOL & $64-17-5$ & 0 & PPB & \\
\hline ACETONE & $67-64-1$ & 7 & PPB & \\
\hline 2-PROPANOL & $67-63-0$ & 0 & PPB & \\
\hline 1,1-DICHLOROETHENE & $75-35-4$ & 0 & PPB & \\
\hline FREON 113 & $76-13-1$ & 0 & PPB & \\
\hline DICHLOROMETHANE & $75-09-2$ & 0 & PPB & \\
\hline 1,1-DICHLOROETHANE & $75-34-3$ & 0 & PPB & \\
\hline 2-BUTANONE & $78-93-3$ & 0 & PPB & \\
\hline N-HEXANE & $110-54-3$ & 0 & PPB & \\
\hline 1,2-DICHLOROETHENE & $156-59-2$ & 0 & PPB & \\
\hline CHLOROFORM & $67-66-3$ & 0 & $\overline{\mathrm{PPB}}$ & \\
\hline TETRAHYDROFURAN & 109-99-9 & 0 & PPB & \\
\hline 1,1,1-TRICHLOROETHANE & $71-55-6$ & 0 & PPB & \\
\hline 1,2-DICHLOROETHANE & $107-06-2$ & 0 & PPB & \\
\hline BENZENE & $71-43-2$ & 0 & PPB & \\
\hline CARBON TETRACHLORIDE & $56-23-5$ & 10 & PPB & \\
\hline CYCLOHEXANE & $110-82-7$ & 0 & PPB & \\
\hline N-BUTANOL & $71-36-3$ & 0 & PPB & \\
\hline TRICHLOROETHENE & $79-01-6$ & 0 & PPB & \\
\hline 4-METHYL-2-PENTANONE & $108-10-1$ & 0 & PPB & \\
\hline TOLUENE & $108-88-3$ & 2 & PPB & \\
\hline 1,1,2-TRICHLOROETHANE & $79-00-5$ & 0 & PPB & \\
\hline TETRACHLOROETHENE & $127-18-4$ & 0 & PPB & \\
\hline CHLOROBENZENE & $108-90-7$ & 0 & PPB & \\
\hline ETHYLBENZENE & $100-41-4$ & 3 & PPB & \\
\hline M-XYLENE & $108-38-3$ & 11 & PPB & \\
\hline STYRENE & $100-42-5$ & 0 & PPB & \\
\hline O-XYLENE & $95-47-6$ & 5 & PPB & \\
\hline 1,1,2,2-TETRACHLORETHANE & $79-34-5$ & 0 & PPB & \\
\hline 1,3,5-TRIMETHYLBENZENE & $108-67-8$ & 1 & PPB & \\
\hline 1,2,4-TRIMETHYLBENZENE & $95-63-6$ & 3 & PPB & \\
\hline 1,3-DICHLOROBENZENE & $541-73-1$ & 0 & PPB & \\
\hline 1,2-DICHLOROBENZENE & $95-50-1$ & 0 & PPB & \\
\hline 1,4-DICHLOROBENZENE & $106-46-7$ & 0 & PPB & \\
\hline
\end{tabular}


HNF-SD-WM-RPT-282 Rev. 0

\section{LIST OF COMPOUNDS TENTATIVELY IDENTIFIED WITH MASS SPECTRAL LIBRARY}

Sample: $\quad 2403 \mathrm{WB}$

File: $\quad$ C:LHPCHEMLLDATA170996_6.D

ID: $\quad$ FT6045_3WB.97A

Compound

CAS\#

Methane, trichlorofluoro-

$000075-69-4$

Methane, tetrachloro-

000056-23-5

2-Pentanone, 4-methyl-

000108-10-1

Benzene, methyl-

000108-88-3

Benzene, ethyl-

$000100-41-4$

Benzene, 1,3-dimethyl-

000108-38-3

3-Heptanone

000106-35-4

ALPHA.-PINENE, (-)-

000080-56-8

Benzene, 1,3,5-trimethyl-

000108-67-8

DELTA.3-Carene

013466-78-9

Benzene, 4-ethyl-1,2-dimethyl-

000934-80-5 
File

: C: $\backslash$ HPCHEM $\backslash 1 \backslash D A T A \backslash 71096$ 6.D

Operator

LAP

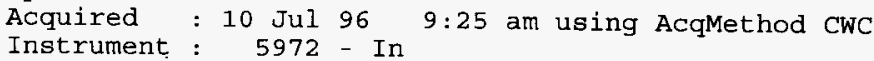

Sample Name: FT6045-3WB.98A

Misc Info : Delta $\mathrm{P}=100$ TORR

vial Number: 1

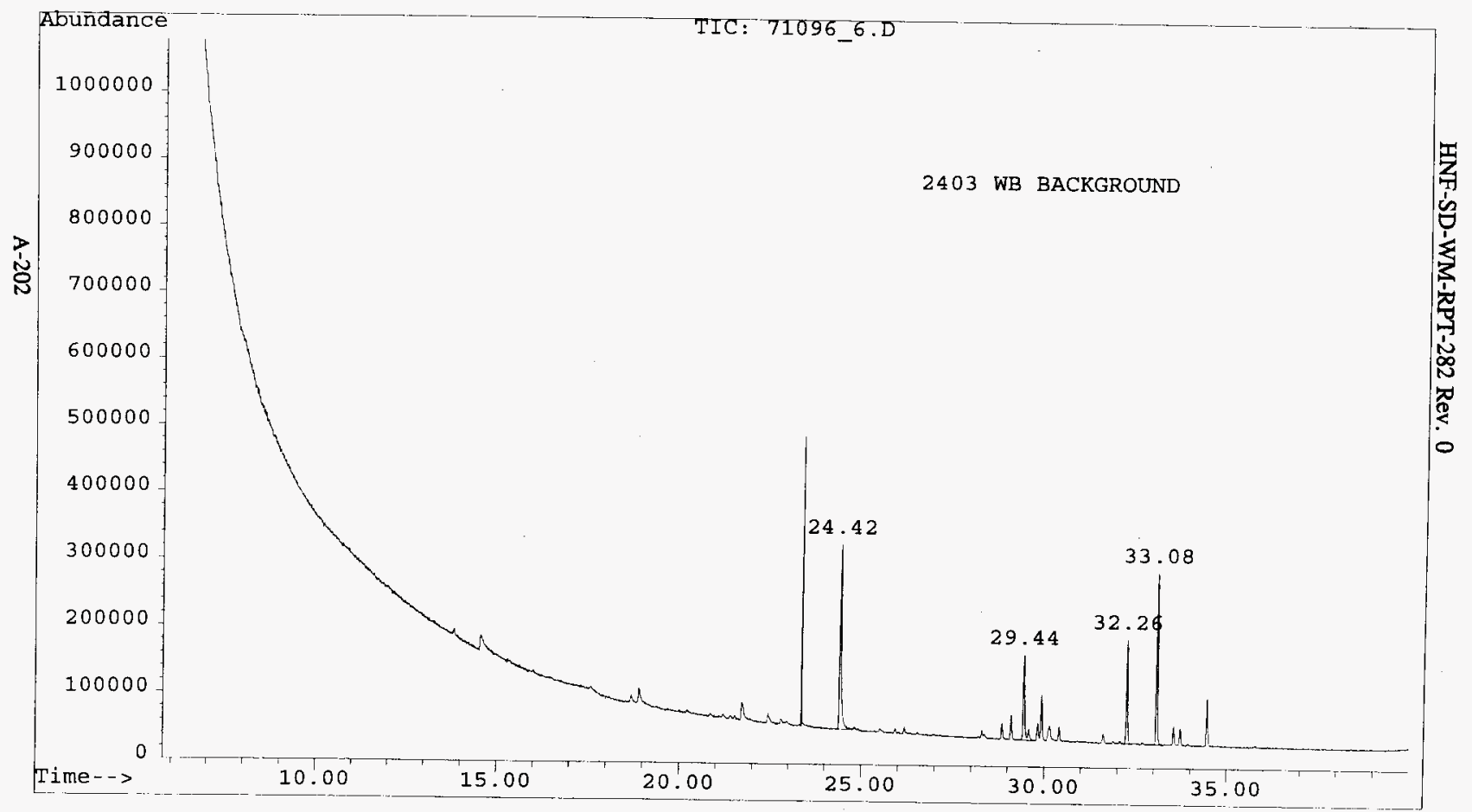


HNF-SD-WM-RPT-282 Rev. 0

CWC_REP.XLS

\title{
ANALYSIS REPORT FOR CWC AIR SAMPLES
}

File Name:

Analyst:

Analysis Date:

Method:

Sample Name:

\author{
71096_6.D \\ LAP \\ 07/10/96 \\ CWC
}

FT6045-3WB.98A

\begin{tabular}{|c|c|c|c|c|}
\hline COMPOUND NAME & CAS \# & Concentration & Units & Comments \\
\hline ISO-BUTANE & $75-28-5$ & $\overline{0}$ & $\overline{\overline{P P B}}$ & \\
\hline VINYL CHLORIDE & $75-01-4$ & 0 & PPB & \\
\hline ETHANOL & $64-17-5$ & 0 & PPB & \\
\hline ACETONE & $67-64-1$ & 0 & PPB & \\
\hline 2-PROPANOL & $67-63-0$ & 0 & PPB & \\
\hline 1,1-DICHLOROETHENE & $75-35-4$ & 0 & PPB & \\
\hline FREON 113 & $76-13-1$ & 0 & PPB & \\
\hline DICHLOROMETHANE & $75-09-2$ & 0 & PPB & \\
\hline 1,1-DICHLOROETHANE & $75-34-3$ & 0 & PPB & \\
\hline 2-BUTANONE & $78-93-3$ & 0 & PPB & \\
\hline N-HEXANE & $110-54-3$ & 0 & PPB & \\
\hline 1,2-DICHLOROETHENE & $156-59-2$ & 0 & PPB & \\
\hline CHLOROFORM & $67-66-3$ & 0 & PPB & \\
\hline TETRAHYDROFURAN & $109-99-9$ & 0 & PPB & \\
\hline 1,1,1-TRICHLOROETHANE & $71-55-6$ & 0 & PPB & \\
\hline 1,2-DICHLOROETHANE & $107-06-2$ & 0 & PPB & \\
\hline BENZENE & $71-43-2$ & 0 & PPB & \\
\hline CARBON TETRACHLORIDE & $56-23-5$ & 0 & $\overline{\text { PPB }}$ & \\
\hline CYCLOHEXANE & $110-82-7$ & 0 & PPB & \\
\hline N-BUTANOL & $71-36-3$ & 0 & PPB & \\
\hline TRICHLOROETHENE & $79-01-6$ & 0 & PPB & \\
\hline 4-METHYL-2-PENTANONE & $108-10-1$ & 0 & PPB & \\
\hline TOLUENE & $108-88-3$ & 0 & PPB & \\
\hline 1,1,2-TRICHLOROETHANE & $79-00-5$ & 0 & PPB & \\
\hline TETRACHLOROETHENE & $127-18-4$ & 0 & PPB & \\
\hline CHLOROBENZENE & $108-90-7$ & 0 & PPB & \\
\hline ETHYLBENZENE & $100-41-4$ & 0 & PPB & \\
\hline M-XYLENE & $108-38-3$ & 0 & PPB & \\
\hline STYRENE & $100-42-5$ & 0 & PPB & \\
\hline O-XYLENE & $95-47-6$ & 2 & PPB & \\
\hline $1,1,2,2$-TETRACHLORETHANE & $79-34-5$ & 0 & PPB & \\
\hline 1,3,5-TRIMETHYLBENZENE & $108-67-8$ & 4 & $\mathrm{PPB}$ & \\
\hline 1,2,4-TRIMETHYLBENZENE & $95-63-6$ & 7 & PPB & \\
\hline 1,3-DICHLOROBENZENE & $541-73-1$ & 0 & PPB & \\
\hline 1,2-DICHLOROBENZENE & $95-50-1$ & 0 & PPB & \\
\hline 1,4-DICHLOROBENZENE & $106-46-7$ & 2 & PPB & \\
\hline
\end{tabular}


HNF-SD-WM-RPT-282 Rev. 0

\section{LIST OF COMPOUNDS TENTATIVELY IDENTIFIED WITH MASS SPECTRAL}

\section{LIBRARY}

$\begin{array}{ll}\text { Sample: } & \text { 2403 WB } \\ \text { File: } & \text { C:UHPCHEM2LDATA171096_6.D } \\ \text { ID: } & \text { FT6045-3WB.98A }\end{array}$

Compound

CAS\#

2-Pentanone, 4-methyl-

000108-10-1

Benzene, ethyl-

$000100-41-4$

Benzene, 1,3-dimethyl-

000108-38-3

3-Heptanone

000106-35-4

Styrene

$000100-42-5$

Benzene, 1,4-dimethyl-

000106-42-3

Ethane, 1,1,2,2-tetrachloro-

000079-34-5

Benzene, 1,2,4-trimethyl-

000095-63-6

Benzene, 1,4-dichloro-

000106-46-7

Benzene, 1,2-dichloro-

000095-50-1 
File

: C: $\backslash$ HPCHEM \1 $\backslash \mathrm{DATA} \backslash 70996-7 . \mathrm{D}$

Operator : MS

Acquired : $9 \mathrm{Jul} 96$ 11:04 am using AcqMethod CWC

Instrument : 5972 - In

Sample Name: FT 6045 3WB.94A

Misc Info : Delta $\mathrm{P}^{-}=100$ TORR

Vial Number: 1

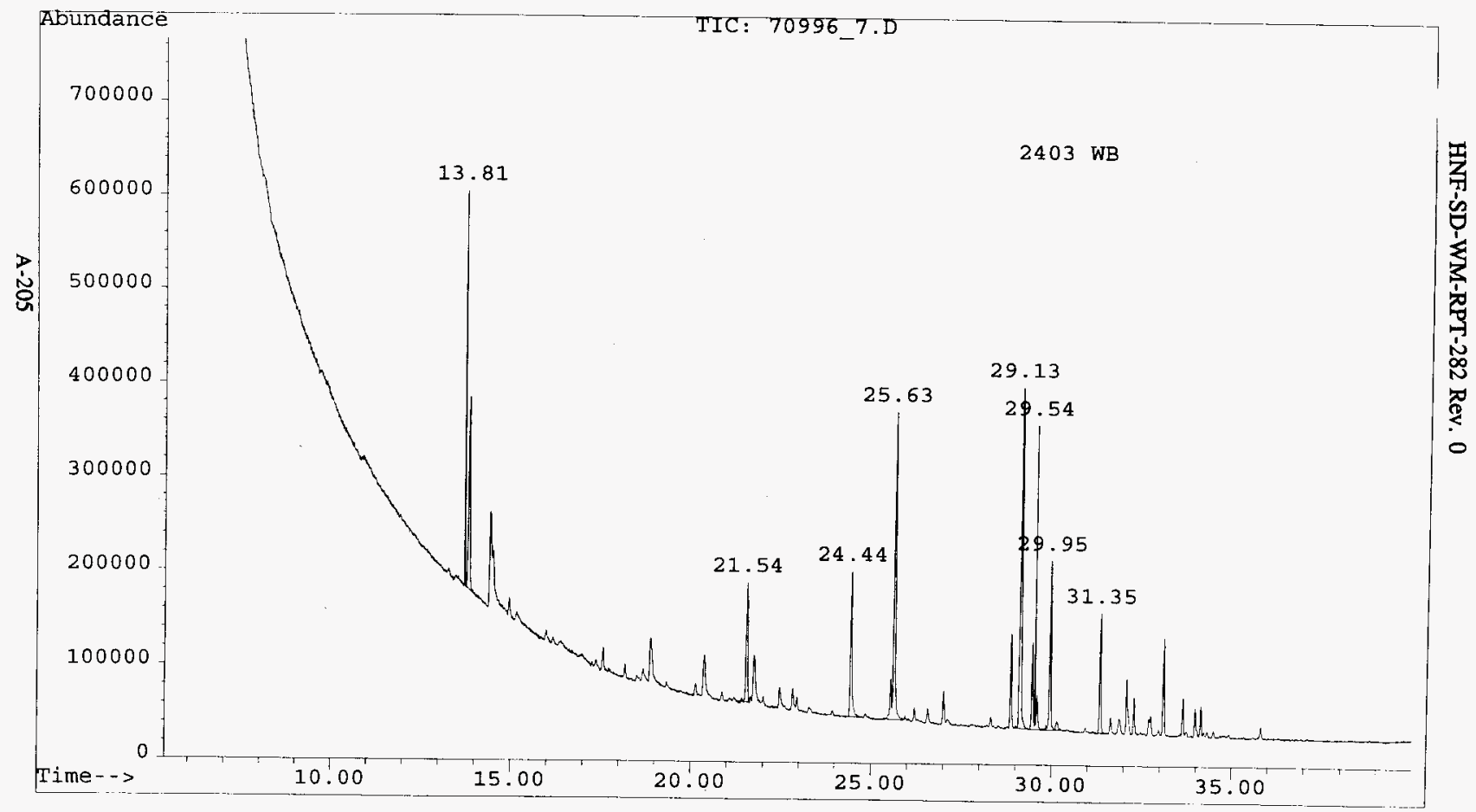


HNF-SD-WM-RPT-282 Rev. 0

CWC_REP.XLS

\section{ANALYSIS REPORT FOR CWC AIR SAMPLES}

File Name:

Analyst:

Analysis Date:

Method:

Sample Name:
70996_7.D

MS

07/09/96

CWC

FT 6045_3WB.94A

\begin{tabular}{|c|c|c|c|c|}
\hline COMPOUND NAME & CAS \# & Concentration & Unifs & Comments \\
\hline ISO-BUTANE & $75-28-5$ & 0 & PPB & \\
\hline VINYL CHLORIDE & $75-01-4$ & 0 & PPB & \\
\hline ETHANOL & $64-17-5$ & 0 & PPB & \\
\hline ACETONE & $67-64-1$ & 5 & PPB & \\
\hline 2-PROPANOL & $67-63-0$ & 0 & PPB & \\
\hline 1,1-DICHLOROETHENE & $75-35-4$ & 0 & PPB & \\
\hline FREON 113 & $76-13-1$ & 0 & PPB & \\
\hline DICHLOROMETHANE & $75-09-2$ & 0 & PPB & \\
\hline 1,1-DICHLOROETHANE & $75-34-3$ & 0 & PPB & \\
\hline 2-BUTANONE & 78-93-3 & 0 & $\mathrm{PPB}$ & \\
\hline N-HEXANE & $110-54-3$ & 0 & PPB & \\
\hline 1.2-DICHLOROETHENE & $156-59-2$ & 0 & PPB & \\
\hline CHLOROFORM & $67-66-3$ & 0 & PPB & \\
\hline TETRAHYDROFURAN & $109-99-9$ & 0 & PPB & \\
\hline 1,1,1-TRICHLOROETHANE & $71-55-6$ & 0 & $\mathrm{PPB}$ & \\
\hline 1,2-DICHLOROETHANE & $107-06-2$ & 0 & PPB & \\
\hline BENZENE & $71-43-2$ & 0 & PPB & \\
\hline CARBON TETRACHLORIDE & $56-23-5$ & 8 & PPB & \\
\hline CYCLOHEXANE & $110-82-7$ & 0 & PPB & \\
\hline N-BUTANOL & $71-36-3$ & 0 & PPB & \\
\hline TRICHLOROETHENE & $79-01-6$ & 0 & PPB & \\
\hline 4-METHYL-2-PENTANONE & $108-10-1$ & 0 & PPB & \\
\hline TOLUENE & $108-88-3$ & 1 & $\overline{\mathrm{PPB}}$ & \\
\hline 1,1,2-TRICHLOROETHANE & $79-00-5$ & 0 & PPB & \\
\hline TETRACHLOROETHENE & $127-18-4$ & 0 & $\mathrm{PPB}$ & \\
\hline CHLOROBENZENE & $108-90-7$ & 0 & PPB & \\
\hline ETHYLBENZENE & $100-41-4$ & 3 & $\mathrm{PPB}$ & \\
\hline M-XYLENE & $108-38-3$ & 11 & PPB & \\
\hline STYRENE & $100-42-5$ & 0 & PPB & \\
\hline O-XYLENE & $95-47-6$ & 5 & PPB & \\
\hline 1,1,2,2-TETRACHLORETHANE & $79-34-5$ & 0 & PPB & \\
\hline 1,3,5-TRIMETHYLBENZENE & $108-67-8$ & 2 & PPB & \\
\hline 1,2,4-TRIMETHYLBENZENE & $95-63-6$ & 3 & PPB & \\
\hline 1,3-DICHLOROBENZENE & $541-73-1$ & 0 & $\overline{\mathrm{PPB}}$ & \\
\hline 1,2-DICHLOROBENZENE & $95-50-1$ & 0 & PPB & \\
\hline 1,4-DICHLOROBENZENE & $106-46-7$ & 0 & PPB & \\
\hline
\end{tabular}


HNF-SD-WM-RPT-282 Rev. 0

\section{LIST OF COMPOUNDS TENTATIVELY IDENTIFIED WITH MASS SPECTRAL LIBRARY}

Sample: $\quad 2403$ WB

File: $\quad$ C:LHPCHEM 2LDATA170996_7.D

ID: FT6045_3WB.94A

Compound

CAS\#

Methane, trichlorofluoro-

$000075-69-4$

Methane, tetrachloro-

000056-23-5

2-Pentanone, 4-methyl-

000108-10-1

Benzene, methyl-

000108-88-3

Acetic acid, 2-methylpropyl ester

000110-19-0

Benzene, ethyl-

$000100-41-4$

Benzene, 1,4-dimethyl-

$000106-42-3$

3-Heptanone

000106-35-4

XYLENE

001330-20-7

ALPHA.-PINENE, (-)-

000080-56-8

Benzene, 1,2,3-trimethyl-

000526-73-8

2-.BETA.-PINENE

000127-91-3

DELTA.3-Carene

013466-78-9

Benzene, 1-ethyl-2,3-dimethyl-

000933-98-2

Cyclohexene, 1-methyl-4-(1-methylethe

005989-27-5 
File

Operator

$\mathrm{C}: \backslash \mathrm{HPCHEM} \backslash 1 \backslash \mathrm{DATA} \backslash \mathrm{CWC} \backslash S A M P L E S \backslash 71096 \_10 . \mathrm{D}$

Acquired

Instrument

12 Mar 153 10:55 pm using AcqMethod CWC

5972 - In

Sample Name: FT 6045 3WB.99A BK

Misc Info : 100 TORR

Vial Number: 1

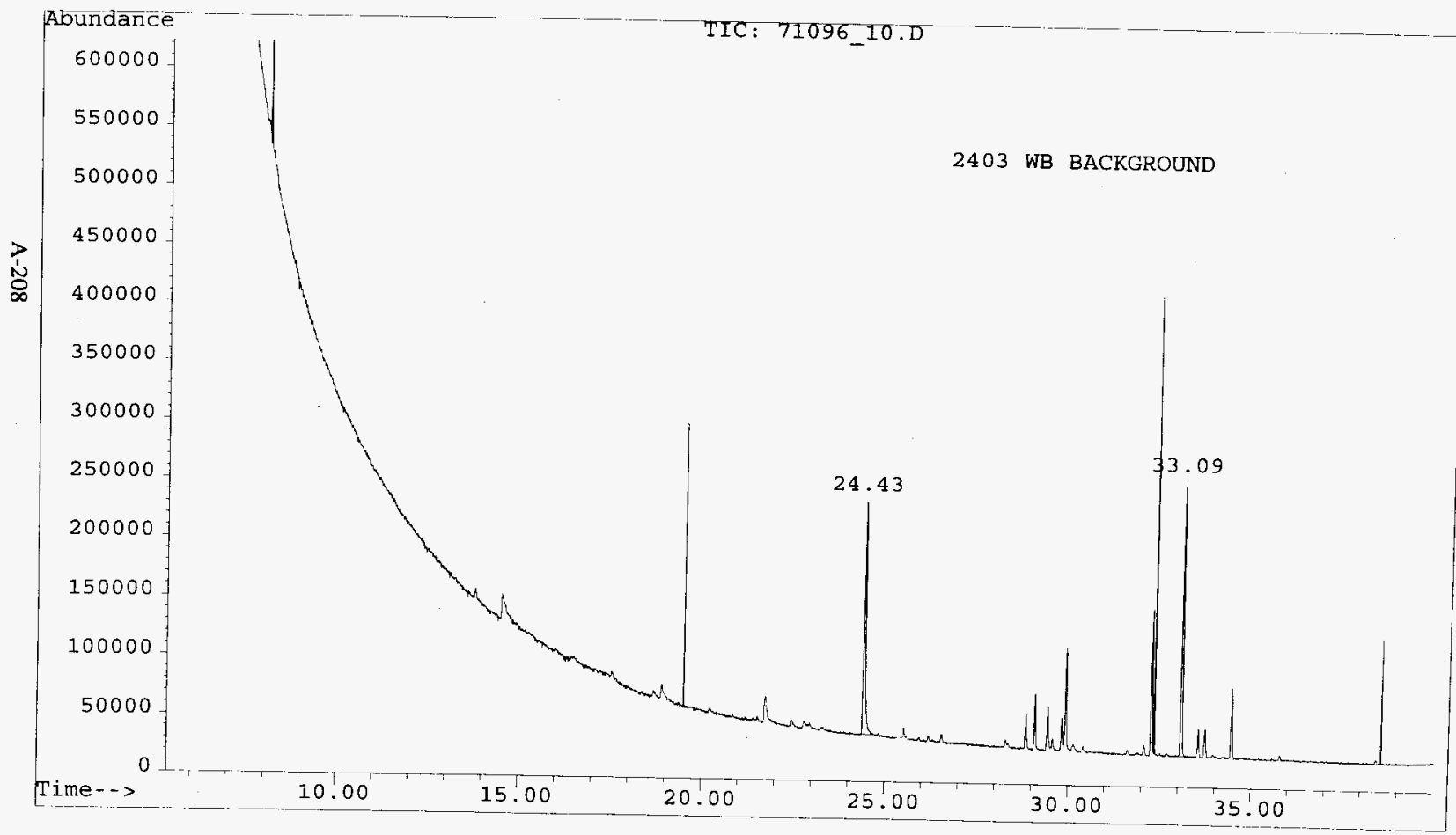


HNF-SD-WM-RPT-282 Rev. 0

CWC_REP.XLS

\section{ANALYSIS REPORT FOR CWC AIR SAMPLES}

File Name:

Analyst:

Analysis Date:

Method:

Sample Name:
71096_10.D

MS

\#VALUE!

CWC

FT 6045_3WB.99A BK

\begin{tabular}{|c|c|c|c|c|}
\hline COMPOUND NAME & CAS \# & Concentration & Units & Comments \\
\hline ISO-BUTANE & $75-28-5$ & $\overline{0}$ & $\overline{\mathrm{PPB}}$ & \\
\hline \begin{tabular}{|l} 
VINYL CHLORIDE \\
\end{tabular} & $75-01-4$ & 0 & PPB & \\
\hline ETHANOL & $64-17-5$ & 0 & PPB & \\
\hline ACETONE & $67-64-1$ & 0 & $\mathrm{PPB}$ & \\
\hline 2-PROPANOL & $67-63-0$ & 0 & PPB & \\
\hline 1,1-DICHLOROETHENE & $75-35-4$ & 0 & PPB & \\
\hline FREON 113 & $76-13-1$ & 0 & PPB & \\
\hline DICHLOROMETHANE & $75-09-2$ & 0 & PPB & \\
\hline 1,1-DICHLOROETHANE & $75-34-3$ & 0 & PPB & \\
\hline 2-BUTANONE & $78-93-3$ & 0 & PPB & \\
\hline N-HEXANE & $110-54-3$ & 0 & PPB & \\
\hline 1,2-DICHLOROETHENE & $156-59-2$ & 0 & PPB & \\
\hline CHLOROFORM & $67-66-3$ & 0 & PPB & \\
\hline TETRAHYDROFURAN & $109-99-9$ & 0 & $\mathrm{PPB}$ & \\
\hline 1,1,1-TRICHLOROETHANE & $71-55-6$ & 0 & PPB & \\
\hline 1,2-DICHLOROETHANE & $107-06-2$ & 0 & PPB & \\
\hline BENZENE & $71-43-2$ & 0 & PPB & \\
\hline CARBON TETRACHLORIDE & $56-23-5$ & 0 & PPB & \\
\hline CYCLOHEXANE & $110-82-7$ & 0 & PPB & \\
\hline N-BUTANOL & $71-36-3$ & 0 & PPB & \\
\hline TRICHLOROETHENE & $79-01-6$ & 0 & PPB & \\
\hline 4-METHYL-2-PENTANONE & $108-10-1$ & 0 & PPB & \\
\hline TOLUENE & $108-88-3$ & 0 & PPB & \\
\hline 1,1,2-TRICHLOROETHANE & $79-00-5$ & 0 & PPB & \\
\hline TETRACHLOROETHENE & $127-18-4$ & 0 & PPB & \\
\hline CHLOROBENZENE & $108-90-7$ & 0 & PPB & \\
\hline ETHYLBENZENE & $100-41-4$ & 1 & PPB & \\
\hline M-XYLENE & $108-38-3$ & 0 & PPB & \\
\hline STYRENE & $100-42-5$ & 0 & PPB & \\
\hline O-XYLENE & $95-47-6$ & 2 & PPB & \\
\hline 1,1,2,2-TETRACHLORETHANE & $79-34-5$ & 0 & PPB & \\
\hline 1,3,5-TRIMETHYLBENZENE & $108-67-8$ & 3 & PPB & \\
\hline 1,2,4-TRIMETHYLBENZENE & $95-63-6$ & 6 & PPB & \\
\hline 1,3-DICHLOROBENZENE & $541-73-1$ & 0 & PPB & \\
\hline 1,2-DICHLOROBENZENE & $95-50-1$ & 0 & PPB & \\
\hline 1,4-DICHLOROBENZENE & $106-46-7$ & 2 & PPB & \\
\hline
\end{tabular}




\section{LIST OF COMPOUNDS TENTATIVELY IDENTIFIED WITH MASS SPECTRAL}

\section{LIBRARY}

Sample: $\quad 2403$ WB BACKGROUND

File:

C:LHPCHEMLLDATA171096_10.D

ID:

FT6045_3WB.99A BK

Compound

CAS\#

2-Pentanone, 4-methyl-

Benzene, ethyl-

XYLENE

3-Heptanone

Styrene

Benzene, 1,3-dimethyl-

Benzene, 1,3,5-trimethyl-

Benzene, 1,2,4-trimethyl-

Benzene, 1,4-dichloro-

Benzene, 1,3-dichloro-
000108-10-1

000100-41-4

001330-20-7

000106-35-4

$000100-42-5$

000108-38-3

000108-67-8

000095-63-6

000106-46-7

000541-73-1 
File

Operator

: C $: \backslash H P C H E M \backslash 1 \backslash D A T A \backslash C W C \backslash S A M P L E S \backslash 71096 \_11 . D$

Acquired

: MS

Instrument

12 Mar 153 11:55 pm using AcqMethod CWC

Sample Name: FT 6045_3WB.96A

Misc Info: 100 TORR

Vial Number: 1

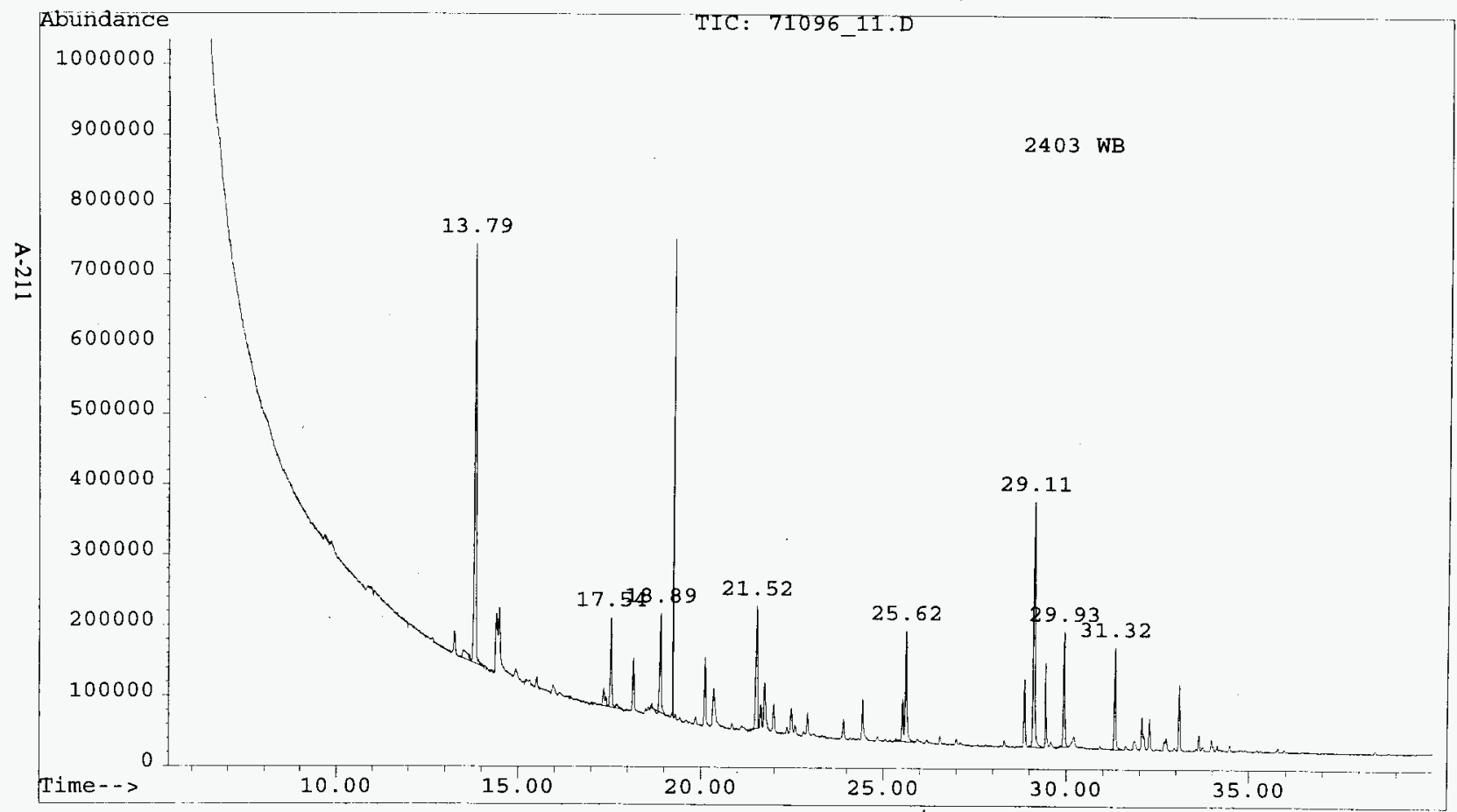


HNF-SD-WM-RPT-282 Rev. 0

CWC_REP.XLS

\title{
ANALYSIS REPORT FOR CWC AIR SAMPLES
}

File Name:

Analyst:

Analysis Date:

Method:

Sample Name:

\author{
71096_11.D \\ MS \\ \#VALUE! \\ CWC \\ FT 6045_3WB.96A
}

\begin{tabular}{|c|c|c|c|c|}
\hline COMPOUND NAME & CAS \# & Concentration & Units & Comments \\
\hline ISO-BUTANE & $75-28-5$ & $\overline{0}$ & PPB & \\
\hline VINYL CHLORIDE & $75-01-4$ & 0 & PPB & \\
\hline ETHANOL & $64-17-5$ & 0 & PPB & \\
\hline ACETONE & $67-64-1$ & 4 & PPB & \\
\hline 2-PROPANOL & $67-63-0$ & 0 & $\overline{\mathrm{PPB}}$ & \\
\hline 1,1-DICHLOROETHENE & $75-35-4$ & 0 & PPB & \\
\hline FREON 113 & $76-13-1$ & 0 & PPB & \\
\hline DICHLOROMETHANE & $75-09-2$ & 0 & PPB & \\
\hline 1,1-DICHLOROETHANE & $75-34-3$ & 0 & PPB & \\
\hline 2-BUTANONE & 78-93-3 & 0 & PPB & \\
\hline N-HEXANE & $110-54-3$ & 3 & $\mathrm{PPB}$ & \\
\hline 1,2-DICHLOROETHENE & $156-59-2$ & 0 & PPB & \\
\hline CHLOROFORM & $67-66-3$ & 0 & PPB & \\
\hline TETRAHYDROFURAN & $109-99-9$ & 0 & PPB & \\
\hline 1,1,1-TRICHLOROETHANE & $71-55-6$ & 0 & PPB & \\
\hline 1,2-DICHLOROETHANE & $107-06-2$ & 0 & PPB & \\
\hline BENZENE & $71-43-2$ & 4 & PPB & \\
\hline CARBON TETRACHLORIDE & $56-23-5$ & 10 & PPB & \\
\hline CYCLOHEXANE & $110-82-7$ & 4 & PPB & \\
\hline N-BUTANOL & $71-36-3$ & 4 & PPB & \\
\hline TRICHLOROETHENE & $79-01-6$ & 0 & PPB & \\
\hline 4-METHYL-2-PENTANONE & $108-10-1$ & 0 & PPB & \\
\hline TOLUENE & $108-88-3$ & 2 & PPB & \\
\hline 1,1,2-TRICHLOROETHANE & $79-00-5$ & 0 & PPB & \\
\hline TETRACHLOROETHENE & $127-18-4$ & 0 & PPB & \\
\hline CHLOROBENZENE & $108-90-7$ & 0 & PPB & \\
\hline ETHYLBENZENE & $100-41-4$ & 3 & PPB & \\
\hline M-XYLENE & $108-38-3$ & 11 & PPB & \\
\hline STYRENE & $100-42-5$ & 0 & PPB & \\
\hline O-XYLENE & $95-47-6$ & 5 & PPB & \\
\hline 1,1,2,2-TETRACHLORETHANE & $79-34-5$ & 0 & PPB & \\
\hline 1,3,5-TRIMETHYLBENZENE & $108-67-8$ & 1 & PPB & \\
\hline 1,2,4-TRIMETHYLBENZENE & $95-63-6$ & 2 & PPB & \\
\hline 1,3-DICHLOROBENZENE & $541-73-1$ & 0 & PPB & \\
\hline 1,2-DICHLOROBENZENE & $95-50-1$ & 0 & PPB & \\
\hline 1,4-DICHLOROBENZENE & $106-46-7$ & $\underline{0}$ & PPB & \\
\hline
\end{tabular}


HNF-SD-WM-RPT-282 Rev. 0

\section{LIST OF COMPOUNDS TENTATIVELY IDENTIFIED WITH MASS SPECTRAL LIBRARY}

Sample: $\quad 2403$ WB

File: $\quad$ C:LHPCHEMLLDATA171096_11.D

ID: $\quad$ FT6045_3WB.96A

Compound

CAS\#

Methane, trichlorofluoro-

$000075-69-4$

Pentane, 2-methyl-

$000107-83-5$

Hexane

000110-54-3

1-Propanol, 2-methyl-

000078-83-1

Methane, tetrachloro-

000056-23-5

Hexane, 3-methyl-

000589-34-4

Cyclohexane, methyl-

000108-87-2

2-Pentanone, 4-methyl-

000108-10-1

Benzene, methyl-

000108-88-3

Acetic acid, 2-methylpropyl ester

000110-19-0

Benzene, ethyl-

000100-41-4

Benzene, 1,3-dimethyl-

000108-38-3

Benzene, 1,4-dimethyl-

$000106-42-3$

ALPHA.-PINENE, (-)-

$000080-56-8$

Benzene, 1,3,5-trimethyl-

$000108-67-8$

1-.beta.-Pinene

$018172-67-3$

Benzene, 1,2,3-trimethyl-

$000526-73-8$

Benzene, 1-methyl-2-(1-methylethyl)-

$000527-84-4$ 
File

$C: \backslash H P C H E M \backslash 1 \backslash D A T A \backslash C W C \backslash S A M P I E S \backslash 71096 \_13 . D$

Operator

MS

Acquired

: 13 Mar 153

Instrument : 5972 - In

Sample Name: FT 6045 3WB.95A

Misc Info: 100 TOR $\bar{R}$

Vial Number: 1

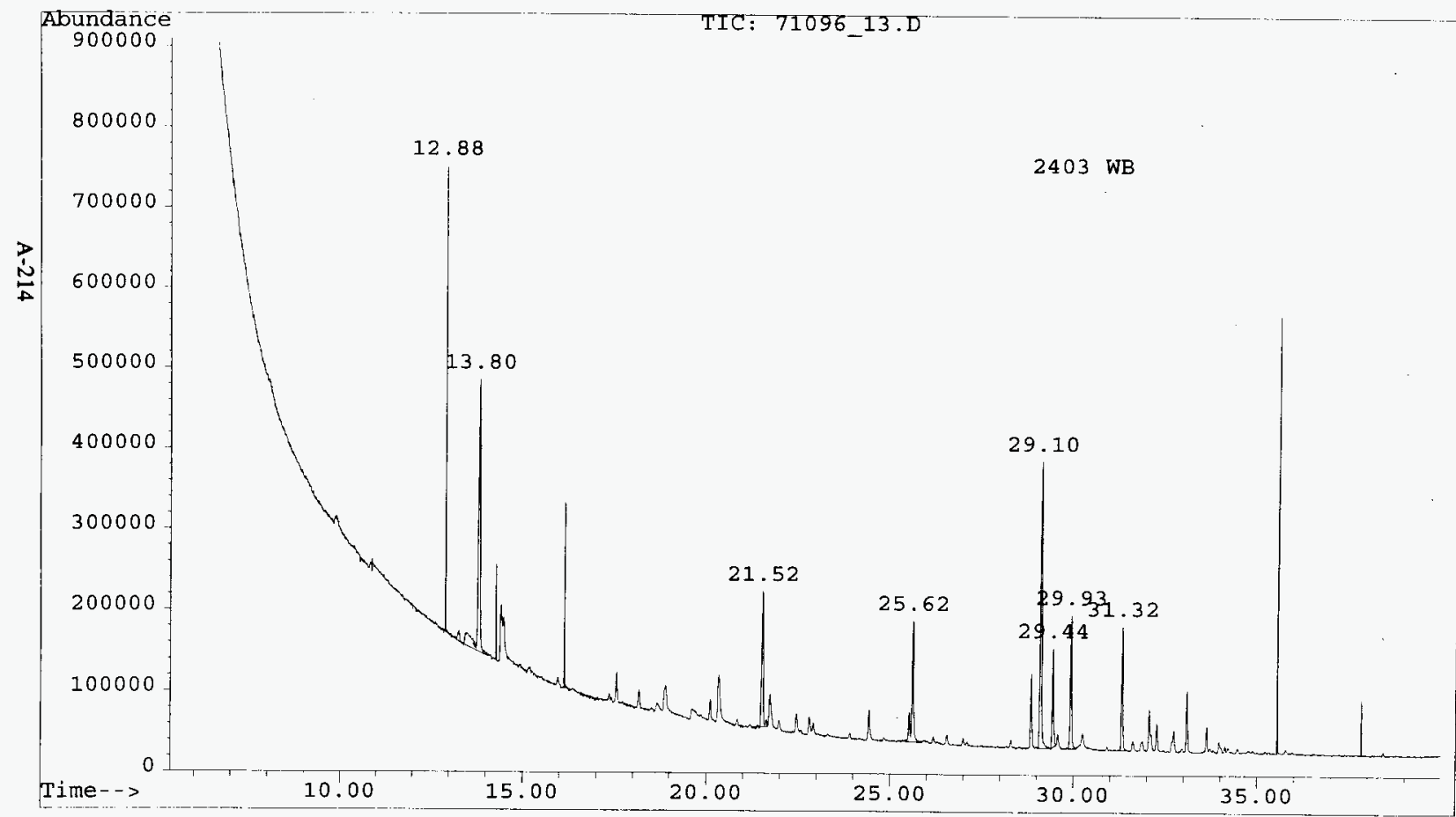


HNF-SD-WM-RPT-282 Rev. 0

CWC_REP.XLS

\section{ANALYSIS REPORT FOR CWC AIR SAMPLES}

File Name:

Analyst:

Analysis Date:

Method:

Sample Name:
71096_13.D

MS

\#VALUE!

CWC

FT 6045_3WB.95A

\begin{tabular}{|c|c|c|c|c|}
\hline COMPOUND NAME & CAS \# & Concentration & Units & Comments \\
\hline ISO-BUTANE & $75-28-5$ & $\overline{0}$ & $\overline{P P B}$ & \\
\hline VINYL CHLORIDE & $75-01-4$ & 0 & PPB & \\
\hline ETHANOL & $64-17-5$ & 0 & PPB & \\
\hline ACETONE & $67-64-1$ & 4 & PPB & \\
\hline 2-PROPANOL & $67-63-0$ & 0 & PPB & \\
\hline 1,1-DICHLOROETHENE & $75-35-4$ & 0 & PPB & \\
\hline FREON 113 & $76-13-1$ & 0 & $\overline{\mathrm{PPB}}$ & \\
\hline DICHLOROMETHANE & $75-09-2$ & 0 & PPB & \\
\hline 1,1-DICHLOROETHANE & $75-34-3$ & 0 & PPB & \\
\hline 2-BUTANONE & 78-93-3 & 0 & PPB & \\
\hline $\mathrm{N}$-HEXANE & $110-54-3$ & 0 & PPB & \\
\hline 1,2-DICHLOROETHENE & $156-59-2$ & 0 & PPB & \\
\hline CHLOROFORM & $67-66-3$ & 0 & PPB & \\
\hline TETRAHYDROFURAN & $109-99-9$ & 0 & PPB & \\
\hline 1,1,1-TRICHLOROETHANE & $71-55-6$ & 0 & $\mathrm{PPB}$ & \\
\hline 1,2-DICHLOROETHANE & $107-06-2$ & 0 & PPB & \\
\hline BENZENE & $71-43-2$ & 0 & $\mathrm{PPB}$ & \\
\hline CARBON TETRACHLORIDE & $56-23-5$ & 13 & PPB & \\
\hline CYCLOHEXANE & $110-82-7$ & 0 & PPB & \\
\hline N-BUTANOL & $71-36-3$ & 0 & PPB & \\
\hline TRICHLOROETHENE & $79-01-6$ & 0 & PPB & \\
\hline 4-METHYL-2-PENTANONE & $108-10-1$ & 0 & PPB & \\
\hline TOLUENE & $108-88-3$ & 1 & PPB & \\
\hline 1,1,2-TRICHLOROETHANE & $79-00-5$ & 0 & PPB & \\
\hline TETRACHLOROETHENE & $127-18-4$ & 0 & PPB & \\
\hline CHLOROBENZENE & $108-90-7$ & 0 & PPB & \\
\hline ETHYLBENZENE & $100-41-4$ & 2 & PPB & \\
\hline M-XYLENE & $108-38-3$ & 11 & PPB & \\
\hline STYRENE & $100-42-5$ & 0 & PPB & \\
\hline O-XYLENE & $95-47-6$ & 5 & PPB & \\
\hline 1,1,2,2-TETRACHLORETHANE & $79-34-5$ & 0 & PPB & \\
\hline 1,3,5-TRIMETHYLBENZENE & $108-67-8$ & 2 & PPB & \\
\hline 1,2,4-TRIMETHYLBENZENE & $95-63-6$ & 2 & PPB & \\
\hline 1,3-DICHLOROBENZENE & $541-73-1$ & 0 & PPB & \\
\hline 1,2-DICHLOROBENZENE & $95-50-1$ & 0 & PPB & \\
\hline 1,4-DICHLOROBENZENE & $106-46-7$ & 0 & PPB & \\
\hline
\end{tabular}


HNF-SD-WM-RPT-282 Rev. 0

\section{LIST OF COMPOUNDS TENTATIVELY IDENTIFIED WITH MASS SPECTRAL}

\section{LIBRARY}

$\begin{array}{ll}\text { Sample: } & \text { 2403 WB } \\ \text { File: } & \text { C:UHPCHEMLLDATA171096_13.D } \\ \text { D: } & \text { FT6045_3WB.95A }\end{array}$

Compound

CAS\#

Methane, trichlorofluoro-

$000075-69-4$

1-Propanol, 2-methyl-

$000078-83-1$

Methane, tetrachloro-

000056-23-5

2-Pentanone, 4-methyl-

000108-10-1

Benzene, methyl-

000108-88-3

Acetic acid, 2-methylpropyl ester

000110-19-0

Benzene, ethyl-

$000100-41-4$

Benzene, 1,4-dimethyl-

$000106-42-3$

3-Heptanone

000106-35-4

Benzene, 1,3-dimethyl-

000108-38-3

ALPHA.-PINENE, (-)-

000080-56-8

Benzene, 1,2,3-trimethyl-

000526-73-8

2-.BETA.-PINENE

000127-91-3

Benzene, 1,2,4-trimethyl-

000095-63-6

DELTA.3-Carene

013466-78-9 
$\begin{array}{ll}\text { File } & : \text { C: } \backslash \text { HPCHEM } \backslash 1 \backslash \text { DATA } \backslash 72296 \_4 . D \\ \text { Operator } & : \text { LAP } \\ \text { Acquired } & : 24 \text { Mar } 15312: 46 \text { pm using AcqMethod CWC } \\ \text { Instrument }: 5972-\text { In } & \end{array}$

Sample Name: FT6045-3WC.86B

Misc Info : 100 TORR

Vial Number: 1

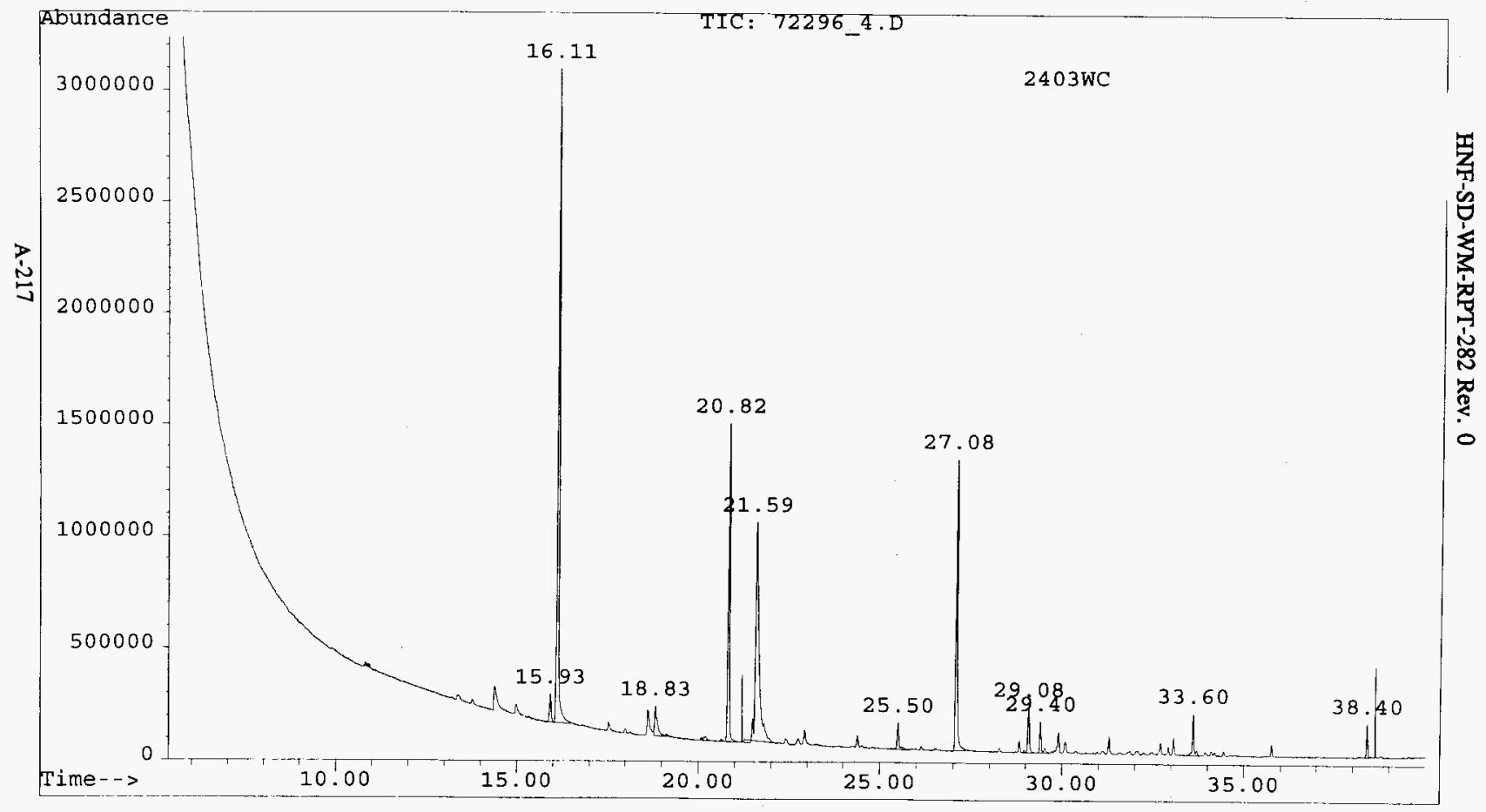


HNF-SD-WM-RPT-282 Rev. 0

CWC_REP.XLS

\section{ANALYSIS REPORT FOR CWC AIR SAMPLES}

File Name:

Analyst:

Analysis Date:

Method:

72296_4.D

Sample Name:

LAP

\#VALUE!

CWC

FT6045-3WC.86B

\begin{tabular}{|c|c|c|c|c|}
\hline COMPOUND NAME & CÁS \# & Concentration & Units & Comments \\
\hline ISO-BUTANE & $75-28-5$ & 0 & $\overline{\mathrm{PPB}}$ & \\
\hline VINYLCHLORIDE & $75-01-4$ & 0 & PPB & \\
\hline ETHANOL & $64-17-5$ & 3 & $\mathrm{PPB}$ & \\
\hline ACETONE & $67-64-1$ & 6 & $\overline{\mathrm{PPB}}$ & \\
\hline 2-PROPANOL & $67-63-0$ & 2 & PPB & \\
\hline 1,1-DICHLOROETHENE & $75-35-4$ & 0 & PPB & \\
\hline FREON 113 & $76-13-1$ & 5 & PPB & \\
\hline DICHLOROMETHANE & $75-09-2$ & 107 & PPB & \\
\hline 1,1-DICHLOROETHANE & $75-34-3$ & 0 & PPB & \\
\hline 2-BUTANONE & $78-93-3$ & 18 & PPB & \\
\hline N-HEXANE & $110-54-3$ & 0 & PPB & \\
\hline 1,2-DICHLOROETHENE & $156-59-2$ & 0 & PPB & \\
\hline CHLOROFORM & $67-66-3$ & 0 & PPB & \\
\hline TETRAHYDROFURAN & $109-99-9$ & 0 & PPB & \\
\hline 1,1,1-TRICHLOROETHANE & $71-55-6$ & 62 & $\mathrm{PPB}$ & \\
\hline 1,2-DICHLOROETHANE & $107-06-2$ & 5 & PPB & \\
\hline BENZENE & $71-43-2$ & 0 & $\overline{\mathrm{PPB}}$ & \\
\hline CARBON TETRACHLORIDE & $56-23-5$ & 8 & PPB & \\
\hline CYCLOHEXANE & $110-82-7$ & 0 & PPB & \\
\hline N-BUTANOL & $71-36-3$ & 96 & $\mathrm{PPB}$ & \\
\hline TRICHLOROETHENE & $79-01-6$ & 0 & PPB & \\
\hline 4-METHYL-2-PENTANONE & $108-10-1$ & 0 & PPB & \\
\hline TOLÜENE & $108-88-3$ & 4 & PPB & \\
\hline 1,1,2-TRICHLOROETHANE & $79-00-5$ & 0 & PPB & \\
\hline TETRACHLOROETHENE & $127-18-4$ & $5 \overline{8}$ & $\overline{\mathrm{PPB}}$ & \\
\hline CHLOROBENZENE & $108-90-7$ & 0 & PPB & \\
\hline ETHYLBENZENE & $100-41-4$ & 1 & PPB & \\
\hline M-XYLENE & $108-38-3$ & 6 & $\overline{P P B}$ & \\
\hline STYRENE & $100-42-5$ & 0 & PPB & \\
\hline O-XYLENE & $95-47-6$ & 3 & $\mathrm{PPB}$ & \\
\hline 1,1,2,2-TETRACHLORETHANE & $79-34-5$ & 0 & PPB & \\
\hline 1,3,5-TRIMETHYLBENZENE & $108-67-8$ & 0 & PPB & \\
\hline 1,2,4-TRIMETHYLBENZENE & $95-63-6$ & 2 & $\mathrm{PPB}$ & \\
\hline 1,3-DICHLOROBENZENE & $541-73-1$ & 0 & $\overline{\mathrm{PPB}}$ & \\
\hline 1,2-DICHLOROBENZENE & $95-50-1$ & 0 & PPB & \\
\hline 1,4-DICHLOROBENZENE & $106-46-7$ & 0 & $\mathrm{PPB}$ & \\
\hline
\end{tabular}


HNF-SD-WM-RPT-282 Rev. 0

\section{LIST OF COMPOUNDS TENTATIVELY WENTIFIED WITH MASS SPECTRAL LIBRARY}

Sample: $\quad 2403$ WC

File: $\quad$ C:LHPCHEML2LATA172296_4.D

ID: $\quad$ FT6045-3WC.86B

Compound

CAS\#

Bicyclo[2.2.1]heptan-2-one, 1,7,7-tri

$013559-66-5$

Methane, dichloro-

000075-09-2

Ethane, 1,1,1-trichloro-

$000071-55-6$

1-Bromobicyclo[4.3.1]decan-8-one

070576-35-1

1-Butanol

$000071-36-3$

Ethene, trichloro-

$000079-01-6$

Benzene, methyl-

000108-88-3

Ethene, tetrachloro-

000127-18-4

Benzene, ethyl-

$000100-41-4$

Benzene, 1,3-dimethyl-

000108-38-3

3-Heptanone

000106-35-4

XYLENE

001330-20-7

ALPHA.-PINENE, (-)-

$000080-56-8$

2-.BETA.-PINENE

000127-91-3

Benzene, 1,3,5-trimethyl-

000108-67-8

DELTA.3-Carene

013466-78-9

Decane

000124-18-5

Dodecane

$000112-40-3$ 
File

Operator

Acquired

Instrument

Sample Name:

: Delta $\mathrm{P}^{-}=100$ TORR

Vial Number: 1

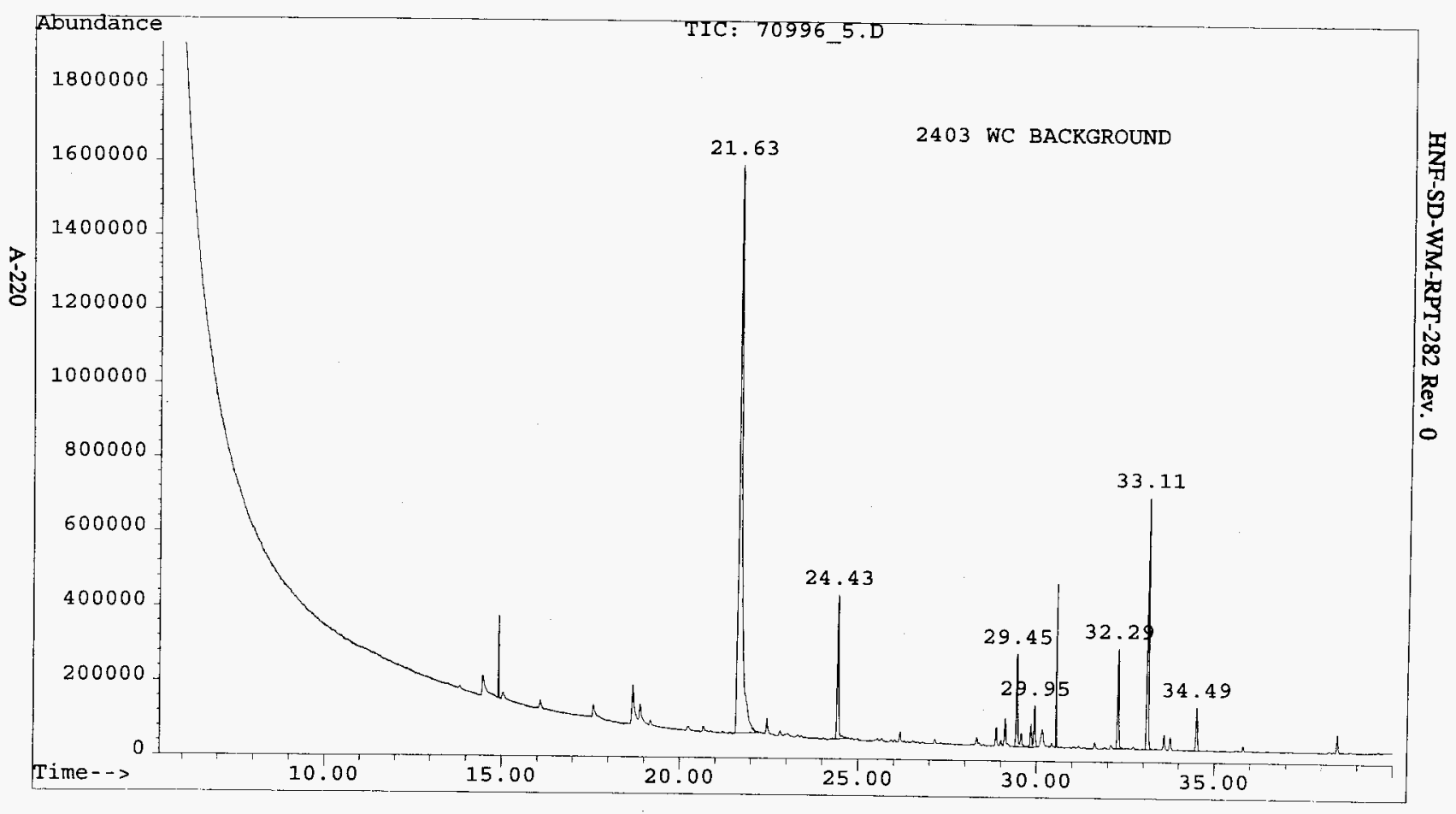


HNF-SD-WM-RPT-282 Rev. 0

CWC_REP.XLS

\section{ANALYSIS REPORT FOR CWC AIR SAMPLES}

File Name:

Analyst:

Analysis Date:

Method:

Sample Name:
70996_5.D

LAP

07/09/96

CWC

FT 6045_3WC.88A BK

\begin{tabular}{|c|c|c|c|c|}
\hline COMPOUND NAME & CAS \# & Concentration & Units & Comments \\
\hline ISO-BUTANE & $75-28-5$ & $\overline{0}$ & PPB & \\
\hline VINYL CHLORIDE & $75-01-4$ & 0 & PPB & \\
\hline ETHANOL & $64-17-5$ & 0 & PPB & \\
\hline ACETONE & $67-64-1$ & 3 & PPB & \\
\hline 2-PROPANOL & $67-63-0$ & 0 & PPB & \\
\hline 1,1-DICHLOROETHENE & $75-35-4$ & 0 & PPB & \\
\hline FREON 113 & $76-13-1$ & 0 & PPB & \\
\hline DICHLOROMETHANE & $75-09-2$ & 0 & PPB & \\
\hline 4,1-DICHLOROETHANE & $75-34-3$ & 0 & PPB & \\
\hline 2-BUTANONE & $78-93-3$ & 0 & PPB & \\
\hline N-HEXANE & $110-54-3$ & 0 & PPB & \\
\hline 1,2-DICHLOROETHENE & $156-59-2$ & 0 & PPB & \\
\hline CHLOROFORM & $67-66-3$ & 0 & PPB & \\
\hline TETRAHYDROFURAN & $109-99-9$ & 0 & PPB & \\
\hline 1,1,1-TRICHLOROETHANE & $71-55-6$ & 0 & PPB & \\
\hline 1,2-DICHLOROETHANE & $107-06-2$ & 0 & PPB & \\
\hline BENZENE & $71-43-2$ & 0 & PPB & \\
\hline CARBON TETRACHLORIDE & $56-23-5$ & 0 & PPB & \\
\hline CYCLOHEXANE & $110-82-7$ & 0 & PPB & \\
\hline N-BUTANOL & $71-36-3$ & 140 & PPB & \\
\hline TRICHLOROETHENE & $79-01-6$ & 0 & PPB & \\
\hline 4-METHYL-2-PENTANONE & $108-10-1$ & 7 & PPB & \\
\hline TOLUENE & $108-88-3$ & 0 & PPB & \\
\hline 1,1,2-TRICHLOROETHANE & $79-00-5$ & 0 & PPB & \\
\hline TETRACHLOROETHENE & $127-18-4$ & 0 & PPB & \\
\hline CHLOROBENZENE & $108-90-7$ & 0 & PPB & \\
\hline ETHYLBENZENE & $100-41-4$ & 1 & PPB & \\
\hline M-XYLENE & $108-38-3$ & 0 & PPB & \\
\hline STYRENE & $100-42-5$ & 2 & PPB & \\
\hline O-XYLENE & $95-47-6$ & 3 & PPB & \\
\hline 1,1,2,2-TETRACHLORETHANE & $79-34-5$ & 0 & PPB & \\
\hline 1,3,5-TRIMETHYLBENZENE & $108-67-8$ & 7 & PPB & \\
\hline 1,2,4-TRIMETHYLBENZENE & $95-63-6$ & 18 & PPB & \\
\hline 1,3-DICHLOROBENZENE & $541-73-1$ & 0 & PPB & \\
\hline 1,2-DICHLOROBENZENE & $95-50-1$ & 0 & PPB & \\
\hline 1,4-DICHLOROBENZENE & $106-46-7$ & 4 & PPB & \\
\hline
\end{tabular}




\section{LIST OF COMPOUNDS TENTATIVELY IDENTIFIED WITH MASS SPECTRAL LIBRARY}

Sample: $\quad 2403$ WC BACKGROUND

File: $\quad$ C:LHPCHEMLDLDATA70996_5.D

ID: $\quad$ FT6045_3WC.88A BK

Compound

CAS\#

Methanamine, N-methyl-

$000124-40-3$

Ethanamine, N-methyl-

000624-78-2

1-Butanol

$000071-36-3$

2-Pentanone, 4-methyl-

000108-10-1

Chlorobenzene-D5

000000-00-0

Benzene, ethyl-

$000100-41-4$

XYLENE

001330-20-7

3-Heptanone

000106-35-4

2-Heptanone

000110-43-0

Styrene

$000100-42-5$

Benzene, 1,4-dimethyl-

000106-42-3

Ethanol, 2-butoxy-

$000111-76-2$

2-Octanamine, N-(1-methylheptyl)-

$005412-92-0$

Benzene, 1,2,3-trimethyl-

000526-73-8

Benzene, 1,4-dichloro-

000106-46-7

Dodecane

$000112-40-3$ 
HNF-SD-WM-RPT-282 Rev. 0

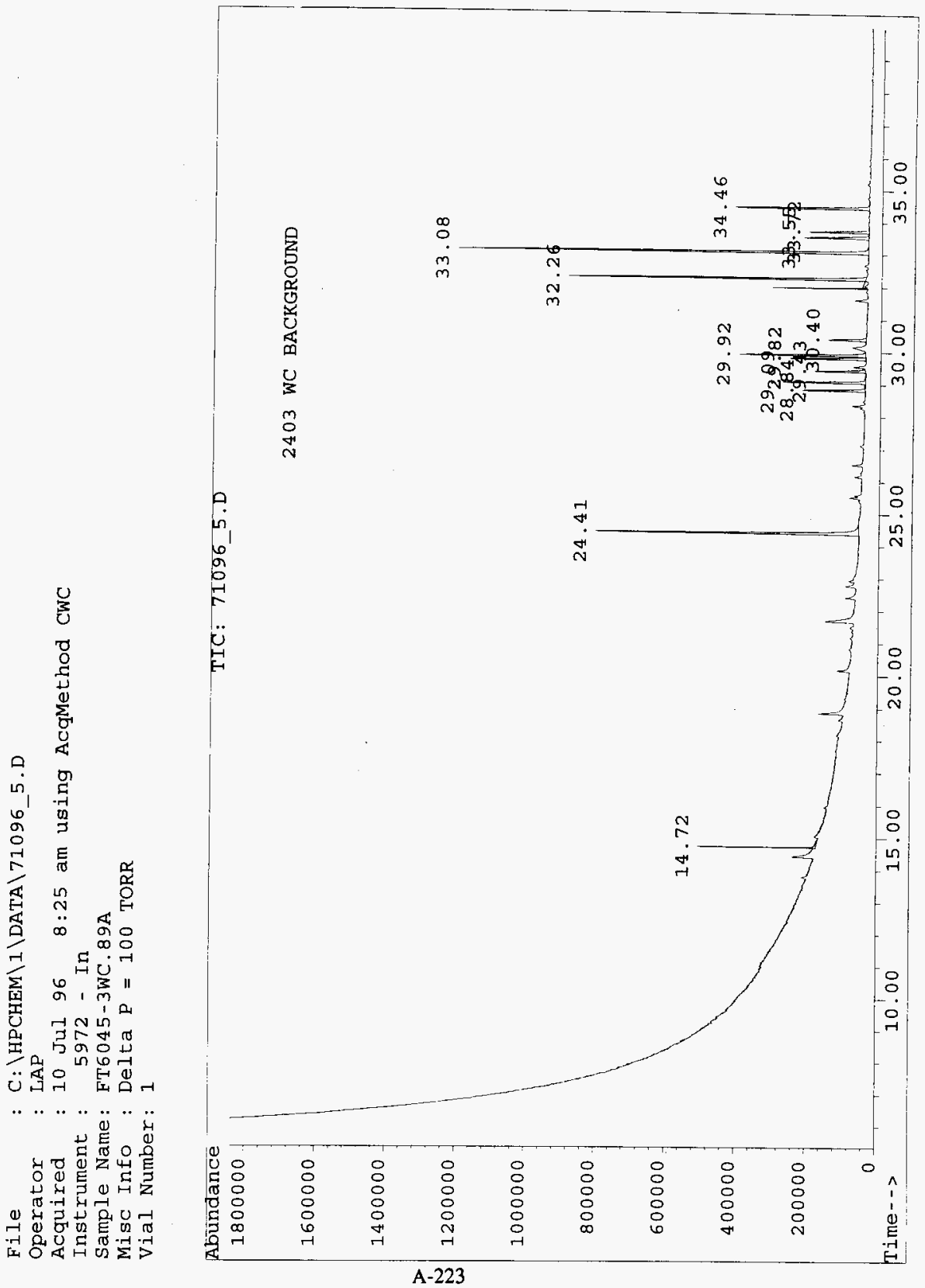


HNF-SD-WM-RPT-282 Rev. 0

CWC_REP.XLS

\section{ANALYSIS REPORT FOR CWC AIR SAMPLES}

File Name:

Analyst:

Analysis Date:

Method:

Sample Name:

\author{
71096_5.D \\ LAP \\ 07/10/96 \\ CWC
}

FT6045-3WC.89A

\begin{tabular}{|c|c|c|c|c|}
\hline COMPOUND NAME & CAS \# & Concentration & Units & Comments \\
\hline ISO-BUTANE & $75-28-5$ & 0 & PPB & \\
\hline VINYL CHLORIDE & $75-01-4$ & 0 & PPB & \\
\hline ETHANOL & $64-17-5$ & 0 & PPB & \\
\hline ACETONE & $67-64-1$ & 0 & PPB & \\
\hline 2-PROPANOL & $67-63-0$ & 0 & PPB & \\
\hline 1,1-DICHLOROETHENE & $75-35-4$ & 0 & PPB & \\
\hline FREON 113 & 76-13-1 & 0 & PPB & \\
\hline DICHLOROMETHANE & $75-09-2$ & 0 & PPB & \\
\hline 1,1-DICHLOROETHANE & $75-34-3$ & 0 & PPB & \\
\hline 2-BUTANONE & $78-93-3$ & 0 & $\overline{P P B}$ & \\
\hline N-HEXANE & $110-54-3$ & 0 & PPB & \\
\hline 1,2-DICHLOROETHENE & $156-59-2$ & 0 & PPB & \\
\hline CHLOROFORM & $67-66-3$ & 0 & PPB & \\
\hline TETRAHYDROFURAN & $109-99-9$ & 0 & PPB & \\
\hline 1,1,1-TRICHLOROETHANE & $71-55-6$ & 0 & PPB & \\
\hline 1,2-DICHLOROETHANE & $107-06-2$ & 0 & $\overline{\mathrm{PPB}}$ & \\
\hline BENZENE & $71-43-2$ & 0 & PPB & \\
\hline CARBON TETRACHLORIDE & $56-23-5$ & 0 & $\overline{\mathrm{PPB}}$ & \\
\hline CYCLOHEXANE & $110-82-7$ & 0 & PPB & \\
\hline N-BUTANOL & $71-36-3$ & 6 & $\mathrm{PPB}$ & \\
\hline TRICHLOROETHENE & $79-01-6$ & $\overline{0}$ & $\mathrm{PPB}$ & \\
\hline 4-METHYL-2-PENTANONE & $108-10-1$ & 17 & $\mathrm{PPB}$ & \\
\hline TOLUENE & $108-88-3$ & 1 & $\mathrm{PPB}$ & \\
\hline 1,1,2-TRICHLOROETHANE & $79-00-5$ & 0 & PPB & \\
\hline TETRACHLOROETHENE & $127-18-4$ & 0 & PPB & \\
\hline CHLOROBENZENE & $108-90-7$ & 0 & PPB & \\
\hline ETHYLBENZENE & $100-41-4$ & 5 & PPB & \\
\hline M-XYLENE & $108-38-3$ & 6 & PPB & \\
\hline STYRENE & $100-42-5$ & 6 & PPB & \\
\hline O-XYLENE & $95-47-6$ & 10 & PPB & \\
\hline $1,1,2,2$-TETRACHLORETHANE & $79-34-5$ & 4 & PPB & \\
\hline 1,3,5-TRIMETHYLBENZENE & $108-67-8$ & 23 & PPB & \\
\hline 1,2,4-TRIMETHYLBENZENE & $95-63-6$ & 31 & PPB & \\
\hline 1,3-DICHLOROBENZENE & $541-73-1$ & 6 & PPB & \\
\hline 1,2-DICHLOROBENZENE & $95-50-1$ & 6 & $\mathrm{PPB}$ & \\
\hline 1,4-DICHLOROBENZENE & $106-46-7$ & 13 & PPB & \\
\hline
\end{tabular}


HNF-SD-WM-RPT-282 Rev. 0

\section{LIST OF COMPOUNDS TENTATIVELY IDENTIFIED WITH MASS SPECTRAL LIBRARY}

Sample: $\quad 2403$ WC

File: $\quad$ C:LHPCHEMI2LDATA171096_5.D

ID: $\quad$ FT6045-3WC.89A

Compound

CAS\#

2-Pentanone, 4-methyl-

000108-10-1

Benzene, methyl-

000108-88-3

Benzene, chloro-

000108-90-7

Benzene, ethyl-

$000100-41-4$

Benzene, 1,4-dimethyi-

000106-42-3

3-Heptanone

000106-35-4

1,3,5,7-Cyclooctatetraene

000629-20-9

XYLENE

001330-20-7

Ethane, 1,1,2,2-tetrachloro-

000079-34-5

Benzene, 1,2,3-trimethyl-

000526-73-8

Benzene, 1,3,5-trimethyl-

000108-67-8

Benzene, 1,3-dichloro-

000541-73-1 
File

: $\mathrm{C}: \backslash$ HPCHEM $\backslash 1 \backslash \mathrm{DATA} \backslash 62596 \_8 . \mathrm{D}$

Operator

LAP

Acquired

25 Jun 96 10:24 am using AcqMethod CWC

Instrument : 5972 - In

Sample Name: FT6045-3WC.84A

Misc Info : 100 TORR

Vial Number: 1

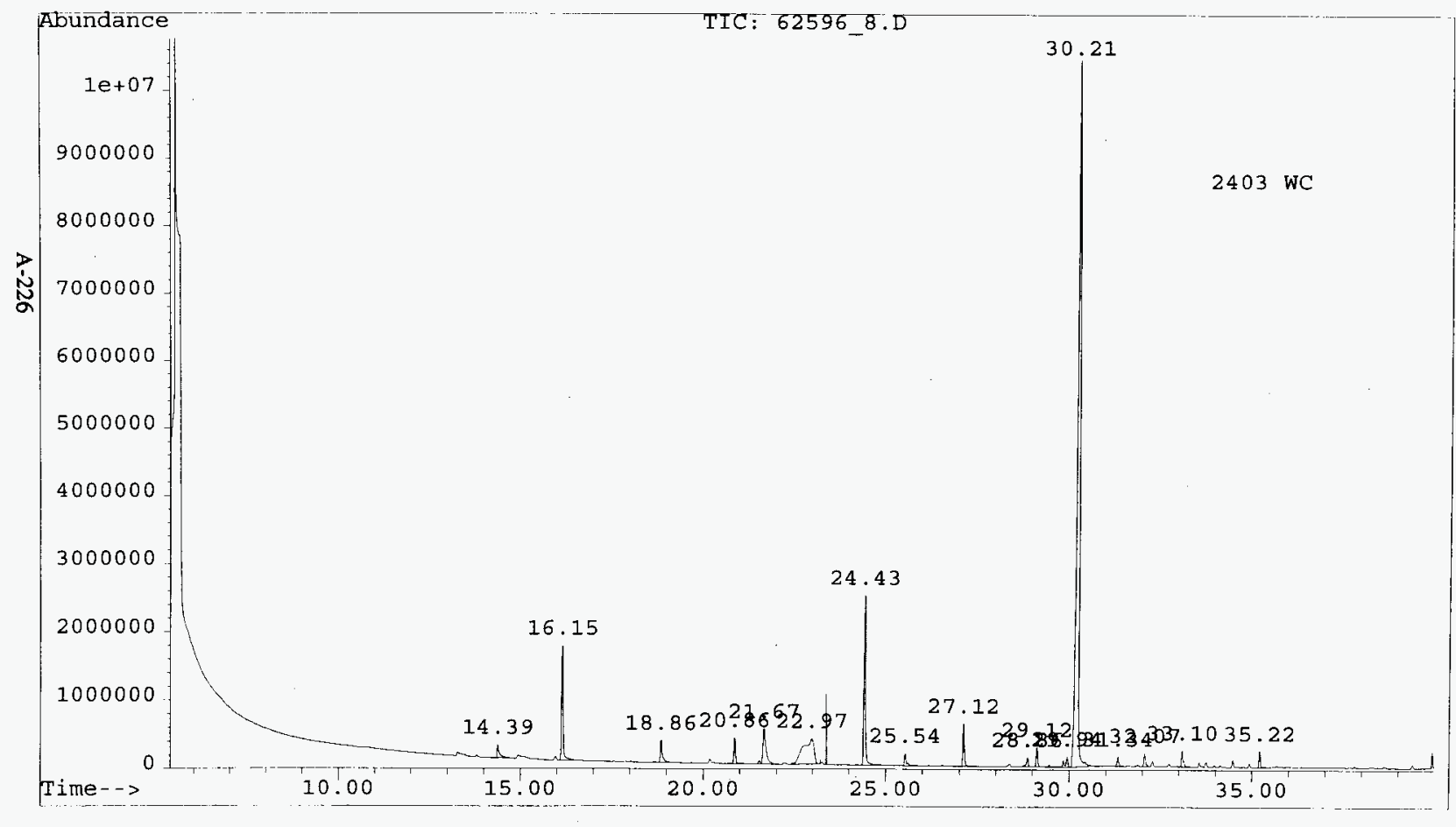


HNF-SD-WM-RPT-282 Rev. 0

CWC_REP.XLS

\section{ANALYSIS REPORT FOR CWC AIR SAMPLES}

File Name:

Analyst:

Analysis Date:

Method:

Sample Name:
62596_8.D

LAP

06/25/96

CWC

FT6045-3WC.84A

\begin{tabular}{|c|c|c|c|c|}
\hline COMPOUND NAME & CAS \# & Concentration & Units & Comments \\
\hline ISO-BUTANE & $75-28-5$ & 0 & PPB & \\
\hline VINYL CHLORIDE & $75-01-4$ & 0 & PPB & \\
\hline ETHANOL & $64-17-5$ & 13 & PPB & \\
\hline ACETONE & $67-64-1$ & 8 & PPB & \\
\hline 2-PROPANOL & $67-63-0$ & 3 & PPB & \\
\hline 1,1-DICHLOROETHENE & $75-35-4$ & 0 & PPB & \\
\hline FREON 113 & $76-13-1$ & $\overline{0}$ & PPB & \\
\hline DICHLOROMETHANE & $75-09-2$ & 57 & PPB & \\
\hline 1,1-DICHLOROETHANE & $75-34-3$ & 0 & PPB & \\
\hline 2-BUTANONE & 78-93-3 & 40 & PPB & \\
\hline N-HEXANE & $110-54-3$ & 0 & PPB & \\
\hline 1,2-DICHLOROETHENE & $156-59-2$ & 0 & PPB & \\
\hline CHLOROFORM & $67-66-3$ & 0 & PPB & \\
\hline TETRAHYDROFURAN & $109-99-9$ & 0 & $\mathrm{PPB}$ & \\
\hline 1,1,1-TRICHLOROETHANE & $71-55-6$ & 16 & PPB & \\
\hline 1,2-DICHLOROETHANE & $107-06-2$ & 0 & PPB & \\
\hline BENZENE & $71-43-2$ & 0 & PPB & \\
\hline CARBON TETRACHLORIDE & $56-23-5$ & 0 & PPB & \\
\hline CYCLOHEXANE & $110-82-7$ & 0 & PPB & \\
\hline N-BUTANOL & $71-36-3$ & 45 & PPB & \\
\hline TRICHLOROETHENE & $79-01-6$ & 0 & PPB & \\
\hline 4-METHYL-2-PENTANONE & $108-10-1$ & 45 & PPB & \\
\hline TOLUENE & $108-88-3$ & 6 & PPB & \\
\hline 1,1,2-TRICHLOROETHANE & $79-00-5$ & 0 & $\overline{\mathrm{PPB}}$ & \\
\hline TETRACHLOROETHENE & $127-18-4$ & 27 & PPB & \\
\hline CHLOROBENZENE & $108-90-7$ & 0 & PPB & \\
\hline ETHYLBENZENE & $100-41-4$ & 4 & PPB & \\
\hline M-XYLENE & $108-38-3$ & 8 & PPB & \\
\hline STYRENE & $100-42-5$ & 2 & PPB & \\
\hline O-XYLENE & $95-47-6$ & 4 & PPB & \\
\hline 1,1,2,2-TETRACHLORETHANE & $79-34-5$ & 0 & PPB & \\
\hline १,3,5-TRIMETHYLBENZENE & $108-67-8$ & 2 & PPB & \\
\hline 1,2,4-TRIMETHYLBENZENE & $95-63-6$ & 6 & PPB & \\
\hline 1,3-DICHLOROBENZENE & $541-73-1$ & 2 & PPB & \\
\hline 1,2-DICHLOROBENZENE & $95-50-1$ & 2 & PPB & \\
\hline 1,4-DICHLOROBENZENE & $106-46-7$ & 3 & PPB & \\
\hline
\end{tabular}




\section{LIST OF COMPOUNDS TENTATIVELY IDENTIFIED WITH MASS SPECTRAL LIBRARY}

Sample: $\quad 2403 \mathrm{WC}$

File: $\quad$ C:LHPCHEMLLDATA162596_8.D

ID: $\quad$ FT6045-3WC.84A

Compound

Methane, dichloro-

2-Butanone

Furan, tetrahydro-

Ethane, 1,1,1-trichloro-

1-Butanol

2-Propanol, 1-methoxy-

2-Pentanone, 4-methyl-

Benzene, methyl-

Ethene, tetrachloro-

Benzene, chloro-

Benzene, ethyl-

Benzene, 1,2-dimethyl-

3-Heptanone

Styrene

Benzene, 1,2-dimethyl-

71

Ethanol, 2-butoxy-

Ethane, 1,1,2,2-tetrachloro-

\section{CAS\#}

000075-09-2

000078-93-3

000109-99-9

$000071-55-6$

000071-36-3

000107-98-2

000108-10-1

000108-88-3

000127-18-4

000108-90-7

$000100-41-4$

$000095-47-6$

000106-35-4

$000100-42-5$

$000095-47-6$

000111-76-2

000079-34-5 
HNF-SD-WM-RPT-282 Rev. 0

\section{LIST OF COMPOUNDS TENTATIVELY IDENTIFLED WITH MASS SPECTRAL LIBRARY}

Sample: $\quad 2403$ WC

File: $\quad$ C:LHPCHEMU2LDATAl62596_8.D

ID: $\quad$ FT6045-3WC.84A

Compound

CAS\#

ALPHA.-PINENE, (-)-

Benzaldehyde

Benzene, 1,2,4-trimethyl-

Benzene, 1,4-dichloro-

Benzene, 1-ethyl-2,3-dimethyl-

dl-Limonene

Benzene, 1,2-dichloro-

Benzenemethanol, .alpha.-methyl-

Ethanone, 1-phenyl-

Benzenemethanol, .alpha.-ethenyl

Benzenemethanol, ar-ethenyl-

1H-Inden-5-ol, 2,3-dihydro-
000080-56-8

000100-52-7

000095-63-6

000106-46-7

000933-98-2

000138-86-3

000095-50-1

000098-85-1

000098-86-2

004393-06-0

030584-69-1

001470-94-6 
File

: C: $\backslash$ HPCHEM \I\DATA $\backslash 62796 \_10 . D$

Operator : MS

Acquired : 27 Jun $96 \quad 4: 53$ pm using AcqMethod CWC

Instrument : 5972 - In

Sample Name: FT 6045-3WC.85A

Misc Info : 100 TORR

Vial Number: 1

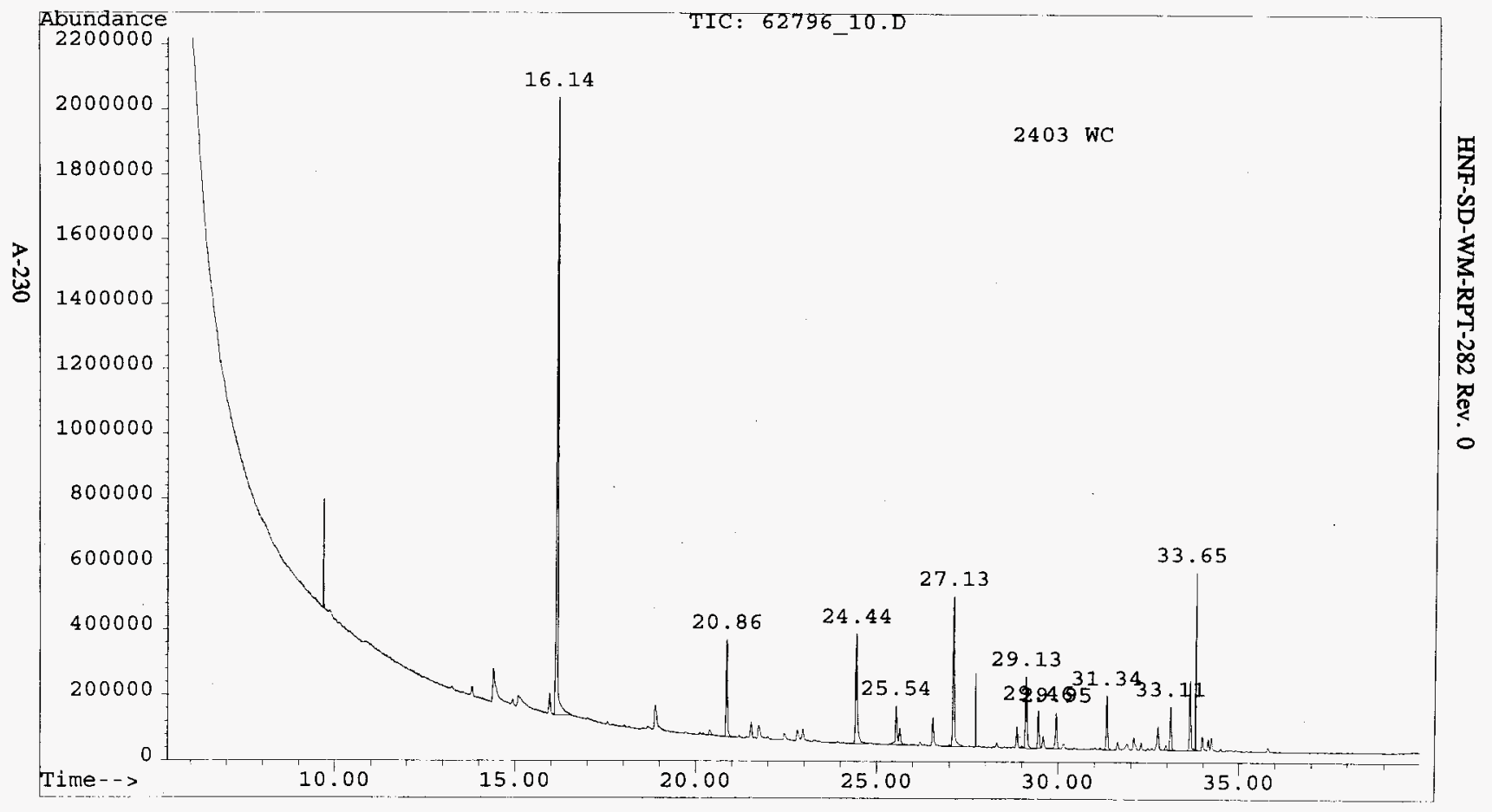




\begin{tabular}{|c|c|c|c|c|}
\hline & gdd & 0 & $L-9 b-901$ & 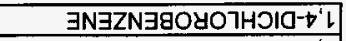 \\
\hline & 8dd & 0 & $1-09-96$ & 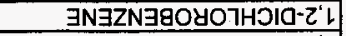 \\
\hline & 8dd & 0 & $1-\varepsilon L-1+G$ & 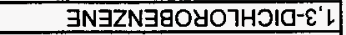 \\
\hline & Edd & $\bar{\varepsilon}$ & $9-\varepsilon 9-96$ & 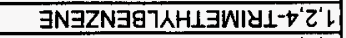 \\
\hline & 8dd & 0 & $8-\angle 9-801$ & 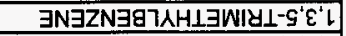 \\
\hline & 8dd & 0 & $S-\neg \varepsilon-6 L$ & $\exists N \forall H \perp \exists Y O=7 H \supset \forall Y \perp \exists \perp-Z^{\prime} Z^{\prime} \iota^{\prime}$ \\
\hline & 8dd & $z$ & $9-\angle t-96$ & Nㅋา스-O \\
\hline & 8dd & 0 & $s-2 t-00 L$ & 킄ㅅㅅㅗ \\
\hline & 8dd & $L$ & $\varepsilon-8 \varepsilon-80 L$ & $\mathrm{BN} 37 \mathrm{X}-\mathrm{W}$ \\
\hline & 8dd & $\bar{z}$ & titrool & 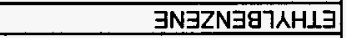 \\
\hline & 8dd & 0 & $\angle-06 \cdot 80 \mathrm{~L}$ & INבZNG9OYO7HO \\
\hline & gdd & 12 & $t-81-\angle 2 L$ & $\exists \mathrm{N} \exists \mathrm{H} \perp \exists \mathrm{OYO} \mathrm{O} \mathrm{H} \mathrm{Y} \forall \mathrm{Y} \perp \exists \perp$ \\
\hline & gdd & 0 & $9-00-62$ & 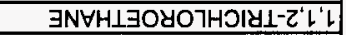 \\
\hline & 8dd & $D$ & $\varepsilon-88-80 L$ & $\exists N \exists \cap 701$ \\
\hline & 8dd & 0 & $1-01-801$ & $\exists N O N \forall \perp N \exists d-Z-7$ ㅅH $\perp \exists N-7$ \\
\hline & 8dd & 0 & $9-10-6 L$ & 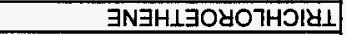 \\
\hline & gdd & 0 & $\varepsilon-9 \varepsilon-1 L$ & 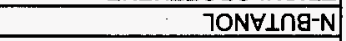 \\
\hline & Edd & 0 & $\angle-28-0 \mathrm{HL}$ & 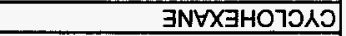 \\
\hline & Edd & 0 & $\mathrm{s- \varepsilon Z-9 \textrm {S }}$ & 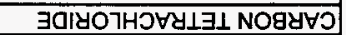 \\
\hline & Edd & 0 & $z-\varepsilon t L L$ & INEZN $\exists$ g \\
\hline & Edd & 0 & $2-90-201$ & 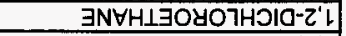 \\
\hline & gdd & 21 & $9-9 s-12$ & 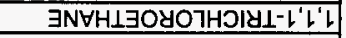 \\
\hline & add & 0 & $6-66-60 \mathrm{~b}$ & 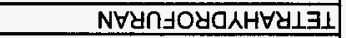 \\
\hline & 8dd & 0 & $\varepsilon-99-29$ & Wप्पO \\
\hline & Add & 0 & $z-69-99 b$ & 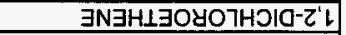 \\
\hline & 8dd & 0 & $\varepsilon-t g-01 L$ & $\exists \mathrm{N} \forall \times \exists \mathrm{H}-\mathrm{N}$ \\
\hline & gdd & 0 & $\varepsilon-\varepsilon 6^{-8 L}$ & 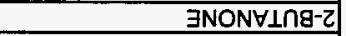 \\
\hline & gdd & 0 & $\varepsilon-t \varepsilon-G L$ & $\exists N \forall H \perp \exists O Y O 7 H \supset I 0-L^{\prime L}$ \\
\hline & gdd & $\varepsilon 9$ & $Z-60-9 L$ & 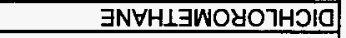 \\
\hline & gdd & 0 & $\frac{L-\varepsilon L-9 L}{L}$ & ELLNOJYH \\
\hline & gdd & 0 & $\nabla G \varepsilon-G L$ & 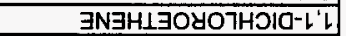 \\
\hline & gdd & $t$ & $0-\varepsilon 9-\angle 9$ & 7ON $\forall d O Z d d-Z$ \\
\hline & gdd & $\underline{G}$ & $1-+69-\angle 9$ & 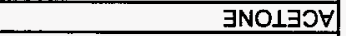 \\
\hline & gdd & 0 & $g-\angle L-t g$ & 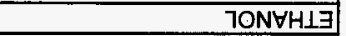 \\
\hline & Add & 0 & $\pm 10-G L$ & 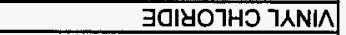 \\
\hline & gdd & 0 & $S-8 z-G L$ & IN $\forall \perp \cap A-O S$ \\
\hline squeulwos & sy!un & บ0ำedques & \# SVO & IWYN aNROdWOS \\
\hline
\end{tabular}

V98.'TME -st09 Lل

OMO

$96 / 2 Z / 90$

SW

$0^{\circ} 0 \mathrm{~L}^{-} 96<29$ :amen əIdues

:poupew

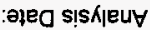
7s אieury

:əweN ㄹ! 
HNF-SD-WM-RPT-282 Rev. 0

\section{LIST OF COMPOUNDS TENTATIVELY IDENTIFIED WITH MASS SPECTRAL LIBRARY}

Sample: $\quad 2403$ WC

File: $\quad$ C:LHPCHEMLLDATA162796_10.D

ID: $\quad$ FT6045-3WC.85A

Compound

CAS\#

Methane, dichloro-

$000075-09-2$

Ethane, 1,1,1-trichloro-

$000071-55-6$

Methane, tetrachloro-

000056-23-5

Pentanal

000110-62-3

Ethene, trichloro-

000079-01-6

2-Pentanone, 4-methyl-

000108-10-1

Benzene, methyl-

000108-88-3

Hexanal

000066-25-1

Ethene, tetrachloro-

000127-18-4

Benzene, ethyl-

$000100-41-4$

Benzene, 1,3-dimethyl-

000108-38-3

3-Heptanone

000106-35-4

2-Heptanone

000110-43-0

Benzene, 1,3-dimethyl-

000108-38-3

ALPHA -PINENE, (-)-

000080-56-8

Benzene, 1,3,5-trimethyl-

000108-67-8

1-beta.-Pinene

018172-67-3 
HNF-SD-WM-RPT-282 Rev. 0

LIST OF COMPOUNDS TENTATIVELY IDENTIFIED WITH MASS SPECTRAL

LIBRARY

Sample: $\quad 2403$ WC (CONTINUED)

File: $\quad$ C:LHPCHEM2LDATAI62796_10.D

ID: $\quad$ FT6045-3WC.85A

Compound

CAS\#

Decane

000124-18-5

Benzene, 1,2,3-trimethyl-

000526-73-8

DELTA.3-Carene

013466-78-9

Benzene, 1-methyl-2-(1-methylethyl)-

000527-84-4

Cyclohexene, 1-methyl-4-(1-methylethe

005989-27-5

Sabinene

003387-41-5 
File

: C $: \backslash H P C H E M \backslash 1 \backslash D A T A \backslash 62796 \_13 . D$

Operator

MS

Acquired

: 27 Jun 96

Instrument : 5972 - In

Sample Name: FT 6045- 3WC.86A

Misc Info : 100 TORR

Vial Number: 1

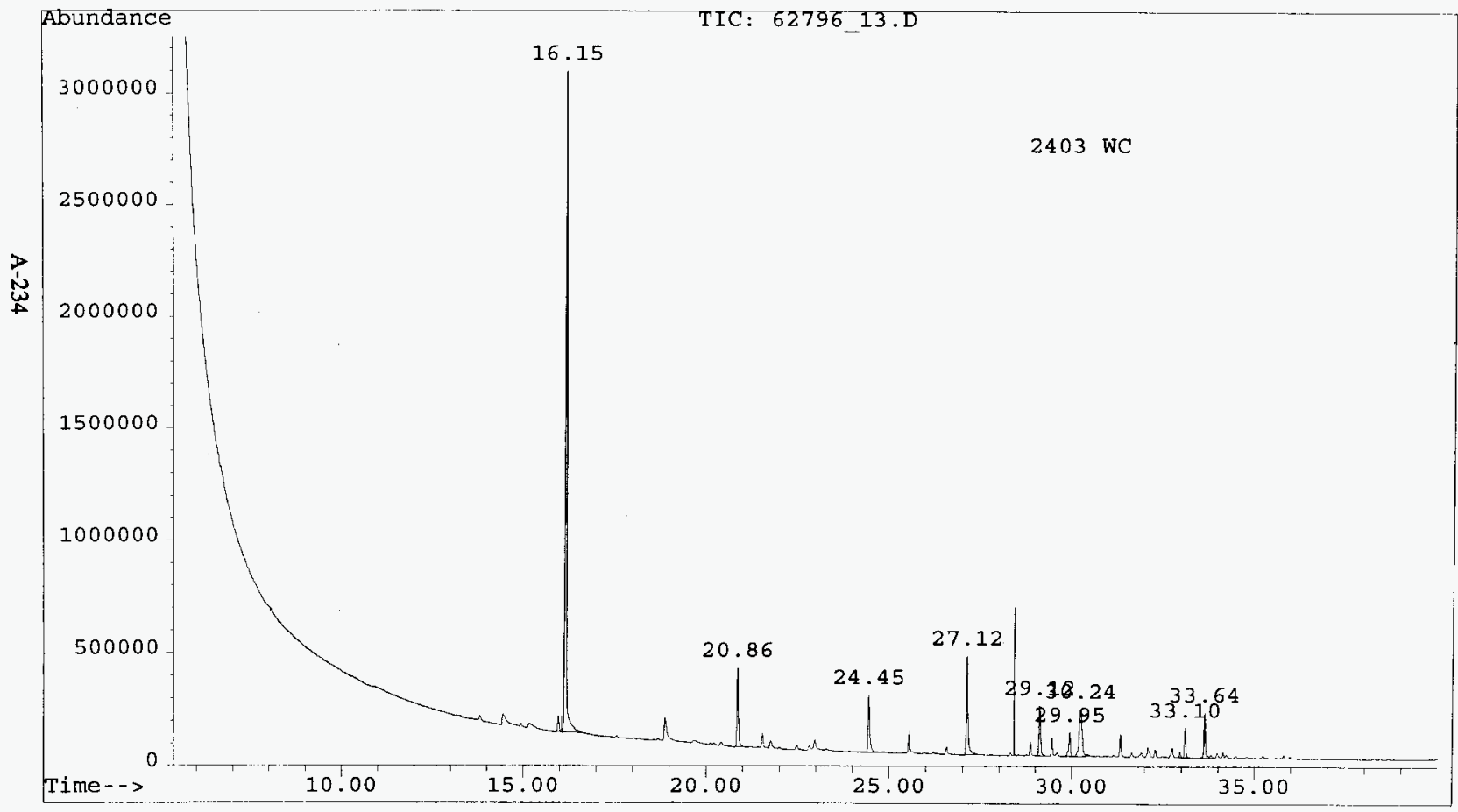


HNF-SD-WM-RPT-282 Rev. 0

CWC_REP.XLS

\section{ANALYSIS REPORT FOR CWC AIR SAMPLES}

File Name:

Analyst:

Analysis Date:

Method:

Sample Name:
62796_13.D

MS

06/27/96

CWC

FT 6045- 3WC.86A

\begin{tabular}{|c|c|c|c|c|}
\hline COMPOUND NAME & CAS \# & Concentration & Units & Comments \\
\hline ISO-BUTANE & $75-28-5$ & 0 & PPB & \\
\hline VINYL CHLORIDE & $75-01-4$ & 0 & PPB & \\
\hline ETHANOL & $64-17-5$ & 0 & PPB & \\
\hline ACETONE & $67-64-1$ & $\underline{0}$ & PPB & \\
\hline 2-PROPANOL & $67-63-0$ & 2 & PPB & \\
\hline 1,1-DICHLOROETHENE & $75-35-4$ & 0 & PPB & \\
\hline FREON 113 & $76-13-1$ & 0 & PPB & \\
\hline DICHLOROMETHANE & $75-09-2$ & 104 & PPB & \\
\hline 1,1-DICHLOROETHANE & $75-34-3$ & 0 & PPB & \\
\hline 2-BUTANONE & $78-93-3$ & 0 & PPB & \\
\hline N-HEXANE & $110-54-3$ & 0 & PPB & \\
\hline 1,2-DICHLOROETHENE & $156-59-2$ & 0 & PPB & \\
\hline CHLOROFORM & $67-66-3$ & 0 & PPB & \\
\hline TETRAHYDROFURAN & $109-99-9$ & 0 & PPB & \\
\hline 1,1,1-TRICHLOROETHANE & $71-55-6$ & 15 & PPB & \\
\hline 1,2-DICHLOROETHANE & $107-06-2$ & 0 & PPB & \\
\hline BENZENE & $71-43-2$ & 0 & PPB & \\
\hline CARBON TETRACHLORIDE & $56-23-5$ & 5 & PPB & \\
\hline CYCLOHEXANE & $110-82-7$ & 0 & PPB & \\
\hline \begin{tabular}{|l} 
N-BUTANOL \\
\end{tabular} & $71-36-3$ & 0 & PPB & \\
\hline TRICHLOROETHENE & $79-01-6$ & 0 & PPB & \\
\hline 4-METHYL-2-PENTANONE & $108-10-1$ & 0 & PPB & \\
\hline TOLUENE & $108-88-3$ & 3 & PPB & \\
\hline 1,1,2-TRICHLOROETHANE & $79-00-5$ & 0 & PPB & \\
\hline TETRACHLOROETHENE & $127-18-4$ & 21 & PPB & \\
\hline CHLOROBENZENE & $108-90-7$ & 0 & PPB & \\
\hline ETHYLBENZENE & $100-41-4$ & 2 & PPB & \\
\hline M-XYLENE & $108-38-3$ & 7 & PPB & \\
\hline STYRENE & $100-42-5$ & 0 & PPB & \\
\hline O-XYLENE & $95-47-6$ & 3 & PPB & \\
\hline $1,1,2,2$-TETRACHLORETHANE & $79-34-5$ & 0 & PPB & \\
\hline 1,3,5-TRIMETHYLBENZENE & $108-67-8$ & 0 & $\mathrm{PPB}$ & \\
\hline 1,2,4-TRIMETHYLBENZENE & $95-63-6$ & 3 & PPB & \\
\hline 1,3-DICHLOROBENZENE & $541-73-1$ & 0 & $\mathrm{PPB}$ & \\
\hline 1,2-DICHLOROBENZENE & $95-50-1$ & 0 & PPB & \\
\hline 1,4-DICHLOROBENZENE & $106-46-7$ & 0 & PPB & \\
\hline
\end{tabular}


HNF-SD-WM-RPT-282 Rev. 0

\section{LIST OF COMPOUNDS TENTATIVELY IDENTIFIED WITH MASS SPECTRAL LIBRARY}

Sample: $\quad 2403 \mathrm{WC}$

File: $\quad$ C:LHPCHEMLIDATAl62796_13.D

ID: $\quad$ FT6045- 3WC.86A

Compound

CAS\#

Methanamine, N-methyl-

000124-40-3

Methane, dichloro-

000075-09-2

Ethane, 1,1,1-trichloro-

$000071-55-6$

METHYL 1,2,3,11,12,12A-HEXAHYDRO-3-OX 060774-49-4

Pentanal

$000110-62-3$

Ethene, trichloro-

000079-01-6

2-Pentanone, 4-methyl-

000108-10-1

Benzene, methyl-

000108-88-3

Ethene, tetrachloro-

$000127-18-4$

Benzene, ethyl-

$000100-41-4$

Benzene, 1,4-dimethyl-

000106-42-3

3-Heptanone

000106-35-4

XYLENE

01330-20-7

Ethanol, 2-butoxy-

000111-76-2

ALPHA.-PINENE, (-)-

000080-56-8

2-BETA.-PINENE

000127-91-3

Benzene, 1,3,5-trimethyl-

000108-67-8 
HNF-SD-WM-RPT-282 Rev. 0

\section{LIST OF COMPOUNDS TENTATIVELY IDENTIFIED WITH MASS SPECTRAL} LIBRARY

Sample: $\quad 2403$ WC (CONTINUED)

File: $\quad$ C:UHPCHEMLDDATA162796_13.D

ID: FT6045- 3WC.86A

Compound

CAS\#

DELTA.3-Carene

013466-78-9

Benzene, 1-methyl-2-(1-methylethyl)-

$000527-84-4$

Bornylene

000464-17-5 
File

$\mathrm{C}: \backslash$ HPCHEM $\backslash 1 \backslash \mathrm{DATA} \backslash 62596 \_15 . \mathrm{D}$

Operator : MS

Acquired : 25 Jun $96 \quad 5: 06 \mathrm{pm}$ using AcqMethod CWC

Instrument : 5972 - In

Sample Name: FT6045-3WC.87A

Misc Info : 100 TORR

Vial Number: 1

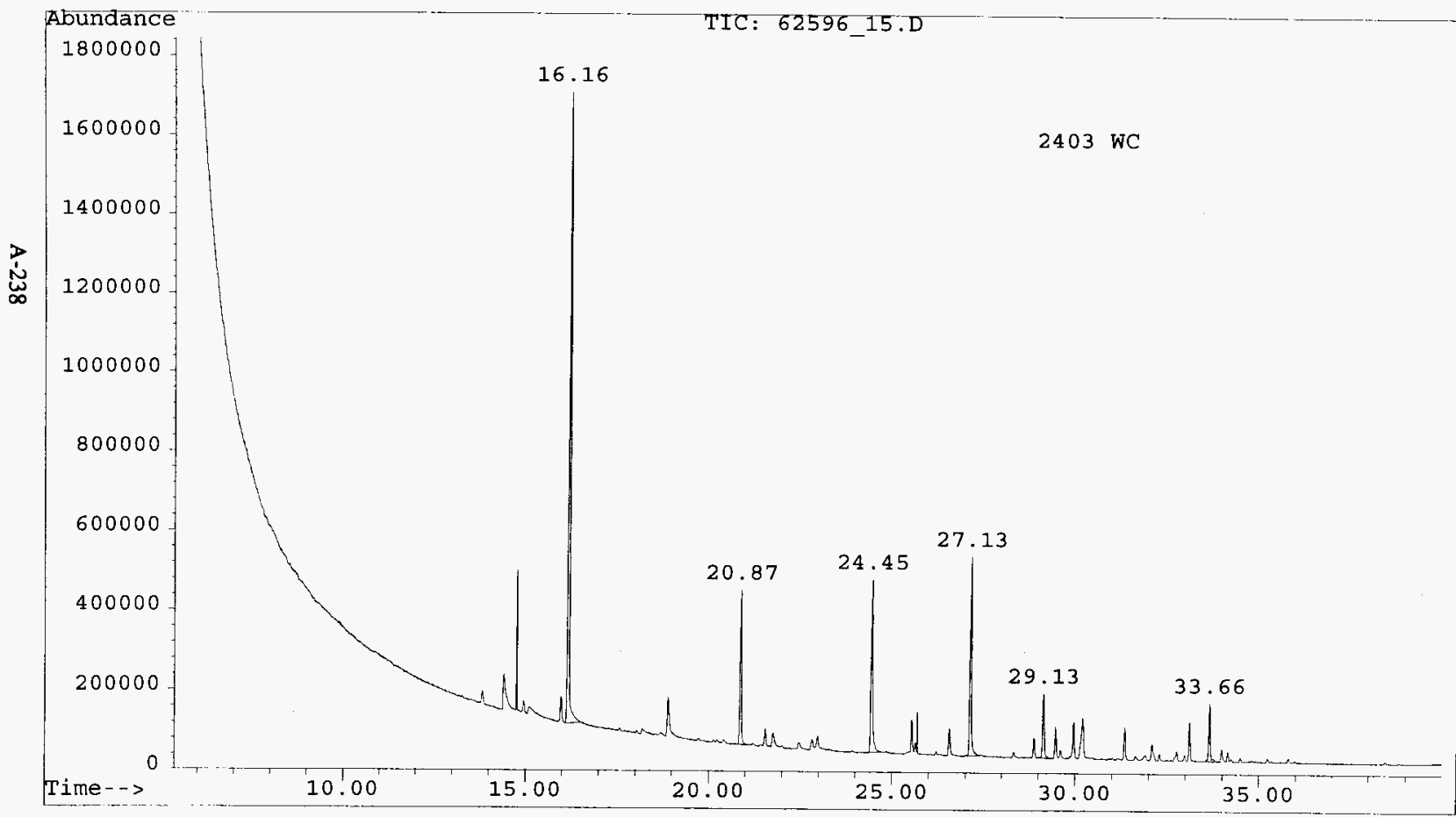


HNF-SD-WM-RPT-282 Rev. 0

CWC_REP.XLS

\section{ANALYSIS REPORT FOR CWC AIR SAMPLES}

File Name:

Analyst:

Analysis Date:

Method:

Sample Name:

\author{
62596_15.D \\ MS \\ 06/25/96 \\ CWC \\ FT6045-3WC.87A
}

\begin{tabular}{|c|c|c|c|c|}
\hline COMPOUND NAME & CAS\# & Concentration & Units & Comments \\
\hline ISO-BUTANE & $75-28-5$ & 0 & $\overline{\text { PPB }}$ & \\
\hline VINYL CHLORIDE & $75-01-4$ & 0 & PPB & \\
\hline ETHANOL & $64-17-5$ & 0 & PPB & \\
\hline ACETONE & $67-64-1$ & 4 & PPB & \\
\hline 2-PROPANOL & $67-63-0$ & 3 & PPB & \\
\hline 1,1-DICHLOROETHENE & $75-35-4$ & 0 & PPB & \\
\hline FREON 113 & $76-13-1$ & 0 & PPB & \\
\hline DICHLOROMETHANE & $75-09-2$ & 50 & PPB & \\
\hline 1,1-DICHLOROETHANE & $75-34-3$ & 0 & PPB & \\
\hline 2-BUTANONE & $78-93-3$ & 0 & PPB & \\
\hline N-HEXANE & $110-54-3$ & 0 & PPB & \\
\hline 1,2-DICHLOROETHENE & $156-59-2$ & 0 & PPB & \\
\hline CHLOROFORM & $67-66-3$ & 0 & PPB & \\
\hline TETRAHYDROFURAN & $109-99-9$ & 0 & PPB & \\
\hline 1,1,1-TRICHLOROETHANE & $71-55-6$ & 16 & PPB & \\
\hline 1,2-DICHLOROETHANE & $107-06-2$ & 0 & PPB & \\
\hline BENZENE & $71-43-2$ & 0 & PPB & \\
\hline CARBON TETRACHLORIDE & $56-23-5$ & 0 & PPB & \\
\hline CYCLOHEXANE & $110-82-7$ & 0 & PPB & \\
\hline N-BUTANOL & $71-36-3$ & 0 & PPB & \\
\hline TRICHLOROETHENE & $79-01-6$ & 0 & PPB & \\
\hline 4-METHYL-2-PENTANONE & $108-10-1$ & 8 & PPB & \\
\hline TOLUENE & $108-88-3$ & 3 & PPB & \\
\hline 1,1,2-TRICHLOROETHANE & $79-00-5$ & 0 & PPB & \\
\hline TETRACHLOROETHENE & $127-18-4$ & 22 & PPB & \\
\hline CHLOROBENZENE & $108-90-7$ & 0 & PPB & \\
\hline ETHYLBENZENE & $100-41-4$ & 1 & PPB & \\
\hline M-XYLENE & $108-38-3$ & 5 & $\mathrm{PPB}$ & \\
\hline STYRENE & $100-42-5$ & 0 & $\mathrm{PPB}$ & \\
\hline O-XYLENE & $95-47-6$ & 2 & PPB & \\
\hline 1,1,2,2-TETRACHLORETHANE & $79-34-5$ & 0 & PPB & \\
\hline 1,3,5-TRIMETHYLBENZENE & $108-67-8$ & 0 & PPB & \\
\hline 1,2,4-TRIMETHYLBENZENE & $95-63-6$ & 2 & PPB & \\
\hline 1,3-DICHLOROBENZENE & $541-73-1$ & 0 & PPB & \\
\hline 1,2-DICHLOROBENZENE & $95-50-1$ & 0 & PPB & \\
\hline 1,4-DICHLOROBENZENE & $106-46-7$ & 0 & PPB & \\
\hline
\end{tabular}


HNF-SD-WM-RPT-282 Rev. 0

\section{LIST OF COMPOUNDS TENTATIVELY IDENTIFIED WITH MASS SPECTRAL LIBRARY}

Sample: $\quad 2403$ WC

File: $\quad$ C:LHPCHEMLLDATAl62596_15.D

ID: $\quad$ FT6045-3WC.87A

Compound

CAS\#

Methane, dichloro-

000075-09-2

Ethane, 1,1,1-trichloro-

$000071-55-6$

1-Bromobicyclo[4.3.1]decan-8-one

070576-35-1

Pentanal

$000110-62-3$

Ethene, trichloro-

000079-01-6

2-Pentanone, 4-methyl-

000108-10-1

Benzene, methyl-

000108-88-3

Hexanal

000066-25-1

Ethene, tetrachloro-

$000127-18-4$

Benzene, ethyl-

$000100-41-4$

Benzene, 1,3-dimethyl-

000108-38-3

3-Heptanone

000106-35-4

ALPHA -PINENE, (-)-

000080-56-8

Benzaldehyde

000100-52-7

Benzene, 1,3,5-trimethyl-

000108-67-8

I- beta.-Pinene

018172-67-3 
HNF-SD-WM-RPT-282 Rev. 0

\section{LIST OF COMPOUNDS TENTATIVELY IENTIFIED WITH MASS SPECTRAL LIBRARY}

Sample: $\quad 2403$ WC (CONTINUED)

File: C:UHPCHEMILDATAI62596_15.D

ID: $\quad$ FT6045-3WC.87A

Compound

CAS\#

Decane

000124-18-5

DELTA.3-Carene

013466-78-9

Benzene, 1-methyl-4-(1-methylethyl)-

000099-87-6

Cyclohexene, 1-methyl-4-(1-methylethe

005989-27-5 
File

: C: $\backslash H P C H E M \backslash 1 \backslash D A T A \backslash 72296 \quad 5 . D$

Operator

LAP

Acquired

: 24 Mar 153

Instrument : 5972 - In

Sample Name: FT6045-3WD.76B

Misc Info : 100 TORR

Vial Number: 1

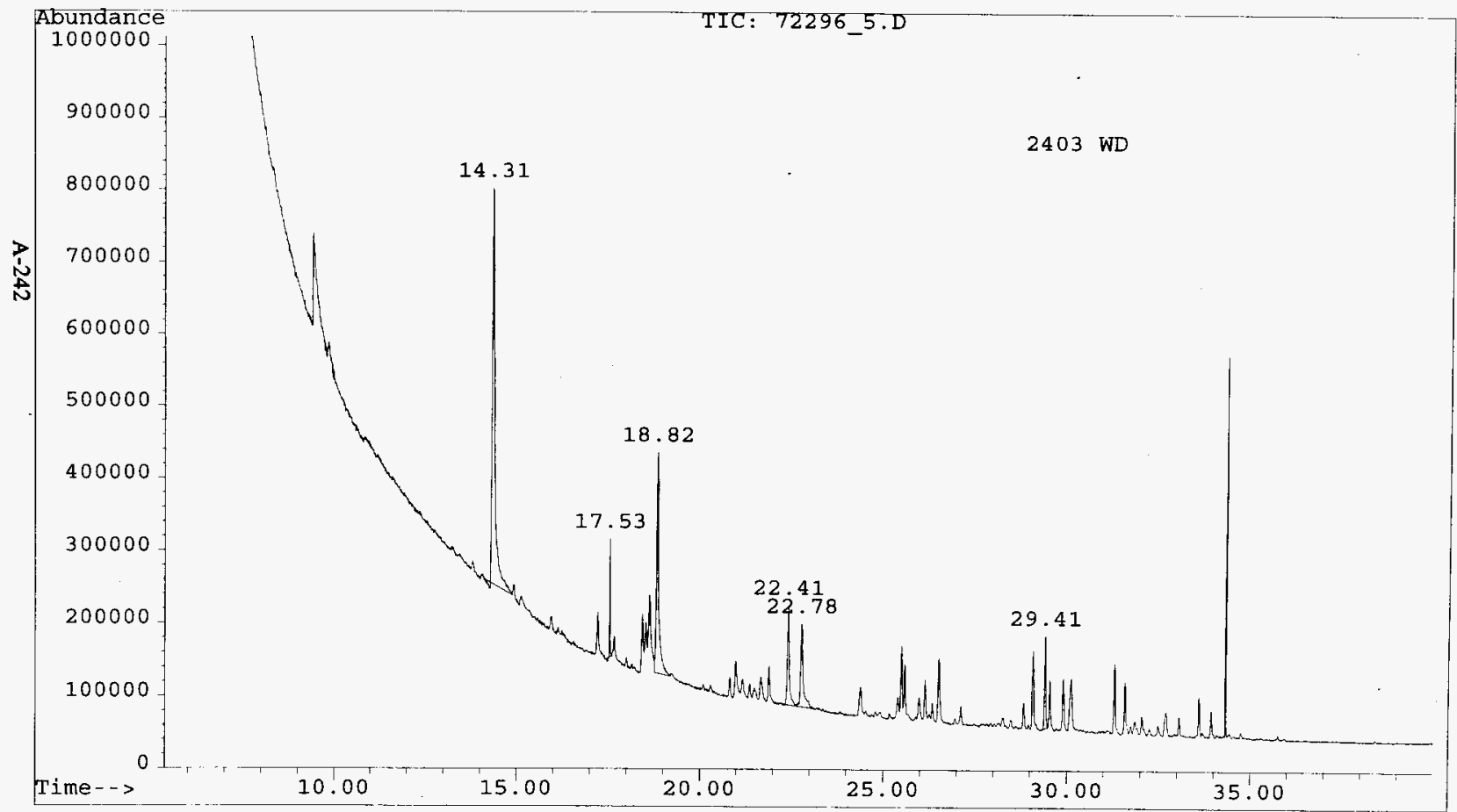




\begin{tabular}{|c|c|c|c|c|}
\hline & gdd & 0 & $L-97-901$ & $\exists N \exists Z N \exists g O Y 07 \mathrm{HOI0-t'L}$ \\
\hline & gdd & 0 & L-0S-96 & $\exists N \exists Z N \exists g O Y O 7 H O I 0-Z^{\prime \prime}$ \\
\hline & gdd & 0 & $1-\varepsilon<-l+g$ & $\exists N \exists Z N \exists 8 O Y O 7 H O I 0-\varepsilon^{\prime} L$ \\
\hline & gdd & 0 & $9-\varepsilon 9-96$ & $\exists N \exists Z N \exists g 7 \wedge H \perp \exists W \mid Y \perp-\nabla^{\prime} Z^{\prime} !$ \\
\hline & gdd & 0 & $8-\angle 9-801$ & $\exists N \exists Z N \exists g 7 \wedge H \perp \exists W \mid \forall \perp-S^{\prime} \varepsilon^{\prime}$ \\
\hline & gdd & 0 & $9-\downarrow \varepsilon-6 L$ & $\exists N \forall H \perp \exists y O 7 H \supset \forall \forall \perp \exists \perp-Z^{\prime} Z^{\prime} \vdash^{\prime}$ \\
\hline & gdd & 0 & $9-2 t-96$ & $\exists N \exists \exists \wedge \times-0$ \\
\hline & gdd & 0 & $s-2 t-001$ & $\exists N \exists y \lambda \perp S$ \\
\hline & gdd & $\varepsilon$ & $\varepsilon-8 \varepsilon-80 l$ & $\exists N \exists 7 \wedge \times-W$ \\
\hline & gdd & 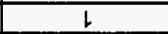 & $b-\downarrow t-00 \downarrow$ & $\exists N \exists Z N \exists g 7 \lambda H \perp \exists$ \\
\hline & gdd & 0 & $\angle-06-80 \mathrm{~L}$ & $\exists N \exists Z N \exists 80807 \mathrm{HO}$ \\
\hline & gdd & $\overline{0}$ & $t-8 L-\angle Z L$ & $\exists N \exists H \perp \exists O У 07 H \supset \forall У \perp \exists \perp$ \\
\hline & gdd & 0 & $9-00-6 L$ & 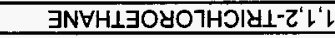 \\
\hline & gdd & $\varepsilon$ & $\varepsilon-88-80 \downarrow$ & $\exists N \exists \cap 70 \perp$ \\
\hline & gdd & 0 & $1-01-801$ & $\exists N O N \forall \perp N \exists d-Z-7 \lambda H \perp \exists W-t$ \\
\hline & gdd & 0 & $9-10-6 L$ & 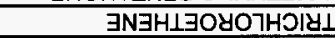 \\
\hline & gdd & 0 & $\varepsilon-9 \varepsilon-1 L$ & $70 N \forall \perp \cap 8-N$ \\
\hline & gdd & 0 & $L-28-01.1$ & $\exists N \forall \times \exists H O 7 \supset \wedge 0$ \\
\hline & gdd & 0 & $\underline{s-\varepsilon z-9 \varsigma}$ & $\exists 01807 H \supset \forall d \perp \exists \perp$ NOgY $\forall 0$ \\
\hline & gdd & 0 & $z-\varepsilon b-t L$ & $\exists \mathrm{N} \exists \mathrm{ZN} \exists \mathrm{G}$ \\
\hline & gdd & 0 & $z-90-\angle O L$ & $\exists N \forall H \perp \exists O Y 07 H \supset \mid 0-Z^{\prime \prime}$ \\
\hline & gdd & 0 & $9-G S-1 L$ & 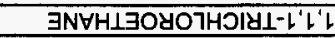 \\
\hline & gdd & 0 & $6-66-60 \mathrm{l}$ & 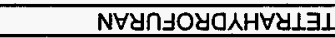 \\
\hline & gdd & 0 & $\varepsilon-99-\angle 9$ & WyOJOYOTHว \\
\hline & gdd & 0 & $2-6 s-99 l$ & $\exists N \exists H \perp \exists O \forall 07 H O \mid a-2^{\prime} \downarrow$ \\
\hline & gdd & 0 & $\varepsilon-t s-0 L L$ & $\exists \mathrm{N} \forall \times \exists \mathrm{H}-\mathrm{N}$ \\
\hline & gdd & $\varepsilon b$ & $\varepsilon-\varepsilon 6-8 L$ & $\exists N O N \forall \perp \cap 8-Z$ \\
\hline & Gdd & 0 & $\varepsilon-\nabla \varepsilon-G L$ & 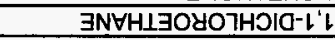 \\
\hline & gdd & 0 & $2-60-9 L$ & $\exists N \forall H \perp \exists W O \forall 07 \mathrm{HDIa}$ \\
\hline & gdd & 0 & $\llcorner-\varepsilon\llcorner-9 L$ & EเLNOJyJ \\
\hline & gdd & 0 & $\nabla-G \varepsilon-9 L$ & $\exists N \exists H \perp \exists O У 07 H ว I 0-L^{\prime}$ \\
\hline & gdd & 0 & $0-\varepsilon 9-\angle 9$ & רON $\forall d O \forall d-Z$ \\
\hline & gdd & $\nabla \varepsilon$ & $1-79-\angle 9$ & $\exists N O \perp \exists O \forall$ \\
\hline & 8dd & 0 & s-Ll-b9 & 7ON $\forall \mathrm{H} \perp \exists$ \\
\hline & gdd & 0 & $010-92$ & 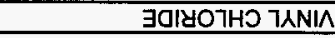 \\
\hline & 8dd & 0 & $9-82-9 L$ & $\exists N \forall \perp n g-O S I$ \\
\hline squeutuos & squn & Loppesuesuos & \# S甘0 & 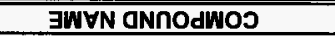 \\
\hline
\end{tabular}

g9LaME-st09LJ

OMO

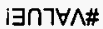

$d \forall 7$

$d g^{-} 96 z Z L$

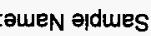
:pounow

:ə]e0 sisর|eu isאieuY :əUEN ㅋ! 
HNF-SD-WM-RPT-282 Rev. 0

\section{LIST OF COMPOUNDS TENTATIVELY IDENTIFIED WITH MASS SPECTRAL LIBRARY}

Sample: $\quad 2403$ WD

File: $\quad$ C:LHPCHEMLLDATAI72296_5.D

ID: $\quad$ FT6045-3WD.76B

Compound

CAS\#

Pentanal

$000110-62-3$

Benzene, methyl-

000108-88-3

Benzene, ethyl-

$000100-41-4$

Benzene, 1,4-dimethyl-

$000106-42-3$

3-Heptanone

000106-35-4

Benzene, 1,3-dimethyl-

000108-38-3

ALPHA.-PINENE, (-)-

000080-56-8

DELTA.3-Carene

013466-78-9

Benzene, methyl(1-methylethyl)-

025155-15-1 
HNF-SD-WM-RPT-282 Rev. 0
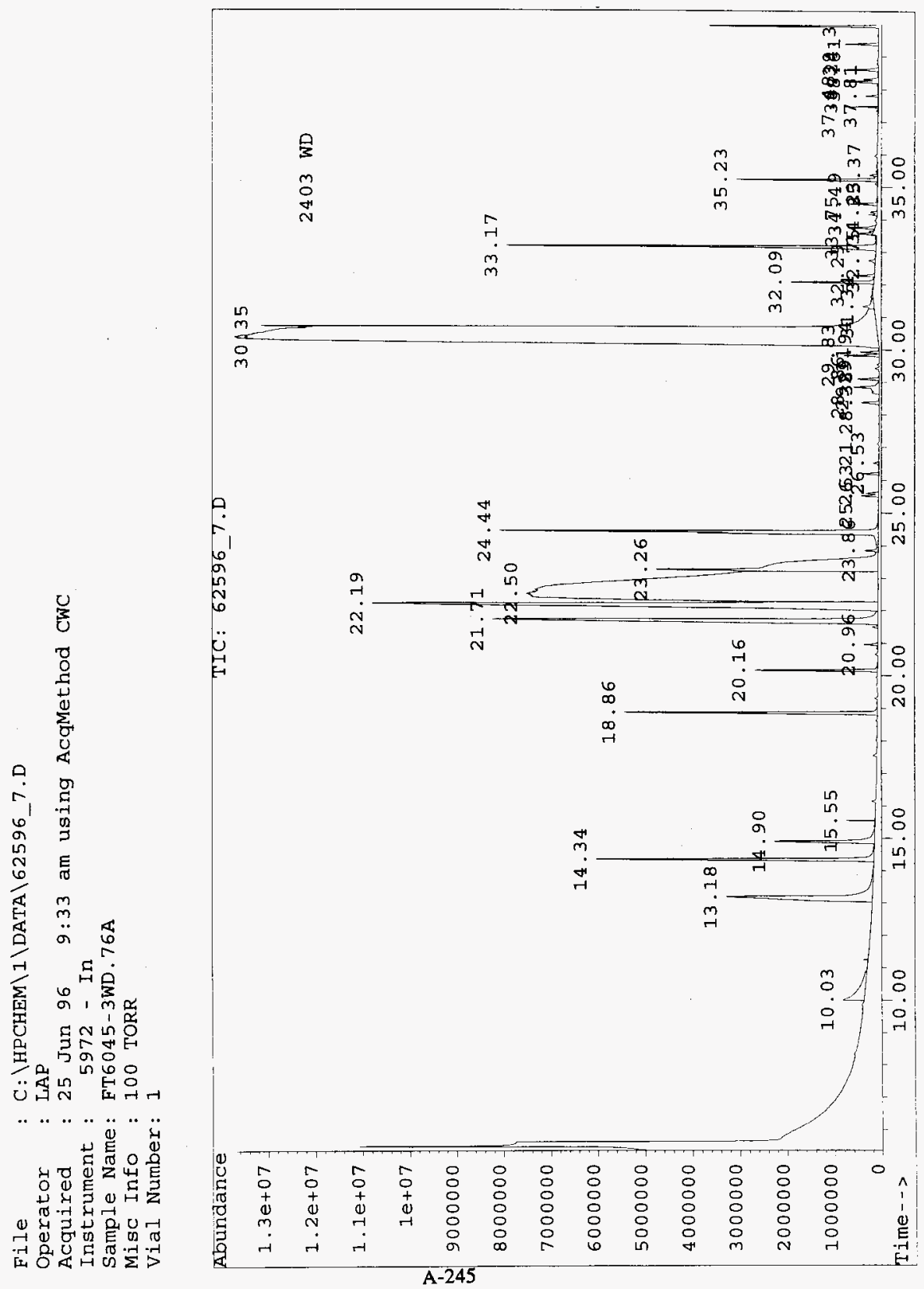
HNF-SD-WM-RPT-282 Rev. 0

CWC_REP.XLS

\section{ANALYSIS REPORT FOR CWC AIR SAMPLES}

File Name:

Analyst:

Analysis Date:

Method:

Sample Name:
62596_7.D

LAP

06/25/96

CWC

FT6045-3WD.76A

\begin{tabular}{|c|c|c|c|c|}
\hline COMPOUND NAME & CAS \# & Concentration & Units & Comments \\
\hline ISO-BUTANE & $75-28-5$ & 0 & PPB & \\
\hline VINYL CHLORIDE & $75-01-4$ & 0 & PPB & \\
\hline ETHANOL & $64-17-5$ & 622 & PPB & \\
\hline ACETONE & $67-64-1$ & 217 & PPB & \\
\hline 2-PROPANOL & $67-63-0$ & 118 & PPB & \\
\hline 1,1-DICHLOROETHENE & $75-35-4$ & 0 & PPB & \\
\hline FREON 113 & $76-13-1$ & 0 & $\overline{P P B}$ & \\
\hline DICHLOROMETHANE & $75-09-2$ & 3 & PPB & \\
\hline 1,1-DICHLOROETHANE & $75-34-3$ & 0 & PPB & \\
\hline 2-BUTANONE & $78-93-3$ & 546 & PPB & \\
\hline N-HEXANE & $110-54-3$ & 32 & PPB & \\
\hline 1,2-DICHLOROETHENE & $156-59-2$ & 0 & PPB & \\
\hline CHLOROFORM & $67-66-3$ & 0 & PPB & \\
\hline TETRAHYDROFURAN & $109-99-9$ & 67 & PPB & \\
\hline 1,1,1-TRICHLOROETHANE & $71-55-6$ & 0 & PPB & \\
\hline 1,2-DICHLOROETHANE & $107-06-2$ & 16 & PPB & \\
\hline BENZENE & $71-43-2$ & 11 & PPB & \\
\hline CARBON TETRACHLORIDE & $56-23-5$ & 0 & PPB & \\
\hline CYCLOHEXANE & $110-82-7$ & 0 & PPB & \\
\hline N-BUTANOL & $71-36-3$ & 746 & PPB & \\
\hline TRICHLOROETHENE & 79-01-6 & 3 & PPB & \\
\hline 4-METHYL-2-PENTANONE & $108-10-1$ & 151 & PPB & \\
\hline TOLUENE & $108-88-3$ & 11 & PPB & \\
\hline 1,1,2-TRICHLOROETHANE & $79-00-5$ & 10 & PPB & \\
\hline TETRACHLOROETHENE & $127-18-4$ & 0 & PPB & \\
\hline CHLOROBENZENE & $108-90-7$ & 7 & PPB & \\
\hline ETHYLBENZENE & $100-41-4$ & 12 & PPB & \\
\hline M-XYLENE & $108-38-3$ & 12 & $\mathrm{PPB}$ & \\
\hline STYRENE & $100-42-5$ & 18 & PPB & \\
\hline O-XYLENE & $95-47-6$ & 10 & PPB & \\
\hline 1,1,2,2-TETRACHLORETHANE & $79-34-5$ & 0 & PPB & \\
\hline 1,3,5-TRIMETHYLBENZENE & $108-67-8$ & 9 & PPB & \\
\hline 1,2,4-TRIMETHYLBENZENE & $95-63-6$ & 17 & PPB & \\
\hline 1,3-DICHLOROBENZENE & $541-73-1$ & 13 & PPB & \\
\hline 1,2-DICHLOROBENZENE & $95-50-1$ & 20 & PPB & \\
\hline 1,4-DICHLOROBENZENE & $106-46-7$ & 18 & PPB & \\
\hline
\end{tabular}


HNF-SD-WM-RPT-282 Rev. 0

\section{LIST OF COMPOUNDS TENTATIVELY IDENTIFIED WITH MASS SPECTRAL LIBRARY}

Sample: $\quad 2403$ WD

File: $\quad$ C:LHPCHEMLLDATAl62596_7.D

ID: $\quad$ FT6045-3WD.76A

Compound

CAS\#

Ethanol

000064-17-5

2-Propanone

000067-64-1

2-Propanol

000067-63-0

Methane, dichloro-

000075-09-2

Ethane, 1,1-dichloro-

000075-34-3

2-Butanone

000078-93-3

Oxirane, 2,3-dimethyl-

003266-23-7

Ethene, 1,2-dichloro-, (Z)-

000156-59-2

Furan, tetrahydro-

000109-99-9

Ethane, 1,2-dichloro-

000107-06-2

Benzene

$000071-43-2$

1-Butanol

000071-36-3

2-Propanol, 1-methoxy-

000107-98-2

2-Pentanone, 4-methyl-

000108-10-1

Benzene, methyl-

000108-88-3

Ethane, 1,1,2-trichloro-

000079-00-5

Hexanal

000066-25-1 
HNF-SD-WM-RPT-282 Rev. 0

\section{LIST OF COMPOUNDS TENTATIVELY IDENTIFIED WITH MASS SPECTRAL LIBRARY}

Sample: $\quad 2403$ WD (CONTINUED)

File: $\quad$ C:LHPCHEML2LDATAl62596_7.D

ID: $\quad$ FT6045-3WD.76A

Compound

CAS\#

Ethene, tetrachloro-

$000127-18-4$

Benzene, chloro-

000108-90-7

Ethanol, 2-[2-(2-butoxyethoxy)ethoxy]

$000143-22-6$

Benzene, ethyl-

000100-41-4

XYLENE

001330-20-7

3-Heptanone

000106-35-4

2-Heptanone

000110-43-0

Styrene

$000100-42-5$

Benzene, 1,2-dimethyl-

$000095-47-6$

Ethanol, 2-butoxy-

$000111-76-2$

ALPHA.-PINENE, (-)-

000080-56-8

Benzaldehyde

$000100-52-7$

Benzene, 1,3,5-trimethyl-

$000108-67-8$

Benzene, 1-methyl-3-(1-methylethyl)-

000535-77-3

beta.-Phellandrene

$000555-10-2$

Benzene, 1,4-dichloro-

$000106-46-7$

Ethanone, 1-phenyl-

000098-86-2 
HNF-SD-WM-RPT-282 Rev. 0

LIST OF COMPOUNDS TENTATIVELY IDENTIFIED WITH MASS SPECTRAL

LIBRARY

Sample: $\quad 2403$ WD (CONTINUED)

File: $\quad$ C:LHPCHEMILLDATAl62596_7.D

ID: $\quad$ FT6045-3WD.76A

Compound CAS\#

Butyl glycol acetate $\quad 000112-07-2$

Benzaldehyde, ethyl- $\quad$ 053951-50-1

2,3-DIMETHYLBENZALDEHYDE 005779-93-1 
File

Operator

Acquired

Instrument

Sample Name: FT6045-3WD.81A bk

Misc Info : 100 TORR

Vial Number: 1

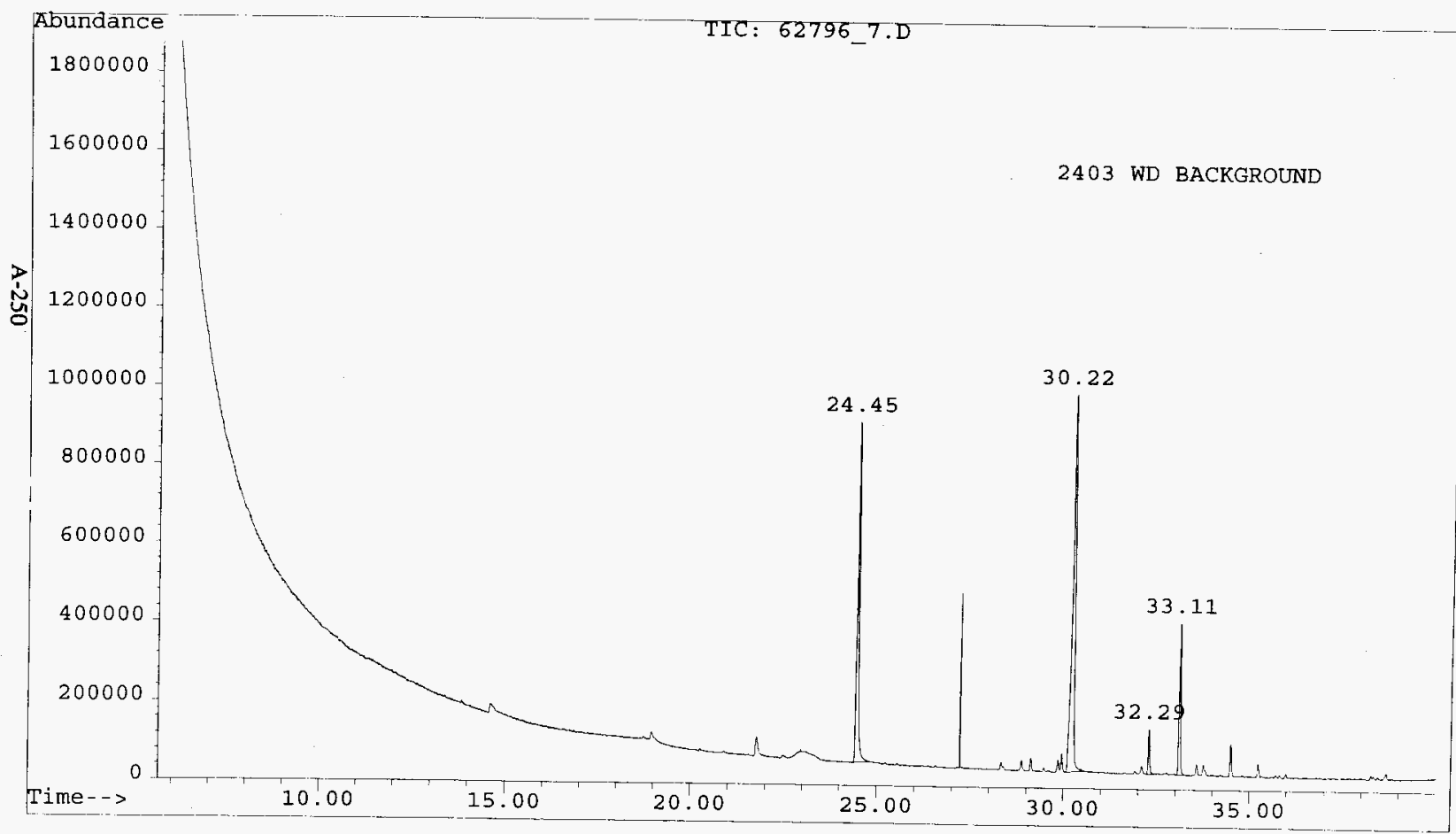


HNF-SD-WM-RPT-282 Rev. 0

CWC_REP.XLS

\section{ANALYSIS REPORT FOR CWC AIR SAMPLES}

File Name:

Analyst:

Analysis Date:

Method:

Sample Name:
62796_7.D

LAP

06/27/96

CWC

FT6045-3WD.81A bk

\begin{tabular}{|c|c|c|c|c|}
\hline COMPOUND NAME & CAS \# & Concentration & Units & Comments \\
\hline ISO-BUTANE & $75-28-5$ & 0 & $\overline{\mathrm{PPB}}$ & \\
\hline VINYL CHLORIDE & $75-01-4$ & 0 & PPB & \\
\hline ETHANOL & $64-17-5$ & 0 & PPB & \\
\hline ACETONE & $67-64-1$ & 0 & PPB & \\
\hline 2-PROPANOL & $67-63-0$ & 0 & PPB & \\
\hline 1,1-DICHLOROETHENE & $75-35-4$ & 0 & PPB & \\
\hline FREON 113 & $76-13-1$ & 0 & PPB & \\
\hline DICHLOROMETHANE & $75-09-2$ & 0 & PPB & \\
\hline 1,1-DICHLOROETHANE & $75-34-3$ & 0 & PPB & \\
\hline 2-BUTANONE & $78-93-3$ & 0 & PPB & \\
\hline N-HEXANE & $110-54-3$ & 0 & PPB & \\
\hline 1,2-DICHLOROETHENE & $156-59-2$ & 0 & PPB & \\
\hline CHLOROFORM & $67-66-3$ & 0 & PPB & \\
\hline TETRAHYDROFURAN & $109-99-9$ & 0 & PPB & \\
\hline 1,1,1-TRICHLOROETHANE & $71-55-6$ & 0 & PPB & \\
\hline 1,2-DICHLOROETHANE & $107-06-2$ & 0 & PPB & \\
\hline BENZENE & $71-43-2$ & 0 & PPB & \\
\hline CARBON TETRACHLORIDE & $56-23-5$ & 0 & PPB & \\
\hline CYCLOHEXANE & $110-82-7$ & 0 & PPB & \\
\hline N-BUTANOL & $71-36-3$ & 0 & PPB & \\
\hline TRICHLOROETHENE & $79-01-6$ & 0 & PPB & \\
\hline 4-METHYL-2-PENTANONE & $108-10-1$ & 20 & PPB & \\
\hline TOLUENE & $108-88-3$ & 0 & PPB & \\
\hline 1,1,2-TRICHLOROETHANE & $79-00-5$ & 0 & PPB & \\
\hline TETRACHLOROETHENE & $127-18-4$ & 0 & PPB & \\
\hline CHLOROBENZENE & $108-90-7$ & 0 & PPB & \\
\hline ETHYLBENZENE & $100-41-4$ & 0 & PPB & \\
\hline M-XYLENE & 108-38-3 & 0 & PPB & \\
\hline STYRENE & $100-42-5$ & 0 & PPB & \\
\hline O-XYLENE & $95-47-6$ & 1 & PPB & \\
\hline 1,1,2,2-TETRACHLORETHANE & $79-34-5$ & 0 & PPB & \\
\hline 1,3,5-TRIMETHYLBENZENE & $108-67-8$ & 3 & PPB & \\
\hline 1,2,4-TRIMETHYLBENZENE & $95-63-6$ & 11 & PPB & \\
\hline 1,3-DICHLOROBENZENE & $541-73-1$ & 0 & PPB & \\
\hline 1,2-DICHLOROBENZENE & $95-50-1$ & 0 & PPB & \\
\hline 1,4-DICHLOROBENZENE & $106-46-7$ & 3 & PPB & \\
\hline
\end{tabular}




\section{LIST OF COMPOUNDS TENTATIVELY IDENTIFIED WITH MASS SPECTRAL LIBRARY}

Sample: 2403 WD BACKGROUND

File: $\quad$ C:LHPCHEML2LDATAl62796_7.D

ID: $\quad$ FT6045-3WD.81A bk

Compound

CAS\#

2-Pentanone, 4-methyl-

$000108-10-1$

Benzene, ethyl-

$000100-41-4$

Benzene, 1,3-dimethyl-

000108-38-3

Styrene

$000100-42-5$

Benzene, 1,2-dimethyl-

000095-47-6

Ethanol, 2-butoxy-

$000111-76-2$

Benzaldehyde

000100-52-7

Benzene, 1,2,4-trimethyl-

000095-63-6

Benzene, 1,2,3-trimethyl-

000526-73-8

Benzene, 1,4-dichloro-

000106-46-7

Benzene, 1,2-dichloro-

000095-50-1

Ethanone, 1-phenyl-

000098-86-2 
File

C : \HPCHEM \1\DATA \72296_8.D

Operator

Acquired

LAP

24 Mar 153

$5972-$ In

Instrument

Sample Name: FT6045-3WD.77B

Misc Info : 100 TORR

Vial Number: 1

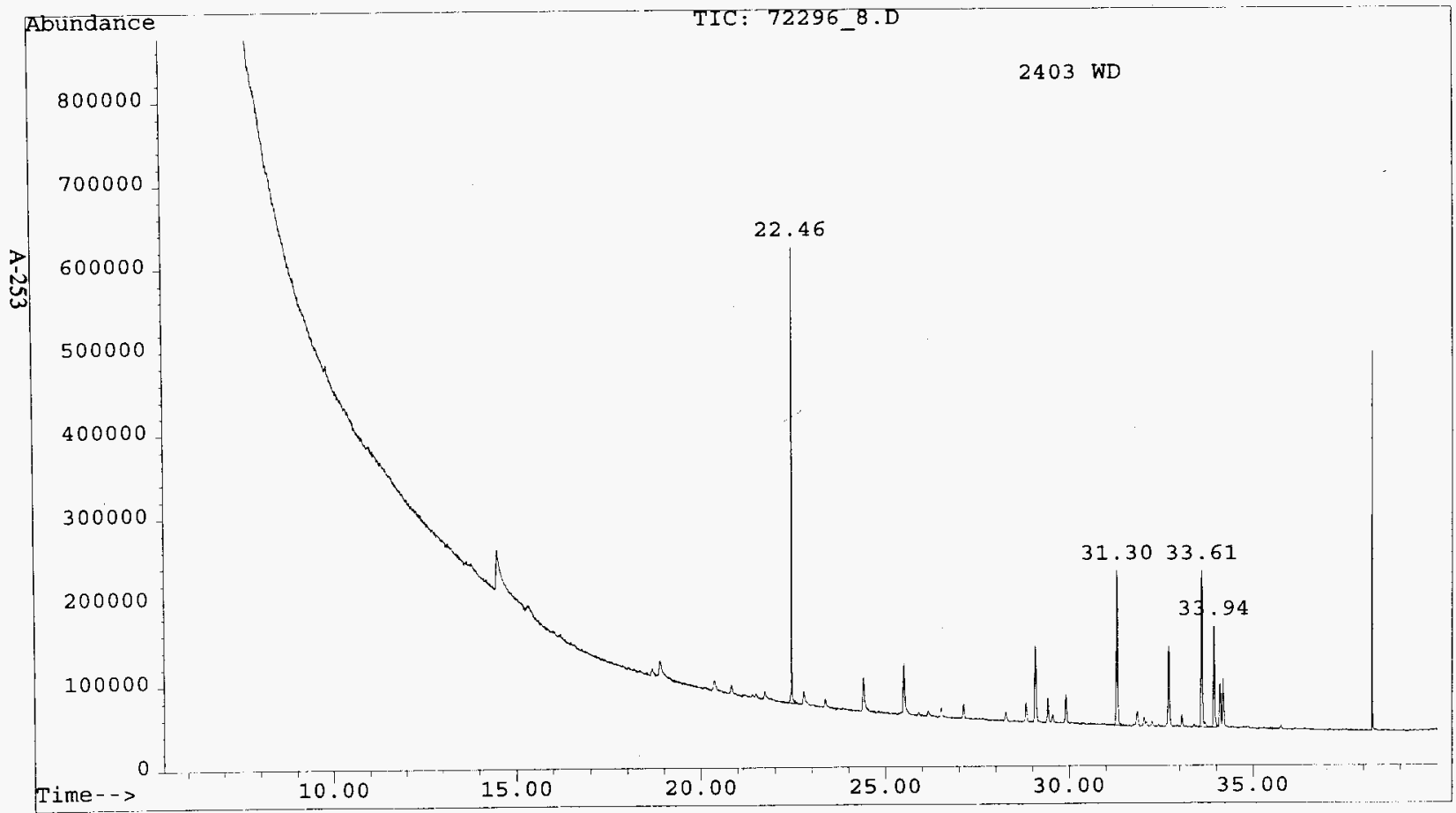


HNF-SD-WM-RPT-282 Rev. 0

CWC_REP.XLS

\section{ANALYSIS REPORT FOR CWC AIR SAMPLES}

File Name:

Analyst:

Analysis Date:

Method:

Sample Name:
72296 8.D

LAP

\#VALUE!

CWC

FT6045-3WD.77B

\begin{tabular}{|c|c|c|c|c|}
\hline COMPOUND NAME & CAS \# & Concentration & Units & Comments \\
\hline ISO-BUTANE & $75-28-5$ & 0 & PPB & \\
\hline VINYL CHLORIDE & $75-01-4$ & 0 & PPB & \\
\hline ETHANOL & $64-17-5$ & 0 & PPB & \\
\hline ACETONE & $67-64-1$ & 0 & PPB & \\
\hline 2-PROPANOL & $67-63-0$ & 0 & PPB & \\
\hline 1,1-DICHLOROETHENE & $75-35-4$ & 0 & PPB & \\
\hline FREON 113 & $76-13-1$ & 0 & PPB & \\
\hline DICHLOROMETHANE & $75-09-2$ & 0 & PPB & \\
\hline 1,1-DICHLOROETHANE & $75-34-3$ & 0 & PPB & \\
\hline 2-BUTANONE & $78-93-3$ & 0 & PPB & \\
\hline N-HEXANE & $110-54-3$ & 0 & PPB & \\
\hline 1,2-DICHLOROETHENE & $156-59-2$ & 0 & PPB & \\
\hline CHLOROFORM & $67-66-3$ & 0 & PPB & \\
\hline TETRAHYDROFURAN & $109-99-9$ & 0 & PPB & \\
\hline 1,1,1-TRICHLOROETHANE & $71-55-6$ & 0 & PPB & \\
\hline 1,2-DICHLOROETHANE & $107-06-2$ & 0 & PPB & \\
\hline BENZENE & $71-43-2$ & 0 & PPB & \\
\hline CARBON TETRACHLORIDE & $56-23-5$ & 0 & PPB & \\
\hline CYCLOHEXANE & $110-82-7$ & 0 & PPB & \\
\hline N-BUTANOL & $71-36-3$ & $\ddot{0}$ & PPB & \\
\hline TRICHLOROETHENE & $79-01-6$ & 0 & PPB & \\
\hline 4-METHYL-2-PENTANONE & $108-10-1$ & 0 & PPB & \\
\hline TOLUENE & $108-88-3$ & 2 & PPB & \\
\hline 1,1,2-TRICHLOROETHANE & $79-00-5$ & 0 & PPB & \\
\hline TETRACHLOROETHENE & $127-18-4$ & 0 & PPB & \\
\hline CHLOROBENZENE & $108-90-7$ & 0 & PPB & \\
\hline ETHYLBENZENE & $100-41-4$ & 2 & PPB & \\
\hline M-XYLENE & $108-38-3$ & 3 & PPB & \\
\hline STYRENE & $100-42-5$ & 0 & PPB & \\
\hline O-XYLENE & $95-47-6$ & 0 & PPB & \\
\hline 1,1,2,2-TETRACHLORETHANE & $79-34-5$ & 0 & PPB & \\
\hline 1,3,5-TRIMETHYLBENZENE & $108-67-8$ & 0 & PPB & \\
\hline 1,2,4-TRIMETHYLBENZENE & $95-63-6$ & 0 & PPB & \\
\hline 1,3-DICHLOROBENZENE & $541-73-1$ & 0 & PPB & \\
\hline 1,2-DICHLOROBENZENE & $95-50-1$ & 0 & PPB & \\
\hline 1,4-DICHLOROBENZENE & $106-46-7$ & 0 & PPB & \\
\hline
\end{tabular}




\section{LIST OF COMPOUNDS TENTATIVELY IDENTIFIED WITH MASS SPECTRAL LIBRARY}

Sample: $\quad 2403$ WD

File: $\quad$ C:LHPCHEML2LDATA172296_8.D

ID: $\quad$ FT6045-3WD.77B

Compound

CAS\#

Benzene, methyl-

000108-88-3

Benzene, 1,2-dimethyl-

$000095-47-6$

Benzene, 1,4-dimethyl-

$000106-42-3$

ALPHA.-PINENE, (-)-

$000080-56-8$

2-.BETA.-PINENE

000127-91-3

DELTA.3-Carene

013466-78-9

Benzene, 1-methyl-2-(1-methylethyl)-

$000527-84-4$

dl-Limonene

000138-86-3

Sabinene

$003387-41-5$ 
File

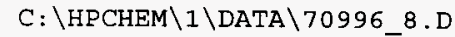

Operator

LAP

Acquired : $9 \mathrm{Jul} 96 \quad 12: 31$ pm using AcqMethod CWC

Instrument : 5972 - In

Sample Name: FT 6045 3WD.83A

Misc Info : Delta $\mathbf{P}^{-}=100 \mathrm{TORR}$

Vial Number: 1

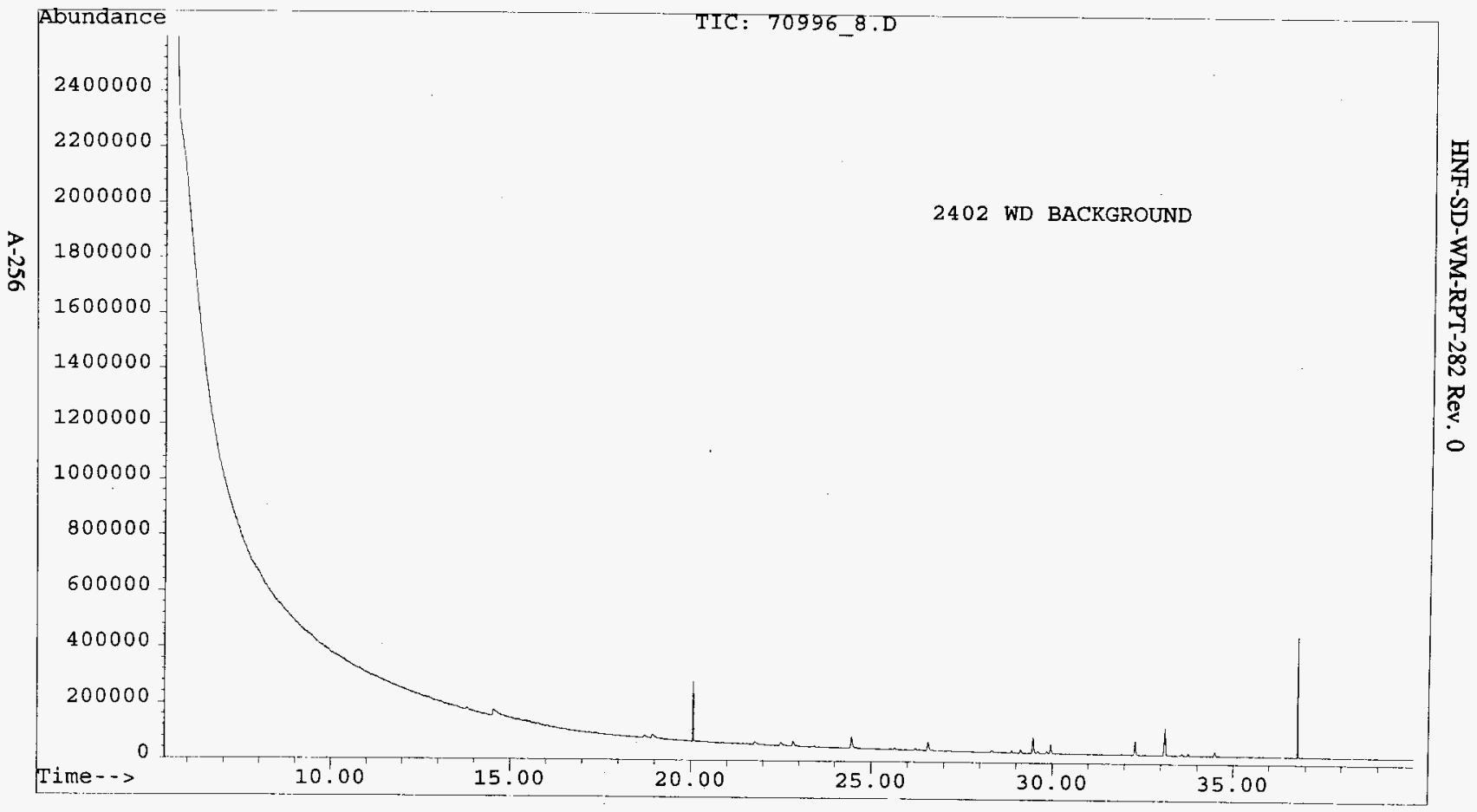


HNF-SD-WM-RPT-282 Rev. 0

CWC_REP.XLS

\section{ANALYSIS REPORT FOR CWC AIR SAMPLES}

File Name:

Analyst:

Analysis Date:

Method:

Sample Name:
70996_8.D

LAP

07/09/96

CWC

FT 6045_3WD.83A

\begin{tabular}{|c|c|c|c|c|}
\hline COMPOUND NAME & CAS \# & Concentration & Units & Comments \\
\hline ISO-BUTANE & $75-28-5$ & $\overline{0}$ & PPB & \\
\hline VINYL CHLORIDE & $75-01-4$ & 0 & PPB & \\
\hline ETHANOL & $64-17-5$ & 0 & $\overline{\mathrm{PPB}}$ & \\
\hline ACETONE & $67-64-1$ & 0 & PPB & \\
\hline 2-PROPANOL & $67-63-0$ & 0 & PPB & \\
\hline 1,1-DICHLOROETHENE & $75-35-4$ & 0 & PPB & \\
\hline FREON 113 & $76-13-1$ & 0 & PPB & \\
\hline DICHLOROMETHANE & $75-09-2$ & 0 & PPB & \\
\hline 1,1-DICHLOROETHANE & $75-34-3$ & 0 & PPB & \\
\hline 2-BUTANONE & $78-93-3$ & 0 & PPB & \\
\hline N-HEXANE & $110-54-3$ & 0 & PPB & \\
\hline 1,2-DICHLOROETHENE & $156-59-2$ & 0 & PPB & \\
\hline CHLOROFORM & $67-66-3$ & 0 & PPB & \\
\hline TETRAHYDROFURAN & $109-99-9$ & 0 & PPB & \\
\hline 1,1,1-TRICHLOROETHANE & $71-55-6$ & 0 & PPB & \\
\hline 1,2-DICHLOROETHANE & $107-06-2$ & 0 & PPB & \\
\hline BENZENE & $71-43-2$ & 0 & PPB & \\
\hline CARBON TETRACHLORIDE & $56-23-5$ & 0 & PPB & \\
\hline CYCLOHEXANE & $110-82-7$ & 0 & PPB & \\
\hline N-BUTANOL & $71-36-3$ & 0 & PPB & \\
\hline TRICHLOROETHENE & $79-01-6$ & 0 & PPB & \\
\hline 4-METHYL-2-PENTANONE & $108-10-1$ & 0 & PPB & \\
\hline TOLUENE & $108-88-3$ & 0 & PPB & \\
\hline 1,1,2-TRICHLOROETHANE & $79-00-5$ & 0 & PPB & \\
\hline TETRACHLOROETHENE & $127-18-4$ & $\overline{0}$ & $\overline{P P B}$ & \\
\hline CHLOROBENZENE & $108-90-7$ & 0 & PPB & \\
\hline ETHYLBENZENE & $100-41-4$ & 0 & PPB & \\
\hline M-XYLENE & $108-38-3$ & 0 & PPB & \\
\hline STYRENE & $100-42-5$ & 0 & PPB & \\
\hline O-XYLENE & $95-47-6$ & 0 & $\mathrm{PPB}$ & \\
\hline $1,1,2,2$-TETRACHLORETHANE & $79-34-5$ & 0 & $\mathrm{PPB}$ & \\
\hline 1,3,5-TRIMETHYLBENZENE & $108-67-8$ & 1 & PPB & \\
\hline 1,2,4-TRIMETHYLBENZENE & $95-63-6$ & 2 & PPB & \\
\hline 1,3-DICHLOROBENZENE & $541-73-1$ & 0 & PPB & \\
\hline 1,2-DICHLOROBENZENE & $95-50-1$ & 0 & $\overline{\mathrm{PPB}}$ & \\
\hline 1,4-DICHLOROBENZENE & $106-46-7$ & 0 & $\overline{\mathrm{PPB}}$ & \\
\hline
\end{tabular}


HNF-SD-WM-RPT-282 Rev. 0

\section{LIST OF COMPOUNDS TENTATIVELY IDENTIFIED WITH MASS SPECTRAL LIBRARY}

Sample: $\quad 2403$ WD

File: $\quad$ C:LHPCHEMLLDATA170996_8.D

ID: FT6045_3WD.83A

Compound

CAS\#

3-Heptanone

000106-35-4

XYLENE

001330-20-7

Benzene, 1,3,5-trimethyl-

$000108-67-8$

Benzene, 1,2,4-trimethyl-

000095-63-6

Benzene, 1,3-dichloro-

000541-73-1 
File

: C: \HPCHEM \1\DATA \62596_9.D

Operator

: LAP

Acquired

Instrument : 5972 - In

Sample Name: FT6045-3WD.80A

Misc Info : 100 TORR

Vial Number: 1

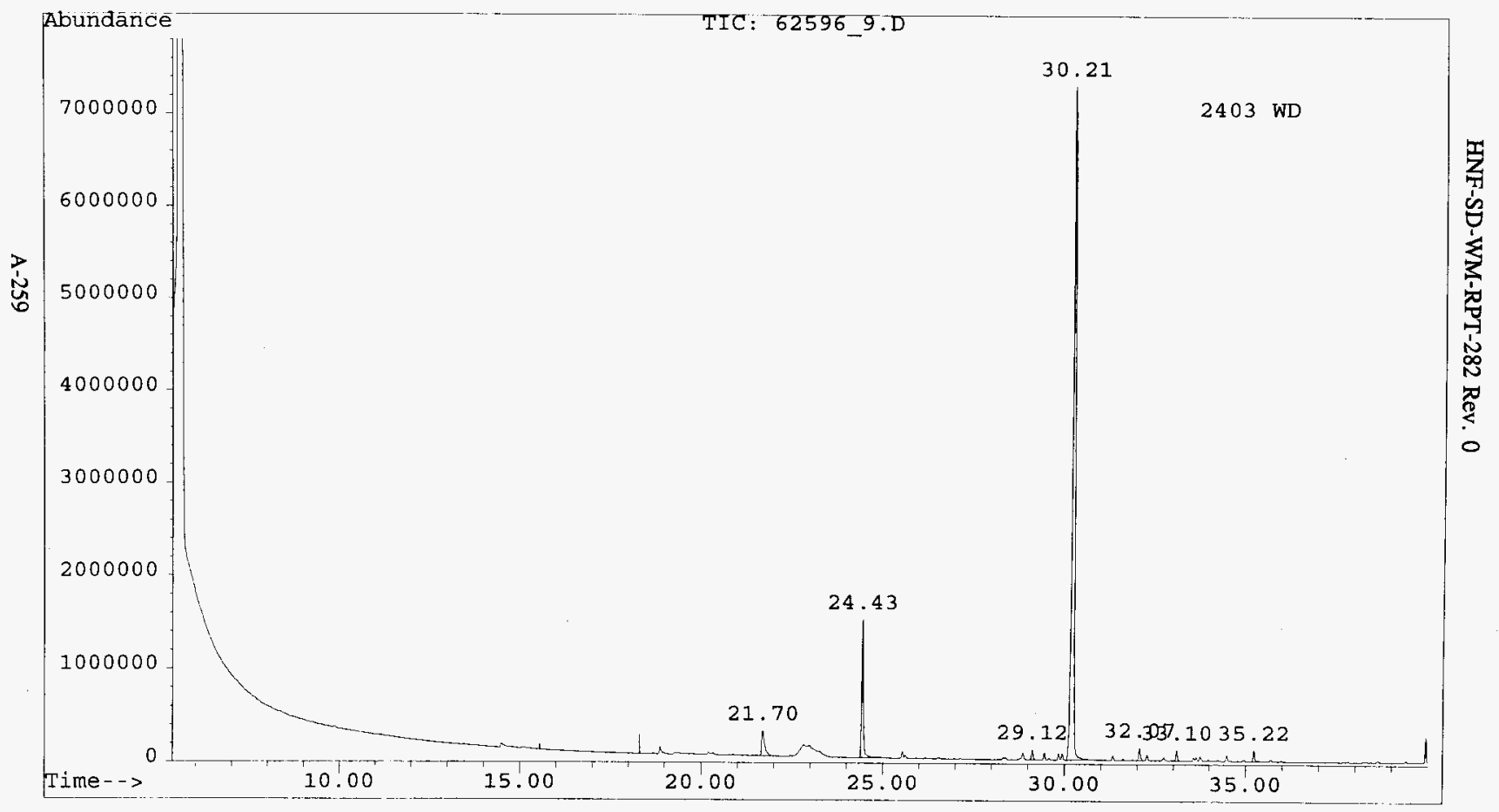


HNF-SD-WM-RPT-282 Rev. 0

CWC_REP.XLS

\section{ANALYSIS REPORT FOR CWC AIR SAMPLES}

File Name:

Analyst:

Analysis Date:

Method:

Sample Name:
62596_9.D

LAP

06/25/96

CWC

FT6045-3WD.80A

\begin{tabular}{|c|c|c|c|c|}
\hline COMPOUND NAME & CAS \# & Concentration & Units & Comments \\
\hline ISO-BUTANE & $75-28-5$ & 0 & PPB & \\
\hline VINYL CHLORIDE & $75-01-4$ & 0 & PPB & \\
\hline ETHANOL & $64-17-5$ & 0 & PPB & \\
\hline ACETONE & $67-64-1$ & 0 & PPB & \\
\hline 2-PROPANOL & $67-63-0$ & 0 & PPB & \\
\hline 1,1-DICHLOROETHENE & $75-35-4$ & 0 & PPB & \\
\hline FREON 113 & $76-13-1$ & 0 & PPB & \\
\hline DICHLOROMETHANE & $75-09-2$ & 0 & PPB & \\
\hline 1,1-DICHLOROETHANE & $75-34-3$ & 0 & PPB & \\
\hline \begin{tabular}{|l|} 
2-BUTANONE \\
\end{tabular} & $78-93-3$ & 0 & PPB & \\
\hline N-HEXANE & $110-54-3$ & 0 & PPB & \\
\hline 1,2-DICHLOROETHENE & $156-59-2$ & 0 & PPB & \\
\hline CHLOROFORM & $67-66-3$ & 0 & PPB & \\
\hline TETRAHYDROFURAN & $109-99-9$ & 0 & PPB & \\
\hline 1,1,1-TRICHLOROETHANE & $71-55-6$ & 0 & PPB & \\
\hline 1,2-DICHLOROETHANE & $107-06-2$ & 0 & PPB & \\
\hline BENZENE & $71-43-2$ & 0 & PPB & \\
\hline CARBON TETRACHLORIDE & $56-23-5$ & 0 & PPB & \\
\hline CYCLOHEXANE & $110-82-7$ & 0 & PPB & \\
\hline N-BUTANOL & $71-36-3$ & 25 & PPB & \\
\hline \begin{tabular}{|l} 
TRICHLOROETHENE \\
\end{tabular} & $79-01-6$ & 0 & PPB & \\
\hline 4-METHYL-2-PENTANONE & $108-10-1$ & 28 & PPB & \\
\hline TOLUENE & 108-88-3 & 3 & PPB & \\
\hline 1,1,2-TRICHLOROETHANE & $79-00-5$ & 0 & $\mathrm{PPB}$ & \\
\hline TETRACHLOROETHENE & $127-18-4$ & 0 & PPB & \\
\hline CHLOROBENZENE & $108-90-7$ & 0 & PPB & \\
\hline ETHYLBENZENE & $100-41-4$ & 2 & PPB & \\
\hline M-XYLENE & $108-38-3$ & 3 & PPB & \\
\hline STYRENE & $100-42-5$ & 2 & PPB & \\
\hline O-XYLENE & $95-47-6$ & 2 & PPB & \\
\hline 1,1,2,2-TETRACHLORETHANE & $79-34-5$ & 0 & PPB & \\
\hline 1,3,5-TRIMETHYLBENZENE & $108-67-8$ & 1 & PPB & \\
\hline 1,2,4-TRIMETHYLBENZENE & $95-63-6$ & 3 & PPB & \\
\hline 1,3-DICHLOROBENZENE & $541-73-1$ & 0 & PPB & \\
\hline 1,2-DICHLOROBENZENE & $95-50-1$ & 0 & PPB & \\
\hline 1,4-DICHLOROBENZENE & $106-46-7$ & 2 & PPB & \\
\hline
\end{tabular}




\section{LIST OF COMPOUNDS TENTATIVELY IDENTIFIED WITH MASS SPECTRAL LIBRARY}

Sample: $\quad 2403$ WD

File: C:LPCHEML2LDATAl62596_9.D

ID: $\quad$ FT6045-3WD.80A

Compound

1-Butanol

2-Pentanone, 4-methyl-

Benzene, methyl-

Benzene, chloro-

Benzene, ethyl-

Benzene, 1,2-dimethyl-

3-Heptanone

Styrene

Ethanol, 2-butoxy-

ALPHA.-PINENE, (-)-

Benzaldehyde

Benzene, 1,2,3-trimethyl-

Benzene, 1,3,5-trimethyl-

Benzene, 1,4-dichloro-

DELTA 3-Carene

Benzene, 4-ethyl-1,2-dimethyl-

Benzenemethanol, .alpha-methyl-

Ethanone, 1-phenyl-

\section{CAS\#}

000071-36-3

000108-10-1

000108-88-3

000108-90-7

$000100-41-4$

000095-47-6

000106-35-4

$000100-42-5$

$000111-76-2$

000080-56-8

$000100-52-7$

000526-73-8

000108-67-8

000106-46-7

013466-78-9

000934-80-5

000098-85-1

000098-86-2 
File

Operator

Acquired

Instrument

\section{2 - In}

Sample Name: FT6045-3WD.77A

Misc Info : 100 TORR

Vial Number: 1

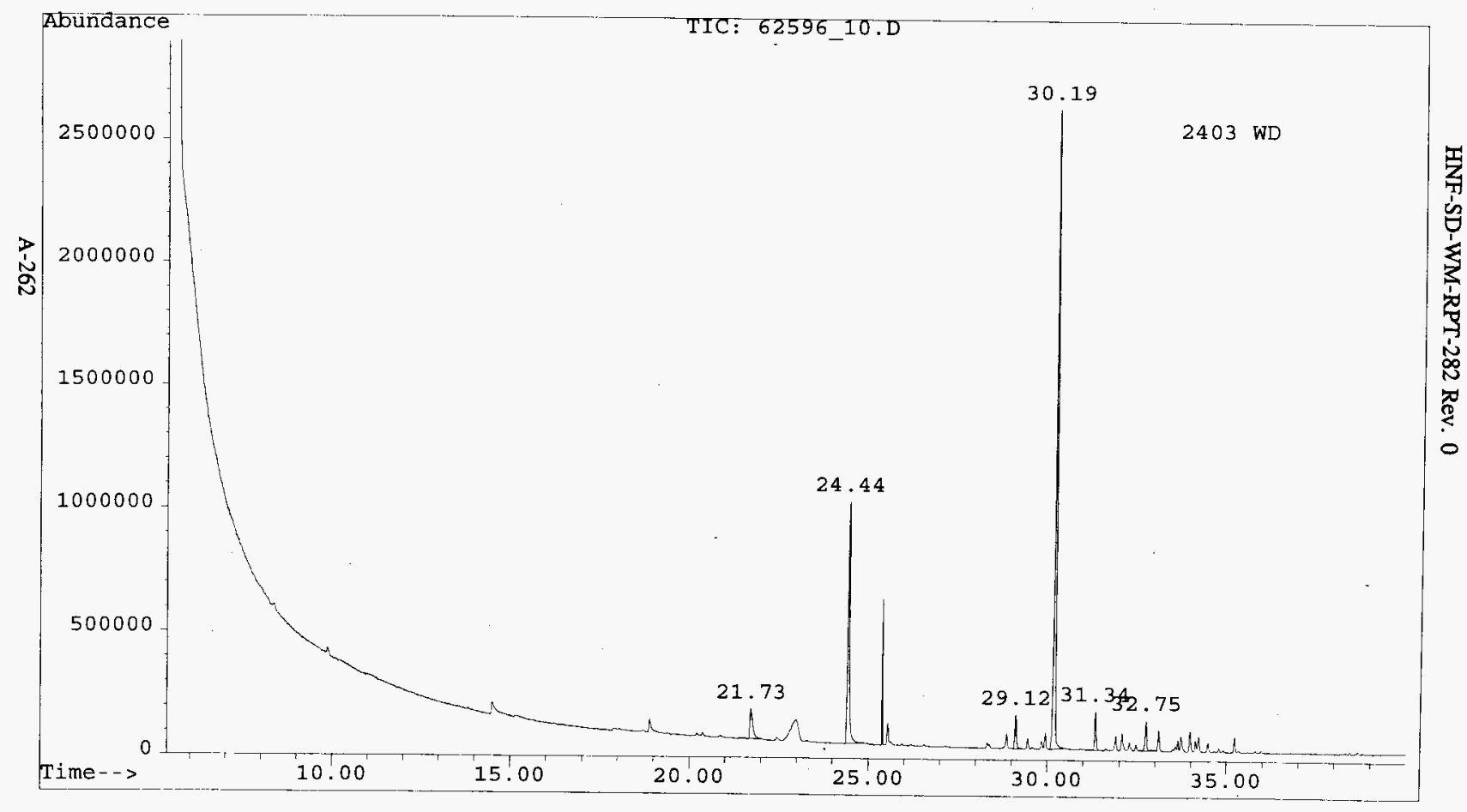


HNF-SD-WM-RPT-282 Rev. 0

CWC_REP.XLS

\section{ANALYSIS REPORT FOR CWC AIR SAMPLES}

File Name:

Anaiyst:

Analysis Date:

Method:

Sample Name
62596_10.D

LAP

06/25/96

CWC

FT6045-3WD.77A

\begin{tabular}{|c|c|c|c|c|}
\hline COMPOUND NAME & CAS \# & Concentration & Units & Comments \\
\hline ISO-BUTANE & $75-28-5$ & $\overline{0}$ & $\overline{\overline{P P B}}$ & \\
\hline VINYL CHLORIDE & $75-01-4$ & 0 & PPB & \\
\hline ETHANOL & $64-17-5$ & 0 & PPB & \\
\hline ACETONE & $67-64-1$ & 0 & PPB & \\
\hline 2-PROPANOL & $67-63-0$ & 0 & PPB & \\
\hline 1,1-DICHLOROETHENE & $75-35-4$ & 0 & PPB & \\
\hline FREON 113 & $76-13-1$ & 0 & PPB & \\
\hline DICHLOROMETHANE & $75-09-2$ & 0 & PPB & \\
\hline 1,1-DICHLOROETHANE & $75-34-3$ & 0 & PPB & \\
\hline 2-BUTANONE & $78-93-3$ & 0 & PPB & \\
\hline N-HEXANE & $110-54-3$ & 0 & $\overline{P P B}$ & \\
\hline 1,2-DICHLOROETHENE & $156-59-2$ & 0 & $\mathrm{PPB}$ & \\
\hline CHLOROFORM & $67-66-3$ & 0 & PPB & \\
\hline TETRAHYDROFURAN & $109-99-9$ & 0 & PPB & \\
\hline 1,1,1-TRICHLOROETHANE & $71-55-6$ & 0 & PPB & \\
\hline 1,2-DICHLOROETHANE & $107-06-2$ & $\overline{0}$ & PPB & \\
\hline BENZENE & $71-43-2$ & 0 & PPB & \\
\hline CARBON TETRACHLORIDE & $56-23-5$ & 0 & PPB & \\
\hline CYCLOHEXANE & $110-82-7$ & 0 & PPB & \\
\hline N-BUTANOL & $71-36-3$ & 10 & PPB & \\
\hline TRICHLOROETHENE & $79-01-6$ & $\overline{0}$ & PPB & \\
\hline 4-METHYL-2-PENTANONE & $108-10-1$ & 19 & PPB & \\
\hline TOLUENE & $108-88-3$ & 3 & PPB & \\
\hline 1,1,2-TRICHLOROETHANE & $79-00-5$ & 0 & PPB & \\
\hline TETRACHLOROETHENE & $127-18-4$ & 0 & PPB & \\
\hline CHLOROBENZENE & $108-90-7$ & 0 & PPB & \\
\hline ETHYLBENZENE & $100-41-4$ & 2 & PPB & \\
\hline M-XYLENE & $108-38-3$ & 4 & PPB & \\
\hline STYRENE & $100-42-5$ & 0 & PPB & \\
\hline O-XYLENE & $95-47-6$ & 2 & PPB & \\
\hline 1,1,2,2-TETRACHLORETHANE & $79-34-5$ & 0 & PPB & \\
\hline 1,3,5-TRIMETHYLBENZENE & $108-67-8$ & 2 & PPB & \\
\hline 1,2,4-TRIMETHYLBENZENE & $95-63-6$ & 2 & PPB & \\
\hline 1,3-DICHLOROBENZENE & $541-73-1$ & 0 & PPB & \\
\hline 1,2-DICHLOROBENZENE & $95-50-1$ & $\overline{0}$ & PPB & \\
\hline 1,4-DICHLOROBENZENE & $106-46-7$ & 0 & PPB & \\
\hline
\end{tabular}




\section{LIST OF COMPOUNDS TENTATIVELY WENTIFIED WITH MASS SPECTRAL, LIBRARY}

Sample:

File:

ID:

Compound

2403 WD

C: $\left(H P C H E M \backslash 2 \backslash D A T A 162596 \_10 . D\right.$

FT6045-3WD.77A
1-Butanol

2-Pentanone, 4-methyl-

Benzene, methyl-

Chlorobenzene-D5

Benzene, ethyl-

Benzene, 1,4-dimethyl-

3-Heptanone

Styrene

Benzene, 1,2-dimethyl-

Ethanol, 2-butoxy-

ALPHA.-PINENE, (-)-

Benzaldehyde

Benzene, 1-ethyl-2,3-dimethyl-

2-.BETA.-PINENE

Benzene, 1,2,3-trimethyl-

Benzene, 1,4-dichloro-

\section{CAS\#}

000071-36-3

000108-10-1

000108-88-3

000000-00-0

$000100-41-4$

000106-42-3

000106-35-4

$000100-42-5$

$000095-47-6$

$000111-76-2$

000080-56-8

000100-52-7

000933-98-2

000127-91-3

000526-73-8

000106-46-7 
HNF-SD-WM-RPT-282 Rev. 0

\section{LIST OF COMPOUNDS TENTATIVELY IDENTIFIED WITH MASS SPECTRAL}

\section{LIBRARY}

Sample: $\quad 2403$ WD

File: $\quad$ C:LHPCHEMLLDATAl62596_10.D

ID: $\quad$ FT6045-3WD.77A

Compound

CAS\#

DELTA 3-Carene

013466-78-9

Benzene, 1-methyl-2-(1-methylethyl)-

$000527-84-4$

Cyclohexene, 1-methyl-4-(1-methylethe

005989-27-5

Sabinene

003387-41-5

Benzene, 1,2-dichloro-

000095-50-1

Ethanone, 1-phenyl-

000098-86-2 


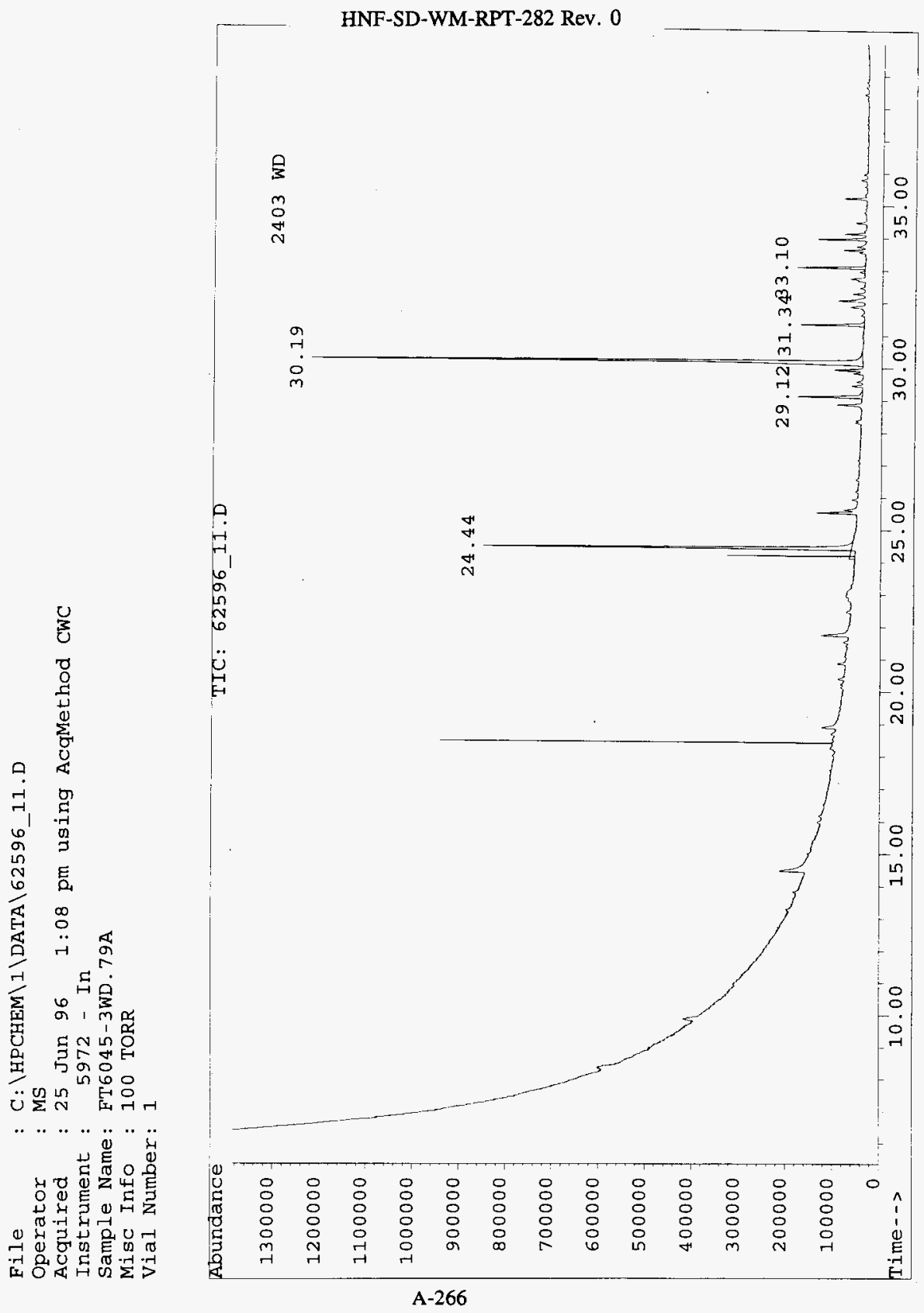


HNF-SD-WM-RPT-282 Rev. 0

CWC_REP.XLS

\section{ANALYSIS REPORT FOR CWC AIR SAMPLES}

File Name:

Analyst:

Analysis Date:

Method:

Sample Name:
62596_11.D

MS

$06 / 25 / 96$

CWC

FT6045-3WD.79A

\begin{tabular}{|c|c|c|c|c|}
\hline COMPOUND NAME & CAS \# & Concentration & Units & Comments \\
\hline ISO-BUTANE & $75-28-5$ & 0 & PPB & \\
\hline VINYL CHLORIDE & $75-01-4$ & 0 & PPB & \\
\hline ETHANOL & $64-17-5$ & 0 & PPB & \\
\hline ACETONE & $67-64-1$ & 0 & PPB & \\
\hline 2-PROPANOL & $67-63-0$ & 0 & PPB & \\
\hline 1,1-DICHLOROETHENE & $75-35-4$ & 0 & PPB & \\
\hline FREON 113 & $76-13-1$ & $\overline{0}$ & PPB & \\
\hline DICHLOROMETHANE & $75-09-2$ & 0 & PPB & \\
\hline 1,1-DICHLOROETHANE & $75-34-3$ & 0 & PPB & \\
\hline 2-BUTANONE & 78-93-3 & 0 & PPB & \\
\hline N-HEXANE & $110-54-3$ & 0 & $\overline{\text { PPB }}$ & \\
\hline 1,2-DICHLOROETHENE & $156-59-2$ & 0 & PPB & \\
\hline CHLOROFORM & $67-66-3$ & 0 & PPB & \\
\hline TETRAHYDROFURAN & $109-99-9$ & 0 & PPB & \\
\hline 1,1,1-TRICHLOROETHANE & $71-55-6$ & 0 & PPB & \\
\hline 1,2-DICHLOROETHANE & $107-06-2$ & 0 & PPB & \\
\hline BENZENE & $71-43-2$ & 0 & PPB & \\
\hline CARBON TETRACHLORIDE & $56-23-5$ & 0 & PPB & \\
\hline CYCLOHEXANE & $110-82-7$ & 0 & PPB & \\
\hline N-BUTANOL & $71-36-3$ & 0 & PPB & \\
\hline TRICHLOROETHENE & $79-01-6$ & 0 & PPB & \\
\hline 4-METHYL-2-PENTANONE & $108-10-1$ & 15 & PPB & \\
\hline TOLUENE & $108-88-3$ & 3 & PPB & \\
\hline 1,1,2-TRICHLOROETHANE & $79-00-5$ & 0 & PPB & \\
\hline TETRACHLOROETHENE & $127-18-4$ & 0 & PPB & \\
\hline CHLOROBENZENE & $108-90-7$ & 0 & PPB & \\
\hline ETHYLBENZENE & $100-41-4$ & 1 & PPB & \\
\hline M-XYLENE & $108-38-3$ & 4 & PPB & \\
\hline STYRENE & $100-42-5$ & 0 & PPB & \\
\hline O-XYLENE & $95-47-6$ & 2 & PPB & \\
\hline 1,1,2,2-TETRACHLORETHANE & $79-34-5$ & 0 & PPB & \\
\hline 1,3,5-TRIMETHYLBENZENE & $108-67-8$ & 2 & $\overline{\mathrm{PPB}}$ & \\
\hline 1,2,4-TRIMETHYLBENZENE & $95-63-6$ & 4 & PPB & \\
\hline 1,3-DICHLOROBENZENE & $541-73-1$ & 0 & PPB & \\
\hline 1,2-DICHLOROBENZENE & $95-50-1$ & 0 & PPB & \\
\hline 1,4-DICHLOROBENZENE & $106-46-7$ & 0 & PPB & \\
\hline
\end{tabular}


HNF-SD-WM-RPT-282 Rev. 0

\section{LIST OF COMPOUNDS TENTATIVELY IDENTIFIED WITH MASS SPECTRAL LIBRARY}

Sample: $\quad 2403$ WD

File: $\quad$ C:LHPCHEMLLDATAl62596_11.D

ID: $\quad$ FT6045-3WD.79A

Compound

CAS\#

1-Butanol

$000071-36-3$

2-Pentanone, 4-methyl-

000108-10-1

Benzene, methyl-

000108-88-3

Benzene, ethyl-

000100-41-4

Benzene, 1,3-dimethyl-

00108-38-3

Styrene

$000100-42-5$

Benzene, 1,3-dimethyl-

000108-38-3

Ethanol, 2-butoxy-

$000111-76-2$

ALPHA.-PINENE, (-)-

000080-56-8

Camphene

000079-92-5

Benzaldehyde

000100-52-7

Benzene, 1,3,5-trimethyl-

000108-67-8

2-.BETA.-PINENE

000127-91-3

Benzene, 1,2,4-trimethyl-

000095-63-6

Benzene, 1,2-dichloro-

000095-50-1

DELTA.3-Carene

013466-78-9

Benzene, 1,2-dichloro-

000095-50-1 
HNF-SD-WM-RPT-282 Rev, 0

\section{LIST OF COMPOUNDS TENTATIVELY IDENTIFIED WITH MASS SPECTRAL LIBRARY}

Sample: $\quad 2403$ WD (CONTINUED)

File: $\quad$ C:LHPCHEMLLDATAI62596_11.D

ID: $\quad$ FT6045-3WD.79A

Compound CAS\#

Benzene, 1-methyl-2-(1-methylethyl)- $\quad$ 000527-84-4

dl-Limonene $\quad 000138-86-3$

Benzene, 1,4-dichloro- $\quad 000106-46-7$

Ethanone, 1-phenyl- 000098-86-2 
File

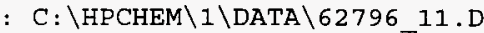

Operator

: MS

Acquired : 27 Jun $96 \quad 6: 17$ pm using AcqMethod CWC

Instrument : 5972 - In

Sample Name: FT 6045- 3WD.75A

Misc Info : 100 TORR

Vial Number: 1

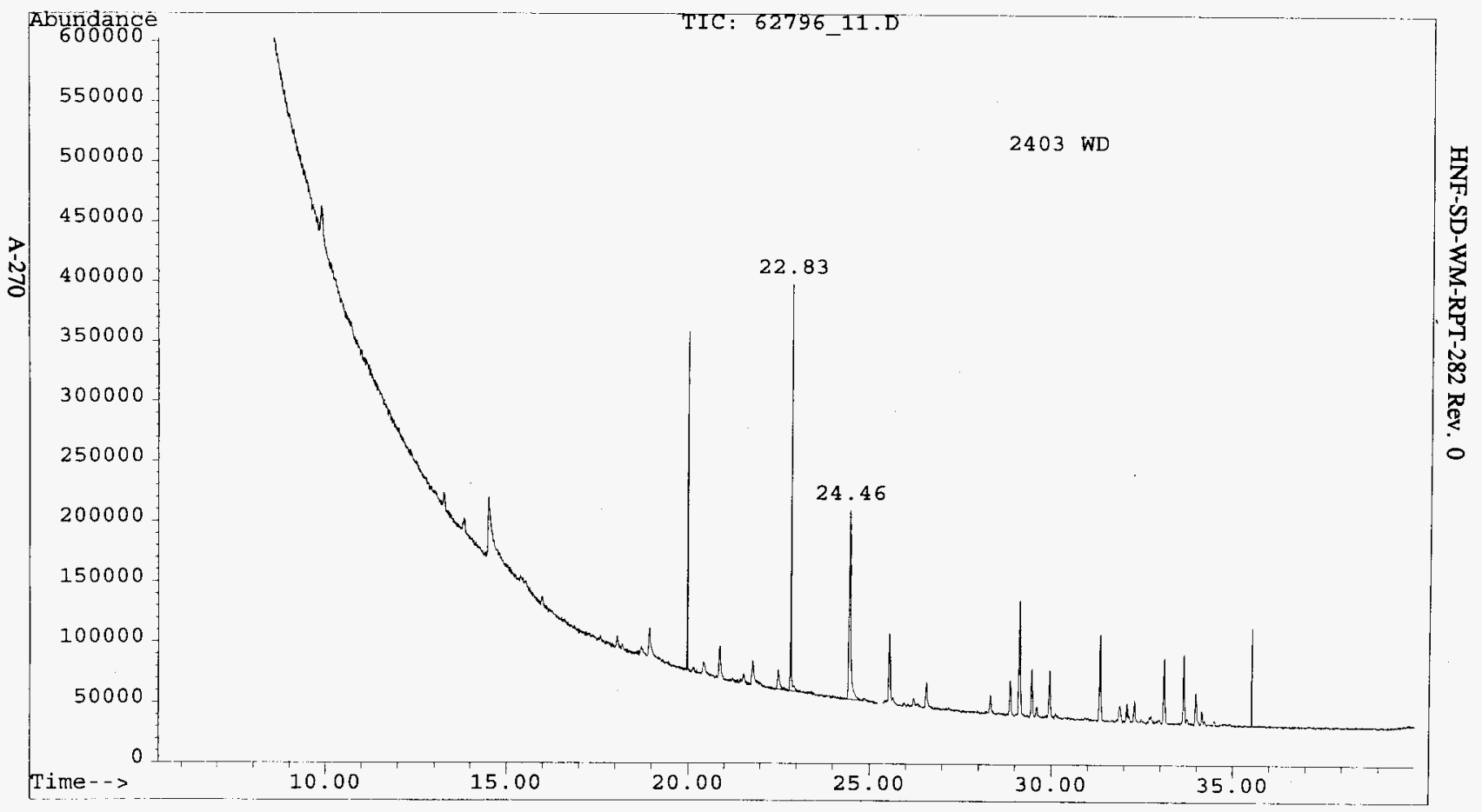


HNF-SD-WM-RPT-282 Rev. 0

CWC_REP.XLS

\section{ANALYSIS REPORT FOR CWC AIR SAMPLES}

File Name:

Anaiyst:

Analysis Date:

Method:

Sample Name:
62796_11.D

MS

06/27/96

CWC

FT 6045- 3WD.75A

\begin{tabular}{|c|c|c|c|c|}
\hline COMPOUND NAME & CAS\# & Concentration & Units & Comments \\
\hline ISO-BUTANE & $75-28-5$ & 0 & PPB & \\
\hline VINYL CHLORIDE & $75-01-4$ & 0 & PPB & \\
\hline ETHANOL & $64-17-5$ & 0 & PPB & \\
\hline ACETONE & $67-64-1$ & 0 & PPB & \\
\hline 2-PROPANOL & $67-63-0$ & 0 & PPB & \\
\hline 1,1-DICHLOROETHENE & $75-35-4$ & 0 & PPB & \\
\hline FREON 113 & $76-13-1$ & 0 & PPB & \\
\hline DICHLOROMETHANE & $75-09-2$ & 0 & PPB & \\
\hline 1,1-DICHLOROETHANE & $75-34-3$ & 0 & PPB & \\
\hline 2-BUTANONE & $78-93-3$ & 0 & PPB & \\
\hline N-HEXANE & $110-54-3$ & 0 & PPB & \\
\hline 1,2-DICHLOROETHENE & $156-59-2$ & 0 & PPB & \\
\hline CHLOROFORM & $67-66-3$ & 0 & PPB & \\
\hline TETRAHYDROFURAN & $109-99-9$ & 0 & PPB & \\
\hline 1,1,1-TRICHLOROETHANE & $71-55-6$ & 0 & $\overline{\mathrm{PPB}}$ & \\
\hline 1,2-DICHLOROETHANE & $107-06-2$ & 0 & PPB & \\
\hline BENZENE & $71-43-2$ & 0 & PPB & \\
\hline CARBON TETRACHLORIDE & $56-23-5$ & 0 & PPB & \\
\hline CYCLOHEXANE & $110-82-7$ & 0 & PPB & \\
\hline N-BUTANOL & $71-36-3$ & 0 & PPB & \\
\hline TRICHLOROETHENE & $79-01-6$ & 0 & PPB & \\
\hline 4-METHYL-2-PENTANONE & $108-10-1$ & 0 & PPB & \\
\hline TOLUENE & $108-88-3$ & 2 & PPB & \\
\hline 1,1,2-TRICHLOROETHANE & $79-00-5$ & 0 & PPB & \\
\hline TETRACHLOROETHENE & $127-18-4$ & 0 & PPB & \\
\hline CHLOROBENZENE & $108-90-7$ & 0 & PPB & \\
\hline ETHYLBENZENE & $100-41-4$ & 2 & PPB & \\
\hline M-XYLENE & $108-38-3$ & 3 & PPB & \\
\hline STYRENE & $100-42-5$ & 0 & PPB & \\
\hline O-XYLENE & $95-47-6$ & 0 & PPB & \\
\hline 1,1,2,2-TETRACHLORETHANE & $79-34-5$ & 0 & PPB & \\
\hline 1,3,5-TRIMETHYLBENZENE & $108-67-8$ & 0 & PPB & \\
\hline 1,2,4-TRIMETHYLBENZENE & $95-63-6$ & 1 & PPB & \\
\hline 1,3-DICHLOROBENZENE & $541-73-1$ & 0 & PPB & \\
\hline 1,2-DICHLOROBENZENE & $95-50-1$ & 0 & PPB & \\
\hline 1,4-DICHLOROBENZENE & $106-46-7$ & 0 & PPB & \\
\hline
\end{tabular}




\section{LIST OF COMPOUNDS TENTATIVELY IDENTIFIED WITH MASS SPECTRAL LIBRARY}

Sample: $\quad 2403$ WD

File: $\quad$ C:LHPCHEMLLDATAl62796_11.D

ID: $\quad$ FT6045-3WD.75A

Compound

CAS\#

2-Pentanone, 4-methyl-

000108-10-1

Benzene, methyl-

000108-88-3

Benzene, ethyl-

$000100-41-4$

Benzene, 1,3-dimethyl-

000108-38-3

3-Heptanone

000106-35-4

XYLENE

001330-20-7

ALPHA.-PINENE, (-)-

000080-56-8

Benzene, 1,2,3-trimethyl-

000526-73-8

Benzene, 1,3,5-trimethyl-

000108-67-8

DELTA.3-Carene

013466-78-9

Benzene, 1-methyl-2-(1-methylethyl)-

$000527-84-4$ 
File

Operator

Acquired

Instrument :

Misc Info : 100 TORR

Vial Number: 1

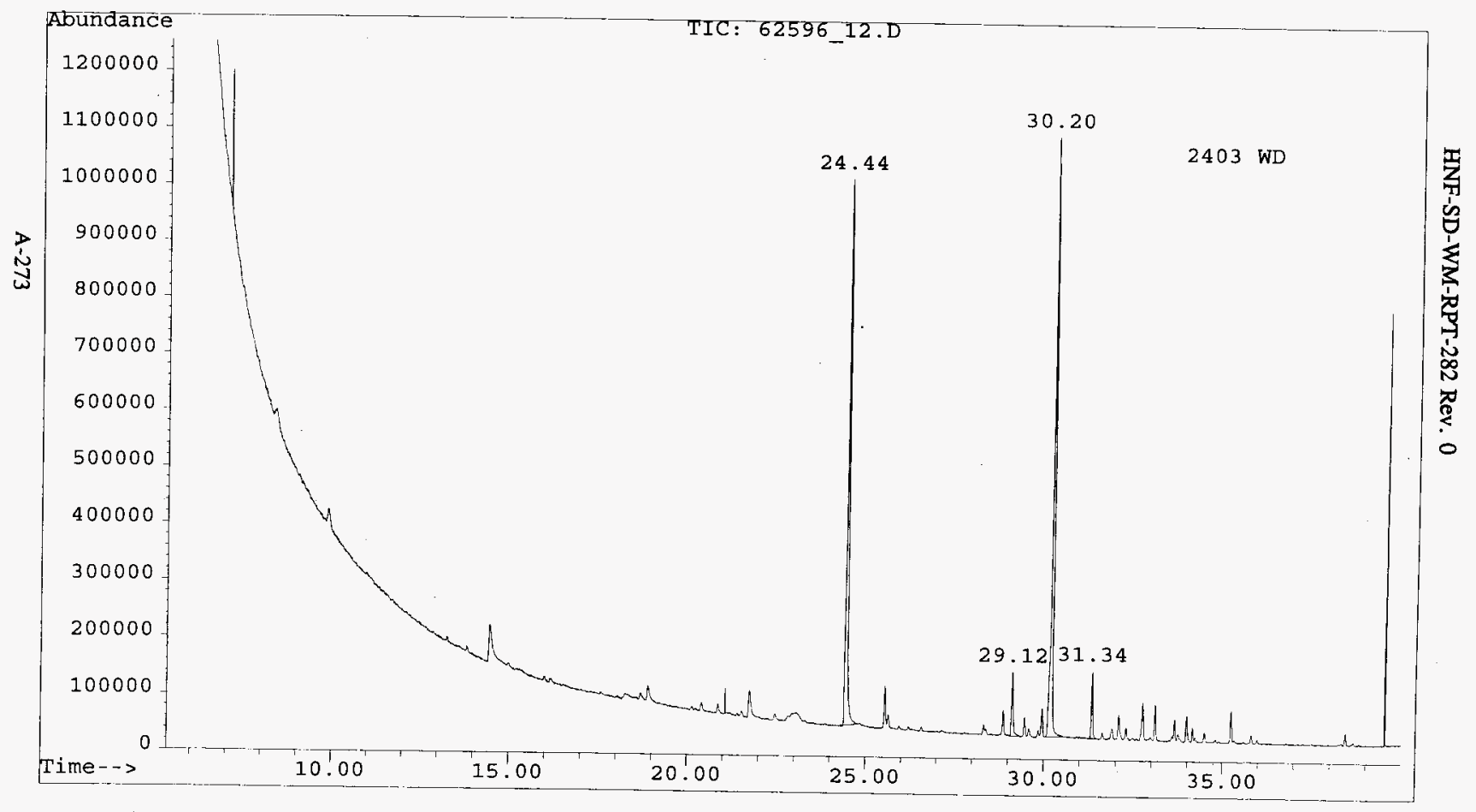


HNF-SD-WM-RPT-282 Rev. 0

CWC_REP.XLS

\section{ANALYSIS REPORT FOR CWC AIR SAMPLES}

File Name:

Analyst:

Analysis Date:

Method:

Sample Name:
62596_12.D

MS

06/25/96

CWC

FT6045-3WD.78A

\begin{tabular}{|c|c|c|c|c|}
\hline COMPOUND NAME & CAS\# & Concentration & Units & Comments \\
\hline ISO-BUTANE & $75-28-5$ & 0 & PPB & \\
\hline VINYL CHLORIDE & $75-01-4$ & 0 & PPB & \\
\hline ETHANOL & $64-17-5$ & 0 & PPB & \\
\hline ACETONE & $67-64-1$ & 4 & PPB & \\
\hline 2-PROPANOL & $67-63-0$ & 0 & PPB & \\
\hline 1,1-DICHLOROETHENE & $75-35-4$ & 0 & PPB & \\
\hline FREON 113 & $7 \overline{76-13-1}$ & 0 & PPB & \\
\hline DICHLOROMETHANE & $75-09-2$ & 0 & PPB & \\
\hline 1,1-DICHLOROETHANE & $75-34-3$ & 0 & PPB & \\
\hline 2-BUTANONE & $78-93-3$ & 0 & PPB & \\
\hline N-HEXANE & $110-54-3$ & 0 & $\mathrm{PPB}$ & \\
\hline 1,2-DICHLOROETHENE & $156-59-2$ & 0 & $\overline{\mathrm{PPB}}$ & \\
\hline CHLOROFORM & $67-66-3$ & 0 & PPB & \\
\hline TETRAHYDROFURAN & $109-99-9$ & 0 & PPB & \\
\hline 1,1,1-TRICHLOROETHANE & $71-55-6$ & 0 & PPB & \\
\hline 1,2-DICHLOROETHANE & $107-06-2$ & 0 & PPB & \\
\hline BENZENE & $71-43-2$ & 0 & PPB & \\
\hline CARBON TETRACHLORIDE & $56-23-5$ & 0 & PPB & \\
\hline CYCLOHEXANE & $110-82-7$ & 0 & PPB & \\
\hline N-BUTANOL & $71-36-3$ & 0 & PPB & \\
\hline TRICHLOROETHENE & $79-01-6$ & 0 & PPB & \\
\hline 4-METHYL-2-PENTANONE & $108-10-1$ & 19 & PPB & \\
\hline TOLUENE & $108-88-3$ & 2 & PPB & \\
\hline 1,1,2-TRICHLOROETHANE & $79-00-5$ & 0 & PPB & \\
\hline TETRACHLOROETHENE & $127-18-4$ & 0 & PPB & \\
\hline CHLOROBENZENE & $108-90-7$ & 0 & PPB & \\
\hline ETHYLBENZENE & $100-41-4$ & 1 & PPB & \\
\hline M-XYLENE & $108-38-3$ & 3 & PPB & \\
\hline STYRENE & $100-42-5$ & 0 & PPB & \\
\hline O-XYLENE & $95-47-6$ & 1 & PPB & \\
\hline 1,1,2,2-TETRACHLORETHANE & $79-34-5$ & 0 & PPB & \\
\hline 1,3,5-TRIMETHYLBENZENE & $108-67-8$ & 0 & PPB & \\
\hline 1,2,4-TRIMETHYLBENZENE & $95-63-6$ & 2 & PPB & \\
\hline 1,3-DICHLOROBENZENE & $541-73-1$ & 0 & PPB & \\
\hline 1,2-DICHLOROBENZENE & $95-50-1$ & 0 & PPB & \\
\hline 1,4-DICHLOROBENZENE & $106-46-7$ & 0 & PPB & \\
\hline
\end{tabular}


HNF-SD-WM-RPT-282 Rev. 0

\section{LIST OF COMPOUNDS TENTATIVELY IDENTIFIED WITH MASS SPECTRAL LIBRARY}

Sample: $\quad 2403$ WD

File: $\quad$ C:IHPCHEMI2LATA162596_12.D

ID: $\quad$ FT6045-3WD.78A

Compound

CAS\#

2-Pentanone, 4-methyl-

000108-10-1

Benzene, methyl-

000108-88-3

Chlorobenzene-D5

000000-00-0

Benzene, ethyl-

$000100-41-4$

Benzene, 1,2-dimethyl-

$000095-47-6$

3-Heptanone

000106-35-4

Ethanol, 2-butoxy-

$000111-76-2$

ALPHA.-PINENE, (-)-

000080-56-8

Benzaldehyde

000100-52-7

Benzene, 1,3,5-trimethyl-

000108-67-8

2-.BETA.-PINENE

$000127-91-3$

Benzene, 1,3,5-trimethyl-

$000108-67-8$

DELTA.3-Carene

013466-78-9

Benzene, 1-methyl-2-(1-methylethyl)-

$000527-84-4$

dl-Limonene

000138-86-3

Benzene, 1,4-dichloro-

000106-46-7

Ethanone, 1-phenyl-

000098-86-2 
File

Operator

Acquired

Instrument

Sample Name: FT6045-3WD.82A BK

Misc Info : 100 TORR

Vial Number: 1

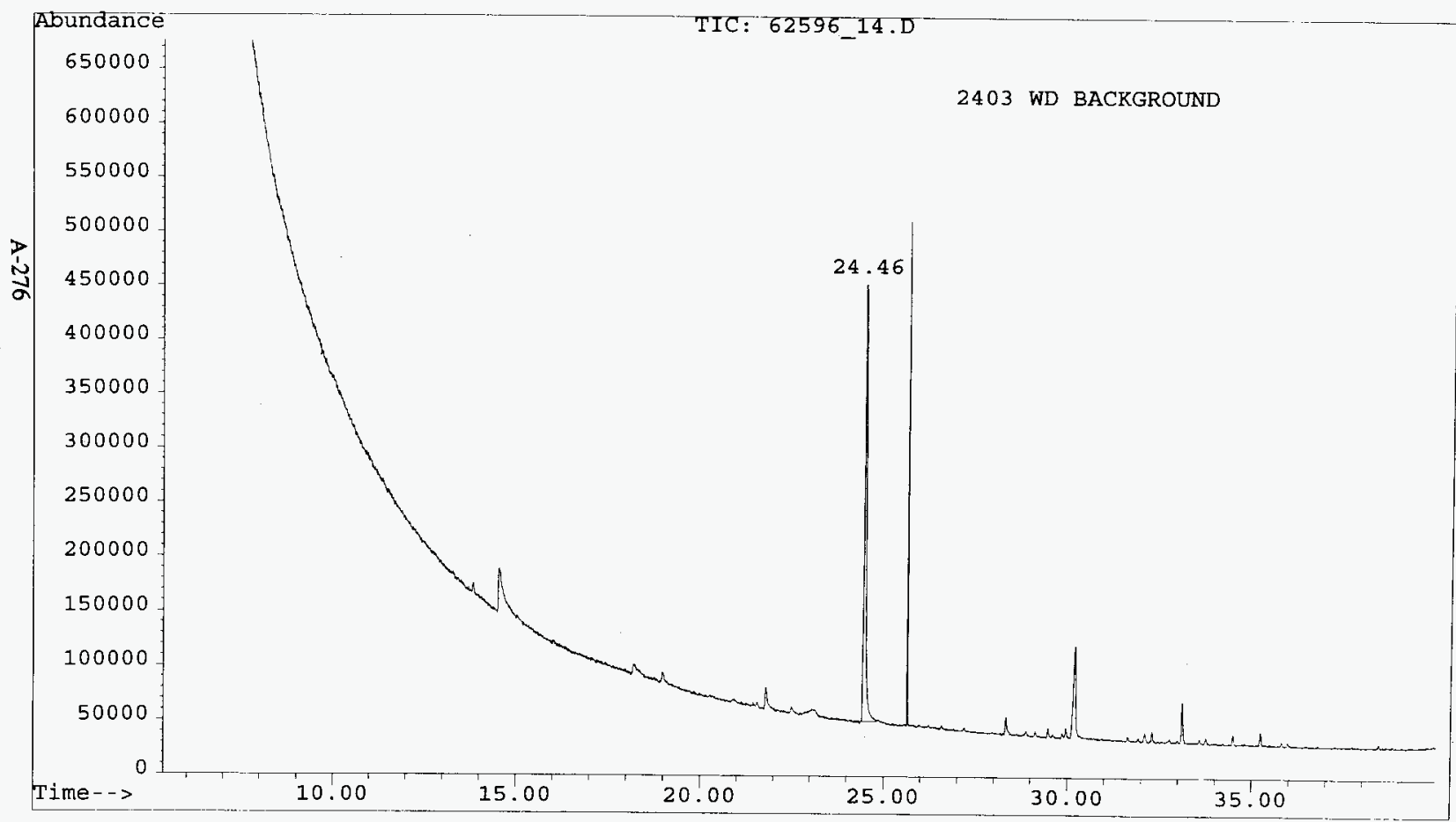


HNF-SD-WM-RPT-282 Rev. 0

CWC_REP.XLS

\section{ANALYSIS REPORT FOR CWC AIR SAMPLES}

File Name:

Analyst:

Analysis Date:

Method:

Sample Name:
62596_14.D

MS

06/25/96

CWC

FT6045-3WD.82A BK

\begin{tabular}{|c|c|c|c|c|}
\hline COMPOUND NAME & CAS \# & Concentration & Units & Comments \\
\hline ISO-BUTANE & $75-28-5$ & $\overline{0}$ & PPB & \\
\hline VINYL CHLORIDE & $75-01-4$ & 0 & PPB & \\
\hline ETHANOL & $64-17-5$ & 0 & PPB & \\
\hline ACETONE & $67-64-1$ & 0 & PPB & \\
\hline 2-PROPANOL & $67-63-0$ & 0 & PPB & \\
\hline 1,1-DICHLOROETHENE & $75-35-4$ & 0 & PPB & \\
\hline FREON 113 & $76-13-1$ & 0 & PPB & \\
\hline DICHLOROMETHANE & $75-09-2$ & 0 & PPB & \\
\hline 1,1-DICHLOROETHANE & $75-34-3$ & 0 & PPB & \\
\hline 2-BUTANONONE & $78-93-3$ & 0 & PPB & \\
\hline N-HEXANE & $110-54-3$ & 0 & PPB & \\
\hline 1,2-DICHLOROETHENE & $156-59-2$ & 0 & PPB & \\
\hline CHLOROFORM & $67-66-3$ & 0 & PPB & \\
\hline TETRAHYDROFURAN & $109-99-9$ & 0 & PPB & \\
\hline 1,1,1-TRICHLOROETHANE & $71-55-6$ & 0 & PPB & \\
\hline 1,2-DICHLOROETHANE & $107-06-2$ & 0 & PPB & \\
\hline BENZENE & $71-43-2$ & 0 & PPB & \\
\hline CARBON TETRACHLORIDE & $56-23-5$ & 0 & PPB & \\
\hline CYCLOHEXANE & $110-82-7$ & 0 & PPB & \\
\hline N-BUTANOL & $71-36-3$ & 0 & PPB & \\
\hline TRICHLOROETHENE & $79-01-6$ & 0 & PPB & \\
\hline 4-METHYL-2-PENTANONE & $108-10-1$ & 9 & PPB & \\
\hline TOLUENE & $108-88-3$ & 0 & PPB & \\
\hline 1,1,2-TRICHLOROETHANE & $79-00-5$ & 0 & PPB & \\
\hline TETRACHLOROETHENE & $127-18-4$ & 0 & PPB & \\
\hline CHLOROBENZENE & $108-90-7$ & 0 & PPB & \\
\hline ETHYLBENZENE & $100-41-4$ & 0 & PPB & \\
\hline M-XYLENE & $108-38-3$ & 0 & PPB & \\
\hline STYRENE & $100-42-5$ & 0 & PPB & \\
\hline O-XYLENE & $95-47-6$ & 0 & PPB & \\
\hline 1,1,2,2-TETRACHLORETHANE & $79-34-5$ & 0 & PPB & \\
\hline 1,3,5-TRIMETHYLBENZENE & $108-67-8$ & 0 & PPB & \\
\hline 1,2,4-TRIMETHYLBENZENE & $95-63-6$ & 0 & PPB & \\
\hline 1,3-DICHLOROBENZENE & $541-73-1$ & 0 & PPB & \\
\hline 1,2-DICHLOROBENZENE & $95-50-1$ & 0 & PPB & \\
\hline 1,4-DICHLOROBENZENE & $106-46-7$ & 0 & PPB & \\
\hline
\end{tabular}


HNF-SD-WM-RPT-282 Rev. 0

LIST OF COMPOUNDS TENTATIVELY IDENTIFIED WITH MASS SPECTRAL LIBRARY

Sample: $\quad 2403$ WD BACKGROUND

File: $\quad$ C:LHPCHEMLLDATA162596_14.D

ID: FT6045-3WD.82A BK

Compound

CAS\#

2-Pentanone, 4-methyl-

$000108-10-1$

Chlorobenzene-D5

$000000-00-0$

Ethanol, 2-butoxy-

$000111-76-2$

Benzene, 1,3,5-trimethyl-

$000108-67-8$ 
HNF-SD-WM-RPT-282 Rev. 0

\section{A.4 CWC LOW FLASHPOINT MODULAR UNITS}


HNF-SD-WM-RPT-282 Rev. 0

This page intentionally left blank. 
File

Operator

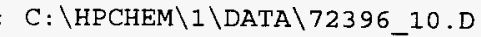

Acquired

: MS

Instrument

25 Mar 153

5972 - In

9:28 pm using AcqMethod CWC

Sample Name: FT6045-01.109A

Misc Info: 100 TORR

Vial Number: 1

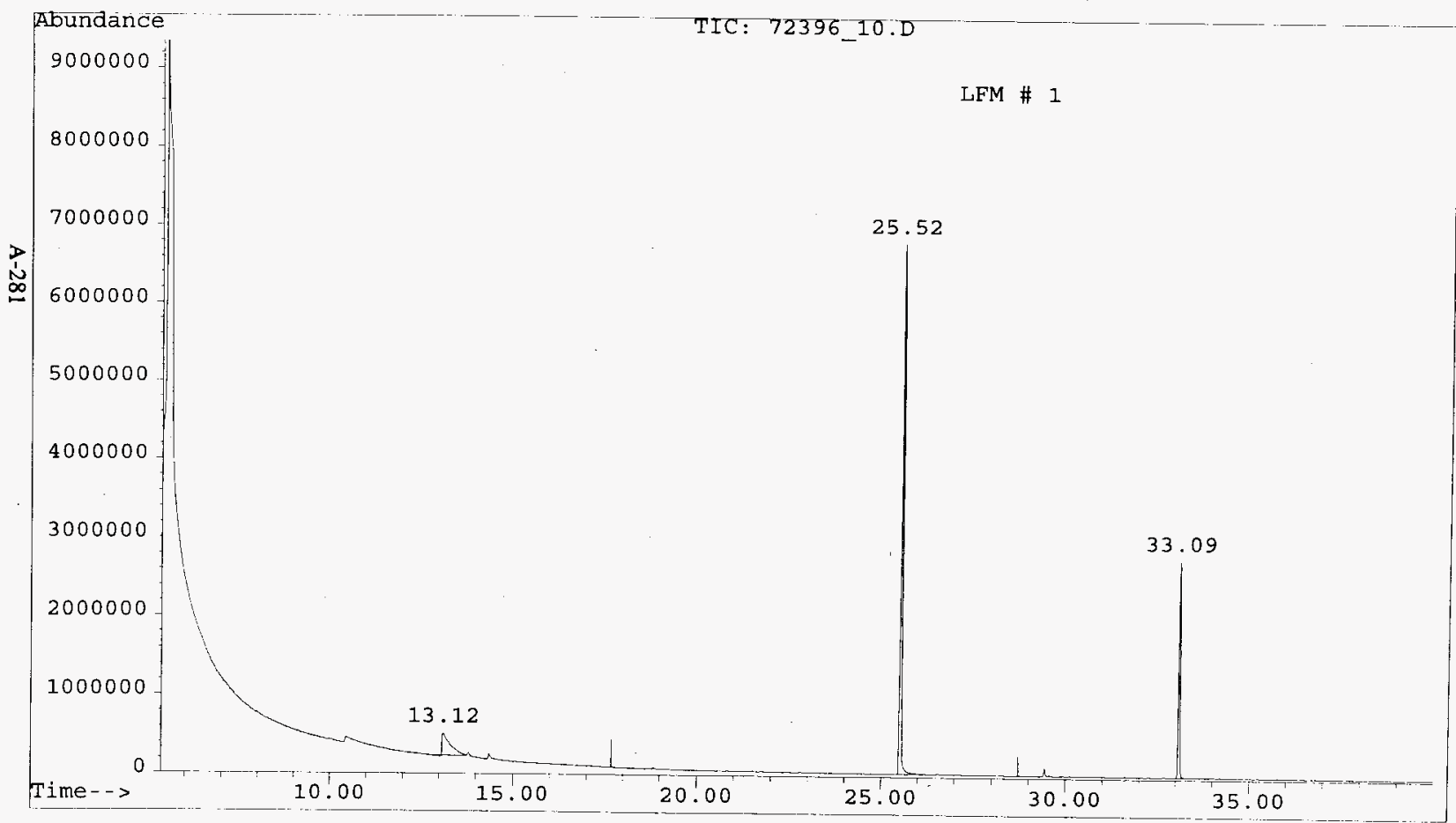


HNF-SD-WM-RPT-282 Rev. 0

CWC_REP.XLS

\section{ANALYSIS REPORT FOR CWC AIR SAMPLES}

File Name:

Analyst:

Analysis Date:

Method:

Sample Name:

\author{
72396_10.D \\ MS \\ \#VALUE! \\ CWC
}

FT6045-01.109A

\begin{tabular}{|c|c|c|c|c|}
\hline COMPOUND NAME & CAS \# & Concentration & Units & Comments \\
\hline ISO-BUTANE & $75-28-5$ & 0 & PPB & \\
\hline VINYL CHLORIDE & $75-01-4$ & 0 & PPB & \\
\hline ETHANOL & $64-17-5$ & 83 & PPB & \\
\hline ACETONE & $67-64-1$ & 3 & PPB & \\
\hline 2-PROPANOL & $67-63-0$ & 0 & PPB & \\
\hline 1,1-DICHLOROETHENE & $75-35-4$ & 0 & PPB & \\
\hline FREON 113 & $76-13-1$ & 0 & PPB & \\
\hline DICHLOROMETHANE & $75-09-2$ & 0 & PPB & \\
\hline 1,1-DICHLOROETHANE & $75-34-3$ & 0 & PPB & \\
\hline 2-BUTANONE & 78-93-3 & 0 & $\overline{\mathrm{PPB}}$ & \\
\hline N-HEXANE & $110-54-3$ & 0 & PPB & \\
\hline 1,2-DICHLOROETHENE & $156-59-2$ & 0 & PPB & \\
\hline CHLOROFORM & $67-66-3$ & 0 & PPB & \\
\hline TETRAHYDROFURAN & $109-99-9$ & 0 & PPB & \\
\hline 1,1,1-TRICHLOROETHANE & $71-55-6$ & 0 & PPB & \\
\hline 1,2-DICHLOROETHANE & $107-06-2$ & 0 & PPB & \\
\hline BENZENE & $71-43-2$ & 0 & PPB & \\
\hline CARBON TETRACHLORIDE & $56-23-5$ & 0 & PPB & \\
\hline CYCLOHEXANE & $110-82-7$ & $\overline{0}$ & PPB & \\
\hline N-BUTANOL & $71-36-3$ & 0 & PPB & \\
\hline TRICHLOROETHENE & $79-01-6$ & 0 & PPB & \\
\hline 4-METHYL-2-PENTANONE & $108-10-1$ & 0 & $\mathrm{PPB}$ & \\
\hline TOLUENE & $108-88-3$ & 239 & PPB & \\
\hline 1,1,2-TRICHLOROETHANE & $79-00-5$ & 0 & PPB & \\
\hline TETRACHLOROETHENE & $127-18-4$ & 0 & PPB & \\
\hline CHLOROBENZENE & $108-90-7$ & 0 & PPB & \\
\hline ETHYLBENZENE & $100-41-4$ & 0 & PPB & \\
\hline M-XYLENE & $108-38-3$ & 0 & PPB & \\
\hline STYRENE & $100-42-5$ & 0 & PPB & \\
\hline O-XYLENE & $95-47-6$ & 0 & PPB & \\
\hline 1,1,2,2-TETRACHLORETHANE & $79-34-5$ & 0 & PPB & \\
\hline 1,3,5-TRIMETHYLBENZENE & $108-67-8$ & 0 & PPB & \\
\hline 1,2,4-TRIMETHYLBENZENE & $95-63-6$ & 76 & PPB & \\
\hline 1,3-DICHLOROBENZENE & $541-73-1$ & 0 & PPB & \\
\hline 1,2-DICHLOROBENZENE & $95-50-1$ & 0 & PPB & \\
\hline 1,4-DICHLOROBENZENE & $106-46-7$ & 0 & PPB & \\
\hline
\end{tabular}


HNF-SD-WM-RPT-282 Rev. 0

LIST OF COMPOUNDS TENTATIVELY IDENTIFIED WITH MASS SPECTRAL LIBRARY

Sample: $\quad$ LFM \# 1

File: $\quad$ C:LHPCHEMLLDATA172396_10.D

ID: $\quad$ FT6045-01.109A

Compound

CAS\#

Ethanol

$000064-17-5$

Benzene, methyl-

000108-88-3

3-Heptanone

000106-35-4

Benzene, 1,2,3-trimethyl-

000526-73-8 
HNF-SD-WM-RPT-282 Rev. 0

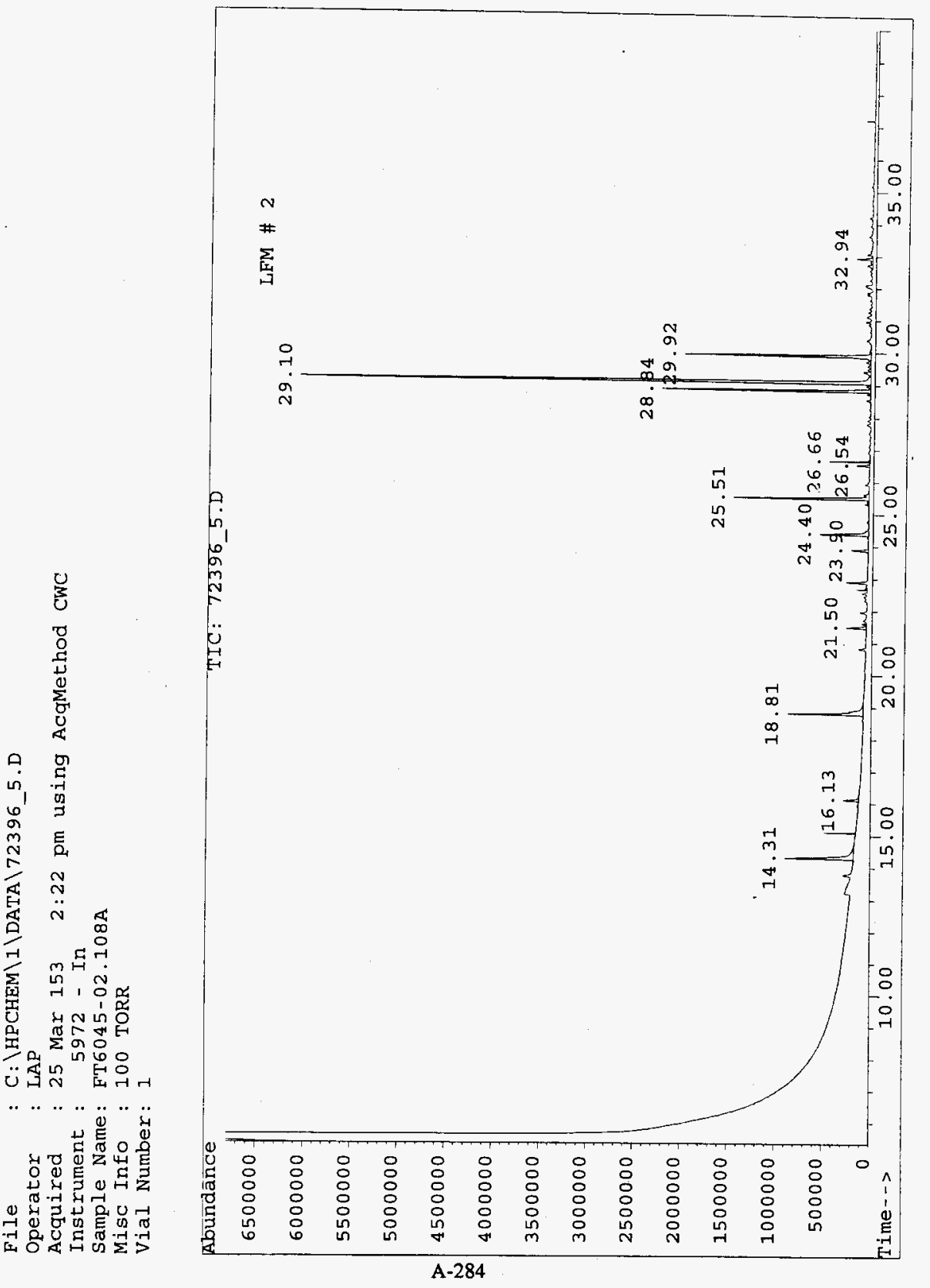


HNF-SD-WM-RPT-282 Rev, 0

CWC_REP.XLS

\section{ANALYSIS REPORT FOR CWC AIR SAMPLES}

File Name:

Analyst:

Analysis Date:

Method:

Sample Name:
72396_5.D

LAP

\#VALUE!

CWC

FT6045-02.108A

\begin{tabular}{|c|c|c|c|c|}
\hline COMPOUND NAME & CAS\# & Concentration & Units & Comments \\
\hline ISO-BUTANE & $75-28-5$ & $\overline{0}$ & PPB & \\
\hline VINYL CHLORIDE & $75-01-4$ & 0 & PPB & \\
\hline ETHANOL & $64-17-5$ & 17 & PPB & \\
\hline ACETONE & $67-64-1$ & 29 & PPB & \\
\hline 2-PROPANOL & $67-63-0$ & 0 & PPB & \\
\hline 1,1-DICHLOROETHENE & $75-35-4$ & 0 & PPB & \\
\hline FREON 113 & $76-13-1$ & 0 & PPB & \\
\hline DICHLOROMETHANE & $75-09-2$ & 6 & PPB & \\
\hline 1,1-DICHLOROETHANE & $75-34-3$ & 0 & PPB & \\
\hline 2-BUTANONE & $78-93-3$ & 107 & PPB & \\
\hline N-HEXANE & $110-54-3$ & 3 & PPB & \\
\hline 1,2-DICHLOROETHENE & $156-59-2$ & 0 & PPB & \\
\hline CHLOROFORM & $67-66-3$ & 0 & $\overline{\text { PPB }}$ & \\
\hline TETRAHYDROFURAN & $109-99-9$ & 0 & PPB & \\
\hline 1,1,1-TRICHLOROETHANE & $71-55-6$ & 3 & PPB & \\
\hline 1,2-DICHLOROETHANE & $107-06-2$ & 0 & $\overline{\text { PPB }}$ & \\
\hline BENZENE & $71-43-2$ & 0 & PPB & \\
\hline CARBON TETRACHLORIDE & $56-23-5$ & 0 & PPB & \\
\hline CYCLOHEXANE & $110-82-7$ & 9 & $\overline{\mathrm{PPB}}$ & \\
\hline N-BUTANOL & $71-36-3$ & 9 & PPB & \\
\hline TRICHLOROETHENE & $79-01-6$ & 0 & PPB & \\
\hline 4-METHYL-2-PENTANONE & $108-10-1$ & 14 & PPB & \\
\hline TOLUENE & $108-88-3$ & 48 & PPB & \\
\hline 1,1,2-TRICHLOROETHANE & $79-00-5$ & 0 & PPB & \\
\hline TETRACHLOROETHENE & $127-18-4$ & 0 & PPB & \\
\hline CHLOROBENZENE & $108-90-7$ & 0 & PPB & \\
\hline ETHYLBENZENE & $100-41-4$ & 65 & PPB & \\
\hline M-XYLENE & $108-38-3$ & 215 & PPB & \\
\hline STYRENE & $100-42-5$ & 1 & PPB & \\
\hline O-XYLENE & $95-47-6$ & 59 & PPB & \\
\hline 1,1,2,2-TETRACHLORETHANE & $79-34-5$ & 0 & PPB & \\
\hline 1,3,5-TRIMETHYLBENZENE & $108-67-8$ & 0 & PPB & \\
\hline 1,2,4-TRIMETHYLBENZENE & $95-63-6$ & 1 & PPB & \\
\hline 1,3-DICHLOROBENZENE & $541-73-1$ & 0 & PPB & \\
\hline 1,2-DICHLOROBENZENE & $95-50-1$ & 0 & PPB & \\
\hline 1,4-DICHLOROBENZENE & $106-46-7$ & 0 & PPB & \\
\hline
\end{tabular}




\section{LIST OF COMPOUNDS TENTATIVELY IDENTIFIED WITH MASS SPECTRAL LIBRARY}

Sample: $\quad$ LFM \# 2

File: $\quad$ C:LHPCHEML2LDATA172396_5.D

ID FT6045-02.108A

Compound

CAS\#

Methane, dichloro-

$000075-09-2$

2-Butanone

$000078-93-3$

Cyclohexane

000110-82-7

Heptane

$000142-82-5$

Cyclohexane, methyl-

000108-87-2

2-Pentanone, 4-methyl-

000108-10-1

Benzene, methyl-

000108-88-3

(Z)-4,4-Dimethyl-2-pentenal

$022597-47-3$

Benzene, ethyl-

000100-41-4

Benzene, 1,3-dimethyl-

000108-38-3

XYLENE

001330-20-7

Cyclohexane, 1,4-dimethyl-

000589-90-2

Decane

000124-18-5

Benzene, 1,2,3-trimethyl-

000526-73-8 
File

Operator

Acquired

Instrument

Sample Name:

Misc Info

Vial Number: 1

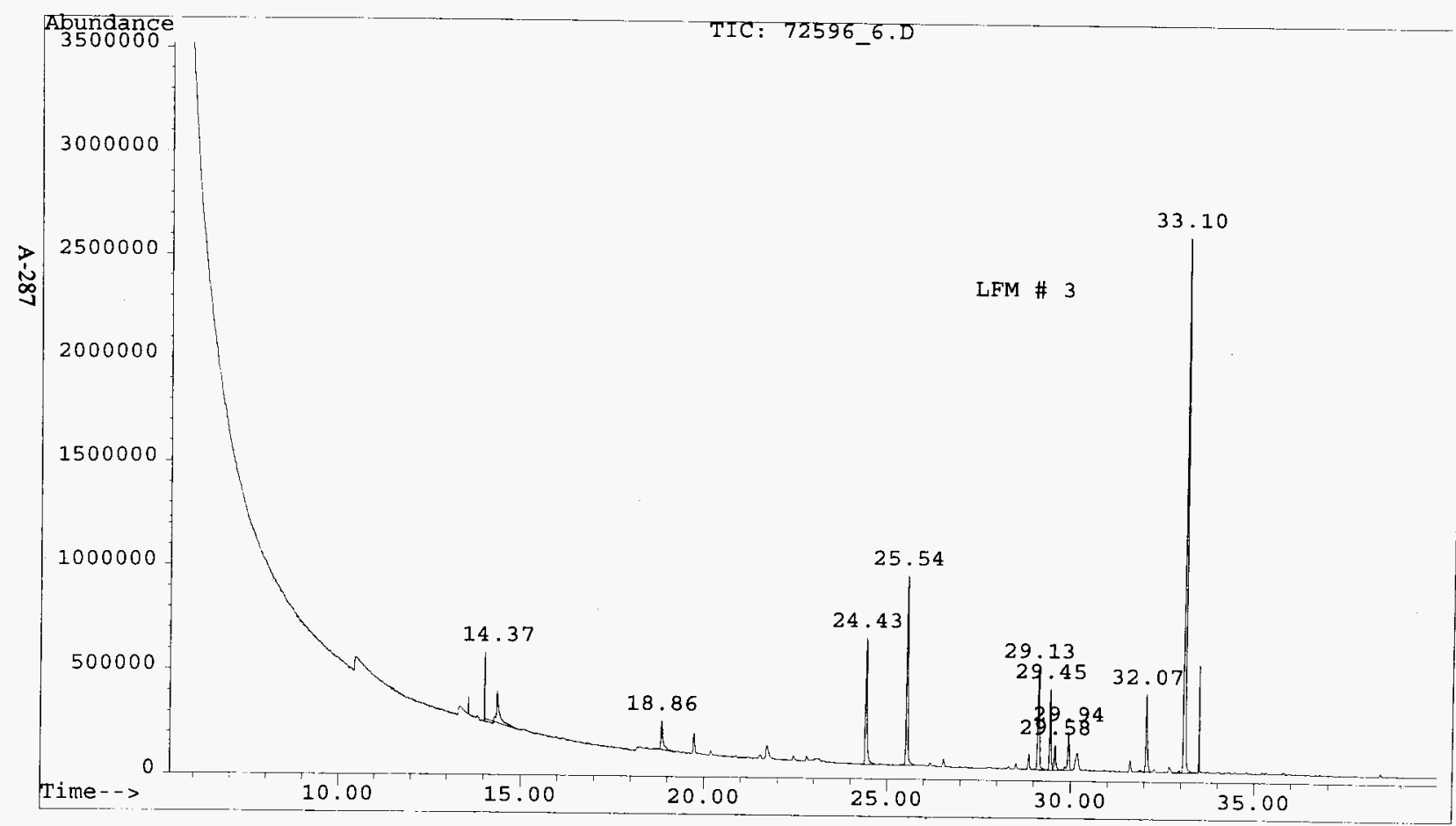


HNF-SD-WM-RPT-282 Rev. 0

CWC_REP.XLS

\section{ANALYSIS REPORT FOR CWC AIR SAMPLES}

File Name:

Analyst:

Analysis Date:

Method:

Sample Name
$725966 . D$

MS

\#VALUE!

CWC

FT 6045_03.120A

\begin{tabular}{|c|c|c|c|c|}
\hline COMPOUND NAME & CÁS\# & \begin{tabular}{|l|} 
Concentration \\
\end{tabular} & Units & Comments \\
\hline ISO-BUTANE & $75-28-5$ & 0 & PPB & \\
\hline VINYL CHLORIDE & $75-01-4$ & 0 & PPB & \\
\hline ETHANOL & $64-17-5$ & 10 & PPB & \\
\hline ACETONE & $67-64-1$ & 5 & PPB & \\
\hline 2-PROPANOL & $67-63-0$ & 0 & PPB & \\
\hline 1,1-DICHLOROETHENE & $75-35-4$ & 0 & PPB & \\
\hline FREON 113 & $76-13-1$ & 0 & PPB & \\
\hline DICHLOROMETHANE & $75-09-2$ & 0 & PPB & \\
\hline 1,1-DICHLOROETHANE & $75-34-3$ & 0 & PPB & \\
\hline 2-BUTANONE & $78-93-3$ & 17 & PPB & \\
\hline $\mathrm{N}$-HEXANE & $110-54-3$ & 0 & $\mathrm{PPB}$ & \\
\hline 1,2-DICHLOROETHENE & $156-59-2$ & 0 & PPB & \\
\hline CHLOROFORM & $67-66-3$ & 4 & PPB & \\
\hline TETRAHYDROFURAN & $109-99-9$ & 0 & $\mathrm{PPB}$ & \\
\hline 1,1,1-TRICHLOROETHANE & $71-55-6$ & 0 & PPB & \\
\hline 1,2-DICHLOROETHANE & $107-06-2$ & 0 & PPB & \\
\hline BENZENE & $71-43-2$ & $\overline{0}$ & PPB & \\
\hline CARBON TETRACHLORIDE & $56-23-5$ & 0 & PPB & \\
\hline CYCLOHEXANE & $110-82-7$ & 0 & PPB & \\
\hline N-BUTANOL & $71-36-3$ & 5 & PPB & \\
\hline TRICHLOROETHENE & $79-01-6$ & 0 & PPB & \\
\hline 4-METHYL-2-PENTANONE & $108-10-1$ & 12 & PPB & \\
\hline TOLUENE & $108-88-3$ & 31 & PPB & \\
\hline 1,1,2-TRICHLOROETHANE & $79-00-5$ & 0 & PPB & \\
\hline TETRACHLOROETHENE & $127-18-4$ & 0 & PPB & \\
\hline CHLOROBENZENE & $108-90-7$ & 0 & PPB & \\
\hline ETHYLBENZENE & $100-41-4$ & 2 & PPB & \\
\hline M-XYLENE & $108-38-3$ & 16 & PPB & \\
\hline STYRENE & $100-42-5$ & 0 & PPB & \\
\hline O-XYLENE & $95-47-6$ & 4 & PPB & \\
\hline 1,1,2,2-TETRACHLORETHANE & $79-34-5$ & 0 & PPB & \\
\hline 1,3,5-TRIMETHYLBENZENE & $108-67-8$ & 7 & PPB & \\
\hline 1,2,4-TRIMETHYLBENZENE & $95-63-6$ & 72 & PPB & \\
\hline 1,3-DICHLOROBENZENE & $541-73-1$ & 0 & PPB & \\
\hline 1,2-DICHLOROBENZENE & $95-50-1$ & 0 & PPB & \\
\hline 1,4-DICHLOROBENZENE & $106-46-7$ & 0 & PPB & \\
\hline
\end{tabular}


HNF-SD-WM-RPT-282 Rev. 0

\section{LIST OF COMPOUNDS TENTATIVELY IDENTIFIED WITH MASS SPECTRAL LIBRARY}

Sample: $\quad$ LFM \# 3

File: $\quad$ C:LHPCHEMI2LDATAl72596_6.D

ID: $\quad$ FT6045_03.120A

Compound

CAS\#

Acetic acid, trichloro-

000076-03-9

2-Pentanone, 4-methyl-

$000108-10-1$

Benzene, methyl-

000108-88-3

Benzene, ethyl-

000100-41-4

Benzene, 1,3-dimethyl-

000108-38-3

3-Heptanone

000106-35-4

2-Heptanone

000110-43-0

Benzene, 1,2-dimethyl-

000095-47-6

Benzaldehyde

000100-52-7

Benzene, 1,3,5-trimethyl-

000108-67-8 
File

Operator

: C $: \backslash H P C H E M \backslash 1 \backslash D A T A \backslash 72496 \quad 7 . D$

Acquired

MS

Instrument

: 26 Mar 153 6:10 pm using AcqMethod CWC

FT6045-04.121B

Misc Info : 100 TORR

Vial Number: 1

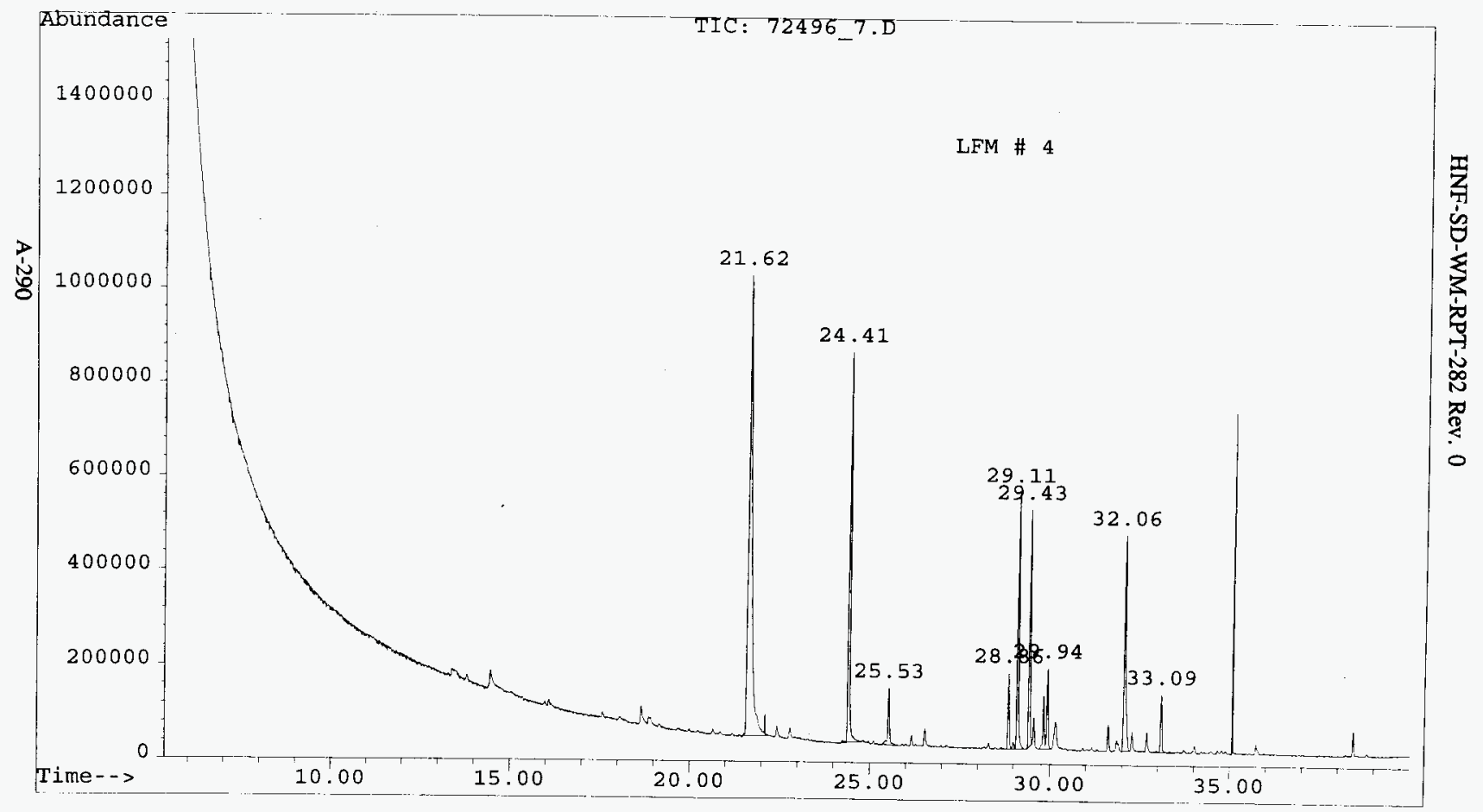


HNF-SD-WM-RPT-282 Rev. 0

CWC_REP.XLS

\section{ANALYSIS REPORT FOR CWC AIR SAMPLES}

File Name:

Analyst:

Analysis Date:

Method:

Sample Name:
72496_7.D

MS

\#VALUE

CWC

FT6045-04.121B

\begin{tabular}{|c|c|c|c|c|}
\hline COMPOUND NAME & CAS \# & Concentration & Units & Comments \\
\hline ISO-BUTANE & $75-28-5$ & 0 & PPB & \\
\hline VINYL CHLORIDE & $75-01-4$ & 0 & PPB & \\
\hline ETHANOL & $64-17-5$ & 0 & PPB & \\
\hline ACETONE & $67-64-1$ & 0 & PPB & \\
\hline 2-PROPANOL & $67-63-0$ & 0 & PPB & \\
\hline 1,1-DICHLOROETHENE & $75-35-4$ & 0 & PPB & \\
\hline FREON 113 & $76-13-1$ & 0 & PPB & \\
\hline DICHLOROMETHANE & $75-09-2$ & 0 & $\overline{\mathrm{PPB}}$ & \\
\hline 1,1-DICHLOROETHANE & $75-34-3$ & 0 & PPB & \\
\hline 2-BUTANONE & $78-93-3$ & 0 & PPB & \\
\hline N-HEXANE & $110-54-3$ & 0 & $\overline{\mathrm{PPB}}$ & \\
\hline 1,2-DICHLOROETHENE & $156-59-2$ & $\overline{0}$ & $\overline{\mathrm{PPB}}$ & \\
\hline CHLOROFORM & $67-66-3$ & 0 & $\overline{\mathrm{PPB}}$ & \\
\hline TETRAHYDROFURAN & $109-99-9$ & 0 & PPB & \\
\hline $1,1,1$-TRICHLOROETHANE & $71-55-6$ & 0 & $\mathrm{PPB}$ & \\
\hline 1,2-DICHLOROETHANE & $107-06-2$ & 0 & PPB & \\
\hline BENZENE & $71-43-2$ & 0 & PPB & \\
\hline CARBON TETRACHLORIDE & $56-23-5$ & 0 & $\mathrm{PPB}$ & \\
\hline CYCLOHEXANE & $110-82-7$ & 0 & $\mathrm{PPB}$ & \\
\hline N-BUTANOL & $71-36-3$ & 89 & $\overline{P P B}$ & \\
\hline TRICHLOROETHENE & $79-01-6$ & $\overline{0}$ & PPB & \\
\hline 4-METHYL-2-PENTANONE & $108-10-1$ & 16 & $\mathrm{PPB}$ & \\
\hline TOLUENE & $108-88-3$ & 4 & PPB & \\
\hline 1,1,2-TRICHLOROETHANE & $79-00-5$ & 0 & $\mathrm{PPB}$ & \\
\hline TETRACHLOROETHENE & $127-18-4$ & 0 & PPB & \\
\hline CHLOROBENZENE & $108-90-7$ & 0 & PPB & \\
\hline ETHYLBENZENE & $100-41-4$ & 4 & PPB & \\
\hline M-XYLENE & $108-38-3$ & 15 & PPB & \\
\hline STYRENE & $100-42-5$ & 3 & PPB & \\
\hline O-XYLENE & $95-47-6$ & 4 & $\overline{\mathrm{PPB}}$ & \\
\hline $1,1,2,2$-TETRACHLORETHANE & $79-34-5$ & 0 & $\mathrm{PPB}$ & \\
\hline 1,3,5-TRIMETHYLBENZENE & $108-67-8$ & 1 & PPB & \\
\hline 1,2,4-TRIMETHYLBENZENE & $95-63-6$ & 3 & PPB & \\
\hline 1,3-DICHLOROBENZENE & $541-73-1$ & 0 & PPB & \\
\hline 1,2-DICHLOROBENZENE & $95-50-1$ & 0 & PPB & \\
\hline 1,4-DICHLOROBENZENE & $106-46-7$ & 0 & PPB & \\
\hline
\end{tabular}


HNF-SD-WM-RPT-282 Rev. 0

\section{LIST OF COMPOUNDS TENTATIVELY IDENTIFIED WITH MASS SPECTRAL LIBRARY}

Sample: $\quad$ LFM \# 4

File: $\quad$ C:LHPCHEMLDLDATA172496_7.D

ID: $\quad$ FT6045-04.121B

Compound

1-Butanol

2-Pentanone, 4-methyl-

Benzene, methyl-

Benzene, ethyl-

Benzene, 1,3-dimethyl-

3-Heptanone

2-Heptanone

1,3,5,7-Cyclooctatetraene

Benzene, 1,2-dimethyl-

Benzaldehyde

Benzene, 1,2,3-trimethyl-

Benzene, I-ethyl-2-methyl-

Benzene, 1,2,4-trimethyl-

Benzene, 1,3,5-trimethyl-

Benzene, 1-ethyl-2,3-dimethyl-

Dodecane
CAS\#

000071-36-3

000108-10-1

000108-88-3

000100-41-4

000108-38-3

000106-35-4

000110-43-0

000629-20-9

000095-47-6

000100-52-7

000526-73-8

$000611-14-3$

000095-63-6

$000108-67-8$

000933-98-2

000112-40-3 
File

Operator

Acquired

Instrument :

Sample Name: FT6045-04.121A

Misc Info : 100 TORR

Vial Number: 1

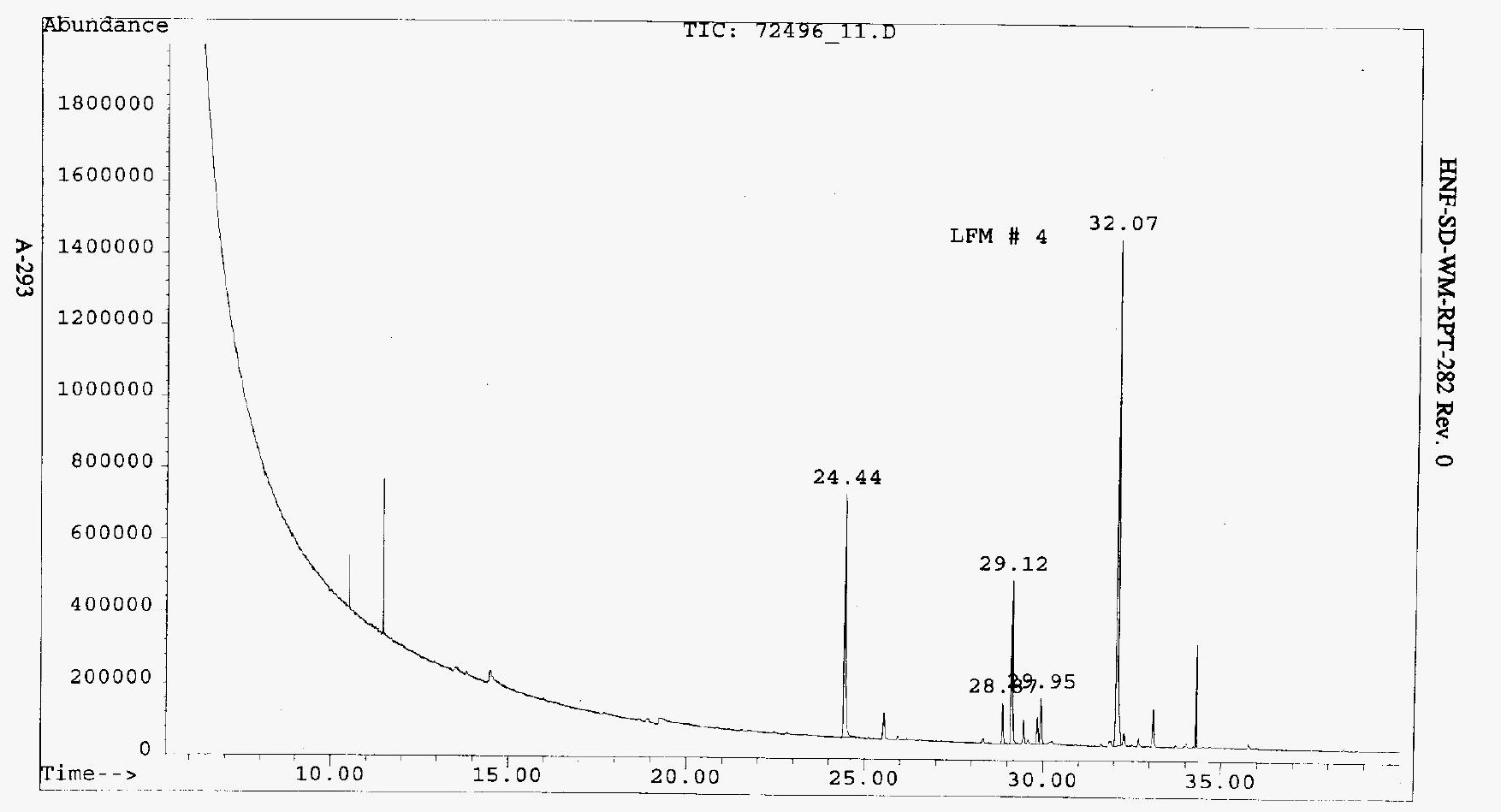


HNF-SD-WM-RPT-282 Rev. 0

CWC_REP.XLS

\section{ANALYSIS REPORT FOR CWC AIR SAMPLES}

File Name:

Analyst:

Analysis Date:

Method:

Sample Name:
72496_11.D

MS

\#VALUE!

CWC

FT6045-04.121A

\begin{tabular}{|c|c|c|c|c|}
\hline COMPOUND NAME & CAS \# & Concentration & Units & Comments \\
\hline ISO-BUTANE & $75-28-5$ & 0 & PPB & \\
\hline VINYL CHLORIDE & $75-01-4$ & 0 & PPB & \\
\hline ETHANOL & $64-17-5$ & 0 & PPB & \\
\hline ACETONE & $67-64-1$ & 0 & PPB & \\
\hline 2-PROPANOL & $67-63-0$ & 0 & PPB & \\
\hline 1,1-DICHLOROETHENE & $75-35-4$ & 0 & PPB & \\
\hline FREON 113 & $76-13-1$ & 0 & PPB & \\
\hline DICHLOROMETHANE & $75-09-2$ & 0 & PPB & \\
\hline 1,1-DICHLOROETHANE & $75-34-3$ & 0 & PPB & \\
\hline 2-BUTANONE & $78-93-3$ & 0 & PPB & \\
\hline N-HEXANE & $110-54-3$ & 0 & PPB & \\
\hline 1,2-DICHLOROETHENE & $156-59-2$ & 0 & PPB & \\
\hline CHLOROFORM & $67-66-3$ & 0 & PPB & \\
\hline TETRAHYDROFURAN & $109-99-9$ & 0 & PPB & \\
\hline 1,1,1-TRICHLOROETHANE & $71-55-6$ & 0 & PPB & \\
\hline 1,2-DICHLOROETHANE & $107-06-2$ & 0 & PPB & \\
\hline BENZENE & $71-43-2$ & 0 & PPB & \\
\hline CARBON TETRACHLORIDE & $56-23-5$ & 0 & PPB & \\
\hline CYCLOHEXANE & $110-82-7$ & 0 & $P P B$ & \\
\hline N-BUTANOL & $71-36-3$ & 0 & PPB & \\
\hline TRICHLOROETHENE & $79-01-6$ & 0 & PPB & \\
\hline 4-METHYL-2-PENTANONE & $108-10-1$ & 13 & PPB & \\
\hline TOLUENE & $108-88-3$ & 3 & PPB & \\
\hline 1,1,2-TRICHLOROETHANE & $79-00-5$ & 0 & PPB & \\
\hline TETRACHLOROETHENE & $127-18-4$ & 0 & $\mathrm{PPB}$ & \\
\hline CHLOROBENZENE & $108-90-7$ & 0 & $\mathrm{PPB}$ & \\
\hline ETHYLBENZENE & $100-41-4$ & 3 & PPB & \\
\hline M-XYLENE & 108-38-3 & 12 & PPB & \\
\hline STYRENE & $100-42-5$ & 2 & PPB & \\
\hline O-XYLENE & $95-47-6$ & 3 & PPB & \\
\hline 1,1,2,2-TETRACHLORETHANE & $79-34-5$ & 0 & PPB & \\
\hline 1,3,5-TRIMETHYLBENZENE & $108-67-8$ & 29 & PPB & \\
\hline 1,2,4-TRIMETHYLBENZENE & $95-63-6$ & 2 & PPB & \\
\hline 1,3-DICHLOROBENZENE & $541-73-1$ & 0 & PPB & \\
\hline 1,2-DICHLOROBENZENE & $95-50-1$ & 0 & PPB & \\
\hline 1,4-DICHLOROBENZENE & $106-46-7$ & 0 & PPB & \\
\hline
\end{tabular}


HNF-SD-WM-RPT-282 Rev. 0

\section{LIST OF COMPOUNDS TENTATIVELY IDENTIFIED WITH MASS SPECTRAL LIBRARY}

Sample: $\quad$ LFM \# 4

File: $\quad$ C:UHPCHEMLLDATA172496_11.D

ID: FT6045-04.121A

Compound

CAS\#

2-Pentanone, 4-methyl-

000108-10-1

Benzene, methyl-

000108-88-3

Benzene, ethyl-

$000100-41-4$

Benzene, 1,3-dimethyl-

000108-38-3

Styrene

$000100-42-5$

Benzene, 1,4-dimethyl-

$000106-42-3$

Benzaldehyde

000100-52-7

Benzene, 1,3,5-trimethyl-

000108-67-8

Benzene, 1,2,3-trimethyl-

000526-73-8 
File

C: $\backslash$ HPCHEM \1 \DATA $\backslash 72396 \_12 . D$

Operator

: MS

Acquired

: 25 Mar 153 11:36 pm using AcqMethod CWC

Instrument : $5972-$ In

Sample Name: FT6045-05.110A

Misc Info : 100 TORR

Vial Number: 1

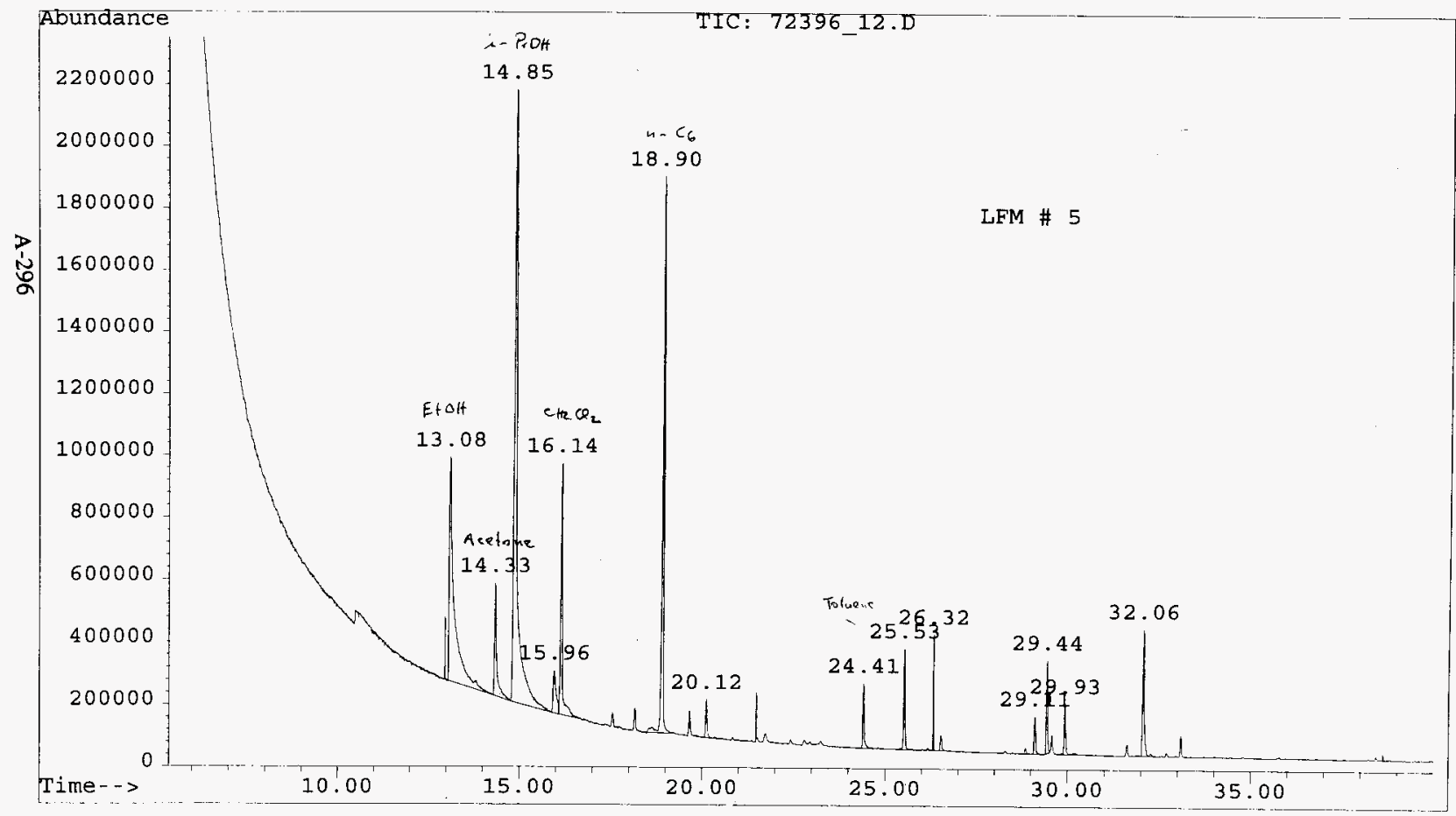


HNF-SD-WM-RPT-282 Rev. 0

CWC_REP.XLS

\section{ANALYSIS REPORT FOR CWC AIR SAMPLES}

File Name:

Analyst:

Analysis Date:

Method:

Sample Name:
72396_12.D

MS

\#VALUE!

CWC

FT6045-05.110A

\begin{tabular}{|c|c|c|c|c|}
\hline COMPOUND NAME & CAS \# & Concentration & Units & Comments \\
\hline ISO-BUTANE & $75-28-5$ & $\overline{0}$ & $\overline{\mathrm{PPB}}$ & \\
\hline VINYL CHLORIDE & $75-01-4$ & 0 & PPB & \\
\hline ETHANOL & $64-17-5$ & 119 & PPB & \\
\hline ACETONE & $67-64-1$ & 14 & PPB & \\
\hline 2-PROPANOL & $67-63-0$ & 122 & PPB & \\
\hline 1,1-DICHLOROETHENE & $75-35-4$ & 0 & PPB & \\
\hline FREON 113 & $76-13-1$ & 0 & PPB & \\
\hline DICHLOROMETHANE & $75-09-2$ & 30 & PPB & \\
\hline 1,1-DICHLOROETHANE & $75-34-3$ & 0 & PPB & \\
\hline 2-BUTANONE & $78-93-3$ & 0 & PPB & \\
\hline N-HEXANE & $110-54-3$ & 48 & PPB & \\
\hline 1,2-DICHLOROETHENE & $156-59-2$ & 0 & PPB & \\
\hline CHLOROFORM & $67-66-3$ & 0 & $\overline{\mathrm{PPB}}$ & \\
\hline TETRAHYDROFURAN & $109-99-9$ & 0 & $\overline{\mathrm{PPB}}$ & \\
\hline 1,1,1-TRICHLOROETHANE & $71-55-6$ & 0 & PPB & \\
\hline 1,2-DICHLOROETHANE & $107-06-2$ & 0 & PPB & \\
\hline BENZENE & $71-43-2$ & 0 & PPB & \\
\hline CARBON TETRACHLORIDE & $56-23-5$ & 0 & PPB & \\
\hline CYCLOHEXANE & $110-82-7$ & 0 & PPB & \\
\hline N-BUTANOL & $71-36-3$ & 0 & PPB & \\
\hline TRICHLOROETHENE & $79-01-6$ & 0 & PPB & \\
\hline 4-METHYL-2-PENTANONE & $108-10-1$ & 0 & PPB & \\
\hline TOLUENE & $108-88-3$ & 10 & PPB & \\
\hline 1,1,2-TRICHLOROETHANE & $79-00-5$ & 0 & PPB & \\
\hline TETRACHLOROETHENE & $127-18-4$ & 0 & PPB & \\
\hline CHLOROBENZENE & $108-90-7$ & 0 & PPB & \\
\hline ETHYLBENZENE & $100-41-4$ & 3 & PPB & \\
\hline M-XYLENE & $108-38-3$ & 4 & PPB & \\
\hline STYRENE & $100-42-5$ & 0 & PPB & \\
\hline O-XYLENE & $95-47-6$ & 2 & PPB & \\
\hline 1,1,2,2-TETRACHLORETHANE & $79-34-5$ & 0 & PPB & \\
\hline 1,3,5-TRIMETHYLBENZENE & $108-67-8$ & 8 & PPB & \\
\hline 1,2,4-TRIMETHYLBENZENE & $95-63-6$ & 2 & PPB & \\
\hline 1,3-DICHLOROBENZENE & $541-73-1$ & 0 & PPB & \\
\hline 1,2-DICHLOROBENZENE & $95-50-1$ & 0 & PPB & \\
\hline 1,4-DICHLOROBENZENE & $106-46-7$ & 0 & PPB & \\
\hline
\end{tabular}


HNF-SD-WM-RPT-282 Rev. 0

LIST OF COMPOUNDS TENTATIVELY IDENTIFIED WITH MASS SPECTRAL LIBRARY

Sample: $\quad$ LFM \# 5

File: $\quad$ C:LHPCHEMLDATA172396_12.D

ID FT6045-05.110A

Compound

CAS\#.

Ethanol

000064-17-5

2-Propanol

000067-63-0

Methane, dichloro-

000075-09-2

Hexane

000110-54-3

Benzene, methyl-

000108-88-3

Benzene, 1,4-dimethyl-

000106-42-3

3-Heptanone

000106-35-4

Benzene, 1,2-dimethyl-

000095-47-6

Benzaldehyde

000100-52-7

Benzene, 1,3,5-trimethyl-

000108-67-8 
File

Operator

: C $: \backslash H P C H E M \backslash 1 \backslash D A T A \backslash 72296 \quad 14 . D$

Acquired

MS

Instrument

: 24 Mar 153 11:51 pm using AcqMethod CWC

FT6045-05.110B

Misc Info : 100 TORR

Vial Number: 1

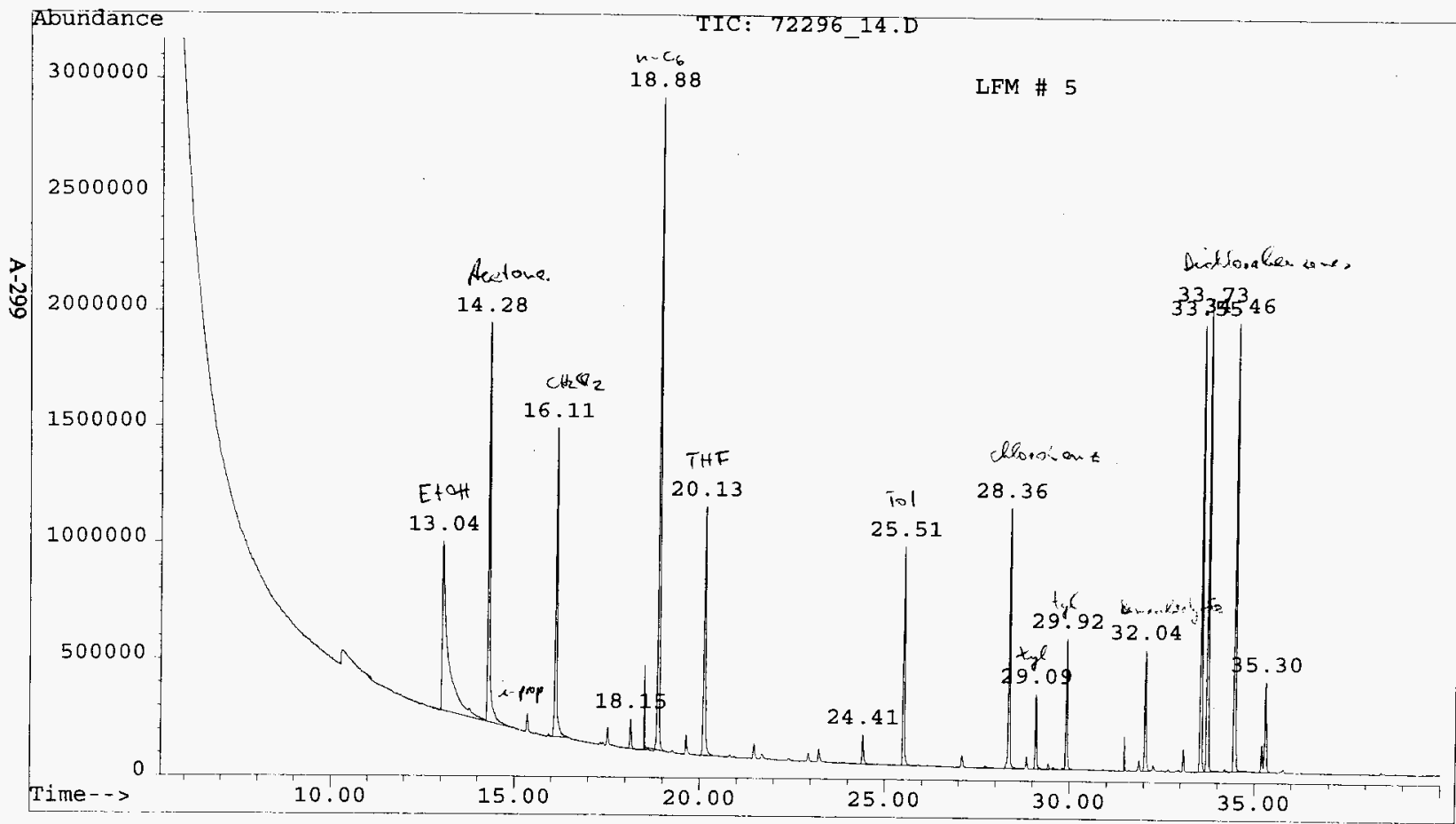


HNF-SD-WM-RPT-282 Rev. 0

CWC_REP.XLS

\section{ANALYSIS REPORT FOR CWC AIR SAMPLES}

File Name:

Analyst:

Analysis Date:

Method:

Sample Name:
72296 14.D

MS

\#VALUE!

CWC

FT6045-05.110B

\begin{tabular}{|c|c|c|c|c|}
\hline COMPOUND NAME & CAS \# & Concentration & Units & Comments \\
\hline ISO-BUTANE & $75-28-5$ & 0 & PPB & \\
\hline VINYL CHLORIDE & $75-01-4$ & 0 & PPB & \\
\hline ETHANOL & $64-17-5$ & 154 & $\mathrm{PPB}$ & \\
\hline ACETONE & $67-64-1$ & 51 & $\mathrm{PPB}$ & \\
\hline 2-PROPANOL & $67-63-0$ & 0 & PPB & \\
\hline 1,1-DICHLOROETHENE & $75-35-4$ & 3 & $\mathrm{PPB}$ & \\
\hline FREON 113 & $76-13-1$ & 0 & $\mathrm{PPB}$ & \\
\hline DICHLOROMETHANE & $75-\overline{09-2}$ & 43 & PPB & \\
\hline 1,1-DICHLOROETHANE & $75-34-3$ & 0 & PPB & \\
\hline 2-BUTANONE & $78-93-3$ & 0 & PPB & \\
\hline N-HEXANE & $110-54-3$ & 78 & PPB & \\
\hline 1,2-DICHLOROETHENE & $156-59-2$ & 0 & PPB & \\
\hline CHLOROFORM & $67-66-3$ & 0 & PPB & \\
\hline TETRAHYDROFURAN & $109-99-9$ & 27 & PPB & \\
\hline 1,1,1-TRICHLOROETHANE & $71-55-6$ & 0 & PPB & \\
\hline 1,2-DICHLOROETHANE & $107-06-2$ & 0 & PPB & \\
\hline BENZENE & $71-43-2$ & 6 & PPB & \\
\hline CARBON TETRACHLORIDE & $56-23-5$ & 0 & PPB & \\
\hline CYCLOHEXANE & $110-82-7$ & 0 & PPB & \\
\hline N-BUTANOL & $71-36-3$ & 0 & PPB & \\
\hline TRICHLOROETHENE & $79-01-6$ & 0 & PPB & \\
\hline 4-METHYL-2-PENTANONE & $108-10-1$ & 0 & PPB & \\
\hline TOLUENE & $108-88-3$ & 27 & PPB & \\
\hline 1,1,2-TRICHLOROETHANE & $79-00-5$ & 0 & PPB & \\
\hline TETRACHLOROETHENE & $127-18-4$ & 0 & PPB & \\
\hline CHLOROBENZENE & $108-90-7$ & 32 & PPB & \\
\hline ETHYLBENZENE & $100-41-4$ & 1 & PPB & \\
\hline M-XYLENE & $108-38-3$ & 8 & PPB & \\
\hline STYRENE & $100-42-5$ & 0 & PPB & \\
\hline O-XYLENE & $95-47-6$ & 16 & PPB & \\
\hline 1,1,2,2-TETRACHLORETHANE & $79-34-5$ & 0 & PPB & \\
\hline 1,3,5-TRIMETHYLBENZENE & $108-67-8$ & 9 & PPB & \\
\hline 1,2,4-TRIMETHYLBENZENE & $95-63-6$ & 2 & PPB & \\
\hline 1,3-DICHLOROBENZENE & $541-73-1$ & 60 & PPB & \\
\hline 1,2-DICHLOROBENZENE & $95-50-1$ & 62 & PPB & \\
\hline 1,4-DICHLOROBENZENE & $106-46-7$ & 59 & PPB & \\
\hline
\end{tabular}


HNF-SD-WM-RPT-282 Rev. 0

\section{LIST OF COMPOUNDS TENTATIVELY IDENTIFIED WITH MASS SPECTRAL}

\section{LIBRARY}

Sample: $\quad$ LFM \# 5

File: $\quad$ C:LHPCHEM 2LDATA172296_14.D

ID: $\quad$ FT6045-05.110B

Compound

CAS\#

Ethanol

000064-17-5

Methane, dichloro-

000075-09-2

Hexane

000110-54-3

Furan, tetrahydro-

000109-99-9

Benzene

$000071-43-2$

Benzene, methyl-

000108-88-3

Benzene, chloro-

000108-90-7

Benzene, 1,2-dimethyl-

$000095-47-6$

Benzene, 1,3-dimethyl-

000108-38-3

Benzene, 1,3-dimethyl-

000108-38-3

Benzaldehyde

000100-52-7

Benzene, 1,3,5-trimethyl-

$000108-67-8$

Benzene, 1,4-dichloro-

000106-46-7

Benzene, 1,4-dichloro-

000106-46-7

Benzene, 1,2-dichloro-

000095-50-1

Ethanone, 1-phenyl-

000098-86-2

Benzaldehyde, 2-methyl-

000529-20-4 


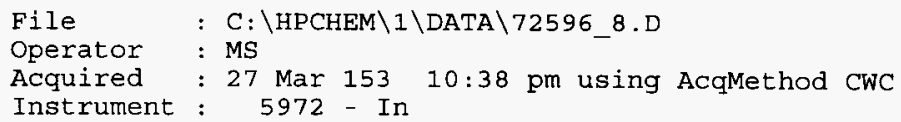

Sample Name: FT 6045 06.122A

MisC Info : 100 TORR

Vial Number: 1

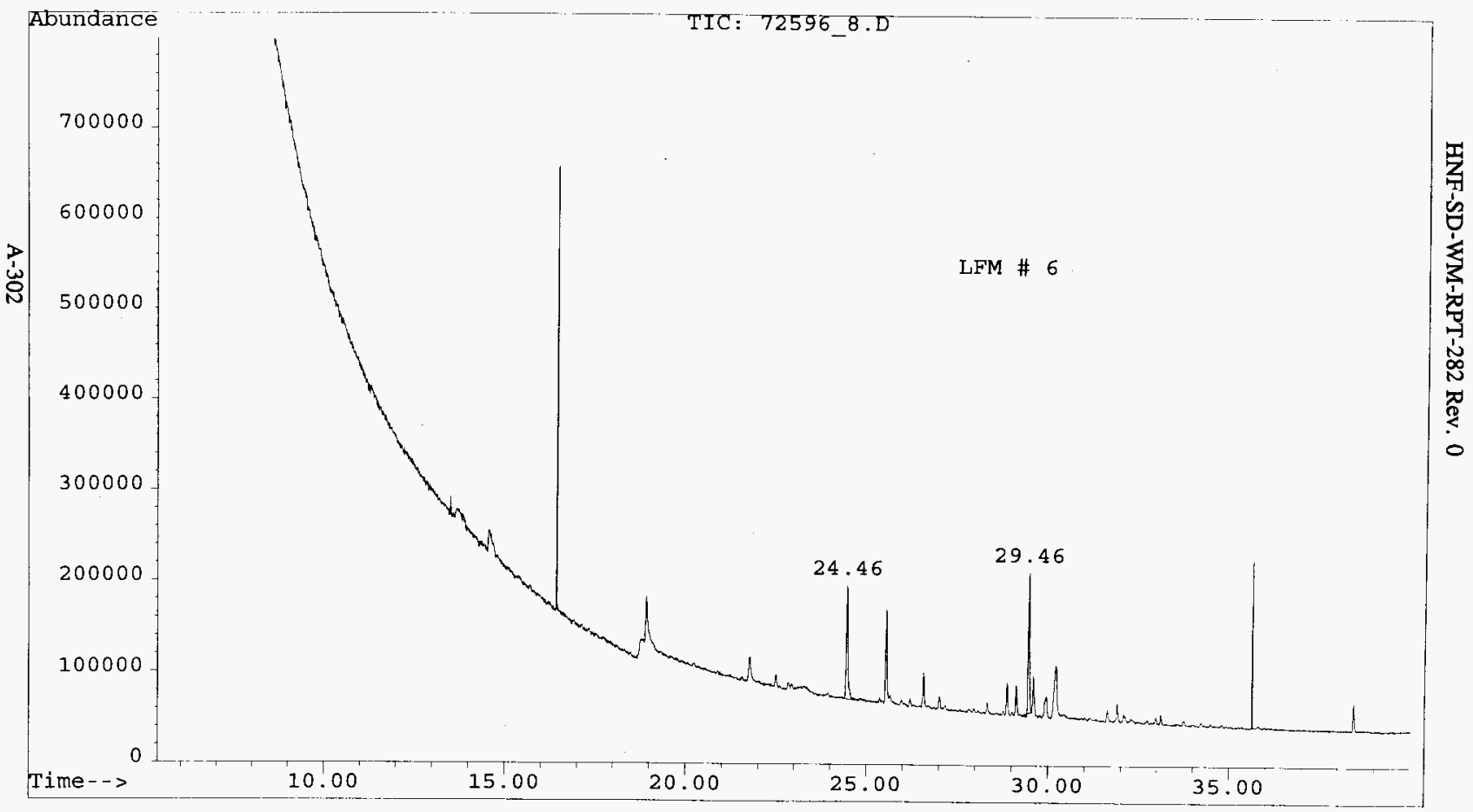


HNF-SD-WM-RPT-282 Rev, 0

CWC_REP.XLS

\section{ANALYSIS REPORT FOR CWC AIR SAMPLES}

File Name:

Analyst:

Analysis Date:

Method:

Sample Name:
72596_8.D

MS

\#VALUE!

CWC

FT 6045_06.122A

\begin{tabular}{|c|c|c|c|c|}
\hline COMPOUND NAME & CAS\# & Concentration & Units & Comments \\
\hline ISO-BUTANE & $75-28-5$ & $\overline{0}$ & $\overline{\mathrm{PPB}}$ & \\
\hline VINYL CHLORIDE & $75-01-4$ & 0 & PPB & \\
\hline ETHANOL & $64-17-5$ & 0 & PPB & \\
\hline ACETONE & $67-64-1$ & 0 & PPB & \\
\hline 2-PROPANOL & $67-63-0$ & 0 & PPB & \\
\hline 1,1-DICHLOROETHENE & $75-35-4$ & 0 & PPB & \\
\hline FREON 113 & $76-13-1$ & 0 & PPB & \\
\hline DICHLOROMETHANE & $75-09-2$ & 0 & PPB & \\
\hline 1,1-DICHLOROETHANE & $75-34-3$ & 0 & PPB & \\
\hline 2-BUTANONE & $78-93-3$ & 0 & $\overline{\mathrm{PPB}}$ & \\
\hline N-HEXANE & $110-54-3$ & 0 & $\mathrm{PPB}$ & \\
\hline 1,2-DICHLOROETHENE & $156-59-2$ & 0 & PPB & \\
\hline CHLOROFORM & $67-66-3$ & 0 & PPB & \\
\hline TETRAHYDROFURAN & $109-99-9$ & 0 & PPB & \\
\hline 1,1,1-TRICHLOROETHANE & $71-55-6$ & 0 & PPB & \\
\hline 1,2-DICHLOROETHANE & $107-06-2$ & 0 & PPB & \\
\hline BENZENE & $71-43-2$ & 0 & PPB & \\
\hline CARBON TETRACHLORIDE & $56-23-5$ & 0 & PPB & \\
\hline CYCLOHEXANE & $110-82-7$ & 0 & PPB & \\
\hline N-BUTANOL & $71-36-3$ & 0 & PPB & \\
\hline TRICHLOROETHENE & $79-01-6$ & 0 & PPB & \\
\hline 4-METHYL-2-PENTANONE & $108-10-1$ & 0 & PPB & \\
\hline TOLUENE & $108-88-3$ & 4 & PPB & \\
\hline 1,1,2-TRICHLOROETHANE & $79-00-5$ & 0 & PPB & \\
\hline TETRACHLOROETHENE & $127-18-4$ & 0 & PPB & \\
\hline CHLOROBENZENE & $108-90-7$ & 0 & $\overline{\mathrm{PPB}}$ & \\
\hline ETHYLBENZENE & $100-41-4$ & 1 & PPB & \\
\hline M-XYYLENE & 108-38-3 & 0 & PPB & \\
\hline STYRENE & $100-42-5$ & 0 & PPB & \\
\hline O-XYLENE & $95-47-6$ & $\overline{0}$ & PPB & \\
\hline 1,1,2,2-TETRACHLORETHANE & $79-34-5$ & 0 & $\mathrm{PPB}$ & \\
\hline 1,3,5-TRIMETHYLBENZENE & $108-67-8$ & 0 & PPB & \\
\hline 1,2,4-TRIMETHYLBENZENE & $95-63-6$ & 0 & PPB & \\
\hline 1,3-DICHLOROBENZENE & $541-73-1$ & 0 & PPB & \\
\hline 1,2-DICHLOROBENZENE & $95-50-1$ & 0 & PPB & \\
\hline 1,4-DICHLOROBENZENE & $106-46-7$ & 0 & PPB & \\
\hline
\end{tabular}


HNF-SD-WM-RPT-282 Rev. 0

LIST OF COMPOUNDS TENTATIVELY IDENTIFIED WITH MASS SPECTRAL LIBRARY

Sample: $\quad$ LFM \# 6

File: $\quad$ C:LHPCHEM2LDATAI72596_8.D

ID: $\quad$ FT6045_06.122A

Compound

CAS \#

Benzene, methyl-

000108-88-3

3-Heptanone

$000106-35-4$ 
HNF-SD-WM-RPT-282 Rev. 0

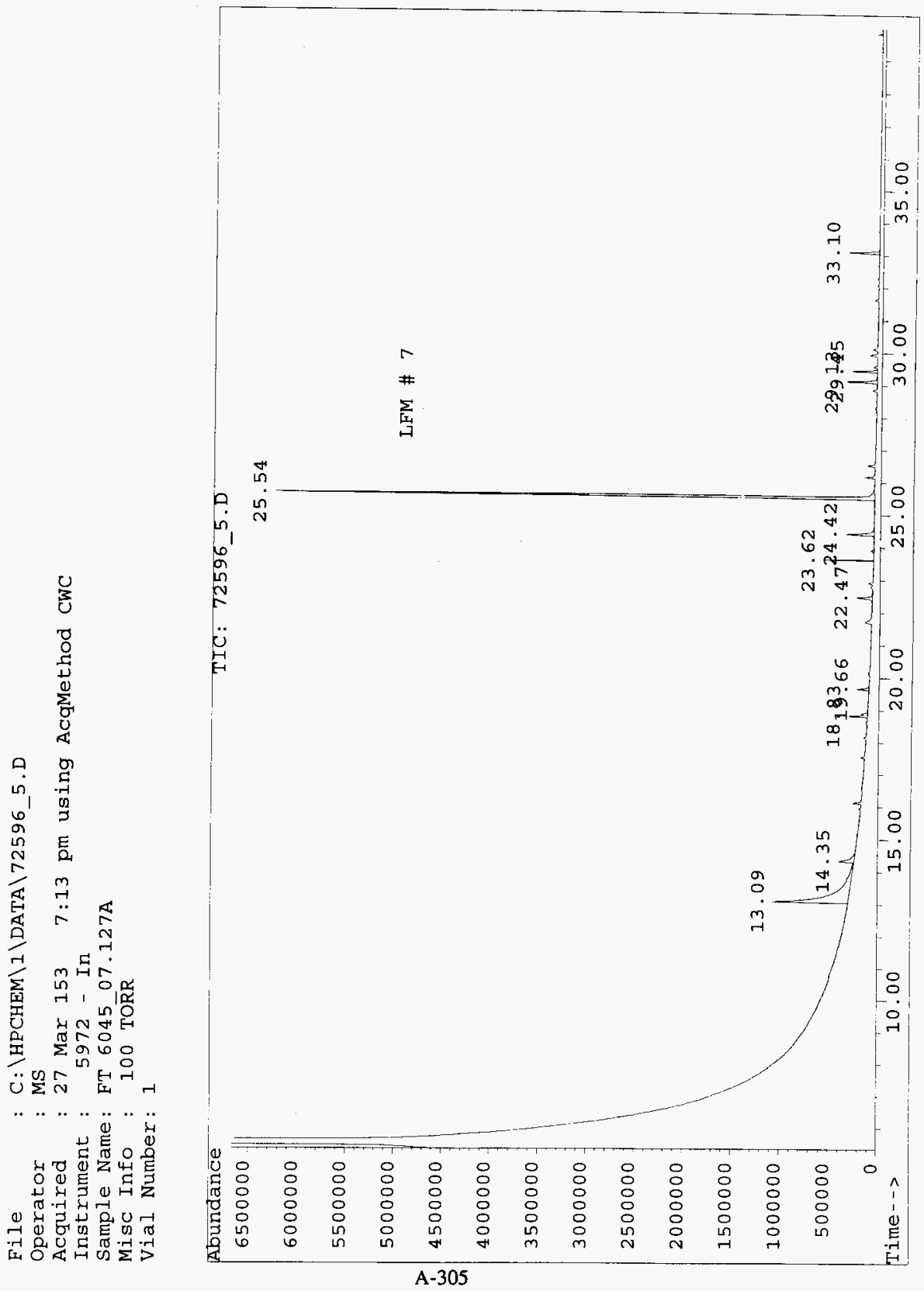


HNF-SD-WM-RPT-282 Rev. 0

CWC_REP.XLS

\section{ANALYSIS REPORT FOR CWC AIR SAMPLES}

File Name:

Analyst:

Analysis Date:

Method:

Sample Name:
72596_5.D

MS

\#VALUE!

CWC

FT 6045_07.127A

\begin{tabular}{|c|c|c|c|c|}
\hline COMPOUND NAME & CAS \# & Concentration & Units & Comments \\
\hline ISO-BUTANE & $75-28-5$ & $\overline{0}$ & PPB & \\
\hline VINYL CHLORIDE & $75-01-4$ & 0 & PPB & \\
\hline ETHANOL & $64-17-5$ & 158 & PPB & \\
\hline ACETONE & $67-64-1$ & 6 & PPB & \\
\hline 2-PROPANOL & $67-63-0$ & 0 & PPB & \\
\hline 1,1-DICHLOROETHENE & $75-35-4$ & 0 & PPB & \\
\hline FREON 113 & $76-13-1$ & 0 & PPB & \\
\hline DICHLOROMETHANE & $75-09-2$ & 3 & PPB & \\
\hline 1,1-DICHLOROETHANE & $75-34-3$ & 0 & PPB & \\
\hline 2-BUTANONE & $78-93-3$ & 21 & PPB & \\
\hline N-HEXANE & $110-54-3$ & 0 & PPB & \\
\hline 1,2-DICHLOROETHENE & $156-59-2$ & 0 & PPB & \\
\hline CHLOROFORM & $67-66-3$ & 0 & PPB & \\
\hline TETRAHYDROFURAN & $109-99-9$ & 0 & PPB & \\
\hline 1,1,1-TRICHLOROETHANE & $71-55-6$ & 0 & PPB & \\
\hline 1,2-DICHLOROETHANE & $107-06-2$ & 0 & PPB & \\
\hline BENZENE & $71-43-2$ & 0 & PPB & \\
\hline CARBON TETRACHLORIDE & $56-23-5$ & 0 & PPB & \\
\hline CYCLOHEXANE & $110-82-7$ & 0 & PPB & \\
\hline N-BUTANOL & $71-36-3$ & 6 & PPB & \\
\hline TRICHLOROETHENE & $79-01-6$ & 0 & PPB & \\
\hline 4-METHYL-2-PENTANONE & $108-10-1$ & 0 & PPB & \\
\hline TOLUENE & $108-88-3$ & 220 & PPB & \\
\hline 1,1,2-TRICHLOROETHANE & $79-00-5$ & 0 & PPB & \\
\hline TETRACHLOROETHENE & $127-18-4$ & 0 & PPB & \\
\hline CHLOROBENZENE & $108-90-7$ & 0 & PPB & \\
\hline ETHYLBENZENE & $100-41-4$ & 1 & PPB & \\
\hline M-XYLENE & $108-38-3$ & 10 & PPB & \\
\hline STYRENE & $100-42-5$ & 0 & PPB & \\
\hline O-XYLENE & $95-47-6$ & 0 & PPB & \\
\hline 1,1,2,2-TETRACHLORETHANE & $79-34-5$ & 0 & PPB & \\
\hline 1,3,5-TRIMETHYLBENZENE & $108-67-8$ & 0 & PPB & \\
\hline 1,2,4-TRIMETHYLBENZENE & $95-63-6$ & 9 & PPB & \\
\hline 1,3-DICHLOROBENZENE & $541-73-1$ & 0 & $\overline{\mathrm{PPB}}$ & \\
\hline 1,2-DICHLOROBENZENE & $95-50-1$ & 0 & PPB & \\
\hline 1,4-DICHLOROBENZENE & $106-46-7$ & 0 & PPB & \\
\hline
\end{tabular}


HNF-SD-WM-RPT-282 Rev. 0

\section{LIST OF COMPOUNDS TENTATIVELY IDENTIFIED WITH MASS SPECTRAL LIBRARY}

Sample: $\quad$ LFM \# 7

File: C:LHPCHEMI2LATA172596_5.D

ID: $\quad$ FT6045_07.127A

Compound

CAS\#

2-Pentanone, 4-methyl-

000108-10-1

Benzene, methyl-

$000108-88-3$

Benzene, ethyl-

$000100-41-4$

Benzene, 1,3-dimethyl-

000108-38-3

3-Heptanone

000106-35-4

Benzene, 1,3,5-trimethyl-

000108-67-8 
File

: C: \HPCHEM $\backslash 1 \backslash D A T A \backslash 72496 \_5 . D$

Operator

LAP

Acquired

: 26 Mar 15

Instrument : 5972 - In

Sample Name: FT6045-08.128A

Misc Info : 100 TORR

Vial Number: 1

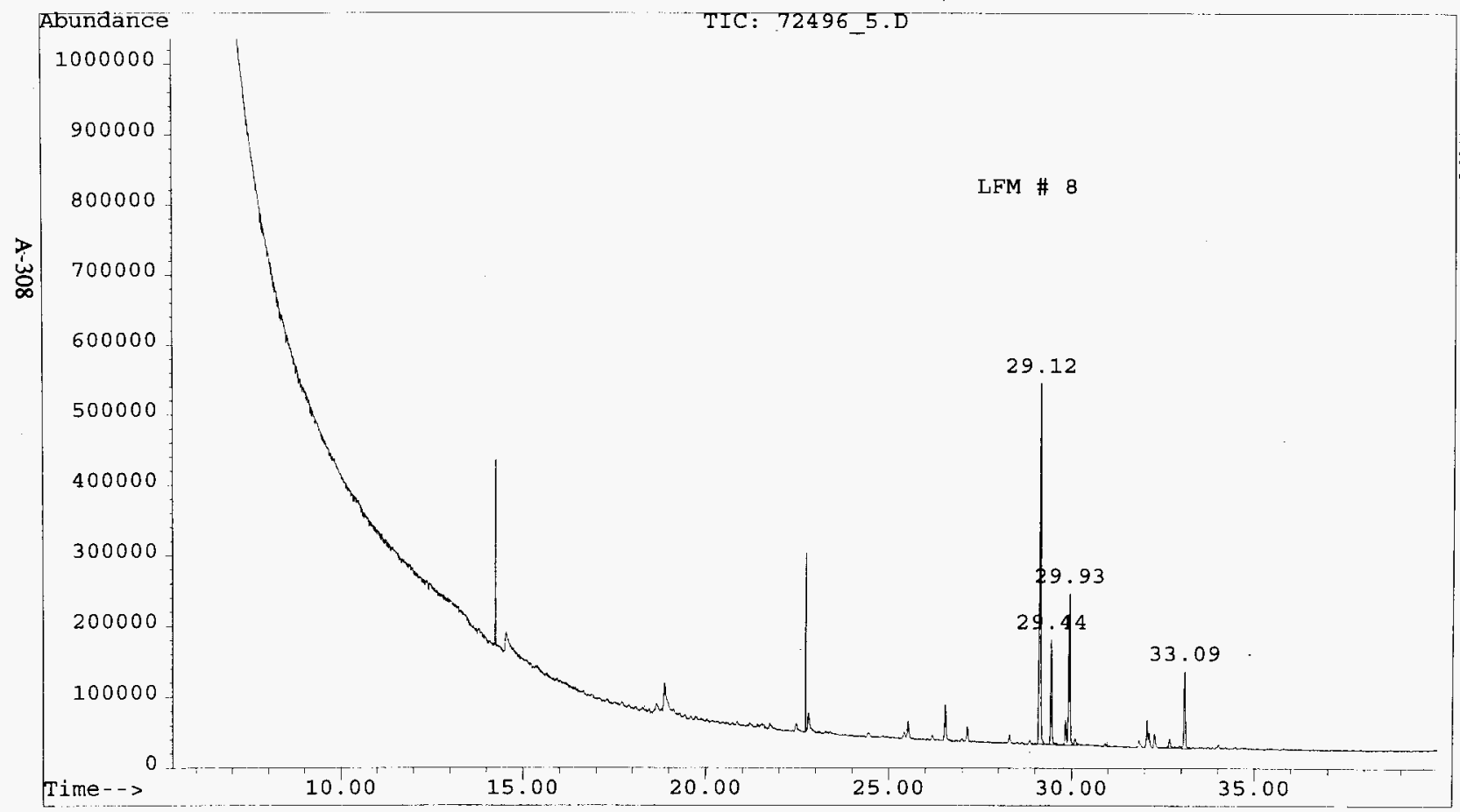


离

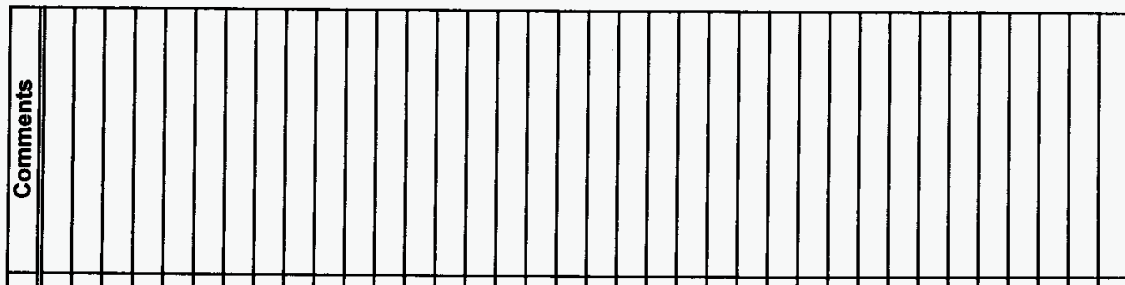

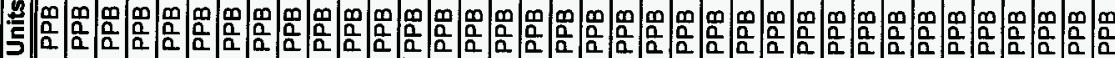

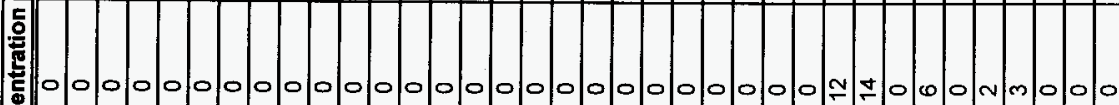

ठั.

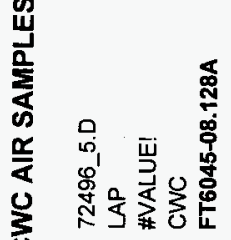

*

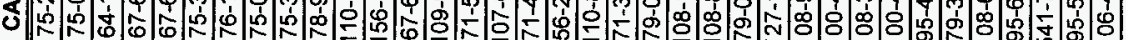


HNF-SD-WM-RPT-282 Rev. 0

\section{LIST OF COMPOUNDS TENTATIVELY IDENTIFIED WITH MASS SPECTRAL}

LIBRARY

Sample: $\quad$ LFM \# 8

File: $\quad$ C:LHPCHEM 2LATA172496_5.D

ID: $\quad$ FT6045-08.128A

Compound

CAS\#

8-Methylisothiazolo[4,5-c]-2,1,3-benz

074801-78-8

Benzene, 1,3-dimethyl-

000108-38-3

3-Heptanone

000106-35-4

Styrene

$000100-42-5$

XYLENE

001330-20-7

Benzene, 1-ethyl-3-methyl-

000620-14-4

Benzene, 1,3,5-trimethyl-

000108-67-8

Benzene, 1,2,3-trimethyl-

000526-73-8 
File

Operator

Acquired

Instrument

Sample Name: FT6045-08.128B

Misc Info : 100 TORR

Vial Number: 1

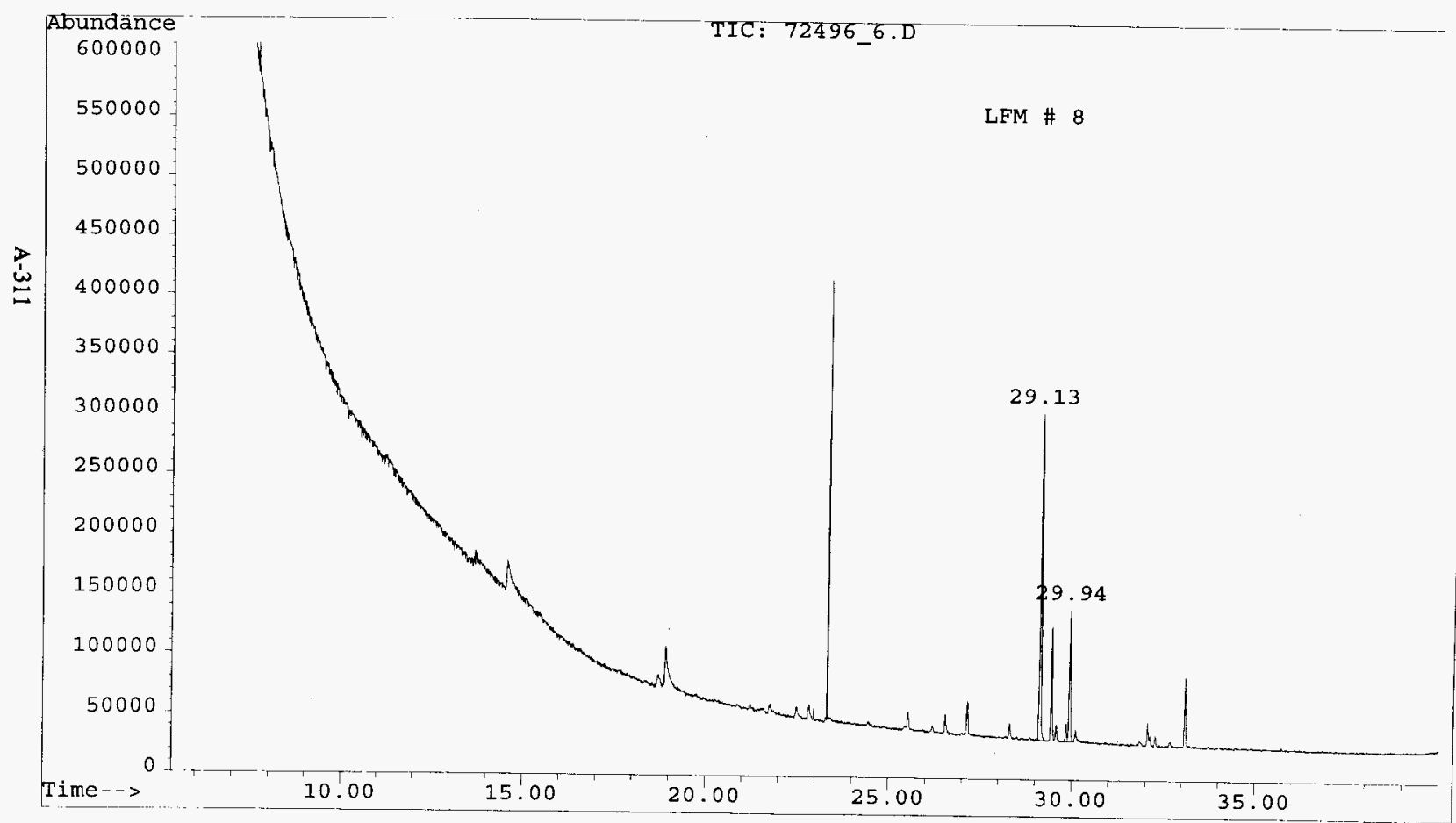


HNF-SD-WM-RPT-282 Rev. 0

CWC_REP.XLS

\section{ANALYSIS REPORT FOR CWC AIR SAMPLES}

File Name:

Analyst:

Analysis Date:

Method:

Sample Name:
72496_6.D

LAP

\#VALUE!

CWC

FT6045-08.128B

\begin{tabular}{|c|c|c|c|c|}
\hline COMPOUND NAME & CAS \# & Concentration & Units & Comments \\
\hline ISO-BUTANE & $75-28-5$ & 0 & PPB & \\
\hline VINYL CHLORIDE & $75-01-4$ & 0 & PPB & \\
\hline ETHANOL & $64-17-5$ & 0 & PPB & \\
\hline ACETONE & $67-64-1$ & 0 & PPB & \\
\hline 2-PROPANOL & $67-63-0$ & 0 & PPB & \\
\hline 1,1-DICHLOROETHENE & $75-35-4$ & 0 & PPB & \\
\hline FREON 113 & $76-13-1$ & 0 & PPB & \\
\hline DICHLOROMETHANE & $75-09-2$ & 0 & PPB & \\
\hline 1,1-DICHLOROETHANE & $75-34-3$ & 0 & PPB & \\
\hline 2-BUTANONE & $78-93-3$ & 0 & PPB & \\
\hline N-HEXANE & $110-54-3$ & 0 & PPB & \\
\hline 1,2-DICHLOROETHENE & $156-59-2$ & 0 & PPB & \\
\hline CHLOROFORM & $67-66-3$ & 0 & PPB & \\
\hline TETRAHYDROFURAN & $109-99-9$ & 0 & PPB & \\
\hline 1,1,1-TRICHLOROETHANE & $71-55-6$ & 0 & PPB & \\
\hline 1,2-DICHLOROETHANE & $107-06-2$ & 0 & PPB & \\
\hline BENZENE & $71-43-2$ & 0 & PPB & \\
\hline CARBON TETRACHLORIDE & $56-23-5$ & 0 & PPB & \\
\hline CYCLOHEXANE & $110-82-7$ & 0 & PPB & \\
\hline N-BUTANOL & $71-36-3$ & 0 & PPB & \\
\hline TRICHLOROETHENE & $79-01-6$ & 0 & PPB & \\
\hline 4-METHYL-2-PENTANONE & $108-10-1$ & 0 & PPB & \\
\hline TOLUENE & $108-88-3$ & 0 & PPB & \\
\hline 1,1,2-TRICHLOROETHANE & $79-00-5$ & 0 & PPB & \\
\hline TETRACHLOROETHENE & $127-18-4$ & 0 & PPB & \\
\hline CHLOROBENZENE & $108-90-7$ & 0 & PPB & \\
\hline ETHYLBENZENE & $100-41-4$ & 6 & PPB & \\
\hline M-XYLENE & $108-38-3$ & 7 & PPB & \\
\hline STYRENE & $100-42-5$ & 0 & PPB & \\
\hline O-XYLENE & $95-47-6$ & 3 & PPB & \\
\hline 1,1,2,2-TETRACHLORETHANE & $79-34-5$ & 0 & PPB & \\
\hline 1,3,5-TRIMETHYLBENZENE & $108-67-8$ & 0 & PPB & \\
\hline 1,2,4-TRIMETHYLBENZENE & $95-63-6$ & 1 & PPB & \\
\hline 1,3-DICHLOROBENZENE & $541-73-1$ & 0 & PPB & \\
\hline 1,2-DICHLOROBENZENE & $95-50-1$ & 0 & PPB & \\
\hline 1,4-DICHLOROBENZENE & $106-46-7$ & 0 & PPB & \\
\hline
\end{tabular}


HNF-SD-WM-RPT-282 Rev. 0

\section{LIST OF COMPOUNDS TENTATIVELY IDENTIFIED WITH MASS SPECTRAL} LIBRARY

Sample: $\quad$ LFM \# 8

File: C:IHPCHEML2LDATA172496_6.D

ID: FT6045-08.128B

Compound

CAS\#

Benzene, 1,4-dimethyl-

$000106-42-3$

3-Heptanone

000106-35-4

Benzene, 1,2-dimethyl-

$000095-47-6$

Benzene, 1,3,5-trimethyl-

$000108-67-8$ 
File

: C: \HPCHEM \I\DATA $72396 \_8 . D$

Operator

: LAP

Acquired

: 25 Mar 15

5972 - In

Sample Name: FT6045-09.117A

Misc Info : 100 TORR

Vial Number: 1

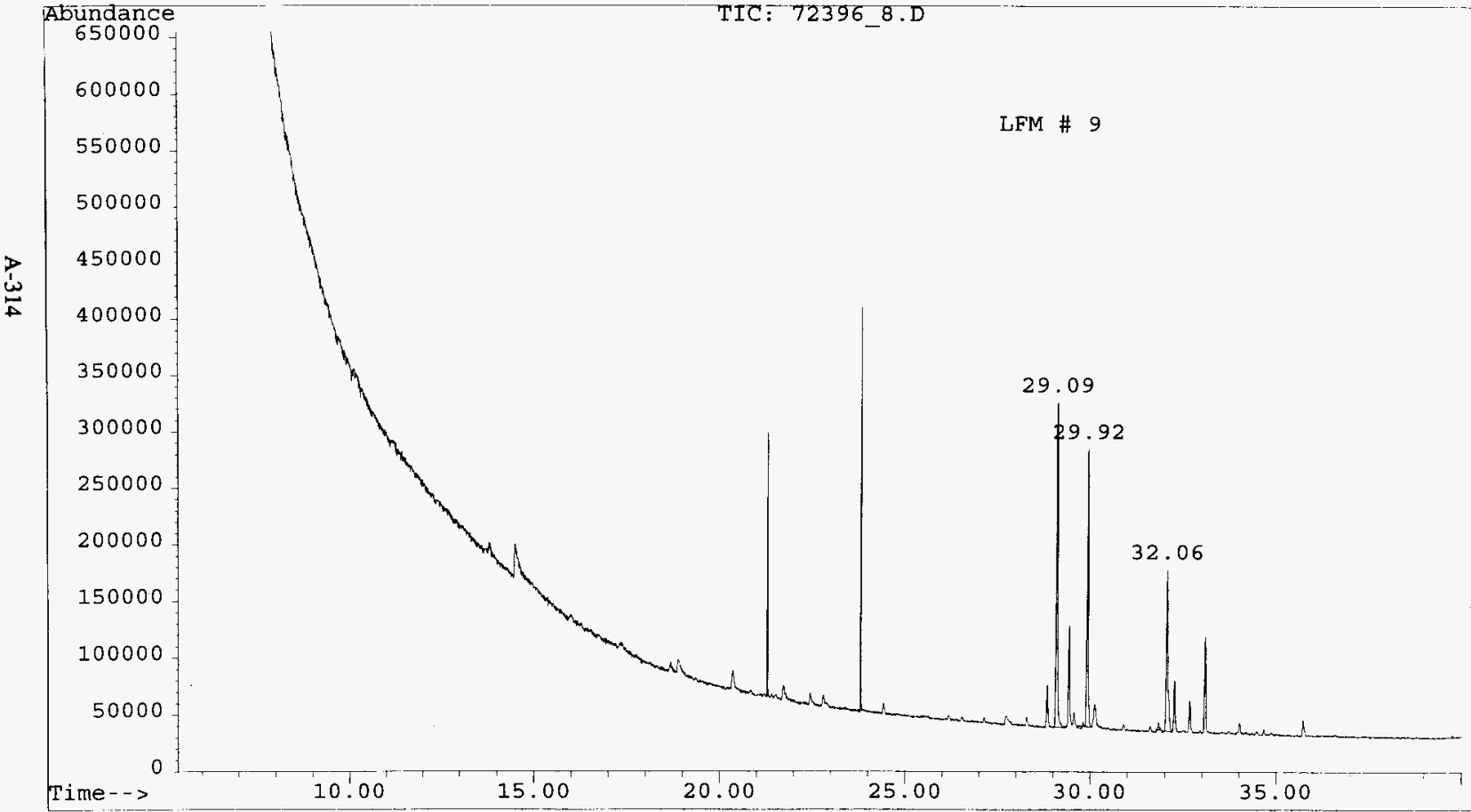


HNF-SD-WM-RPT-282 Rev. 0

CWC_REP.XLS

\section{ANALYSIS REPORT FOR CWC AIR SAMPLES}

File Name:

Analyst:

Analysis Date:

Method:

Sample Name:
72396 8.D

LAP

\#VALUE!

CWC

FT6045-09.117A

\begin{tabular}{|c|c|c|c|c|}
\hline COMPOUND NAME & CAS \# & Concentration & Units & Comments \\
\hline ISO-BUTANE & $75-28-5$ & 0 & PPB & \\
\hline VINYL CHLORIDE & $75-01-4$ & 0 & PPB & \\
\hline ETHANOL & $64-17-5$ & 0 & PPB & \\
\hline ACETONE & $67-64-1$ & 0 & PPB & \\
\hline 2-PROPANOL & $67-63-0$ & 0 & PPB & \\
\hline 1,1-DICHLOROETHENE & $75-35-4$ & 0 & PPB & \\
\hline FREON 113 & $76-13-1$ & 0 & PPB & \\
\hline DICHLOROMETHANE & $75-09-2$ & 0 & PPB & \\
\hline 1,1-DICHLOROETHANE & $75-34-3$ & 0 & PPB & \\
\hline 2-BUTANONE & $78-93-3$ & 0 & PPB & \\
\hline N-HEXANE & $110-54-3$ & 0 & PPB & \\
\hline 1,2-DICHLOROETHENE & $156-59-2$ & 0 & PPB & \\
\hline CHLOROFORM & $67-66-3$ & 0 & PPB & \\
\hline TETRAHYDROFURAN & $109-99-9$ & 0 & PPB & \\
\hline 1,1,1-TRICHLOROETHANE & $71-55-6$ & 0 & PPB & \\
\hline 1,2-DICHLOROETHANE & $107-06-2$ & 0 & $\overline{\mathrm{PPB}}$ & \\
\hline BENZENE & $71-43-2$ & 0 & PPB & \\
\hline CARBON TETRACHLORIDE & $56-23-5$ & 0 & PPB & \\
\hline CYCLOHEXANE & $110-82-7$ & 0 & PPB & \\
\hline N-BUTANOL & $71-36-3$ & 0 & $\overline{\text { PPB }}$ & \\
\hline TRICHLOROETHENE & $79-01-6$ & 0 & PPB & \\
\hline 4-METHYL-2-PENTANONE & $108-10-1$ & 0 & PPB & \\
\hline TOLUENE & $108-88-3$ & 0 & PPB & \\
\hline 1,1,2-TRICHLOROETHANE & $79-00-5$ & 0 & PPB & \\
\hline TETRACHLOROETHENE & $127-18-4$ & 0 & PPB & \\
\hline CHLOROBENZENE & $108-90-7$ & 0 & PPB & \\
\hline ETHYLBENZENE & $100-41-4$ & 1 & PPB & \\
\hline M-XYLENE & $108-38-3$ & 8 & PPB & \\
\hline STYRENE & $100-42-5$ & 0 & PPB & \\
\hline O-XYLENE & $95-47-6$ & 7 & PPB & \\
\hline 1,1,2,2-TETRACHLORETHANE & $79-34-5$ & 0 & PPB & \\
\hline 1,3,5-TRIMETHYLBENZENE & $108-67-8$ & 1 & PPB & \\
\hline 1,2,4-TRIMETHYLBENZENE & $95-63-6$ & 2 & PPB & \\
\hline 1,3-DICHLOROBENZENE & $541-73-1$ & 0 & PPB & \\
\hline 1,2-DICHLOROBENZENE & $95-50-1$ & 0 & PPB & \\
\hline 1,4-DICHLOROBENZENE & $106-46-7$ & 0 & PPB & \\
\hline
\end{tabular}


HNF-SD-WM-RPT-282 Rev. 0

\section{LIST OF COMPOUNDS TENTATIVELY IDENTIFIED WITH MASS SPECTRAL LIBRARY}

\section{Sample: $\quad$ LFM \# 9}

File: $\quad$ C:LHPCHEMI2LDATA172396_8.D

ID: $\quad$ FT6045-09.117A

Compound

\section{CAS\#}

Benzene, ethyl-

$000100-41-4$

Benzene, 1,4-dimethyl-

$000106-42-3$

3-Heptanone

000106-35-4

Benzene, 1,3-dimethyl-

000108-38-3

Ethanone, 1-phenyl-

000098-86-2

Benzene, 1,2,3-trimethyl-

000526-73-8

Benzene, 1,3,5-trimethyl-

000108-67-8 
File

: C: \HPCHEM \I\DATA $\backslash 72396 \_13 . \mathrm{D}$

Operator : MS

Acquired : 26 Mar $153 \quad 12: 35$ am using AcqMethod CWC

Instrument : 5972 - In

Sample Name: FT6045-10.116A

Misc Info : 100 TORR

Vial Number: 1

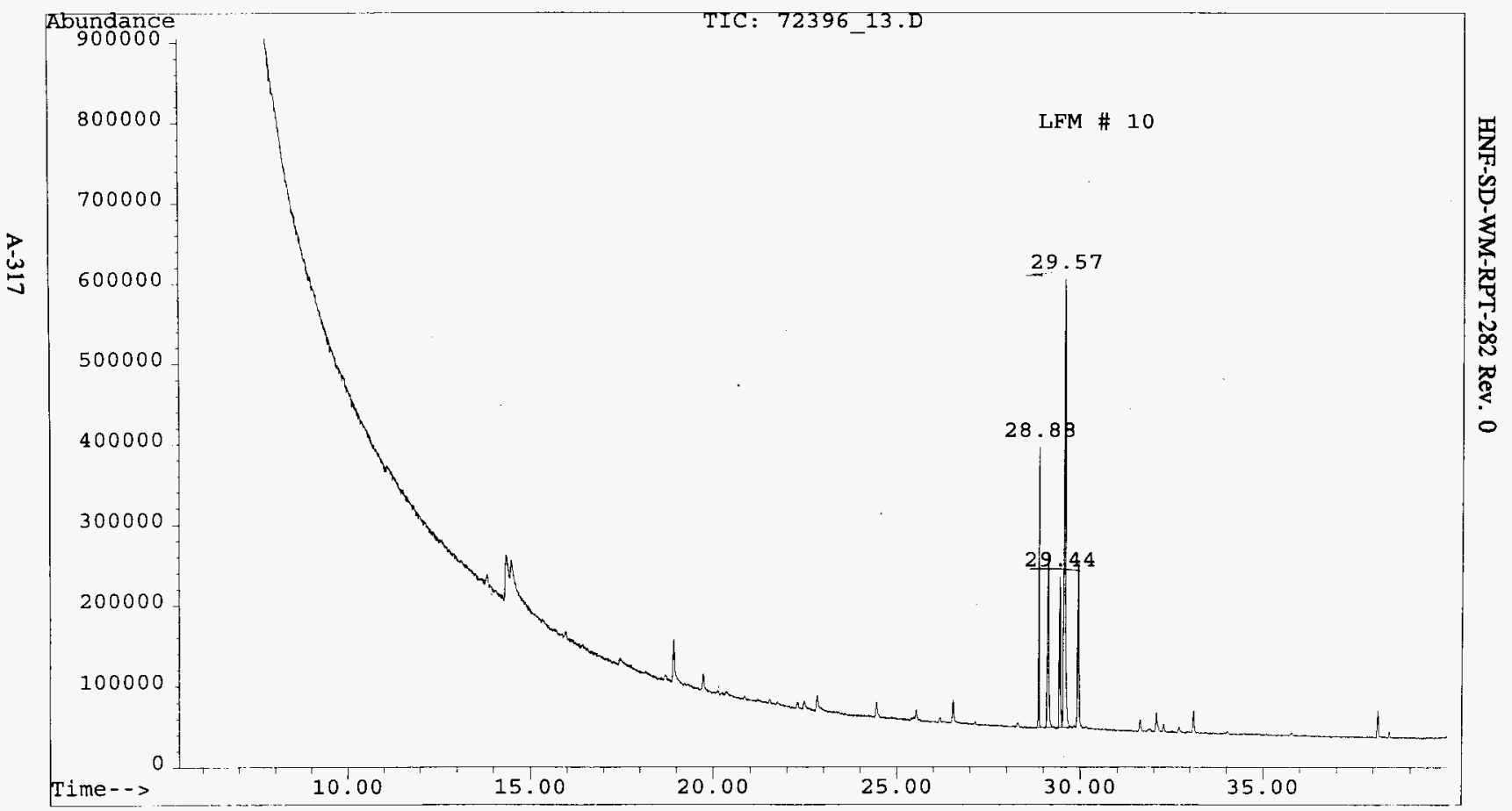


HNF-SD-WM-RPT-282 Rev. 0

CWC_REP.XLS

\section{ANALYSIS REPORT FOR CWC AIR SAMPLES}

File Name:

Analyst:

Analysis Date:

Method:

Sample Name:
72396_13.D

MS

\#VALUE!

CWC

FT6045-10.116A

\begin{tabular}{|c|c|c|c|c|}
\hline COMPOUND NAME & CAS \# & Concentration & Units & Comments \\
\hline ISO-BUTANE & $75-28-5$ & 0 & PPB & \\
\hline VINYL CHLORIDE & $75-01-4$ & 0 & PPB & \\
\hline ETHANOL & $64-17-5$ & 0 & PPB & \\
\hline ACETONE & $67-64-1$ & 0 & PPB & \\
\hline 2-PROPANOL & $67-63-0$ & 0 & PPB & \\
\hline 1,1-DICHLOROETHENE & $75-35-4$ & 0 & PPB & \\
\hline FREON 113 & $76-13-1$ & 0 & PPB & \\
\hline DICHLOROMETHANE & $75-09-2$ & 0 & PPB & \\
\hline 1,1-DICHLOROETHANE & $75-34-3$ & 0 & PPB & \\
\hline 2-BUTANONE & $78-93-3$ & 0 & PPB & \\
\hline N-HEXANE & $110-54-3$ & 0 & PPB & \\
\hline 1,2-DICHLOROETHENE & $356-59-2$ & 0 & PPB & \\
\hline CHLOROFORM & $67-66-3$ & 0 & PPB & \\
\hline TETRAHYDROFURAN & $109-99-9$ & 0 & PPB & \\
\hline 1,1,1-TRICHLOROETHANE & $71-55-6$ & 0 & PPB & \\
\hline 1,2-DICHLOROETHANE & $107-06-2$ & 0 & $\overline{P P B}$ & \\
\hline BENZENE & $71-43-2$ & 0 & PPB & \\
\hline CARBON TETRACHLORIDE & $56-23-5$ & 0 & PPB & \\
\hline CYCLOHEXANE & $110-82-7$ & 0 & PPB & \\
\hline N-BUTANOL & $71-36-3$ & 0 & PPB & \\
\hline TRICHLOROETHENE & $79-01-6$ & 0 & PPB & \\
\hline 4-METHYL-2-PENTANONE & $108-10-1$ & 0 & PPB & \\
\hline TOLUENE & $108-88-3$ & 0 & PPB & \\
\hline 1,1,2-TRICHLOROETHANE & $79-00-5$ & 0 & PPB & \\
\hline TETRACHLOROETHENE & $127-18-4$ & 0 & PPB & \\
\hline CHLOROBENZENE & $108-90-7$ & 0 & $\overline{\mathrm{PPB}}$ & \\
\hline ETHYLBENZENE & $100-41-4$ & 6 & PPB & \\
\hline M-XYLENE & $108-38-3$ & 7 & PPB & \\
\hline STYRENE & $100-42-5$ & 0 & PPB & \\
\hline O-XYLENE & $95-47-6$ & 5 & PPB & \\
\hline 1,1,2,2-TETRACHLORETHANE & $79-34-5$ & 0 & PPB & \\
\hline $1,3,5$-TRIMETHYLBENZENE & $108-67-8$ & 0 & PPB & \\
\hline 1,2,4-TRIMETHYLBENZENE & $95-63-6$ & 0 & PPB & \\
\hline 1,3-DICHLOROBENZENE & $541-73-1$ & 0 & PPB & \\
\hline 1,2-DICHLOROBENZENE & $95-50-1$ & 0 & PPB & \\
\hline 1,4-DICHLOROBENZENE & $106-46-7$ & 0 & $\overline{\mathrm{PPB}}$ & \\
\hline
\end{tabular}


HNF-SD-WM-RPT-282 Rev. 0

\section{LIST OF COMPOUNDS TENTATIVELY IDENTIFIED WITH MASS SPECTRAL LIBRARY}

Sample: $\quad$ LFM \# 10

File: $\quad$ C:LHPCHEMLIDATAI72396_13.D

ID: $\quad$ FT6045-10.116A

Compound

CAS\#

XYLENE

001330-20-7

3-Heptanone

$000106-35-4$

2-Heptanone

000110-43-0

Benzene, 1,4-dimethyl-

$000106-42-3$ 
File

: C: $\backslash$ HPCHEM $\backslash 1 \backslash D A T A \backslash 72296 \_16 . D$

Operator

MS

Acquired : 25 Mar $153 \quad 1: 45$ am using AcqMethod CWC

Instrument : 5972 - In

Sample Name: FT6045-10.116B

Misc Info : 100 TORR

Vial Number: 1

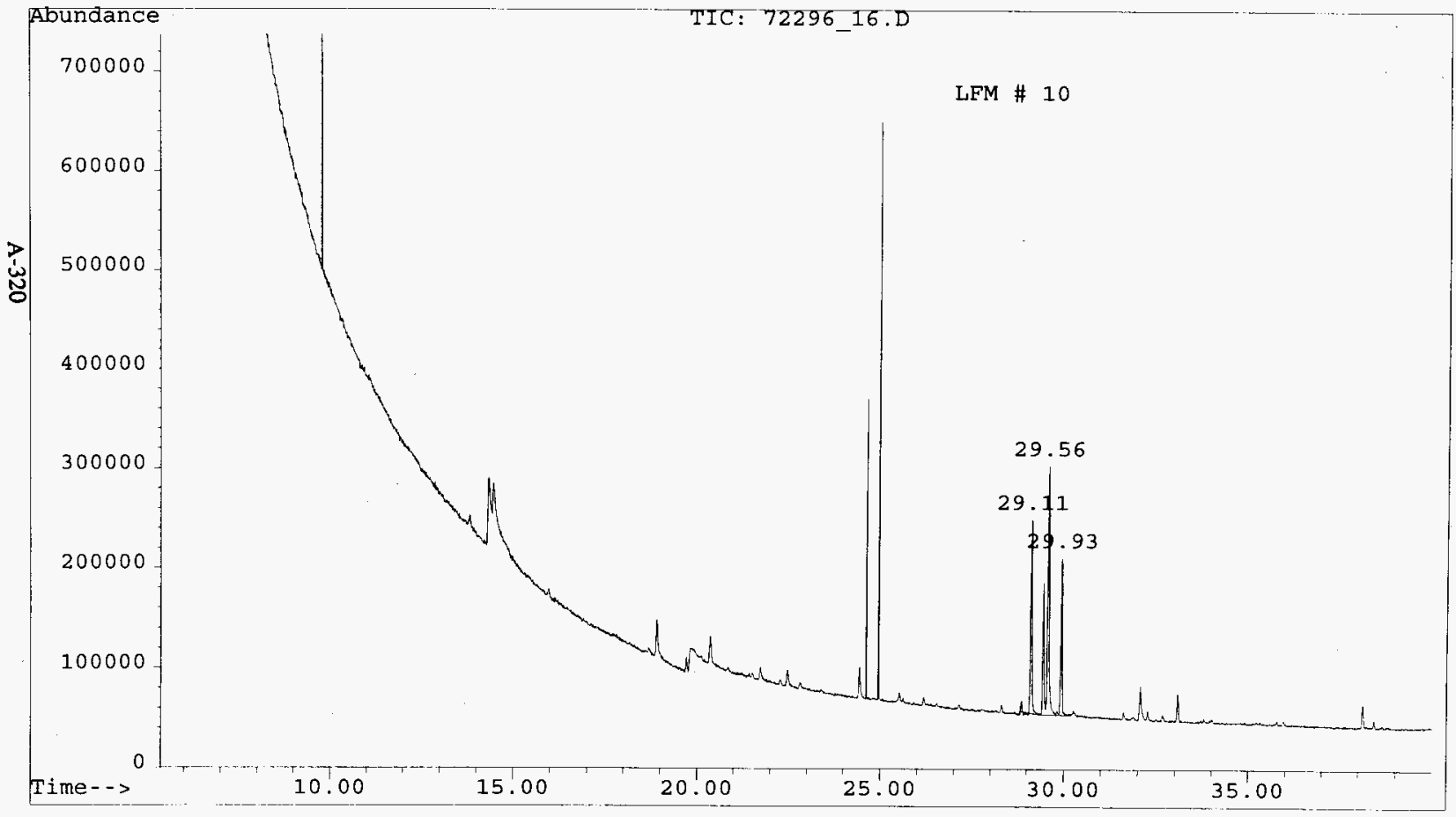


HNF-SD-WM-RPT-282 Rev. 0

CWC_REP.XLS

\section{ANALYSIS REPORT FOR CWC AIR SAMPLES}

File Name:

Analyst:

Analysis Date:

Method:

Sample Name:
72296_16.D

MS

\#VALUE!

CWC

FT6045-10.116B

\begin{tabular}{|c|c|c|c|c|}
\hline COMPOUND NAME & CAS\# & Concentration & Units & Comments \\
\hline ISO-BUTANE & $75-28-5$ & 0 & PPB & \\
\hline VINYL CHLORIDE & $75-01-4$ & 0 & PPB & \\
\hline ETHANOL & $64-17-5$ & 0 & PPB & \\
\hline ACETONE & $67-64-1$ & 0 & PPB & \\
\hline 2-PROPANOL & $67-63-0$ & 0 & PPB & \\
\hline 1,1-DICHLOROETHENE & $75-35-4$ & 0 & PPB & \\
\hline FREON 113 & $76-13-1$ & 0 & PPB & \\
\hline DICHLOROMETHANE & $75-09-2$ & 0 & PPB & \\
\hline 1,1-DICHLOROETHANE & $75-34-3$ & 0 & PPB & \\
\hline 2-BUTANONE & $78-93-3$ & 0 & PPB & \\
\hline N-HEXANE & $110-54-3$ & 0 & PPB & \\
\hline 1,2-DICHLOROETHENE & $156-59-2$ & 0 & PPB & \\
\hline CHLOROFORM & $67-66-3$ & 0 & PPB & \\
\hline TETRAHYDROFURAN & $109-99-9$ & 0 & PPB & \\
\hline 1,1,1-TRICHLOROETHANE & $71-55-6$ & 0 & PPB & \\
\hline 1,2-DICHLOROETHANE & $107-06-2$ & 0 & PPB & \\
\hline BENZENE & $71-43-2$ & 0 & PPB & \\
\hline CARBON TETRACHLORIDE & $56-23-5$ & 0 & PPB & \\
\hline CYCLOHEXANE & $110-82-7$ & 0 & PPB & \\
\hline N-BUTANOL & $71-36-3$ & $\overline{0}$ & PPB & \\
\hline TRICHLOROETHENE & $79-01-6$ & 0 & PPB & \\
\hline 4-METHYL-2-PENTANONE & $108-10-1$ & 0 & PPB & \\
\hline TOLUENE & $108-88-3$ & 0 & PPB & \\
\hline 1,1,2-TRICHLOROETHANE & $79-00-5$ & 0 & PPB & \\
\hline TETRACHLOROETHENE & $127-18-4$ & 0 & PPB & \\
\hline CHLOROBENZENE & $108-90-7$ & 0 & PPB & \\
\hline ETHYLBENZENE & $100-41-4$ & 5 & PPB & \\
\hline M-XYLENE & $108-38-3$ & 6 & PPB & \\
\hline STYRENE & $100-42-5$ & 0 & $\mathrm{PPB}$ & \\
\hline O-XYLENE & $95-47-6$ & 4 & PPB & \\
\hline 1,1,2,2-TETRACHLORETHANE & $79-34-5$ & 0 & $\mathrm{PPB}$ & \\
\hline 1,3,5-TRIMETHYLBENZENE & $108-67-8$ & 0 & PPB & \\
\hline 1,2,4-TRIMETHYLBENZENE & $95-63-6$ & 0 & PPB & \\
\hline 1,3-DICHLOROBENZENE & $541-73-1$ & 0 & PPB & \\
\hline 1,2-DICHLOROBENZENE & $95-50-1$ & 0 & PPB & \\
\hline 1,4-DICHLOROBENZENE & $106-46-7$ & 0 & PPB & \\
\hline
\end{tabular}


HNF-SD-WM-RPT-282 Rev. 0

\section{LIST OF COMPOUNDS TENTATIVELY IDENTIFIED WITH MASS SPECTRAL LIBRARY}

$\begin{array}{ll}\text { Sample } & \text { LFM \# 10 } \\ \text { File } & \text { C:HPCHEMLDATA172296_16.D } \\ \text { ID: } & \text { FT6045-10,116B }\end{array}$

Compound

CAS\#

Benzene, 1,4-dimethyl-

$000106-42-3$

2-Heptanone

000110-43-0

Benzene, 1,2-dimethyl-

$000095-47-6$ 
File

C : \HPCHEM \I\DATA $\backslash 72396$ 9.D

Operator

MS

Acquired

: 25 Mar 153 5972 - In

Instrument

Sample Name: FT6045-11.115A

Misc Info : 100 TORR

Vial Number: 1

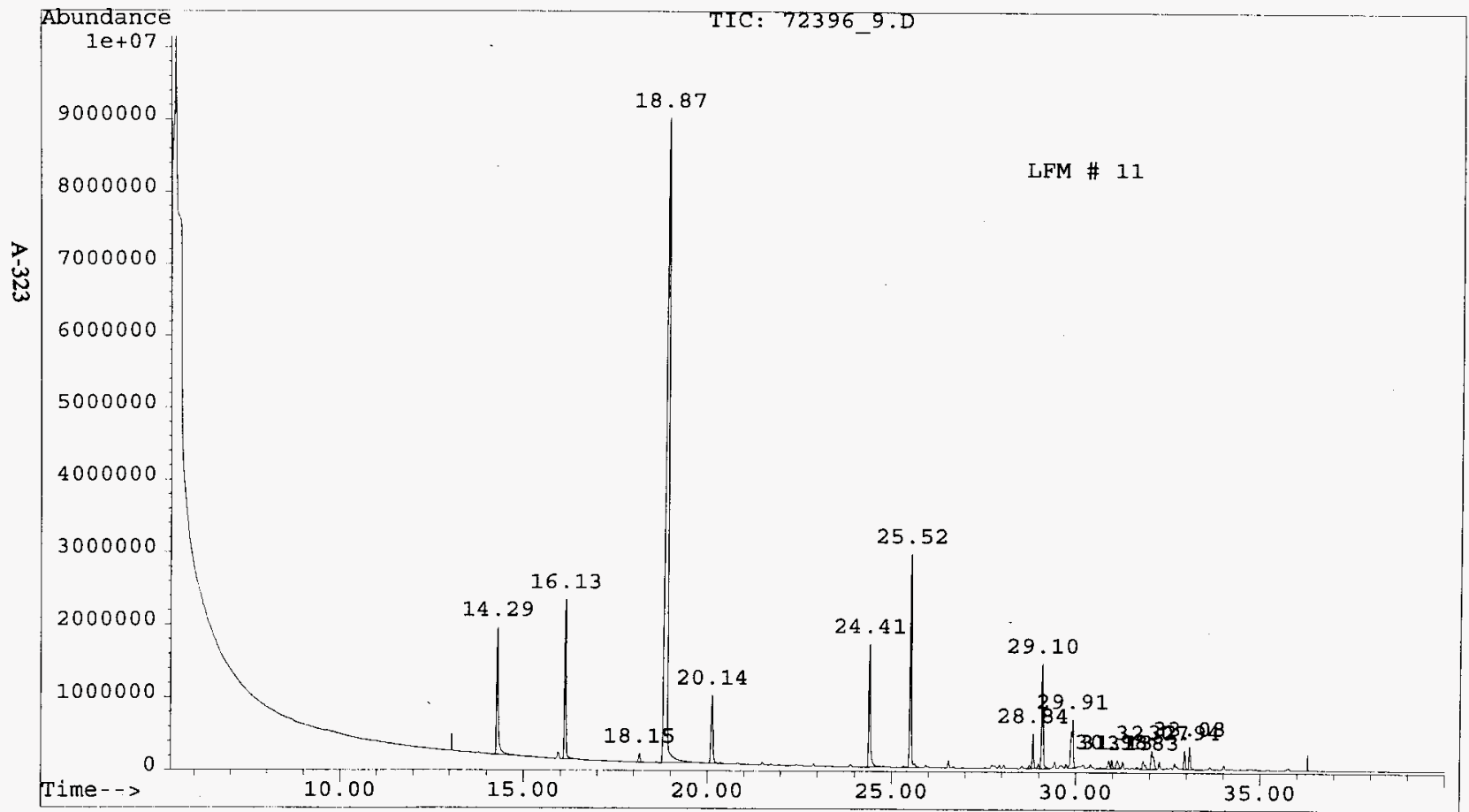


HNF-SD-WM-RPT-282 Rev. 0

CWC_REP.XLS

ANALYSIS REPORT FOR CWC AIR SAMPLES

File Name:

Analyst:

72396_9.D

Analysis Date:

MS

Method:

\#VALUE!

Sample Name:

CWC

FT6045-11.115A

\begin{tabular}{|c|c|c|c|c|}
\hline COMPOUND NAME & CAS \# & Concentration & Units & Comments \\
\hline ISO-BUTANE & $75-28-5$ & 0 & PPB & \\
\hline VINYL CHLORIDE & $75-01-4$ & 0 & PPB & \\
\hline ETHANOL & $64-17-5$ & 0 & $\overline{\mathrm{PPB}}$ & \\
\hline ACETONE & $67-64-1$ & 54 & PPB & \\
\hline 2-PROPANOL & $67-63-0$ & 0 & PPB & \\
\hline 1,1-DICHLOROETHENE & $75-35-4$ & 0 & PPB & \\
\hline FREON 113 & $76-13-1$ & 4 & PPB & \\
\hline DICHLOROMETHANE & $75-09-2$ & 82 & PPB & \\
\hline 1,1-DICHLOROETHANE & $75-34-3$ & 0 & PPB & \\
\hline 2-BUTANONE & 78-93-3 & 1465 & PPB & \\
\hline N-HEXANE & $110-54-3$ & 123 & PPB & \\
\hline 1,2-DICHLOROETHENE & $156-59-2$ & 0 & PPB & \\
\hline CHLOROFORM & $67-66-3$ & 0 & PPB & \\
\hline TETRAHYDROFURAN & $109-99-9$ & 25 & PPB & \\
\hline 1,1,1-TRICHLOROETHANE & $71-55-6$ & 0 & $\overline{\mathrm{PPB}}$ & \\
\hline 1,2-DICHLOROETHANE & $107-06-2$ & 0 & PPB & \\
\hline BENZENE & $71-43-2$ & 0 & PPB & \\
\hline CARBON TETRACHLORIDE & $56-23-5$ & 0 & PPB & \\
\hline CYCLOHEXANE & $110-82-7$ & 0 & PPB & \\
\hline N-BUTANOL & $71-36-3$ & 0 & PPB & \\
\hline TRICHLOROETHENE & $79-01-6$ & 0 & PPB & \\
\hline 4-METHYL-2-PENTANONE & $108-10-1$ & 41 & PPB & \\
\hline TOLUENE & $108-88-3$ & 96 & PPB & \\
\hline 1,1,2-TRICHLOROETHANE & $79-00-5$ & 0 & PPB & \\
\hline TETRACHLOROETHENE & $127-18-4$ & 0 & PPB & \\
\hline CHLOROBENZENE & $108-90-7$ & 0 & PPB & \\
\hline ETHYLBENZENE & $100-41-4$ & 14 & PPB & \\
\hline M-XYLENE & $108-38-3$ & 46 & PPB & \\
\hline STYRENE & $100-42-5$ & 0 & PPB & \\
\hline O-XYLENE & $95-47-6$ & 19 & PPB & \\
\hline $1,1,2,2$-TETRACHLORETHANE & $79-34-5$ & 0 & PPB & \\
\hline 1,3,5-TRIMETHYLBENZENE & $108-67-8$ & 3 & PPB & \\
\hline 1,2,4-TRIMETHYLBENZENE & $95-63-6$ & 8 & PPB & \\
\hline 1,3-DICHLOROBENZENE & $541-73-1$ & 0 & PPB & \\
\hline 1,2-DICHLOROBENZENE & $95-50-1$ & 0 & PPB & \\
\hline 1,4-DICHLOROBENZENE & $106-46-7$ & 0 & $\mathrm{PPB}$ & \\
\hline
\end{tabular}


HNF-SD-WM-RPT-282 Rev. 0

\section{LIST OF COMPOUNDS TENTATIVELY IDENTIFIED WITH MASS SPECTRAL LIBRARY}

Sample: $\quad$ LFM \# 11

File: C:LHPCHEMLDATA172396_9.D

ID: FT6045-11.115A

Compound

CAS\#

2-Propanone

000067-64-1

Methane, dichloro-

$000075-09-2$

2-Butanone

000078-93-3

Furan, tetrahydro-

000109-99-9

2-Pentanone, 4-methyl-

000108-10-1

Benzene, methyl-

000108-88-3

Benzene, ethyl-

000100-41-4

Benzene, 1,4-dimethyl-

$000106-42-3$

Benzene, 1,3-dimethyl-

000108-38-3

Benzene, 1,3,5-trimethyl-

000108-67-8

Benzene, l-ethyl-2-methyl-

000611-14-3

Decane

000124-18-5

Benzene, 1,2,3-trimethyl-

000526-73-8

Benzene, 1,2,4-trimethyl-

000095-63-6 
File

Operator

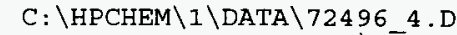

Acquired

: LAP

Instrument

26 Mar 153 2:32 pm using AcqMethod CWC

Sample Name: FT6045-12.129A

Misc Info : 100 TORR

Vial Number: 1

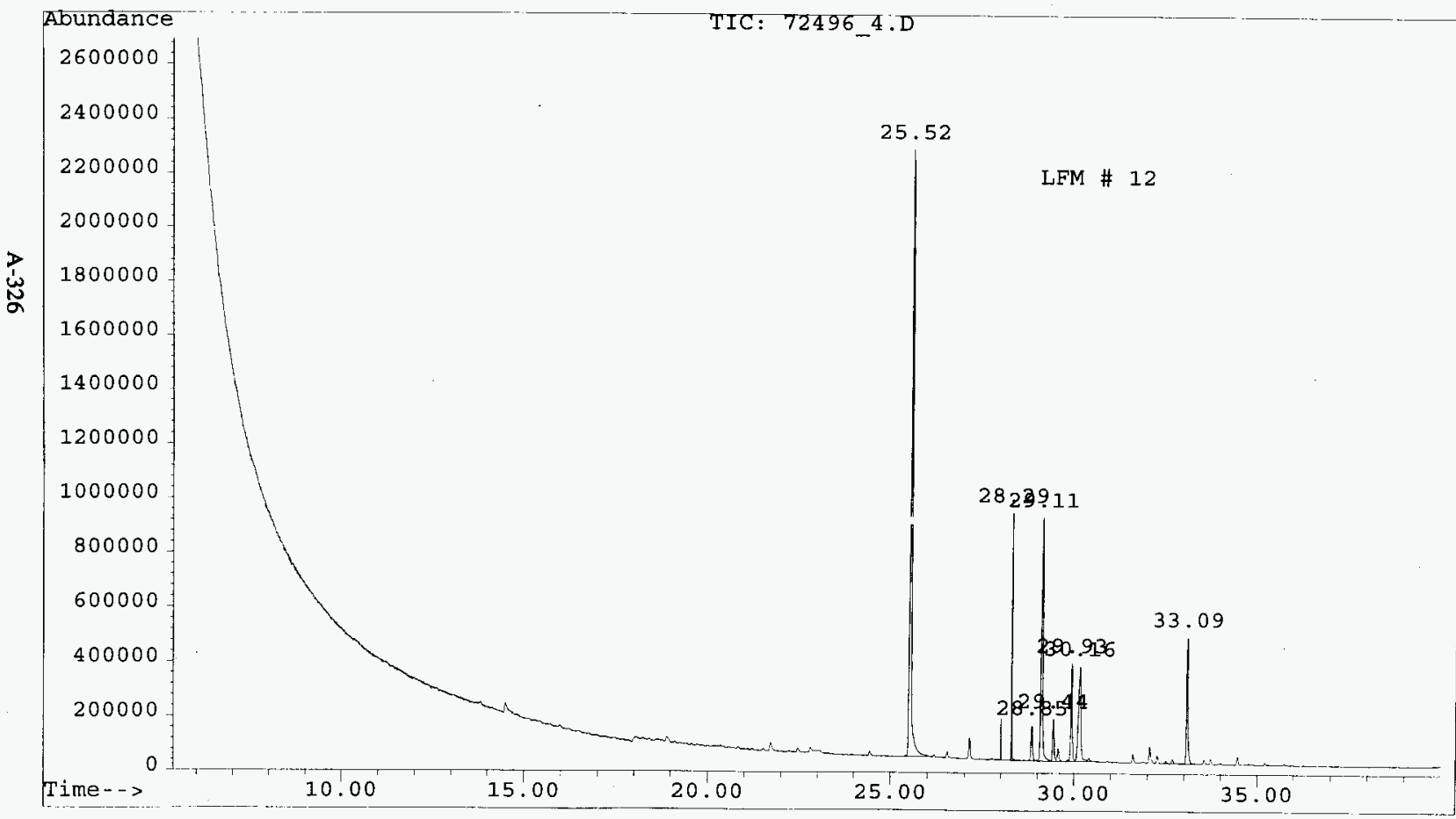


HNF-SD-WM-RPT-282 Rev. 0

CWC_REP.XLS

\section{ANALYSIS REPORT FOR CWC AIR SAMPLES}

File Name:

Analyst:

Analysis Date:

Method:

Sample Name:
72496_4.D

LAP

\#VALUE!

CWC

FT6045-12.129A

\begin{tabular}{|c|c|c|c|c|}
\hline COMPOUND NAME & CAS \# & Concentration & Units & Comments \\
\hline ISO-BUTANE & $75-28-5$ & 0 & PPB & \\
\hline VINYL CHLORIDE & $75-01-4$ & 0 & $\overline{\mathrm{PPB}}$ & \\
\hline ETHANOL & $64-17-5$ & 0 & PPB & \\
\hline ACETONE & $67-64-1$ & 0 & PPB & \\
\hline 2-PROPANOL & $67-63-0$ & 0 & $\overline{\mathrm{PPB}}$ & \\
\hline 1,1-DICHLOROETHENE & $75-35-4$ & 0 & PPB & \\
\hline FREON 113 & $76-13-1$ & 0 & PPB & \\
\hline DICHLOROMETHANE & $75-09-2$ & 0 & PPB & \\
\hline 1,1-DICHLOROETHANE & $75-34-3$ & 0 & $\overline{\mathrm{PPB}}$ & \\
\hline 2-BUTANONE & $78-93-3$ & 0 & PPB & \\
\hline N-HEXANE & $110-54-3$ & $\overline{0}$ & $\overline{P P B}$ & \\
\hline 1,2-DICHLOROETHENE & $156-59-2$ & 0 & PPB & \\
\hline CHLOROFORM & $67-66-3$ & 0 & $\overline{\text { PPB }}$ & \\
\hline TETRAHYDROFURAN & $109-99-9$ & 0 & PPB & \\
\hline 1,1,1-TRICHLOROETHANE & $71-55-6$ & 0 & PPB & \\
\hline 1,2-DICHLOROETHANE & 107-06-2 & 0 & $\overline{\mathrm{PPB}}$ & \\
\hline BENZENE & $71-43-2$ & 0 & $\overline{\mathrm{PPB}}$ & \\
\hline CARBON TETRACHLORIDE & $56-23-5$ & 0 & PPB & \\
\hline CYCLOHEXANE & $110-82-7$ & 0 & PPB & \\
\hline N-BUTANOL & $71-36-3$ & 0 & $\mathrm{PPB}$ & \\
\hline TRICHLOROETHENE & $79-01-6$ & 0 & PPB & \\
\hline 4-METHYL-2-PENTANONE & $108-10-1$ & 0 & PPB & \\
\hline TOLUENE & $108-88-3$ & 83 & PPB & \\
\hline 1,1,2-TRICHLOROETHANE & $79-00-5$ & 0 & PPB & \\
\hline TETRACHLOROETHENE & $127-18-4$ & 0 & PPB & \\
\hline CHLOROBENZENE & $108-90-7$ & 0 & PPB & \\
\hline ETHYLBENZENE & $100-41-4$ & 4 & PPB & \\
\hline M-XYLENE & $108-38-3$ & 29 & PPB & \\
\hline STYRENE & $100-42-5$ & 0 & PPB & \\
\hline O-XYLENE & $95-47-6$ & 10 & PPB & \\
\hline $1,1,2,2$-TETRACHLORETHANE & $79-34-5$ & 0 & PPB & \\
\hline 1,3,5-TRIMETHYLBENZENE & $108-67-8$ & 2 & PPB & \\
\hline 1,2,4-TRIMETHYLBENZENE & $95-63-6$ & 13 & PPB & \\
\hline 1,3-DICHLOROBENZENE & $541-73-1$ & 0 & $\overline{\mathrm{PPB}}$ & \\
\hline 1,2-DICHLOROBENZENE & $95-50-1$ & 0 & PPB & \\
\hline 1,4-DICHLOROBENZENE & $106-46-7$ & 0 & PPB & \\
\hline
\end{tabular}


HNF-SD-WM-RPT-282 Rev. 0

\section{LIST OF COMPOUNDS TENTATIVELY IDENTIFIED WITH MASS SPECTRAL LIBRARY}

Sample: $\quad$ LFM \# 12

File: $\quad$ C:IHPCHEMI2LDATA172496_4.D

ID: $\quad$ FT6045-12.129A

Compound

CAS\#

Benzene, methyl-

$000108-88-3$

Cyclobutanol

002919-23-5

Benzene, ethyl-

000100-41-4

Benzene, 1,3-dimethyl-

000108-38-3

3-Heptanone

000106-35-4

Benzene, 1,2-dimethyl-

000095-47-6

Benzaldehyde

000100-52-7

Benzene, 1,2,4-trimethyl-

000095-63-6 


\section{File}

Operator Acquired

: C: $\backslash$ HPCHEM $\backslash 1 \backslash D A T A \backslash 72496 \_8 . D$

: MS

Instrument : 5972 - In

Sample Name: FT6045-13.130A

Misc Info : 100 TORR

Vial Number: 1

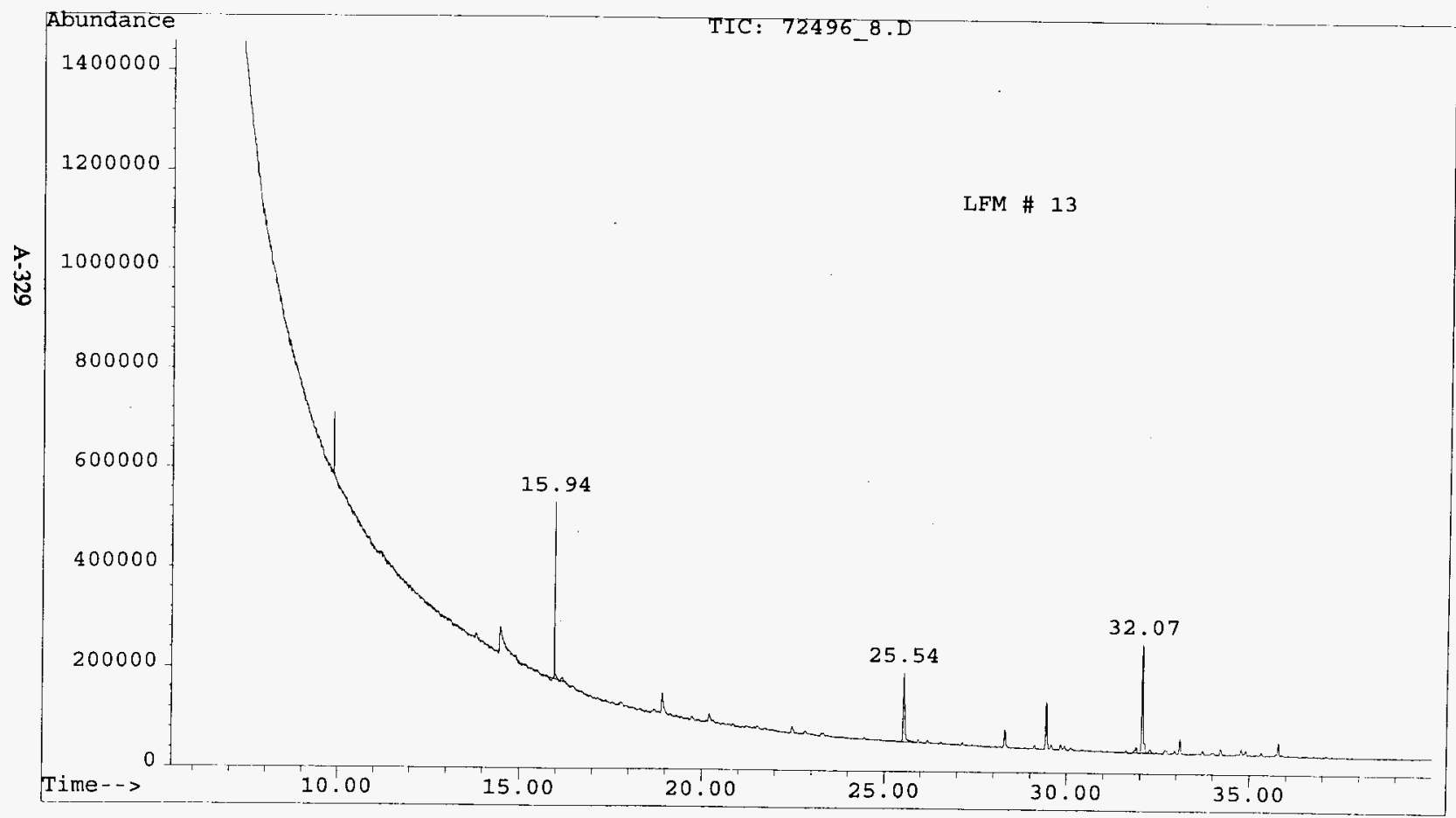


HNF-SD-WM-RPT-282 Rev. 0

CWC_REP.XLS

\section{ANALYSIS REPORT FOR CWC AIR SAMPLES}

File Name:

Analyst:

Analysis Date:

Method:

Sample Name:
72496_8.D

MS

\#VALUE!

CWC

FT6045-13.130A

\begin{tabular}{|c|c|c|c|c|}
\hline COMPOUND NAME & CAS \# & Concentration & Units & Comments \\
\hline ISO-BUTANE & $75-28-5$ & 0 & PPB & \\
\hline VINYL CHLORIDE & $75-01-4$ & 0 & PPB & \\
\hline ETHANOL & $64-17-5$ & 0 & PPB & \\
\hline ACETONE & $67-64-1$ & 0 & PPB & \\
\hline 2-PROPANOL & $67-63-0$ & 0 & PPB & \\
\hline 1,1-DICHLOROETHENE & $75-35-4$ & 0 & PPB & \\
\hline FREON 113 & $76-13-1$ & 0 & PPB & \\
\hline DICHLOROMETHANE & $75-09-2$ & 0 & PPB & \\
\hline 1,1-DICHLOROETHANE & $75-34-3$ & 0 & PPB & \\
\hline 2-BUTANONE & $78-93-3$ & 0 & PPB & \\
\hline N-HEXANE & $110-54-3$ & 0 & PPB & \\
\hline 1,2-DICHLOROETHENE & $156-59-2$ & 0 & PPB & \\
\hline CHLOROFORM & $67-66-3$ & 0 & PPB & \\
\hline TETRAHYDROFURAN & $109-99-9$ & 0 & PPB & \\
\hline 1,1,1-TRICHLOROETHANE & $71-55-6$ & 0 & PPB & \\
\hline 1,2-DICHLOROETHANE & $107-06-2$ & 0 & PPB & \\
\hline BENZENE & $71-43-2$ & $\overline{0}$ & PPB & \\
\hline CARBON TETRACHLORIDE & $56-23-5$ & 0 & PPB & \\
\hline CYCLOHEXANE & $110-82-7$ & 0 & PPB & \\
\hline N-BUTANOL & $71-36-3$ & 0 & PPB & \\
\hline TRICHLOROETHENE & $79-01-6$ & 0 & PPB & \\
\hline 4-METHYL-2-PENTANONE & $108-10-1$ & 0 & PPB & \\
\hline TOLUENE & $108-88-3$ & 5 & PPB & \\
\hline 1,1,2-TRICHLOROETHANE & $79-00-5$ & 0 & PPB & \\
\hline TETRACHLOROETHENE & $127-18-4$ & 0 & PPB & \\
\hline CHLOROBENZENE & $108-90-7$ & 0 & $\overline{\mathrm{PPB}}$ & \\
\hline ETHYLBENZENE & $100-41-4$ & 0 & PPB & \\
\hline M-XYLENE & $108-38-3$ & 0 & PPB & \\
\hline STYRENE & $100-42-5$ & 0 & PPB & \\
\hline O-XYLENE & $95-47-6$ & 0 & PPB & \\
\hline 1,1,2,2-TETRACHLORETHANE & $79-34-5$ & 0 & PPB & \\
\hline 1,3,5-TRIMETHYLBENZENE & $108-67-8$ & 5 & $\mathrm{PPB}$ & \\
\hline 1,2,4-TRIMETHYLBENZENE & $95-63-6$ & 0 & $\mathrm{PPB}$ & \\
\hline 1,3-DICHLOROBENZENE & $541-73-1$ & 0 & PPB & \\
\hline 1,2-DICHLOROBENZENE & $95-50-1$ & 0 & PPB & \\
\hline 1,4-DICHLOROBENZENE & $106-46-7$ & 0 & PPB & \\
\hline
\end{tabular}


HNF-SD-WM-RPT-282 Rev. 0

\section{LIST OF COMPOUNDS TENTATIVELY IDENTIFIED WITH MASS SPECTRAL LIBRARY}

Sample: $\quad$ LFM \# 13

File: C:IHPCHEM 2WDATA172496_8.D

ID: FT6045-13.130A

Compound CAS\#

Benzene, methyl- $\quad$ 000108-88-3

$\begin{array}{ll}\text { Benzaldehyde } & 000100-52-7\end{array}$ 
: C: \HPCHEM \1\DATA $\backslash 72396 \_14 . D$

Operator : MS

Acquired : 26 Mar 153 $1: 31$ am using AcqMethod CWC

Instrument : 5972 - In

Sample Name: FT6045-13.130B

Misc Info : 100 TORR

Vial Number: 1

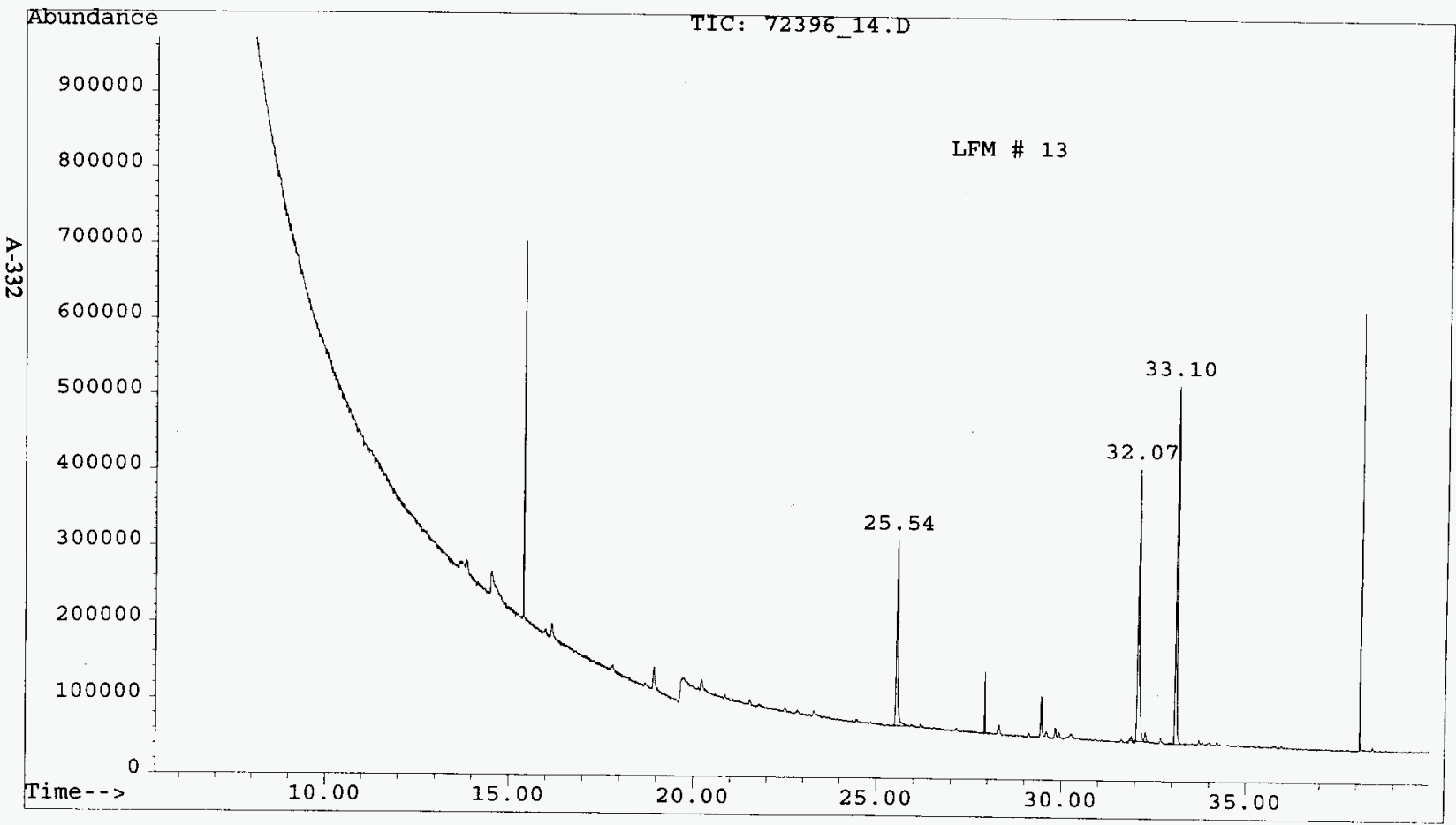


HNF-SD-WM-RPT-282 Rev. 0

CWC_REP.XLS

\section{ANALYSIS REPORT FOR CWC AIR SAMPLES}

File Name:

Analyst:

72396_14.D

Analysis Date:

MS

Method:

\#VALUE!

Sample Name:

CWC

FT6045-13.130B

\begin{tabular}{|c|c|c|c|c|}
\hline COMPOUND NAME & CAS \# & Concentration & Units & Comments \\
\hline ISO-BUTANE & $75-28-5$ & 0 & PPB & \\
\hline VINYL CHLORIDE & $75-01-4$ & 0 & PPB & \\
\hline ETHANOL & $64-17-5$ & 0 & PPB & \\
\hline ACETONE & $67-64-1$ & 0 & PPB & \\
\hline 2-PROPANOL & $67-63-0$ & 0 & PPB & \\
\hline 1,1-DICHLOROETHENE & $75-35-4$ & 0 & PPB & \\
\hline FREON 113 & $76-13-1$ & 0 & PPB & \\
\hline DICHLOROMETHANE & $75-09-2$ & 0 & PPB & \\
\hline 1,1-DICHLOROETHANE & $75-34-3$ & 0 & PPB & \\
\hline 2-BUTANONE & $78-93-3$ & 0 & PPB & \\
\hline N-HEXANE & $110-54-3$ & 0 & PPB & \\
\hline 1,2-DICHLOROETHENE & $156-59-2$ & 0 & PPB & \\
\hline CHLOROFORM & $67-66-3$ & 0 & PPB & \\
\hline TETRAHYDROFURAN & $109-99-9$ & 0 & PPB & \\
\hline 1,1,1-TRICHLOROETHANE & $71-55-6$ & 0 & PPB & \\
\hline 1,2-DICHLOROETHANE & $107-06-2$ & 0 & PPB & \\
\hline BENZENE & $71-43-2$ & 0 & PPB & \\
\hline CARBON TETRACHLORIDE & $56-23-5$ & 0 & PPB & \\
\hline CYCLOHEXANE & $110-82-7$ & 0 & $\overline{\mathrm{PPB}}$ & \\
\hline N-BUTANOL & $71-36-3$ & 0 & PPB & \\
\hline TRICHLOROETHENE & $79-01-6$ & 0 & PPB & \\
\hline 4-METHYL-2-PENTANONE & $108-10-1$ & 0 & $\overline{\mathrm{PPB}}$ & \\
\hline TOLUENE & $108-88-3$ & 8 & PPB & \\
\hline 1,1,2-TRICHLOROETHANE & $79-00-5$ & 0 & PPB & \\
\hline TETRACHLOROETHENE & $127-18-4$ & 0 & PPB & \\
\hline CHLOROBENZENE & $108-90-7$ & 0 & PPB & \\
\hline ETHYLBENZENE & $100-41-4$ & 0 & $\mathrm{PPB}$ & \\
\hline M-XYLENE & $108-38-3$ & $\overline{0}$ & PPB & \\
\hline STYRENE & $100-42-5$ & 0 & PPB & \\
\hline O-XYLENE & $95-47-6$ & 0 & PPB & \\
\hline 1,1,2,2-TETRACHLORETHANE & $79-34-5$ & 0 & PPB & \\
\hline 1,3,5-TRIMETHYLBENZENE & $108-67-8$ & 9 & PPB & \\
\hline 1,2,4-TRIMETHYLBENZENE & $95-63-6$ & 12 & PPB & \\
\hline 1,3-DICHLOROBENZENE & $541-73-1$ & 0 & PPB & \\
\hline 1,2-DICHLOROBENZENE & $95-50-1$ & 0 & PPB & \\
\hline 1,4-DICHLOROBENZENE & $106-46-7$ & 0 & PPB & \\
\hline
\end{tabular}


HNF-SD-WM-RPT-282 Rev. 0

\section{LIST OF COMPOUNDS TENTATIVELY IDENTIFIED WITH MASS SPECTRAL LIBRARY}

\section{Sample: $\quad$ LFM \# 13}

File $\quad$ C:IHPCHEML2LDATA172396_14.D

ID: $\quad$ FT6045-13.130B

Compound CAS\#

Benzene, methyl-

000108-88-3

Benzaldehyde

000100-52-7

Benzene, 1,2,3-trimethyl-

$000526-73-8$ 
HNF-SD-WM-RPT-282 Rev. 0

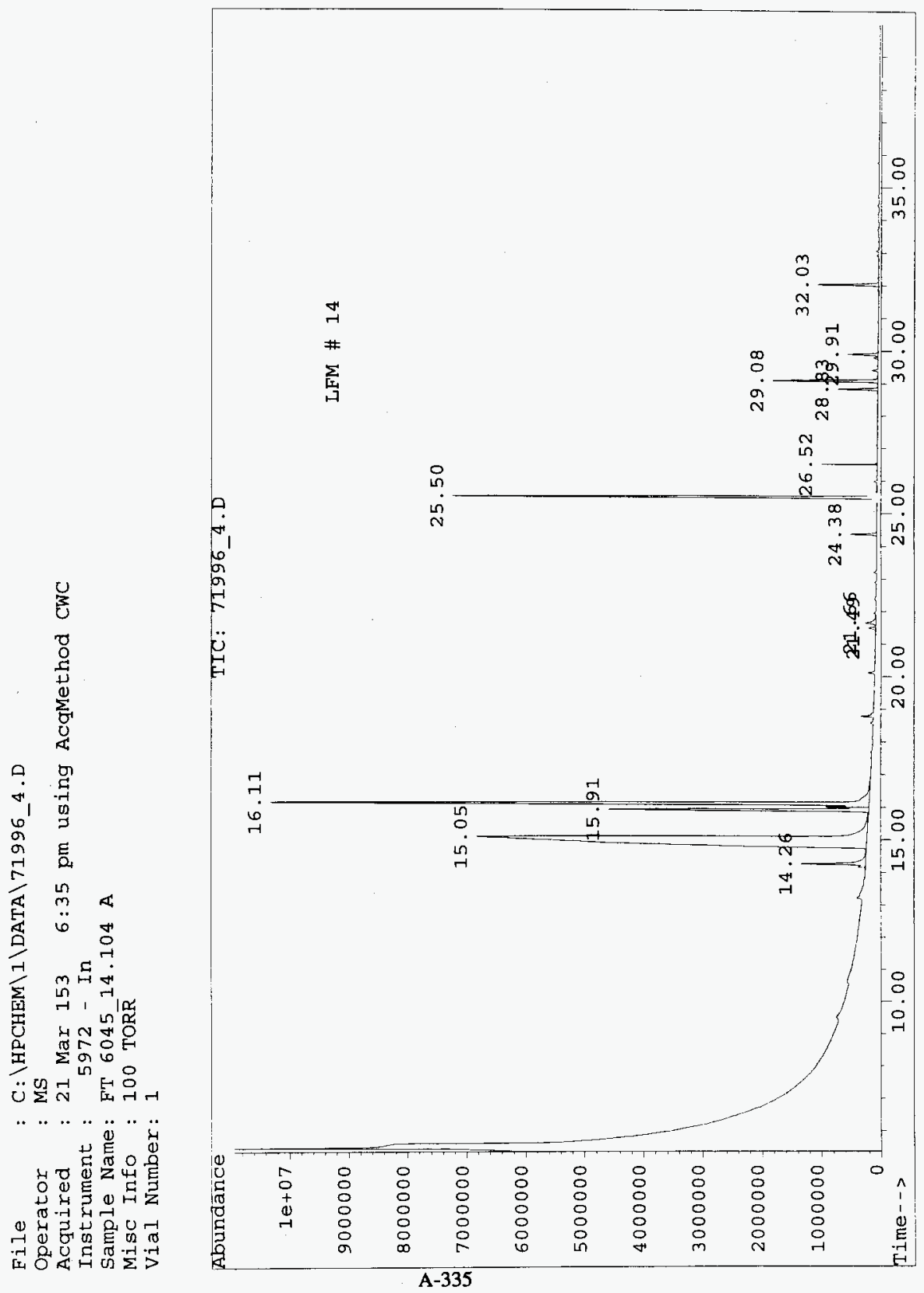


HNF-SD-WM-RPT-282 Rev. 0

CWC_REP.XLS

\section{ANALYSIS REPORT FOR CWC AIR SAMPLES}

File Name:

Analyst:

Analysis Date:

Method:

Sample Name:
71996_4.D

MS

\#VALUE!

CWC

FT 6045_14.104 A

\begin{tabular}{|c|c|c|c|c|}
\hline COMPOUND NAME & CAS \# & Concentration & Units & Comments \\
\hline ISO-BUTANE & $75-28-5$ & $\overline{0}$ & PPB & \\
\hline VINYL CHLORIDE & $75-01-4$ & 0 & PPB & \\
\hline ETHANOL & $64-17-5$ & 19 & PPB & \\
\hline ACETONE & $67-64-1$ & 34 & PPB & \\
\hline 2-PROPANOL & $67-63-0$ & 1055 & PPB & \\
\hline 1,1-DICHLOROETHENE & $75-35-4$ & 0 & PPB & \\
\hline FREON 113 & $76-13-1$ & 190 & PPB & \\
\hline DICHLOROMETHANE & $75-09-2$ & 479 & PPB & \\
\hline 1,1-DICHLOROETHANE & $75-34-3$ & 0 & PPB & \\
\hline 2-BUTANONE & 78-93-3 & 20 & PPB & \\
\hline N-HEXANE & $110-54-3$ & 0 & PPB & \\
\hline 1,2-DICHLOROETHENE & $156-59-2$ & 0 & PPB & \\
\hline CHLOROFORM & $67-66-3$ & 0 & PPB & \\
\hline TETRAHYDROFURAN & $109-99-9$ & 0 & PPB & \\
\hline 1,1,1-TRICHLOROETHANE & $71-55-6$ & 0 & PPB & \\
\hline 1,2-DICHLOROETHANE & $107-06-2$ & 0 & PPB & \\
\hline BENZENE & $71-43-2$ & 0 & PPB & \\
\hline CARBON TETRACHLORIDE & $56-23-5$ & 8 & PPB & \\
\hline CYCLOHEXANE & $110-82-7$ & 0 & PPB & \\
\hline N-BUTANOL & $71-36-3$ & 0 & PPB & \\
\hline TRICHLOROETHENE & $79-01-6$ & 0 & PPB & \\
\hline 4-METHYL-2-PENTANONE & $108-10-1$ & 7 & PPB & \\
\hline TOLUENE & $108-88-3$ & 247 & PPB & \\
\hline 1,1,2-TRICHLOROETHANE & $79-00-5$ & 0 & PPB & \\
\hline TETRACHLOROETHENE & $127-18-4$ & 0 & PPB & \\
\hline CHLOROBENZENE & $108-90-7$ & 0 & PPB & \\
\hline ETHYLBENZENE & $100-41-4$ & 19 & PPB & \\
\hline M-XYLENE & $108-38-3$ & 55 & PPB & \\
\hline STYRENE & $100-42-5$ & 1 & PPB & \\
\hline O-XYLENE & $95-47-6$ & 14 & PPB & \\
\hline 1,1,2,2-TETRACHLORETHANE & $79-34-5$ & 0 & PPB & \\
\hline 1,3,5-TRIMETHYLBENZENE & $108-67-8$ & 17 & PPB & \\
\hline 1,2,4-TRIMETHYLBENZENE & $95-63-6$ & 1 & PPB & \\
\hline 1,3-DICHLOROBENZENE & $541-73-1$ & 0 & PPB & \\
\hline 1,2+DICHLOROBENZENE & $95-50-1$ & 0 & PPB & \\
\hline 1,4-DICHLOROBENZENE & $106-46-7$ & 0 & PPB & \\
\hline
\end{tabular}




\section{LIST OF COMPOUNDS TENTATIVELY IDENTIFIED WITH MASS SPECTRAL LIBRARY}

Sample: $\quad$ LFM \# 14

File: $\quad$ C:LHPCHEMI2LDATA171996_4.D

ID: FT6045_14.104 A

Compound

CAS\#

2-Propanol

000067-63-0

Ethane, 1,1,2-trichloro-1,2,2-trifluo

$000076-13-1$

Methane, dichloro-

$000075-09-2$

METHYL 1,2,3,11,12,12A-HEXAHYDRO-3-OX 060774-49-4

2-Pentanone, 4-methyl-

000108-10-1

Benzene, methyl-

000108-88-3

Benzene, ethyl-

$000100-41-4$

Benzene, 1,2-dimethyl-

000095-47-6

Styrene

$000100-42-5$

Benzaldehyde

000100-52-7

Benzene, 1,2,4-trimethyl-

000095-63-6 
File

$: C: \backslash$ HPCHEM $\backslash 1 \backslash D A T A \backslash 71996 \_5 . D$

Operator : MS

Acquired : 21 Mar $153 \quad 7: 34 \mathrm{pm}$ using AcqMethod CWC

Instrument : 5972 - In

Sample Name: FT 6045_14.103 A

Misc Info: 100 TOR $\bar{R}$

Vial Number: 1

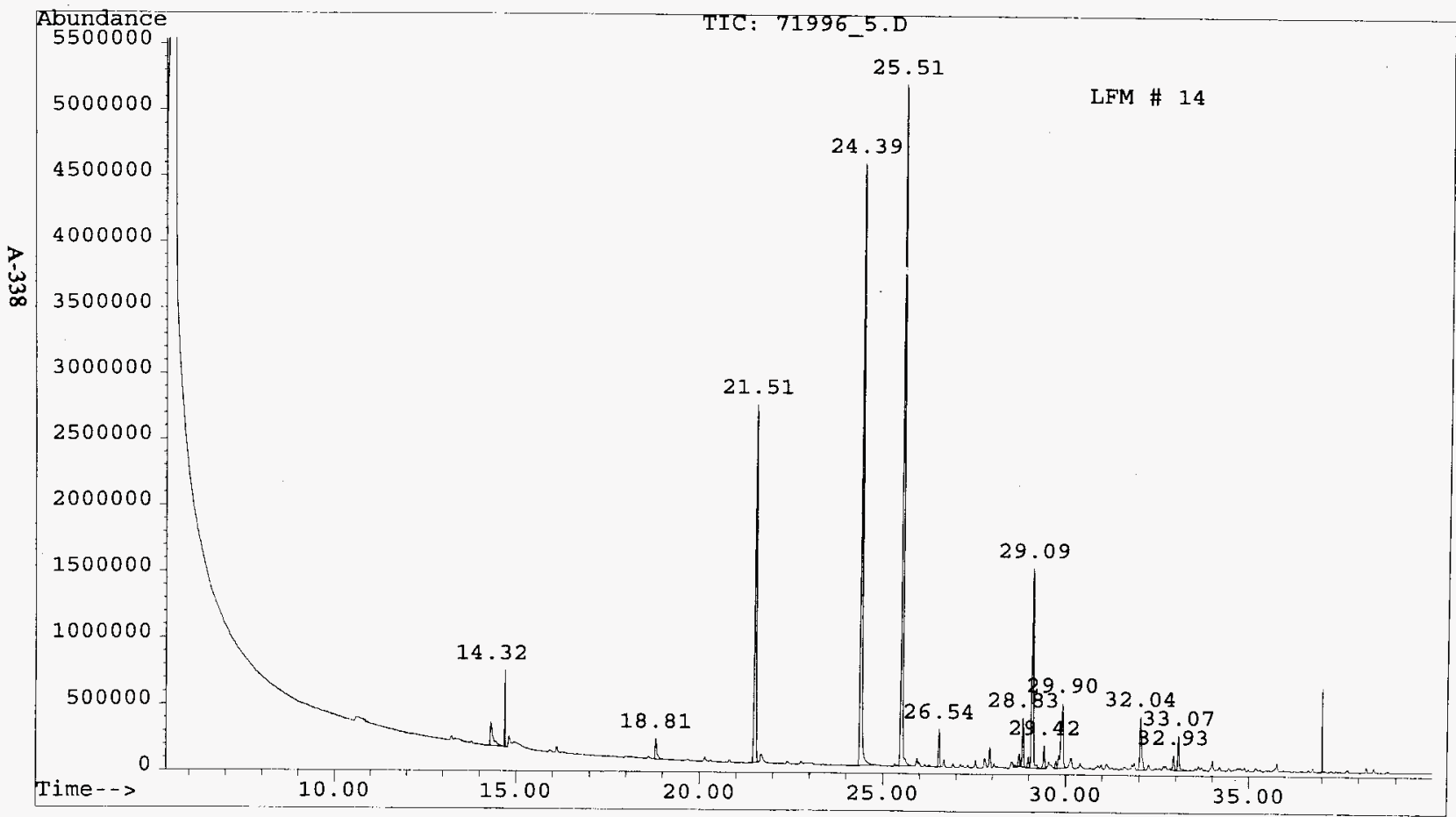


HNF-SD-WM-RPT-282 Rev. 0

CWC_REP.XLS

\section{ANALYSIS REPORT FOR CWC AIR SAMPLES}

File Name:

Analyst:

Analysis Date:

Method:

Sample Name:
71996_5.D

MS

\#VALUE!

CWC

FT 6045_14.103 A

\begin{tabular}{|c|c|c|c|c|}
\hline COMPOUND NAME & CAS \# & Concentration & Units & Comments \\
\hline ISO-BUTANE & $75-28-5$ & 0 & PPB & \\
\hline VINYL CHLORIDE & $75-01-4$ & 0 & PPB & \\
\hline ETHANOL & $64-17-5$ & 0 & PPB & \\
\hline ACETONE & $67-64-1$ & 7 & PPB & \\
\hline 2-PROPANOL & $67-63-0$ & 8 & PPB & \\
\hline 1,1-DICHLOROETHENE & $75-35-4$ & 0 & PPB & \\
\hline FREON 113 & $76-13-1$ & 0 & PPB & \\
\hline DICHLOROMETHANE & $75-09-2$ & 0 & PPB & \\
\hline 1,1-DICHLOROETHANE & $75-34-3$ & 0 & PPB & \\
\hline 2-BUTANONE & $78-93-3$ & 16 & PPB & \\
\hline N-HEXANE & $110-54-3$ & 0 & $\mathrm{PPB}$ & \\
\hline 1,2-DICHLOROETHENE & $156-59-2$ & 0 & PPB & \\
\hline CHLOROFORM & $67-66-3$ & 0 & PPB & \\
\hline TETRAHYDROFURAN & $109-99-9$ & 0 & PPB & \\
\hline 1,1,1-TRICHLOROETHANE & $71-55-6$ & 0 & PPB & \\
\hline 1,2-DICHLOROETHANE & $107-06-2$ & 0 & PPB & \\
\hline BENZENE & $71-43-2$ & 0 & PPB & \\
\hline CARBON TETRACHLORIDE & $56-23-5$ & 224 & PPB & \\
\hline CYCLOHEXANE & $110-82-7$ & 37 & PPB & \\
\hline N-BUTANOL & $71-36-3$ & 0 & $\overline{\mathrm{PPB}}$ & \\
\hline TRICHLOROETHENE & $79-01-6$ & 0 & PPB & \\
\hline 4-METHYL-2-PENTANONE & $108-10-1$ & 82 & PPB & \\
\hline TOLUENE & $108-88-3$ & 168 & PPB & \\
\hline 1,1,2-TRICHLOROETHANE & $79-00-5$ & 0 & PPB & \\
\hline TETRACHLOROETHENE & $127-18-4$ & 0 & PPB & \\
\hline CHLOROBENZENE & $108-90-7$ & 0 & PPB & \\
\hline ETHYLBENZENE & $100-41-4$ & 11 & PPB & \\
\hline M-XYLENE & $108-38-3$ & 44 & PPB & \\
\hline STYRENE & $100-42-5$ & 2 & PPB & \\
\hline O-XYLENE & $95-47-6$ & 12 & PPB & \\
\hline 1,1,2,2-TETRACHLORETHANE & $79-34-5$ & 0 & PPB & \\
\hline 1,3,5-TRIMETHYLBENZENE & $108-67-8$ & 8 & $\overline{\mathrm{PPB}}$ & \\
\hline 1,2,4-TRIMETHYLBENZENE & $95-63-6$ & 7 & PPB & \\
\hline 1,3-DICHLOROBENZENE & $541-73-1$ & 0 & PPB & \\
\hline 1,2-DICHLOROBENZENE & $95-50-1$ & 0 & PPB & \\
\hline 1,4-DICHLOROBENZENE & $106-46-7$ & 0 & PPB & \\
\hline
\end{tabular}




\section{LIST OF COMPOUNDS TENTATIVELY IDENTIFIED WITH MASS SPECTRAL LIBRARY}

Sample: $\quad$ LFM \# 14

File: $\quad$ C:UHPCHEMLWDATA171996_5.D

ID: $\quad$ FT6045_14.103 A

Compound

CAS\#

TRIDEUTEROACETONITRILE

$002206-26-0$

Methane, tetrachloro-

000056-23-5

2-Pentanone, 4-methyl-

000108-10-1

Benzene, methyl-

000108-88-3

(Z)-4,4-Dimethyl-2-pentenal

022597-47-3

Octane

000111-65-9

Cyclohexane, 1,4-dimethyl-

000589-90-2

Cyclohexane, ethyl-

001678-91-7

Benzene, ethyl-

$000100-41-4$

Benzene, 1,2-dimethyl-

000095-47-6

3-Heptanone

000106-35-4

Benzaldehyde

000100-52-7

Benzene, 1,2,3-trimethyl-

000526-73-8

Decane

000124-18-5

Benzene, 1,2,4-trimethyl-

000095-63-6

Undecane

001120-21-4

Benzene, 4-ethyl-1,2-dimethyl-

000934-80-5 
HNF-SD-WM-RPT-282 Rev. 0
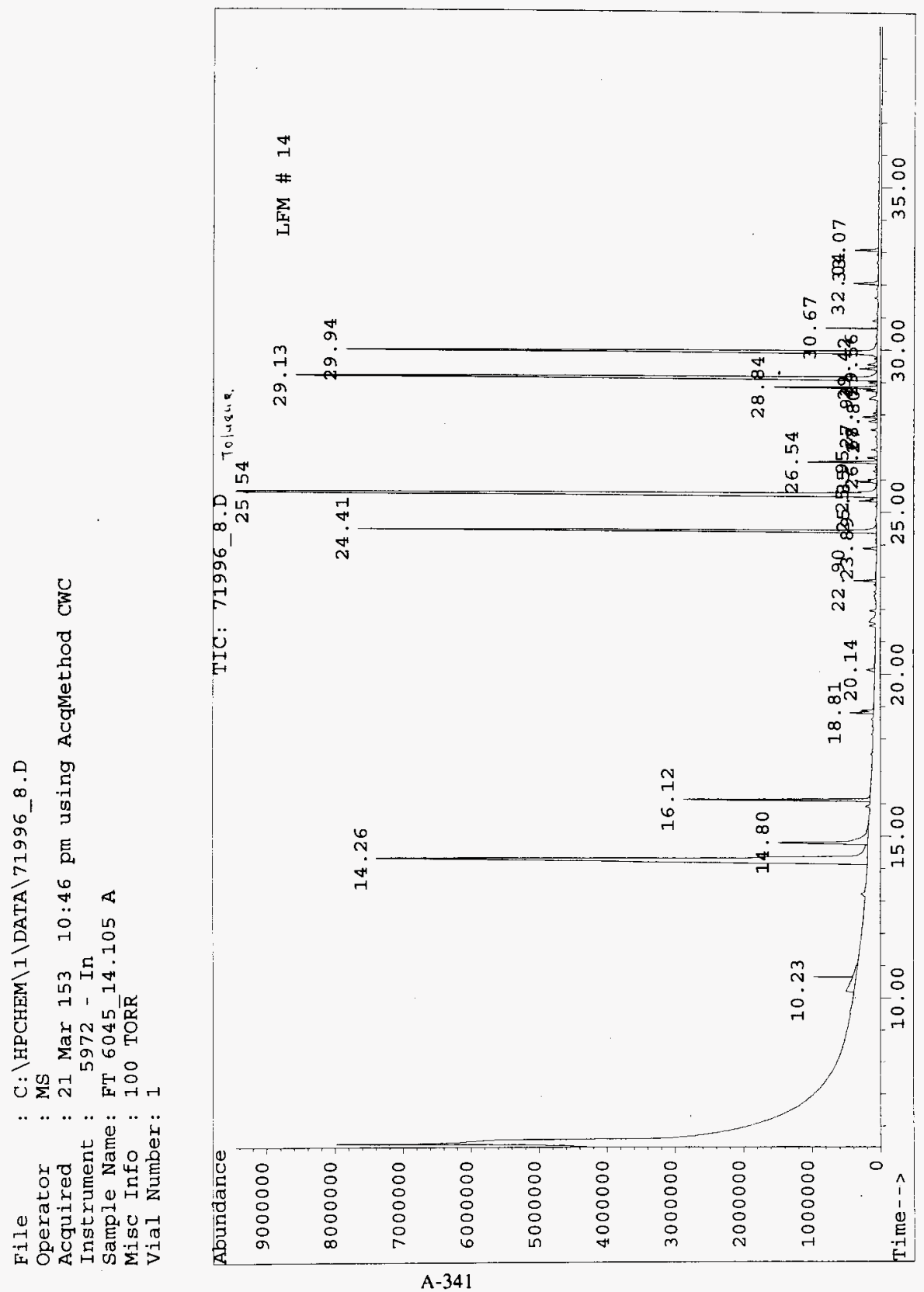
HNF-SD-WM-RPT-282 Rev. 0

CWC_REP.XLS

\title{
ANALYSIS REPORT FOR CWC AIR SAMPLES
}

File Name:

Analyst:

Analysis Date:

Method:

Sample Name:

\author{
71996_8.D \\ MS \\ \#VALUE! \\ CWC \\ FT 6045_14.105 A
}

\begin{tabular}{|c|c|c|c|c|}
\hline COMPOUND NAME & CAS \# & Concentration & Units & Comments \\
\hline ISO-BUTANE & $75-28-5$ & $\overline{0}$ & PPB & \\
\hline VINYL CHLORIDE & $75-01-4$ & 0 & PPB & \\
\hline ETHANOL & $64-17-5$ & 0 & PPB & \\
\hline ACETONE & $67-64-1$ & 61 & PPB & \\
\hline 2-PROPANOL & $67-63-0$ & 65 & PPB & \\
\hline 1,1-DICHLOROETHENE & $75-35-4$ & 0 & PPB & \\
\hline FREON 113 & $76-13-1$ & 0 & PPB & \\
\hline DICHLOROMETHANE & $75-09-2$ & 87 & PPB & \\
\hline 1,1-DICHLOROETHANE & $75-34-3$ & 0 & PPB & \\
\hline 2-BUTANONE & $78-93-3$ & 30 & PPB & \\
\hline N-HEXANE & $110-54-3$ & 5 & PPB & \\
\hline 1,2-DICHLOROETHENE & $156-59-2$ & 0 & PPB & \\
\hline CHLOROFORM & $67-66-3$ & 0 & PPB & \\
\hline TETRAHYDROFURAN & $109-99-9$ & 0 & PPB & \\
\hline 1,1,1-TRICHLOROETHANE & $71-55-6$ & 0 & PPB & \\
\hline 1,2-DICHLOROETHANE & $107-06-2$ & $\overline{0}$ & PPB & \\
\hline BENZENE & $71-43-2$ & 0 & PPB & \\
\hline CARBON TETRACHLORIDE & $56-23-5$ & 4 & PPB & \\
\hline CYCLOHEXANE & $110-82-7$ & 0 & PPB & \\
\hline N-BUTANOL & $71-36-3$ & 8 & PPB & \\
\hline TRICHLOROETHENE & $79-01-6$ & 0 & PPB & \\
\hline 4-METHYL-2-PENTANONE & $108-10-1$ & 147 & PPB & \\
\hline TOLUENE & $108-88-3$ & 507 & PPB & \\
\hline 1,1,2-TRICHLOROETHANE & $79-00-5$ & 0 & PPB & \\
\hline TETRACHLOROETHENE & $127-18-4$ & 0 & PPB & \\
\hline CHLOROBENZENE & $108-90-7$ & 0 & PPB & \\
\hline ETHYLBENZENE & $100-41-4$ & 42 & PPB & \\
\hline M-XYLENE & $108-38-3$ & 329 & PPB & \\
\hline STYRENE & $100-42-5$ & 6 & PPB & \\
\hline O-XYLENE & $95-47-6$ & 273 & PPB & \\
\hline 1,1,2,2-TETRACHLORETHANE & $79-34-5$ & 0 & PPB & \\
\hline 1,3,5-TRIMETHYLBENZENE & $108-67-8$ & 7 & PPB & \\
\hline 1,2,4-TRIMETHYLBENZENE & $95-63-6$ & 9 & PPB & \\
\hline 1,3-DICHLOROBENZENE & 541-73-1 & 0 & PPB & \\
\hline 1,2-DICHLOROBENZENE & $95-50-1$ & 0 & PPB & \\
\hline 1,4-DICHLOROBENZENE & $106-46-7$ & 0 & PPB & \\
\hline
\end{tabular}


HNF-SD-WM-RPT-282 Rev. 0

\section{LIST OF COMPOUNDS TENTATIVELY IDENTIFIED WITH MASS SPECTRAL LIBRARY}

Sample: $\quad$ LFM \# 14

File: $\quad$ C:LHPCHEMLLDATA171996_8.D

ID: FT6045_14.105 A

Compound

CAS\#

Glycidol

000556-52-5

2-Propanol

000067-63-0

Methane, dichloro-

000075-09-2

Hexane

000110-54-3

Heptane

000142-82-5

Cyclohexane, methyl-

000108-87-2

2-Pentanone, 4-methyl-

000108-10-1

Heptane, 2-methyl-

000592-27-8

Benzene, methyl-

000108-88-3

Cyclohexane, 1,3-dimethyl-, cis-

000638-04-0

Octane

000111-65-9

CYCLOHEXANE, 1,2-DIMETHYL-

002207-01-4

Cyclohexane, 1,4-dimethyl-

000589-90-2

Cyclohexane, ethyl-

001678-91-7

Cyclohexane, 1,1,3-trimethyl-

003073-66-3

Benzene, ethyl-

000100-41-4

Octane, 3-methyl-

002216-33-3

Benzene, 1,2-dimethyl-

000095-47-6 
HNF-SD-WM-RPT-282 Rev. 0

\section{LIST OF COMPOUNDS TENTATIVELY IDENTIFIED WITH MASS SPECTRAL LIBRARY}

Sample: $\quad$ LFM \# 14 (CONTINUED)

File:

C:LHPCHEM12IDATAI71996_8.D

ID: FT6045_14.105 A

Compound

CAS\#

3-Heptanone

000106-35-4

Benzene, 1-ethyl-4-methyl-

000622-96-8

Benzaldehyde

000100-52-7

Benzene, 1,2,4-trimethyl-

000095-63-6 


\section{File}

Operator

Acquired

Instrument

Sample Name: FT6045-14 103

Misc Info : 100 TORR

Vial Number: 1

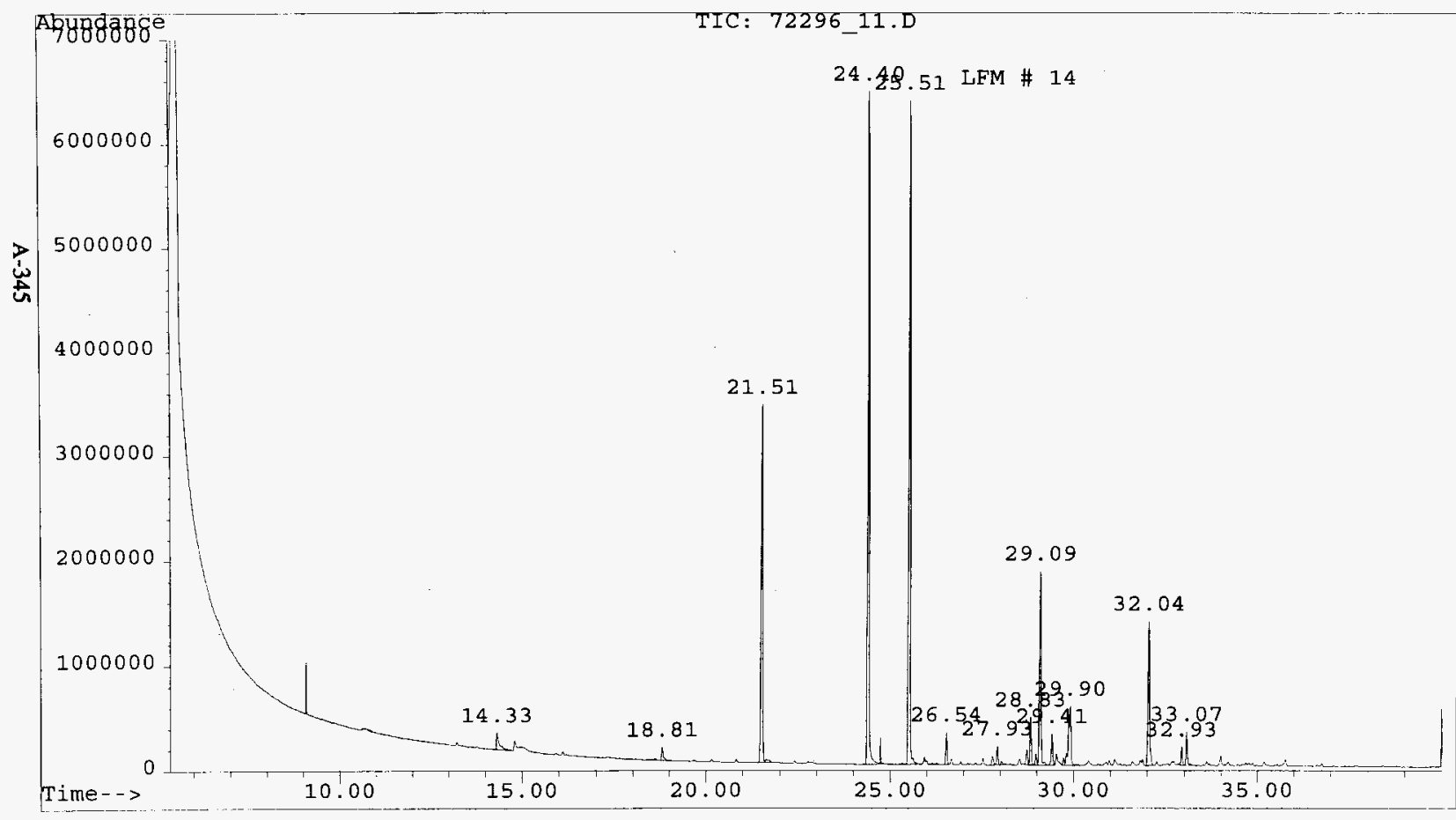


HNF-SD-WM-RPT-282 Rev. 0

CWC_REP.XLS

\section{ANALYSIS REPORT FOR CWC AIR SAMPLES}

File Name:

Analyst:

Analysis Date:

Method:

Sample Name:

\author{
72296_11.D \\ MS \\ \#VALUE! \\ CWC \\ FT6045-14.103B
}

\begin{tabular}{|c|c|c|c|c|}
\hline COMPOUND NAME & CAS \# & Concentration & Units & Comments \\
\hline ISO-BUTANE & $75-28-5$ & 0 & PPB & \\
\hline VINYL CHLORIDE & $75-01-4$ & 0 & PPB & \\
\hline ETHANOL & $64-17-5$ & 0 & PPB & \\
\hline ACETONE & $67-64-1$ & 7 & PPB & \\
\hline 2-PROPANOL & $67-63-0$ & 8 & PPB & \\
\hline 1,1-DICHLOROETHENE. & $75-35-4$ & 0 & PPB & \\
\hline FREON 113 & $76-13-1$ & 0 & PPB & \\
\hline DICHLOROMETHANE & $75-09-2$ & 0 & PPB & \\
\hline 1,1-DICHLOROETHANE & $75-34-3$ & 0 & PPB & \\
\hline 2-BUTANONE & $78-93-3$ & 14 & PPB & \\
\hline N-HEXANE & $110-54-3$ & 0 & PPB & \\
\hline 1,2-DICHLOROETHENE & $156-59-2$ & 0 & PPB & \\
\hline CHLOROFORM & $67-66-3$ & 0 & PPB & \\
\hline TETRAHYDROFURAN & $109-99-9$ & 0 & PPB & \\
\hline 1,1,1-TRICHLOROETHANE & $71-55-6$ & 0 & PPB & \\
\hline 1,2-DICHLOROETHANE & $107-06-2$ & 0 & PPB & \\
\hline BENZENE & $71-43-2$ & 0 & PPB & \\
\hline CARBON TETRACHLORIDE & $56-23-5$ & 288 & PPB & \\
\hline CYCLOHEXANE & $110-82-7$ & 47 & PPB & \\
\hline N-BUTANOL & $71-36-3$ & 0 & PPB & \\
\hline TRICHLOROETHENE & $79-01-6$ & 0 & PPB & \\
\hline 4-METHYL-2-PENTANONE & $108-10-1$ & 127 & PPB & \\
\hline TOLUENE & $108-88-3$ & 203 & $\mathrm{PPB}$ & \\
\hline 1,1,2-TRICHLOROETHANE & $79-00-5$ & 0 & PPB & \\
\hline TETRACHLOROETHENE & $127-18-4$ & 0 & PPB & \\
\hline CHLOROBENZENE & $108-90-7$ & 0 & PPB & \\
\hline ETHYLBENZENE & $100-41-4$ & 12 & PPB & \\
\hline M-XYLENE & 108-38-3 & 56 & PPB & \\
\hline STYRENE & $100-42-5$ & 2 & PPB & \\
\hline O-XYLENE & $95-47-6$ & 15 & PPB & \\
\hline 1,1,2,2-TETRACHLORETHANE & $79-34-5$ & 0 & PPB & \\
\hline 1,3,5-TRIMETHYLBENZENE & $108-67-8$ & 25 & PPB & \\
\hline 1,2,4-TRIMETHYLBENZENE & $95-63-6$ & 8 & PPB & \\
\hline 1,3-DICHLOROBENZENE & $541-73-1$ & 0 & PPB & \\
\hline 1,2-DICHLOROBENZENE & $95-50-1$ & 0 & PPB & \\
\hline 1,4-DICHLOROBENZENE & $106-46-7$ & 0 & PPB & \\
\hline
\end{tabular}


HNF-SD-WM-RPT-282 Rev. 0

\section{LIST OF COMPOUNDS TENTATIVELY IDENTIFIED WITH MASS SPECTRAL LIBRARY}

$\begin{array}{ll}\text { Sample: } & \text { LFM \# 14 } \\ \text { File: } & \text { C:UHPCHEMLLDATA172296_11.D } \\ \text { ID: } & \text { FT6045-14.103B }\end{array}$

Compound

CAS\#

Methane, tetrachloro-

000056-23-5

2-Pentanone, 4-methyl-

000108-10-1

Benzene, methyl-

000108-88-3

Cyclohexane, 1,3-dimethyl-, cis-

000638-04-0

Octane

000111-65-9

Cyclohexane, 1,4-dimethyl-

$000589-90-2$

Cyclohexane, ethyl-

001678-91-7

Benzene, ethyl-

$000100-41-4$

Benzene, 1,3-dimethyl-

000108-38-3

3-Heptanone

$000106-35-4$

Benzaldehyde

000100-52-7

Benzene, 1,3,5-trimethyl-

$000108-67-8$

Cyclohexane, 1,4-dimethyl-

$000589-90-2$

Decane

000124-18-5

Benzene, 1,2,3-trimethyl-

000526-73-8

Benzene, 1,2,4-trimethyl-

000095-63-6

3-.alpha.-Cumyl-1,3,4-oxadiazolidine- 060104-03-2 
HNF-SD-WM-RPT-282 Rev. 0

U

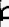

a

$r$
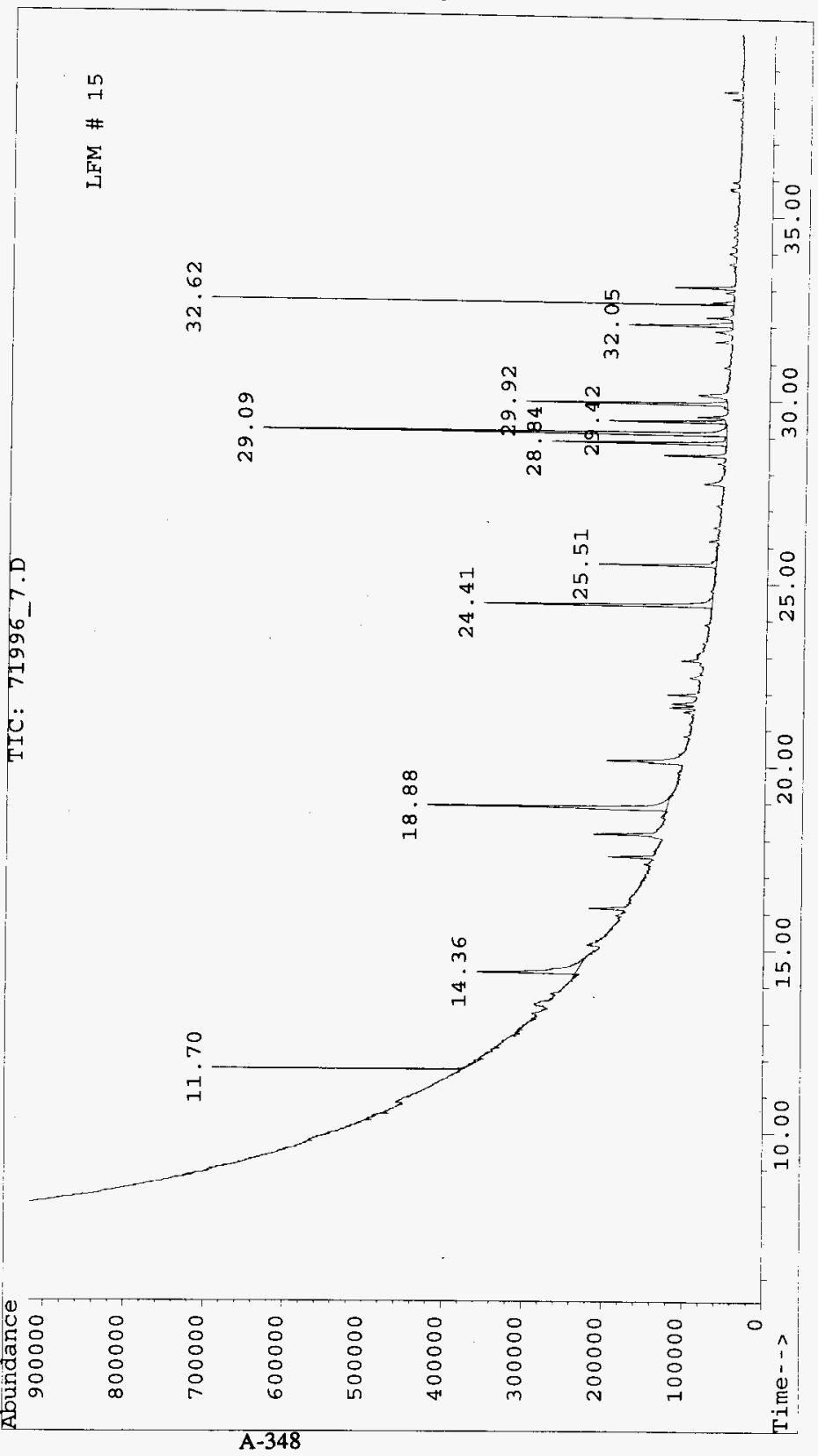

0 
HNF-SD-WM-RPT-282 Rev. 0

CWC_REP.XLS

\section{ANALYSIS REPORT FOR CWC AIR SAMPLES}

File Name:

Analyst:

Analysis Date:

Method:

Sample Name:
71996_7.D

MS

\#VALUE!

CWC

FT 6045_15.102 A

\begin{tabular}{|c|c|c|c|c|}
\hline COMPOUND NAME & CAS \# & Concentration & Units & Comments \\
\hline ISO-BUTANE & $75-28-5$ & 0 & PPB & \\
\hline VINYL CHLORIDE & $75-01-4$ & 0 & PPB & \\
\hline ETHANOL & $64-17-5$ & 0 & PPB & \\
\hline ACETONE & $67-64-1$ & 5 & PPB & \\
\hline 2-PROPANOL & $67-63-0$ & 0 & PPB & \\
\hline 1,1-DICHLOROETHENE & $75-35-4$ & 0 & PPB & \\
\hline FREON 113 & $76-13-1$ & 0 & PPB & \\
\hline DICHLOROMETHANE & $75-09-2$ & 0 & PPB & \\
\hline 1,1-DICHLOROETHANE & $75-34-3$ & 0 & PPB & \\
\hline 2-BUTANONE & $78-93-3$ & 0 & PPB & \\
\hline N-HEXANE & $110-54-3$ & 8 & PPB & \\
\hline 1,2-DICHLOROETHENE & $156-59-2$ & 0 & PPB & \\
\hline CHLOROFORM & $67-66-3$ & 0 & PPB & \\
\hline TETRAHYDROFURAN & $109-99-9$ & 0 & PPB & \\
\hline 1,1,1-TRICHLOROETHANE & $71-55-6$ & 0 & PPB & \\
\hline 1,2-DICHLOROETHANE & $107-06-2$ & 0 & PPB & \\
\hline BENZENE & $71-43-2$ & 0 & PPB & \\
\hline CARBON TETRACHLORIDE & $56-23-5$ & 0 & PPB & \\
\hline CYCLOHEXANE & $110-82-7$ & 0 & PPB & \\
\hline N-BUTANOL & $71-36-3$ & 0 & PPB & \\
\hline TRICHLOROETHENE & $79-01-6$ & 0 & PPB & \\
\hline 4-METHYL-2-PENTANONE & $108-10-1$ & 0 & PPB & \\
\hline TOLUENE & $108-88-3$ & 5 & PPB & \\
\hline 1,1,2-TRICHLOROETHANE & $79-00-5$ & 0 & PPB & \\
\hline TETRACHLOROETHENE & $127-18-4$ & 0 & PPB & \\
\hline CHLOROBENZENE & $108-90-7$ & 0 & PPB & \\
\hline ETHYLBENZENE & $100-41-4$ & 6 & PPB & \\
\hline M-XYLENE & $108-38-3$ & 17 & PPB & \\
\hline STYRENE & $100-42-5$ & 0 & PPB & \\
\hline O-XYLENE & $95-47-6$ & 7 & PPB & \\
\hline 1,1,2,2-TETRACHLORETHANE & $79-34-5$ & 0 & PPB & \\
\hline 1,3,5-TRIMETHYLBENZENE & $108-67-8$ & 3 & PPB & \\
\hline 1,2,4-TRIMETHYLBENZENE & $95-63-6$ & 2 & PPB & \\
\hline 1,3-DICHLOROBENZENE & $541-73-1$ & 0 & PPB & \\
\hline 1,2-DICHLOROBENZENE & $95-50-1$ & 0 & PPB & \\
\hline 1,4-DICHLOROBENZENE & $106-46-7$ & 0 & PPB & \\
\hline
\end{tabular}




\section{LIST OF COMPOUNDS TENTATIVELY IDENTIFIED WITH MASS SPECTRAL LIBRARY}

Sample: $\quad$ LFM \# 15

File: $\quad$ C:LHPCHEM2LDATA171996_7.D

ID: FT6045_15.102 A

Compound

CAS\#

Octanal

000124-13-0

2-Pentanone, 4-methyl-

000108-10-1

Benzene, methyl-

000108-88-3

Benzene, ethyl-

$000100-41-4$

XYLENE

001330-20-7

Benzene, 1,3-dimethyl-

000108-38-3

Benzaldehyde

000100-52-7

Benzene, 1,3,5-trimethyl-

$000108-67-8$ 
File

operator

: D : \CWC \SAMPLES $\backslash 42396 \_8 . D$

Acquired

: MS

Instrument

Misc Info : delta $p=50$ torr

Vial Number: 1

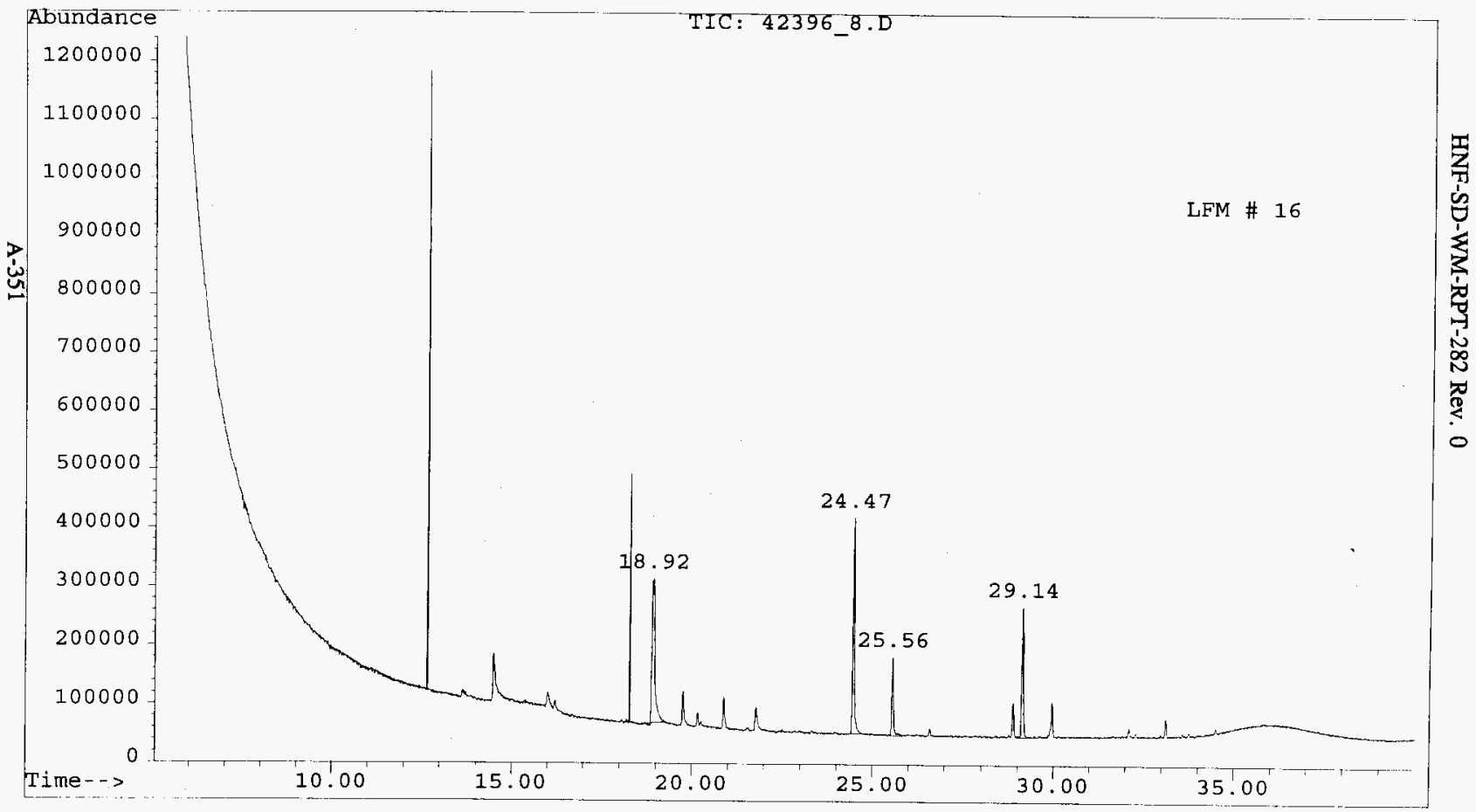


HNF-SD-WM-RPT-282 Rev. 0

CWC_REP.XLS

\section{ANALYSIS REPORT FOR CWC AIR SAMPLES}

File Name:

Analyst:

42396_8.D

Analysis Date:

MS

Method:

04/23/96

Sample Name:

CWC

FT6045-16.09B

\begin{tabular}{|c|c|c|c|c|}
\hline COMPOUND NAME & CAS\# & Concentration & Units & Comments \\
\hline ISO-BUTANE & $75-28-5$ & 0 & $\overline{\mathrm{PPB}}$ & \\
\hline VINYL CHLORIDE & $75-01-4$ & 0 & PPB & \\
\hline ETHANOL & $64-17-5$ & 0 & $\overline{\mathrm{PPB}}$ & \\
\hline ACETONE & $67-64-1$ & 3 & PPB & \\
\hline 2-PROPANOL & $67-63-0$ & 0 & PPB & \\
\hline 1,1-DICHLOROETHENE & $75-35-4$ & 0 & PPB & \\
\hline FREON 113 & $76-13-1$ & 0 & PPB & \\
\hline DICHLOROMETHANE & $75-09-2$ & 0 & PPB & \\
\hline 1,1-DICHLOROETHANE & $75-34-3$ & 0 & PPB & \\
\hline 2-BUTANONE & $78-93-3$ & 32 & PPB & \\
\hline N-HEXANE & $110-54-3$ & 6 & PPB & \\
\hline 1,2-DICHLOROETHENE & $156-59-2$ & 0 & PPB & \\
\hline CHLOROFORM & $67-66-3$ & 2 & PPB & \\
\hline TETRAHYDROFURAN & $109-99-9$ & 0 & PPB & \\
\hline 1,1,1-TRICHLOROETHANE & $71-55-6$ & 3 & $\overline{\text { PPB }}$ & \\
\hline 1,2-DICHLOROETHANE & $107-06-2$ & 0 & PPB & \\
\hline BENZENE & $71-43-2$ & 0 & PPB & \\
\hline CARBON TETRACHLORIDE & $56-23-5$ & 0 & PPB & \\
\hline CYCLOHEXANE & $110-82-7$ & 0 & $\overline{\mathrm{PPB}}$ & \\
\hline N-BUTANOL & $71-36-3$ & 0 & $\overline{P P B}$ & \\
\hline TRICHLOROETHENE & $79-01-6$ & $\overline{0}$ & PPB & \\
\hline 4-METHYL-2-PENTANONE & $108-10-1$ & 8 & PPB & \\
\hline TOLUENE & $108-88-3$ & 4 & PPB & \\
\hline 1,1,2-TRICHLOROETHANE & $79-00-5$ & 0 & PPB & \\
\hline TETRACHLOROETHENE & $127-18-4$ & 0 & PPB & \\
\hline CHLOROBENZENE & $108-90-7$ & 0 & PPB & \\
\hline ETHYLBENZENE & $100-41-4$ & 2 & $\overline{\text { PPB }}$ & \\
\hline M-XYLENE & $108-38-3$ & 7 & PPB & \\
\hline STYRENE & $100-42-5$ & $\overline{0}$ & $\overline{P P B}$ & \\
\hline O-XYLENE & $95-47-6$ & 2 & PPB & \\
\hline 1,1,2,2-TETRACHLORETHANE & $79-34-5$ & 0 & PPB & \\
\hline 1,3,5-TRIMETHYLBENZENE & $108-67-8$ & 0 & PPB & \\
\hline 1,2,4-TRIMETHYLBENZENE & $95-63-6$ & 0 & PPB & \\
\hline 1,3-DICHLOROBENZENE & $541-73-1$ & 0 & PPB & \\
\hline 1,2-DICHLOROBENZENE & $95-50-1$ & 0 & $\overline{\mathrm{PPB}}$ & \\
\hline 1,4-DICHLOROBENZENE & $106-46-7$ & 0 & PPB & \\
\hline
\end{tabular}




\section{LIST OF COMPOUNDS TENTATIVELY WENTIFIED WITH MASS SPECTRAL LIBRARY}

Sample: $\quad$ LFM \# 16

File: $\quad$ D:ICWC $142396 \_8 . D$

Sample Name: FT6045-16.09B

Compound

CAS\#

Methane, dichloro-

000075-09-2

Ethane, 1,1,1-trichloro-

$000071-55-6$

2-Pentanone, 4-methyl-

000108-10-1

Benzene, methyl-

000108-88-3

Benzene, ethyl-

000100-41-4

Benzene, 1,4-dimethyl-

$000106-42-3$

Benzene, 1,2-dimethyl-

000095-47-6

Benzaldehyde

00100-52-7

Benzene, 1,3,5-trimethyl-

000108-67-8 
HNF-SD-WM-RPT-282 Rev. 0

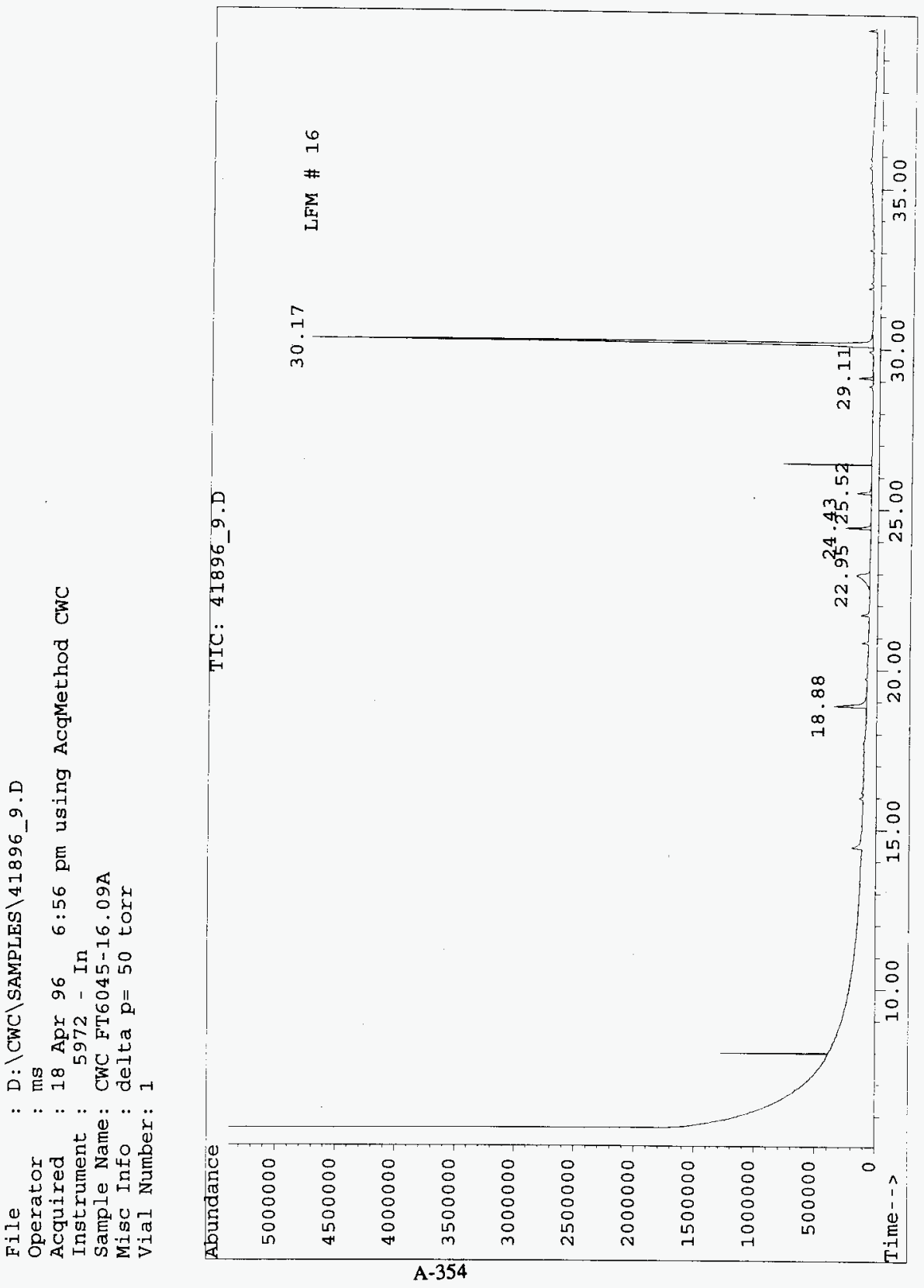


HNF-SD-WM-RPT-282 Rev. 0

CWC_REP.XLS

\section{ANALYSIS REPORT FOR CWC AIR SAMPLES}

File Name:

Analyst:

Analysis Date:

Method:

Sample Name:
41896_9.D

$\mathrm{ms}$

04/18/96

CWC

CWC FT6045-16.09A

\begin{tabular}{|c|c|c|c|c|}
\hline COMPOUND NAME & CAS \# & Concentration & Units & Comments \\
\hline ISO-BUTANE & $75-28-5$ & $\overline{0}$ & $\overline{P P B}$ & \\
\hline VINYL CHLORIDE & $75-01-4$ & 0 & PPB & \\
\hline ETHANOL & $64-17-5$ & 0 & PPB & \\
\hline ACETONE & $67-64-1$ & 4 & $\overline{\text { PPB }}$ & \\
\hline 2-PROPANOL & $67-63-0$ & 0 & PPB & \\
\hline 1,1-DICHLOROETHENE & $75-35-4$ & 0 & PPB & \\
\hline FREON 113 & $76-13-1$ & 0 & PPB & \\
\hline DICHLOROMETHANE & $75-09-2$ & 0 & PPB & \\
\hline 1,1-DICHLOROETHANE & $75-34-3$ & 0 & PPB & \\
\hline 2-BUTANONE & $78-93-3$ & 37 & PPB & \\
\hline N-HEXANE & $110-54-3$ & 4 & PPB & \\
\hline 1,2-DICHLOROETHENE & $156-59-2$ & 0 & PPB & \\
\hline CHLOROFORM & $67-66-3$ & 0 & PPB & \\
\hline TETRAHYDROFURAN & $109-99-9$ & 0 & PPB & \\
\hline 1,1,1-TRICHLOROETHANE & $71-55-6$ & 2 & PPB & \\
\hline 1,2-DICHLOROETHANE & $107-06-2$ & 0 & PPB & \\
\hline BENZENE & $71-43-2$ & 0 & PPB & \\
\hline CARBON TETRACHLORIDE & $56-23-5$ & 0 & PPB & \\
\hline CYCLOHEXANE & $110-82-7$ & 0 & PPB & \\
\hline N-BUTANOL & $71-36-3$ & 0 & PPB & \\
\hline TRICHLOROETHENE & $79-01-6$ & 0 & PPB & \\
\hline 4-METHYL-2-PENTANONE & $108-10-1$ & 0 & PPB & \\
\hline TOLUENE & $108-88-3$ & 4 & PPB & \\
\hline 1,1,2-TRICHLOROETHANE & $79-00-5$ & 0 & PPB & \\
\hline TETRACHLOROETHENE & $127-18-4$ & 0 & PPB & \\
\hline CHLOROBENZENE & $108-90-7$ & 0 & PPB & \\
\hline ETHYLBENZENE & $100-41-4$ & 3 & PPB & \\
\hline M-XYLENE & $108-38-3$ & 3 & PPB & \\
\hline STYRENE & $100-42-5$ & 0 & PPB & \\
\hline O-XYLENE & $95-47-6$ & 0 & PPB & \\
\hline 1,1,2,2-TETRACHLORETHANE & $79-34-5$ & 0 & PPB & \\
\hline 1,3,5-TRIMETHYLBENZENE & $108-67-8$ & 0 & $\overline{\mathrm{PPB}}$ & \\
\hline 1,2,4-TRIMETHYLBENZENE & $95-63-6$ & 0 & $\overline{\mathrm{PPB}}$ & \\
\hline 1,3-DICHLOROBENZENE & $541-73-1$ & 0 & $\overline{\mathrm{PPB}}$ & \\
\hline 1,2-DICHLOROBENZENE & $95-50-1$ & 0 & PPB & \\
\hline 1,4-DICHLOROBENZENE & $106-46-7$ & 0 & PPB & \\
\hline
\end{tabular}


HNF-SD-WM-RPT-282 Rev. 0

\section{LIST OF COMPOUNDS TENTATIVELY IDENTIFIED WITH MASS SPECTRAL LIBRARY}

$\begin{array}{ll}\text { Sample: } & \text { LFM \# 16 } \\ \text { File: } & \text { D:ICWC\41896_9.D } \\ \text { ID } & \text { CWC FT6045-16.09A }\end{array}$

Compound

CAS\#

2-Butanone

000078-93-3

Ethane, 1,1,1-trichloro-

$000071-55-6$

1-Butanol

000071-36-3

Ethane, 1,1-diethoxy-

00105-57-7

2-Pentanone, 4-methyl-

000108-10-1

Benzene, methyl-

000108-88-3

Benzene, ethyl-

$000100-41-4$

Benzene, 1,2-dimethyl-

$000095-47-6$

Benzene, 1,4-dimethyl-

000106-42-3

Ethanol, 2-butoxy-

000111-76-2

Benzaldehyde

000100-52-7

Benzene, 1,3,5-trimethyl-

000108-67-8 
File

: D : \CWC \SAMPLES $\backslash 42296-9 . \mathrm{D}$

operator

Acquired

: 22 Apr 96

$4: 18 \mathrm{pm}$ using AcqMethod CWC

Instrument : 5972 - In

Sample Name: FT6045-17.05A

Misc Info : delta $p=50$ torr

Vial Number: 1
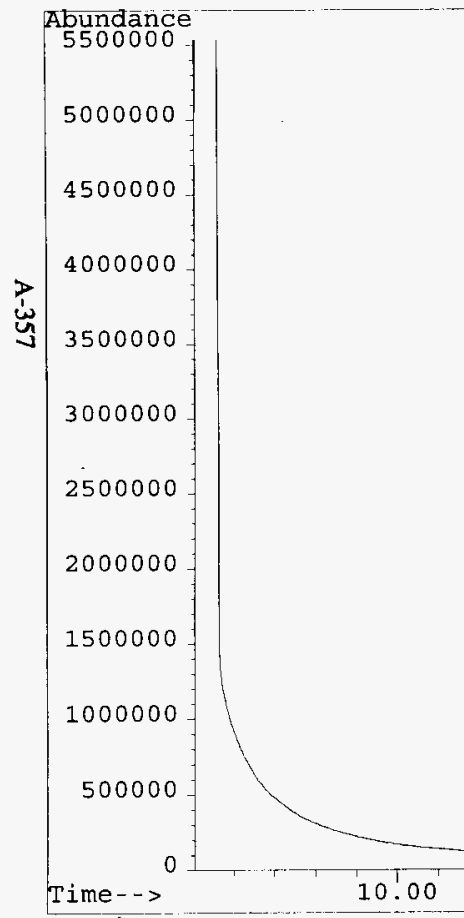

TIC: $42296-9 . \mathrm{D}$

Time-->

10.00

15.00

20.00

24.53

24.43

32.05

LFM \# 17

$\frac{1}{25.00}$ 
HNF-SD-WM-RPT-282 Rev. 0

CWC_REP.XLS

\section{ANALYSIS REPORT FOR CWC AIR SAMPLES}

File Name:

Analyst:

Analysis Date:

Method:

Sample Name:
42296-9.D

$\mathrm{ms}$

04/22/96

CWC

FT6045-17.05A

\begin{tabular}{|c|c|c|c|c|}
\hline COMPOUND NAME & CAS \# & Concentration & Units & Comments \\
\hline ISO-BUTANE & $75-28-5$ & $\overline{0}$ & PPB & \\
\hline VINYL CHLORIDE & $75-01-4$ & 0 & PPB & \\
\hline ETHANOL & $64-17-5$ & 0 & PPB & \\
\hline ACETONE & $67-64-1$ & 0 & PPB & \\
\hline 2-PROPANOL & $67-63-0$ & 0 & PPB & \\
\hline 1,1-DICHLOROETHENE & $75-35-4$ & $\overline{0}$ & PPB & \\
\hline FREON 113 & $76-13-1$ & 0 & PPB & \\
\hline DICHLOROMETHANE & $75-09-2$ & 0 & PPB & \\
\hline 1,1-DICHLOROETHANE & $75-34-3$ & 0 & PPB & \\
\hline 2-BUTANONE & $78-93-3$ & 0 & PPB & \\
\hline N-HEXANE & $110-54-3$ & 0 & PPB & \\
\hline 1,2-DICHLOROETHENE & $156-59-2$ & 0 & PPB & \\
\hline CHLOROFORM & $67-66-3$ & 0 & PPB & \\
\hline TETRAHYDROFURAN & $109-99-9$ & 0 & PPB & \\
\hline 1,1,1-TRICHLOROETHANE & $71-55-6$ & 0 & $\overline{\mathrm{PPB}}$ & \\
\hline 1,2-DICHLOROETHANE & $107-06-2$ & 0 & PPB & \\
\hline BENZENE & $71-43-2$ & 0 & PPB & \\
\hline CARBON TETRACHLORIDE & $56-23-5$ & 0 & PPB & \\
\hline CYCLOHEXANE & $110-82-7$ & 0 & PPB & \\
\hline N-BUTANOL & $71-36-3$ & 5 & PPB & \\
\hline TRICHLOROETHENE & $79-01-6$ & 0 & PPB & \\
\hline 4-METHYL-2-PENTANONE & $108-10-1$ & 0 & PPB & \\
\hline TOLUENE & $108-88-3$ & 14 & PPB & \\
\hline 1,1,2-TRICHLOROETHANE & $79-00-5$ & 0 & PPB & \\
\hline TETRACHLOROETHENE & $127-18-4$ & 0 & PPB & \\
\hline CHLOROBENZENE & $108-90-7$ & 0 & PPB & \\
\hline ETHYLBENZENE & $100-41-4$ & 0 & PPB & \\
\hline M-XYLENE & $108-38-3$ & 0 & PPB & \\
\hline STYRENE & $100-42-5$ & 0 & PPB & \\
\hline O-XYLENE & $95-47-6$ & 0 & PPB & \\
\hline 1,1,2,2-TETRACHLORETHANE & $79-34-5$ & 0 & PPB & \\
\hline 1,3,5-TRIMETHYLBENZENE & $108-67-8$ & 10 & PPB & \\
\hline 1,2,4-TRIMETHYLBENZENE & $95-63-6$ & 2 & PPB & \\
\hline 1,3-DICHLOROBENZENE & $541-73-1$ & 0 & PPB & \\
\hline 1,2-DICHLOROBENZENE & $95-50-1$ & 0 & PPB & \\
\hline 1,4-DICHLOROBENZENE & $106-46-7$ & 0 & PPB & \\
\hline
\end{tabular}




\section{LIST OF COMPOUNDS TENTATIVELY IDENTIFIED WITH MASS SPECTRAL LIBRARY}

Sample: $\quad$ LFM \# 17

Sample Name: FT6045-17.05A

Search Libraries: $\quad$ C:DATABASEIWILEY138.L Minimum Quality: 70

C:IDATABASEINBS54K.L Minimum Quality: 70

Unknown Spectrum: Apex

Integration Params: VOA.E

Pk\# RT Area\% Library/ID $\quad$ Ref\# CAS\# Qual

$221.681 .66 \mathrm{C}:$ DATABASEIWILEY138.L

1-Butanol

$116550000071-36-378$

524.432 .44 C:LATABASEIWILEY138.L

2-Pentanone, 4-methyl-

$118019000108-10-191$

$625.52 \quad 4.65$ C:DATABASEIWILEY138.L Benzene, methyl-

$117430000108-88-395$

729.110 .23 C:DATABASEIWILEY138.L Benzene, 1,3-dimethyl- $\quad 118579$ 000108-38-3 91

$930.1979 .36 \mathrm{C}:$ :DATABASEIWILEY138.L Ethanol, 2-butoxy$119793000111-76-283$

$1032.056 .81 \mathrm{C}:$ :DATABASEIWILEY138.L Benzaldehyde

$118546000100-52-797$

$1233.080 .79 \mathrm{C}:$ DATABASEIWILEY138.L Benzene, 1,3,5-trimethyl- $\quad 120037$ 000108-67-895

$1334.470 .13 \mathrm{C}:$ DATABASEIWILEY138.L Benzene, 1,4-dichloro$123085000106-46-797$

$1435.210 .78 \mathrm{C}:$ :DATABASEIWILEY138.L Ethanone, 1-phenyl- 
File

Operator

Acquired

Instrument :

Name: ft6045-18.03a

Misc Info : rerun, delta $p=50$ torr

Vial Number: 1

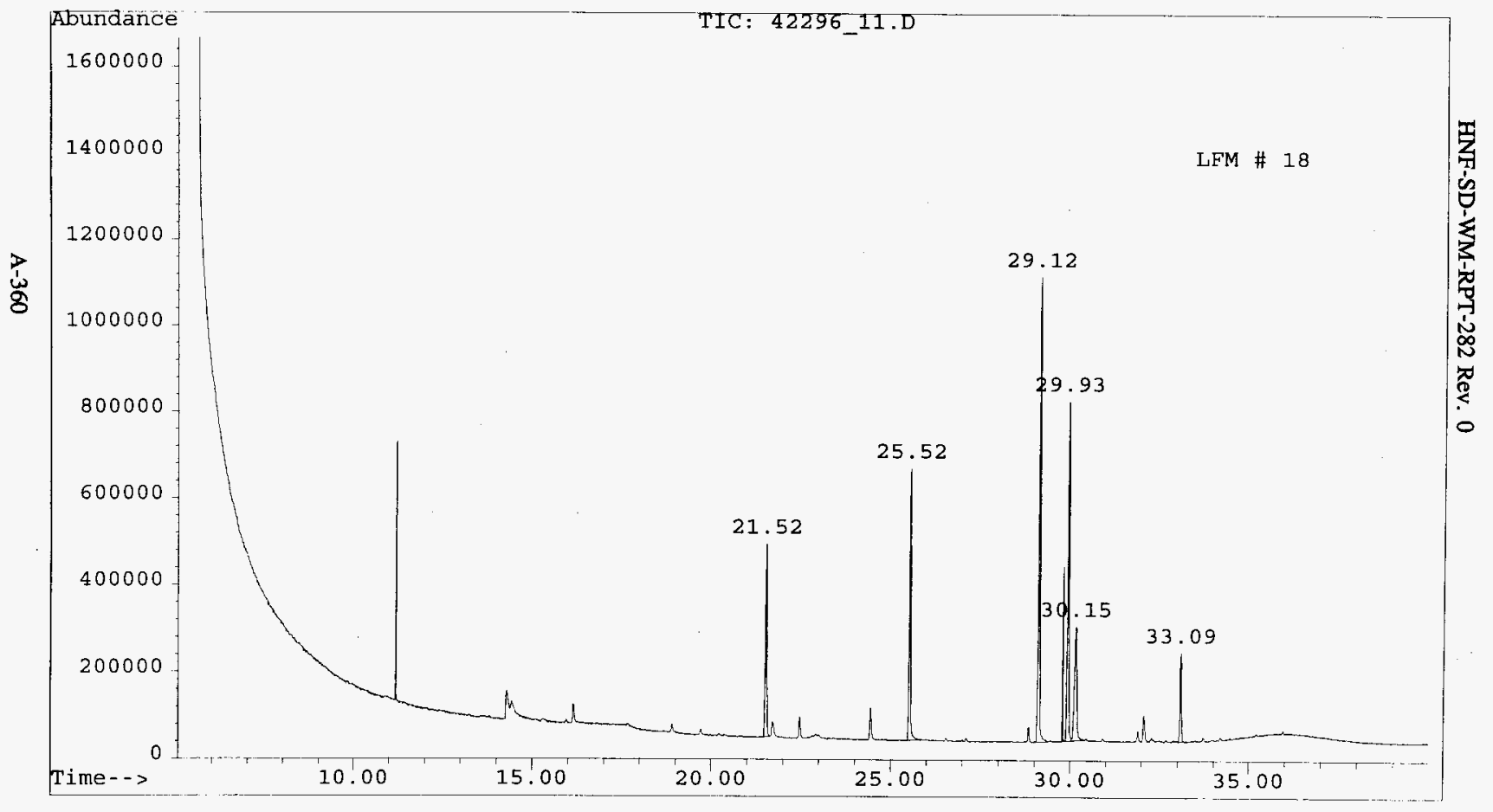


HNF-SD-WM-RPT-282 Rev. 0

CWC_REP.XLS

\section{ANALYSIS REPORT FOR CWC AIR SAMPLES}

File Name:

Analyst:

Analysis Date:

Method:

Sample Name:

\author{
42296_11.D \\ $\mathrm{ms}$ \\ 04/22/96 \\ CWC
}

ft6045-18.03a

\begin{tabular}{|c|c|c|c|c|}
\hline COMPOUND NAME & CAS \# & Concentration & Units & Comments \\
\hline ISO-BUTANE & $75-28-5$ & 0 & PPB & \\
\hline VINYL CHLORIDE & $75-01-4$ & 0 & $\mathrm{PPB}$ & \\
\hline ETHANOL & $64-17-5$ & 0 & $\mathrm{PPB}$ & \\
\hline ACETONE & $67-64-1$ & 0 & PPB & \\
\hline 2-PROPANOL & $67-63-0$ & 0 & PPB & \\
\hline 1,1-DICHLOROETHENE & $75-35-4$ & 0 & PPB & \\
\hline FREON 113 & $76-13-1$ & 0 & PPB & \\
\hline DICHLOROMETHANE & $75-09-2$ & $\overline{0}$ & PPB & \\
\hline 1,1-DICHLOROETHANE & $75-34-3$ & 0 & PPB & \\
\hline 2-BUTANONE & $78-93-3$ & 0 & PPB & \\
\hline N-HEXANE & $110-54-3$ & 0 & PPB & \\
\hline 1,2-DICHLOROETHENE & $156-59-2$ & 0 & PPB & \\
\hline CHLOROFORM & $67-66-3$ & 0 & PPB & \\
\hline TETRAHYDROFURAN & $109-99-9$ & 0 & PPB & \\
\hline 1,1,1-TRICHLOROETHANE & $71-55-6$ & 0 & PPB & \\
\hline 1,2-DICHLOROETHANE & $107-06-2$ & 0 & PPB & \\
\hline BENZENE & $71-43-2$ & 0 & PPB & \\
\hline CARBON TETRACHLORIDE & $56-23-5$ & 21 & PPB & \\
\hline CYCLOHEXANE & $110-82-7$ & 12. & PPB & \\
\hline N-BUTANOL & $71-36-3$ & 10 & PPB & \\
\hline TRICHLOROETHENE & $79-01-6$ & 0 & $\mathrm{PPB}$ & \\
\hline 4-METHYL-2-PENTANONE & $108-10-1$ & 0 & PPB & \\
\hline TOLUENE & $108-88-3$ & 20 & PPB & \\
\hline 1,1,2-TRICHLOROETHANE & $79-00-5$ & 0 & PPB & \\
\hline TETRACHLOROETHENE & $127-18-4$ & 0 & PPB & \\
\hline CHLOROBENZENE & $108-90-7$ & 0 & PPB & \\
\hline ETHYLBENZENE & $100-41-4$ & 1 & PPB & \\
\hline M-XYLENE & $108-38-3$ & 28 & PPB & \\
\hline STYRENE & $100-42-5$ & 0 & PPB & \\
\hline O-XYLENE & $95-47-6$ & 23 & PPB & \\
\hline 1,1,2,2-TETRACHLORETHANE & $79-34-5$ & 0 & PPB & \\
\hline 1,3,5-TRIMETHYLBENZENE & $108-67-8$ & 0 & PPB & \\
\hline 1,2,4-TRIMETHYLBENZENE & $95-63-6$ & 5 & PPB & \\
\hline 1,3-DICHLOROBENZENE & $541-73-1$ & 0 & PPB & \\
\hline 1,2-DICHLOROBENZENE & $95-50-1$ & 0 & PPB & \\
\hline 1,4-DICHLOROBENZENE & $106-46-7$ & 0 & PPB & \\
\hline
\end{tabular}


HNF-SD-WM-RPT-282 Rev. 0

\section{LIST OF COMPOUNDS TENTATIVELY IDENTIFIED WITH MASS SPECTRAL LIBRARY}

Sample: $\quad$ LFM \# 18

Sample Name: ft6045-18.03a

Search Libraries: C:IDATABASEIWILEY138.L Minimum Quality: 70

C:DATABASELNBS54K.L Minimum Quality: 70

Unknown Spectrum: Apex

Integration Params: VOA.E

Pk\# RT Area\% Library/DD Ref\# CAS\# Qual

$4 \quad 16.15 \quad 1.18 \mathrm{C}:$ IDATABASEIWILEY138.L Methane, dichloro-

116807 000075-09-2 95

721.72 1.32 C:LATABASEIWILEY138.L 1-Butanol

$116552000071-36-372$

$924.441 .82 \mathrm{C}:$ IDATABASEIWILEY138.L 2-Pentanone, 4-methyl-

118019 000108-10-1 83

1025.5314 .02 C:DATABASEIWILEY138.L Benzene, methyl-

$117430000108-88-395$

$1128850.90 \mathrm{C}:$ DDATABASEIWILEY138.L Benzene, ethyl-

$118560000100-41-494$

1229.1223 .67 C: DATABASEIWILEY138.L Benzene, 1,2-dimethyl-

$118564000095-47-697$

$1429.9316 .70 \mathrm{C}:$ DATABASEIWILEY138.L Benzene, 1,2-dimethyl-

$118564000095-47-697$

1530.159 .64 C:IDATABASEIWILEY138.L Ethanol, 2-butoxy-

$3837000111-76-283$

1732.061 .51 C:DATABASEIWILEY138.L Benzaldehyde $118546000100-52-796$

$1833.094 .23 \mathrm{C}:$ DATABASEIWILEY138.L Benzene, 1,2,3-trimethyl-

120022 000526-73-895 
File

: D : \CWC \SAMPLES \42296-7.D

Operator

: ms

Acquired

Instrument

Sample Name: FT6045-19.02

Misc Info : delta $\mathrm{p}=50$ torr

vial Number: 1

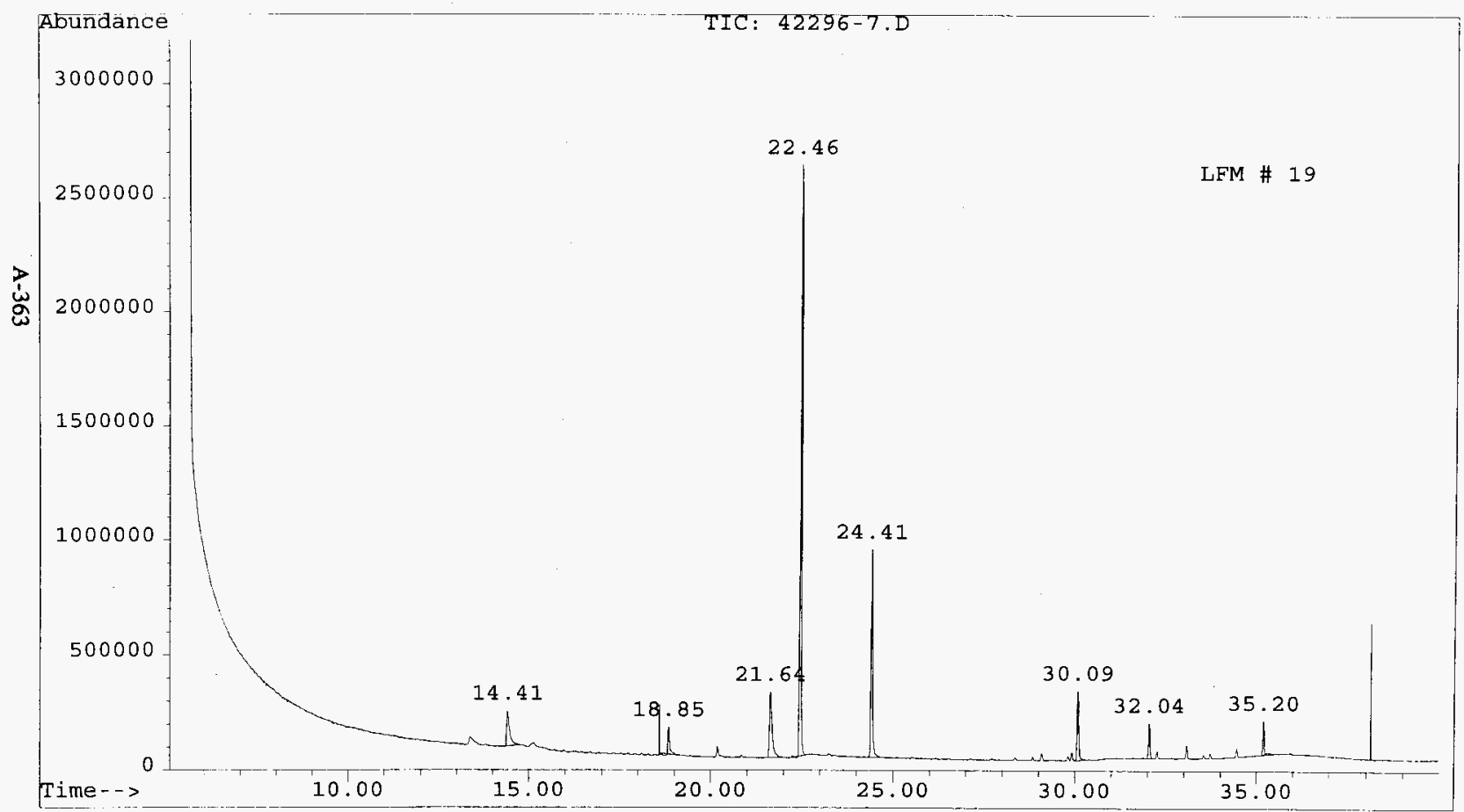


HNF-SD-WM-RPT-282 Rev. 0

CWC_REP.XLS

\section{ANALYSIS REPORT FOR CWC AIR SAMPLES}

File Name:

Analyst:

Analysis Date:

Method:

Sample Name:
42296-7.D

$\mathrm{ms}$

04/22/96

CWC

FT6045-19.02B

\begin{tabular}{|c|c|c|c|c|}
\hline COMPOUND NAME & CAS \# & Concentration & Units & Comments \\
\hline ISO-BUTANE & $75-28-5$ & 0 & PPB & \\
\hline VINYL CHLORIDE & $75-01-4$ & 0 & PPB & \\
\hline ETHANOL & $64-17-5$ & 6 & PPB & \\
\hline ACETONE & $67-64-1$ & 7 & PPB & \\
\hline 2-PROPANOL & $67-63-0$ & 0 & PPB & \\
\hline 1,1-DICHLOROETHENE & $75-35-4$ & 0 & $\mathrm{PPB}$ & \\
\hline FREON 113 & $76-13-1$ & 0 & PPB & \\
\hline DICHLOROMETHANE & $75-09-2$ & 0 & PPB & \\
\hline 1,1-DICHLOROETHANE & $75-34-3$ & 0 & PPB & \\
\hline 2-BUTANONE & $78-93-3$ & 13 & PPB & \\
\hline N-HEXANE & $110-54-3$ & 0 & PPB & \\
\hline 1,2-DICHLOROETHENE & $156-59-2$ & 0 & PPB & \\
\hline CHLOROFORM & $67-66-3$ & $\overline{0}$ & $\mathrm{PPB}$ & \\
\hline TETRAHYDROFURAN & $109-99-9$ & 0 & $\overline{\mathrm{PPB}}$ & \\
\hline 1,1,1-TRICHLOROETHANE & $71-55-6$ & 0 & PPB & \\
\hline 1,2-DICHLOROETHANE & $107-06-2$ & 0 & PPB & \\
\hline BENZENE & $71-43-2$ & 0 & PPB & \\
\hline CARBON TETRACHLORIDE & $56-23-5$ & 0 & PPB & \\
\hline CYCLOHEXANE & $110-82-7$ & 0 & PPB & \\
\hline N-BUTANOL & $71-36-3$ & 20 & PPB & \\
\hline TRICHLOROETHENE & $79-01-6$ & 0 & PPB & \\
\hline 4-METHYL-2-PENTANONE & $108-10-1$ & 16 & PPB & \\
\hline TOLUENE & $108-88-3$ & 0 & PPB & \\
\hline 1,1,2-TRICHLOROETHANE & $79-00-5$ & 0 & PPB & \\
\hline TETRACHLOROETHENE & $127-18-4$ & 0 & PPB & \\
\hline CHLOROBENZENE & $108-90-7$ & 0 & PPB & \\
\hline ETHYLBENZENE & $100-41-4$ & 0 & PPB & \\
\hline M-XYLENE & $108-38-3$ & 0 & PPB & \\
\hline STYRENE & $100-42-5$ & 0 & PPB & \\
\hline O-XYLENE & $95-47-6$ & 0 & PPB & \\
\hline 1,1,2,2-TETRACHLORETHANE & $79-34-5$ & 0 & PPB & \\
\hline 1,3,5-TRIMETHYLBENZENE & $108-67-8$ & 2 & PPB & \\
\hline 1,2,4-TRIMETHYLBENZENE & $95-63-6$ & 1 & PPB & \\
\hline 1,3-DICHLOROBENZENE & $541-73-1$ & 0 & PPB & \\
\hline 1,2-DICHLOROBENZENE & $95-50-1$ & 0 & PPB & \\
\hline 1,4-DICHLOROBENZENE & $106-46-7$ & 0 & PPB & \\
\hline
\end{tabular}




\section{LIST OF COMPOUNDS TENTATIVELY DENTIFIED WITH MASS SPECTRAL LIBRARY}

Sample: $\quad$ LFM \# 19

Sample Name: FT6045-19.02B

Search Libraries: $\quad$ C:LDATABASEIWILEY138.L Minimum Quality: 70

C:DATABASEINBS54K.L Minimum Quality: 70

Unknown Spectrum: Apex

Integration Params: VOA.E

Pk\# RT Area\% L Library/ID Ref\# CAS\# Qual

620.18 1.14 C:DATABASEIWILEY138.L

Furan, tetrahydro- $\quad 116444$ 000109-99-9 80

721.649 .07 C:DATABASEIWILEY138.L 1-Butanol

$116556000071-36-391$

822.4640 .86 C:LATABASEIWILEY138.L 2-Propanol, 1-methoxy-

$47000107-98-272$

924.40 16.10 C:DATABASEIWILEY138.L

2-Pentanone, 4-methyl-

$118019000108-10-191$

$1028.84 \quad 0.25$ C:DATABASE $\mid$ WILEY138.L Benzene, ethyl- $\quad 118561000100-41-490$

1129.090 .59 C:DATABASElWILEY138.L Benzene, 1,3-dimethyl-

$118579000108-38-395$

1229.820 .37 C:IDATABASEIWILEY138.L Styrene

$118481000100-42-594$

$1329.920 .56 \mathrm{C}:$ DATABASE WWILEY138.L Benzene, 1,4-dimethyl-

$118587000106-42-393$

1430.095 .62 C:IDATABASEIWILEY138.L Ethanol, 2-butoxy-

$119793000111-76-272$

$15 \quad 32.05 \quad 2.54$ C:LATABASEIWILEY138.L Benzaldehyde

$118545000100-52-796$ 
HNF-SD-WM-RPT-282 Rev. 0

Sample $\quad$ LFM \# 19 (continued)

Pk\# RT Area\% Library/ID Ref\# CAS\# Qual

$1632.260 .51 \mathrm{C}:$ DATABASEIWILEY138.L

Benzene, 1,3,5-trimethyl- $\quad 120037$ 000108-67-8 90

1733.07 1.00 C:LDATABASEIWILEY138.L

Benzene, 1,3,5-trimethyl- $\quad 120037$ 000108-67-8 95

1833.540 .35 C:LDATABASEIWILEY138.L

Benzene, 1,4-dichloro- $\quad 123085$ 000106-46-7 95

$1933.730 .40 \mathrm{C}$ :DATABASEIWILEY138.L

Benzene, 1,4-dichloro-

$123085000106-46-796$

$2034.460 .61 \mathrm{C}:$ DATABASEIWILEY138.L

Benzene, 1,2-dichloro- 11151 000095-50-1 97

$21 \quad 35.20 \quad 2.37$ C:LATABASEIWLEY138.L

Ethanone, 1-phenyl-

$119989000098-86-297$ 


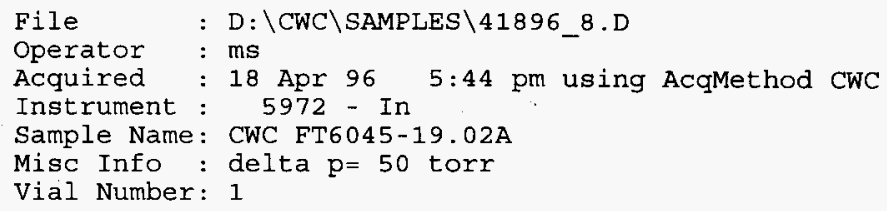

Vial Number: 1

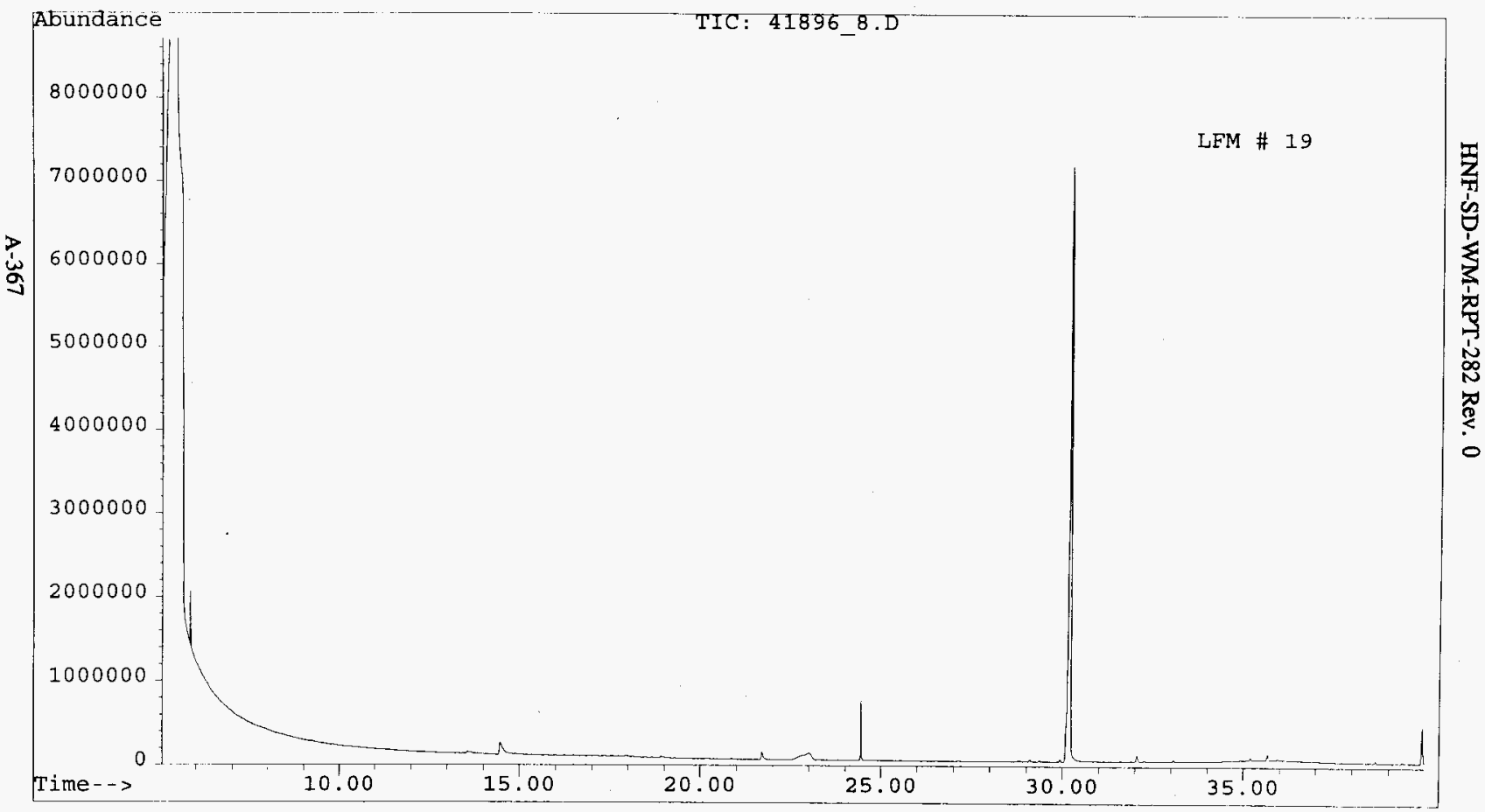




\begin{tabular}{|c|c|c|c|c|}
\hline & gdd & 0 & $L-9 t-90 L$ & 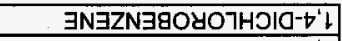 \\
\hline & 8dd & 0 & $1-0 s-96$ & 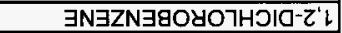 \\
\hline & gdd & 0 & $L-\varepsilon L-l \nabla G$ & $\exists N \exists Z N \exists g O y 07 \mathrm{HOIO- \varepsilon ^{ \prime } \iota}$ \\
\hline & gdd & 0 & $9-89-96$ & $\exists N \exists Z N \exists B 7 \lambda H \perp \exists W I Y \perp \nabla^{\prime} Z^{\prime} \downarrow$ \\
\hline & gdd & 1 & $8-\angle 9-801$ & 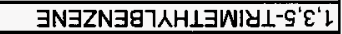 \\
\hline & gdd & 0 & $\mathrm{G}-\triangleright \varepsilon-6 L$ & $\exists N \forall H \perp \exists y 07 H O \forall \& \perp \exists \perp-Z^{\prime} Z^{\prime} ! '\llcorner$ \\
\hline & gdd & 0 & $9-2 b-96$ & $\exists N \exists 7 \wedge \times-0$ \\
\hline & gdd & 0 & s-Zt-0OL & $\exists N \exists y \lambda \perp S$ \\
\hline & 9dd & 0 & $\varepsilon-8 \varepsilon-80 \mathrm{l}$ & $\exists \mathrm{N} \exists 7 \mathrm{XX}-\mathrm{W}$ \\
\hline & 8dd & 0 & $t-L-00 L$ & $\exists \mathrm{N} \exists Z \mathrm{~N} \exists \mathrm{g} \backslash \mathrm{H} \mathrm{H} \perp \exists$ \\
\hline & gdd & 0 & $L-06-801$ & $\exists N \exists Z N \exists B O У O 7 H O$ \\
\hline & gdd & 0 & $t-8 l-\angle Z l$ & $\exists N \exists H \perp \exists 0$ ㅇำ \\
\hline & gdd & 0 & $9-00-6 L$ & $\exists N \forall H \perp \exists O \forall O 7 H O I Y \perp-Z^{\prime} L^{\prime} L$ \\
\hline & gdd & 0 & $\varepsilon-88-80 \mathrm{l}$ & $\exists \mathrm{N} \exists \cap 7 \mathrm{I}$ \\
\hline & gdd & 0 & $L-0 L-80 L$ & $\exists N O N \forall \perp N \exists d-Z-7 \lambda H \perp \exists W-t$ \\
\hline & gdd & 0 & $9-10-6 L$ & $\exists N \exists H \perp \exists O Y O 7 \mathrm{HOI} \perp$ \\
\hline & Gdd & $\underline{g}$ & $\varepsilon-9 \varepsilon-L L$ & TON $\forall \perp \cap 9-N$ \\
\hline & 8dd & 0 & $L-28-0 L L$ & $\exists N \forall \times \exists$ ヨดาว人ว \\
\hline & gdd & 0 & s- $\varepsilon ट-99$ & 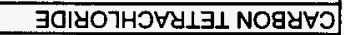 \\
\hline & gdd & 0 & $z-\varepsilon+l L$ & $\exists N \exists Z N \exists G$ \\
\hline & gdd & 0 & $z-90-\angle O L$ & $\exists N \forall H \perp \exists O Y 07 \mathrm{HOI}-\tau^{\prime \prime}$ \\
\hline & Edd & 0 & $9-G 9-L L$ & $\exists N \forall H \perp \exists O Y 07 H O I Y \perp-L^{\prime} L^{\prime} l$ \\
\hline & gdd & 0 & $6-66-60 l$ & 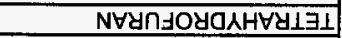 \\
\hline & gdd & 0 & $\varepsilon-99-\angle 9$ & WYOJOYOTHO \\
\hline & gdd & 0 & $z-6 s-9 s L$ & $\exists N \exists H \perp \exists O Y O 7 H \supset 10-Z^{\prime} \downarrow$ \\
\hline & 8dd & 0 & $\varepsilon-t S-0 \downarrow l$ & $\exists \mathrm{N} \forall \times \exists \mathrm{H}^{-\mathrm{N}}$ \\
\hline & 8dd & 0 & $\varepsilon-\varepsilon 6-8 L$ & $\exists N O N \forall \perp \cap Q-Z$ \\
\hline & 8dd & 0 & $\varepsilon-\downarrow \varepsilon-s L$ & $\exists N \forall H \perp \exists O Y O 7 H O I 0-\downarrow !$ \\
\hline & gdd & 0 & $z-60-S L$ & $\exists \mathrm{N} \forall \mathrm{H} \perp \exists$ WOYOTHOIO \\
\hline & gdd & 0 & $L-\varepsilon L-9 L$ & عนเ NOتYJ \\
\hline & gdd & 0 & $\nabla-S \varepsilon-S L$ & $\exists N \exists H \perp \exists O Y 07 \mathrm{HOIO-l' \downarrow )}$ \\
\hline & Edd & 0 & $0-\varepsilon 9-\angle 9$ & 7ONVdOQdd-Z \\
\hline & gdd & 6 & $1-+9-\angle 9$ & בNOLIOV \\
\hline & gdd & 0 & $S=\angle l-t 9$ & $70 N \forall H \perp \exists$ \\
\hline & gdd & 0 & $t-10-s L$ & 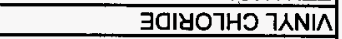 \\
\hline & 8dd & 0 & $S-8 Z-S L$ & $\exists N \forall \perp \cap 9-O S$ \\
\hline squeumos & syun & 4o!̣มquesuos & \# S\&J & ヨWVN aNNOdWOS \\
\hline
\end{tabular}

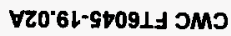

OMO

$96 / 8 \mathrm{~L} / \mathrm{t} \mathrm{O}$

sw

व.8-968L
:aweN әृdues

:poupaw

:әңе s! sরאeur

7Sאןeur

:aureN 키버 
HNF-SD-WM-RPT-282 Rev. 0

\section{LIST OF COMPOUNDS TENTATIVELY IDENTIFIED WITH MASS SPECTRAL LIBRARY}

Sample: $\quad$ LFM \# 19

Sample Name: CWC FT6045-19.02A

Search Libraries: C:IDATABASEIWILEY138.L Minimum Quality: 70

C:LDATABASEINBS54K.L Minimum Quality: 70

Unknown Spectrum: Apex

Integration Params: VOA.E

Pk\# RT Area\% Library/ID Ref\# CAS\# Qual

$521.71 \quad 1.21 \mathrm{C}:$ DATABASEIWILEY138.L 1-Butanol 116551 000071-36-386

623.014 .96 C:DATABASEIWILEY138.L Ethane, 1,1-diethoxy-

$119799000105-57-778$

$724.441 .69 \mathrm{C}:$ IDATABASEIWILEY138.L 2-Pentanone, 4-methyl-

118023 000108-10-1 72

$829.110 .29 \mathrm{C}:$ DATABASEIWILEY138.L Benzene, 1,4-dimethyl-

$118587000106-42-393$

$1029.93 \quad 0.25$ C:IDATABASEIWILEY138.L Benzene, 1,2-dimethyl-

I18564 000095-47-690

1130.1982 .12 C:DATABASEIWILEY 138.L Ethanol, 2-butoxy-

$119793000111-76-283$

$1232.05 \quad 0.67 \mathrm{C}$.DATABASEIWILEY138.L Benzaldehyde $118545000100-52-796$

1635.210 .41 C:IDATABASEIWILEY138.L Ethanone, 1-phenyl-

$119986000098-86-294$ 
File

Operator

Acquired

Instrument

Sample Name: CWC FT6045-20.01A

Misc Info : delta $p=50$ torr

Vial Number: 1

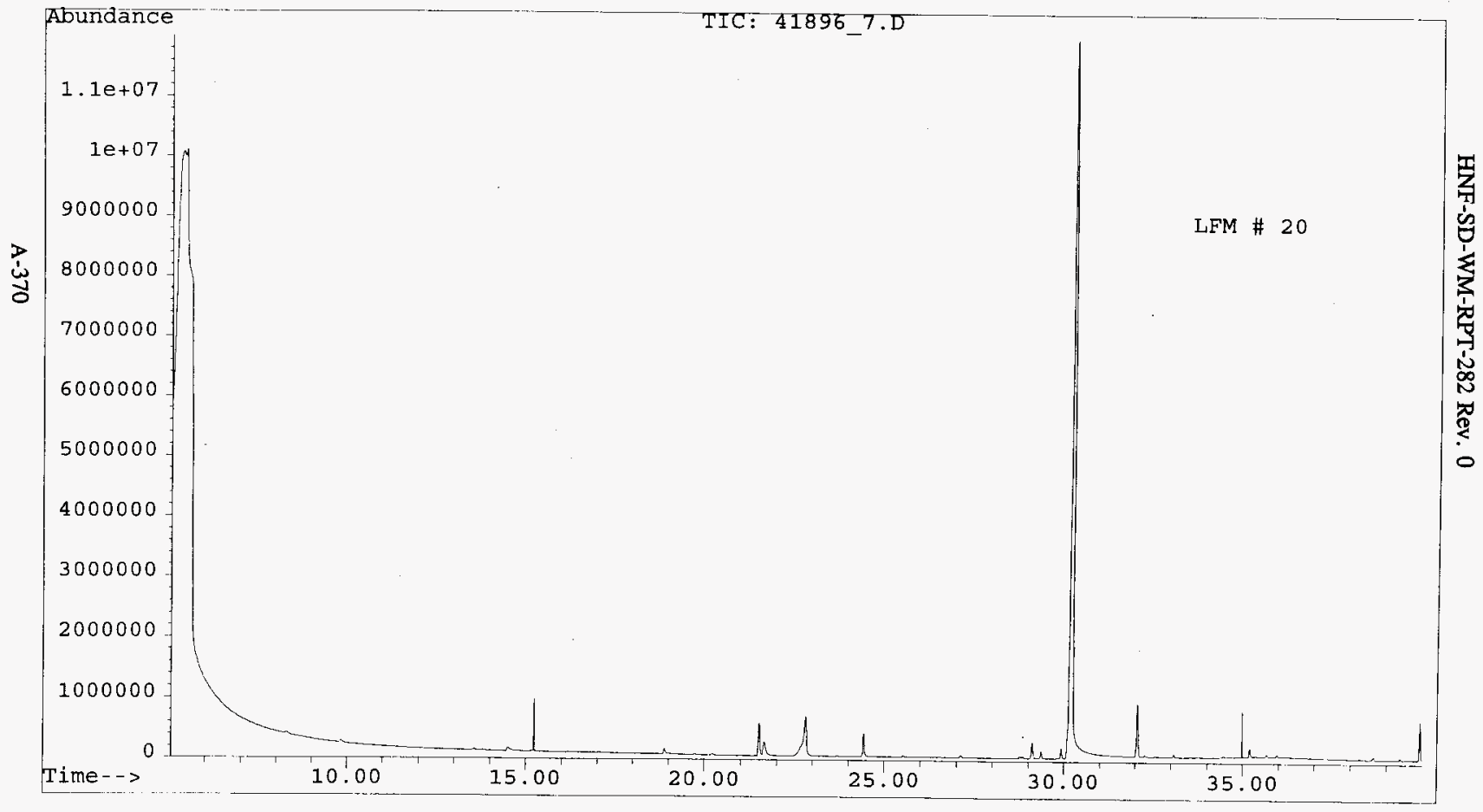


HNF-SD-WM-RPT-282 Rev. 0

CWC_REP.XLS

\section{ANALYSIS REPORT FOR CWC AIR SAMPLES}

File Name:

Analyst:

Analysis Date:

Method:

Sample Name:

\author{
41896_7.D \\ $\mathrm{ms}$ \\ 04/18/96 \\ CWC \\ CWC FT6045-20.01A
}

\begin{tabular}{|c|c|c|c|c|}
\hline COMPOUND NAME & CAS \# & Concentration & Units & Comments \\
\hline ISO-BUTANE & $75-28-5$ & 0 & PPB & \\
\hline VINYL CHLORIDE & $75-01-4$ & 0 & PPB & \\
\hline ETHANOL & $64-17-5$ & 0 & PPB & \\
\hline ACETONE & $67-64-1$ & 0 & PPB & \\
\hline 2-PROPANOL & $67-63-0$ & 0 & PPB & \\
\hline 1,1-DICHLOROETHENE & $75-35-4$ & 0 & PPB & \\
\hline FREON 113 & $76-13-1$ & 0 & $\mathrm{PPB}$ & \\
\hline DICHLOROMETHANE & $75-09-2$ & 0 & PPB & \\
\hline 1,1-DICHLOROETHANE & $75-34-3$ & 0 & PPB & \\
\hline 2-BUTANONE & $78-93-3$ & 1 & PPB & \\
\hline N-HEXANE & $110-54-3$ & 0 & PPB & \\
\hline 1,2-DICHLOROETHENE & $156-59-2$ & 0 & PPB & \\
\hline CHLOROFORM & $67-66-3$ & 0 & PPB & \\
\hline TETRAHYDROFURAN & $109-99-9$ & $\overline{0}$ & PPB & \\
\hline 1,1,1-TRICHLOROETHANE & $71-55-6$ & 0 & PPB & \\
\hline 1,2-DICHLOROETHANE & $107-06-2$ & 0 & PPB & \\
\hline BENZENE & $71-43-2$ & 0 & PPB & \\
\hline CARBON TETRACHLORIDE & $56-23-5$ & 0 & PPB & \\
\hline CYCLOHEXANE & $110-82-7$ & 2 & PPB & \\
\hline N-BUTANOL & $71-36-3$ & 2 & PPB & \\
\hline TRICHLOROETHENE & $79-01-6$ & 0 & PPB & \\
\hline 4-METHYL-2-PENTANONE & $108-10-1$ & 1 & PPB & \\
\hline TOLUENE & $108-88-3$ & 0 & PPB & \\
\hline 1,1,2-TRICHLOROETHANE & $79-00-5$ & 0 & PPB & \\
\hline TETRACHLOROETHENE & $127-18-4$ & 0 & PPB & \\
\hline CHLOROBENZENE & $108-90-7$ & 0 & $\overline{\mathrm{PPB}}$ & \\
\hline ETHYLBENZENE & $100-41-4$ & 0 & PPB & \\
\hline M-XYLENE & $108-38-3$ & 1 & PPB & \\
\hline STYRENE & $100-42-5$ & 0 & PPB & \\
\hline O-XYLENE & $95-47-6$ & 0 & PPB & \\
\hline 1,1,2,2-TETRACHLORETHANE & $79-34-5$ & 0 & PPB & \\
\hline 1,3,5-TRIMETHYLBENZENE & $108-67-8$ & 1 & PPB & \\
\hline 1,2,4-TRIMETHYLBENZENE & $95-63-6$ & 0 & PPB & \\
\hline 1,3-DICHLOROBENZENE & 541-73-1 & 0 & PPB & \\
\hline 1,2-DICHLOROBENZENE & $95-50-1$ & 0 & PPB & \\
\hline 1,4-DICHLOROBENZENE & $106-46-7$ & 0 & PPB & \\
\hline
\end{tabular}




\section{LIST OF COMPOUNDS TENTATIVELY IDENTIFIED WITH MASS SPECTRAL LIBRARY}

Sample: $\quad$ LFM \# 20

Sample Name: CWC FT6045-20.01A

Search Libraries: $\quad$ C:LDATABASEIWILEY138.L Minimum Quality: 70

C:IDATABASEINBS54K.L Minimum Quality: 70

Unknown Spectrum: Apex

Integration Params: VOA.E

Pk\# RT Area\% Library/ID Ref\# CAS\# Qual

921.522 .03 C:DATABASEIWILEY138.L Cyclohexane

$116928000110-82-787$

1021.661 .57 C:DATABASEIWILEY138.L 1-Butanol

$116556000071-36-390$

$1122.825 .63 \mathrm{C}:$ DATABASEIWILEY 138.L 2-Propanol, 1-methoxy-

$347000107-98-272$

$1224.421 .32 \mathrm{C}:$ DATABASEIWILEY 138.L 2-Pentanone, 4-methyl-

$118023000108-10-187$

$\begin{array}{lll}13 & 25.53 & 0.09 \\ C\end{array}$ Benzene, methyl-

117432 000108-88-391

$1628.850 .11 \mathrm{C}:$ DATABASEIWILEY138.L Benzene, ethyl-

$118558000100-41-490$

$1729.120 .97 \mathrm{C}:$ DATABASE\WILEY138.L Benzene, 1,3-dimethyl-

$118578000108-38-397$

1929.93 0.55 C:LDATABASEIWILEY138.L Benzene, 1,3-dimethyl-

$118576000108-38-394$

2030.2179 .13 C:DATABASEIWILEY138.L Ethanol, 2-butoxy-

$119793000111-76-283$ 
HNF-SD-WM-RPT-282 Rev. 0

Sample: $\quad$ LFM $\# \mathbf{2 0}$ (continued)

Pk\# RT Area\% Library/ID Ref\# CAS\# Qual

2232.053 .01 C:DATABASEIWILEY138.L

Benzaldehyde $\quad 118546000100-52-797$

$2433.080 .21 \mathrm{C}:$ DATABASEIWILEY138.L

Benzene, 1,2,4-trimethyl- $\quad 120030$ 000095-63-691

2634.47 0.1 I C:IDATABASEIWLEY138.L

Benzene, 1,2-dichloro- 11151 000095-50-1 95

$28 \quad 35.21 \quad 0.48$ C:LDATABASEIWILEY138.L Ethanone, 1-phenyl-

$119988000098-86-296$

3035.670 .17 C:IDATABASEIWILEY138.L

Benzenemethanol, alpha,, alpha.-dime 121923 000617-94-7 90 
File

Operator

$\mathrm{D}: \backslash \mathrm{CWC} \backslash S A M P L E S \backslash 41996 \_10 . \mathrm{D}$

Acquired

Instrument

19 Apr $96 \quad 3: 33$ pm using AcqMethod CWC

Misc Info: delta $p=50$ torr

Vial Number: 1

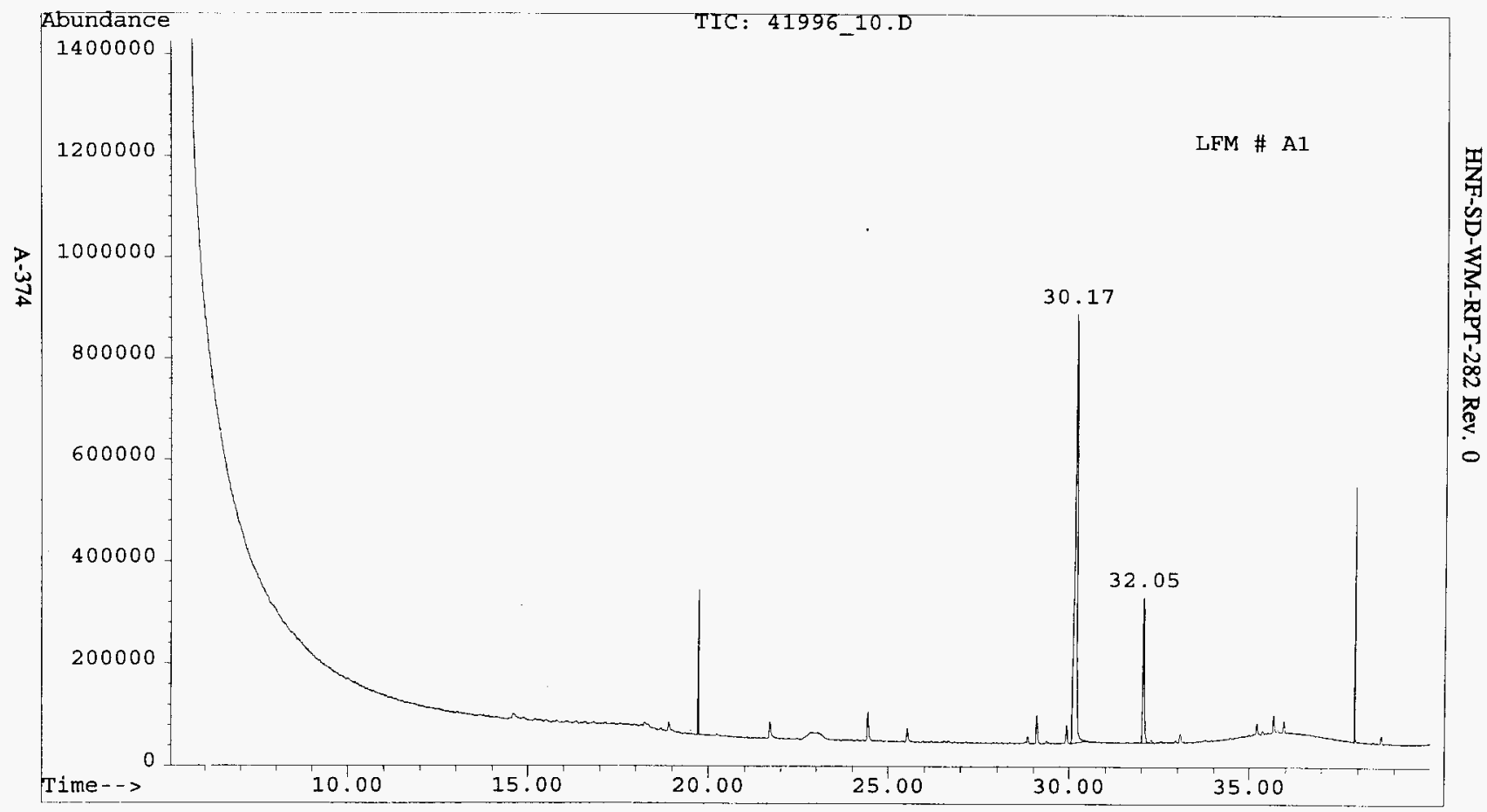


HNF-SD-WM-RPT-282 Rev. 0

CWC_REP.XLS

\section{ANALYSIS REPORT FOR CWC AIR SAMPLES}

File Name:

Analyst:

Analysis Date:

Method:

Sample Name:
41996_10.D

MS

04/19/96

CWC

FT6045-A1.11A

\begin{tabular}{|c|c|c|c|c|}
\hline COMPOUND NAME & CAS \# & Concentration & Units & Comments \\
\hline ISO-BUTANE & $75-28-5$ & $\overline{0}$ & PPB & \\
\hline VINYL CHLORIDE & $75-01-4$ & 0 & PPB & \\
\hline ETHANOL & $64-17-5$ & 0 & PPB & \\
\hline ACETONE & $67-64-1$ & 0 & PPB & \\
\hline 2-PROPANOL & $67-63-0$ & 0 & PPB & \\
\hline 1,1-DICHLOROETHENE & $75-35-4$ & 0 & PPB & \\
\hline FREON 113 & $76-13-1$ & 0 & PPB & \\
\hline DICHLOROMETHANE & $75-09-2$ & 0 & PPB & \\
\hline 1,1-DICHLOROETHANE & $75-34-3$ & 0 & PPB & \\
\hline 2-BUTANONE & $78-93-3$ & 0 & PPB & \\
\hline N-HEXANE & $110-54-3$ & 0 & PPB & \\
\hline 1,2-DICHLOROETHENE & $156-59-2$ & 0 & PPB & \\
\hline CHLOROFORM & $67-66-3$ & 0 & PPB & \\
\hline TETRAHYDROFURAN & $109-99-9$ & 0 & PPB & \\
\hline 1,1,1-TRICHLOROETHANE & $71-55-6$ & 0 & PPB & \\
\hline 1,2-DICHLOROETHANE & $107-06-2$ & 0 & PPB & \\
\hline BENZENE & $71-43-2$ & 0 & PPB & \\
\hline CARBON TETRACHLORIDE & $56-23-5$ & 0 & PPB & \\
\hline CYCLOHEXANE & $110-82-7$ & 0 & PPB & \\
\hline N-BUTANOL & $71-36-3$ & 0 & PPB & \\
\hline TRICHLOROETHENE & $79-01-6$ & 0 & PPB & \\
\hline 4-METHYL-2-PENTANONE & $108-10-1$ & 0 & PPB & \\
\hline TOLUENE & $108-88-3$ & 0 & PPB & \\
\hline 1,1,2-TRICHLOROETHANE & $79-00-5$ & 0 & PPB & \\
\hline TETRACHLOROETHENE & $127-18-4$ & 0 & PPB & \\
\hline CHLOROBENZENE & $108-90-7$ & 0 & PPB & \\
\hline ETHYLBENZENE & $100-41-4$ & 1 & PPB & \\
\hline M-XYLENE & $108-38-3$ & 0 & PPB & \\
\hline STYRENE & $100-42-5$ & $\overline{0}$ & PPB & \\
\hline O-XYLENE & $95-47-6$ & 0 & PPB & \\
\hline 1,1,2,2-TETRACHLORETHANE & $79-34-5$ & 0 & PPB & \\
\hline 1,3,5-TRIMETHYLBENZENE & $108-67-8$ & 5 & PPB & \\
\hline 1,2,4-TRIMETHYLBENZENE & $95-63-6$ & 0 & PPB & \\
\hline 1,3-DICHLOROBENZENE & $541-73-1$ & 0 & PPB & \\
\hline 1,2-DICHLOROBENZENE & $95-50-1$ & 0 & PPB & \\
\hline 1,4-DICHLOROBENZENE & $106-46-7$ & 0 & PPB & \\
\hline
\end{tabular}




\section{LIST OF COMPOUNDS TENTATIVELY IDENTIFIED WITH MASS SPECTRAL LIBRARY}

Sample: $\quad$ LFM A1

Sample Name: FT6045-A1.11A

Search Libraries: $\quad$ C:IDATABASEIWILEY138.L Minimum Quality: 70

C:IDATABASELNBS54K.L $\quad$ Minimum Quality: 70

Unknown Spectrum: Apex

Integration Params: VOA.E

Pk\# RT Area\% Library/ID Ref\# CAS\# Qual

$625.53 \quad 1.42 \mathrm{C}:$ DATABASEIWILEY138.L

Benzene, methyl-

$117433000108-88-391$

$728.840 .96 \mathrm{C}:$ DATABASEIWILEY138.L Benzene, ethyl- $\quad 118561000100-41-486$

$829.102 .70 \mathrm{C}:$ DATABASEIWILEY138.L Benzene, 1,4-dimethyl-

$118586000106-42-395$

$929.921 .79 \mathrm{C}:$ LATABASEIWILEY138.L XYLENE

$118580001330-20-795$

1030.1760 .14 C:LDATABASEIWLEY138.L Ethanol, 2-butoxy-

$119793000111-76-283$

$1132.0513 .09 \mathrm{C}:$ DATABASEIWILEY138.L Benzaldehyde

$118545000100-52-797$

$1233.070 .96 \mathrm{C}:$ IDATABASEIWILEY138.L Benzene, 1,3,5-trimethyl- $\quad 120037$ 000108-67-8 86

1335.202 .04 C:IDATABASEIWILEY138.L 1-Propanone, 2-bromo-1-phenyl-

$40090002114-00-372$ 
File

D $: \backslash C W C \backslash S A M P L E S \backslash 42396$ - 9.

Operator : MS

Acquired : 23 Apr $96 \quad 2: 18$ pm using AcqMethod CWC

Instrument : 5972 - In

Sample Name: FT6045-A1.11B

Misc Info : delta $p=50$ torr

Vial Number: 1

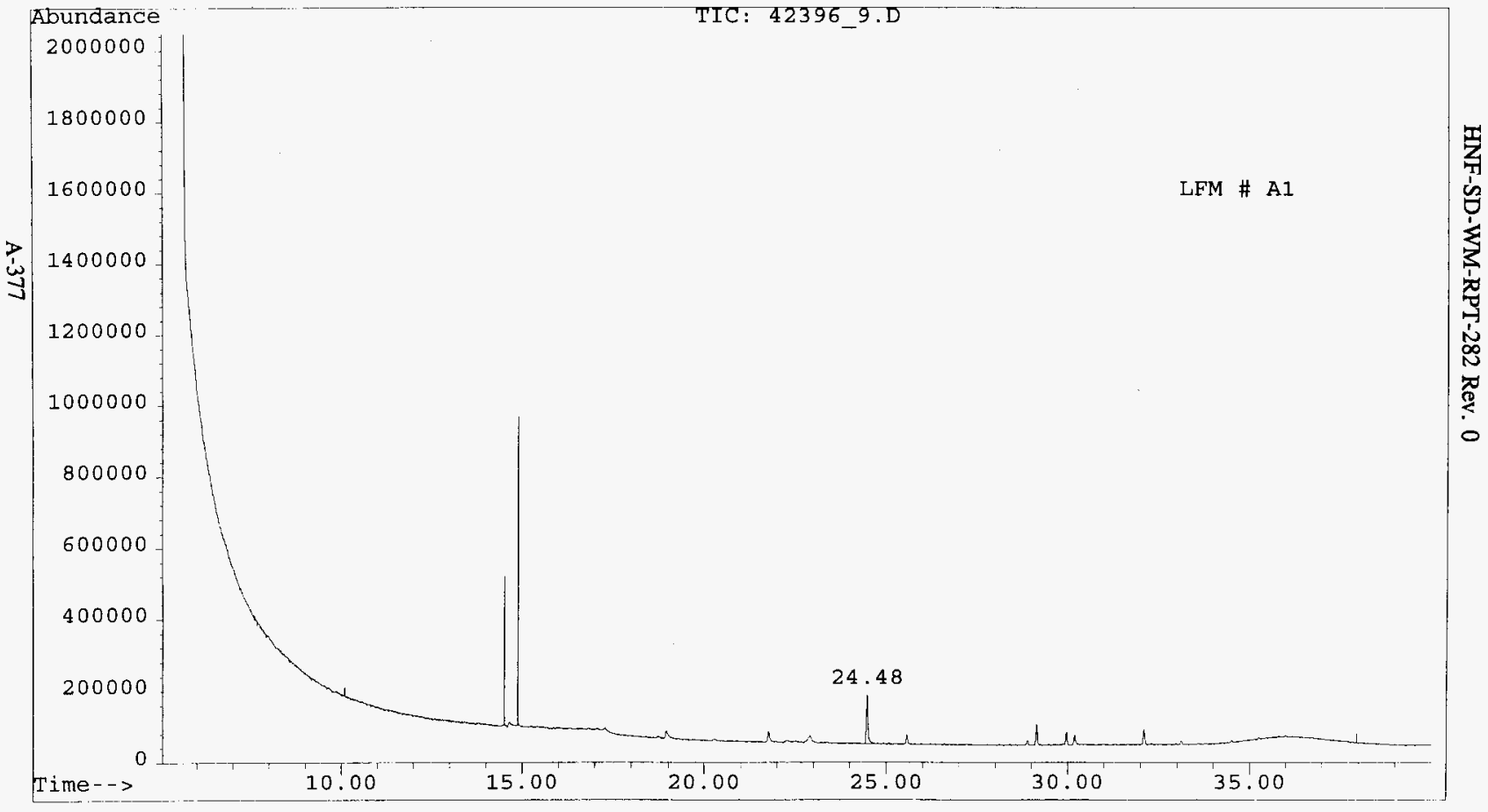


HNF-SD-WM-RPT-282 Rev. 0

CWC_REP.XLS

\section{ANALYSIS REPORT FOR CWC AIR SAMPLES}

File Name:

Analyst:

Analysis Date:

Method:

Sample Name:
42396_9.D

MS

04/23/96

CWC

FT6045-A1.11B

\begin{tabular}{|c|c|c|c|c|}
\hline COMPOUND NAME & CAS \# & Concentration & Units & Comments \\
\hline ISO-BUTANE & $75-28-5$ & 0 & PPB & \\
\hline VINYL CHLORIDE & $75-01-4$ & 0 & PPB & \\
\hline ETHANOL & $64-17-5$ & 0 & PPB & \\
\hline ACETONE & $67-64-1$ & 0 & PPB & \\
\hline 2-PROPANOL & $67-63-0$ & 0 & PPB & \\
\hline 1,1-DICHLOROETHENE & $75-35-4$ & 0 & PPB & \\
\hline FREON 113 & $76-13-1$ & 0 & PPB & \\
\hline DICHLOROMETHANE & $75-09-2$ & 0 & PPB & \\
\hline 1,1-DICHLOROETHANE & $75-34-3$ & 0 & PPB & \\
\hline 2-BUTANONE & $78-93-3$ & 0 & PPB & \\
\hline N-HEXANE & $110-54-3$ & 0 & $\overline{\mathrm{PPB}}$ & \\
\hline 1,2-DICHLOROETHENE & $156-59-2$ & 0 & $\overline{\mathrm{PPB}}$ & \\
\hline CHLOROFORM & $67-66-3$ & 0 & PPB & \\
\hline TETRAHYDROFURAN & $109-99-9$ & 0 & PPB & \\
\hline 1,1,1-TRICHLOROETHANE & $71-55-6$ & 0 & PPB & \\
\hline 1,2-DICHLOROETHANE & 107-06-2 & $\overline{0}$ & PPB & \\
\hline BENZENE & $71-43-2$ & 0 & PPB & \\
\hline CARBON TETRACHLORIDE & $56-23-5$ & 0 & PPB & \\
\hline CYCLOHEXANE & $110-82-7$ & 0 & PPB & \\
\hline N-BUTANOL & $71-36-3$ & 0 & PPB & \\
\hline TRICHLOROETHENE & $79-01-6$ & 0 & PPB & \\
\hline 4-METHYL-2-PENTANONE & $108-10-1$ & 0 & PPB & \\
\hline TOLUENE & $108-88-3$ & 0 & PPB & \\
\hline 1,1,2-TRICHLOROETHANE & $79-00-5$ & 0 & PPB & \\
\hline TETRACHLOROETHENE & $127-18-4$ & 0 & PPB & \\
\hline CHLOROBENZENE & $108-90-7$ & 0 & PPB & \\
\hline ETHYLBENZENE & $100-41-4$ & 1 & PPB & \\
\hline M-XYLENE & $108-38-3$ & 0 & PPB & \\
\hline STYRENE & $100-42-5$ & 0 & PPB & \\
\hline O-XYLENE & $95-47-6$ & 0 & PPB & \\
\hline 1,1,2,2-TETRACHLORE'THANE & $79-34-5$ & 0 & PPB & \\
\hline 1,3,5-TRIMETHYLBENZENE & $108-67-8$ & 0 & PPB & \\
\hline 1,2,4-TRIMETHYLBENZENE & $95-63-6$ & 0 & PPB & \\
\hline 1,3-DICHLOROBENZENE & 541-73-1 & 0 & PPB & \\
\hline 1,2-DICHLOROBENZENE & $95-50-1$ & 0 & PPB & \\
\hline 1,4-DICHLOROBENZENE & $106-46-7$ & 0 & PPB & \\
\hline
\end{tabular}




\section{LIST OF COMPOUNDS TENTATIVELY IDENTIFIED WITH MASS SPECTRAL LIBRARY}

Sample: $\quad$ LFM A1

Sample Name: FT6045-A1.11B

Search Libraries: C:LATABASEIWILEY138.L Minimum Quality: 70

C:DATABASELNBS54K.L Minimum Quality: 70

Unknown Spectrum: Apex

Integration Params: VOA.E

Pk\# RT Area\% Library/ID Ref\# CAS\# Qual

724.48 18.30 C:LDATABASEIWILEY138.L 2-Pentanone, 4-methyl- $\quad 118019000108-10-187$

$825.573 .74 \mathrm{C}:$ DATABASEIWILEY138.L Benzene, methyl-

117430 000108-88-391

929.147 .36 C:LDATABASEIWILEY138.L XYLENE

$118580001330-20-795$

1029.974 .08 C:IDATABASEIWILEY138.L Benzene, 1,4-dimethyl-

$118587000106-42-393$

1232.105 .41 C:LDATABASEIWILEY138.L Benzaldehyde $118545000100-52-793$ 
HNF-SD-WM-RPT-282 Rev. 0

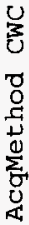

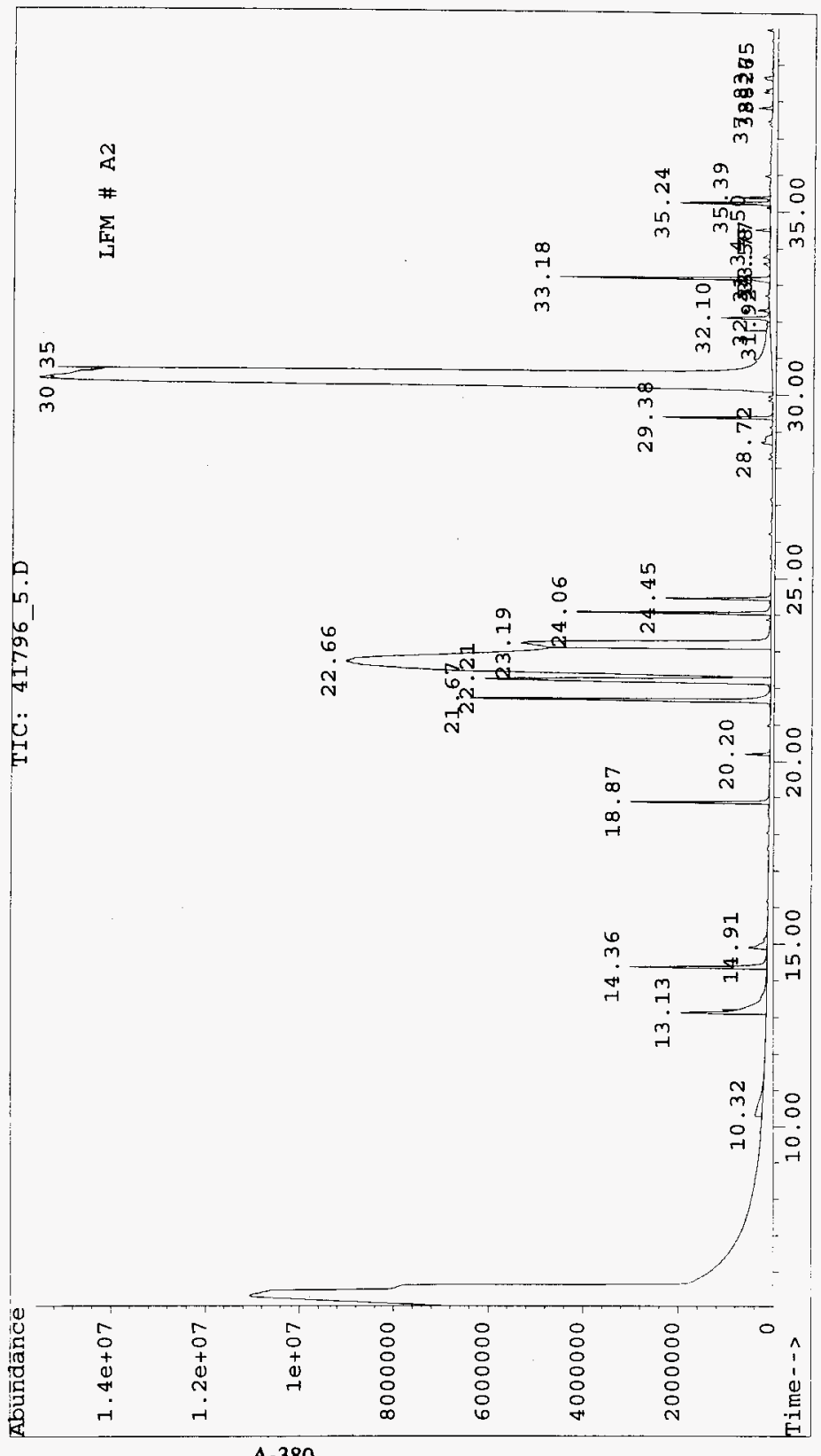

A-380 
HNF-SD-WM-RPT-282 Rev. 0

CWC_REP.XLS

\section{ANALYSIS REPORT FOR CWC AIR SAMPLES}

File Name:

Analyst:

Analysis Date:

Method:

Sample Name:
41796_5.D

$\mathrm{ms}$

04/17/96

CWC

CWC 6045-A2.10A

\begin{tabular}{|c|c|c|c|c|}
\hline COMPOUND NAME & CAS \# & Concentration & Units & Comments \\
\hline ISO-BUTANE & $75-28-5$ & 0 & PPB & \\
\hline VINYL CHLORIDE & $75-01-4$ & 0 & PPB & \\
\hline ETHANOL & $64-17-5$ & 319 & PPB & \\
\hline ACETONE & $67-64-1$ & 91 & PPB & \\
\hline 2-PROPANOL & $67-63-0$ & 30 & PPB & \\
\hline 1,1-DICHLOROETHENE & $75-35-4$ & 0 & PPB & \\
\hline FREON 113 & $76-13-1$ & 0 & $\overline{\mathrm{PPB}}$ & \\
\hline DICHLOROMETHANE & $75-09-2$ & 0 & PPB & \\
\hline 1,1-DICHLOROETHANE & $75-34-3$ & 0 & PPB & \\
\hline 2-BUTANONE & $78-93-3$ & 265 & PPB & \\
\hline N-HEXANE & $110-54-3$ & 15 & PPB & \\
\hline 1,2-DICHLOROETHENE & $156-59-2$ & 0 & $\overline{\mathrm{PPB}}$ & \\
\hline CHLOROFORM & $67-66-3$ & 0 & PPB & \\
\hline TETRAHYDROFURAN & $109-99-9$ & 13 & PPB & \\
\hline 1,1,1-TRICHLOROETHANE & $71-55-6$ & 0 & PPB & \\
\hline 1,2-DICHLOROETHANE & $107-06-2$ & 4 & PPB & \\
\hline BENZENE & $71-43-2$ & 0 & PPB & \\
\hline CARBON TETRACHLORIDE & $56-23-5$ & 0 & PPB & \\
\hline CYCLOHEXANE & $110-82-7$ & 0 & PPB & \\
\hline N-BUTANOL & $71-36-3$ & 353 & $\mathrm{PPB}$ & \\
\hline TRICHLOROETHENE & $79-01-6$ & 0 & PPB & \\
\hline 4-METHYL-2-PENTANONE & $108-10-1$ & 33 & PPB & \\
\hline TOLUENE & $108-88-3$ & 0 & PPB & \\
\hline 1,1,2-TRICHLOROETHANE & $79-00-5$ & 0 & PPB & \\
\hline TETRACHLOROETHENE & $127-18-4$ & 0 & PPB & \\
\hline CHLOROBENZENE & $108-90-7$ & 0 & PPB & \\
\hline ETHYLBENZENE & $100-41-4$ & 1 & PPB & \\
\hline M-XYLENE & $108-38-3$ & 0 & PPB & \\
\hline STYRENE & $100-42-5$ & 2 & PPB & \\
\hline O-XYLENE & $95-47-6$ & 2 & PPB & \\
\hline 1,1,2,2-TETRACHLORETHANE & $79-34-5$ & 0 & PPB & \\
\hline 1,3,5-TRIMETHYLBENZENE & $108-67-8$ & 4 & PPB & \\
\hline 1,2,4-TRIMETHYLBENZENE & $95-63-6$ & 5 & $\mathrm{PPB}$ & \\
\hline 1,3-DICHLOROBENZENE & $541-73-1$ & 5 & PPB & \\
\hline 1,2-DICHLOROBENZENE & $95-50-1$ & 5 & $\mathrm{PPB}$ & \\
\hline 1,4-DICHLOROBENZENE & $106-46-7$ & 9 & PPB & \\
\hline
\end{tabular}




\section{LIST OF COMPOUNDS TENTATIVELY IDENTIFIED WITH MASS SPECTRAL LIBRARY}

\section{Sample: $\quad$ LFM A2}

Sample Name: CWC 6045-A2.10A

Search Libraries: $\quad$ C:LDATABASEIWILEY138.L $\quad$ Minimum Quality: 70 C:IDATABASEWBS54K.L Minimum Quality: 70

Unknown Spectrum: Apex

Integration Params: VOA.E

Pk\# RT Area\% Library/ID $\quad$ Ref\# CAS\# Qual

$2 \quad 13.121 .61 \mathrm{C}:$ DATABASEIWILEY138.L Ethanol

$115955000064-17-590$

$314.36 \quad 1.06 \mathrm{C}:$ DATABASEIWILEY138.L 2-Propanone

$116053000067-64-172$

$4 \quad 14.91 \quad 0.27 \mathrm{C}:$ DATABASEIWILEY138.L 2-Propanol

$116151000067-63-080$

$7 \quad 16.180 .01 \mathrm{C}:$ DATABASEIWILEY138.L Methane, dichloro-

116807 000075-09-2 91

$13 \quad 18.87 \quad 0.85 \mathrm{C}:$ DATABASEIWILEY $138 . \mathrm{L}$ 2-Butanone

$116394000078-93-386$

$1419.330 .01 \mathrm{C}:$ DATABASEIWILEY138.L Ethene, 1,2-dichloro-, ( $\mathrm{Z}$ )-

$117549000156-59-296$

$1520.20 \quad 0.17$ C:LDATABASEIWILEY138.L Furan, tetrahydro-

116445 000109-99-9 91

1620.990 .02 C:DATABASEIWILEY138.L Ethane, 1,2-dichloro-

$117658000107-06-294$

$1721.530 .01 \mathrm{C}:$ DATABASEIWILEY138.L Benzene $116687000071-43-276$

1821.672 .56 C:LATABASEIWILEY138.L 1-Butanol 
HNF-SD-WM-RPT-282 Rev. 0

Sample: $\quad$ LFM A2 (continued)

Pk\# RT Area\% Library/ID Ref\# CAS\# Qual

1922.214 .01 C:IDATABASEIWILEY138.L

2-Propanol, 1-methoxy-

347 000107-98-2 83

$2022.6732 .77 \mathrm{C}:$ DATABASEIWILEY138.L

2-Propanol, 1-methoxy-

$347000107-98-283$

$2123.196 .90 \mathrm{C}:$ :DATABASEIWILEY138.L 2-Propanol, 1-methoxy-

$347000107-98-283$

2524.450 .64 C:IDATABASEIWILEY138.L 2-Pentanone, 4-methyl-

118023 000108-10-191

$2625.56 \quad 0.01 \mathrm{C}:$ :DATABASEIWILEY138.L Benzene, methyl-

$117430000108-88-390$

$2725.62 \quad 0.01 \mathrm{C}:$ DATABASEIWILEY138.L Ethane, 1,1,2-trichloro-

$121295000079-00-597$

$3228.40 \quad 0.02$ C:IDATABASEIWILEY138.L Benzene, chloro-

$118974000108-90-794$

3529.130 .02 C:IDATABASEIWILEY138.L Benzene, 1,2-dimethyl-

118564 000095-47-695

3729.850 .02 C:IDATABASEIWILEY138.L Styrene

$118479000100-42-595$

3829.960 .03 C:IDATABASEIWILEY138.L

Benzene, 1,2-dimethyl-

$118571000095-47-694$

3930.3441 .38 C:DATABASEIWILEY138.L Ethanol, 2-butoxy-

$119793000111-76-278$

$4432.10 \quad 0.45$ C:IDATABASEIWILEY138.L Benzaldehyde

$118545000100-52-796$

4532.310 .18 C:DATABASEIWILEY138.L Benzene, 1,2,4-trimethyl-

$120030000095-63-683$

$4933.590 .11 \mathrm{C}:$ DATABASEIWILEY138.L Benzene, 1,4-dichloro-

$123082000106-46-797$ 
HNF-SD-WM-RPT-282 Rev. 0

Sample: $\quad$ LFM A2 (continued)

Pk\# RT Area\% $\quad$ Library/ID $\quad$ Ref\# CAS\# Qual

$5033.770 .10 \mathrm{C}:$ :DATABASEIWILEY138.L Benzene, 1,4-dichloro-

$123082000106-46-797$

$53 \quad 34.50 \quad 0.10 \mathrm{C}:$ :DATABASEIWILEY138.L Benzene, 1,2-dichloro-

$11151000095-50-198$

5635.240 .55 C:IDATABASEIWILEY138.L Ethanone, 1-phenyl-

$119986000098-86-296$

6638.260 .06 C:LDATABASEIWILEY138.L Benzaldehyde, ethyl-

$7377053951-50-187$

6738.330 .05 C:IDATABASEIWILEY138.L 2,3-DIMETHYLBENZALDEHYDE 7378 005779-93-1 87 
File

: D : \CWC \SAMPLES $\backslash 41896 \quad 5 . \mathrm{D}$

Operator

: LAP

Acquired

: 18 Apr 9

Instrument : 5972 - In

Sample Name: CWC 6045 A2.10A

Misc Info : delta $p=50$ torr

Vial Number: 1

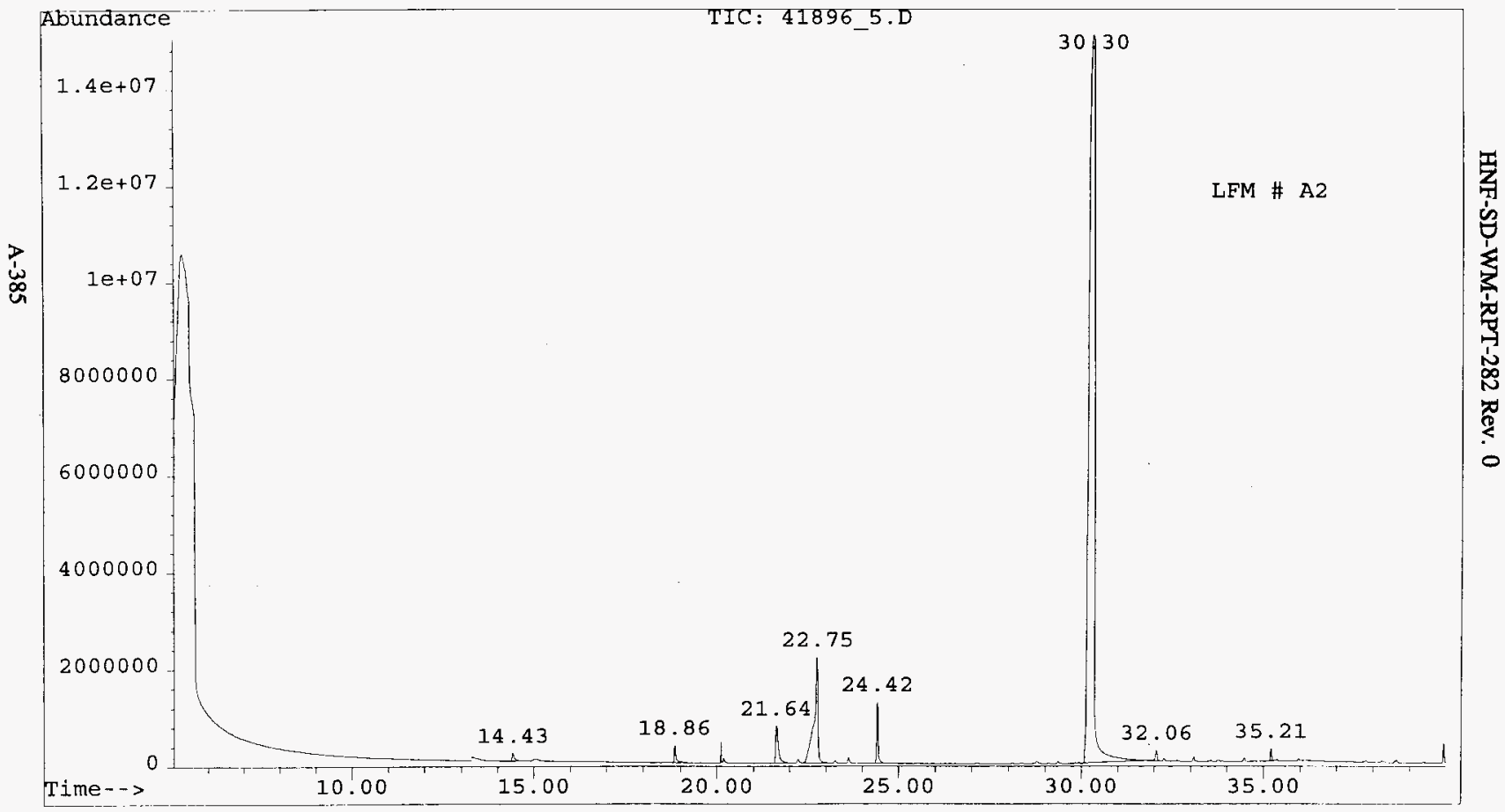


HNF-SD-WM-RPT-282 Rev. 0

CWC_REP.XLS

\section{ANALYSIS REPORT FOR CWC AIR SAMPLES}

File Name:

Analyst:

Analysis Date:

Method:

Sample Name:

\author{
41896_5.D \\ LAP \\ 04/18/96 \\ CWC \\ CWC 6045_A2.10A
}

\begin{tabular}{|c|c|c|c|c|}
\hline COMPOUND NAME & CAS \# & Concentration & Units & Comments \\
\hline ISO-BUTANE & $75-28-5$ & 0 & PPB & \\
\hline VINYL CHLORIDE & $75-01-4$ & 0 & PPB & \\
\hline ETHANOL & $64-17-5$ & 18 & PPB & \\
\hline ACETONE & $67-64-1$ & 6 & $\overline{\mathrm{PPB}}$ & \\
\hline 2-PROPANOL & $67-63-0$ & 6 & PPB & \\
\hline 1,1-DICHLOROETHENE & $75-35-4$ & 0 & PPB & \\
\hline FREON 113 & $76-13-1$ & 0 & PPB & \\
\hline DICHLOROMETHANE & $75-09-2$ & 0 & PPB & \\
\hline 1,1-DICHLOROETHANE & $75-34-3$ & 0 & PPB & \\
\hline 2-BUTANONE & $78-93-3$ & 39 & PPB & \\
\hline N-HEXANE & $110-54-3$ & 2 & PPB & \\
\hline 1,2-DICHLOROETHENE & $156-59-2$ & 0 & PPB & \\
\hline CHLOROFORM & $67-66-3$ & 0 & PPB & \\
\hline TETRAHYDROFURAN & $109-99-9$ & 0 & PPB & \\
\hline 1,1,1-TRICHLOROETHANE & $71-55-6$ & 0 & PPB & \\
\hline 1,2-DICHLOROETHANE & $107-06-2$ & 0 & PPB & \\
\hline BENZENE & $71-43-2$ & 0 & $\overline{P P B}$ & \\
\hline CARBON TETRACHLORIDE & $56-23-5$ & 0 & PPB & \\
\hline CYCLOHEXANE & $110-82-7$ & 0 & PPB & \\
\hline N-BUTANOL & $71-36-3$ & 59 & PPB & \\
\hline TRICHLOROETHENE & $79-01-6$ & 0 & PPB & \\
\hline 4-METHYL-2-PENTANONE & $108-10-1$ & 21 & PPB & \\
\hline TOLUENE & $108-88-3$ & 0 & PPB & \\
\hline 1,1,2-TRICHLOROETHANE & $79-00-5$ & 0 & PPB & \\
\hline TETRACHLOROETHENE & $127-18-4$ & 0 & PPB & \\
\hline CHLOROBENZENE & $108-90-7$ & 0 & PPB & \\
\hline ETHYLBENZENE & $100-41-4$ & 0 & PPB & \\
\hline M-XYLENE & $108-38-3$ & 0 & PPB & \\
\hline STYRENE & $100-42-5$ & 0 & PPB & \\
\hline O-XYLENE & $95-47-6$ & 0 & PPB & \\
\hline 1,1,2,2-TETRACHLORETHANE & $79-34-5$ & 0 & PPB & \\
\hline 1,3,5-TRIMETHYLBENZENE & $108-67-8$ & 1 & PPB & \\
\hline 1,2,4-TRIMETHYLBENZENE & $95-63-6$ & 2 & PPB & \\
\hline 1,3-DICHLOROBENZENE & $541-73-1$ & 0 & PPB & \\
\hline 1,2-DICHLOROBENZENE & $95-50-1$ & 0 & PPB & \\
\hline 1,4-DICHLOROBENZENE & $106-46-7$ & 2 & PPB & \\
\hline
\end{tabular}




\section{LIST OF COMPOUNDS TENTATIVELY IDENTIFIED WITH MASS SPECTRAL LIBRARY}

Sample: $\quad$ LFM A2

Sample Name: CWC 6045-A2.10A

Search Libraries: $\quad$ C:IDAT ABASEIWILEY138.L Minimum Quality: 70

C:IDATABASEWBS54K.L Minimum Quality: 70

Unknown Spectrum: Apex

Integration Params: VOA.E

Pk\# RT Area\% $\quad$ Library/ID $\quad$ Ref\# CAS\# Qual

$213.12 \quad 1.61 \mathrm{C}:$ DATABASEIWILEY138.L Ethanol

$115955000064-17-590$

$314.361 .06 \mathrm{C}:$ DATABASEIWILEY138.L 2-Propanone

$116053000067-64-172$

$4 \quad 14.910 .27$ C:LATABASEIWILEY 138.L 2-Propanol

$116151000067-63-080$

$7 \quad 16.18 \quad 0.01$ C:LDATABASEIWILEY138.L

Methane, dichloro-

$116807000075-09-291$

$13 \quad 18.87 \quad 0.85$ C:IDATABASEIWILEY138.L

2-Butanone

$116394000078-93-386$

$14 \quad 19.330 .01 \mathrm{C}:$ DATABASEIWILEY138.L

Ethene, 1,2-dichloro-, (Z)-

$117549000156-59-296$

1520.200 .17 C:LDATABASEIWILEY138.L Furan, tetrahydro-

$116445000109-99-991$

1620.990 .02 C:DATABASEIWLEY138.L

Ethane, 1,2-dichloro-

$117658000107-06-294$

$1721.53 \quad 0.01$ C:DATABASEIWILEY138.L

Benzene

$116687000071-43-276$

1821.672 .56 C:DATABASE IWLEY138.L

1-Butanol

$116556000071-36-391$ 
HNF-SD-WM-RPT-282 Rev. 0

Sample: $\quad$ LFM A2 (continued)

Pk\# RT Area\% Library/ID Ref\# CAS\# Qual

$1922.214 .01 \mathrm{C}:$ DATABASEIWILEY138.L 2-Propanol, 1-methoxy-

$347000107-98-283$

2022.6732 .77 C:IDATABASEIWILEY $138 . \mathrm{L}$ 2-Propanol, 1-methoxy$347000107-98-283$

$2123.196 .90 \mathrm{C}:$ :DATABASEIWLEY138.L 2-Propanol, 1-methoxy-

$347000107-98-283$

$2524.45 \quad 0.64$ C:IDATABASEIWILEY138.L 2-Pentanone, 4-methyl118023 000108-10-1 91

2625.560 .01 C:LDATABASEIWILEY138.L Benzene, methyl$117430000108-88-390$

2725.620 .01 C:IDATABASEIWILEY138.L Ethane, 1,1,2-trichloro$121295000079-00-597$

$3228.40 \quad 0.02 \mathrm{C}:$ DATABASEIWILEY138.L Benzene, chloro118974 000108-90-7 94

$3529.130 .02 \mathrm{C}:$ DATABASEIWILEY138.L Benzene, 1,2-dimethyl$118564000095-47-695$

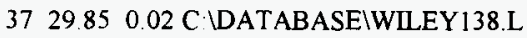
Styrene $118479000100-42-595$

$3829.96 \quad 0.03$ C:IDATABASEIWLEY138.L Benzene, 1,2-dimethyl$118571000095-47-694$

3930.3441 .38 C:DATABASEIWILEY138.L Ethanol, 2-butoxy$119793000111-76-278$

$4432.10 \quad 0.45$ C:DATABASEIWILEY 138.L Benzaldehyde $118545000100-52-796$

$45 \quad 32.310 .18 \mathrm{C}:$ :DATABASEIWILEY138.L Benzene, 1,2,4-trimethyl$120030000095-63-683$ $4933.590 .11 \mathrm{C}:$ DATABASEIWILEY138.L Benzene, 1,4-dichloro$123082000106-46-797$ 
HNF-SD-WM-RPT-282 Rev. 0

Sample: LFM A2 (continued)

Pk\# RT Area\% Library/ID Ref\# CAS\# Qual

$5033.770 .10 \mathrm{C}:$ :DATABASEIWILEY138.L

Benzene, 1,4-dichloro- $\quad 123082$ 000106-46-7 97

$53 \quad 34.50 \quad 0.10$ C:IDATABASEIWILEY138.L Benzene, 1,2-dichloro-

$11151000095-50-198$

$56 \quad 35.24 \quad 0.55$ C:IDATABASEIWILEY138.L Ethanone, 1-phenyl-

$119986000098-86-296$

6638.260 .06 C:IDATABASEIWILEY138.L Benzaldehyde, ethyl$7377053951-50-187$

6738.330 .05 C: DATABASEIWILEY $138 . \mathrm{L}$ 2,3-DIMETHYLBENZALDEHYDE 7378 005779-93-1 87 
File

Operator

Acquired

Instrument

Sample Name: FT $6045-A 2$ In

Misc Info : DELTA $P=50$ TORR

Vial Number: 1

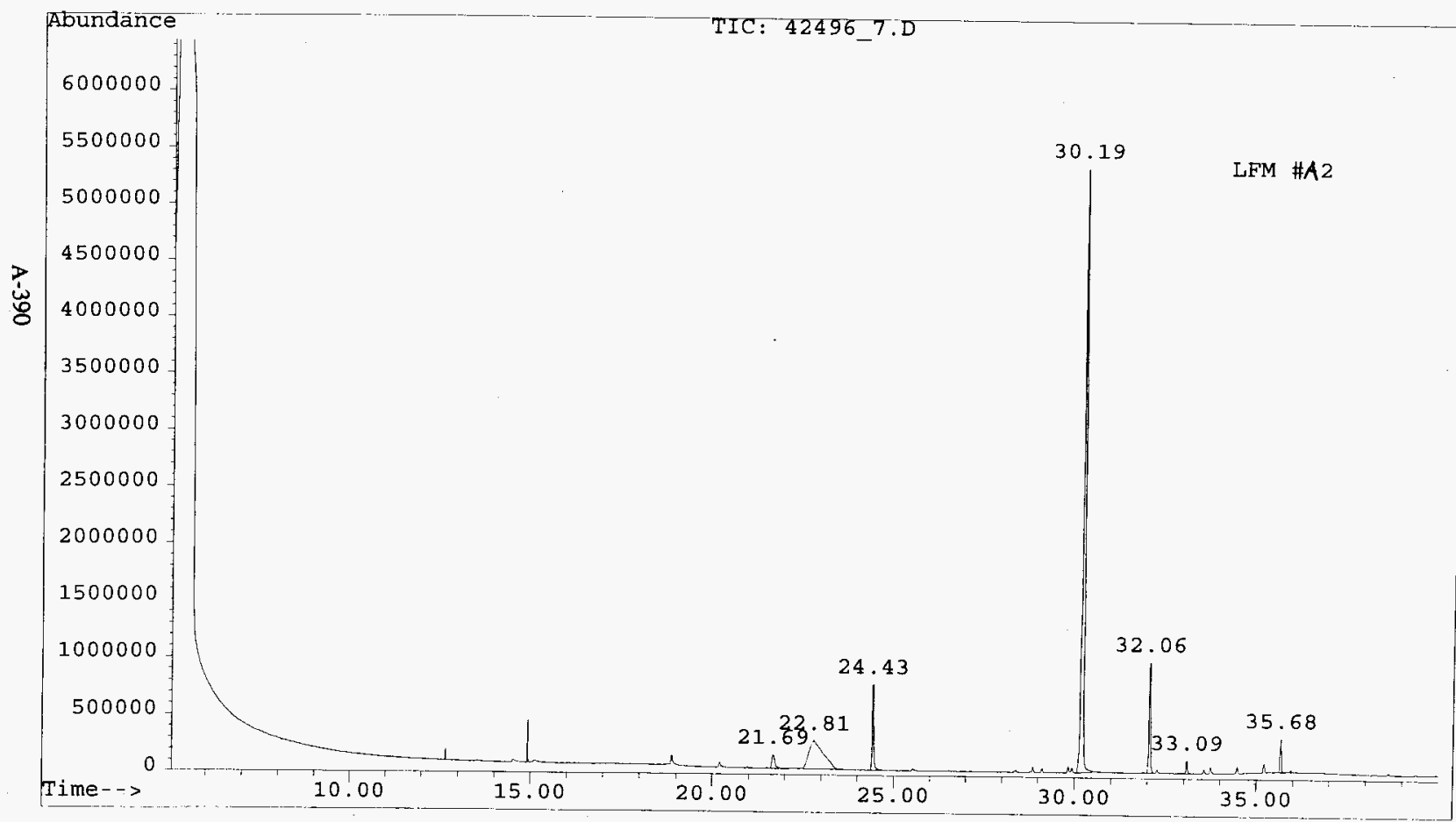


HNF-SD-WM-RPT-282 Rev. 0

CWC_REP.XLS

\section{ANALYSIS REPORT FOR CWC AIR SAMPLES}

File Name:

Analyst:

Analysis Date:

Method:

Sample Name:
42496_7.D

MS

04/24/96

CWC

FT 6045-A2.10A

\begin{tabular}{|c|c|c|c|c|}
\hline COMPOUND NAME & CAS\# & Concentration & Units & Comments \\
\hline ISO-BUTANE & $75-28-5$ & 0 & PPB & \\
\hline VINYL CHLORIDE & $75-01-4$ & 0 & PPB & \\
\hline ETHANOL & $64-17-5$ & 0 & PPB & \\
\hline ACETONE & $67-64-1$ & 0 & PPB & \\
\hline 2-PROPANOL & $67-63-0$ & 0 & PPB & \\
\hline 1,1-DICHLOROETHENE & $75-35-4$ & 0 & PPB & \\
\hline FREON 113 & $76-13-1$ & 0 & PPB & \\
\hline DICHLOROMETHANE & $75-09-2$ & 0 & PPB & \\
\hline 1,1-DICHLOROETHANE & $75-34-3$ & 0 & PPB & \\
\hline 2-BUTANONE & $78-93-3$ & 0 & PPB & \\
\hline N-HEXANE & $110-54-3$ & 0 & PPB & \\
\hline 1,2-DICHLOROETHENE & $156-59-2$ & 0 & PPB & \\
\hline CHLOROFORM & $67-66-3$ & 0 & PPB & \\
\hline TETRAHYDROFURAN & $109-99-9$ & 0 & PPB & \\
\hline 1,1,1-TRICHLOROETHANE & $71-55-6$ & 0 & PPB & \\
\hline 1,2-DICHLOROETHANE & $107-06-2$ & 0 & PPB & \\
\hline BENZENE & $71-43-2$ & 0 & PPB & \\
\hline CARBON TETRACHLORIDE & $56-23-5$ & 0 & PPB & \\
\hline CYCLOHEXANE & $110-82-7$ & 0 & PPB & \\
\hline N-BUTANOL & $71-36-3$ & 10 & $\overline{\mathrm{PPB}}$ & \\
\hline TRICHLOROETHENE & $79-01-6$ & 0 & PPB & \\
\hline 4-METHYL-2-PENTANONE & $108-10-1$ & 16 & PPB & \\
\hline TOLUENE & $108-88-3$ & 0 & PPB & \\
\hline 1,1,2-TRICHLOROETHANE & $79-00-5$ & 0 & PPB & \\
\hline TETRACHLOROETHENE & $127-18-4$ & 0 & PPB & \\
\hline CHLOROBENZENE & $108-90-7$ & 0 & PPB & \\
\hline ETHYLBENZENE & $100-41-4$ & 1 & PPB & \\
\hline M-XYLENE & $108-38-3$ & 0 & PPB & \\
\hline STYRENE & $100-42-5$ & 2 & PPB & \\
\hline O-XYLENE & $95-47-6$ & 0 & PPB & \\
\hline 1,1,2,2-TETRACHLORETHANE & $79-34-5$ & 0 & $\mathrm{PPB}$ & \\
\hline 1,3,5-TRIMETHYLBENZENE & $108-67-8$ & 17 & PPB & \\
\hline 1,2,4-TRIMETHYLBENZENE & $95-63-6$ & 3 & PPB & \\
\hline 1,3-DICHLOROBENZENE & $541-73-1$ & 0 & PPB & \\
\hline 1,2-DICHLOROBENZENE & $95-50-1$ & 0 & PPB & \\
\hline 1,4-DICHLOROBENZENE & $106-46-7$ & 2 & PPB & \\
\hline
\end{tabular}


HNF-SD-WM-RPT-282 Rev. 0

\section{LIST OF COMPOUNDS TENTATIVELY DENTIFIED WITH MASS SPECTRAL LIBRARY}

Sample: $\quad$ LFM A2

Sample Name: FT 6045-A2.10A

Search Libraries: $\quad$ C:LDATABASEIWILEY138.L $\quad$ Minimum Quality: 70

C:DATABASEINBS54K.L Minimum Quality: 70

Unknown Spectrum: Apex

Integration Params: VOA.E

Pk\# RT Area\% Library/ID $\quad$ Ref\# CAS\# Qual

$621.691 .94 \mathrm{C}:$ DATABASE WWLEY138.L 1-Butanol

$116554000071-36-390$

722.8018 .55 C:IDATABASEIWILEY138.L 2-Propanol, 1-methoxy-

$347000107-98-278$

$824.435 .91 \mathrm{C}:$ DATABASEIWILEY138.L 2-Pentanone, 4-methyl-

$118019000108-10-191$

$1028.38 \quad 0.16 \mathrm{C}:$ DATABASEIWILEY138.L Benzene, chloro-

$118974000108-90-793$

1128.860 .40 C:DATABASEIWILEY138.L Benzene, ethyl-

$118560000100-41-494$

$1229.110 .28 \mathrm{C}$.DATABASE Benzene, 1,4-dimethyl-

$118587000106-42-395$

$1329.83 \quad 0.43 \mathrm{C}:$ DATABASE\WILEY138.L Styrene

$118481000100-42-596$

$1429.93 \quad 0.33 \mathrm{C}:$ DATABASEIWILEY138.L XYLENE

$118580001330-20-795$

15 30.20 56.55 C:DATABASEIWILEY138.L Ethanol, 2-butoxy-

$119793000111-76-286$

$1632.067 .18 \mathrm{C}:$ DATABASE $\backslash$ WILEY138.L Benzaldehyde

$118546000100-52-796$ 
HNF-SD-WM-RPT-282 Rev. 0

Sample: $\quad$ LFM A2 (continued)

Pk\# RT Area\% Library/ID Ref\# CAS\# Qual

$1732.270 .26 \mathrm{C}:$ :DATABASEIWILEY138.L

Benzene, 1,3,5-trimethyl- $\quad 120037$ 000108-67-8 92

1833.090 .85 C:LDATABASEIWILEY138.L

Benzene, 1,2,3-trimethyl- $\quad 4145$ 000526-73-8 95

1933.560 .30 C:LATABASEIWILEY138.L

Benzene, 1,4-dichloro-

$123085000106-46-797$

$2033.740 .47 \mathrm{C}:$ :DATABASEIWILEY138.L

Benzene, 1,4-dichloro-

$123085000106-46-797$

$2134.47 \quad 0.40 \mathrm{C}:$ :DATABASEIWILEY138.L

Benzene, I,4-dichloro- $\quad 11154$ 000106-46-7 98

$2235.210 .61 \mathrm{C}:$ DATABASEIWILEY138.L

Ethanone, I-phenyl- 119985 000098-86-2 94

2335.682 .03 C:IDATABASEIWILEY 138.L

Benzenemethanol, .alpha.,.alpha.-dime 121926 000617-94-7 93 
File operator Acquired Instrument

Sample Name

Misc Info : r
Vial Number: 1

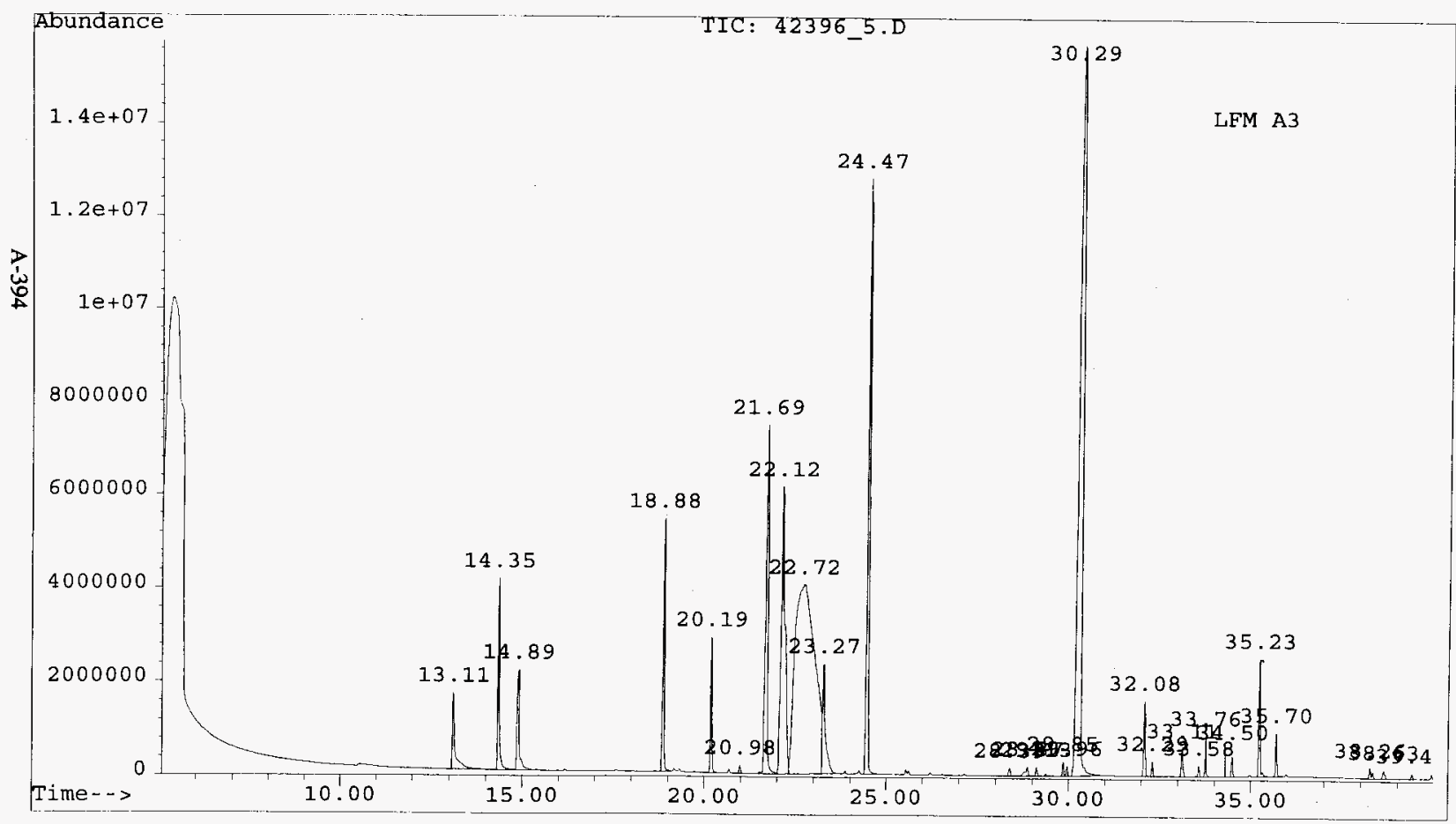

$D: \backslash C W C \backslash S A M P L E S \backslash 42396$

1 ap

23 Apr 96 8:54 am using AcqMethod CWC

5972 - In

FT6045-A3.07B 
HNF-SD-WM-RPT-282 Rev. 0

CWC_REP.XLS

\section{ANALYSIS REPORT FOR CWC AIR SAMPLES}

File Name:

Analyst:

Analysis Date:

Method:

Sample Name:
42396_5.D

lap

04/23/96

CWC

FT6045-A3.07B

\begin{tabular}{|c|c|c|c|c|}
\hline COMPOUND NAME & CAS \# & Concentration & Units & Comments \\
\hline ISO-BUTANE & $75-28-5$ & 0 & PPB & \\
\hline VINYL CHLORIDE & $75-01-4$ & 0 & PPB & \\
\hline ETHANOL & $64-17-5$ & 175 & $\overline{P P B}$ & \\
\hline ACETONE & $67-64-1$ & 123 & PPB & \\
\hline 2-PROPANOL & $67-63-0$ & 100 & PPB & \\
\hline 1,1-DICHLOROETHENE & $75-35-4$ & 0 & PPB & \\
\hline FREON 113 & $76-13-1$ & 0 & PPB & \\
\hline DICHLOROMETHANE & $75-09-2$ & 0 & PPB & \\
\hline 1,1-DICHLOROETHANE & $75-34-3$ & 0 & PPB & \\
\hline 2-BUTANONE & $78-93-3$ & 523 & PPB & \\
\hline N-HEXANE & $110-54-3$ & 29 & PPB & \\
\hline 1,2-DICHLOROETHENE & $156-59-2$ & 0 & $\overline{P P B}$ & \\
\hline CHLOROFORM & $67-66-3$ & 0 & PPB & \\
\hline TETRAHYDROFURAN & $109-99-9$ & 76 & PPB & \\
\hline 1,1,1-TRICHLOROETHANE & $71-55-6$ & 0 & $\overline{\mathrm{PPB}}$ & \\
\hline 1,2-DICHLOROETHANE & $107-06-2$ & 10 & PPB & \\
\hline BENZENE & $71-43-2$ & 4 & PPB & \\
\hline CARBON TETRACHLORIDE & $56-23-5$ & 0 & $\mathrm{PPB}$ & \\
\hline CYCLOHEXANE & $110-82-7$ & 0 & PPB & \\
\hline N-BUTANOL & $71-36-3$ & 477 & PPB & \\
\hline TRICHLOROETHENE & $79-01-6$ & 0 & PPB & \\
\hline 4-METHYL-2-PENTANONE & $108-10-1$ & 318 & PPB & \\
\hline TOLUENE & $108-88-3$ & 3 & PPB & \\
\hline 1,1,2-TRICHLOROETHANE & $79-00-5$ & 0 & PPB & \\
\hline TETRACHLOROETHENE & $127-18-4$ & 0 & $\overline{\text { PPB }}$ & \\
\hline CHLOROBENZENE & $108-90-7$ & 3 & PPB & \\
\hline ETHYLBENZENE & $100-41-4$ & 4 & PPB & \\
\hline M-XYLENE & $108-38-3$ & 5 & PPB & \\
\hline STYRENE & $100-42-5$ & 8 & PPB & \\
\hline O-XYLENE & $95-47-6$ & 6 & $\mathrm{PPB}$ & \\
\hline 1,1,2,2-TETRACHLORETHANE & $79-34-5$ & 2 & PPB & \\
\hline 1,3,5-TRIMETHYLBENZENE & $108-67-8$ & 8 & $\overline{\mathrm{PPB}}$ & \\
\hline 1,2,4-TRIMETHYLBENZENE & $95-63-6$ & 16 & $\overline{\mathrm{PPB}}$ & \\
\hline 1,3-DICHLOROBENZENE & $541-73-1$ & 7 & PPB & \\
\hline 1,2-DICHLOROBENZENE & $95-50-1$ & 10 & PPB & \\
\hline 1,4-DICHLOROBENZENE & $106-46-7$ & 13 & PPB & \\
\hline
\end{tabular}




\section{LIST OF COMPOUNDS TENTATIVELY IDENTIFIED WITH MASS SPECTRAL LIBRARY}

Sample: $\quad$ LFM A3

Sample Name: FT6045-A3.07B

Search Libraries: $\quad$ C:LDATABASEIWTLEY138.L $\quad$ Minimum Quality: 70

C:IDATABASEINBS54K.L $\quad$ Minimum Quality: 70

Unknown Spectrum: Apex

Integration Params: VOAE

Pk\# RT Area\% Library/ID

Ref\# CAS\# Qual

$213.111 .81 \mathrm{C}:$ IDATABASEIWILEY138.L Ethanol

$115963000064-17-590$

314.352 .61 C:WATABASEIWILEY138.L 2-Propanone

$116051000067-64-172$

$414.891 .90 \mathrm{C}:$ DATABASEIWILEY138.L 2-Propanol

$116152000067-63-090$

$6 \quad 16.170 .02$ C:DATABASE|WILEY138.L Methane, dichloro-

$116807000075-09-293$

$9 \quad 18.88$ 3.32 C:LDATABASE $\mid W I L E Y 138 . L$ 2-Butanone

$116391000078-93-386$

$10 \quad 19.18 \quad 0.07$ C:LDATABASEIWILEY $138 . \mathrm{L}$ Oxirane, 2,3-dimethyl-

$116439003266-23-772$

$11 \quad 19.320 .08$ C:WDATABASEIWLEY138.L Ethene, 1,2-dichloro-, (Z)-

$536000156-59-296$

$1219.74 \quad 0.02$ C:IDATABASEIWLEY138.L Acetic acid, trichloro-

$125012000076-03-983$

$1320.191 .55 \mathrm{C}:$ :DATABASEIWILEY138.L Furan, tetrahydro-

116445 000109-99-9 91

1520.980 .10 C:LDATABASEIWILEY138.L

Ethane, 1,2-dichloro-

$117655000107-06-296$ 
Sample: LFM A3 (continued)

Pk\# RT Area\% Library/ID Ref\# CAS\# Qual

1621.520 .03 C:DATABASEIWILEY138.L

Benzene

$116687000071-43-294$

1721.706 .43 C:IDATABASEIWLEY138.L 1-Butanol

$116556000071-36-391$

1922.7130 .54 C:DATABASEIWILEY138.L 2-Propanol, 1-methoxy-

347 000107-98-2 74

2324.479 .01 C:IDATABASEIWILEY138.L 2-Pentanone, 4-methyl-

$118021000108-10-191$

$2525.55 \quad 0.05$ C:LDATABASEIWILEY138.L Benzene, methyl-

$117429000108-88-381$

$2625.620 .04 \mathrm{C}$ :DATABASEIWILEY138.L Ethane, 1,1,2-trichloro-

$121295000079-00-599$

3128.880 .24 C:IDATABASEIWILEY138.L Benzene, ethyl-

$118560000100-41-494$

3229.130 .11 C:LDATABASEIWILEY 138.L Benzene, 1,3-dimethyl-

$118578000108-38-395$

$3429.85 \quad 0.17$ C:DATABASEIWILEY138.L Styrene

$118481000100-42-596$

3529.960 .11 C:IDATABASEIWILEY138.L

Benzene, 1,2-dimethyl-

$118571000095-47-695$

$3630.3024 .86 \mathrm{C}$. LATABASEIWILEY138.L Ethanol, 2-butoxy$119793000111-76-278$

3732.080 .88 C:IDATABASEIWILEY138.L Benzaldehyde $118546000100-52-796$

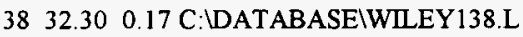
Benzene, 1,3,5-trimethyl-

$120037000108-67-895$ 
HNF-SD-WM-RPT-282 Rev. 0

Sample: $\quad$ LFM A3 (continued)

Pk\# RT Area\% Library/ID Ref\# CAS\# Qual

$3933.110 .38 \mathrm{C}:$ DATABASEIWILEY138.L

Benzene, 1,3,5-trimethyl- $\quad 120037$ 000108-67-8 95

$4033.58 \quad 0.12$ C IDATABASEIWILEY138.L

Benzene, 1,3-dichloro- $\quad 123079$ 000541-73-1 98

4133.760 .24 C:IDATABASEIWILEY138.L

Benzene, 1,4-dichloro-

$123085000106-46-798$

$4334.490 .24 \mathrm{C}:$ :DATABASEIWILEY138.L

Benzene, 1,3-dichloro- $\quad 123079$ 000541-73-1 98

$4434.98 \quad 0.04$ C:IDATABASEIWILEY138.L

Benzenemethanol, alpha.-methyl- $\quad 120232$ 000098-85-1 90

$45 \quad 35.23 \quad$ 1.40 C:LDATABASEIWREY138.L

Ethanone, 1-phenyl-

$4126000098-86-295$

$4635.700 .51 \mathrm{C}:$ DATABASEIWILEY138.L

Benzenemethanol, .alpha..,alpha.-dime 121924 000617-94-7 83

$4938.25 \quad 0.12 \mathrm{C}:$ IDATABASEIWILEY $138 . \mathrm{L}$

Benzaldehyde, ethyl-

$7377053951-50-191$

5038.330 .08 C:LDATABASEIWILEY138.L 2,3-DIMETHYLBENZALDEHYDE 7378 005779-93-1 91

5138.620 .14 C:LDATABASEIWILEY138.L

2,3-DIMETHYLBENZALDEHYDE 7378 005779-93-1 90

5239.410 .06 C:WDATABASEIWLEY138.L

Benzaldehyde, ethyl- 121568 053951-50-1 83 

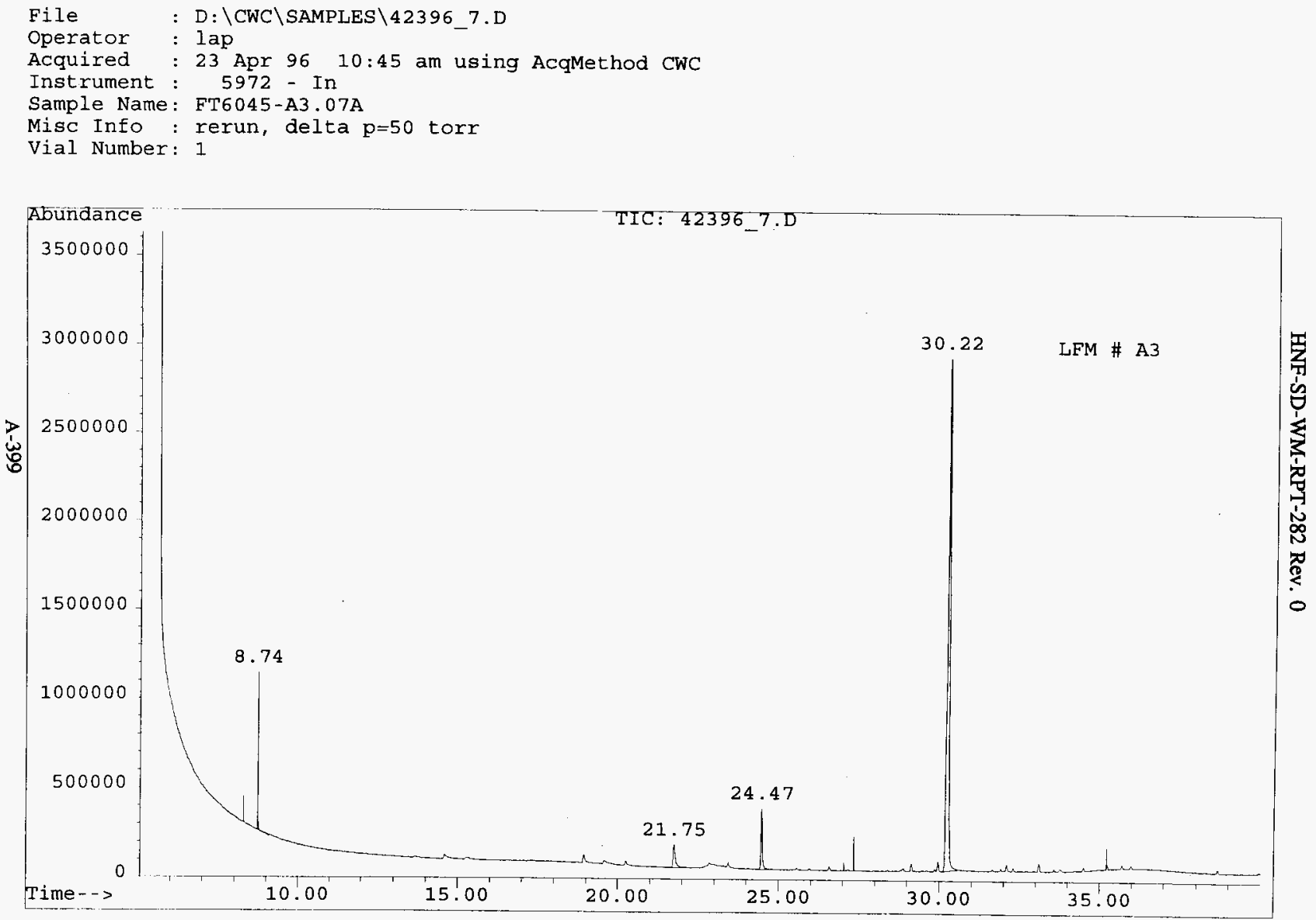
HNF-SD-WM-RPT-282 Rev. 0

CWC_REP.XLS

\section{ANALYSIS REPORT FOR CWC AIR SAMPLES}

File Name:

Analyst:

Analysis Date:

Method:

Sample Name:
42396_7.D

lap

04/23/96

CWC

FT6045-A3.07A

\begin{tabular}{|c|c|c|c|c|}
\hline COMPOUND NAME & CAS \# & Concentration & Units & Comments \\
\hline ISO-BUTANE & $75-28-5$ & $\overline{0}$ & $\overline{\mathrm{PPB}}$ & \\
\hline VINYL CHLORIDE & $75-01-4$ & 0 & $\mathrm{PPB}$ & \\
\hline ETHANOL & $64-17-5$ & 0 & PPB & \\
\hline ACETONE & $67-64-1$ & 0 & PPB & \\
\hline 2-PROPANOL & $67-63-0$ & 0 & PPB & \\
\hline 1,1-DICHLOROETHENE & $75-35-4$ & 0 & PPB & \\
\hline FREON 113 & $76-13-1$ & 0 & PPB & \\
\hline DICHLOROMETHANE & $75-09-2$ & 0 & PPB & \\
\hline 1,1-DICHLOROETHANE & $75-34-3$ & 0 & PPB & \\
\hline 2-BUTANONE & $78-93-3$ & 0 & PPB & \\
\hline N-HEXANE & $110-54-3$ & 0 & PPB & \\
\hline 1,2-DICHLOROETHENE & $156-59-2$ & 0 & PPB & \\
\hline CHLOROFORM & $67-66-3$ & 0 & PPB & \\
\hline TETRAHYDROFURAN & $109-99-9$ & 0 & PPB & \\
\hline 1,1,1-TRICHLOROETHANE & $71-55-6$ & 0 & PPB & \\
\hline 1,2-DICHLOROETHANE & $107-06-2$ & 0 & PPB & \\
\hline BENZENE & $71-43-2$ & 0 & PPB & \\
\hline CARBON TETRACHLORIDE & $56-23-5$ & 0 & PPB & \\
\hline CYCLOHEXANE & $110-82-7$ & 0 & PPB & \\
\hline N-BUTANOL & $71-36-3$ & 8 & $\overline{\mathrm{PPB}}$ & \\
\hline TRICHLOROETHENE & $79-01-6$ & 0 & PPB & \\
\hline 4-METHYL-2-PENTANONE & $108-10-1$ & 7 & PPB & \\
\hline TOLUENE & $108-88-3$ & 0 & PPB & \\
\hline 1,1,2-TRICHLOROETHANE & $79-00-5$ & 0 & PPB & \\
\hline TETRACHLOROETHENE & $127-18-4$ & 0 & PPB & \\
\hline CHLOROBENZENE & $108-90-7$ & 0 & PPB & \\
\hline ETHYLBENZENE & $100-41-4$ & 1 & PPB & \\
\hline M-XYLENE & $108-38-3$ & 0 & PPB & \\
\hline STYRENE & $100-42-5$ & 0 & PPB & \\
\hline O-XYLENE & $95-47-6$ & 0 & PPB & \\
\hline 1,1,2,2-TETRACHLORETHANE & $79-34-5$ & 0 & PPB & \\
\hline 1,3,5-TRIMETHYLBENZENE & $108-67-8$ & 0 & PPB & \\
\hline 1,2,4-TRIMETHYLBENZENE & $95-63-6$ & 0 & PPB & \\
\hline 1,3-DICHLOROBENZENE & $541-73-1$ & 0 & PPB & \\
\hline 1,2-DICHLOROBENZENE & $95-50-1$ & 0 & PPB & \\
\hline 1,4-DICHLOROBENZENE & $106-46-7$ & 0 & PPB & \\
\hline
\end{tabular}




\section{LIST OF COMPOUNDS TENTATIVELY IDENTIFIED WITH MASS SPECTRAL LIBRARY}

Sample: LFM A3

Sample Name: FT6045-A3.07A

Search Libraries: $\quad$ C:DATABASEIWLEY138.L $\quad$ Minimum Quality: 70 C:LATABASELNBS4K.L $\quad$ Minimum Quality: 70

Pk\# RT Area\% Library/D $\quad$ Ref\# CAS\# Qual

621.753 .78 C:LDATABASEIWLEY138.L
1-Butanol
$116550000071-36-390$

9. 24.476 .25 C:DATABASEIWILEY138.L

2-Pentanone, 4-methyl-

$118021000108-10-191$

1228.880 .52 C:IDATABASEIWILEY138.L Benzene, ethyl-

$118561000100-41-472$

1329.140 .90 C:LDATABASEIWLEY $138 . \mathrm{L}$ Benzene, 1,3-dimethyl-

$118578000108-38-395$

1529.961 .00 C:DATABASEIWILEY138.L. Benzene, 1,3-dimethyl-

$118576000108-38-392 \quad$

1630.2275 .35 C:IDATABASEIWILEY138.L Ethanol, 2-butoxy-

$119793000111-76-278$

1832.090 .82 C:LDATABASEIWILEY138.L Benzaldehyde

$118546000100-52-796$

1932.300 .35 C:WATABASEIWILEY138.L Benzene, 1,3,5-trimethyl-

$120037000108-67-890$

2033.110 .90 C:WATABASEIWILEY138.L Benzene, 1,3,5-trimethyl-

120037 000108-67-8 95

2133.760 .47 C:IDATABASEIWILEY138.L Benzene, 1,4-dichloro-

$123085000106-46-796$

$2234.50 \quad 0.69$ C:LDATABASEIWILEY138.L

Benzene, 1,4-dichloro-

$123085000106-46-797$ 


\section{File}

Operator

Acquired

Instrument

Inste

FT6045-A3.07A

Misc Info : delta $\mathrm{p}=50$ torr

Vial Number: 1

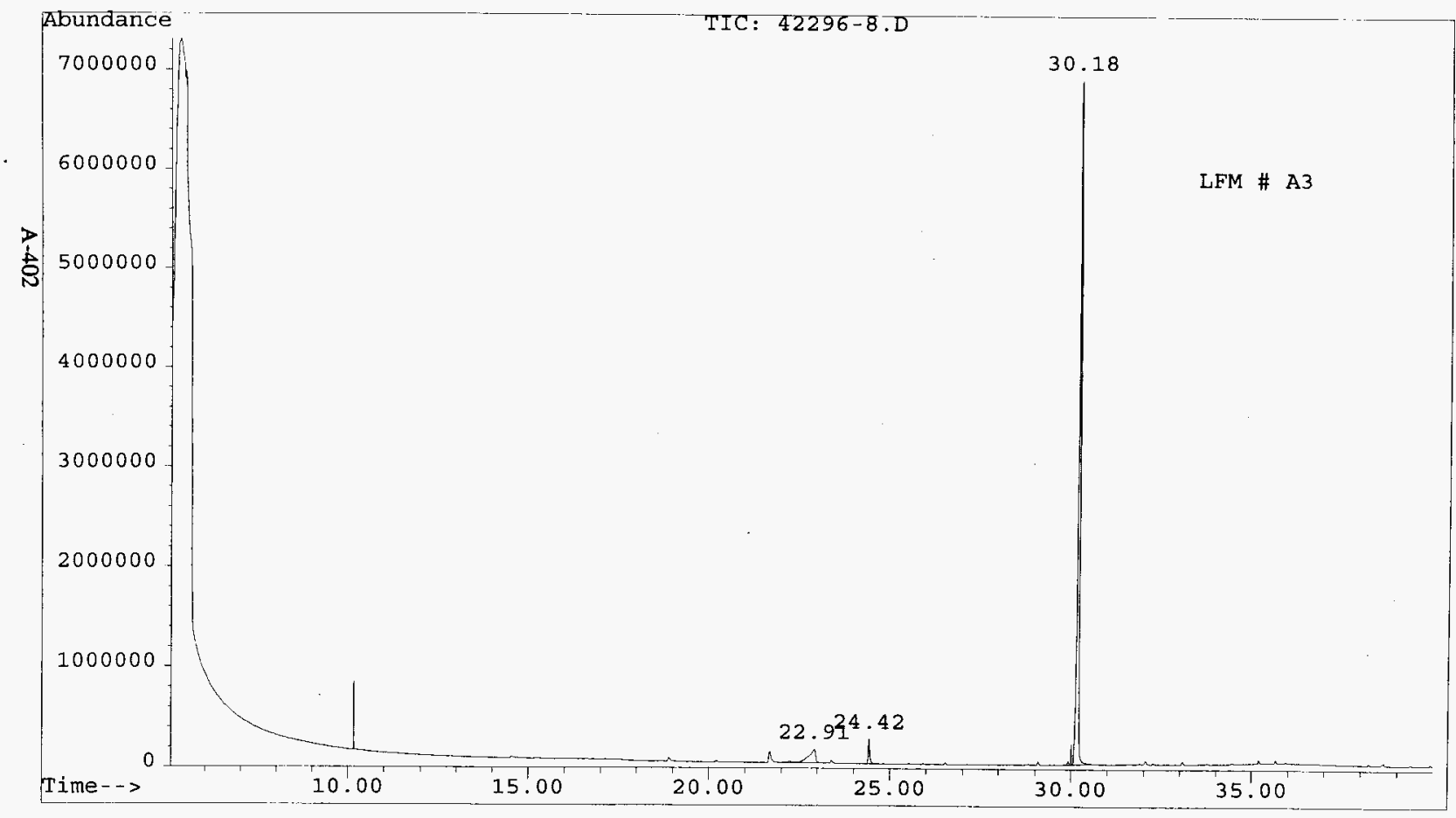


HNF-SD-WM-RPT-282 Rev. 0

CWC_REP.XLS

\section{ANALYSIS REPORT FOR CWC AIR SAMPLES}

File Name:

Analyst:

Analysis Date:

Method:

Sample Name:
42296-8.D

$\mathrm{ms}$

04/22/96

CWC

FT6045-A3.07A

\begin{tabular}{|c|c|c|c|c|}
\hline COMPOUND NAME & CAS \# & Concentration & Units & Comments \\
\hline ISO-BUTANE & $75-28-5$ & 0 & PPB & \\
\hline VINYL CHLORIDE & $75-01-4$ & 0 & PPB & \\
\hline ETHANOL & $64-17-5$ & 0 & PPB & \\
\hline ACETONE & $67-64-1$ & 0 & PPB & \\
\hline 2-PROPANOL & $67-63-0$ & 0 & PPB & \\
\hline 1,1-DICHLOROETHENE & $75-35-4$ & 0 & PPB & \\
\hline FREON 113 & $76-13-1$ & 0 & PPB & \\
\hline DICHLOROMETHANE & $75-09-2$ & 0 & PPB & \\
\hline 1,1-DICHLOROETHANE & $75-34-3$ & 0 & PPB & \\
\hline 2-BUTANONE & $78-93-3$ & 0 & PPB & \\
\hline N-HEXANE & $110-54-3$ & 0 & PPB & \\
\hline 1,2-DICHLOROETHENE & 156-59-2 & 0 & PPB & \\
\hline CHLOROFORM & $67-66-3$ & 0 & PPB & \\
\hline TETRAHYDROFURAN & $109-99-9$ & 0 & PPB & \\
\hline 1,1,1-TRICHLOROETHANE & $71-55-6$ & 0 & PPB & \\
\hline 1,2-DICHLOROETHANE & $107-06-2$ & 0 & PPB & \\
\hline BENZENE & $71-43-2$ & 0 & PPB & \\
\hline CARBON TETRACHLORIDE & $56-23-5$ & 0 & PPB & \\
\hline CYCLOHEXANE & $110-82-7$ & 0 & PPB & \\
\hline N-BUTANOL & $71-36-3$ & 7 & PPB & \\
\hline TRICHLOROETHENE & $79-01-6$ & 0 & PPB & \\
\hline 4-METHYL-2-PENTANONE & $108-10-1$ & 0 & PPB & \\
\hline TOLUENE & $108-88-3$ & 0 & PPB & \\
\hline 1,1,2-TRICHLOROETHANE & $79-00-5$ & 0 & PPB & \\
\hline TETRACHLOROETHENE & $127-18-4$ & 0 & PPB & \\
\hline CHLOROBENZENE & $108-90-7$ & 0 & PPB & \\
\hline ETHYLBENZENE & $100-41-4$ & 0 & PPB & \\
\hline M-XYLENE & $108-38-3$ & 0 & PPB & \\
\hline STYRENE & $100-42-5$ & 0 & PPB & \\
\hline O-XYLENE & $95-47-6$ & 0 & PPB & \\
\hline 1,1,2,2-TETRACHLORETHANE & $79-34-5$ & 0 & PPB & \\
\hline 1,3,5-TRIMETHYLBENZENE & $108-67-8$ & 0 & PPB & \\
\hline 1,2,4-TRIMETHYLBENZENE & $95-63-6$ & 0 & PPB & \\
\hline 1,3-DICHLOROBENZENE & $541-73-1$ & 0 & PPB & \\
\hline 1,2-DICHLOROBENZENE & $95-50-1$ & 0 & PPB & \\
\hline 1,4-DICHLOROBENZENE & $106-46-7$ & 0 & $\overline{\mathrm{PPB}}$ & \\
\hline
\end{tabular}




\section{LIST OF COMPOUNDS TENTATIVELY IDENTIFIED WITH MASS SPECTRAL}

LIBRARY

Sample: $\quad$ LFM A3

Sample Name: FT6045-A3.07A

Search Libraries: $\quad$ C:DATABASEIWILEY138.L Minimum Quality: 70 C:DATABASELNBS54K.L Minimum Quality: 70

Unknown Spectrum: Apex

Integration Params: VOA.E

Pk\# RT Area\% Library/ID $\quad$ Ref\# CAS\# Qual

$\begin{array}{lll}5 & 21.68 \quad 1.72 \mathrm{C}: \text { |DATABASE } \backslash W I L E Y 138 . L\end{array}$ 1-Butanol

$116551000071-36-386$

722.92 6.26 C:LDATABASEIWILEY138.L

2-Propanol, 1-methoxy-

$347000107-98-278$

924.422 .57 C:LDATABASEIWILEY 138.L

2-Pentanone, 4-methyl-

$118019000108-10-190$

$1129.100 .41 \mathrm{C}:$ DATABASEIWILEY138.L

Benzene, 1,2-dimethyl-

$118564000095-47-695$

1229.920 .40 C:LATABASEIWILEY 138.L

Benzene, 1,2-dimethyl-

$118571000095-47-693$

1430.18 82.00 C:IDATABASEIWILEY138.L Ethanol, 2-butoxy-

$119793000111-76-283$

$1632.05 \quad 0.42 \mathrm{C}:$ WATABASEIWILEY138.L Benzaldehyde

$118545000100-52-793$

$1732.27 \quad 0.16 \mathrm{C}:$ DATABASEIWILEY 138.L

Benzene, 1,3,5-trimethyl- $\quad 120037000108-67-880$

$1833.08 \quad 0.35$ C:IDATABASEIWILEY138.L Benzene, 1,3,5-trimethyl- $\quad 120037$ 000108-67-8 95

$1934.46 \quad 0.19$ C:LDATABASEIWILEY138.L

Benzene, 1,4-dichloro-

$123085000106-46-797$ 
HNF-SD-WM-RPT-282 Rev. 0

Sample: $\quad$ LFM A3

Pk\# RT Area\% Library/ID Ref\# CAS\# Qual

$2035.20 \quad 0.49$ C:LDATABASEIWILEY 138.L

Ethanone, 1-phenyl-

$119989000098-86-294$ 
File

: D: \CWC \SAMPLES $\backslash 41996 \_12 . \mathrm{D}$

Operator

MS

Acquired

19 Apr 96 5972 - In

Sample Name: FT6045-A4.06A

Misc Info: delta $\mathrm{p}=50$ torr

Vial Number: 1

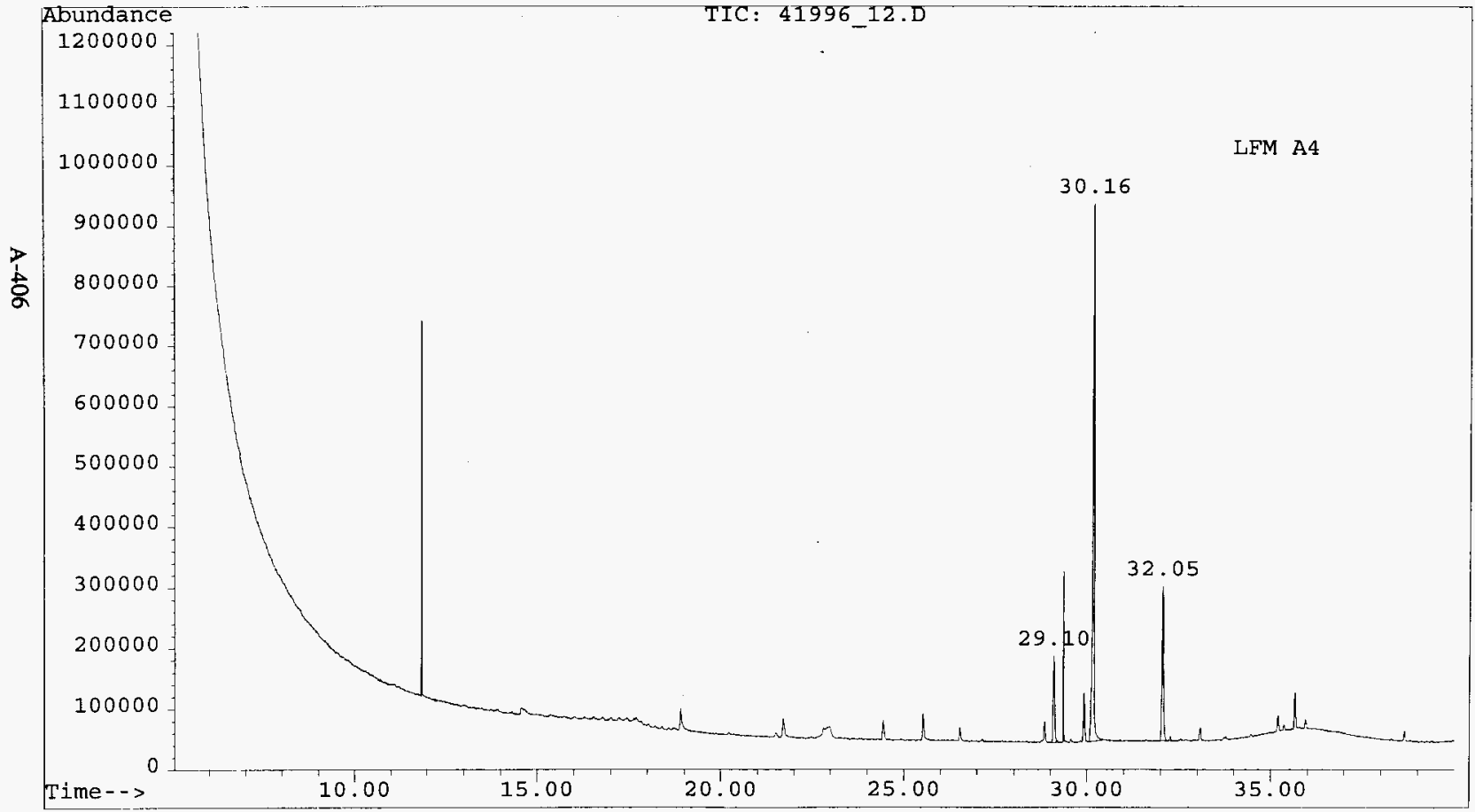


HNF-SD-WM-RPT-282 Rev. 0

CWC_REP.XLS

\section{ANALYSIS REPORT FOR CWC AIR SAMPLES}

File Name:

Analyst:

Analysis Date:

Method:

Sample Name:

\author{
41996_12.D \\ MS \\ 04/19/96 \\ CWC
}

FT6045-A4.06A

\begin{tabular}{|c|c|c|c|c|}
\hline COMPOUND NAME & CAS \# & Concentration & Units & Comments \\
\hline ISO-BUTANE & $75-28-5$ & 0 & PPB & \\
\hline VINYL CHLORIDE & $75-01-4$ & 0 & PPB & \\
\hline ETHANOL & $64-17-5$ & 0 & PPB & \\
\hline ACETONE & $67-64-1$ & 0 & PPB & \\
\hline 2-PROPANOL & $67-63-0$ & 0 & PPB & \\
\hline 1,1-DICHLOROETHENE & $75-35-4$ & 0 & PPB & \\
\hline FREON 113 & $76-13-1$ & 0 & PPB & \\
\hline DICHLOROMETHANE & $75-09-2$ & 0 & PPB & \\
\hline 1,1-DICHLOROETHANE & $75-34-3$ & 0 & PPB & \\
\hline 2-BUTANONE & $78-93-3$ & 0 & PPB & \\
\hline N-HEXANE & $110-54-3$ & 0 & PPB & \\
\hline 1,2-DICHLOROETHENE & $156-59-2$ & 0 & PPB & \\
\hline CHLOROFORM & $67-66-3$ & 0 & PPB & \\
\hline TETRAHYDROFURAN & $109-99-9$ & 0 & PPB & \\
\hline 1,1,1-TRICHLOROETHANE & $71-55-6$ & 0 & PPB & \\
\hline 1,2-DICHLOROETHANE & $107-06-2$ & 0 & PPB & \\
\hline BENZENE & $71-43-2$ & 0 & PPB & \\
\hline CARBON TETRACHLORIDE & $56-23-5$ & 0 & PPB & \\
\hline CYCLOHEXANE & $110-82-7$ & 0 & PPB & \\
\hline N-BUTANOL & $71-36-3$ & 0 & PPB & \\
\hline TRICHLOROETHENE & $79-01-6$ & 0 & PPB & \\
\hline 4-METHYL-2-PENTANONE & $108-10-1$ & 0 & PPB & \\
\hline TOLUENE & $108-88-3$ & 2 & PPB & \\
\hline 1,1,2-TRICHLOROETHANE & $79-00-5$ & 0 & PPB & \\
\hline TETRACHLOROETHENE & $127-18-4$ & 0 & PPB & \\
\hline CHLOROBENZENE & $108-90-7$ & 0 & PPB & \\
\hline ETHYLBENZENE & $100-41-4$ & 1 & PPB & \\
\hline M-XYLENE & $108-38-3$ & 4 & PPB & \\
\hline STYRENE & $100-42-5$ & 0 & PPB & \\
\hline O-XYLENE & $95-47-6$ & 2 & PPB & \\
\hline 1,1,2,2-TETRACHLORETHANE & $79-34-5$ & 0 & PPB & \\
\hline 1,3,5-TRIMETHYLBENZENE & $108-67-8$ & 4 & PPB & \\
\hline 1,2,4-TRIMETHYLBENZENE & $95-63-6$ & 0 & PPB & \\
\hline 1,3-DICHLOROBENZENE & $541-73-1$ & 0 & PPB & \\
\hline 1,2-DICHLOROBENZENE & $95-50-1$ & 0 & PPB & \\
\hline 1,4-DICHLOROBENZENE & $106-46-7$ & 0 & PPB & \\
\hline
\end{tabular}




\section{LIST OF COMPOUNDS TENTATIVELY IDENTIFIED WITH MASS SPECTRAL LIBRARY}

Sample: $\quad$ LFM A4

Sample Name: FT6045-A4.06A

Misc Info : delta $\mathrm{p}=50$ torr

Vial Number: 1

Search Libraries: $\quad$ C:LDATABASEIWILEY138.L Minimum Quality: 70

C:DATABASELNBS54K.L Minimum Quality: 70

Unknown Spectrum: Apex

Integration Params: VOA.E

Pk\# RT Area\% Library/mD Ref\# CAS\# Qual

725.532 .30 C:DATABASEIWILEY138.L Benzene, methyl-

$117429000108-88-392$

$928.851 .78 \mathrm{C}:$ DATABASEIWILEY138.L Benzene, ethyl- $\quad 118561000100-41-490$

$1029.107 .10 \mathrm{C}:$ DATABASEIWILEY138.L Benzene, 1,3-dimethyl-

$118578000108-38-397$

1229.933 .59 C:LDATABASEIWILEY138.L XYLENE

$118580001330-20-795$

1330.1643 .88 C:LDATABASEIWILEY138.L Ethanol, 2-butoxy-

$119793000111-76-283$

1432.0512 .54 C:DATABASEIWILEY138.L Benzaldehyde

$118545000100-52-797$

$1533.081 .42 \mathrm{C}:$ :DATABASEIWILEY138.L Benzene, 1,3,5-trimethyl-

$120037000108-67-890$

$1635.21 \quad 1.83 \mathrm{C}:$ :DATABASEIWILEY138.L

Ethanone, 1-phenyl-

$119988000098-86-293$ 